Supporting Information for:

\title{
Asymmetric Markovnikov Hydroaminocarbonylation of Alkenes Enabled by Palladium-Monodentate Phosphoramidite Catalysis
}

Ya-Hong Yao, Hui-Yi Yang, Ming Chen, Fei Wu, Xing-Xing Xu, Zheng-Hui Guan*

Key Laboratory of Synthetic and Natural Molecule of Ministry of Education, Department of Chemistry \& Materials Science, Northwest University, Xi'an 710127, P. R. China

Email: guanzhh@,nwu.edu.cn

\section{CONTENTS}

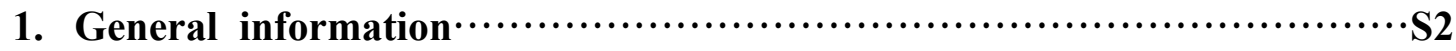

2. Typical procedure for the Pd-catalyzed asymmetric Markovnikov

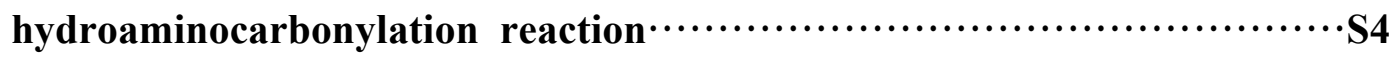

3. General procedures for the synthesis of the racemic amides $\cdots \cdots \cdots \cdots \cdots \cdots$, S4

4. The procedure for hydrolysis of amides for the synthesis of $(S)$-ibuprofen (6),

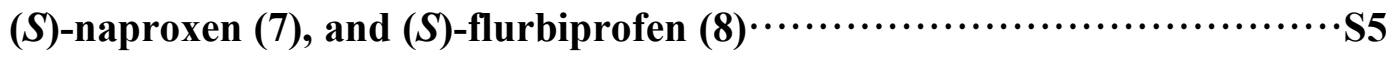

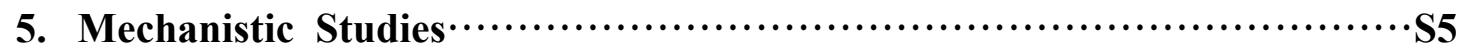

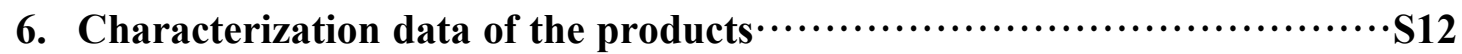

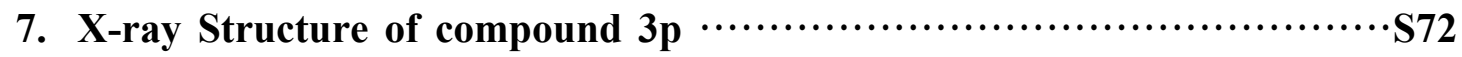

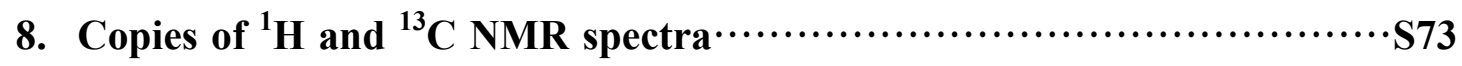




\section{General Information.}

\section{Chemicals}

Chemicals were commercially purchased from Adamas-beta, Energy Chemical, Aladdin, Daicel Chiral Technologies (China) Co., etc, and directly used without further purification unless otherwise stated. $\mathrm{PdI}_{2}$ was purchased from laajoo. Aniline- $\mathrm{D}_{7},>98$ atom\% D were purchased from $J \& K$ Scientific Ltd. Anhydrious THF was distilled from sodium/benzophenone once the indicator had turned a persistent blue color. Phosphoramidite ligands (L4-L16) were prepared according to our previous report (Chen, M.; Wang, X.; Yang, P.; Kou, X.; Ren, Z.-H.; Guan, Z.-H. Angew. Chem. Int. Ed. 2020, 59, 12199-12205).

L16: ${ }^{1} \mathrm{H}$ NMR $\left(400 \mathrm{MHz}, \mathrm{CDCl}_{3}\right) \delta 7.31(\mathrm{~d}, J=8.4 \mathrm{~Hz}, 2 \mathrm{H}), 7.18(\mathrm{~d}, J=8.4 \mathrm{~Hz}, 2 \mathrm{H})$, $7.14(\mathrm{~s}, 1 \mathrm{H}), 7.08(\mathrm{~s}, 1 \mathrm{H}), 6.81-6.75(\mathrm{~m}, 6 \mathrm{H}), 6.69(\mathrm{~d}, J=8.4 \mathrm{~Hz}, 2 \mathrm{H}), 6.49$ (t, $J=7.6$ $\mathrm{Hz}, 2 \mathrm{H}), 5.54(\mathrm{~s}, 2 \mathrm{H}), 3.66(\mathrm{~d}, J=6.4 \mathrm{~Hz}, 2 \mathrm{H}), 3.64-3.62(\mathrm{~m}, 1 \mathrm{H}), 3.56-3.52(\mathrm{~m}, 1 \mathrm{H})$, 2.93-2.82 (m, 4H), 2.77-2.71 (m, 2H), 2.54-2.43 (m, 2H), 2.06-2.01 (m, 2H), 1.95-1.80 (m, 6H), 1.70-1.68 (m, 2H), 1.02-0.97 (m, 12H); ${ }^{13} \mathrm{C}$ NMR (101 MHz, $\left.\mathrm{CDCl}_{3}\right) \delta 158.6,158.4,149.6,144.69,144.65,143.9,137.0,134.6,134.2,132.2$, $131.41,131.39,130.9,130.7,130.5,130.44,130.42,130.2,130.12,130.11,130.06$, 130.0, 129.4, 124.0, 122.6, 115.5, 114.1, 114.0, 74.3, 74.2, 29.3, 29.1, 28.3, 28.2, 27.9, 27.7, 23.0, 22.88, 22.86, 22.7, 19.3. HRMS calcd. (ESI) $\mathrm{m} / \mathrm{z}$ for $\mathrm{C}_{52} \mathrm{H}_{52} \mathrm{NNaO}_{5} \mathrm{P}$ : $[\mathrm{M}+\mathrm{Na}]^{+} 824.3475$, found: 824.3482 .

\section{Chromatography}

Analytical thin-layer chromatography (TLC) was carried out with silica gel pre-coated glass plates (TLC-Silica gel GF254, coating thickness: 0.20-0.25 mm, particle size: 10-40 $\mu \mathrm{m}$ ) purchased from Xinnuo Chemical (Yantai, China). The TLC was visualized with a UV lamp (254 or $365 \mathrm{~nm}$ ).

Column chromatography was carried out on silica gel (200-300 mesh) purchased from Xinnuo Chemicals (Yantai, China) with technical grade solvents as the eluent. All the yields referred to spectroscopically and chromatographically pure compounds.

\section{Nuclear Magnetic Resonance (NMR) Spectroscopy}

${ }^{1} \mathrm{H}$ NMR spectra were recorded on Bruker AVANCE III instrument (400 or 600 $\mathrm{MHz}$ spectrometer). The analytical sample was dissolved in an appropriate deuterated solvent. The employed deuterated solvent and the measuring frequency are indicated 
in each ${ }^{1} \mathrm{H}$ NMR data. Chemical shifts are reported in parts per million (ppm) with the solvent resonance as the internal reference $\left(\mathrm{CDCl}_{3} \delta 7.26\right.$, DMSO- $\left.d^{6} \delta 2.54\right)$. The following abbreviations (or combinations thereof) were used to explain multiplicities: $\mathrm{s}=$ singlet, $\mathrm{d}=$ doublet, $\mathrm{t}=$ triplet, $\mathrm{q}=$ quartet, $\mathrm{m}=$ multiplet, $\mathrm{b}=$ broad. Coupling constants, J were reported in Hertz unit (Hz).

${ }^{13} \mathrm{C}$ NMR spectra were recorded on Bruker AVANCE III instrument (400 or 600 $\mathrm{MHz}$ spectrometer). The employed deuterated solvent and the measuring frequency are both indicated in each ${ }^{13} \mathrm{C}$ NMR data. Chemical shifts are reported in ppm with the solvent resonance as the internal reference $\left(\mathrm{CDCl}_{3} \delta 77.0\right)$.

\section{High Resolution Mass Spectrometry (HRMS)}

HRMS were recorded on a quadrupole time-of-flight mass spectrometer (MicroTof-Q II mass spectrometer, Bruker Daltonics) using electrospray ionization-time of flight (ESI-TOF) at the Instrumental Analysis Center of Northwest University. The calculated values are based on the most abundant isotope.

\section{Optical Rotations}

Optical rotations were measured with a WZZ-2S automatic polarimeter purchased from Shanghai INESA Physico-Optical instrument company using a sodium lamp (sodium D line, $\lambda=589 \mathrm{~nm}$ ) in the indicated solvent at the indicated temperature. The measurements were carried out in a $1.5 \mathrm{~mL}$ cell (50 mm length) with concentrations $(\mathrm{g} / 100 \mathrm{~mL})$ reported in the corresponding solvent. The optical rotation values $([\alpha] \mathrm{D})$ were reported at a given temperature $\left({ }^{\circ} \mathrm{C}\right)$ in $\mathrm{deg} . \mathrm{mL} \mathrm{g}-1 \mathrm{dm}-1$.

\section{High Performance Liquid Chromatography (HPLC)}

HPLC analysis was performed on a thermos scientific UitiMate 3000 using Chiralpak columns IG or IH (Daicel Chiral Reagent Company). The solvents ( $n$-hexane and iso-propanol, HPLC-grade) used as the eluent were purchased from Oceanpak. The column type and the eluent (a mixture of $n$-hexane and iso-propanol) are indicated for each experiment.

\section{X-ray Crystallography}

X-ray crystallography was performed on a BRUKERSMA RTAPEXIICCD diffractometer at Instrumental Analysis Center of Northwest University. 


\section{Typical procedure for the Pd-catalyzed asymmetric Markovnikov}

hydroaminocarbonylation reaction

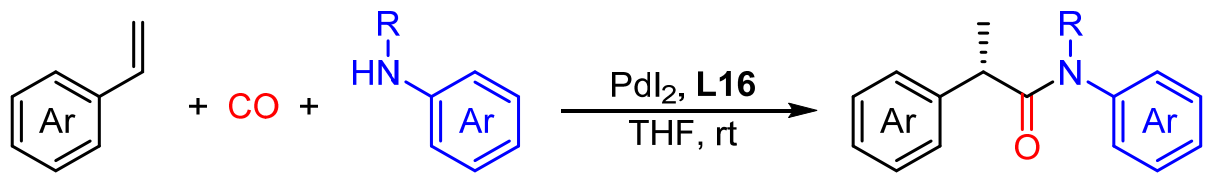

To a $5.0 \mathrm{~mL}$ glass vessel was added styrene $(0.15 \mathrm{mmol}, 1.5$ equiv $)$, aniline ( 0.1 mmol, 1.0 equiv), $\mathrm{PdI}_{2}$ (0.01 mmol, $\left.10 \mathrm{~mol} \%\right)$, $\mathbf{L 1 6}$ (0.011 mmol, $\left.11 \mathrm{~mol} \%\right)$, and THF $(1.0 \mathrm{~mL})$. The glass vessel was then put into an autoclave. The autoclave was evacuated and backfilled with CO for three times in a well-ventilated fume hood, and then pressurized to $50 \mathrm{~atm}$ of $\mathrm{CO}$. The reaction mixture in autoclave was stirred at room temperature for $72 \mathrm{~h}$ under $50 \mathrm{~atm}$ of $\mathrm{CO}$. Then, the $\mathrm{CO}$ in autoclave was carefully released in a well-ventilated fume hood. The ratio of branched and linear amides was determined by GC-MS analysis of the crude products. The reaction mixture was concentrated and purified by column chromatography on silica gel (eluent: petroleum ether/ethyl acetate $=10 / 1$ ) to afford the desired amide products.

\section{General procedures for the synthesis of racemic amides}

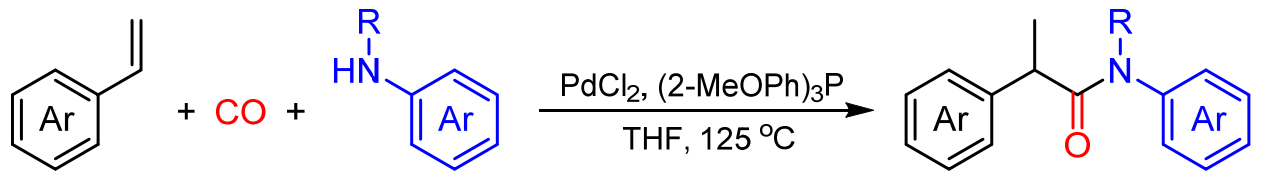

To a $5.0 \mathrm{~mL}$ glass vessel was added $\mathrm{PdCl}_{2}(5 \mathrm{~mol} \%),(2-\mathrm{MeOPh})_{3} \mathrm{P}(10 \mathrm{~mol} \%)$, aniline ( $0.2 \mathrm{mmol}, 1.0$ equiv), styrene (5.0 equiv) and THF ( $2 \mathrm{~mL})$. The vessel was placed in an autoclave. The autoclave was evacuated and backfilled with $\mathrm{CO}$ for three times and pressurized to $50 \mathrm{~atm}$ of $\mathrm{CO}$. The reaction mixture in autoclave was stirred at $125{ }^{\circ} \mathrm{C}$ for $2 \mathrm{~h}$. After cooling down to the room temperature, the $\mathrm{CO}$ was carefully released in a well-ventilated fume hood. The reaction mixture was concentrated and purified by column chromatography on silica gel (eluent: petroleum ether/ethyl acetate $=10 / 1)$ to give the racemic amides. 


\section{The procedure for hydrolysis of amides for the synthesis of (S)-ibuprofen (6),}

$(S)$-naproxen (7), and (S)-flurbiprofen (8)

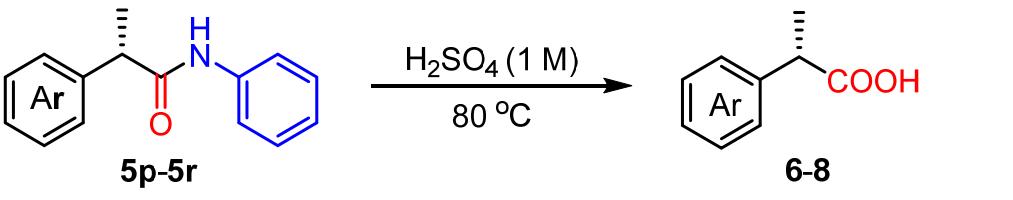<smiles>CC(C)Cc1ccc([C@@H](C)C(=O)O)cc1</smiles>

ibuprofen, 6<smiles>COc1ccc2cc([C@@H](C)C(=O)O)ccc2c1</smiles>

naproxen, 7<smiles>C[C@@H](C(=O)O)c1ccc(-c2ccccc2)c(F)c1</smiles>

A solution of compounds 5p-5r in $1 \mathrm{M} \mathrm{H}_{2} \mathrm{SO}_{4}(\mathrm{aq}, 1.0 \mathrm{~mL}$ ) and 1,4-dioxane (1.0 $\mathrm{mL}$ ) was stirred at $80{ }^{\circ} \mathrm{C}$ for $8 \mathrm{~h}$. After the reaction completed, the reaction was cooled down to room temperature. The mixture was extracted with $\mathrm{CH}_{2} \mathrm{Cl}_{2}(20 \mathrm{~mL} \times 3)$. The organic layers was dried over sodium sulfate and concentrated. The residue was then purified by column chromatography on silica gel (eluent: petroleum ether/ethyl acetate $=8 / 1)$ to give the corresponding $(S)$-ibuprofen (6), $(S)$-naproxen (7), and (S)-flurbiprofen (8).

\section{Mechanistic studies}

\subsection{Preparation of 1a- $\mathrm{D}_{2}$}

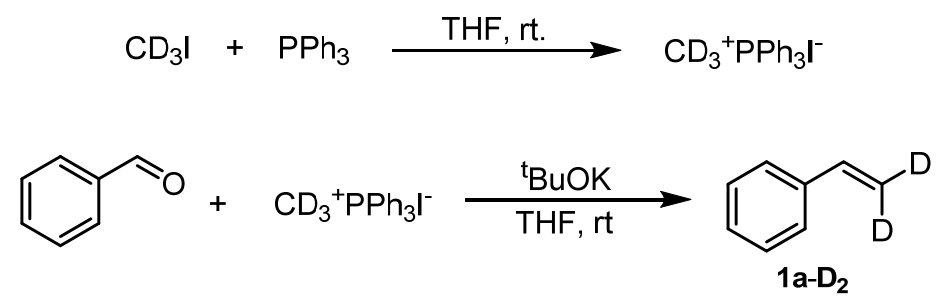

To a dry and stirred round bottomed flask which charged with $\mathrm{PPh}_{3}(1.0 \mathrm{mmol}, 1$ equiv), anhydrous THF (5 mL) was added $\mathrm{CD}_{3} \mathrm{I}(1.2 \mathrm{mmol}, 1.2$ equiv) dropwise at room temperature. After completed the reaction (about $6 \mathrm{~h}$ ), the mixture was filtered by a Buchner funnel and washed by THF. The methyl- $\mathrm{D}_{3}$-triphenylphosphonium iodide was obtained in $87 \%$ yield (354 mg).

To a $25 \mathrm{~mL}$ flask were added methyl- $\mathrm{D}_{3}$-triphenylphosphonium iodide (0.6 
mmol, 1.2 equiv), ${ }^{t} \mathrm{BuOK}$ ( $0.6 \mathrm{mmol}, 1.2$ equiv) and anhydrous THF ( $\left.7 \mathrm{~mL}\right)$. The mixture was stirred at room temperature for $1 \mathrm{~h}$. Then, a solution of benzaldehyde ( $0.5 \mathrm{mmol}, 1.0$ equiv) in anhydrous THF $(1 \mathrm{~mL})$ was added to the flask. The reaction was stirred at room temperature for overnight. After completion of the reaction, the mixture was diluted with $\mathrm{CH}_{2} \mathrm{Cl}_{2}(5 \mathrm{~mL} \times 3)$ and washed with brine $(5 \mathrm{~mL})$. The organic layers was dried over $\mathrm{Na}_{2} \mathrm{SO}_{4}$ and concentrated in vacuo. The crude product was purified by column chromatography on silica gel (petroleum ether as the eluent) to afford the pure $\mathbf{1} \mathbf{a}-\mathbf{D}_{\mathbf{2}}$ in $82 \%$ yield $(43.5 \mathrm{mg})$.

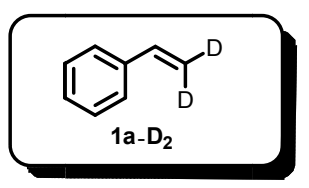

1a-D $\mathbf{D}_{2}:{ }^{1} \mathrm{H}$ NMR $\left(400 \mathrm{MHz}, \mathrm{CDCl}_{3}\right) \delta 7.41(\mathrm{~d}, J=7.2 \mathrm{~Hz}, 2 \mathrm{H}), 7.33(\mathrm{t}, J=7.2$ $\mathrm{Hz}, 2 \mathrm{H}), 7.25(\mathrm{t}, J=7.2 \mathrm{~Hz}, 1 \mathrm{H}), 5.74(\mathrm{dd}, J=17.2,3.2 \mathrm{~Hz}, 0.10 \mathrm{H}), 5.24(\mathrm{dd}, J=$ 11.2, $3.2 \mathrm{~Hz}, 0.11 \mathrm{H})$.

${ }^{1} \mathrm{H}$ NMR (400 MHz, $\mathrm{CDCl}_{3}$ ) of compound $\mathbf{1 a - \mathbf { D } _ { 2 }}$
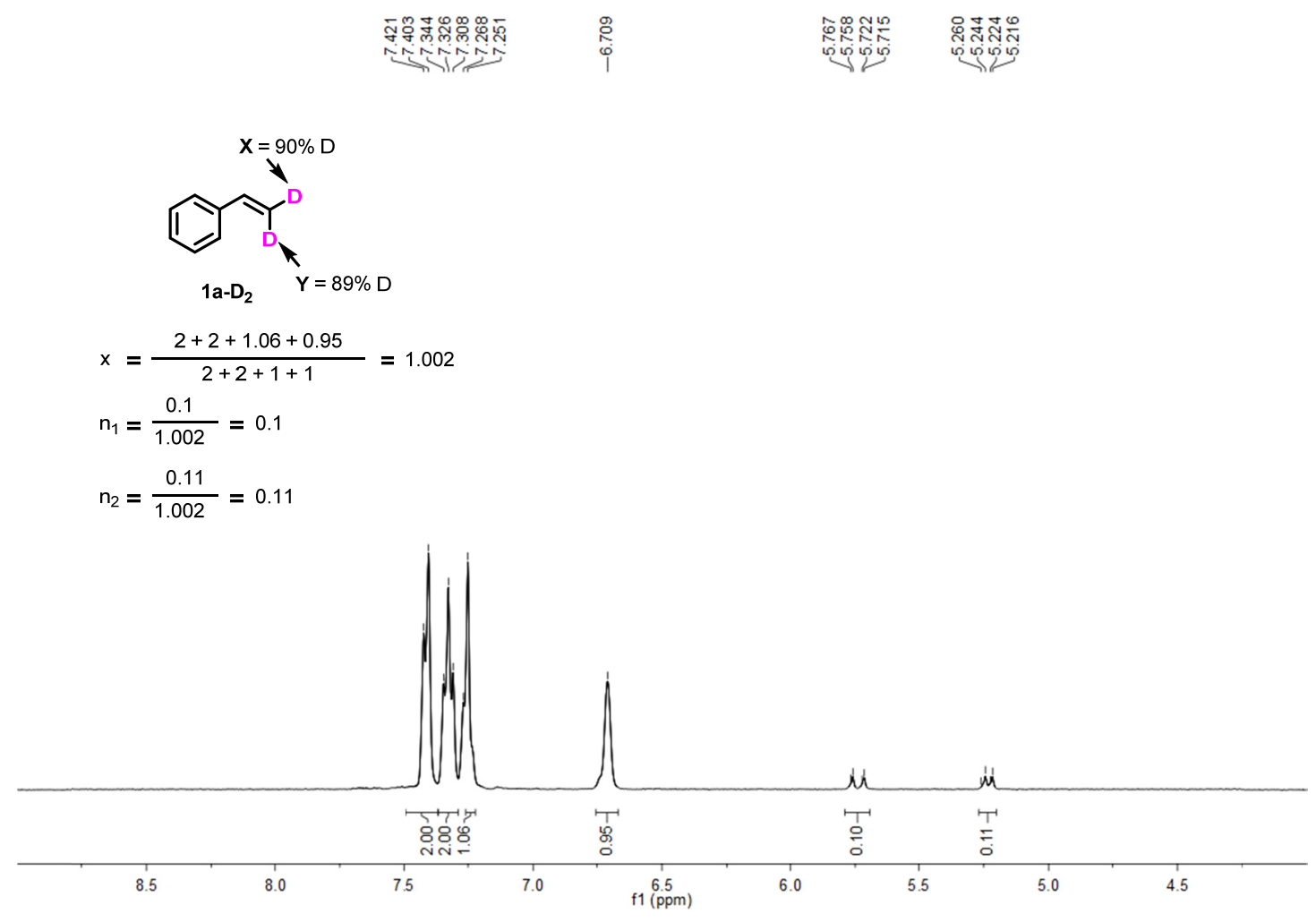


\subsection{Deuterium-labeling studies with $1 \mathrm{a}-\mathrm{D}_{2}$ and $2 \mathrm{a}$ under standard conditions}

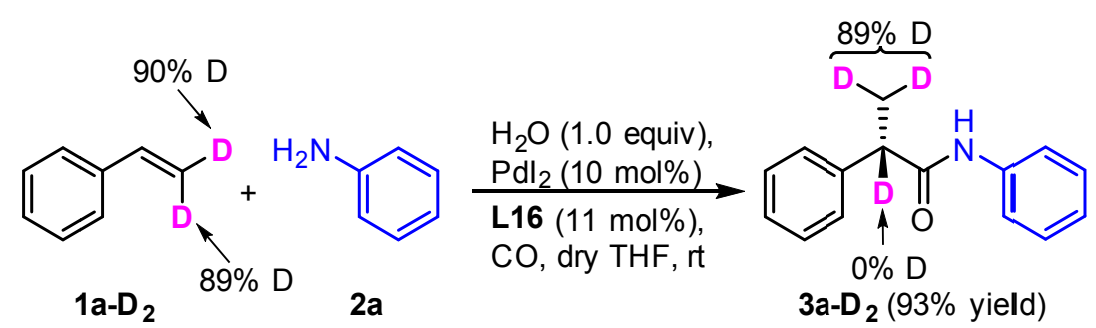

To a dry glass vessel were added $\mathrm{PdI}_{2}(0.01 \mathrm{mmol}, 10 \mathrm{~mol} \%), \mathbf{L 1 6}$ (0.011 mmol, $11 \mathrm{~mol} \%)$, 1a-D $\mathbf{2}$ (0.15 mmol, 1.5 equiv), 2a ( $0.1 \mathrm{mmol}, 1.0$ equiv), $\mathrm{H}_{2} \mathrm{O}$ ( 1 equiv) and anhydrous THF $(1.0 \mathrm{~mL})$. The glass vessel was put into an autoclave. The autoclave was evacuated and backfilled with $\mathrm{CO}$ for three times in a well-ventilated fume hood, and then pressurized to $50 \mathrm{~atm}$ of $\mathrm{CO}$. The reaction mixture in autoclave was stirred at room temperature for $72 \mathrm{~h}$ under $50 \mathrm{~atm}$ of $\mathrm{CO}$. Then, the $\mathrm{CO}$ in autoclave was carefully released in a well-ventilated fume hood. The reaction mixture was concentrated and purified by column chromatography on silica gel (eluent: petroleum ether/ethyl acetate $=10 / 1)$ to afford the $\mathbf{3} \mathbf{a}-\mathbf{D}_{\mathbf{2}}$ in $93 \%$ yield. The 89 atom $\%$ $\mathrm{D}$ in $\mathbf{3} \mathbf{a}-\mathbf{D}_{2}$ was determined by ${ }^{1} \mathrm{H}$ NMR.

${ }^{1} \mathrm{H}$ NMR (400 MHz, $d^{6}$-DMSO) of compound 3a

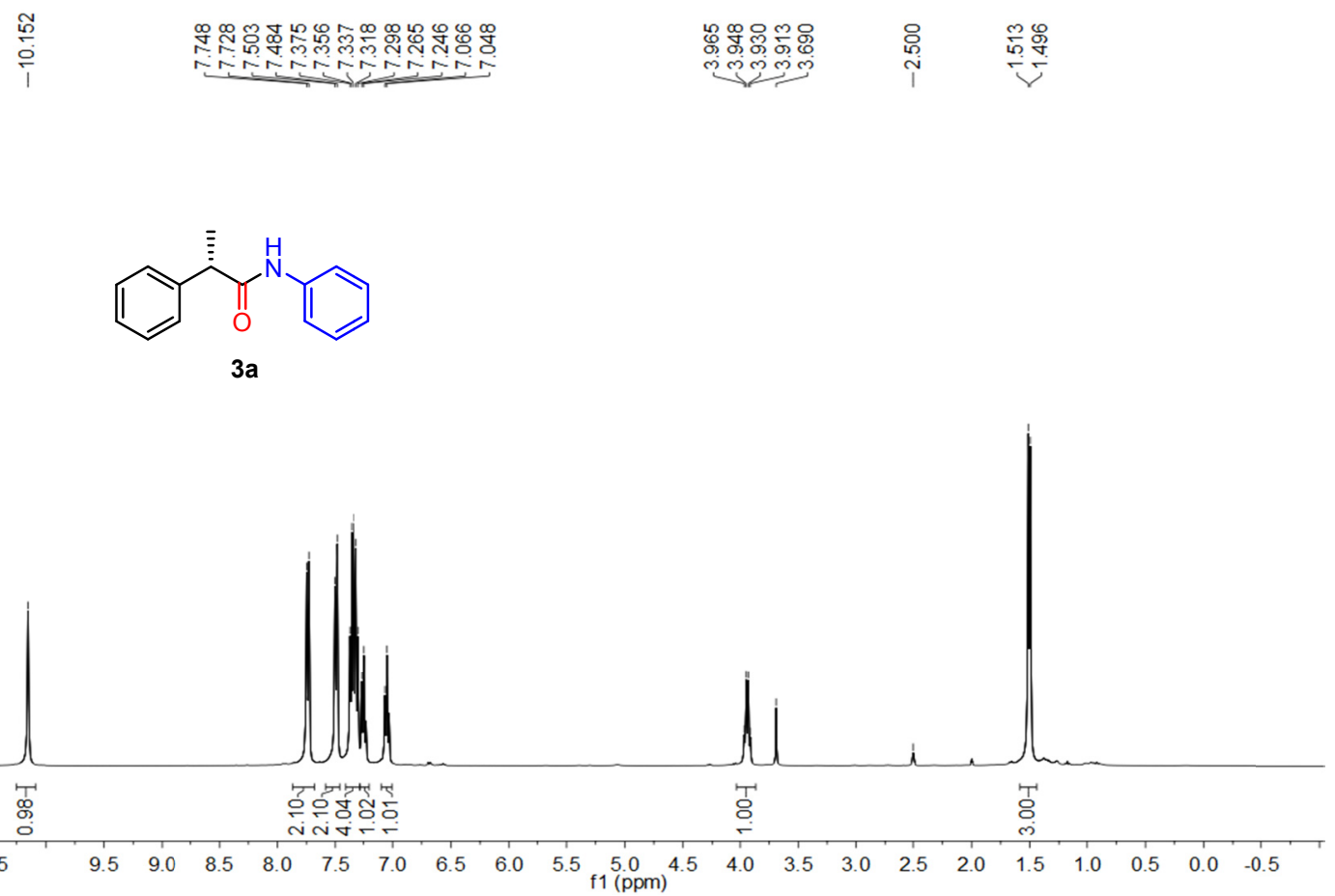


${ }^{1} \mathrm{H}$ NMR (400 MHz, $d^{6}$-DMSO) of compound $\mathbf{3 a -}-\mathbf{D}_{2}$
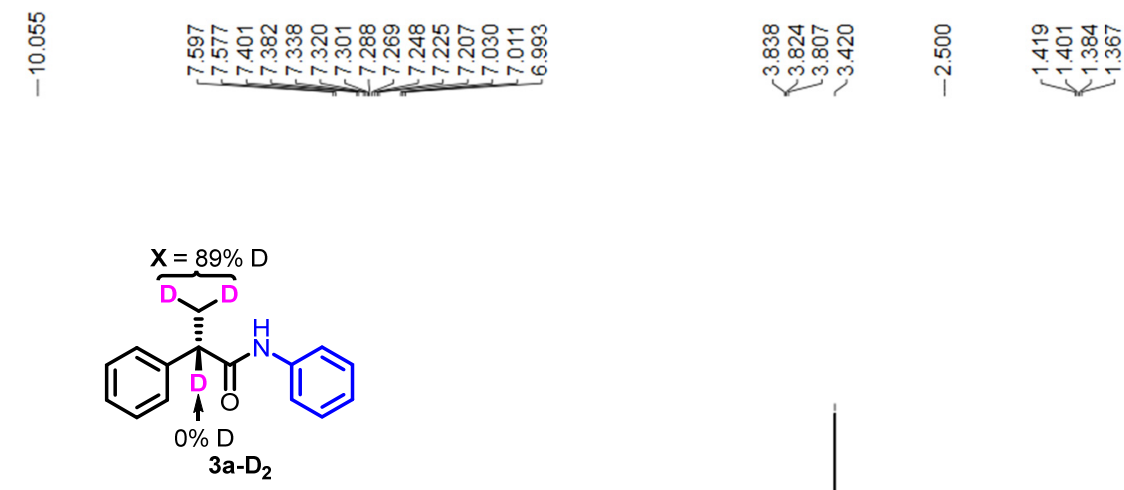

$x=\frac{2+2.01+4.99+1.0+1.0}{2+2+5+1+1}=1.000$

$\mathrm{n}_{1}=\frac{1.22}{1.000}=1.22$

\subsection{Deuterium-labeling studies with aniline- $\mathrm{D}_{7}$ and $\mathrm{D}_{2} \mathrm{O}$ under standard}

\section{conditions}

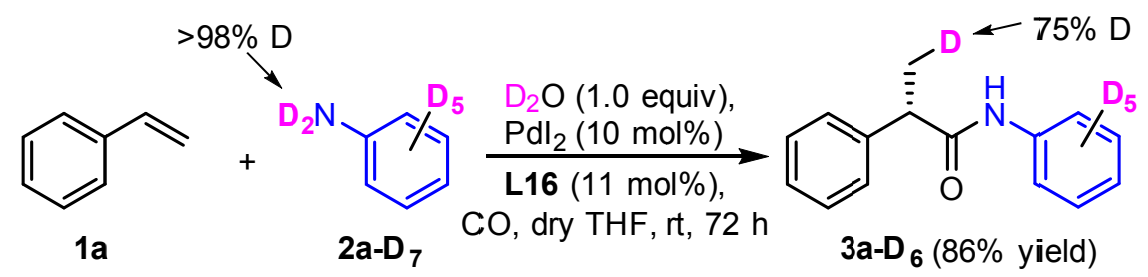

To a dry glass vessel were added $\mathrm{PdI}_{2}(0.01 \mathrm{mmol}, 10 \mathrm{~mol} \%), \mathbf{L 1 6}$ (0.011 mmol, $11 \mathrm{~mol} \%)$, $1 \mathrm{a}\left(0.15 \mathrm{mmol}, 1.5\right.$ equiv), $\mathbf{2 a - \mathbf { D } _ { 7 }}(>98$ atom\% D) $(0.1 \mathrm{mmol}, 1.0$ equiv) (purchased from $J \& K$ Scientific Ltd.), $\mathrm{D}_{2} \mathrm{O}(0.1 \mathrm{mmol}, 1.0$ equiv) and anhydrous THF $(1.0 \mathrm{~mL})$ in a glovebox. The glass vessel was put into an autoclave. The autoclave was evacuated and backfilled with $\mathrm{CO}$ for three times in a well-ventilated fume hood, and then pressurized to $50 \mathrm{~atm}$ of $\mathrm{CO}$. The reaction mixture in autoclave was stirred at room temperature for $72 \mathrm{~h}$ under $50 \mathrm{~atm}$ of $\mathrm{CO}$. Then, the $\mathrm{CO}$ in autoclave was carefully released in a well-ventilated fume hood. The reaction mixture was 
concentrated and purified by column chromatography on silica gel (eluent: petroleum ether/ethyl acetate $=10 / 1$ ) to afford the $\mathbf{3 a}_{\mathbf{a}} \mathbf{D}_{\mathbf{6}}$ in $86 \%$ yield. The $\mathbf{3} \mathbf{a}-\mathbf{D}_{\mathbf{6}}$ with 75 atom \% D on the methyl group was determined by ${ }^{1} \mathrm{H}$ NMR as following. .

${ }^{1} \mathrm{H}$ NMR (400 MHz, $d^{6}$-DMSO) of compound 3a-D

$\stackrel{1}{i}$<smiles>O=C(Nc1ccccc1)C(COCCOCCO)c1ccccc1</smiles>

$x=\frac{1.04+2.00+2.02+1.00+1.02}{1+2+2+1+1}=1.011$

$\mathrm{n}=\frac{2.27}{1.011}=2.25$

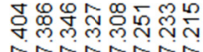

Nininis

$75 \% \mathrm{D}$

$1.00+1.02-1.011$
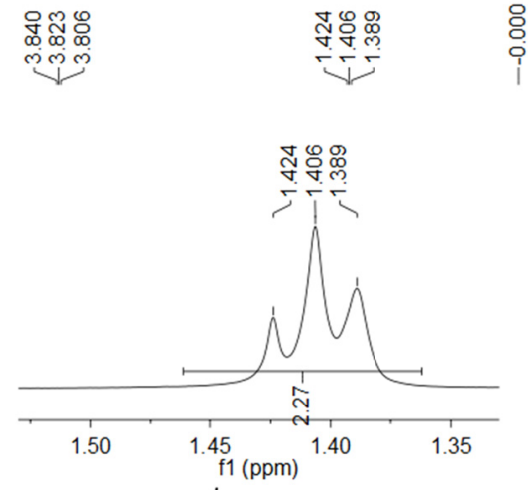

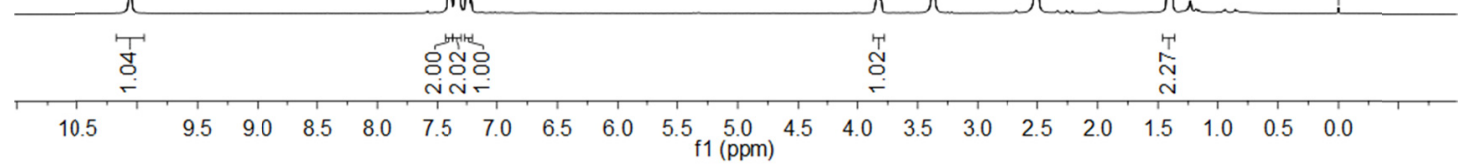

\subsection{Deuterium-labeling studies with aniline- $\mathrm{D}_{7}$ and $\mathrm{H}_{2} \mathrm{O}$ under standard}

\section{conditions}

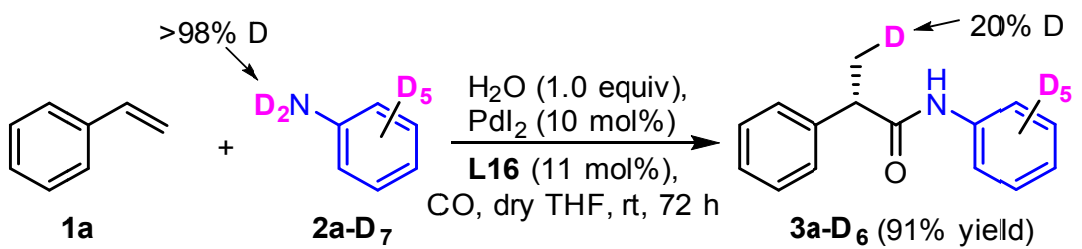

To a dry glass vessel were added $\mathrm{PdI}_{2}(0.01 \mathrm{mmol}, 10 \mathrm{~mol} \%), \mathbf{L 1 6}$ (0.011 mmol, $11 \mathrm{~mol} \%)$, $1 \mathrm{a}(0.15 \mathrm{mmol}, 1.5$ equiv), 2a-D 7 ( $>98$ atom\% D) (0.1 mmol, 1.0 equiv) (purchased from $J \& K$ Scientific Ltd.), $\mathrm{H}_{2} \mathrm{O}(0.1 \mathrm{mmol}, 1.0$ equiv) and anhydrous THF $(1.0 \mathrm{~mL})$ in a glovebox. The glass vessel was put into an autoclave. The autoclave was evacuated and backfilled with CO for three times in a well-ventilated fume hood, and 
then pressurized to $50 \mathrm{~atm}$ of $\mathrm{CO}$. The reaction mixture in autoclave was stirred at room temperature for $72 \mathrm{~h}$ under $50 \mathrm{~atm}$ of $\mathrm{CO}$. Then, the $\mathrm{CO}$ in autoclave was carefully released in a well-ventilated fume hood. The reaction mixture was concentrated and purified by column chromatography on silica gel (eluent: petroleum ether/ethyl acetate $=10 / 1)$ to afford the $\mathbf{3} \mathbf{a}_{-}-\mathbf{D}_{\mathbf{6}}$ in $91 \%$ yield. The $\mathbf{3} \mathbf{a}-\mathbf{D}_{\mathbf{6}}$ with 20 atom $\%$ D on the methyl group was determined by ${ }^{1} \mathrm{H}$ NMR as following.

${ }^{1} \mathrm{H}$ NMR (400 MHz, $d^{6}$-DMSO) of compound 3a-D 6

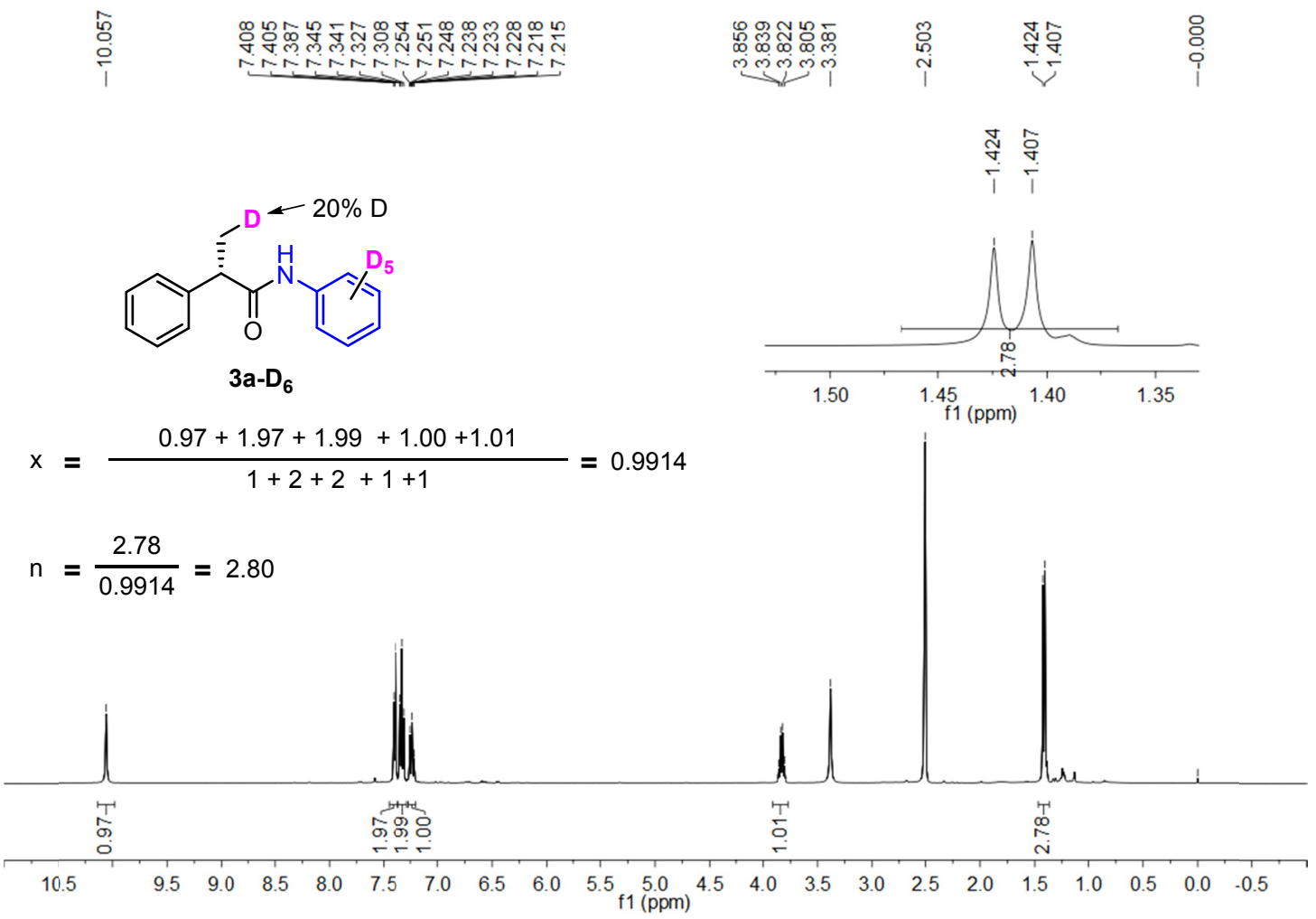

\subsection{Deuterium-labeling studies with aniline and $\mathrm{D}_{2} \mathrm{O}$ under standard conditions}

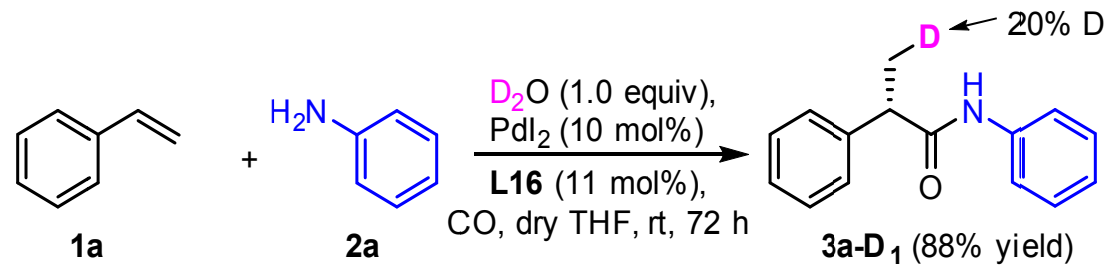

To a dry glass vessel were added $\mathrm{PdI}_{2}(0.01 \mathrm{mmol}, 10 \mathrm{~mol} \%), \mathbf{L 1 6}$ (0.011 mmol, $11 \mathrm{~mol} \%)$, $1 \mathrm{a}$ ( $0.15 \mathrm{mmol}, 1.5$ equiv), $2 \mathrm{a}\left(0.1 \mathrm{mmol}, 1.0\right.$ equiv), $\mathrm{D}_{2} \mathrm{O}(0.1 \mathrm{mmol}, 1.0$ equiv) and anhydrous THF (1.0 mL) in a glovebox. The glass vessel was put into an 
autoclave. The autoclave was evacuated and backfilled with $\mathrm{CO}$ for three times in a well-ventilated fume hood, and then pressurized to $50 \mathrm{~atm}$ of $\mathrm{CO}$. The reaction mixture in autoclave was stirred at room temperature for $72 \mathrm{~h}$ under 50 atm of $\mathrm{CO}$. Then, the CO in autoclave was carefully released in a well-ventilated fume hood. The reaction mixture was concentrated and purified by column chromatography on silica gel (eluent: petroleum ether/ethyl acetate $=10 / 1$ ) to afford the $\mathbf{3 a - \mathbf { D } _ { 1 }}$ in $88 \%$ yield. The 3a-D $\mathbf{1}$ with 20 atom $\%$ D on the methyl group was determined by ${ }^{1} \mathrm{H}$ NMR as following.

${ }^{1} \mathrm{H}$ NMR (400 MHz, $d^{6}$-DMSO) of compound 3a-D

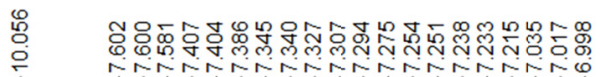

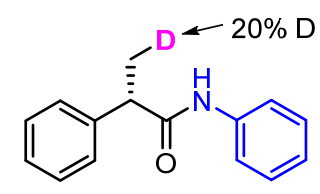

$3 a-D_{1}$

$x=\frac{0.99+2.07+2.01+5.28+1.00+1.03}{1+2+2+5+1+1}=1.032$

$\mathrm{n}=\frac{2.89}{1.032}=2.80$
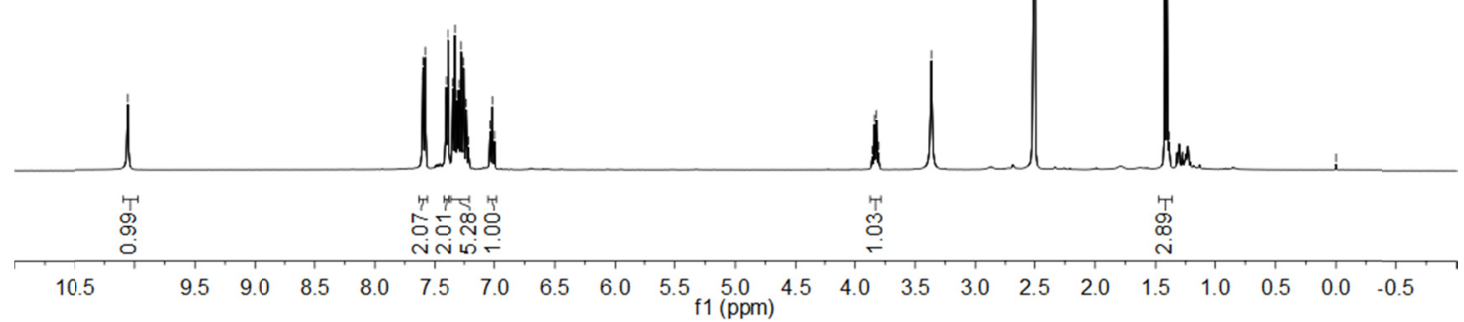


\section{Characterization data of the products}

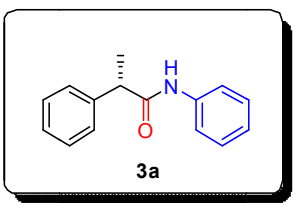

\section{(S)-N, 2-diphenylpropanamide}

$98 \%$ yield, $\mathrm{b} / \mathrm{l}>99: 1, \mathrm{ee}=94 \%,[\alpha] \mathrm{D}^{20}=+65.9\left(\mathrm{c}=1.8, \mathrm{CHCl}_{3}\right),{ }^{1} \mathrm{H} \mathrm{NMR}(400$ $\left.\mathrm{MHz}, \mathrm{CDCl}_{3}\right) \delta 7.42(\mathrm{~d}, J=7.6 \mathrm{~Hz}, 2 \mathrm{H}), 7.38-7.35(\mathrm{~m}, 3 \mathrm{H}), 7.32-7.23(\mathrm{~m}, 4 \mathrm{H}), 7.05(\mathrm{t}$, $J=7.2 \mathrm{~Hz}, 1 \mathrm{H}), 3.71(\mathrm{q}, J=7.2 \mathrm{~Hz}, 1 \mathrm{H}), 1.58(\mathrm{~d}, J=7.2 \mathrm{~Hz}, 3 \mathrm{H}) ;{ }^{13} \mathrm{C} \mathrm{NMR}(101 \mathrm{MHz}$, $\left.\mathrm{CDCl}_{3}\right) \delta 172.4,140.9,137.8,129.0,128.8,127.6,127.5,124.2,119.7,47.9,18.5$. HRMS calcd. (ESI) $\mathrm{m} / \mathrm{z}$ for $\mathrm{C}_{15} \mathrm{H}_{15} \mathrm{NNaO}:[\mathrm{M}+\mathrm{Na}]^{+} 248.1046$, found: 248.1047 .

Chiral HPLC (Chiralpak IG, 4.6x250 mm; $5 \mu \mathrm{L}$, hexane/isopropanol $=90 / 10$, flow $1.0 \mathrm{~mL} / \mathrm{min}, 254 \mathrm{~nm}$ ): $\mathrm{t}_{\mathrm{R}}=12.21$ (major), $\mathrm{t}_{\mathrm{R}}=14.43 \mathrm{~min}$ (minor).

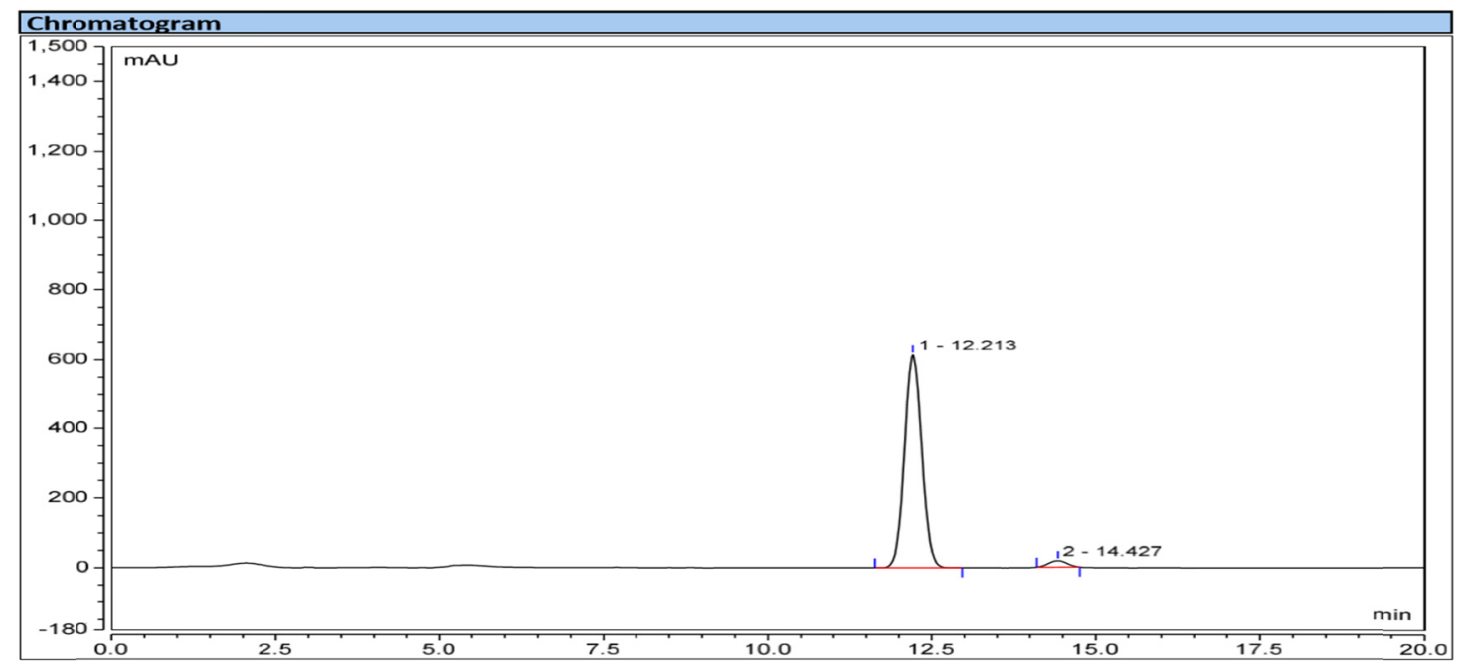

\begin{tabular}{|c|c|c|c|c|c|c|}
\hline \multicolumn{9}{|l|}{ Integration Results } \\
\hline No. & Peak Name & $\begin{array}{c}\text { Retention Time } \\
\text { min }\end{array}$ & $\begin{array}{c}\text { Height } \\
\text { mAU }\end{array}$ & $\begin{array}{c}\text { Area } \\
\text { mAU*min }\end{array}$ & $\begin{array}{c}\text { Relative Height } \\
\%\end{array}$ & $\begin{array}{c}\text { Relative Area } \\
\%\end{array}$ \\
\hline 1 & & 12.213 & 614.647 & 188.669 & 97.04 & 96.88 \\
2 & & 14.427 & 18.776 & 6.082 & 2.96 & 3.12 \\
\hline
\end{tabular}

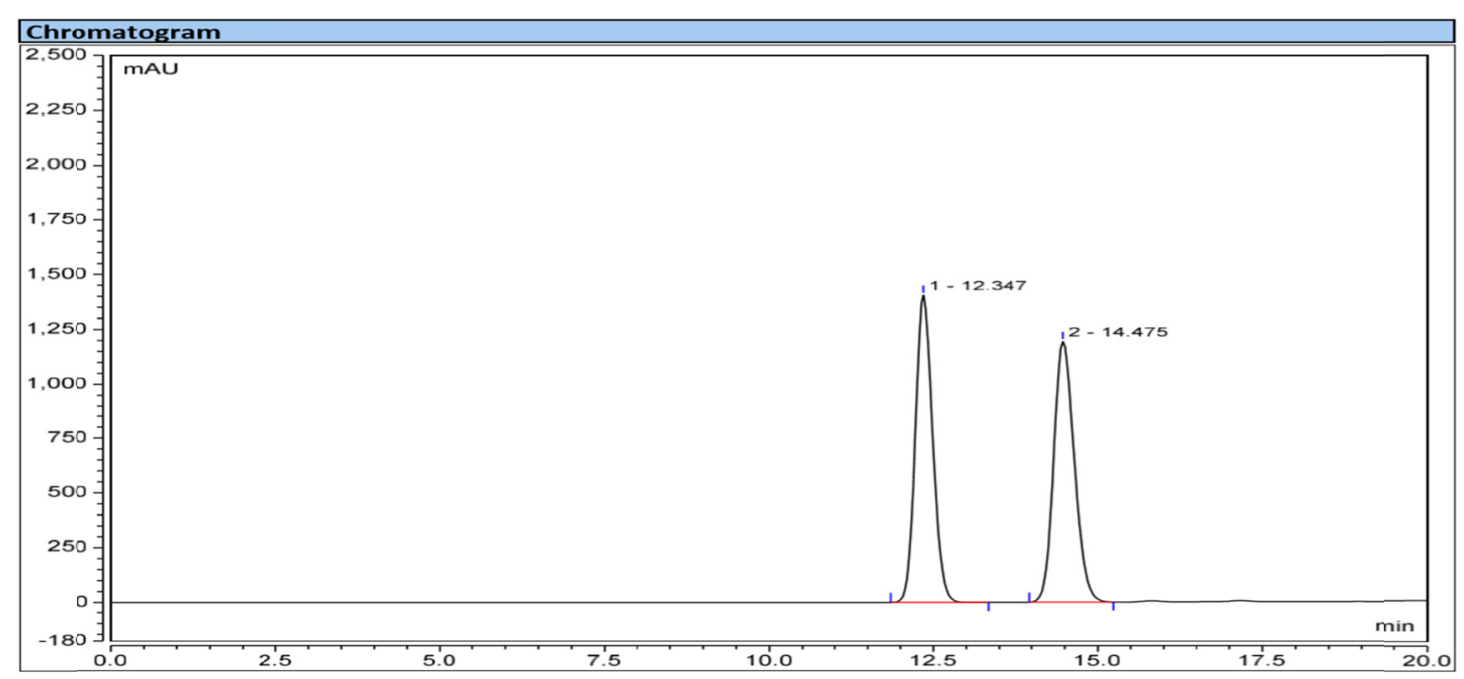

\begin{tabular}{|c|c|c|c|c|c|c|}
\hline \multicolumn{9}{|l|}{ Integration Results } \\
\hline No. & Peak Name & $\begin{array}{c}\text { Retention Time } \\
\text { min }\end{array}$ & $\begin{array}{c}\text { Height } \\
\text { mAU }\end{array}$ & $\begin{array}{c}\text { Area } \\
\text { mAU*min }\end{array}$ & $\begin{array}{c}\text { Relative Height } \\
\%\end{array}$ & $\begin{array}{c}\text { Relative Area } \\
\%\end{array}$ \\
\hline 1 & & 12.347 & 1404.856 & 427.792 & 54.06 & 49.82 \\
2 & & 14.475 & 1193.872 & 430.849 & 45.94 & 50.18 \\
\hline
\end{tabular}




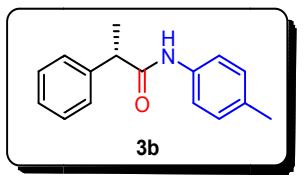

\section{(S)-2-phenyl- $N$-(p-tolyl)propanamide}

$81 \%$ yield, $\mathrm{b} / \mathrm{l}>99: 1, \mathrm{ee}=98 \%,[\alpha] \mathrm{D}^{18}=-165.2\left(\mathrm{c}=0.6, \mathrm{CHCl}_{3}\right),{ }^{1} \mathrm{H}$ NMR $(400$ $\left.\mathrm{MHz}, \mathrm{CDCl}_{3}\right) \delta 7.40-7.33(\mathrm{~m}, 4 \mathrm{H}), 7.32-7.26(\mathrm{~m}, 3 \mathrm{H}), 7.16(\mathrm{~s}, 1 \mathrm{H}), 7.05(\mathrm{~d}, J=8.2$ $\mathrm{Hz}, 2 \mathrm{H}), 3.69(\mathrm{q}, J=7.2 \mathrm{~Hz}, 1 \mathrm{H}), 2.27(\mathrm{~s}, 3 \mathrm{H}), 1.57(\mathrm{~d}, J=7.2 \mathrm{~Hz}, 3 \mathrm{H}) ;{ }^{13} \mathrm{C} \mathrm{NMR}$ $\left(151 \mathrm{MHz}, \mathrm{CDCl}_{3}\right) \delta 172.2,141.0,135.3,133.8,129.3,129.0,127.6,127.4,119.8$, 47.9, 20.8, 18.5. HRMS calcd. (ESI) $\mathrm{m} / \mathrm{z}$ for $\mathrm{C}_{16} \mathrm{H}_{17} \mathrm{NNaO}$ : $[\mathrm{M}+\mathrm{Na}]^{+}$262.1202, found: 262.1202 .

Chiral HPLC (Chiralpak IG, 4.6x250 mm; $5 \mu \mathrm{L}$, hexane/isopropanol = 90/10, flow $1.0 \mathrm{~mL} / \mathrm{min}, 254 \mathrm{~nm}$ ): $\mathrm{t}_{\mathrm{R}}=17.75 \mathrm{~min}$ (major), $\mathrm{t}_{\mathrm{R}}=26.55 \mathrm{~min}$ (minor).

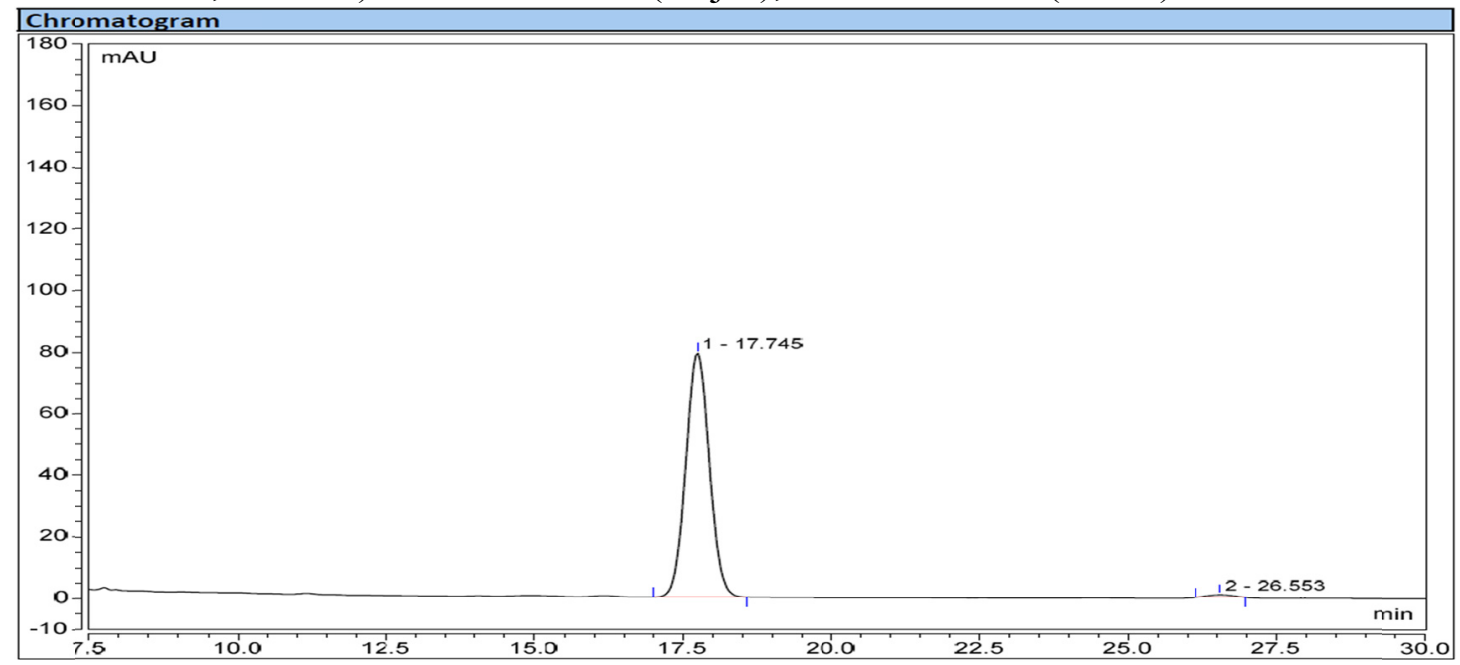

\begin{tabular}{|c|c|c|c|c|c|c|}
\hline \multicolumn{7}{|c|}{ Integration Results } \\
\hline No. & Peak Name & $\begin{array}{c}\text { Retention Time } \\
\text { min }\end{array}$ & $\begin{array}{l}\text { Height } \\
\text { mAU }\end{array}$ & $\begin{array}{c}\text { Area } \\
\text { mAU*min }\end{array}$ & $\begin{array}{c}\text { Relative Height } \\
\%\end{array}$ & $\begin{array}{c}\text { Relative Area } \\
\%\end{array}$ \\
\hline $\begin{array}{l}1 \\
2\end{array}$ & & $\begin{array}{l}17.745 \\
26.553\end{array}$ & $\begin{array}{c}79.419 \\
0.885\end{array}$ & $\begin{array}{c}36.215 \\
0.427\end{array}$ & $\begin{array}{c}98.90 \\
1.10\end{array}$ & $\begin{array}{c}98.84 \\
1.16\end{array}$ \\
\hline
\end{tabular}

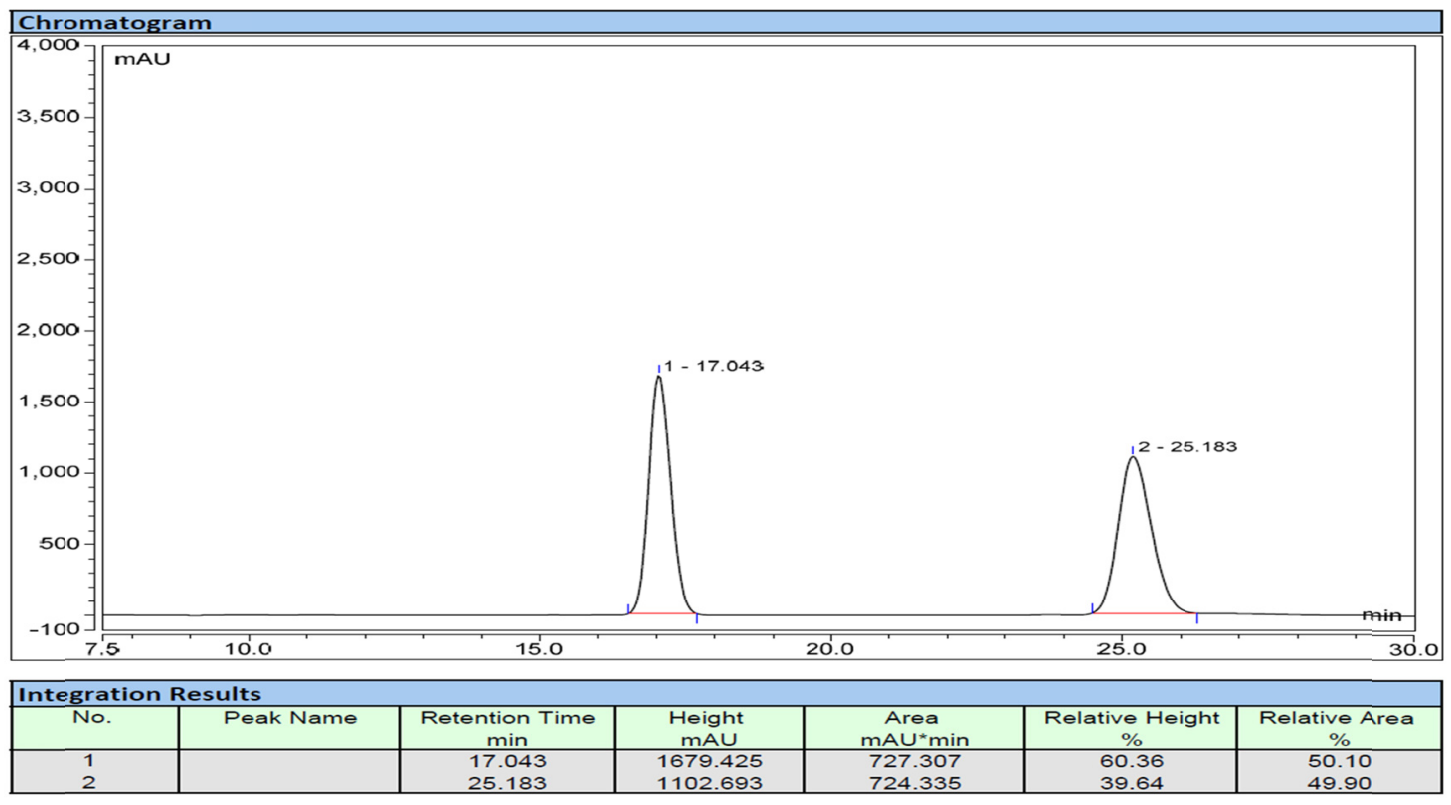




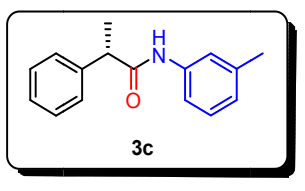

\section{(S)-2-phenyl- $N$-(m-tolyl)propanamide}

97\% yield, $\mathrm{b} / \mathrm{l}>99: 1$, ee $\left.=92 \%,[\alpha] \mathrm{D}^{20}=+75.5\left(\mathrm{c}=1.8, \mathrm{CHCl}_{3}\right)\right) .{ }^{1} \mathrm{H}$ NMR $(400$ $\left.\mathrm{MHz}, \mathrm{CDCl}_{3}\right) \delta 7.86(\mathrm{~d}, J=8.0 \mathrm{~Hz}, 1 \mathrm{H}), 7.40-7.39(\mathrm{~m}, 4 \mathrm{H}), 7.34-7.32(\mathrm{~m}, 1 \mathrm{H}), 7.17(\mathrm{td}$, $J=8.0,1.2 \mathrm{~Hz}, 1 \mathrm{H}), 7.07$ (d, $J=6.8 \mathrm{~Hz}, 1 \mathrm{H}), 7.00(\mathrm{t}, J=7.2 \mathrm{~Hz}, 1 \mathrm{H}), 6.88(\mathrm{~s}, 1 \mathrm{H}), 3.78$ $(\mathrm{q}, J=7.2 \mathrm{~Hz}, 1 \mathrm{H}), 1.87(\mathrm{~s}, 3 \mathrm{H}), 1.64(\mathrm{~d}, J=6.8 \mathrm{~Hz}, 3 \mathrm{H}),{ }^{13} \mathrm{C} \mathrm{NMR}\left(151 \mathrm{MHz}, \mathrm{CDCl}_{3}\right)$ $\delta$ 172.4, 141.0, 138.7, 137.8, 129.0, 128.6, 127.6, 127.4, 124.9, 120.4, 116.8, 47.9, 21.3, 18.5. HRMS calcd. (ESI) $\mathrm{m} / \mathrm{z}$ for $\mathrm{C}_{16} \mathrm{H}_{17} \mathrm{NNaO}$ : [M+Na] $]^{+} 262.1202$, found: 262.1198 .

Chiral HPLC (Chiralpak IG, 4.6x250 mm; $5 \mu \mathrm{L}$, hexane/isopropanol = 90/10, flow $1.0 \mathrm{~mL} / \mathrm{min}, 254 \mathrm{~nm}$ ): $t_{\mathrm{R}}=9.79 \min$ (major), $\mathrm{t}_{\mathrm{R}}=11.35 \mathrm{~min}$ (minor).
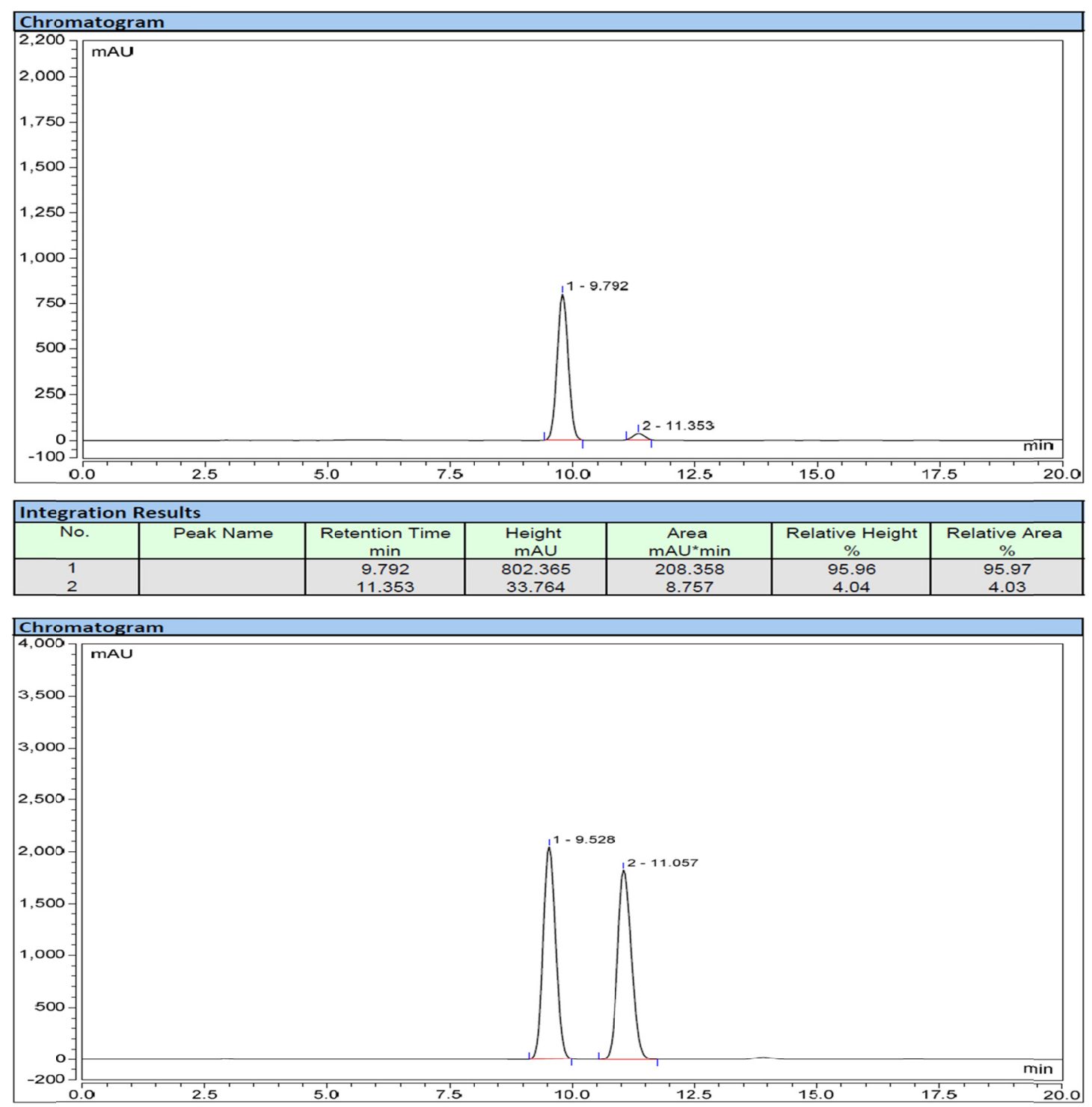

\begin{tabular}{|c|c|c|c|c|c|c|}
\hline \multicolumn{9}{|l|}{ Integration Results } \\
\hline No. & Peak Name & $\begin{array}{c}\text { Retention Time } \\
\text { min }\end{array}$ & $\begin{array}{c}\text { Height } \\
\text { mAU }\end{array}$ & $\begin{array}{c}\text { Area } \\
\text { mAU*min }\end{array}$ & $\begin{array}{c}\text { Relative Height } \\
\%\end{array}$ & $\begin{array}{c}\text { Relative Area } \\
\%\end{array}$ \\
\hline 1 & & 9.528 & 2044.300 & 602.181 & 52.82 & 49.86 \\
2 & & 11.057 & 1825.869 & 605.572 & 47.18 & 50.14 \\
\hline
\end{tabular}




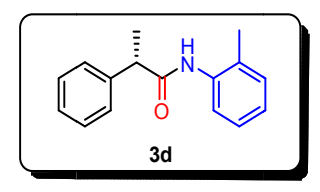

\section{(S)-2-phenyl- $N$-(o-tolyl)propanamide}

97\% yield, $\mathrm{b} / 1>99: 1, \mathrm{ee}=91 \%,[\alpha] \mathrm{D}^{18}=+24.1\left(\mathrm{c}=1.3, \mathrm{CHCl}_{3}\right) .{ }^{1} \mathrm{H}$ NMR $(400$ $\left.\mathrm{MHz}, \mathrm{CDCl}_{3}\right) \delta 7.67$ (d, $\left.J=8.0 \mathrm{~Hz}, 1 \mathrm{H}\right), 7.35(\mathrm{~s}, 1 \mathrm{H}), 7.33-7.21(\mathrm{~m}, 5 \mathrm{H}), 7.10-6.94$ (m, 3H), $3.71(\mathrm{q}, J=7.2 \mathrm{~Hz}, 1 \mathrm{H}), 1.85(\mathrm{~s}, 3 \mathrm{H}), 1.54(\mathrm{~d}, J=7.2 \mathrm{~Hz}, 3 \mathrm{H}) ;{ }^{13} \mathrm{C}$ NMR $\left(151 \mathrm{MHz}, \mathrm{CDCl}_{3}\right) \delta 172.3,141.0,135.6,130.2,129.1,128.3,127.8,127.6,126.6$, 124.8, 122.2, 47.9, 18.0, 17.0. HRMS calcd. (ESI) $\mathrm{m} / \mathrm{z}$ for $\mathrm{C}_{16} \mathrm{H}_{17} \mathrm{NNaO}:[\mathrm{M}+\mathrm{Na}]^{+}$ 262.1202, found: 262.1200 .

Chiral HPLC (Chiralpak IG, 4.6x250 mm; $5 \mu \mathrm{L}$, hexane/isopropanol = 90/10, flow $1.0 \mathrm{~mL} / \mathrm{min}, 254 \mathrm{~nm}$ ): $\mathrm{t}_{\mathrm{R}}=14.43 \mathrm{~min}$ (major), $\mathrm{t}_{\mathrm{R}}=18.45 \mathrm{~min}$ (minor).

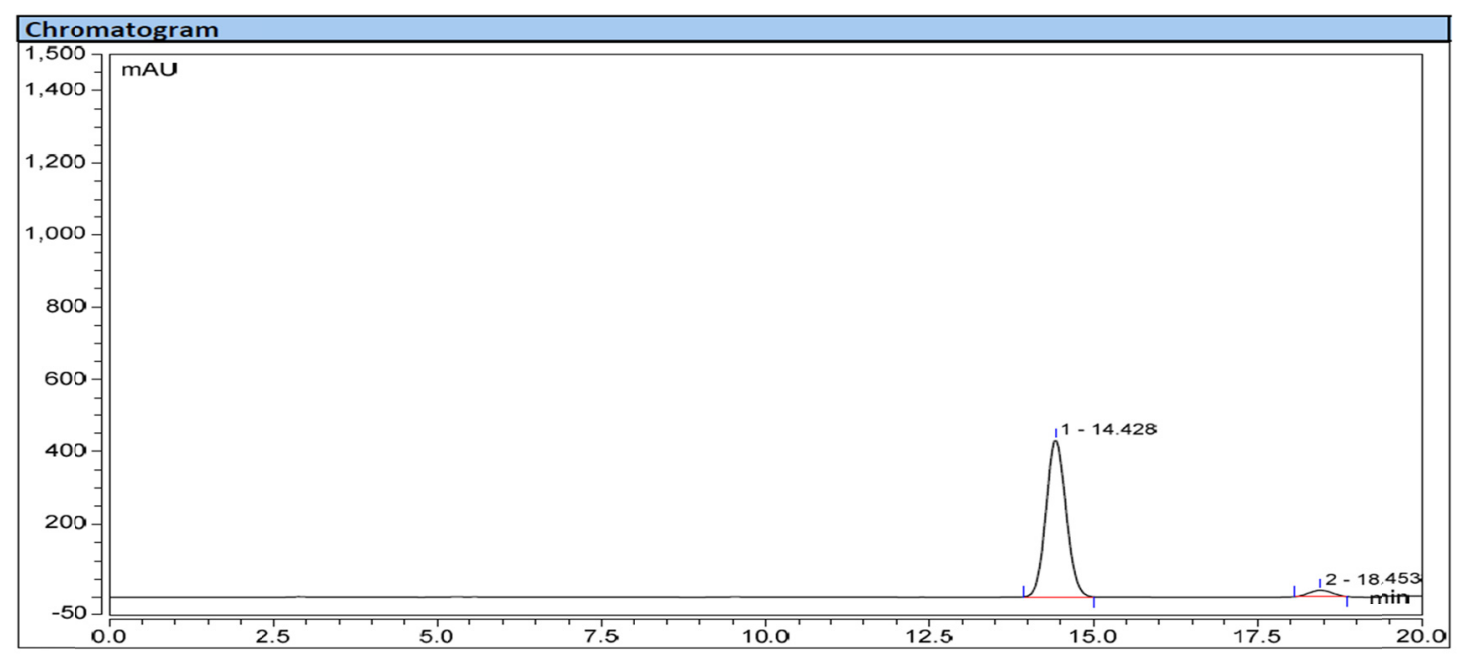

\begin{tabular}{|c|c|c|c|c|c|c|}
\hline \multicolumn{9}{|l|}{ Integration Results } \\
\hline No. & Peak Name & $\begin{array}{c}\text { Retention Time } \\
\text { min }\end{array}$ & $\begin{array}{c}\text { Height } \\
\text { mAU }\end{array}$ & $\begin{array}{c}\text { Area } \\
\text { mAU*min }\end{array}$ & $\begin{array}{c}\text { Relative Height } \\
\%\end{array}$ & $\begin{array}{c}\text { Relative Area } \\
\%\end{array}$ \\
\hline 1 & & 14.428 & 431.106 & 151.942 & 96.06 & 95.54 \\
2 & & 18.453 & 17.689 & 7.087 & 3.94 & 4.46 \\
\hline
\end{tabular}

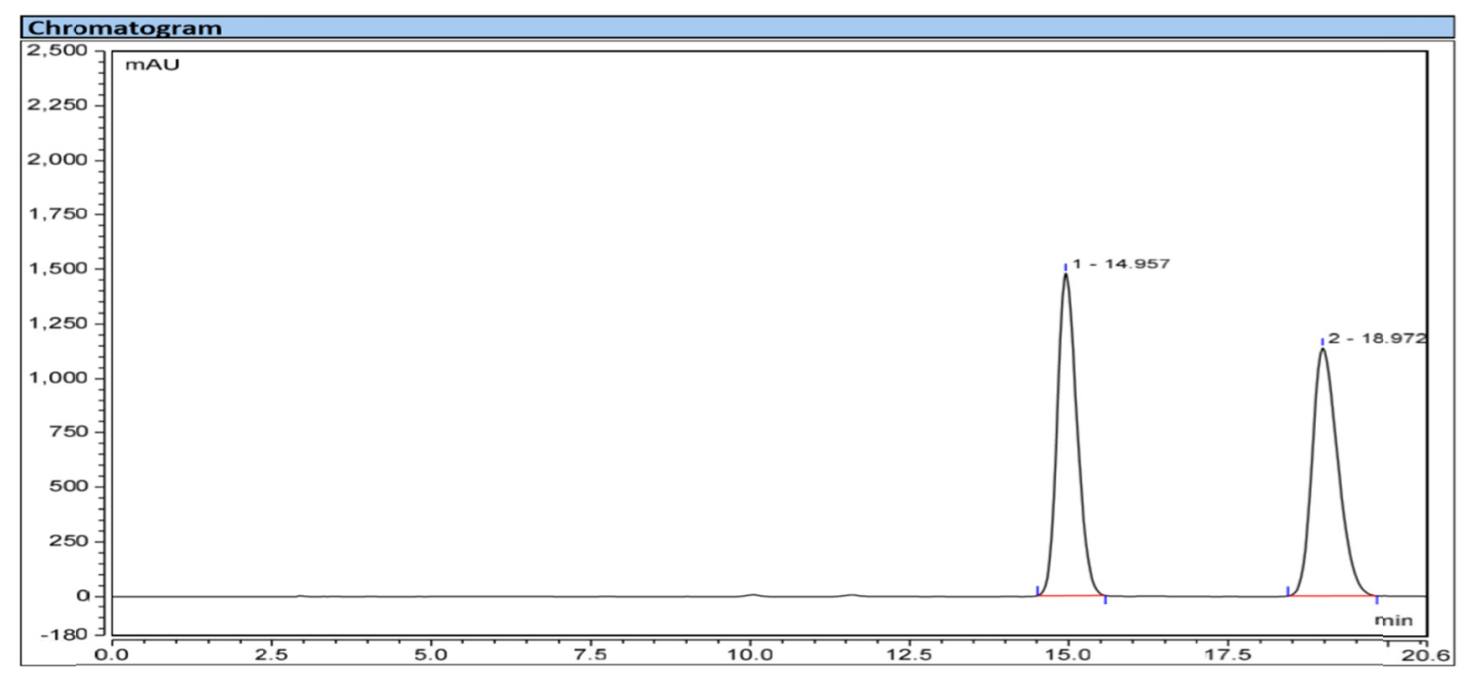

\begin{tabular}{|c|c|c|c|c|c|c|}
\hline \multicolumn{9}{|l|}{ Integration Results } \\
\hline No. & Peak Name & $\begin{array}{c}\text { Retention Time } \\
\text { min }\end{array}$ & $\begin{array}{c}\text { Height } \\
\text { mAU }\end{array}$ & $\begin{array}{c}\text { Area } \\
\text { mAU*min }\end{array}$ & $\begin{array}{c}\text { Relative Height } \\
\%\end{array}$ & $\begin{array}{c}\text { Relative Area } \\
\%\end{array}$ \\
\hline 1 & & 14.957 & 1476.115 & 523.164 & 56.47 & 49 \\
2 & & 18.972 & 1138.049 & 526.312 & 43.53 & 50.15 \\
\hline
\end{tabular}




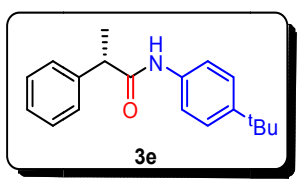

\section{(S)- $N$-(4-(tert-butyl)phenyl)-2-phenylpropanamide}

$84 \%$ yield, $\mathrm{b} / 1>99: 1, \mathrm{ee}=94 \%,[\alpha] \mathrm{D}^{18}=+27.2\left(\mathrm{c}=1.5, \mathrm{CHCl}_{3}\right) .{ }^{1} \mathrm{H} \mathrm{NMR}(600$ $\left.\mathrm{MHz}, \mathrm{CDCl}_{3}\right) \delta$ 7.36-7.30 (m, 6H), 7.28-7.25 (m, 3H), $7.09(\mathrm{~s}, 1 \mathrm{H}), 3.70(\mathrm{q}, J=7.2$ $\mathrm{Hz}, 1 \mathrm{H}), 1.58(\mathrm{~d}, J=6.6 \mathrm{~Hz}, 3 \mathrm{H}), 1.27(\mathrm{~s}, 9 \mathrm{H}) ;{ }^{13} \mathrm{C} \mathrm{NMR}\left(151 \mathrm{MHz}, \mathrm{CDCl}_{3}\right) \delta 172.2$, 147.2, 141.0, 135.2, 129.1, 127.7, 127.5, 125.7, 119.5, 48.0, 34.3, 31.3, 18.5. HRMS calcd. (ESI) m/z for $\mathrm{C}_{19} \mathrm{H}_{23} \mathrm{NNaO}$ : [M+Na] 304.1672 , found: 304.1677 .

Chiral HPLC (Chiralpak IG, 4.6x250 mm; $5 \mathrm{~L}$, hexane/isopropanol $=90 / 10$, flow $1.0 \mathrm{~mL} / \mathrm{min}, 254 \mathrm{~nm}$ ): $\mathrm{t}_{\mathrm{R}}=12.71 \mathrm{~min}$ (major), $\mathrm{t}_{\mathrm{R}}=13.66 \mathrm{~min}$ (minor).

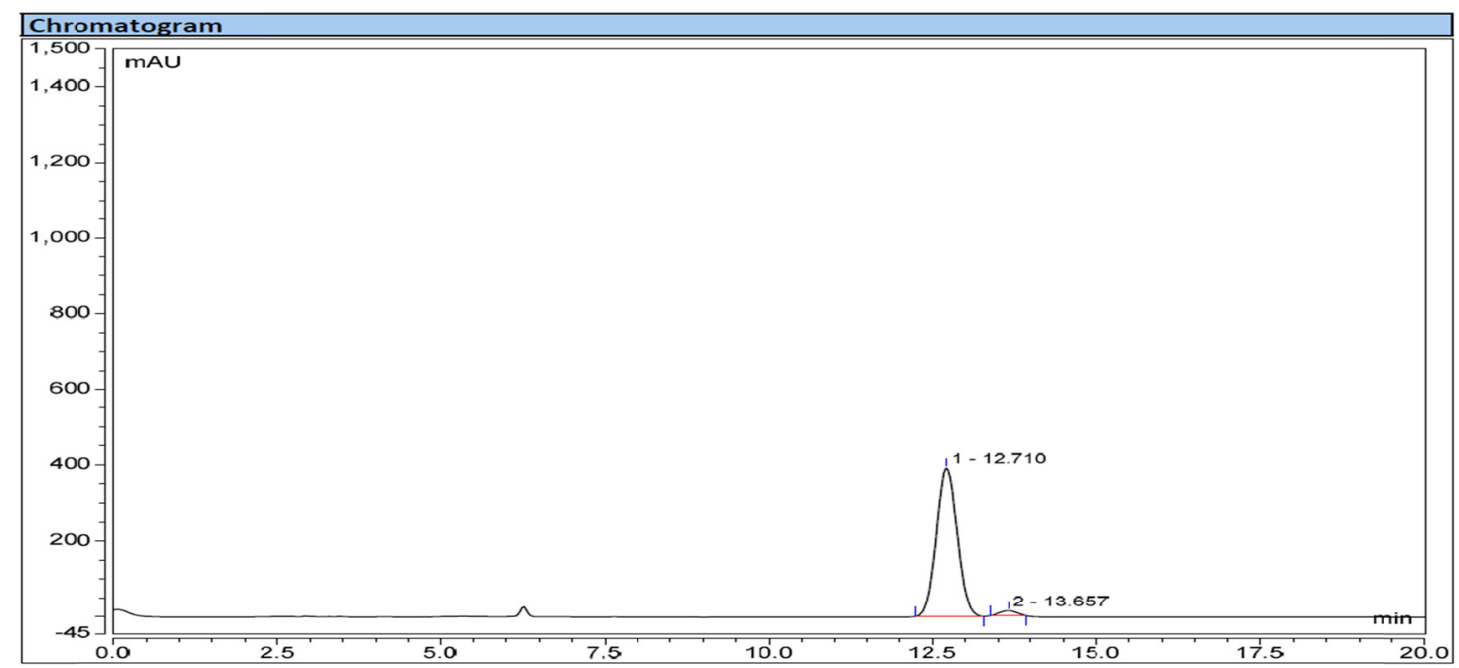

\begin{tabular}{|c|c|c|c|c|c|c|}
\hline \multicolumn{9}{|l|}{ Integration Results } \\
\hline No. & Peak Name & $\begin{array}{c}\text { Retention Time } \\
\text { min }\end{array}$ & $\begin{array}{c}\text { Height } \\
\text { mAU }\end{array}$ & $\begin{array}{c}\text { Area } \\
\text { mAU*min }\end{array}$ & $\begin{array}{c}\text { Relative Height } \\
\%\end{array}$ & $\begin{array}{c}\text { Relative Area } \\
\%\end{array}$ \\
\hline 1 & & 12.710 & 391.444 & 144.948 & 96.68 & 97.18 \\
2 & & 13.657 & 13.427 & 4.205 & 3.32 & 2.82 \\
\hline
\end{tabular}

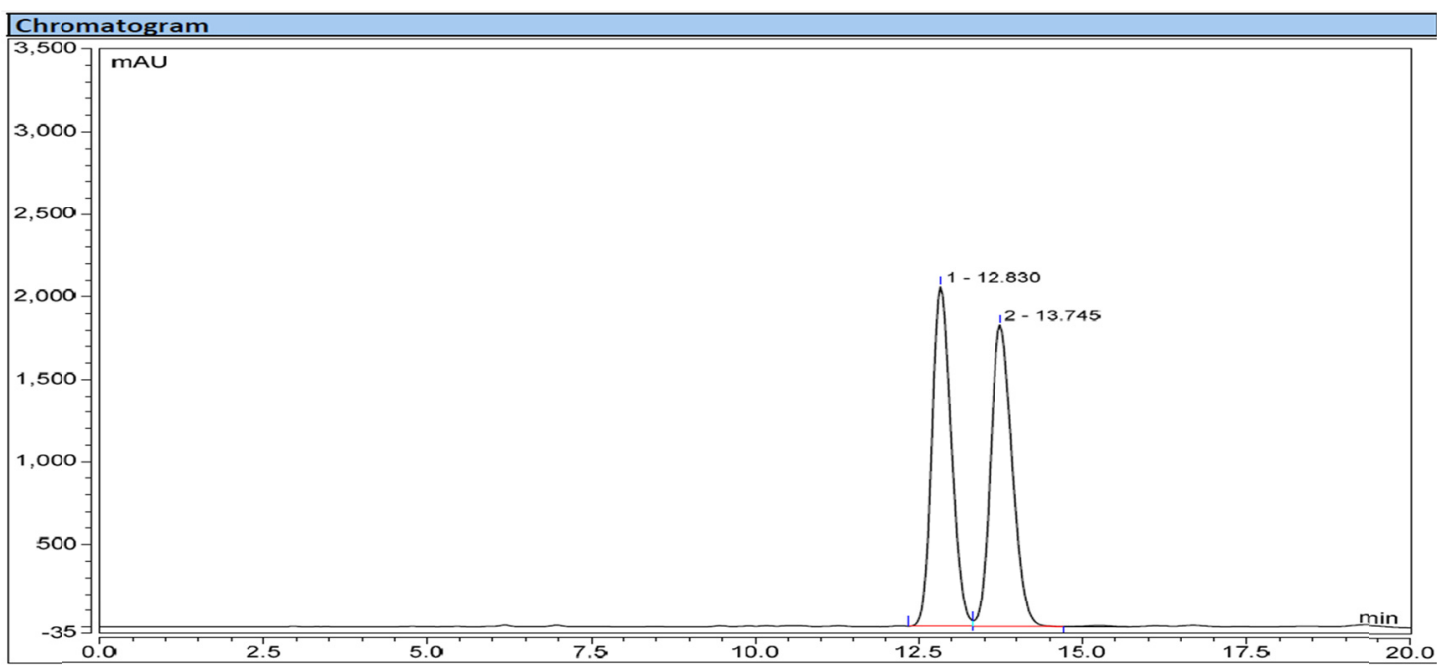

\begin{tabular}{|c|c|c|c|c|c|c|}
\hline \multicolumn{9}{|l|}{ Integration Results } \\
\hline No. & Peak Name & $\begin{array}{c}\text { Retention Time } \\
\text { min }\end{array}$ & $\begin{array}{c}\text { Height } \\
\text { mAU }\end{array}$ & $\begin{array}{c}\text { Area } \\
\text { mAU*min }\end{array}$ & $\begin{array}{c}\text { Relative Height } \\
\%\end{array}$ & $\begin{array}{c}\text { Relative Area } \\
\%\end{array}$ \\
\hline 1 & & 12.830 & 2057.400 & 705.096 & 52.92 & 49.85 \\
2 & & 13.745 & 1830.506 & 709.367 & 47.08 & 50.15 \\
\hline
\end{tabular}




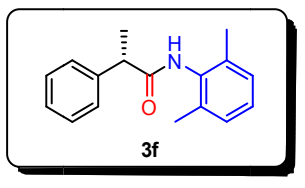

\section{(S)-N-(2,6-dimethylphenyl)-2-phenylpropanamide}

$93 \%$ yield, $\mathrm{b} / \mathrm{l}>99: 1, \mathrm{ee}=92 \%,[\alpha] \mathrm{D}^{18}=-18.6\left(\mathrm{c}=1.2, \mathrm{CHCl}_{3}\right) .{ }^{1} \mathrm{H}$ NMR $(400$ $\left.\mathrm{MHz}, \mathrm{CDCl}_{3}\right)^{1} \mathrm{H}$ NMR $\left(400 \mathrm{MHz}, \mathrm{CDCl}_{3}\right) \delta$ 7.47-7.37 (m, 4H), 7.35-7.29 (m, $\left.1 \mathrm{H}\right)$, 7.07-6.95 (m, 3H), 6.52 (s, 1H), 3.81 (q, $J=7.2 \mathrm{~Hz}, 1 \mathrm{H}), 2.05$ (s, 6H), 1.65 (d, $J=7.2$ $\mathrm{Hz}, 3 \mathrm{H}) ;{ }^{13} \mathrm{C}$ NMR $\left(101 \mathrm{MHz}, \mathrm{CDCl}_{3}\right) \delta 172.5,141.2,135.3,133.7,129.1,128.1$, 127.7, 127.6, 127.1, 47.3, 18.1, 18.0. HRMS calcd. (ESI) $\mathrm{m} / \mathrm{z}$ for $\mathrm{C}_{17} \mathrm{H}_{19} \mathrm{NNaO}$ : $[\mathrm{M}+\mathrm{Na}]^{+}$276.1359, found: 276.1360 .

Chiral HPLC (Chiralpak IG, 4.6x250 mm; $5 \mu \mathrm{L}$, hexane/isopropanol $=85 / 15$, flow $1.0 \mathrm{~mL} / \mathrm{min}, 254 \mathrm{~nm}$ ): $\mathrm{t}_{\mathrm{R}}=8.61 \mathrm{~min}$ (major), $\mathrm{t}_{\mathrm{R}}=11.48 \mathrm{~min}$ (minor).
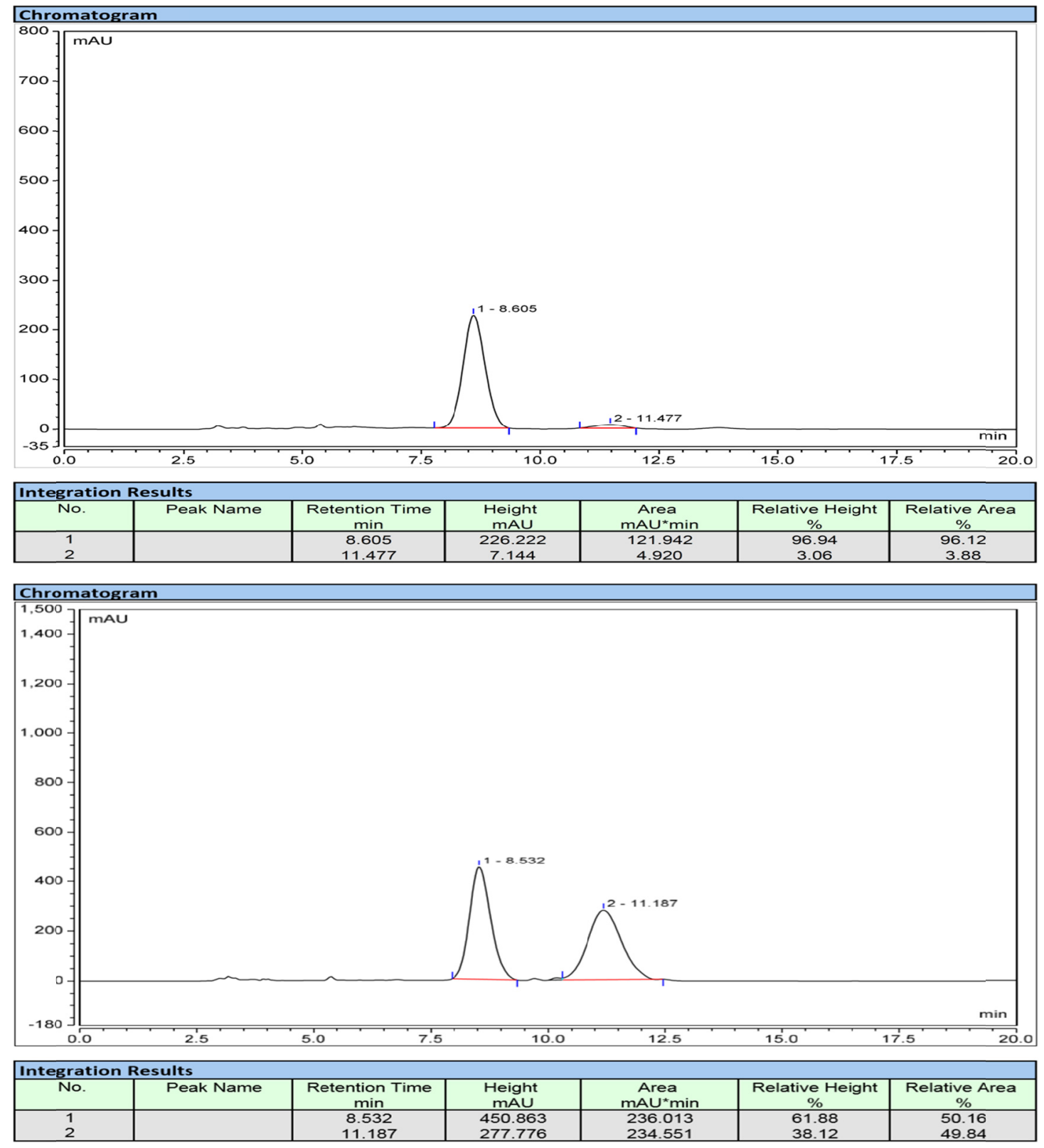


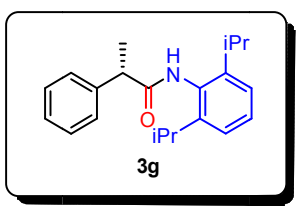

\section{(S)-N-(2,6-diisopropylphenyl)-2-phenylpropanamide}

$62 \%$ yield, $\mathrm{b} / \mathrm{l}>99: 1, \mathrm{ee}=93 \%,[\alpha] \mathrm{D}^{20}=-23.6\left(\mathrm{c}=1.8, \mathrm{CHCl}_{3}\right) .{ }^{1} \mathrm{H}$ NMR $(600$ $\left.\mathrm{MHz}, \mathrm{CDCl}_{3}\right) \delta$ 7.44-7.39 (m, 4H), 7.33-7.31 (m, 1H), 7.25-7.21 (m, 1H), $7.09(\mathrm{~d}, J=$ $7.8 \mathrm{~Hz}, 2 \mathrm{H}), 6.45$ (s, 1H), 3.83 (q, $J=7.2 \mathrm{~Hz}, 1 \mathrm{H}), 2.80(\mathrm{~m}, 2 \mathrm{H}), 1.66(\mathrm{~d}, J=7.2 \mathrm{~Hz}$, $3 \mathrm{H}), 1.07(\mathrm{~d}, J=6.6 \mathrm{~Hz}, 12 \mathrm{H}) ;{ }^{13} \mathrm{C}$ NMR $\left(151 \mathrm{MHz}, \mathrm{CDCl}_{3}\right) \delta 173.3,146.1,141.2$, 131.2, 129.0, 128.1, 127.6, 127.5, 123.2, 47.4, 28.5, 23.4, 23.3, 17.7. HRMS calcd. (ESI) $\mathrm{m} / \mathrm{z}$ for $\mathrm{C}_{21} \mathrm{H}_{27} \mathrm{NNaO}$ : [M+Na] ${ }^{+} 332.1985$, found: 332.1980 .

Chiral HPLC (Chiralpak IF, 4.6x250 mm; $5 \mu \mathrm{L}$, hexane/isopropanol $=85 / 15$, flow $1.0 \mathrm{~mL} / \mathrm{min}, 254 \mathrm{~nm}$ ): $\mathrm{t}_{\mathrm{R}}=4.87 \mathrm{~min}$ (major), $\mathrm{t}_{\mathrm{R}}=5.26 \mathrm{~min}$ (minor)
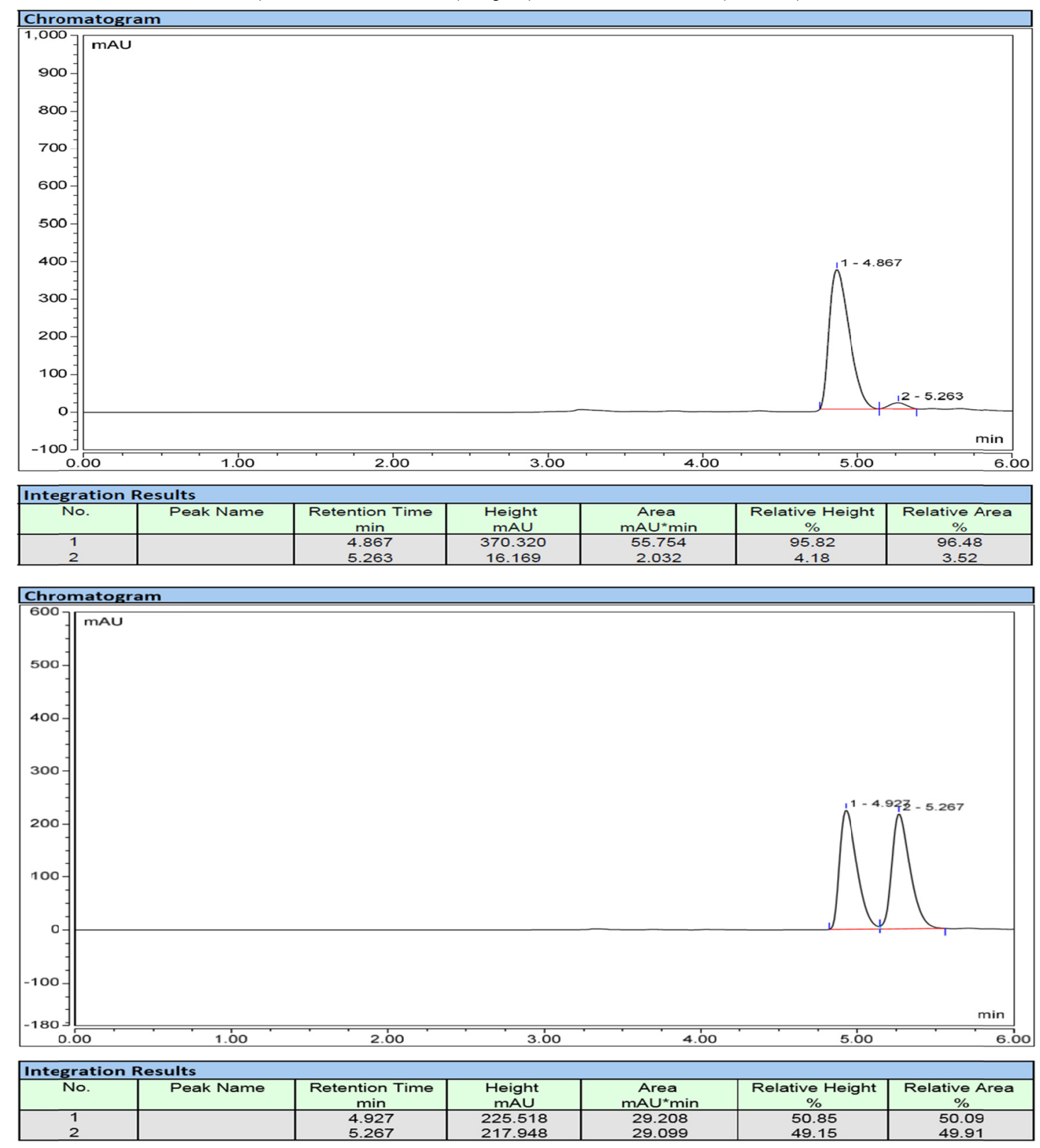


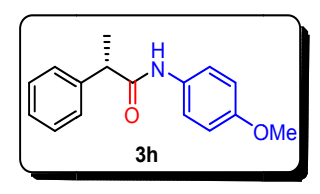

\section{(S)-N-(4-methoxyphenyl)-2-phenylpropanamide}

$73 \%$ yield, $\mathrm{b} / 1>99: 1, \mathrm{ee}=98 \%$, $[\alpha] \mathrm{D}^{20}=-31.9\left(\mathrm{c}=1.8, \mathrm{CHCl}_{3}\right) .{ }^{1} \mathrm{H}$ NMR $(400$ $\left.\mathrm{MHz}, \mathrm{CDCl}_{3}\right) \delta$ 7.42-7.34 (m, 4H), 7.33-7.27 (m, 3H), $7.06(\mathrm{~s}, 1 \mathrm{H}), 6.83-6.76(\mathrm{~m}, 2 \mathrm{H})$, $3.75(\mathrm{~s}, 3 \mathrm{H}), 3.69(\mathrm{q}, J=7.2 \mathrm{~Hz}, 1 \mathrm{H}), 1.59(\mathrm{~d}, J=7.2 \mathrm{~Hz}, 3 \mathrm{H}) ;{ }^{13} \mathrm{C}$ NMR $(101 \mathrm{MHz}$, $\left.\mathrm{CDCl}_{3}\right) \delta 172.1,156.3,141.1,131.0,129.1,127.7,127.5,121.6,114.0,55.4,47.9$, 18.6. HRMS calcd. (ESI) $\mathrm{m} / \mathrm{z}$ for $\mathrm{C}_{16} \mathrm{H}_{17} \mathrm{NNaO}_{2}:[\mathrm{M}+\mathrm{Na}]^{+}$278.1152, found: 278.1159 .

Chiral HPLC (Chiralpak IF, 4.6x250 mm; $5 \mu \mathrm{L}$, hexane/isopropanol $=90 / 10$, flow $1.0 \mathrm{~mL} / \mathrm{min}, 254 \mathrm{~nm}$ ): $\mathrm{t}_{\mathrm{R}}=17.95$ (major), $\mathrm{t}_{\mathrm{R}}=21.42 \mathrm{~min}$ (minor).

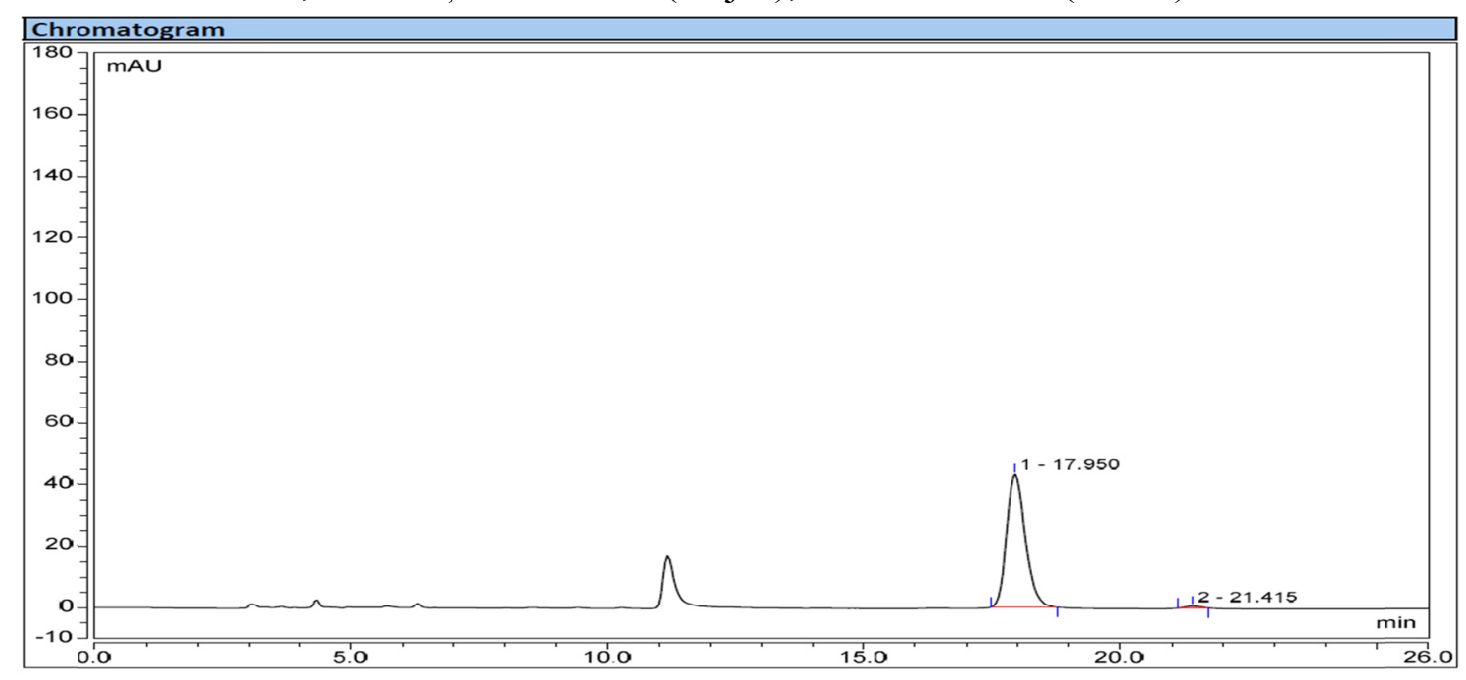

\begin{tabular}{|c|c|c|c|c|c|c|}
\hline Integration Results & \multicolumn{9}{c|}{$\begin{array}{c}\text { Area } \\
\text { No. }\end{array}$} & Peak Name & $\begin{array}{c}\text { Retention Time } \\
\text { min }\end{array}$ & $\begin{array}{c}\text { Height } \\
\text { mAU }\end{array}$ & $\begin{array}{c}\text { Relative Height } \\
\text { mAU*min }\end{array}$ & $\begin{array}{c}\text { Relative Area } \\
\%\end{array}$ \\
\hline 1 & & 17.950 & 43.192 & 18.323 & 98.70 & 98 \\
2 & & 21.415 & 0.571 & 0.203 & 1.30 & 1.10 \\
\hline
\end{tabular}

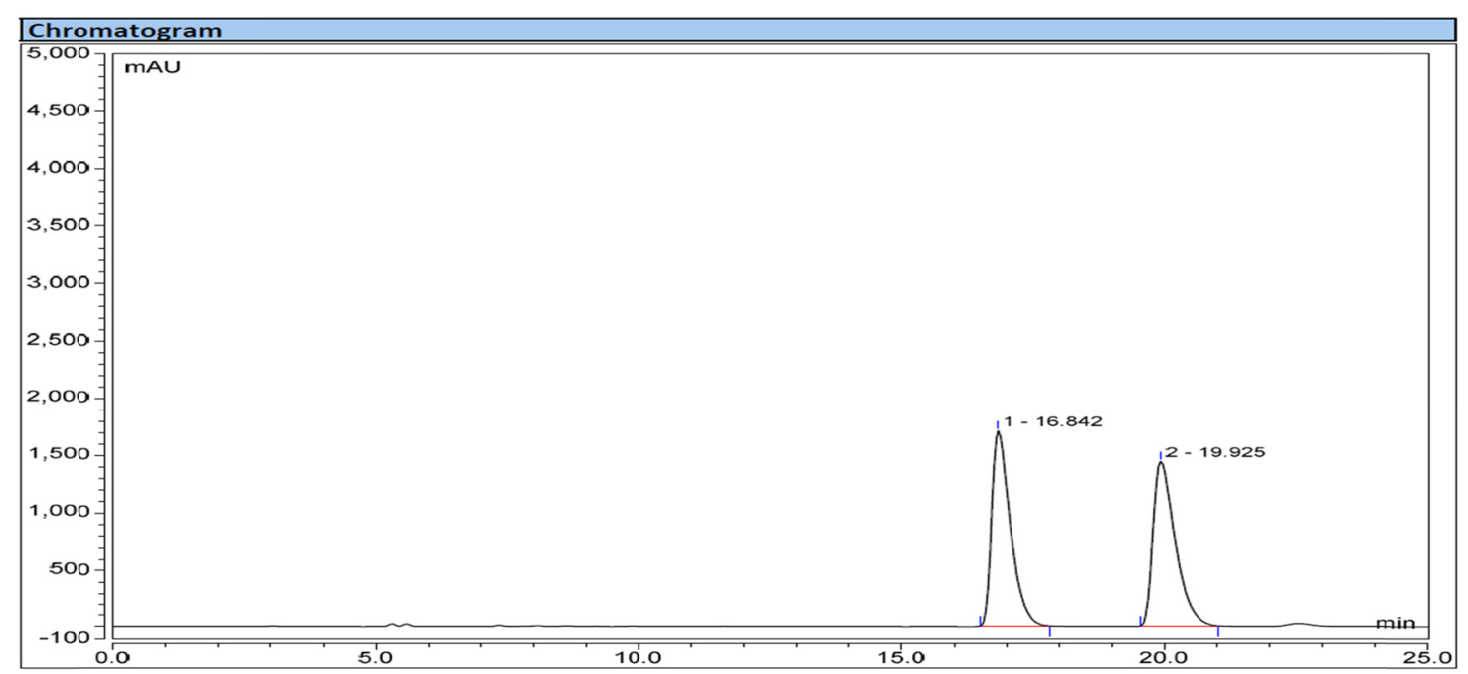

\begin{tabular}{|c|c|c|c|c|c|c|}
\hline \multicolumn{9}{|c|}{ Integration Results } \\
\hline No. & Peak Name & $\begin{array}{c}\text { Retention Time } \\
\text { min }\end{array}$ & $\begin{array}{c}\text { Height } \\
\text { mAU }\end{array}$ & $\begin{array}{c}\text { Area } \\
\text { mAU*min }\end{array}$ & $\begin{array}{c}\text { Relative Height } \\
\%\end{array}$ & $\begin{array}{c}\text { Relative Area } \\
\%\end{array}$ \\
\hline 1 & & 16.842 & 1710.979 & 711.779 & 54.28 & 50.12 \\
2 & & 19.925 & 1441.410 & 708.274 & 45.72 & 49.88 \\
\hline
\end{tabular}




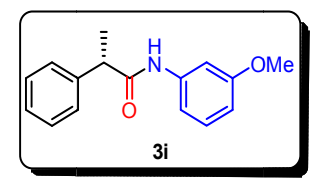

\section{(S)-N-(3-methoxyphenyl)-2-phenylpropanamide}

98\% yield, b/1 > 99:1, ee $=92 \%,[\alpha] \mathrm{D}^{17}=+44.7\left(\mathrm{c}=2.0, \mathrm{CHCl}_{3}\right) .{ }^{1} \mathrm{H}$ NMR $(600$ $\mathrm{MHz}, \mathrm{CDCl}_{3}$ ) $\delta 7.47(\mathrm{~s}, 1 \mathrm{H}), 7.33-7.28(\mathrm{~m}, 5 \mathrm{H}), 7.27-7.22(\mathrm{~m}, 2 \mathrm{H}), 7.11(\mathrm{t}, J=8.4$ $\mathrm{Hz}, 1 \mathrm{H}), 6.85(\mathrm{~d}, J=6.6 \mathrm{~Hz}, 1 \mathrm{H}), 6.60(\mathrm{dd}, J=8.4,1.8 \mathrm{~Hz}, 1 \mathrm{H}), 3.74-3.67(\mathrm{~m}, 4 \mathrm{H})$, $1.55(\mathrm{~d}, J=7.2 \mathrm{~Hz}, 3 \mathrm{H}) ;{ }^{13} \mathrm{C}$ NMR $\left(151 \mathrm{MHz}, \mathrm{CDCl}_{3}\right) \delta 172.6,160.0,140.8,139.1$, 129.4, 129.0, 128.5, 127.5, 111.8, 110.2, 105.4, 55.1, 47.9, 18.5. HRMS calcd. (ESI) $\mathrm{m} / \mathrm{z}$ for $\mathrm{C}_{16} \mathrm{H}_{17} \mathrm{NNaO}_{2}:[\mathrm{M}+\mathrm{Na}]^{+}$278.1152, found: 278.1152 .

Chiral HPLC (Chiralpak IG, 4.6x250 mm; $5 \mu \mathrm{L}$, hexane/isopropanol = 90/10, flow $1.0 \mathrm{~mL} / \mathrm{min}, 254 \mathrm{~nm}$ ): $\mathrm{t}_{\mathrm{R}}=15.14 \mathrm{~min}$ (major), $\mathrm{t}_{\mathrm{R}}=18.91 \mathrm{~min}$ (minor).

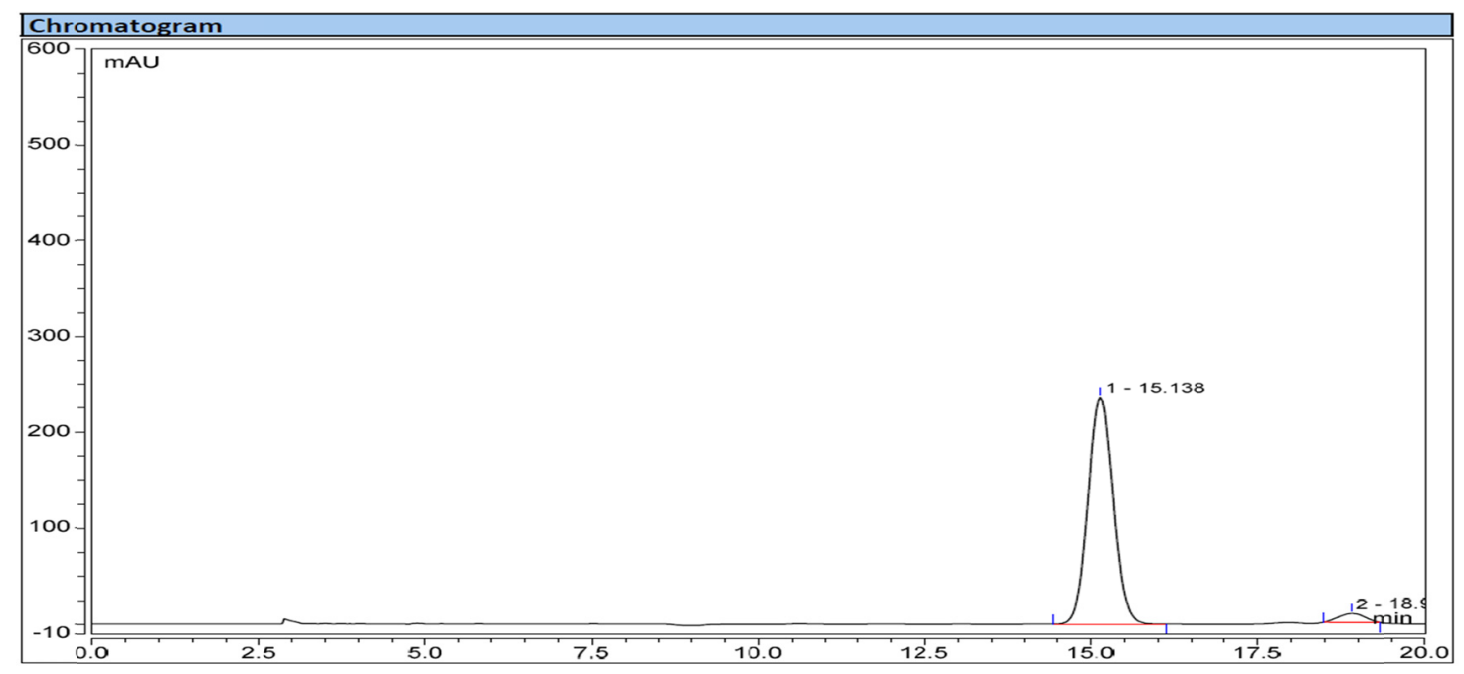

\begin{tabular}{|c|c|c|c|c|c|c|}
\hline \multicolumn{9}{|l|}{ Integration Results } \\
\hline No. & Peak Name & $\begin{array}{c}\text { Retention Time } \\
\text { min }\end{array}$ & $\begin{array}{c}\text { Height } \\
\text { mAU }\end{array}$ & $\begin{array}{c}\text { Area } \\
\text { mAU*min }\end{array}$ & $\begin{array}{c}\text { Relative Height } \\
\%\end{array}$ & $\begin{array}{c}\text { Relative Area } \\
\%\end{array}$ \\
\hline 1 & & 15.138 & 236.746 & 102.483 & 96.17 & 95.98 \\
2 & & 18.907 & 9.439 & 4.291 & 3.83 & 4.02 \\
\hline
\end{tabular}

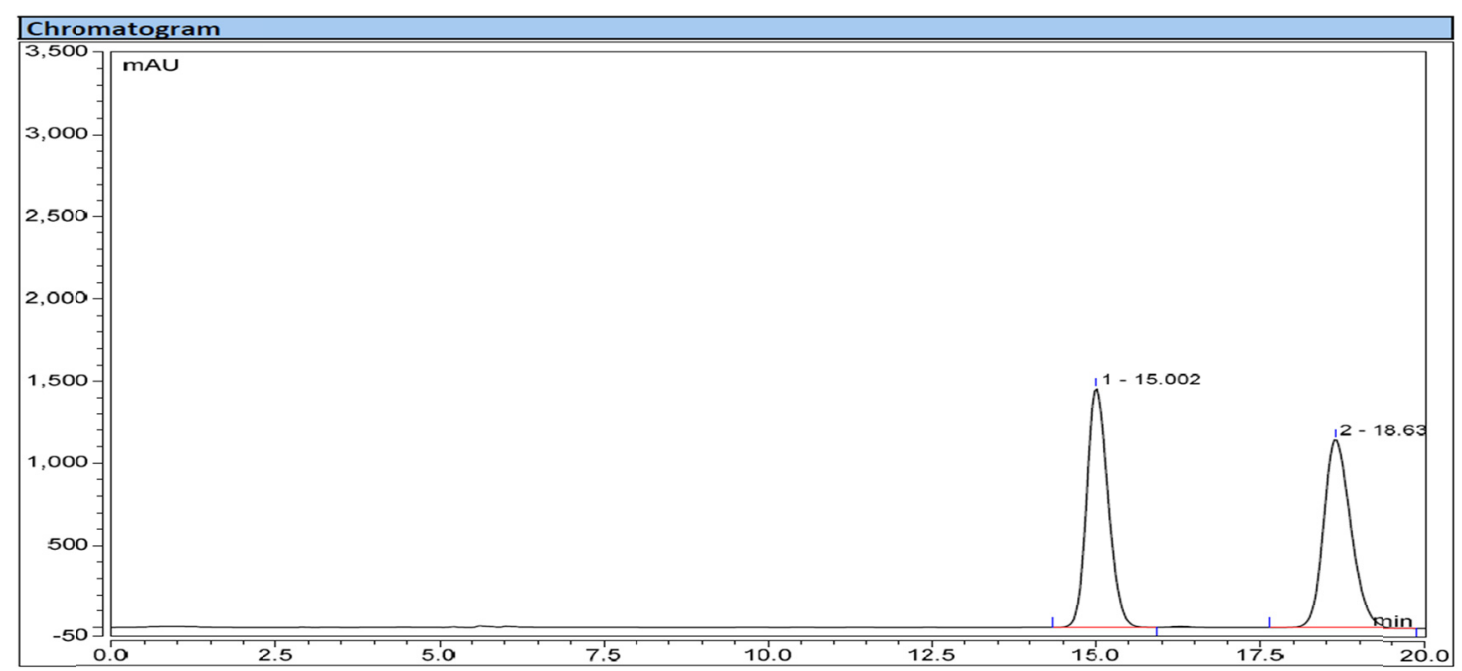

\begin{tabular}{|c|c|c|c|c|c|c|}
\hline \multicolumn{9}{|l|}{ Integration Results } \\
\hline No. & Peak Name & $\begin{array}{c}\text { Retention Time } \\
\text { min }\end{array}$ & $\begin{array}{c}\text { Height } \\
\text { mAU }\end{array}$ & $\begin{array}{c}\text { Area } \\
\text { mAU*min }\end{array}$ & $\begin{array}{c}\text { Relative Height } \\
\%\end{array}$ & $\begin{array}{c}\text { Relative Area } \\
\%\end{array}$ \\
\hline 1 & & 15.002 & 1457.977 & 546.991 & 56.03 & 49.98 \\
2 & & 18.635 & 1144.342 & 547.504 & 43.97 & 50.02 \\
\hline
\end{tabular}




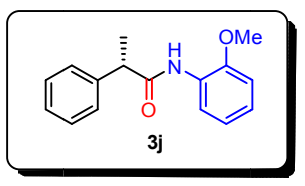

\section{(S)-N-(2-methoxyphenyl)-2-phenylpropanamide}

95\% yield, $\mathrm{b} / \mathrm{l}>99: 1, \mathrm{ee}=91 \%,[\alpha] \mathrm{D}^{17}=+26.8\left(\mathrm{c}=1.8, \mathrm{CHCl}_{3}\right) .{ }^{1} \mathrm{H}$ NMR $(600$ $\left.\mathrm{MHz}, \mathrm{CDCl}_{3}\right) \delta 8.35(\mathrm{~d}, J=7.8 \mathrm{~Hz}, 1 \mathrm{H}), 7.77(\mathrm{~s}, 1 \mathrm{H}), 7.37-7.34(\mathrm{~m}, 3 \mathrm{H}), 7.31-7.27$ (m, 2H), 6.97 (t, $J=7.8 \mathrm{~Hz}, 1 \mathrm{H}), 6.91(\mathrm{t}, J=7.8 \mathrm{~Hz}, 1 \mathrm{H}), 6.77(\mathrm{~d}, J=8.4 \mathrm{~Hz}, 1 \mathrm{H})$, $3.74(\mathrm{q}, J=7.2 \mathrm{~Hz}, 1 \mathrm{H}), 3.69(\mathrm{~d}, J=1.8 \mathrm{~Hz}, 3 \mathrm{H}), 1.61(\mathrm{~d}, J=7.2 \mathrm{~Hz}, 3 \mathrm{H}) ;{ }^{13} \mathrm{C}$ NMR $\left(151 \mathrm{MHz}, \mathrm{CDCl}_{3}\right) \delta 172.1,147.8,140.9,128.9,128.5,127.7,127.3,123.5,121.0$, 119.4, 109.9, 55.6, 48.3, 18.3. HRMS calcd. (ESI) $\mathrm{m} / \mathrm{z}$ for $\mathrm{C}_{16} \mathrm{H}_{17} \mathrm{NNaO}_{2}:[\mathrm{M}+\mathrm{Na}]^{+}$: 278.1152, found: 278.1149 .

Chiral HPLC (Chiralpak IG, 4.6x250 mm; $5 \mu \mathrm{L}$, hexane/isopropanol = 90/10, flow $1.0 \mathrm{~mL} / \mathrm{min}, 254 \mathrm{~nm}$ ): $\mathrm{t}_{\mathrm{R}}=16.06 \mathrm{~min}$ (major), $\mathrm{t}_{\mathrm{R}}=18.60 \mathrm{~min}$ (minor).
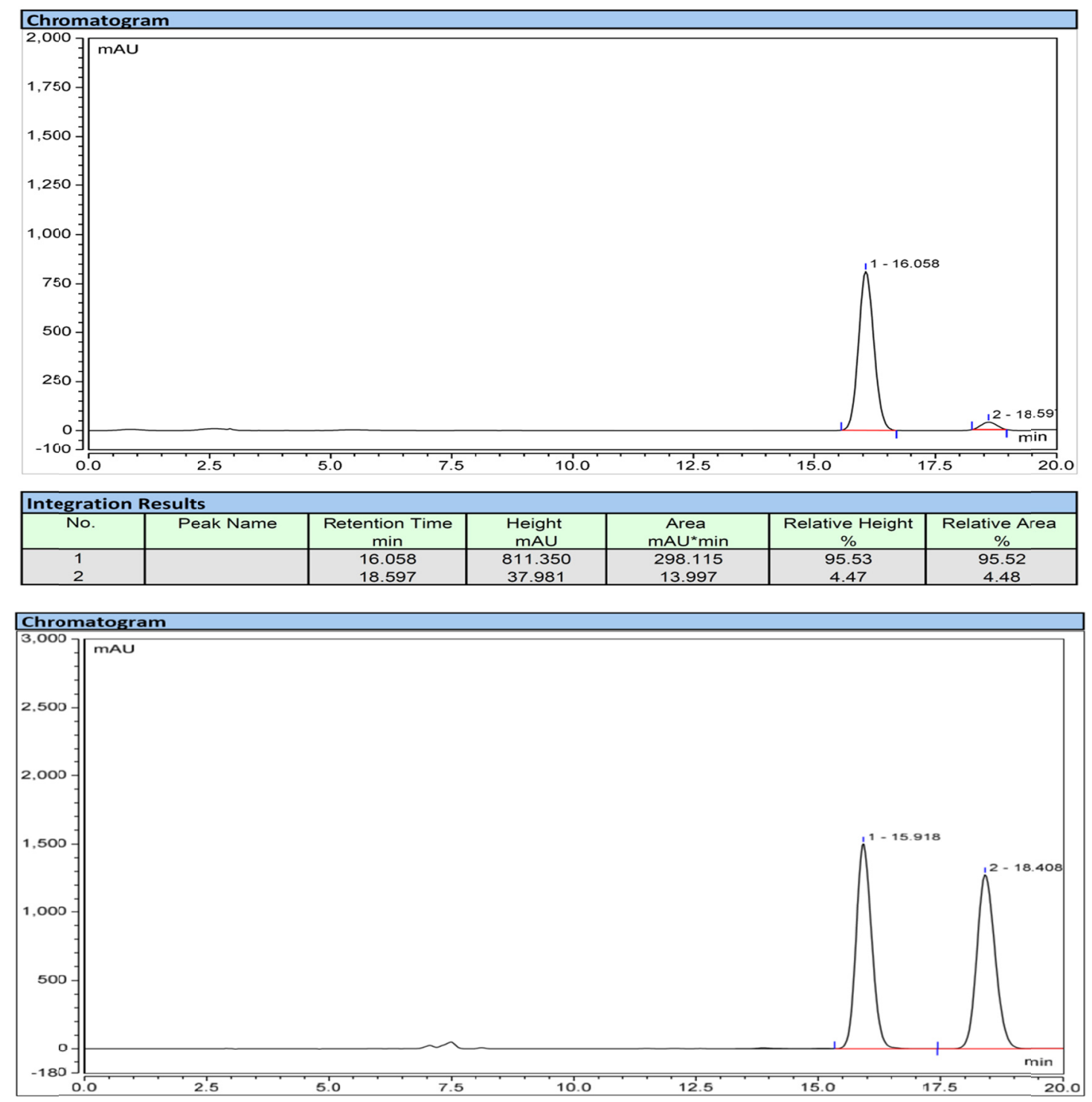

\begin{tabular}{|c|c|c|c|c|c|c|}
\hline \multicolumn{9}{|l|}{ Integration Results } \\
\hline No. & Peak Name & $\begin{array}{c}\text { Retention Time } \\
\text { min }\end{array}$ & $\begin{array}{c}\text { Height } \\
\text { mAU }\end{array}$ & $\begin{array}{c}\text { Area } \\
\text { mAU*min }\end{array}$ & $\begin{array}{c}\text { Relative Height } \\
\%\end{array}$ & $\begin{array}{c}\text { Relative Area } \\
\%\end{array}$ \\
\hline 1 & & 15.918 & 1502.009 & 550.075 & 54.04 & 50.02 \\
2 & & 18.408 & 1277.547 & 549.613 & 45.96 & 49.98 \\
\hline
\end{tabular}




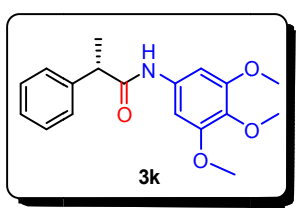

\section{(S)-2-phenyl- $N$-(3,4,5-trimethoxyphenyl)propanamide}

$87 \%$ yield, $\mathrm{b} / 1>99: 1, \mathrm{ee}=93 \%,[\alpha] \mathrm{D}^{16}=+45.6\left(\mathrm{c}=1.9, \mathrm{CHCl}_{3}\right) .{ }^{1} \mathrm{H}$ NMR $(400$ $\left.\mathrm{MHz}, \mathrm{CDCl}_{3}\right) \delta$ 7.40-7.35 (m, 4H), 7.33-7.30 (m, 1H), $7.12(\mathrm{~s}, 1 \mathrm{H}), 6.76(\mathrm{~d}, J=2.0$ $\mathrm{Hz}, 2 \mathrm{H}), 3.79-3.78(\mathrm{~m}, 9 \mathrm{H}), 3.70(\mathrm{q}, J=7.2 \mathrm{~Hz}, 1 \mathrm{H}), 1.59(\mathrm{~d}, J=7.2 \mathrm{~Hz}, 3 \mathrm{H}) ;{ }^{13} \mathrm{C}$ NMR $\left(101 \mathrm{MHz}, \mathrm{CDCl}_{3}\right) \delta 172.3,153.2,140.8,134.5,134.1,129.1,127.6,127.5$, 97.2, 60.9, 56.0, 48.1, 18.6. HRMS calcd. (ESI) $\mathrm{m} / \mathrm{z}$ for $\mathrm{C}_{18} \mathrm{H}_{21} \mathrm{NNaO}_{4}:[\mathrm{M}+\mathrm{Na}]^{+}$ 338.1363, found: 338.1367 .

Chiral HPLC (Chiralpak IG, 4.6x250 mm; $5 \mu \mathrm{L}$, hexane/isopropanol $=85 / 15$, flow $1.0 \mathrm{~mL} / \mathrm{min}, 254 \mathrm{~nm}$ ): $\mathrm{t}_{\mathrm{R}}=15.33 \mathrm{~min}$ (major) , $\mathrm{t}_{\mathrm{R}}=17.35 \mathrm{~min}$ (minor)

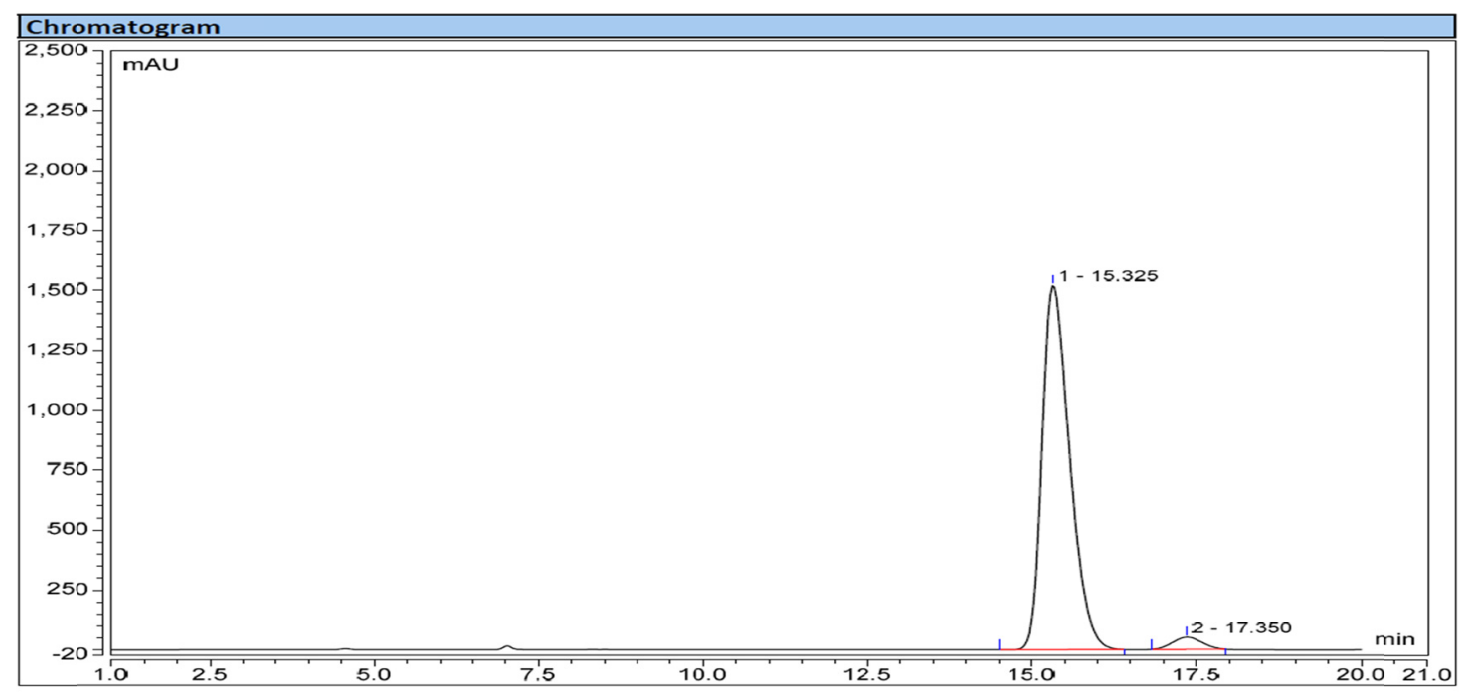

\begin{tabular}{|c|c|c|c|c|c|c|}
\hline \multicolumn{9}{|l|}{ Integration Results } \\
\hline No. & Peak Name & $\begin{array}{c}\text { Retention Time } \\
\text { min }\end{array}$ & $\begin{array}{c}\text { Height } \\
\text { mAU }\end{array}$ & $\begin{array}{c}\text { Area } \\
\text { mAU*min }\end{array}$ & $\begin{array}{c}\text { Relative Height } \\
\%\end{array}$ & $\begin{array}{c}\text { Relative Area } \\
\%\end{array}$ \\
\hline 1 & & 15.325 & 1520.144 & 735.932 & 96.76 & 96.57 \\
2 & & 17.350 & 50.867 & 26.122 & 3.24 & 3.43 \\
\hline
\end{tabular}

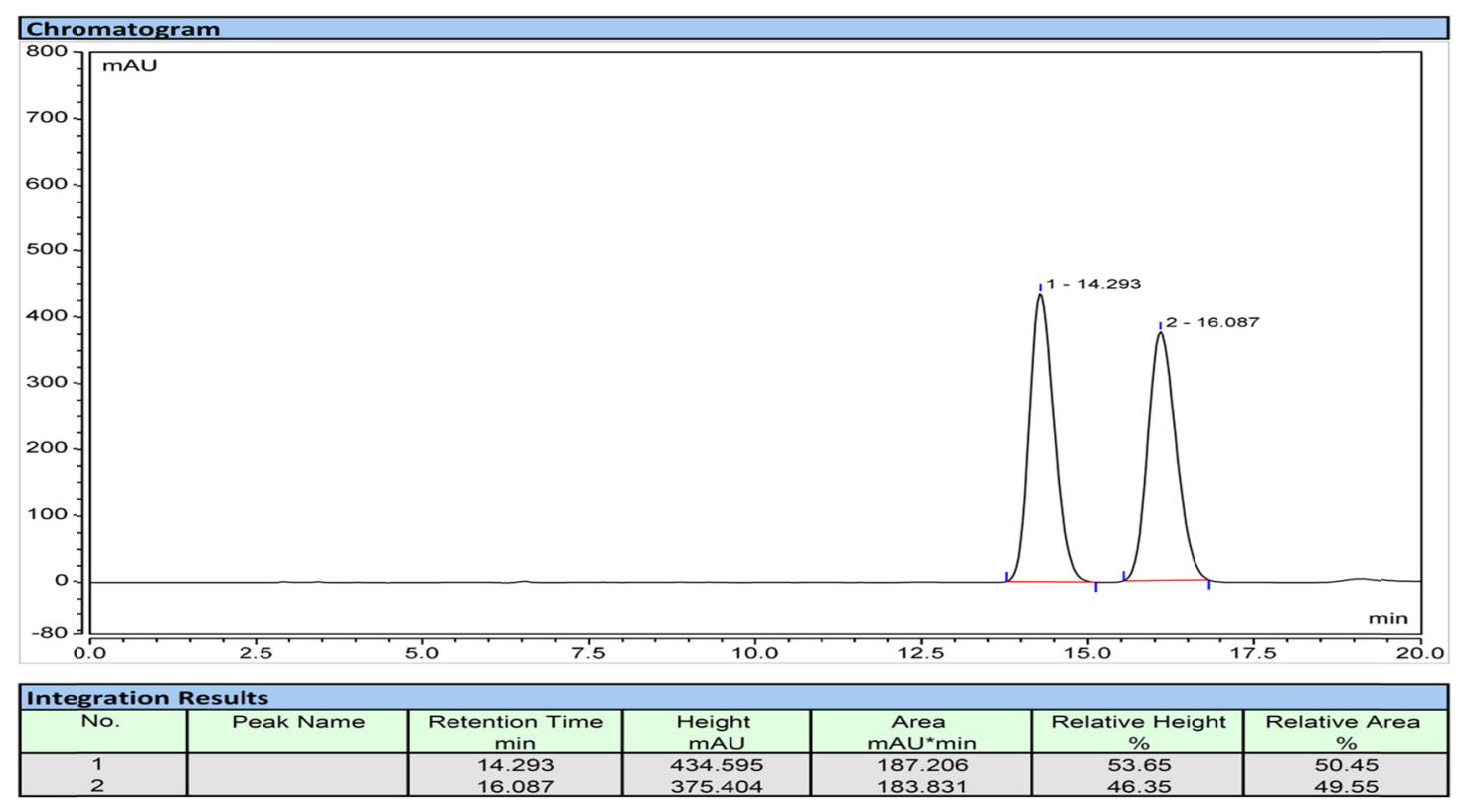




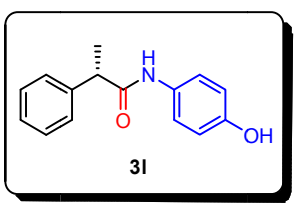

\section{(S)-N-(4-hydroxyphenyl)-2-phenylpropanamide}

$72 \%$ yield, $\mathrm{b} / \mathrm{l}>99: 1, \mathrm{ee}=88 \%,[\alpha] \mathrm{D}^{18}=-106.8\left(\mathrm{c}=0.6, \mathrm{CHCl}_{3}\right) .{ }^{1} \mathrm{H}$ NMR $(400$ $\mathrm{MHz}, d^{6}$-DMSO) $\delta 9.78(\mathrm{~s}, 1 \mathrm{H}), 9.15(\mathrm{~s}, 1 \mathrm{H}), 7.38-7.29(\mathrm{~m}, 6 \mathrm{H}), 7.22(\mathrm{t}, J=7.2 \mathrm{~Hz}$, $1 \mathrm{H}), 6.65(\mathrm{~d}, J=8.4 \mathrm{~Hz}, 2 \mathrm{H}), 3.76(\mathrm{q}, J=7.2 \mathrm{~Hz}, 1 \mathrm{H}), 1.39(\mathrm{~d}, \mathrm{~J}=7.2 \mathrm{~Hz}, 3 \mathrm{H}) ;{ }^{13} \mathrm{C}$ NMR (101 MHz, $d^{6}$-DMSO) $\delta 171.4,153.2,142.1,130.9,128.3,127.2,126.6,120.8$, 115.0, 45.7, 18.7. HRMS calcd. (ESI) $\mathrm{m} / \mathrm{z}$ for $\mathrm{C}_{15} \mathrm{H}_{15} \mathrm{NNaO}_{2}$ : $[\mathrm{M}+\mathrm{Na}]^{+}$264.0995, found: 264.0997.

Chiral HPLC (Chiralpak IB N-5, 4.6x250 mm; $5 \mu \mathrm{L}$, hexane/isopropanol $=85 / 15$, flow $1.0 \mathrm{~mL} / \mathrm{min}, 254 \mathrm{~nm}$ ): $\mathrm{t}_{\mathrm{R}}=14.47$ (major), $\mathrm{t}_{\mathrm{R}}=20.60 \mathrm{~min}$ (minor).
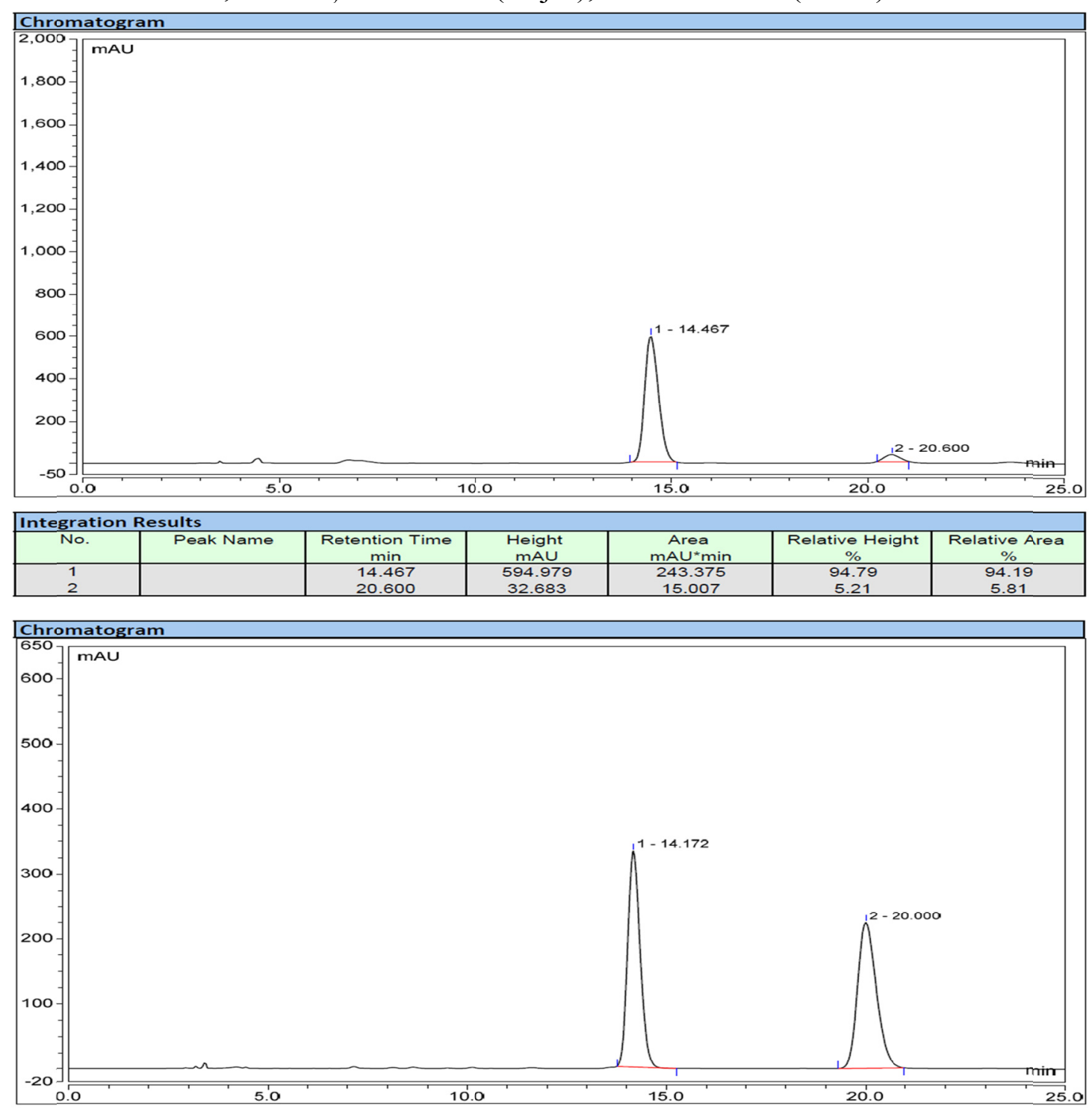

\begin{tabular}{|c|c|c|c|c|c|c|}
\hline \multicolumn{9}{|l|}{ Integration Results } \\
\hline No. & Peak Name & $\begin{array}{c}\text { Retention Time } \\
\text { min }\end{array}$ & $\begin{array}{c}\text { Height } \\
\text { mAU }\end{array}$ & $\begin{array}{c}\text { Area } \\
\text { mAU*min }\end{array}$ & $\begin{array}{c}\text { Relative Height } \\
\%\end{array}$ & $\begin{array}{c}\text { Relative Area } \\
\%\end{array}$ \\
\hline 1 & & 14.172 & 333.226 & 120.987 & 59.78 & 49.70 \\
2 & & 20.000 & 224.191 & 122.425 & 40.22 & 50.30 \\
\hline
\end{tabular}




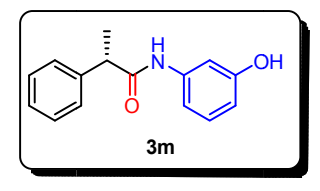

\section{(S)-N-(3-hydroxyphenyl)-2-phenylpropanamide}

$91 \%$ yield, $\mathrm{b} / \mathrm{l}>99: 1, \mathrm{ee}=94 \%,[\alpha] \mathrm{D}^{19}=+15.5\left(\mathrm{c}=1.1, \mathrm{CHCl}_{3}\right) .{ }^{1} \mathrm{H} \mathrm{NMR}(400$ $\left.\mathrm{MHz}, \mathrm{CDCl}_{3}\right) \delta 7.87(\mathrm{~s}, 1 \mathrm{H}), 7.53-7.16(\mathrm{~m}, 6 \mathrm{H}), 7.05(\mathrm{t}, J=8.0 \mathrm{~Hz}, 1 \mathrm{H}), 6.63(\mathrm{~d}, J=$ $8.0 \mathrm{~Hz}, 1 \mathrm{H}), 6.36(\mathrm{~d}, J=8.0 \mathrm{~Hz}, 1 \mathrm{H}), 3.74(\mathrm{q}, J=7.2 \mathrm{~Hz}, 1 \mathrm{H}), 1.60(\mathrm{~d}, J=7.2 \mathrm{~Hz}$, $3 \mathrm{H}) ;{ }^{13} \mathrm{C}$ NMR $\left(101 \mathrm{MHz}, \mathrm{CDCl}_{3}\right) \delta 173.8,157.4,140.1,138.3,129.6,129.2,127.7$, 127.6, 112.1, 110.7, 107.4, 48.1, 18.2. HRMS calcd. (ESI) $\mathrm{m} / \mathrm{z}$ for $\mathrm{C}_{15} \mathrm{H}_{15} \mathrm{NNaO}_{2}$ : $[\mathrm{M}+\mathrm{Na}]^{+}$264.0995, found: 264.0994 .

Chiral HPLC (Chiralpak IG, 4.6x250 mm; $5 \mu \mathrm{L}$, hexane/isopropanol $=85 / 15$, flow $1.0 \mathrm{~mL} / \mathrm{min}, 254 \mathrm{~nm}$ ): $\mathrm{t}_{\mathrm{R}}=14.33 \mathrm{~min}$ (major), $\mathrm{t}_{\mathrm{R}}=17.41 \mathrm{~min}$ (minor).

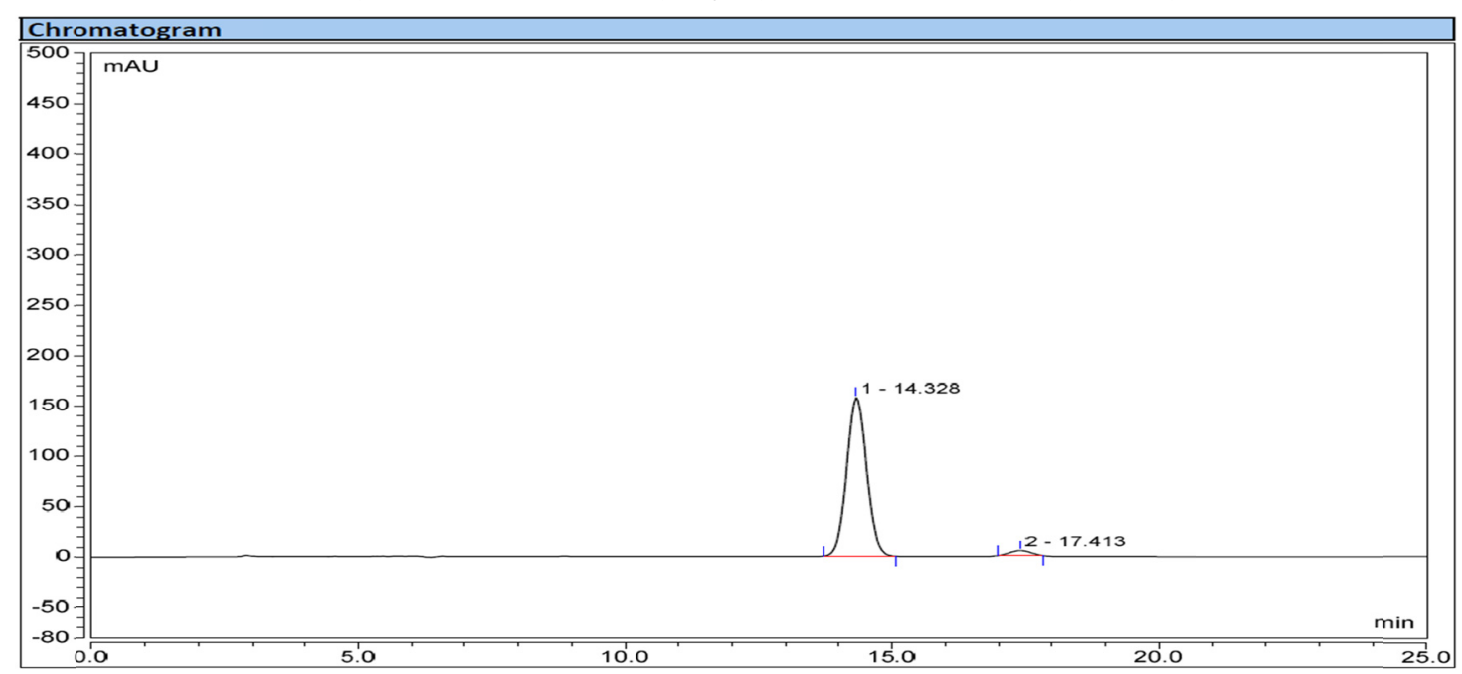

\begin{tabular}{|c|c|c|c|c|c|c|}
\hline Integration Results & \multicolumn{9}{|c|}{ Relative Height } & $\begin{array}{c}\text { Relative Area } \\
\%\end{array}$ \\
\hline No. & Peak Name & $\begin{array}{c}\text { Retention Time } \\
\text { min }\end{array}$ & $\begin{array}{c}\text { Height } \\
\text { mAU }\end{array}$ & $\begin{array}{c}\text { Area } \\
\text { mAU*min }\end{array}$ & $\begin{array}{c}\text { Relat } \\
\%\end{array}$ \\
\hline 1 & & 14.328 & 157.314 & 68.521 & 96.89 & 96.75 \\
2 & & 17.413 & 5.043 & 2.302 & 3.11 & 3.25 \\
\hline
\end{tabular}

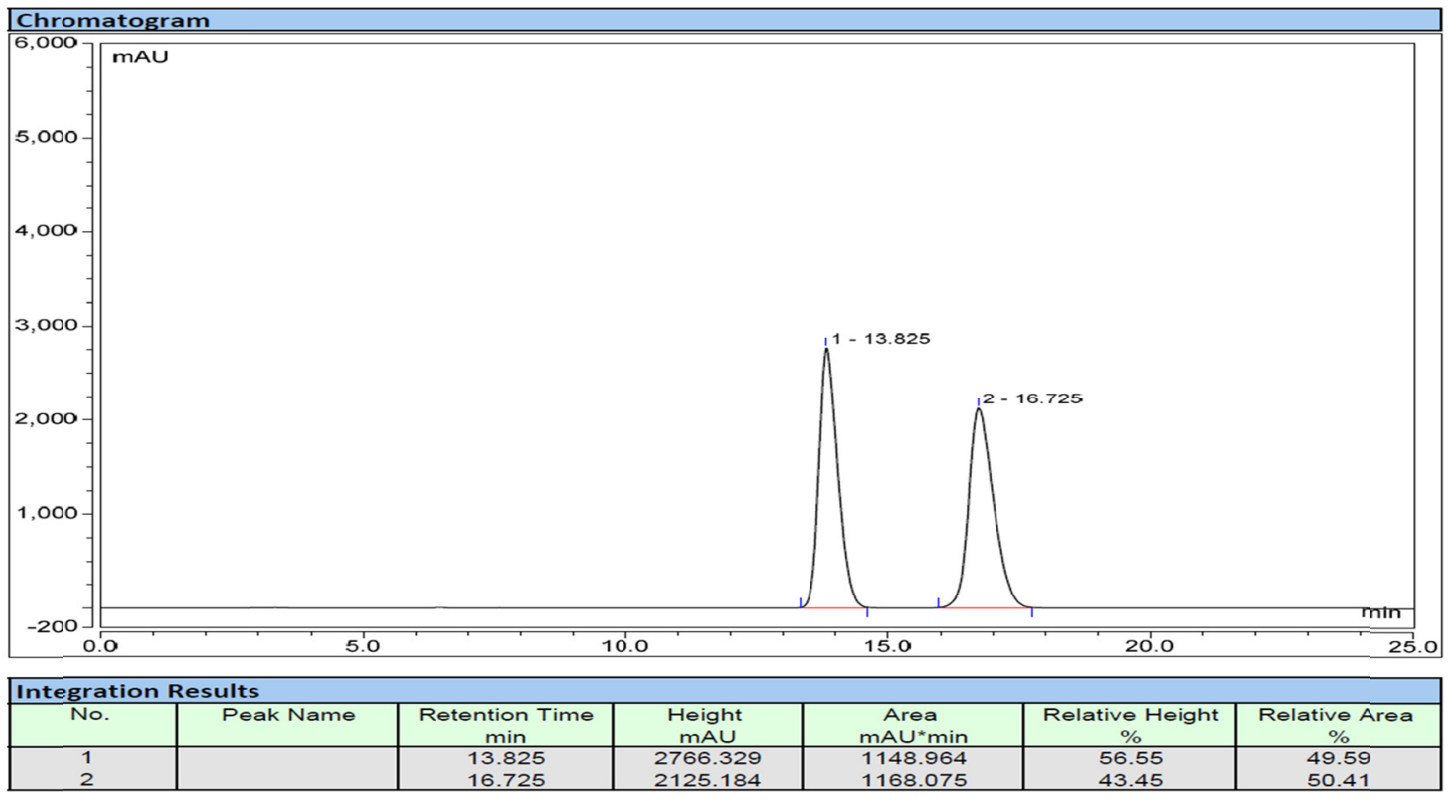




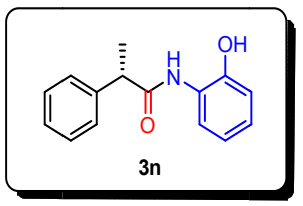

\section{(S)-N-(2-hydroxyphenyl)-2-phenylpropanamide}

$85 \%$ yield, $\mathrm{b} / \mathrm{l}>99: 1$, ee $=95 \%$ ee, $[\alpha] \mathrm{D}^{18}=+8.9\left(\mathrm{c}=4.6, \mathrm{CHCl}_{3}\right) .{ }^{1} \mathrm{H} \mathrm{NMR}(400$ $\mathrm{MHz}, d^{6}$-DMSO) $\delta 9.78(\mathrm{~s}, 1 \mathrm{H}), 9.16(\mathrm{~s}, 1 \mathrm{H}), 7.46-7.28(\mathrm{~m}, 6 \mathrm{H}), 7.22-7.20(\mathrm{~m}, 1 \mathrm{H})$, $6.65(\mathrm{dd}, J=8.8,2.0 \mathrm{~Hz}, 2 \mathrm{H}), 3.75(\mathrm{q}, J=6.8 \mathrm{~Hz}, 1 \mathrm{H}), 1.38(\mathrm{~d}, J=6.8 \mathrm{~Hz}, 3 \mathrm{H}) ;{ }^{13} \mathrm{C}$ NMR (100 MHz, $d^{6}$-DMSO) $\delta 171.4,153.2,142.1,130.9,128.3,127.2,126.6,120.9$, 115.0, 45.7, 18.6. HRMS calcd. (ESI) $\mathrm{m} / \mathrm{z}$ for $\mathrm{C}_{15} \mathrm{H}_{15} \mathrm{NNaO}_{2}$ : $[\mathrm{M}+\mathrm{Na}]^{+}$264.0995, found: 264.0999 .

Chiral HPLC (Chiralpak IB N-5, 4.6x250 mm; $5 \mu \mathrm{L}$, hexane/isopropanol = 85/15, flow $1.0 \mathrm{~mL} / \mathrm{min}, 254 \mathrm{~nm}$ ): $\mathrm{t}_{\mathrm{R}}=6.93 \mathrm{~min}$ (major), $\mathrm{t}_{\mathrm{R}}=9.55 \mathrm{~min}$ (minor).

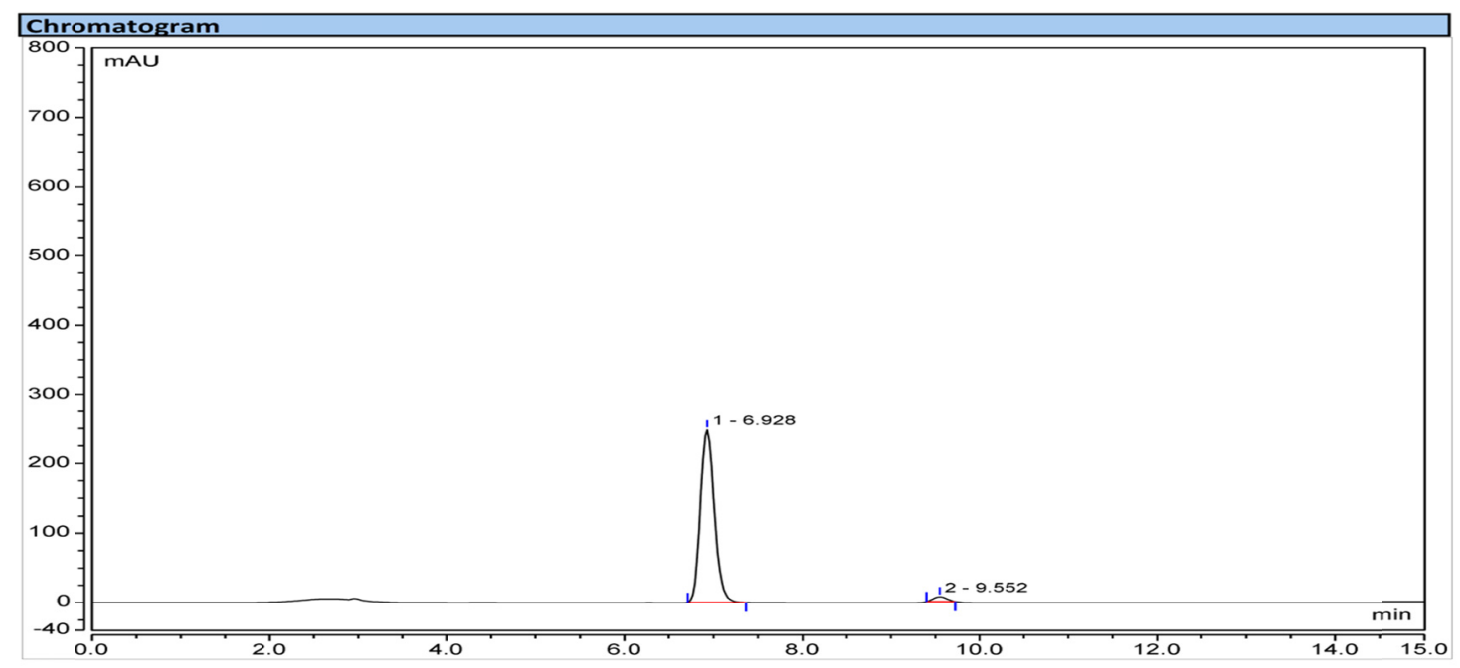

\begin{tabular}{|c|c|c|c|c|c|c|}
\hline Integration Results & \multicolumn{3}{c|}{$\begin{array}{c}\text { Relative Height } \\
\%\end{array}$} & $\begin{array}{c}\text { Relative Area } \\
\%\end{array}$ \\
\hline No. & Peak Name & $\begin{array}{c}\text { Retention Time } \\
\text { min }\end{array}$ & $\begin{array}{c}\text { Height } \\
\text { mAU }\end{array}$ & $\begin{array}{c}\text { Area } \\
\text { mAU*min }\end{array}$ & $\begin{array}{c}\text { Rel } \\
\%\end{array}$ \\
\hline 1 & & 6.928 & 247.939 & 45.166 & 97.26 & 97.31 \\
2 & & 9.552 & 6.977 & 1.248 & 2.74 & 2.69 \\
\hline
\end{tabular}

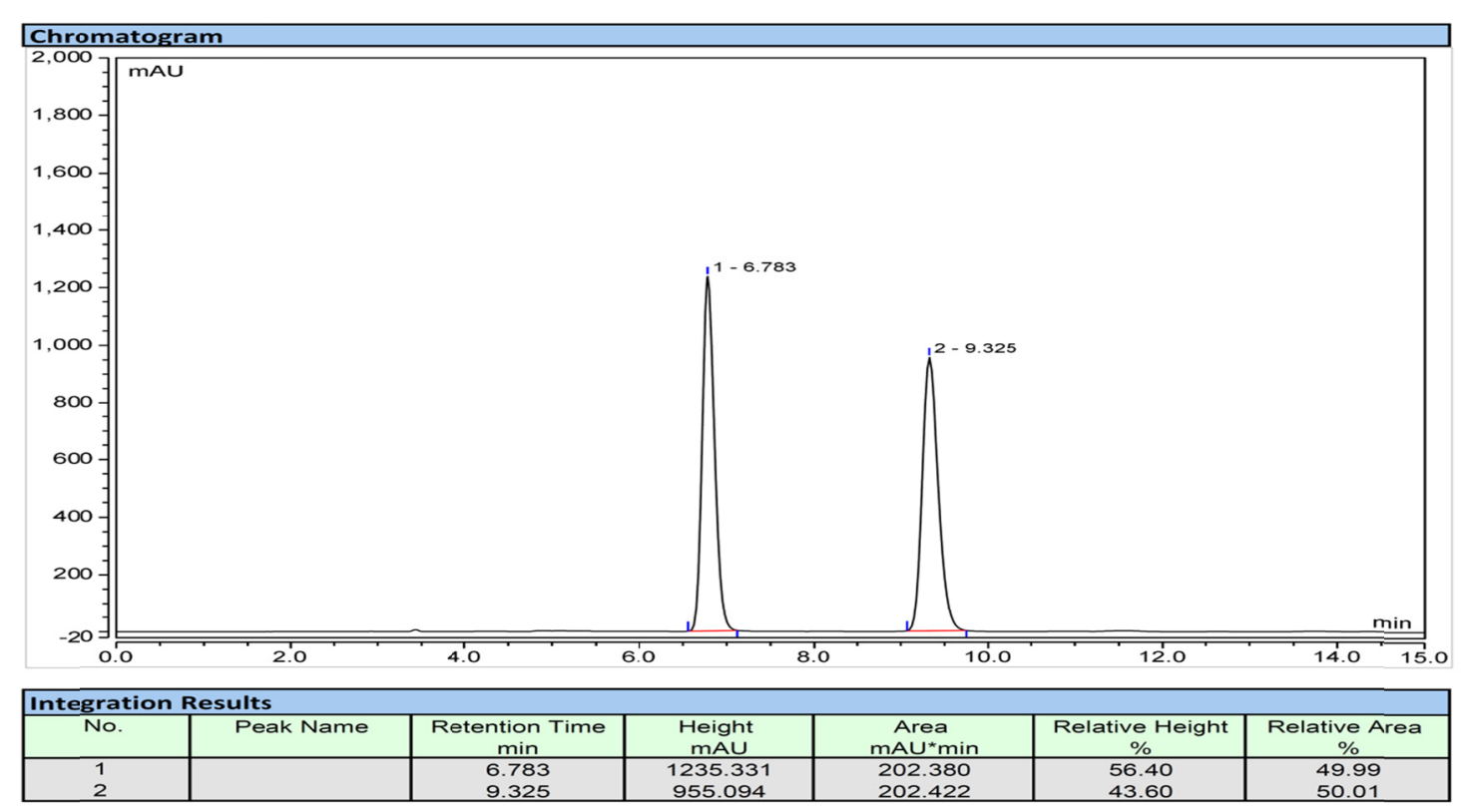




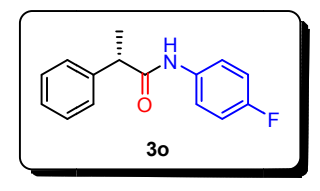

\section{(S)- $N$-(4-fluorophenyl)-2-phenylpropanamide}

$92 \%$ yield, $\mathrm{b} / \mathrm{l}>99: 1$, ee $=90 \%,[\alpha] \mathrm{D}^{18}=+48.6\left(\mathrm{c}=1.2, \mathrm{CHCl}_{3}\right) .{ }^{1} \mathrm{H}$ NMR $(600$ $\left.\mathrm{MHz}, \mathrm{CDCl}_{3}\right) \delta$ 7.44-7.33 (m, 6H), 7.32-7.27 (m, 1H), $6.93(\mathrm{t}, J=8.4 \mathrm{~Hz}, 2 \mathrm{H}), 3.70$ $(\mathrm{q}, J=7.2 \mathrm{~Hz}, 1 \mathrm{H}), 1.58(\mathrm{~d}, J=7.2 \mathrm{~Hz}, 3 \mathrm{H}) ;{ }^{13} \mathrm{C}$ NMR $\left(151 \mathrm{MHz}, \mathrm{CDCl}_{3}\right) \delta 172.4$, $159.3(\mathrm{~d}, J=242.0 \mathrm{~Hz}), 140.8,133.8(\mathrm{~d}, J=2.6 \mathrm{~Hz}), 129.1,127.6,127.6,121.7$ (d, $J=$ $7.6 \mathrm{~Hz}), 115.4(\mathrm{~d}, J=22.5 \mathrm{~Hz}), 47.9,18.5 ;{ }^{19} \mathrm{~F}$ NMR $\left(376 \mathrm{MHz}, \mathrm{CDCl}_{3}\right) \delta-117.73$. HRMS calcd. (ESI) m/z for $\mathrm{C}_{15} \mathrm{H}_{14} \mathrm{FNNaO}$ : [M+Na] ${ }^{+} 266.0952$, found: 266.0952 .

Chiral HPLC (Chiralpak IG, 4.6x250 mm; $5 \mu \mathrm{L}$, hexane/isopropanol $=90 / 10$, flow $1.0 \mathrm{~mL} / \mathrm{min}, 254 \mathrm{~nm}$ ): $t_{\mathrm{R}}=10.11$ (major), $\mathrm{t}_{\mathrm{R}}=11.62 \mathrm{~min}$ (minor).

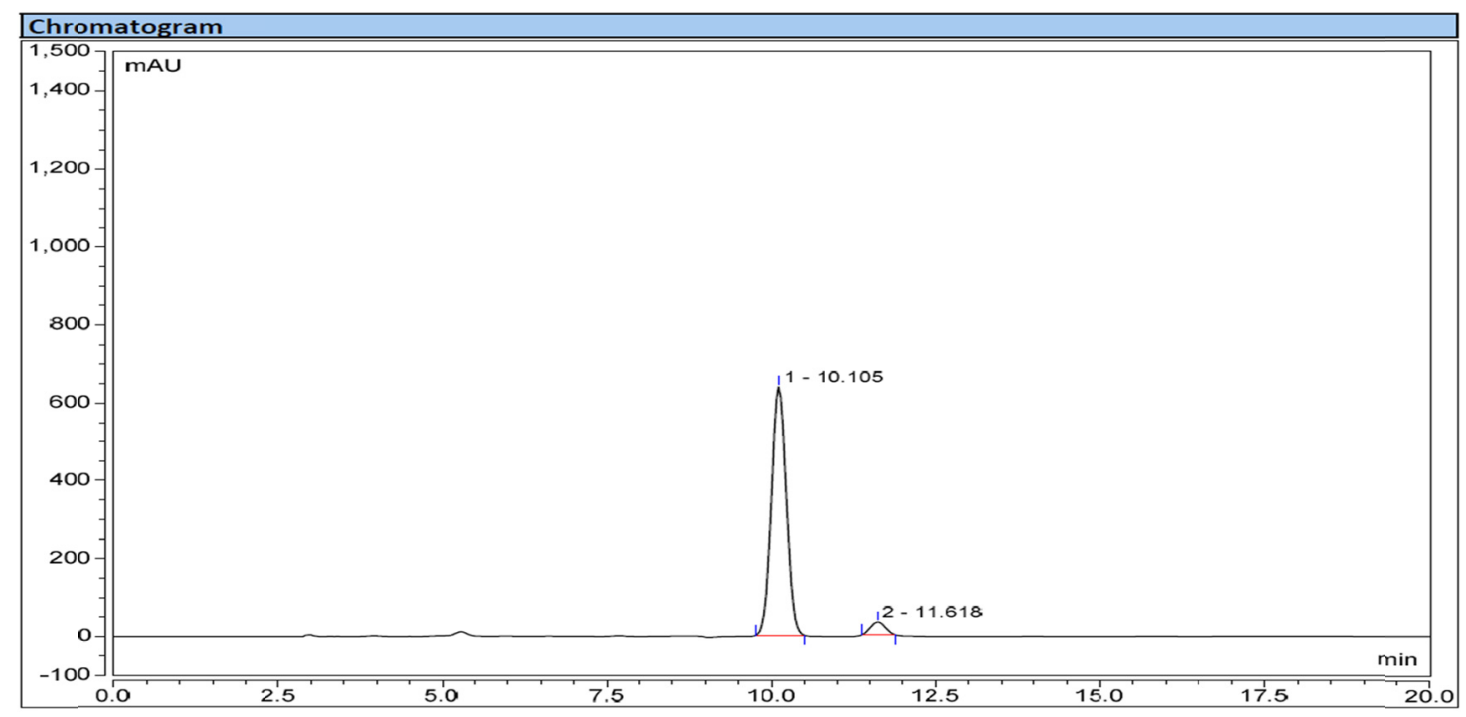

\begin{tabular}{|c|c|c|c|c|c|c|}
\hline \multicolumn{9}{|l|}{ Integration Results } \\
\hline No. & Peak Name & $\begin{array}{c}\text { Retention Time } \\
\text { min }\end{array}$ & $\begin{array}{c}\text { Height } \\
\text { mAU }\end{array}$ & $\begin{array}{c}\text { Area } \\
\text { mAU*min }\end{array}$ & $\begin{array}{c}\text { Relative Height } \\
\%\end{array}$ & $\begin{array}{c}\text { Relative Area } \\
\%\end{array}$ \\
\hline 1 & & 10.105 & 638.696 & 166.605 & 95.05 & 95 \\
2 & & 11.618 & 33.238 & 8.658 & 4.95 & 4.94 \\
\hline
\end{tabular}

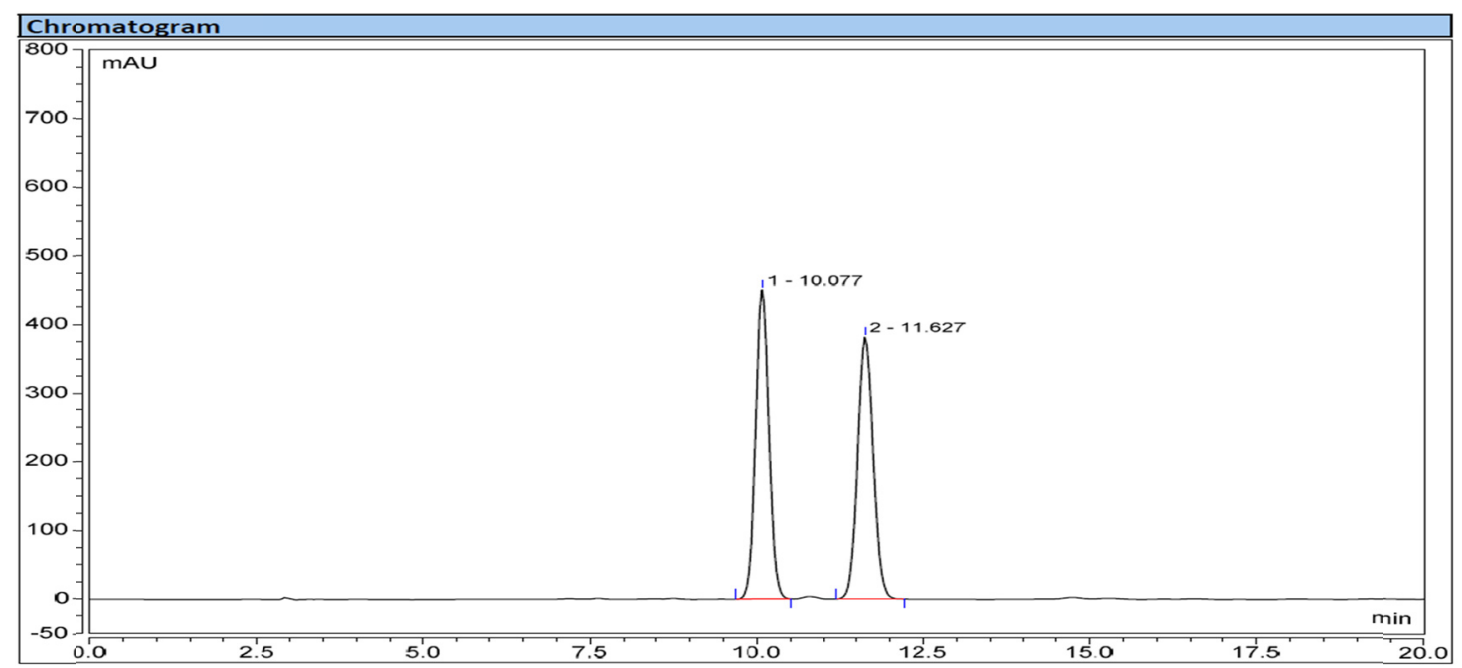

\begin{tabular}{|c|c|c|c|c|c|c|}
\hline \multicolumn{9}{|c|}{ Integration Results } \\
\hline No. & Peak Name & $\begin{array}{c}\text { Retention Time } \\
\text { min }\end{array}$ & $\begin{array}{c}\text { Height } \\
\text { mAU }\end{array}$ & $\begin{array}{c}\text { Area } \\
\text { mAU*min }\end{array}$ & $\begin{array}{c}\text { Relative Height } \\
\%\end{array}$ & $\begin{array}{c}\text { Relative Area } \\
\%\end{array}$ \\
\hline 1 & & 10.077 & 449.610 & 103.816 & 54.07 & 49.97 \\
2 & & 11.627 & 381.932 & 103.930 & 45.93 & 50.03 \\
\hline
\end{tabular}




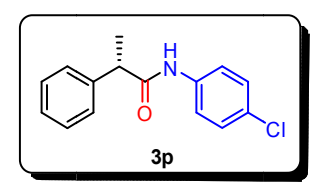

\section{(S)-N-(4-chlorophenyl)-2-phenylpropanamide}

$86 \%$ yield, b/1 $>99: 1$, ee $=92 \%,[\alpha] \mathrm{D}^{19}=+36.5\left(\mathrm{c}=2.0, \mathrm{CHCl}_{3}\right) .{ }^{1} \mathrm{H}$ NMR $(400$ $\left.\mathrm{MHz}, \mathrm{CDCl}_{3}\right) \delta$ 7.47-7.29 (m, 7H), 7.02-6.90 (m, 3H), $3.71(\mathrm{q}, J=7.2 \mathrm{~Hz}, 1 \mathrm{H}), 1.60$ $(\mathrm{d}, J=7.2 \mathrm{~Hz}, 3 \mathrm{H}) .{ }^{13} \mathrm{C} \mathrm{NMR}\left(101 \mathrm{MHz}, \mathrm{CDCl}_{3}\right) \delta 172.8,140.6,136.3,129.1,128.9$, 128.7, 127.5, 127.4, 121.3, 47.6, 18.4. HRMS calcd. (ESI) $\mathrm{m} / \mathrm{z}$ for $\mathrm{C}_{15} \mathrm{H}_{14} \mathrm{ClNNaO}$ : $[\mathrm{M}+\mathrm{Na}]^{+} 282.0656$, found: 282.0662 .

Chiral HPLC (Chiralpak IG, 4.6x250 mm; $5 \mu \mathrm{L}$, hexane/isopropanol $=90 / 10$, flow $1.0 \mathrm{~mL} / \mathrm{min}, 254 \mathrm{~nm}$ ): $\mathrm{t}_{\mathrm{R}}=10.93$ (major), $\mathrm{t}_{\mathrm{R}}=14.40 \mathrm{~min}$ (minor).

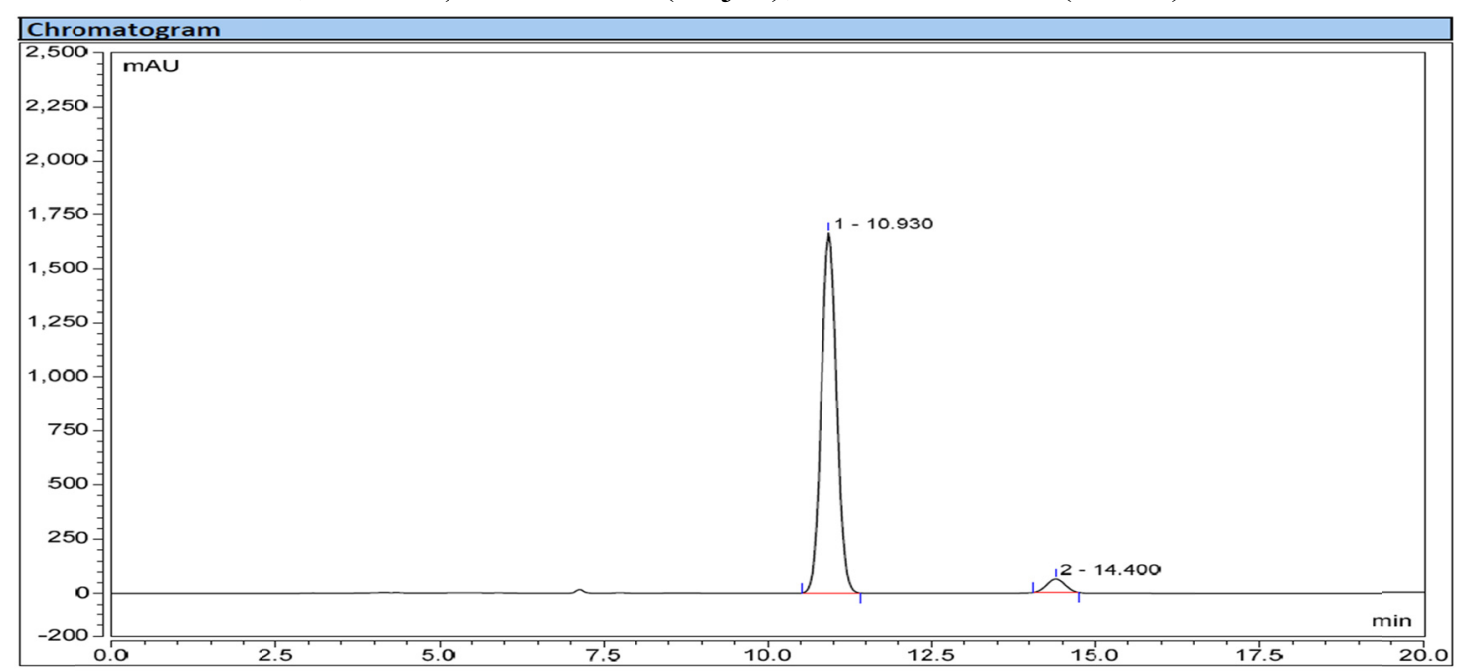

\begin{tabular}{|c|c|c|c|c|c|c|}
\hline \multicolumn{9}{|c|}{ Integration Results } \\
\hline No. & Peak Name & $\begin{array}{c}\text { Retention Time } \\
\text { min }\end{array}$ & $\begin{array}{c}\text { Height } \\
\text { mAU }\end{array}$ & $\begin{array}{c}\text { Area } \\
\text { mAU*min }\end{array}$ & $\begin{array}{c}\text { Relative Height } \\
\%\end{array}$ & $\begin{array}{c}\text { Relative Area } \\
\%\end{array}$ \\
\hline 1 & & 10.930 & 1662.423 & 460.584 & 96.38 & 95.82 \\
2 & & 14.400 & 62.488 & 20.079 & 3.62 & 4.18 \\
\hline
\end{tabular}

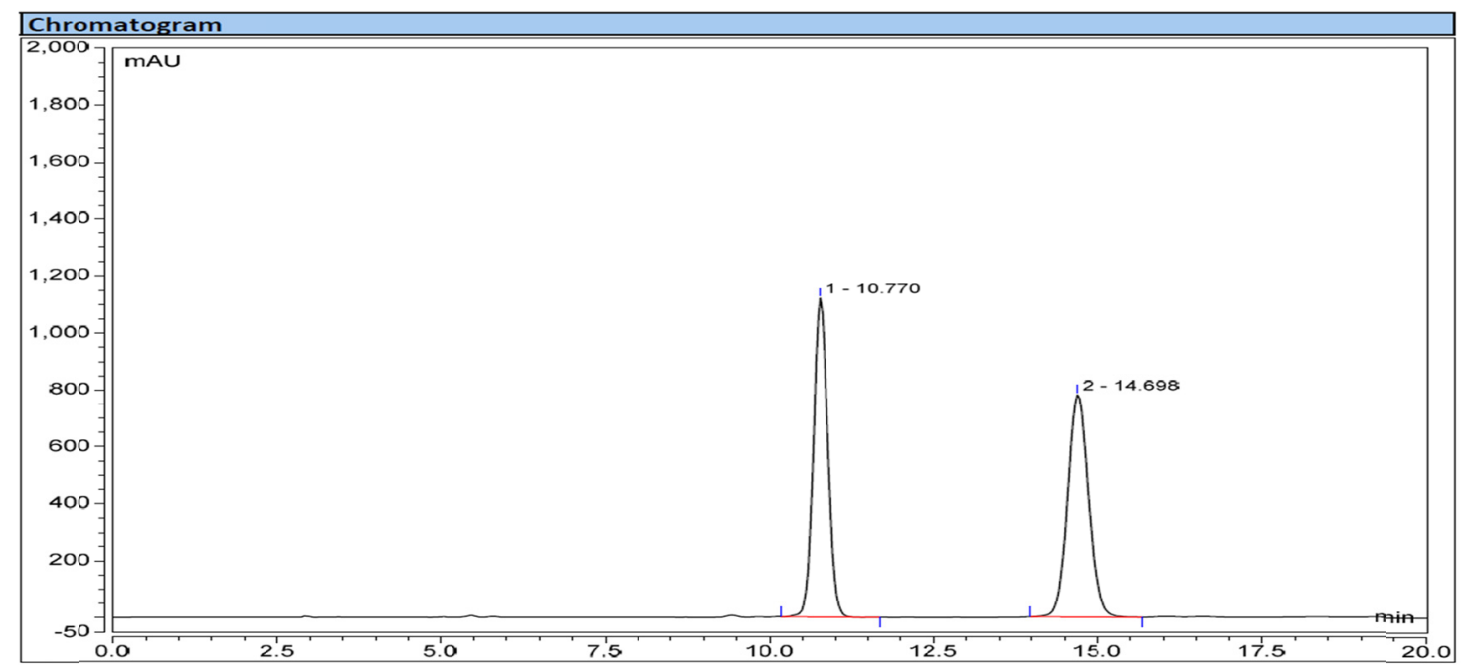

\begin{tabular}{|c|c|c|c|c|c|c|}
\hline \multicolumn{9}{|l|}{ Integration Results } \\
\hline No. & Peak Name & $\begin{array}{c}\text { Retention Time } \\
\text { min }\end{array}$ & $\begin{array}{c}\text { Height } \\
\text { mAU }\end{array}$ & $\begin{array}{c}\text { Area } \\
\text { mAU*min }\end{array}$ & $\begin{array}{c}\text { Relative Height } \\
\%\end{array}$ & $\begin{array}{c}\text { Relative Area } \\
\%\end{array}$ \\
\hline 1 & & 10.770 & 1120.966 & 286.983 & 58.92 & 49.93 \\
2 & & 14.698 & 781.612 & 287.815 & 41.08 & 50.07 \\
\hline
\end{tabular}




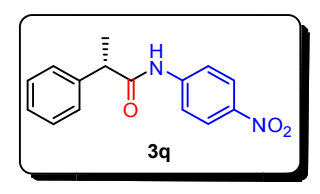

\section{(S)-N-(4-nitrophenyl)-2-phenylpropanamide}

$56 \%$ yield, b/1 $>99: 1$, ee $=85 \%$, $[\alpha] \mathrm{D}^{20}=+45.2\left(\mathrm{c}=1.5, \mathrm{CHCl}_{3}\right) .{ }^{1} \mathrm{H}$ NMR $(600$ $\left.\mathrm{MHz}, \mathrm{CDCl}_{3}\right) \delta 8.02(\mathrm{~d}, J=9.0 \mathrm{~Hz}, 2 \mathrm{H}), 7.70(\mathrm{~s}, 1 \mathrm{H}), 7.53(\mathrm{~d}, J=9.0 \mathrm{~Hz}, 2 \mathrm{H})$, 7.30-7.20 (m, 5H), 3.69 (q, $J=7.2 \mathrm{~Hz}, 1 \mathrm{H}), 1.50(\mathrm{~d}, J=7.2 \mathrm{~Hz}, 3 \mathrm{H}) ;{ }^{13} \mathrm{C}$ NMR $(101$ $\left.\mathrm{MHz}, \mathrm{CDCl}_{3}\right) \delta 173.0,143.8,143.3,140.1,129.2,127.8,127.5,124.9,119.0,48.1$, 18.5. HRMS calcd. (ESI) $\mathrm{m} / \mathrm{z}$ for $\mathrm{C}_{15} \mathrm{H}_{14} \mathrm{~N}_{2} \mathrm{NaO}_{3}$ : $[\mathrm{M}+\mathrm{Na}]^{+}$293.0897, found: 293.0899.

Chiral HPLC (Chiralpak IG, 4.6x250 mm; $5 \mu \mathrm{L}$, hexane/isopropanol $=90 / 10$, flow $1.0 \mathrm{~mL} / \mathrm{min}, 254 \mathrm{~nm}$ ): $\mathrm{t}_{\mathrm{R}}=9.07 \mathrm{~min}$ (major), $\mathrm{t}_{\mathrm{R}}=10.97 \mathrm{~min}$ (minor).
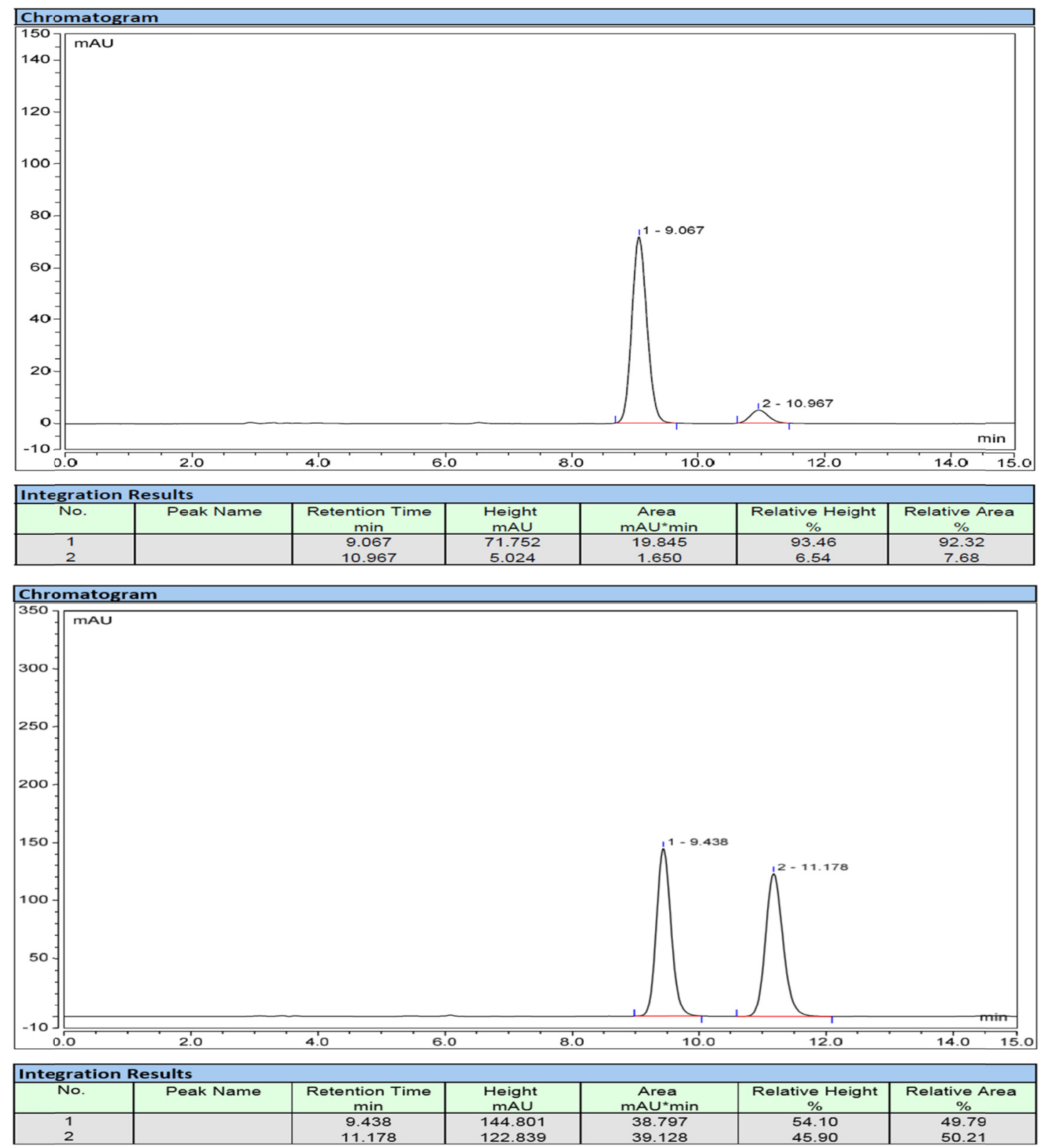


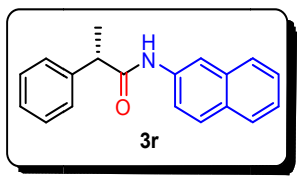

\section{(S)- $N$-(naphthalen-2-yl)-2-phenylpropanamide}

$96 \%$ yield, $\mathrm{b} / \mathrm{l}>99: 1, \mathrm{ee}=89 \%,[\alpha] \mathrm{D}^{20}=+80.6\left(\mathrm{c}=2.0, \mathrm{CHCl}_{3}\right) .{ }^{1} \mathrm{H}$ NMR $(400$ $\left.\mathrm{MHz}, \mathrm{CDCl}_{3}\right) \delta 8.16(\mathrm{~s}, 1 \mathrm{H}), 7.73-7.68(\mathrm{~m}, 3 \mathrm{H}), 7.43-7.29(\mathrm{~m}, 9 \mathrm{H}), 3.76(\mathrm{q}, J=7.2$ $\mathrm{Hz}, 1 \mathrm{H}), 1.62(\mathrm{~d}, J=7.2 \mathrm{~Hz}, 3 \mathrm{H}) ;{ }^{13} \mathrm{C} \mathrm{NMR}\left(101 \mathrm{MHz}, \mathrm{CDCl}_{3}\right) \delta 172.6,140.9,135.2$, 133.7, 130.5, 129.1, 128.6, 127.7, 127.57, 127.56, 127.4, 126.4, 124.9, 119.7, 116.5, 48.1, 18.6. HRMS calcd. (ESI) $\mathrm{m} / \mathrm{z}$ for $\mathrm{C}_{19} \mathrm{H}_{17} \mathrm{NNaO}$ : $[\mathrm{M}+\mathrm{Na}]^{+}$298.1202, found: 298.1201.

Chiral HPLC (Chiralpak IG, 4.6x250 mm; $5 \mu \mathrm{L}$, hexane/isopropanol $=85 / 15$, flow $1.0 \mathrm{~mL} / \mathrm{min}, 254 \mathrm{~nm}$ ): $\mathrm{t}_{\mathrm{R}}=13.55 \mathrm{~min}$ (major), $\mathrm{t}_{\mathrm{R}}=23.79 \min$ (minor)
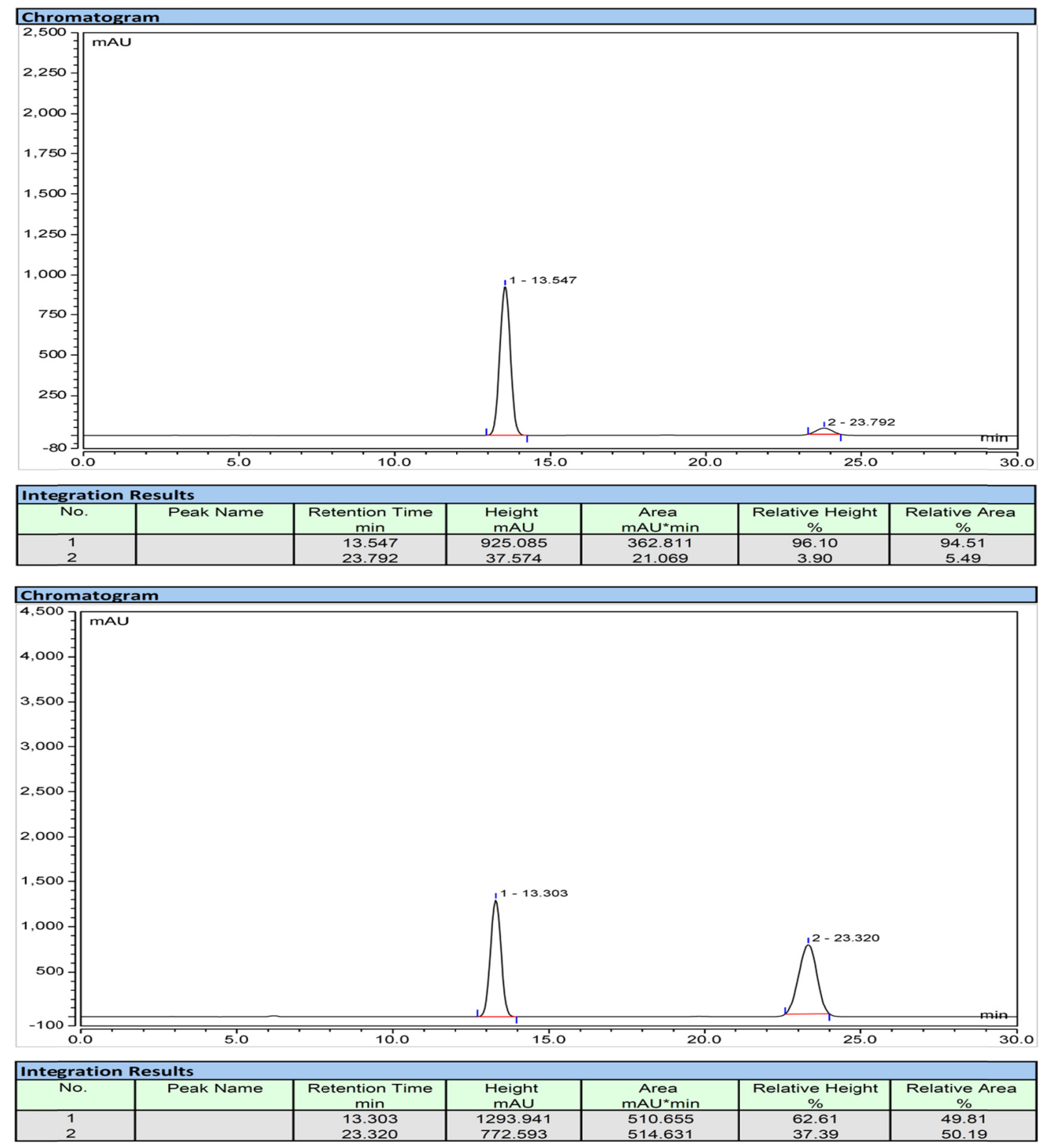


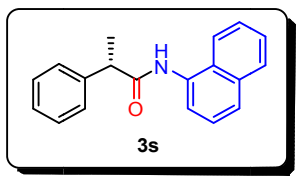

\section{(S)-N-(naphthalen-1-yl)-2-phenylpropanamide}

95\% yield, b/1 $>99: 1$, ee $=90 \%$ ee, $[\alpha] \mathrm{D}^{16}=-5.4\left(\mathrm{c}=1.9, \mathrm{CHCl}_{3}\right) .{ }^{1} \mathrm{H}$ NMR $(400$ $\left.\mathrm{MHz}, \mathrm{CDCl}_{3}\right) \delta 7.78(\mathrm{~d}, J=7.2 \mathrm{~Hz}, 1 \mathrm{H}), 7.74(\mathrm{~d}, J=8.2 \mathrm{~Hz}, 1 \mathrm{H}), 7.72-7.63(\mathrm{~m}, 1 \mathrm{H})$, $7.56(\mathrm{~d}, J=8.2 \mathrm{~Hz}, 1 \mathrm{H}), 7.38-7.34(\mathrm{~m}, 5 \mathrm{H}), 7.34-7.30(\mathrm{~m}, 2 \mathrm{H}), 7.28(\mathrm{~d}, J=4.0 \mathrm{~Hz}, 2 \mathrm{H})$, $3.82(\mathrm{q}, J=7.2 \mathrm{~Hz}, 1 \mathrm{H}), 1.59(\mathrm{~d}, J=7.3 \mathrm{~Hz}, 3 \mathrm{H}),{ }^{13} \mathrm{C} \mathrm{NMR}\left(151 \mathrm{MHz}, \mathrm{CDCl}_{3}\right) \delta 173.0$, 141.1, 133.9, 132.1, 129.1, 128.5, 127.8, 127.5, 127.1, 126.0, 125.7, 125.51, 125.47, 120.6, 120.3, 47.7, 18.2. HRMS calcd. (ESI) $\mathrm{m} / \mathrm{z}$ for $\mathrm{C}_{19} \mathrm{C}_{17} \mathrm{NNaO}$ : $[\mathrm{M}+\mathrm{Na}]^{+} 298.1202$, found: 298.1205 .

Chiral HPLC (Chiralpak IG, 4.6x250 mm; $5 \mu \mathrm{L}$, hexane/isopropanol = 90/10, flow $1.0 \mathrm{~mL} / \mathrm{min}, 254 \mathrm{~nm}$ ): $\mathrm{t}_{\mathrm{R}}=18.64 \mathrm{~min}$ (major), $\mathrm{t}_{\mathrm{R}}=24.69 \mathrm{~min}$ (minor).

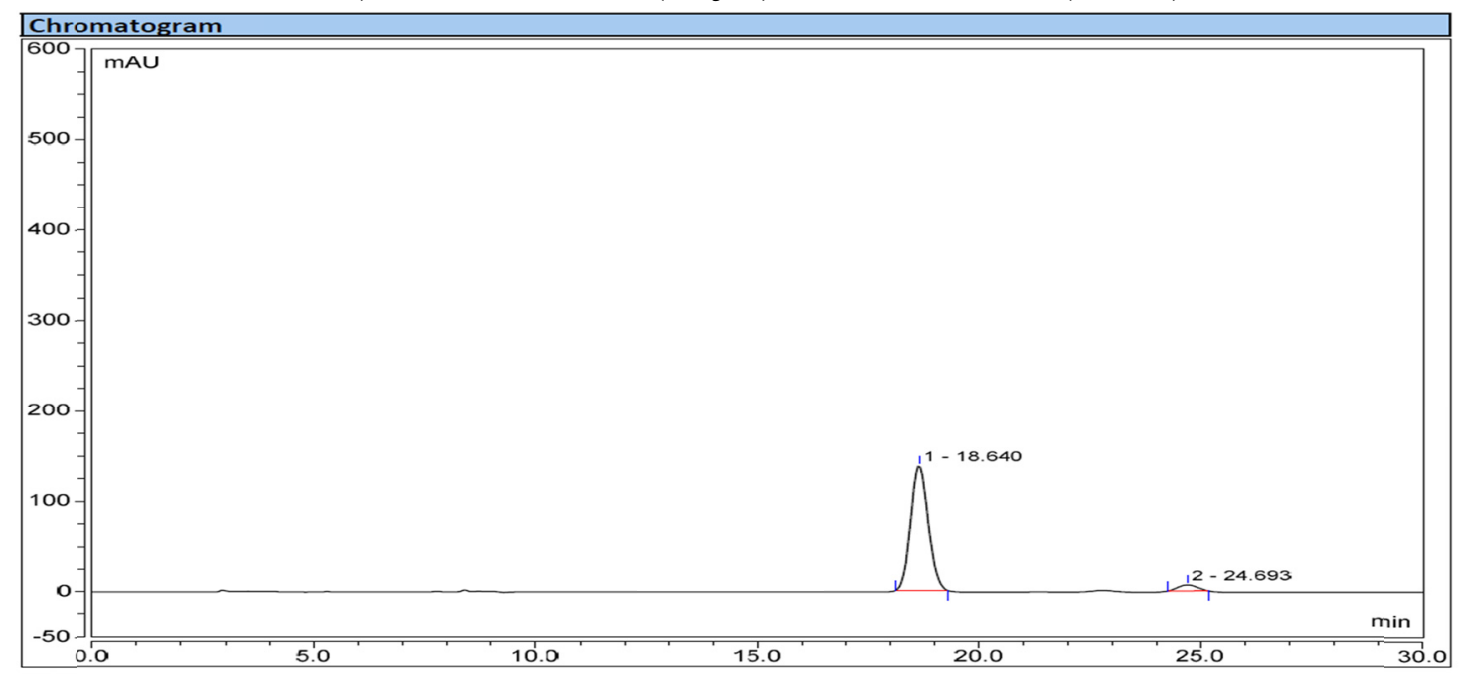

\begin{tabular}{|c|c|c|c|c|c|c|}
\hline \multicolumn{7}{|c|}{ Integration Results } \\
\hline No. & Peak Name & $\begin{array}{c}\text { Retention Time } \\
\text { min }\end{array}$ & $\begin{array}{c}\text { Height } \\
\text { mAU }\end{array}$ & $\begin{array}{c}\text { Area } \\
m A U^{*} \min \end{array}$ & $\begin{array}{c}\text { Relative Height } \\
\%\end{array}$ & $\begin{array}{c}\text { Relative Area } \\
\%\end{array}$ \\
\hline 1 & & 18.640 & 137.778 & 63.448 & 95.38 & 94.97 \\
\hline
\end{tabular}

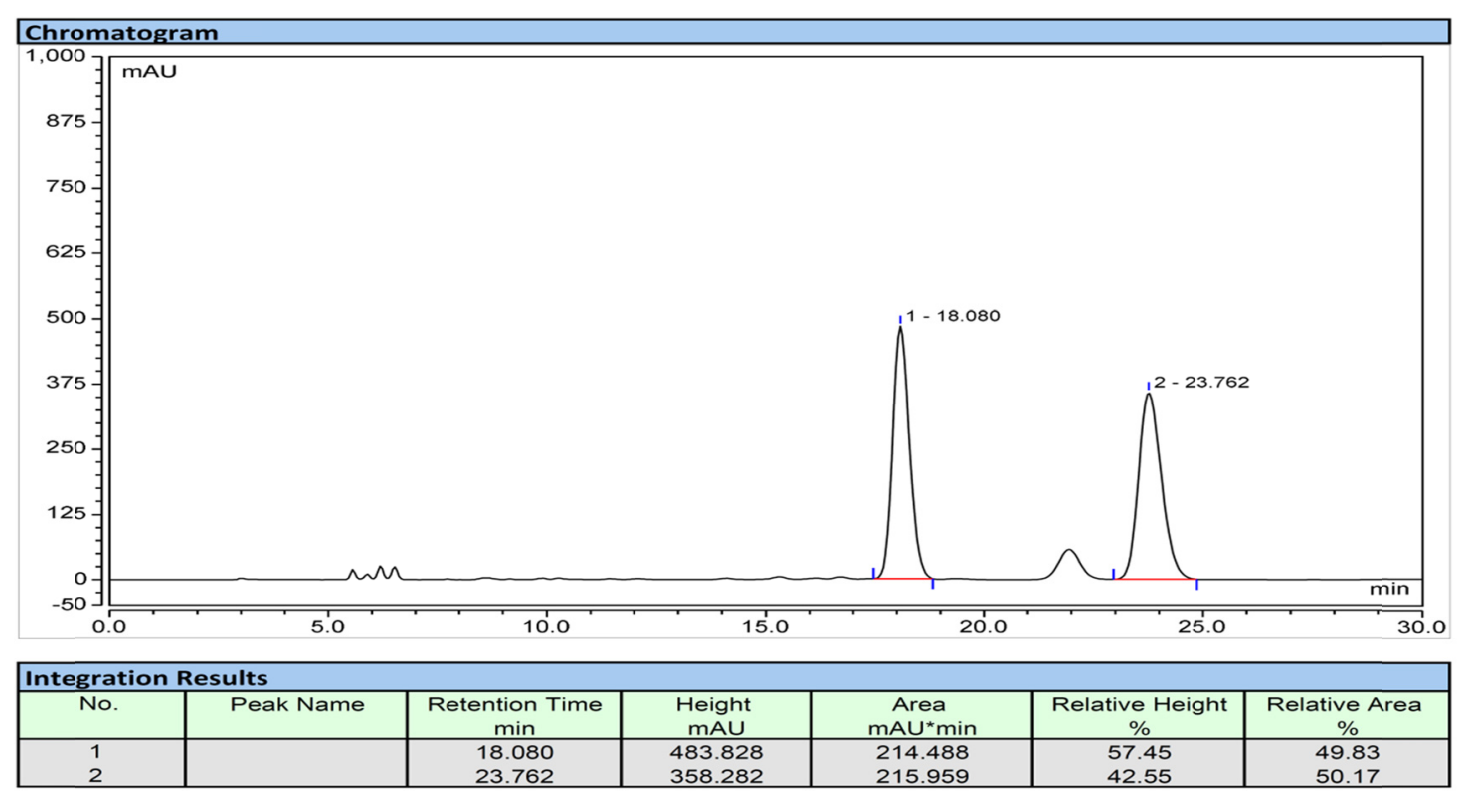




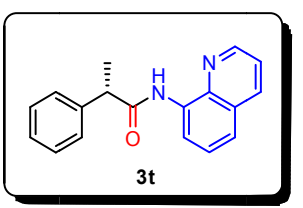

\section{(S)-2-phenyl- $N$-(quinolin-8-yl)propanamide}

$96 \%$ yield, b/1 > 99:1, ee $=89 \%,[\alpha] \mathrm{D}^{18}=+54.7\left(\mathrm{c}=1.4, \mathrm{CHCl}_{3}\right) .{ }^{1} \mathrm{H}$ NMR $(600$ $\left.\mathrm{MHz}, \mathrm{CDCl}_{3}\right) \delta 9.88(\mathrm{~s}, 1 \mathrm{H}), 9.05-8.74(\mathrm{~m}, 1 \mathrm{H}), 8.70-8.62(\mathrm{~m}, 1 \mathrm{H}), 8.24-7.91(\mathrm{~m}, 1 \mathrm{H})$, 7.48-7.44 (m, 3H), 7.41-7.39 (m, 1H), 7.36 (t, $J=7.2 \mathrm{~Hz}, 2 \mathrm{H}), 7.33-7.31$ (m, 1H), 7.27 $(\mathrm{t}, J=7.8 \mathrm{~Hz}, 1 \mathrm{H}), 3.91(\mathrm{q}, J=7.2 \mathrm{~Hz}, 1 \mathrm{H}), 1.68(\mathrm{~d}, J=7.2 \mathrm{~Hz}, 3 \mathrm{H}) ;{ }^{13} \mathrm{C}$ NMR $(151$ $\left.\mathrm{MHz}, \mathrm{CDCl}_{3}\right) \delta 172.6,148.0,141.0,138.3,136.1,134.4,128.8,127.7,127.6,127.2$, 127.2, 121.4, 121.3, 116.2, 48.5, 18.5. HRMS calcd. (ESI) $\mathrm{m} / \mathrm{z}$ for $\mathrm{C}_{18} \mathrm{H}_{16} \mathrm{~N}_{2} \mathrm{NaO}$ : $[\mathrm{M}+\mathrm{Na}]^{+}$299.1155, found: 299.1151 .

Chiral HPLC (Chiralpak IB N-5, 4.6x250 mm; $5 \mu \mathrm{L}$, hexane/isopropanol $=80 / 20$, flow $1.0 \mathrm{~mL} / \mathrm{min}, 254 \mathrm{~nm}$ ): $\mathrm{t}_{\mathrm{R}}=6.53 \mathrm{~min}$ (major), $\mathrm{t}_{\mathrm{R}}=11.22 \mathrm{~min}$ (minor)

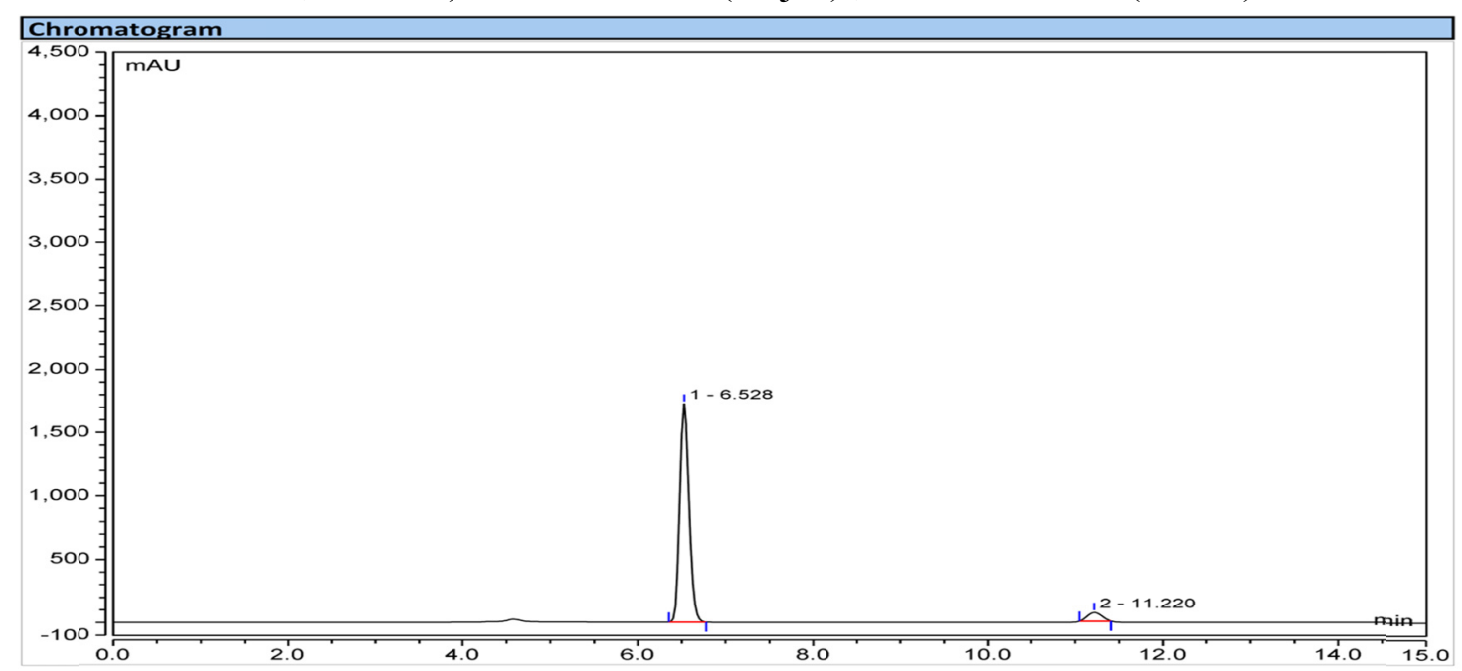

\begin{tabular}{|c|c|c|c|c|c|c|}
\hline \multicolumn{9}{|c|}{ Integration Results } \\
\hline No. & Peak Name & $\begin{array}{c}\text { Retention Time } \\
\text { min }\end{array}$ & $\begin{array}{c}\text { Height } \\
\text { mAU }\end{array}$ & $\begin{array}{c}\text { Area } \\
\text { mAU*min }\end{array}$ & $\begin{array}{c}\text { Relative Height } \\
\%\end{array}$ & $\begin{array}{c}\text { Relative Area } \\
\%\end{array}$ \\
\hline 1 & & 6.528 & 1724.987 & 211.652 & 96.20 & 94.32 \\
2 & & 11.220 & 68.093 & 12.747 & 3.80 & 5.68 \\
\hline
\end{tabular}

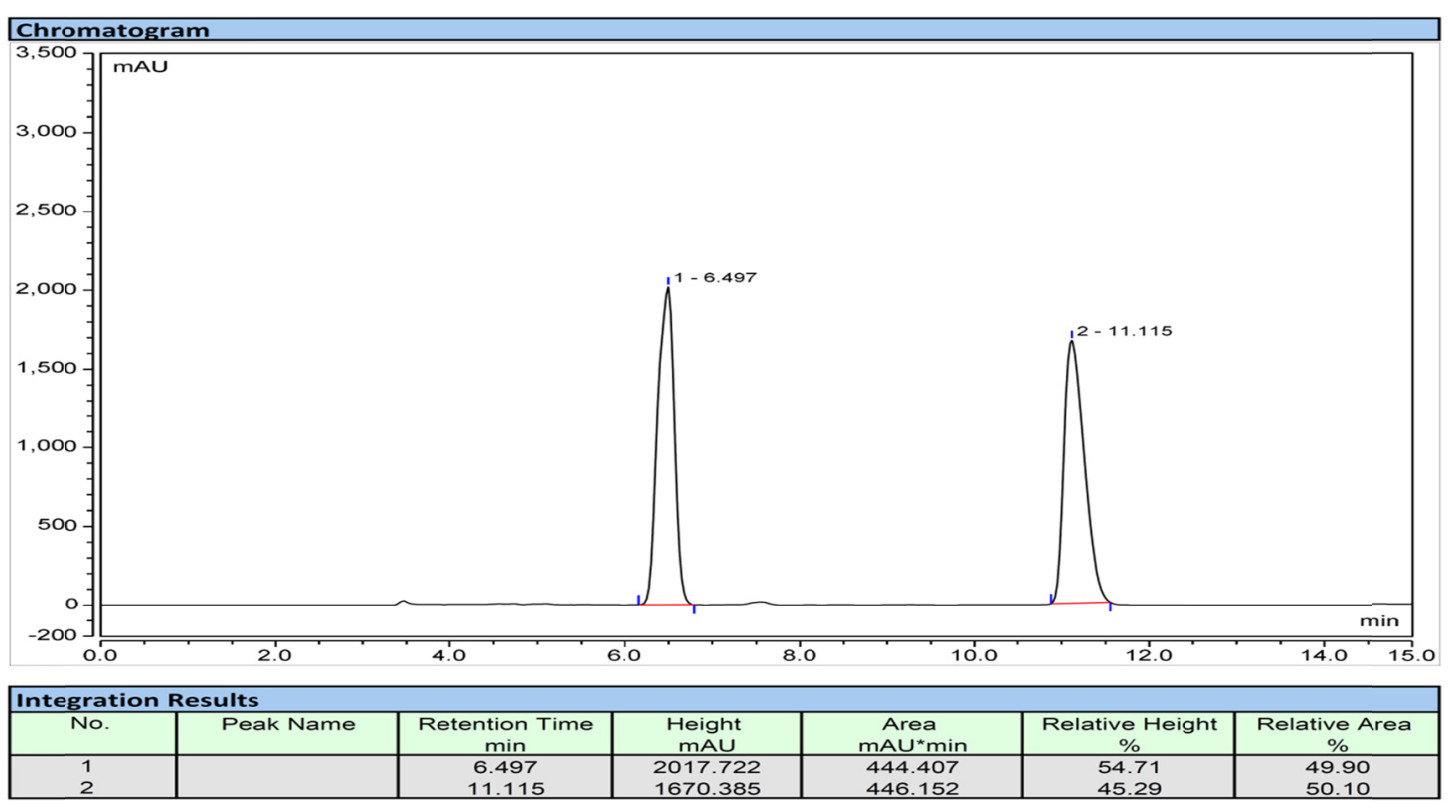




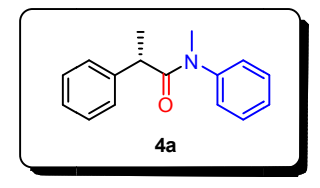

\section{(S)- $N$-methyl- $N$,2-diphenylpropanamide}

97\% yield, b/1 > 99:1, ee $=91 \%,[\alpha] \mathrm{D}^{18}=+26.1\left(\mathrm{c}=1.5, \mathrm{CHCl}_{3}\right) .{ }^{1} \mathrm{H}$ NMR $(400$ $\left.\mathrm{MHz}, \mathrm{CDCl}_{3}\right) \delta$ 7.34-7.31 (m, 3H), 7.23-7.11 (m, 3H), 7.09-6.89 (m, 4H), 3.64 (q, $J=$ $6.8 \mathrm{~Hz}, 1 \mathrm{H}), 3.23(\mathrm{~s}, 3 \mathrm{H}), 1.39(\mathrm{~d}, J=6.8 \mathrm{~Hz}, 3 \mathrm{H}) ;{ }^{13} \mathrm{C} \mathrm{NMR}\left(101 \mathrm{MHz}, \mathrm{CDCl}_{3}\right) \delta$ 173.8, 143.6, 141.8, 129.3, 128.1, 127.63, 127.60, 127.3, 126.4, 42.9, 37.5, 20.1. HRMS calcd. (ESI) m/z for $\mathrm{C}_{16} \mathrm{H}_{17} \mathrm{NNaO}$ : [M+Na] 262.1202 , found: 262.1205 .

Chiral HPLC (Chiralpak IG, 4.6×250 mm; $5 \mu \mathrm{L}$, hexane/isopropanol = 90/10, flow $1.0 \mathrm{~mL} / \mathrm{min}, 254 \mathrm{~nm}$ ): $\mathrm{t}_{\mathrm{R}}=8.65 \mathrm{~min}$ (major), $\mathrm{t}_{\mathrm{R}}=9.46 \mathrm{~min}$ (minor).
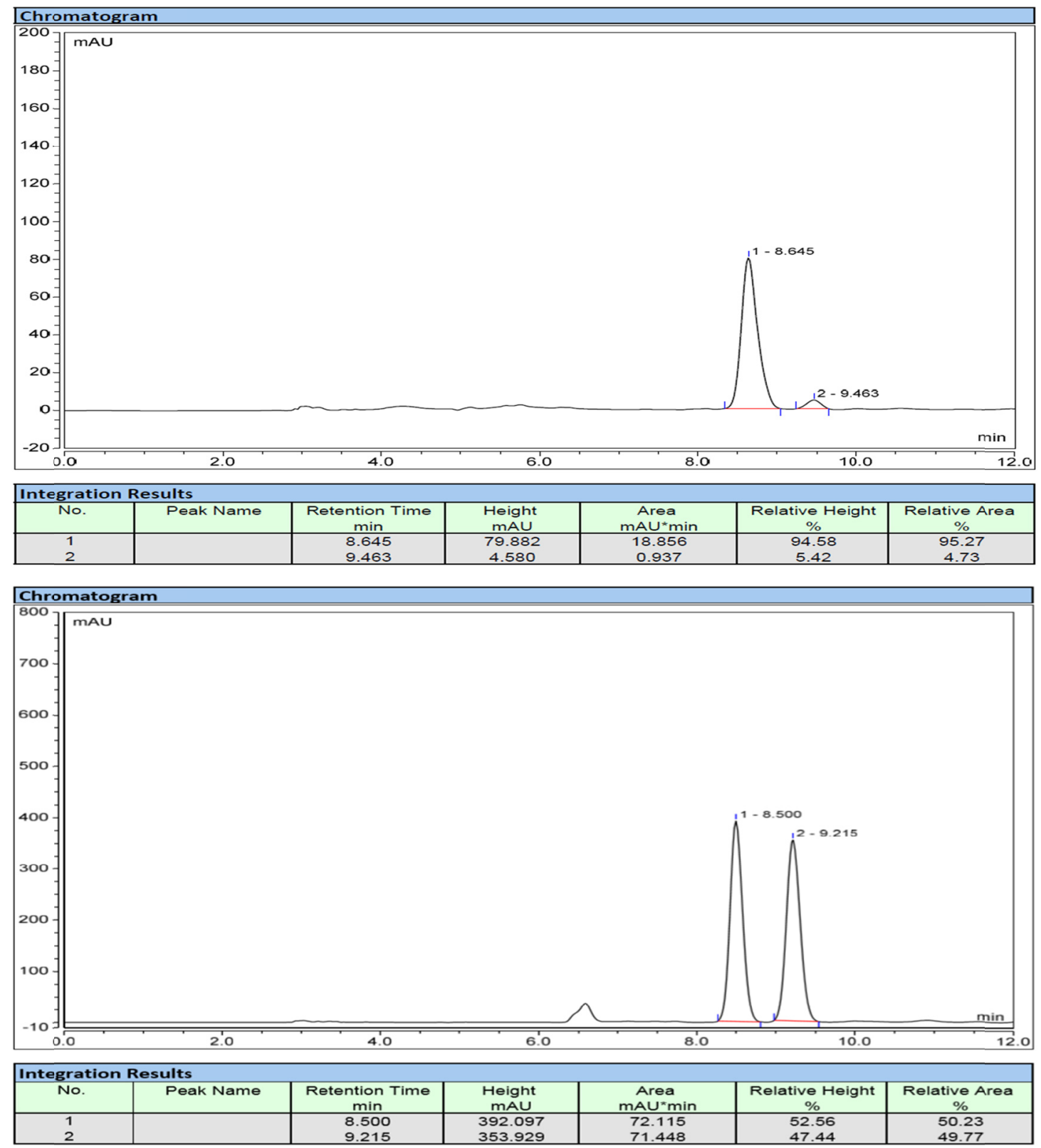


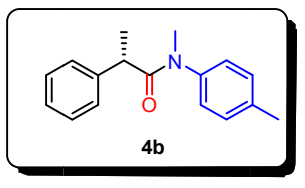

(S)- $N$-methyl-2-phenyl- $N$-(p-tolyl)propanamide

$90 \%$ yield, b:1 $>99: 1$, ee $=94 \%$ ee, $[\alpha] \mathrm{D}^{18}=+32.4\left(\mathrm{c}=1.2, \mathrm{CHCl}_{3}\right) .{ }^{1} \mathrm{H}$ NMR $(400$ $\left.\mathrm{MHz}, \mathrm{CDCl}_{3}\right) \delta 7.24-7.11(\mathrm{~m}, 5 \mathrm{H}), 7.05(\mathrm{~d}, J=6.8 \mathrm{~Hz}, 2 \mathrm{H}), 6.87(\mathrm{~d}, J=7.2 \mathrm{~Hz}, 2 \mathrm{H})$, $3.65(\mathrm{q}, J=7.2 \mathrm{~Hz}, 1 \mathrm{H}), 3.21(\mathrm{~s}, 3 \mathrm{H}), 2.37(\mathrm{~s}, 3 \mathrm{H}), 1.38(\mathrm{~d}, J=7.2 \mathrm{~Hz}, 3 \mathrm{H}) ;{ }^{13} \mathrm{C}$ NMR $\left(101 \mathrm{MHz}, \mathrm{CDCl}_{3}\right) \delta 174.0,141.9,141.1,137.6,130.0,128.2,127.4,127.3,126.4$, 42.7, 37.6, 20.9, 20.1. HRMS calcd. (ESI) $\mathrm{m} / \mathrm{z}$ for $\mathrm{C}_{17} \mathrm{H}_{19} \mathrm{NNaO}$ : $[\mathrm{M}+\mathrm{Na}]^{+} 276.1359$, found: 276.1358 .

Chiral HPLC (Chiralpak IG, 4.6x250 mm; $5 \mu \mathrm{L}$, hexane/isopropanol = 90/10, flow $1.0 \mathrm{~mL} / \mathrm{min}, 254 \mathrm{~nm}$ ): $\mathrm{t}_{\mathrm{R}}=12.81 \mathrm{~min}$ (major), $\mathrm{t}_{\mathrm{R}}=14.76 \mathrm{~min}$ (minor).
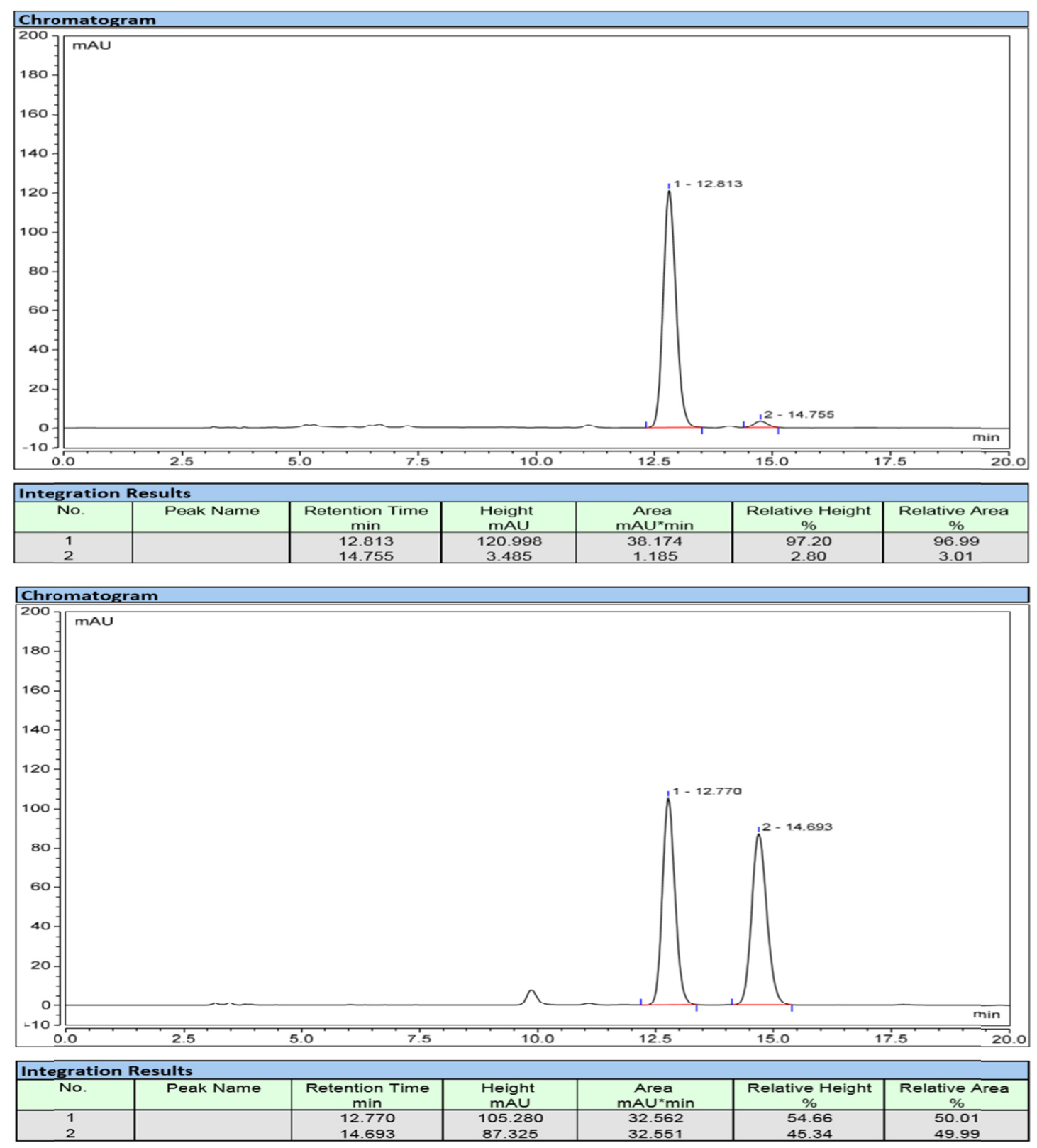


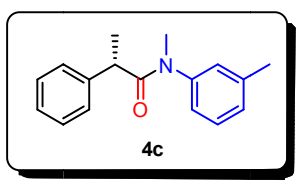

(S)- $N$-methyl-2-phenyl- $N$-(m-tolyl)propanamide

$94 \%$ yield, $\mathrm{b} / \mathrm{l}>99: 1, \mathrm{ee}=95 \%,[\alpha] \mathrm{D}^{18}=+56.4\left(\mathrm{c}=1.7, \mathrm{CHCl}_{3}\right) .{ }^{1} \mathrm{H}$ NMR $(400$ $\left.\mathrm{MHz}, \mathrm{CDCl}_{3}\right) \delta 7.28-7.13(\mathrm{~m}, 5 \mathrm{H}), 7.05(\mathrm{~d}, J=6.8 \mathrm{~Hz}, 2 \mathrm{H}), 6.84(\mathrm{~d}, J=6.0 \mathrm{~Hz}, 1 \mathrm{H})$, $6.73(\mathrm{~s}, 1 \mathrm{H}), 3.65(\mathrm{q}, J=6.8 \mathrm{~Hz}, 1 \mathrm{H}), 3.25(\mathrm{~s}, 3 \mathrm{H}), 2.31(\mathrm{~s}, 3 \mathrm{H}), 1.42(\mathrm{~d}, J=7.2 \mathrm{~Hz}$, $3 \mathrm{H}) ;{ }^{13} \mathrm{C}$ NMR $\left(101 \mathrm{MHz}, \mathrm{CDCl}_{3}\right) \delta 173.9,143.5,142.0,139.4,129.1,128.5,128.4$, 128.2, 127.4, 126.4, 124.5, 43.1, 37.5, 21.0, 20.2. HRMS calcd. (ESI) $\mathrm{m} / \mathrm{z}$ for $\mathrm{C}_{17} \mathrm{H}_{19} \mathrm{NNaO}:[\mathrm{M}+\mathrm{Na}]^{+} 276.1359$, found: 276.1366 .

Chiral HPLC (Chiralpak IG, 4.6x250 mm; $5 \mu \mathrm{L}$, hexane/isopropanol = 90/10, flow $1.0 \mathrm{~mL} / \mathrm{min}, 254 \mathrm{~nm}$ ): $\mathrm{t}_{\mathrm{R}}=11.86 \min$ (major), $\mathrm{t}_{\mathrm{R}}=13.03 \mathrm{~min}$ (minor).

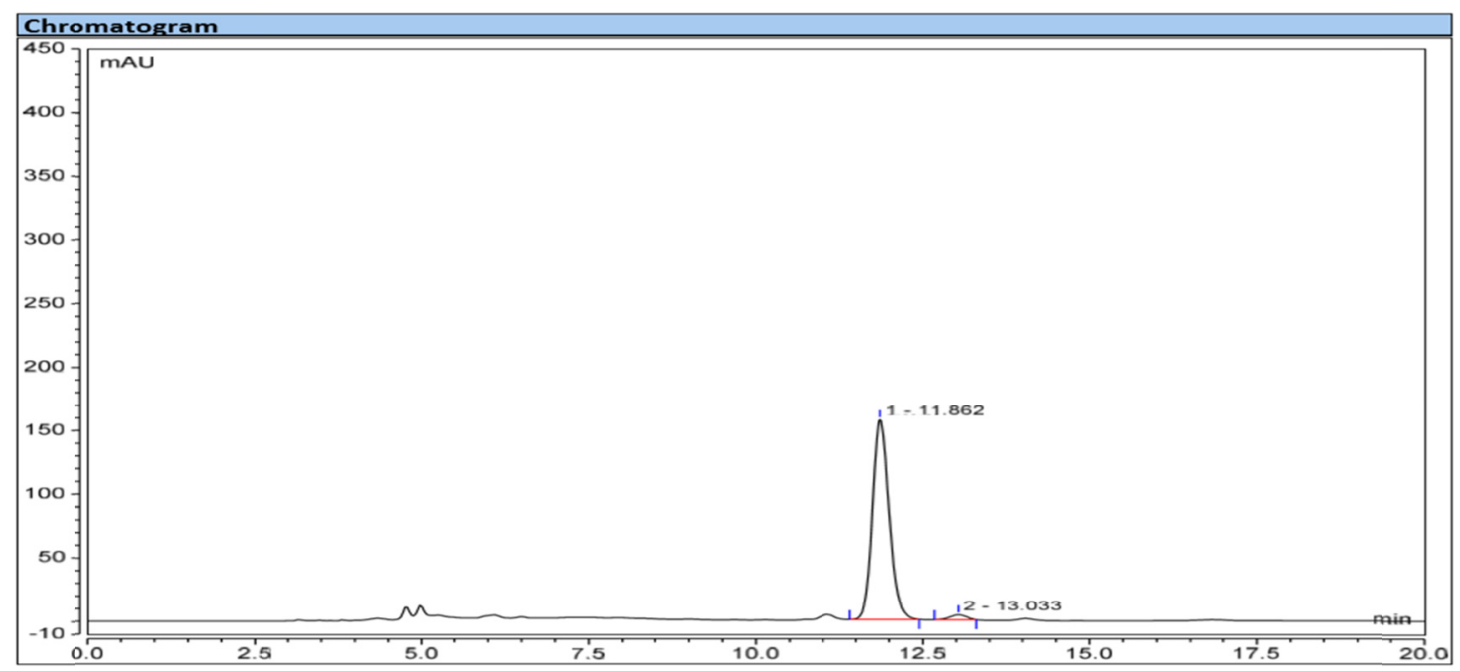

\begin{tabular}{|c|c|c|c|c|c|c|}
\hline \multicolumn{7}{|c|}{ Integration Results } \\
\hline No. & Peak Name & $\begin{array}{c}\text { Retention Time } \\
\text { min }\end{array}$ & $\begin{array}{c}\text { Height } \\
\text { mAU }\end{array}$ & $\begin{array}{c}\text { Area } \\
\text { mAU*min }\end{array}$ & $\begin{array}{c}\text { Relative Height } \\
\%\end{array}$ & $\begin{array}{c}\text { Relative Area } \\
\%\end{array}$ \\
\hline $\begin{array}{l}1 \\
2\end{array}$ & & $\begin{array}{l}11.862 \\
13.033\end{array}$ & $\begin{array}{c}157.150 \\
3.844\end{array}$ & $\begin{array}{c}45.699 \\
1.094\end{array}$ & $\begin{array}{l}97.61 \\
2.39\end{array}$ & $\begin{array}{l}97.66 \\
2.34\end{array}$ \\
\hline
\end{tabular}

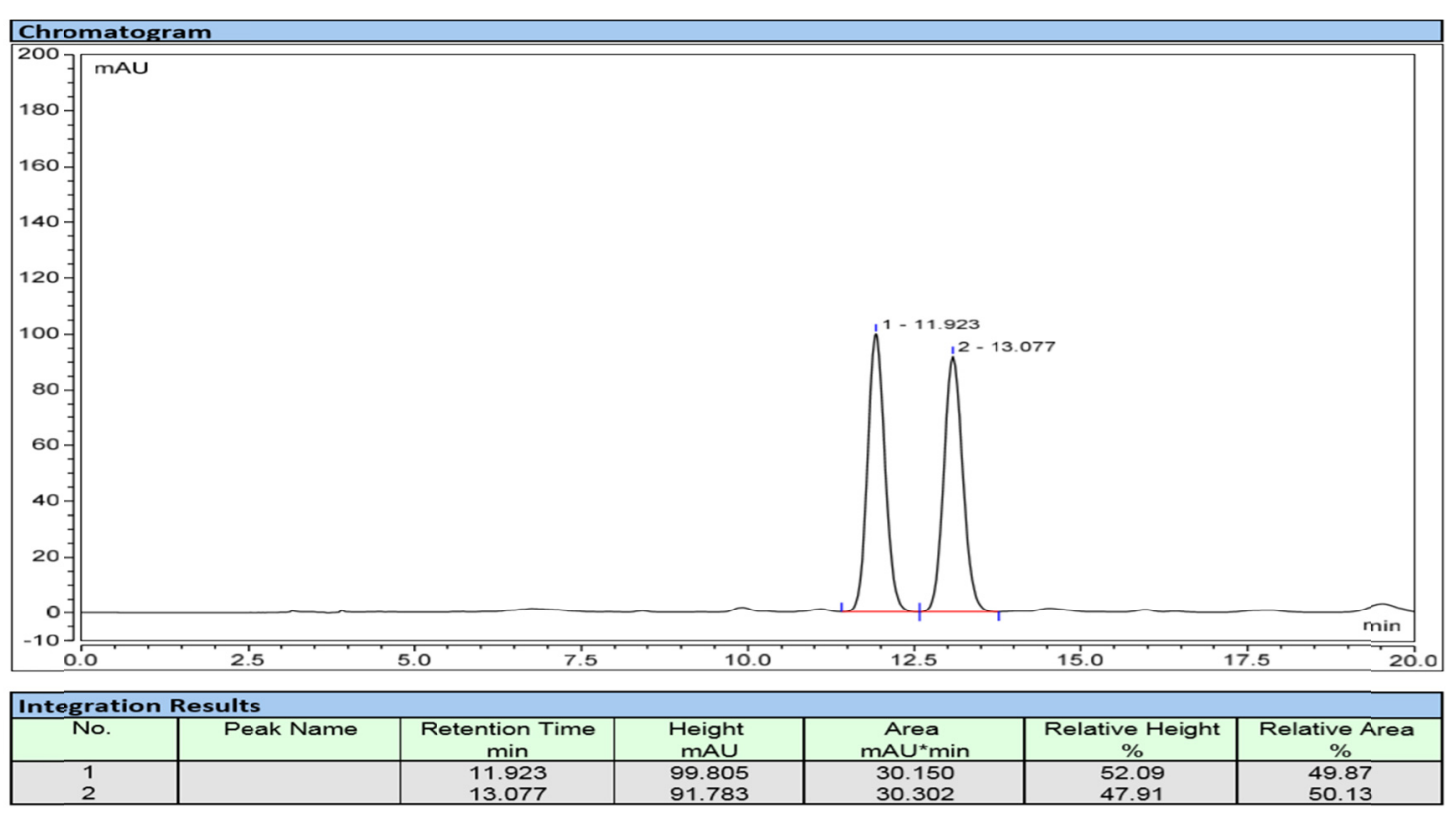




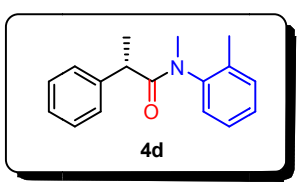

(S)- $N$-methyl-2-phenyl- $N$-(o-tolyl)propanamide

$87 \%$ yield, $\mathrm{b} / \mathrm{l}>99: 1, \mathrm{ee}=98 \%,[\alpha] \mathrm{D}^{18}=-13.2\left(\mathrm{c}=0.7, \mathrm{CHCl}_{3}\right) .{ }^{1} \mathrm{H}$ NMR $(400$ $\left.\mathrm{MHz}, \mathrm{CDCl}_{3}\right) \delta$ 7.32-7.24 (m, 2H), 7.20-7.11 (m, 4H), 7.06-6.98 (m, 1.5H), 6.91-6.89 $(\mathrm{m}, 1 \mathrm{H}), 6.59-6.58(\mathrm{~m}, 0.5 \mathrm{H}), 3.59(\mathrm{q}, J=6.8 \mathrm{~Hz}, 0.5 \mathrm{H}), 3.35(\mathrm{q}, J=6.8 \mathrm{~Hz}, 0.5 \mathrm{H})$, $3.14(\mathrm{~d}, J=9.2 \mathrm{~Hz}, 3 \mathrm{H}), 2.29(\mathrm{~s}, 1.5 \mathrm{H}), 1.53(\mathrm{~s}, 1.5 \mathrm{H}), 1.39(\mathrm{~d}, J=6.8 \mathrm{~Hz}, 3 \mathrm{H}) ;{ }^{13} \mathrm{C}$ NMR (101 MHz, $\mathrm{CDCl}_{3}$,) $\delta 173.92,173.85,142.1,142.0,141.6,141.1,136.6,135.2$, $131.4,131.0,128.9,128.3,128.21,128.16,128.1,127.6,127.4,127.0,126.9,126.54$, 126.48, 43.2, 43.1, 36.1, 20.6, 20.1, 17.3, 16.5. HRMS calcd. (ESI) $\mathrm{m} / \mathrm{z}$ for $\mathrm{C}_{17} \mathrm{H}_{19} \mathrm{NNaO}:[\mathrm{M}+\mathrm{Na}]^{+} 276.1359$, found: 276.1351 .

Chiral HPLC (Chiralpak OJ-H, 4.6x250 mm; $5 \mu \mathrm{L}$, hexane/isopropanol $=98 / 2$, flow $0.7 \mathrm{~mL} / \mathrm{min}, 220 \mathrm{~nm}$ ): $\mathrm{t}_{\mathrm{R}}=16.90 \min$ (major), $\mathrm{t}_{\mathrm{R}}=30.79 \mathrm{~min}$ (minor).
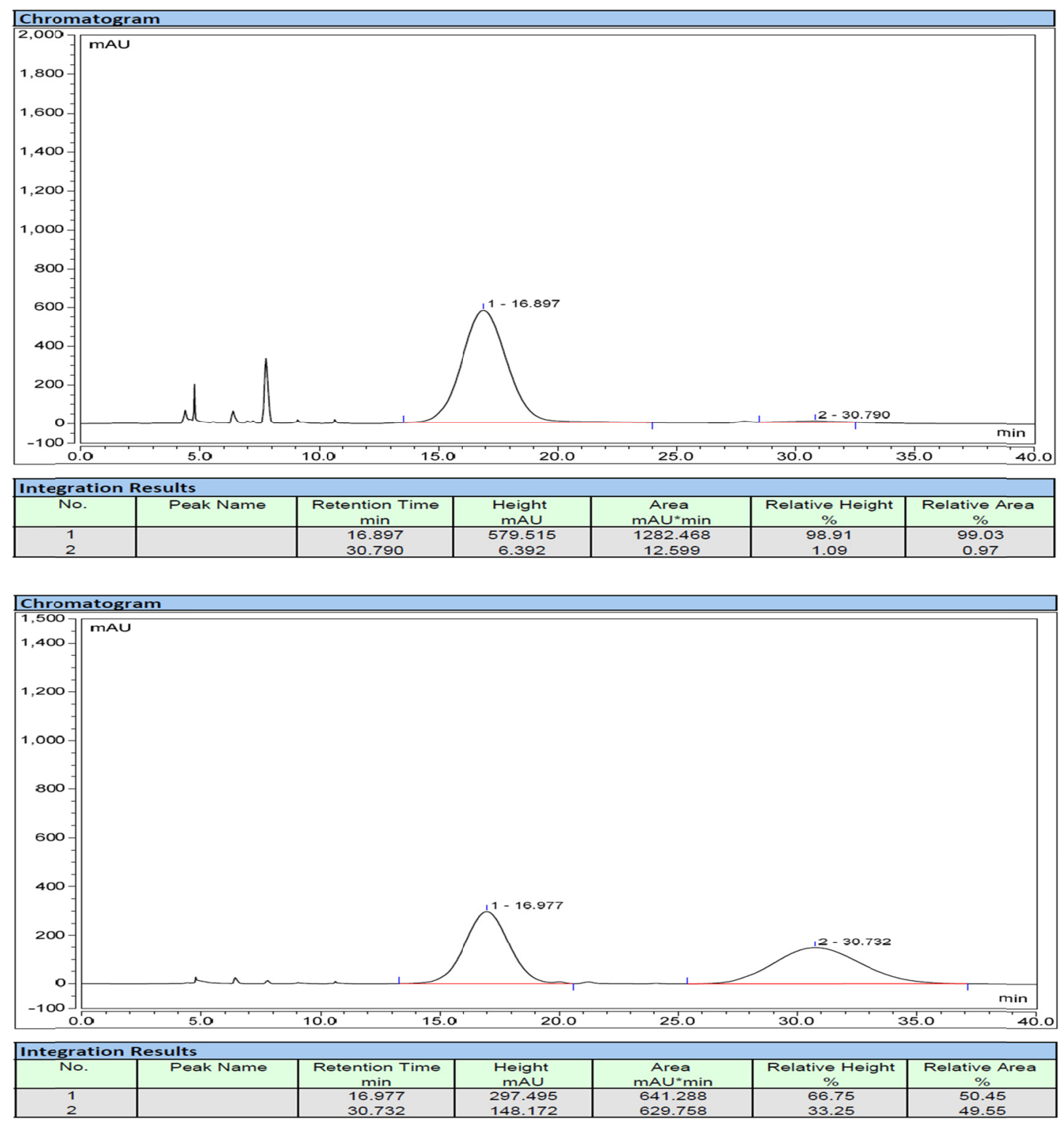


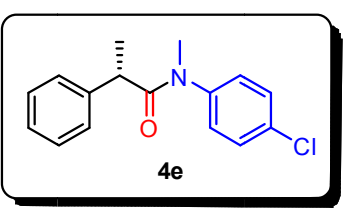

(S)-N-(4-chlorophenyl)- $\boldsymbol{N}$-methyl-2-phenylpropanamide

$95 \%$ yield, b/l $>99: 1$, ee $=90 \%,[\alpha] \mathrm{D}^{18}=+32.5\left(\mathrm{c}=1.3, \mathrm{CHCl}_{3}\right) .{ }^{1} \mathrm{H}$ NMR $(400$

$\left.\mathrm{MHz}, \mathrm{CDCl}_{3}\right){ }^{1} \mathrm{H}$ NMR $\left(400 \mathrm{MHz}, \mathrm{CDCl}_{3}\right) \delta 7.30(\mathrm{~d}, J=8.4 \mathrm{~Hz}, 2 \mathrm{H}), 7.24-7.17(\mathrm{~m}$, $3 \mathrm{H}$ ), 7.01 (d, $J=6.4 \mathrm{~Hz}, 2 \mathrm{H}), 6.90$ (d, $J=7.2 \mathrm{~Hz}, 2 \mathrm{H}), 3.59$ (q, $J=6.8 \mathrm{~Hz}, 1 \mathrm{H}), 3.21$ (s, $3 \mathrm{H}), 1.39(\mathrm{~d}, J=6.8 \mathrm{~Hz}, 3 \mathrm{H}) ;{ }^{13} \mathrm{C} \mathrm{NMR}\left(101 \mathrm{MHz}, \mathrm{CDCl}_{3}\right) \delta 173.8,142.2,141.7,133.6$, 129.6, 129.2, 128.4, 127.4, 126.7, 43.3, 37.7, 20.3. HRMS calcd. (ESI) $\mathrm{m} / \mathrm{z}$ for $\mathrm{C}_{16} \mathrm{H}_{16} \mathrm{ClNNaO}:[\mathrm{M}+\mathrm{Na}]^{+} 296.0813$, found: 296.0821.

Chiral HPLC (Chiralpak IG, 4.6x250 mm; $5 \mu \mathrm{L}$, hexane/isopropanol = 90/10, flow $1.0 \mathrm{~mL} / \mathrm{min}, 254 \mathrm{~nm}$ ): $t_{\mathrm{R}}=7.14 \mathrm{~min}$ (major), $\mathrm{t}_{\mathrm{R}}=7.58 \mathrm{~min}$ (minor).
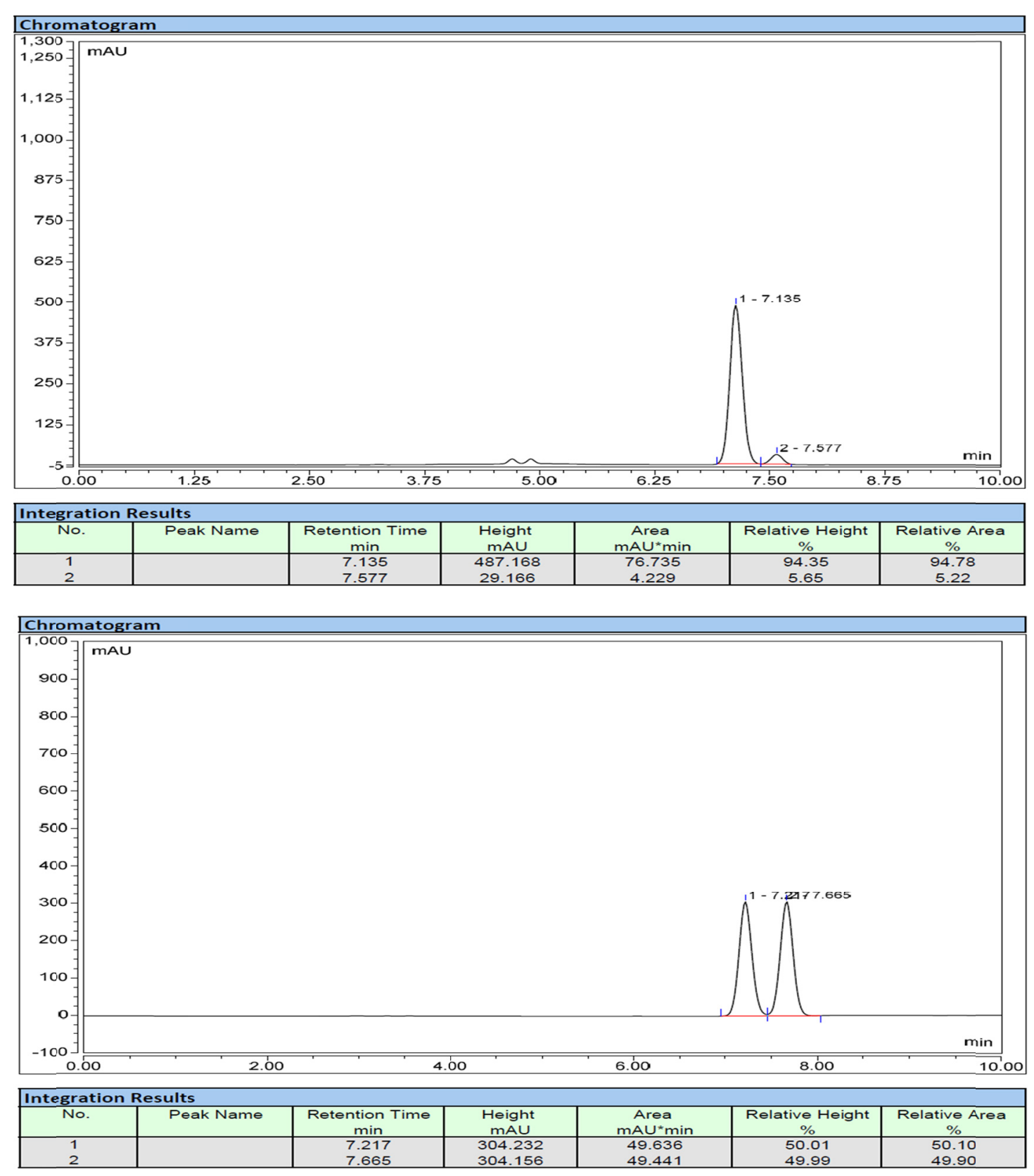


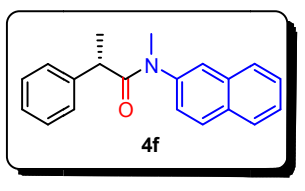

(S)- $N$-methyl- $N$-(naphthalen-2-yl)-2-phenylpropanamide

$91 \%$ yield, $\mathrm{b} / \mathrm{l}>99: 1, \mathrm{ee}=93 \%,[\alpha] \mathrm{D}^{18}=+37.1\left(\mathrm{c}=1.6, \mathrm{CHCl}_{3}\right) .{ }^{1} \mathrm{H} \mathrm{NMR}(400$ $\left.\mathrm{MHz}, \mathrm{CDCl}_{3}\right) \delta$ 7.88-7.86 (m, 1H), $7.83(\mathrm{~d}, J=8.8 \mathrm{~Hz}, 1 \mathrm{H}), 7.71-7.69(\mathrm{~m}, 1 \mathrm{H})$, 7.55-7.50 (m, 2H), $7.39(\mathrm{~s}, 1 \mathrm{H}), 7.19-7.17(\mathrm{~m}, 3 \mathrm{H}), 7.12-7.10(\mathrm{~m}, 1 \mathrm{H}), 7.01-6.99(\mathrm{~m}$, $2 \mathrm{H}), 3.66(\mathrm{q}, J=6.8 \mathrm{~Hz}, 1 \mathrm{H}), 3.32(\mathrm{~s}, 3 \mathrm{H}), 1.40(\mathrm{~d}, J=6.8 \mathrm{~Hz}, 3 \mathrm{H}) ;{ }^{13} \mathrm{C}$ NMR $(101 \mathrm{MHz}$, $\left.\mathrm{CDCl}_{3}\right) \delta 174.1,142.0,141.0,133.4,132.4,129.6,128.3,127.8,127.7,127.6,126.8$, 126.64, 126.60, 126.4, 125.6, 43.3, 37.7, 20.4. HRMS calcd. (ESI) $\mathrm{m} / \mathrm{z}$ for $\mathrm{C}_{20} \mathrm{H}_{19} \mathrm{NNaO}$ : $[\mathrm{M}+\mathrm{Na}]^{+} 312.1359$, found: 312.1362 .

Chiral HPLC (Chiralpak IG, 4.6x250 mm; $5 \mu \mathrm{L}$, hexane/isopropanol = 90/10, flow $1.0 \mathrm{~mL} / \mathrm{min}, 254 \mathrm{~nm}$ ): $\mathrm{t}_{\mathrm{R}}=14.72 \mathrm{~min}$ (major), $\mathrm{t}_{\mathrm{R}}=16.32 \mathrm{~min}$ (minor).
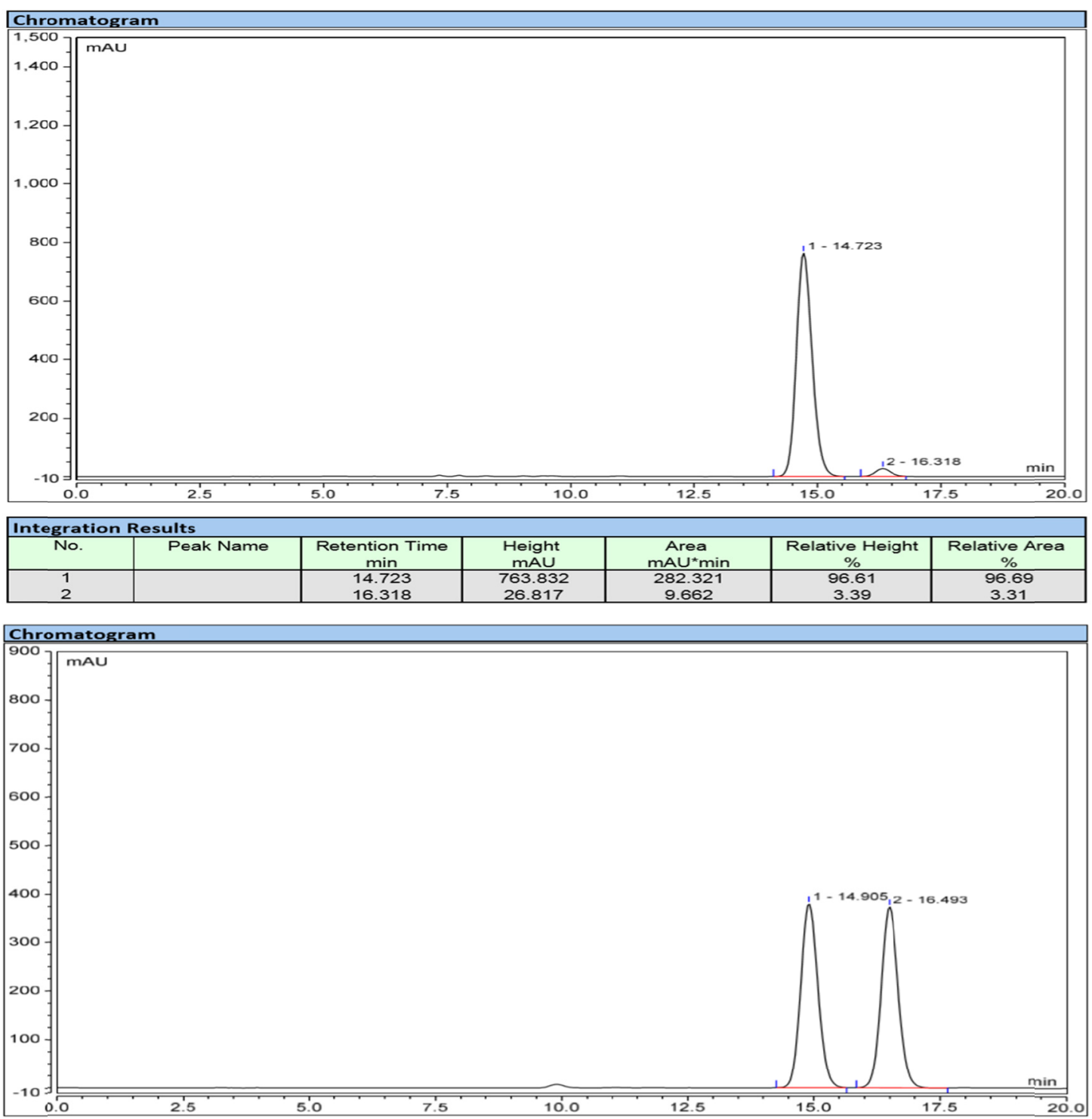

\begin{tabular}{|c|c|c|c|c|c|c|}
\hline Integration Results & \multicolumn{5}{|c|}{} \\
\hline No. & Peak Name & $\begin{array}{c}\text { Retention Time } \\
\text { min }\end{array}$ & $\begin{array}{c}\text { Height } \\
\text { mAU }\end{array}$ & $\begin{array}{c}\text { Area } \\
\text { mAUmin }\end{array}$ & $\begin{array}{c}\text { Relative Height } \\
\%\end{array}$ & $\begin{array}{c}\text { Relative Area } \\
\%\end{array}$ \\
\hline 1 & & 14.905 & 380.794 & 150.020 & 50.39 & 50.04 \\
2 & & 16.493 & 374.839 & 149.777 & 49.61 & 49.96 \\
\hline
\end{tabular}




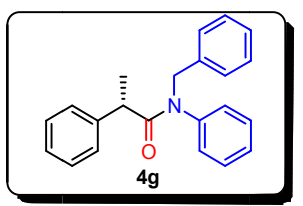

\section{(S)- $N$-benzyl- $N$,2-diphenylpropanamide}

$87 \%$ yield, $\mathrm{b} / \mathrm{l}>99: 1, \mathrm{ee}=85 \%,[\alpha] \mathrm{D}^{19}=-2.9\left(\mathrm{c}=0.7, \mathrm{CHCl}_{3}\right) .{ }^{1} \mathrm{H}$ NMR $(600$ $\left.\mathrm{MHz}, \mathrm{CDCl}_{3}\right) \delta 7.51-7.11(\mathrm{~m}, 11 \mathrm{H}), 7.01(\mathrm{~d}, J=7.4 \mathrm{~Hz}, 2 \mathrm{H}), 6.74(\mathrm{~s}, 2 \mathrm{H}), 4.89-4.81(\mathrm{~m}$, $2 \mathrm{H}), 3.61(\mathrm{q}, J=6.6 \mathrm{~Hz}, 1 \mathrm{H}), 1.41(\mathrm{~d}, J=6.6 \mathrm{~Hz}, 3 \mathrm{H}) ;{ }^{13} \mathrm{C} \mathrm{NMR}\left(151 \mathrm{MHz}, \mathrm{CDCl}_{3}\right) \delta$ 173.7, 141.9, 141.8, 137.5, 129.1, 128.8, 128.6, 128.24, 128.19, 127.8, 127.5, 127.1, 126.5, 53.2, 43.4, 20.3. HRMS calcd. (ESI) $\mathrm{m} / \mathrm{z}$ for $\mathrm{C}_{22} \mathrm{H}_{21} \mathrm{NNaO}$ : [M+Na] $]^{+} 338.1515$, found: 338.1518 .

Chiral HPLC (Chiralpak IG, 4.6x250 mm; $5 \mu \mathrm{L}$, hexane/isopropanol = 90/10, flow $1.0 \mathrm{~mL} / \mathrm{min}, 254 \mathrm{~nm}$ ): $\mathrm{t}_{\mathrm{R}}=8.67 \mathrm{~min}$ (major), $\mathrm{t}_{\mathrm{R}}=11.52 \mathrm{~min}$ (minor).

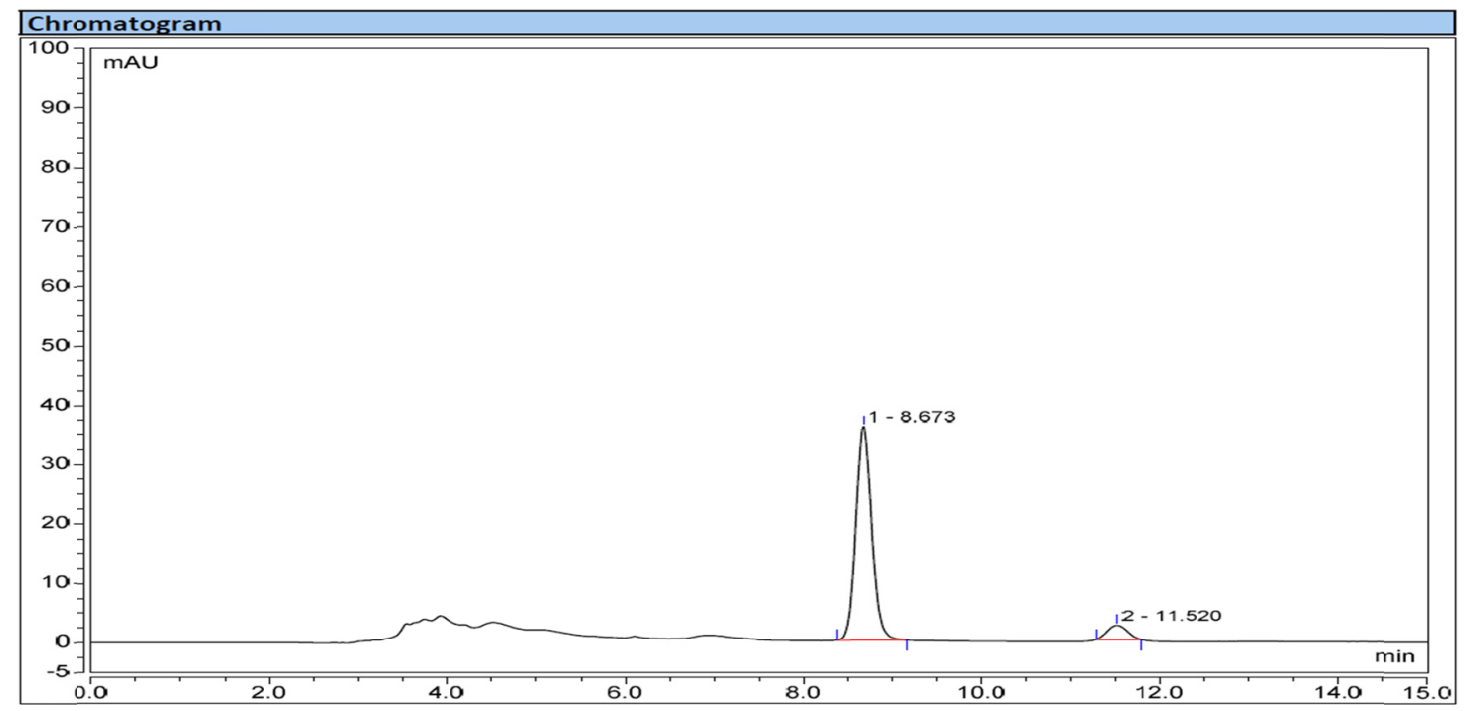

\begin{tabular}{|c|c|c|c|c|c|c|}
\hline \multicolumn{9}{|l|}{ Integration Results } \\
\hline No. & Peak Name & $\begin{array}{c}\text { Retention Time } \\
\text { min }\end{array}$ & $\begin{array}{c}\text { Height } \\
\text { mAU }\end{array}$ & $\begin{array}{c}\text { Area } \\
\text { mAU*min }\end{array}$ & $\begin{array}{c}\text { Relative Height } \\
\%\end{array}$ & $\begin{array}{c}\text { Relative Area } \\
\%\end{array}$ \\
\hline 1 & & 8.673 & 36.095 & 7.485 & 93.72 & 92.38 \\
2 & & 11.520 & 2.420 & 0.618 & 6.28 & 7.62 \\
\hline
\end{tabular}

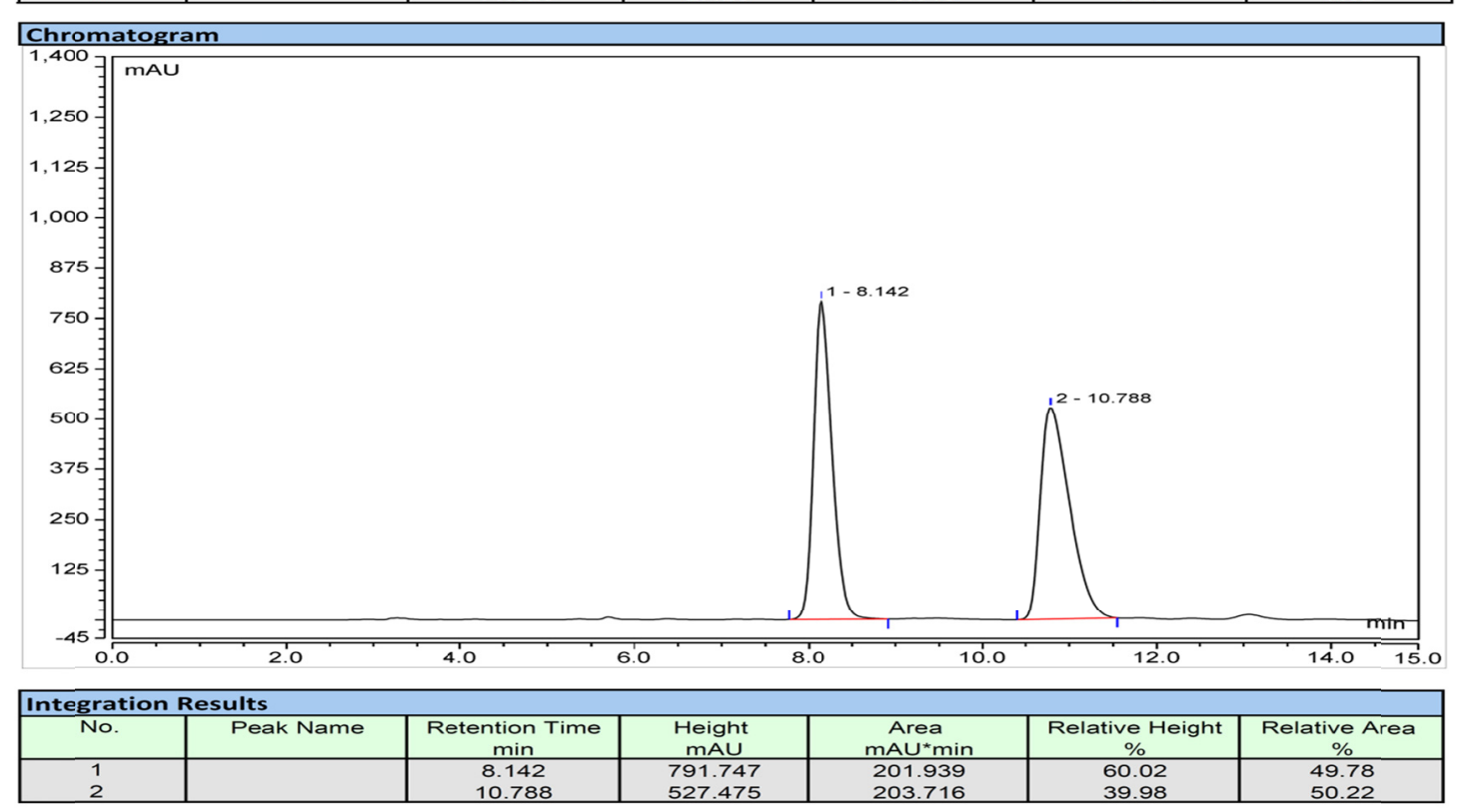




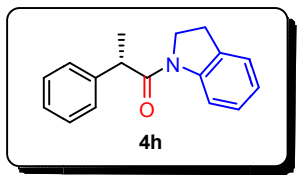

\section{(S)-1-(indolin-1-yl)-2-phenylpropan-1-one}

$95 \%$ yield, $\mathrm{b} / \mathrm{l}>99: 1, \mathrm{ee}=92 \%,[\alpha] \mathrm{D}^{18}=+84.6\left(\mathrm{c}=1.8, \mathrm{CHCl}_{3}\right) .{ }^{1} \mathrm{H}$ NMR $(600$ $\left.\mathrm{MHz}, \mathrm{CDCl}_{3}\right) \delta 8.33(\mathrm{~d}, J=8.0 \mathrm{~Hz}, 1 \mathrm{H}), 7.47-7.32(\mathrm{~m}, 4 \mathrm{H}), 7.27-7.122(\mathrm{~m}, 1 \mathrm{H})$, 7.21-7.15 (m, 1H), $7.11(\mathrm{~d}, J=7.2 \mathrm{~Hz}, 1 \mathrm{H}), 6.98(\mathrm{t}, J=7.2 \mathrm{~Hz}, 1 \mathrm{H}), 4.22-4.03(\mathrm{~m}, 1 \mathrm{H})$, 3.92-3.81 (m, $1 \mathrm{H}), 3.80-3.65(\mathrm{~m}, 1 \mathrm{H}), 3.12-3.07(\mathrm{~m}, 1 \mathrm{H}), 3.04-2.86(\mathrm{~m}, 1 \mathrm{H}), 1.52(\mathrm{~d}, J$ $=6.8 \mathrm{~Hz}, 3 \mathrm{H}) ;{ }^{13} \mathrm{C} \mathrm{NMR}\left(151 \mathrm{MHz}, \mathrm{CDCl}_{3}\right) \delta 171.9,143.3,141.1,131.0,128.9,127.5$, 126.9, 124.4, 123.6, 120.8, 117.2, 47.6, 46.2, 27.9, 20.4. HRMS calcd. (ESI) $\mathrm{m} / \mathrm{z}$ for $\mathrm{C}_{17} \mathrm{H}_{17} \mathrm{NNaO}:[\mathrm{M}+\mathrm{Na}]^{+}$274.1202, found: 274.1206.

Chiral HPLC (Chiralpak IG, 4.6x250 mm; $5 \mu \mathrm{L}$, hexane/isopropanol = 90/10, flow $1.0 \mathrm{~mL} / \mathrm{min}, 254 \mathrm{~nm}$ ): $\mathrm{t}_{\mathrm{R}}=14.01 \mathrm{~min}$ (major), $\mathrm{t}_{\mathrm{R}}=16.14 \mathrm{~min}$ (minor).
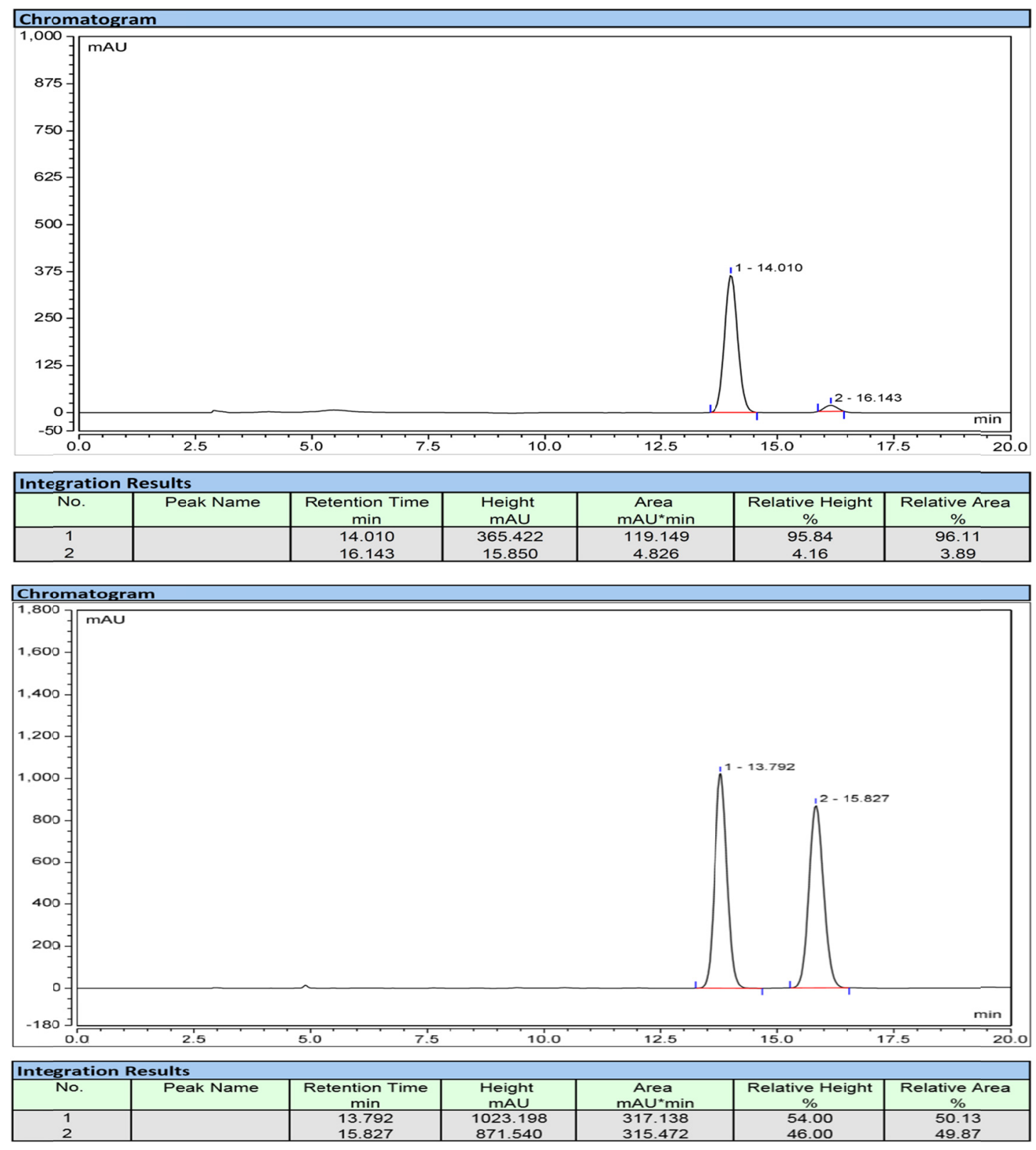


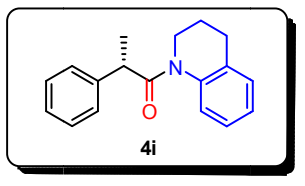

\section{(S)-1-(3,4-dihydroquinolin-1(2H)-yl)-2-phenylpropan-1-one}

$91 \%$ yield, $\mathrm{b} / 1>99: 1, \mathrm{ee}=90 \%,[\alpha] \mathrm{D}^{16}=+14.1\left(\mathrm{c}=1.4, \mathrm{CHCl}_{3}\right) .{ }^{1} \mathrm{H}$ NMR $(600$ $\left.\mathrm{MHz}, \mathrm{CDCl}_{3}\right) \delta$ 7.66-6.64 (m, 10H), $4.22(\mathrm{~s}, 1 \mathrm{H}), 3.85(\mathrm{~s}, 1 \mathrm{H}), 3.61(\mathrm{q}, J=7.2,1 \mathrm{H})$, 2.64-2.48 (m, 1H), $2.30(\mathrm{~s}, 1 \mathrm{H}), 2.07-1.67(\mathrm{~m}, 2 \mathrm{H}), 1.48(\mathrm{~d}, J=7.2 \mathrm{~Hz}, 3 \mathrm{H}) ;{ }^{13} \mathrm{C} \mathrm{NMR}$ $\left(151 \mathrm{MHz}, \mathrm{CDCl}_{3}\right) \delta 174.0,141.7,139.3,128.4,128.3,127.3,126.6,125.9,125.6$, 125.0, 42.9, 26.2, 23.8, 20.4. HRMS calcd. (ESI) $\mathrm{m} / \mathrm{z}$ for $\mathrm{C}_{18} \mathrm{H}_{19} \mathrm{NNaO}$ : $[\mathrm{M}+\mathrm{Na}]^{+}$ 288.1359, found: 288.1364 .

Chiral HPLC (Chiralpak IB N-5, 4.6x250 mm; $5 \mu \mathrm{L}$, hexane/isopropanol = 95/5, flow $1.0 \mathrm{~mL} / \mathrm{min}, 254 \mathrm{~nm}$ ): $\mathrm{t}_{\mathrm{R}}=7.00 \mathrm{~min}$ (major), $\mathrm{t}_{\mathrm{R}}=7.40 \mathrm{~min}$ (minor).
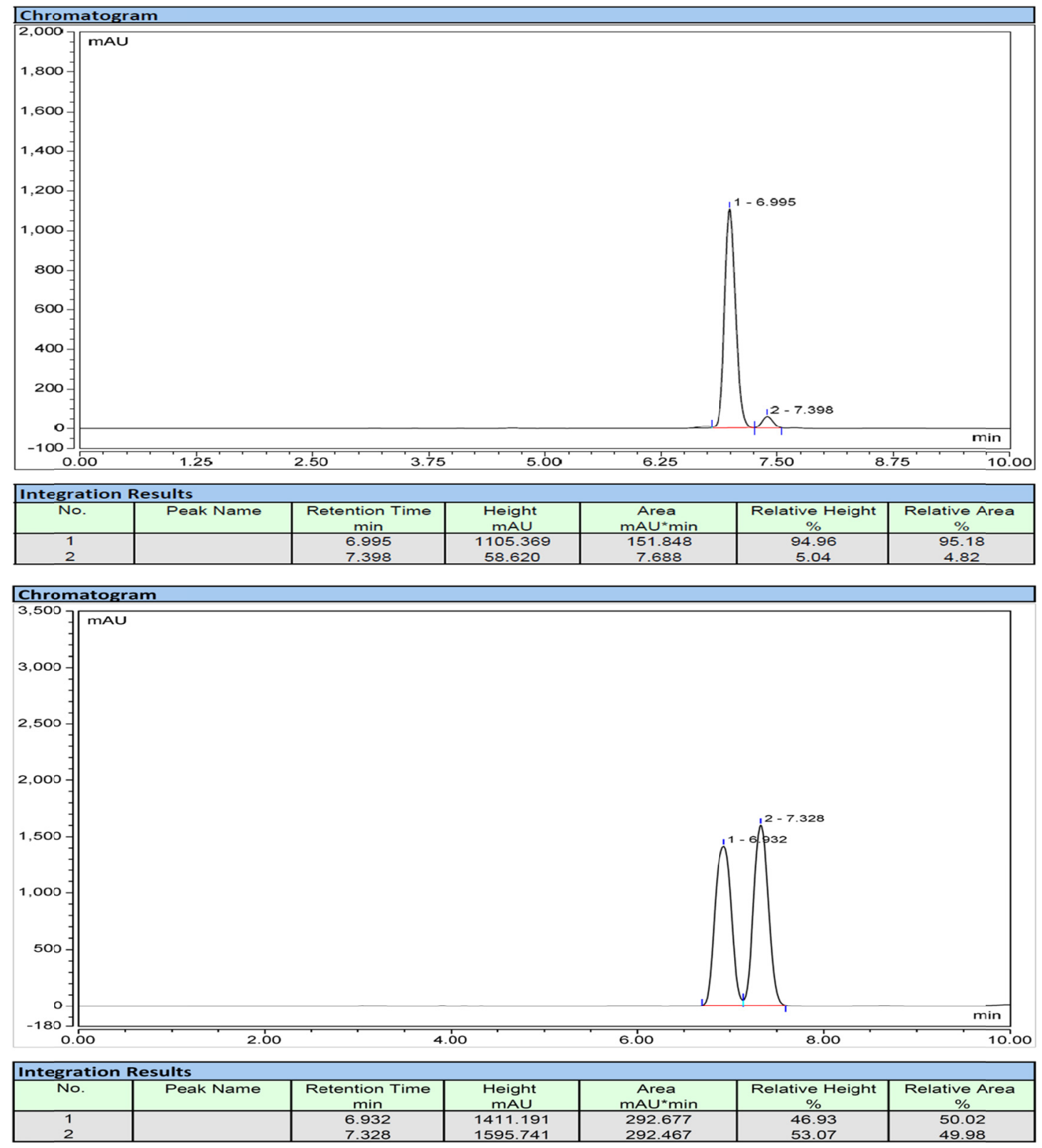


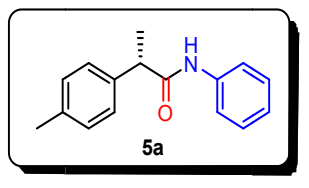

\section{(S)-N-phenyl-2-(p-tolyl)propanamide}

$94 \%$ yield, $\mathrm{b} />99: 1, \mathrm{ee}=94 \%,[\alpha] \mathrm{D}^{19}=+49.0\left(\mathrm{c}=1.6, \mathrm{CHCl}_{3}\right) .{ }^{1} \mathrm{H}$ NMR $(400$ $\left.\mathrm{MHz}, \mathrm{CDCl}_{3}\right) \delta 7.41(\mathrm{~d}, J=8.0 \mathrm{~Hz}, 2 \mathrm{H}), 7.27-7.23(\mathrm{~m}, 4 \mathrm{H}), 7.17(\mathrm{~d}, J=8.0 \mathrm{~Hz}, 3 \mathrm{H})$, 7.05 (t, $J=7.2 \mathrm{~Hz}, 1 \mathrm{H}), 3.68(\mathrm{q}, J=7.2 \mathrm{~Hz}, 1 \mathrm{H}), 2.34(\mathrm{~s}, 3 \mathrm{H}), 1.57$ (d, $J=7.2 \mathrm{~Hz}, 3 \mathrm{H})$; ${ }^{13} \mathrm{C}$ NMR $\left(151 \mathrm{MHz}, \mathrm{CDCl}_{3}\right) \delta 172.8,137.91,137.86,137.0,129.6,128.7,127.4$, 124.0, 119.8, 47.3, 20.9, 18.5. HRMS calcd. (ESI) $\mathrm{m} / \mathrm{z}$ for $\mathrm{C}_{16} \mathrm{H}_{17} \mathrm{NNaO}$ : $[\mathrm{M}+\mathrm{Na}]^{+}$ 262.1202, found: 262.1204 .

Chiral HPLC (Chiralpak IG, 4.6x250 mm; $5 \mu \mathrm{L}$, hexane/isopropanol = 90/10, flow $1.0 \mathrm{~mL} / \mathrm{min}, 254 \mathrm{~nm}$ ): $\mathrm{t}_{\mathrm{R}}=13.39 \mathrm{~min}($ major$), \mathrm{t}_{\mathrm{R}}=16.84 \mathrm{~min}$ (minor).
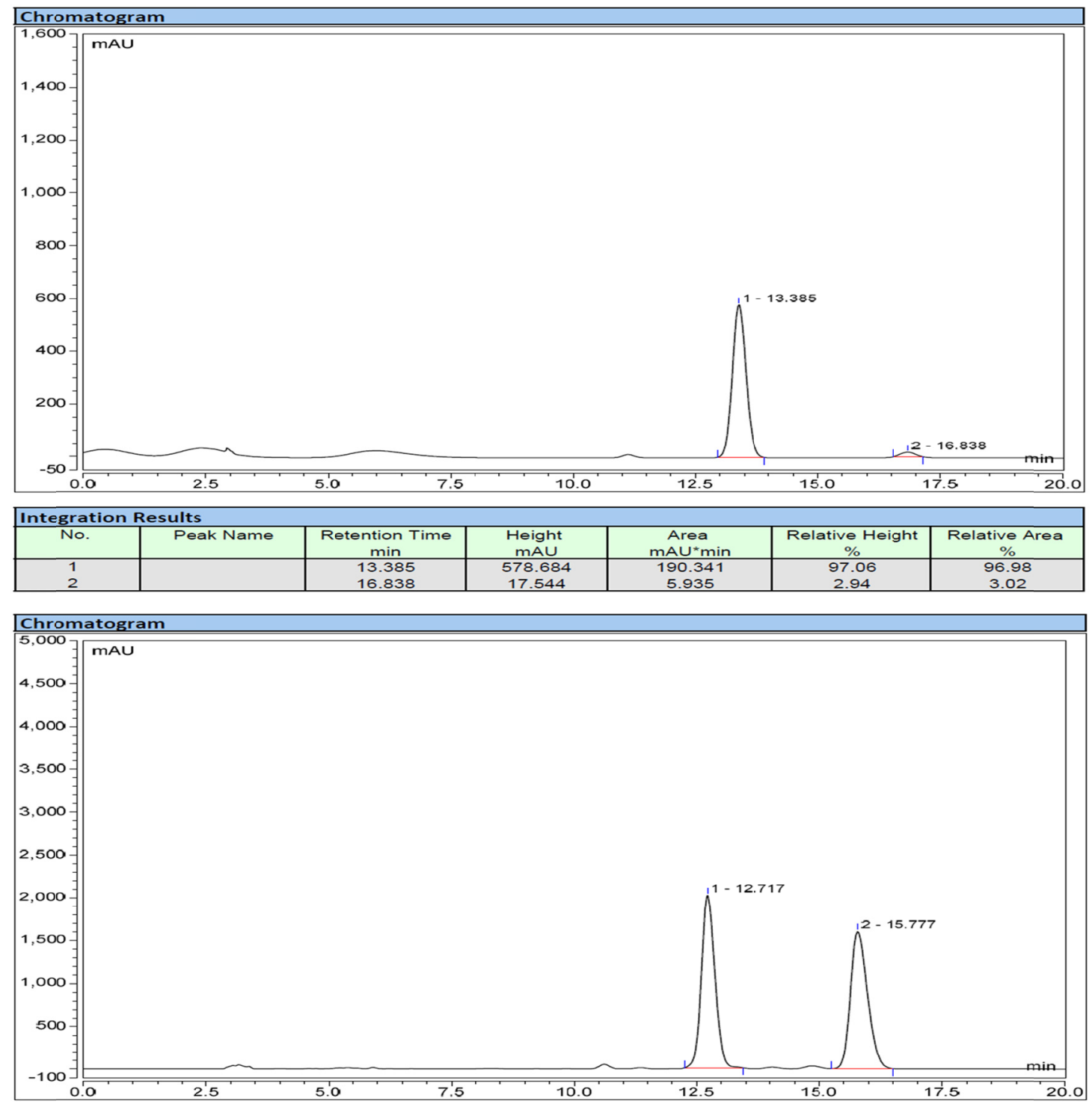

\begin{tabular}{|c|c|c|c|c|c|c|}
\hline Integration Results & \multicolumn{9}{|c|}{ Reight } \\
\hline No. & Peak Name & $\begin{array}{c}\text { Retention Time } \\
\text { min }\end{array}$ & $\begin{array}{c}\text { Area } \\
\text { mAU }\end{array}$ & $\begin{array}{c}\text { Relative Height } \\
\%\end{array}$ & $\begin{array}{c}\text { Relative Area } \\
\%\end{array}$ \\
\hline 1 & & 12.717 & 2015.368 & 643.756 & 55.75 & 50.08 \\
2 & & 15.777 & 1599.369 & 641.621 & 44.25 & 49.92 \\
\hline
\end{tabular}




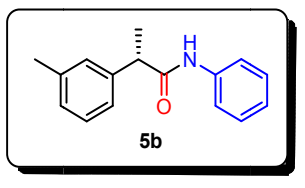

\section{(S)- $N$-phenyl-2-(m-tolyl)propanamide}

97\% yield, b/1 $>99: 1$, ee $=94 \%,[\alpha] \mathrm{D}^{17}=+61.1\left(\mathrm{c}=1.5, \mathrm{CHCl}_{3}\right) .{ }^{1} \mathrm{H}$ NMR $(400$ $\left.\mathrm{MHz}, \mathrm{CDCl}_{3}\right) \delta 7.42(\mathrm{~d}, J=8.0 \mathrm{~Hz}, 2 \mathrm{H}), 7.27-7.23(\mathrm{~m}, 3 \mathrm{H}), 7.20(\mathrm{~s}, 1 \mathrm{H}), 7.16-7.10$ (m, 3H), 7.05 (t, $J=7.6 \mathrm{~Hz}, 1 \mathrm{H}), 3.67$ (q, $J=7.2 \mathrm{~Hz}, 1 \mathrm{H}), 2.35$ (s, 3H), 1.57 (d, $J=$ $7.2 \mathrm{~Hz}, 3 \mathrm{H}) ;{ }^{13} \mathrm{C}$ NMR $\left(101 \mathrm{MHz}, \mathrm{CDCl}_{3}\right) \delta 172.4,140.8,138.8,137.9,129.0,128.8$, $128.35,128.28,124.7,124.1,119.6,48.0,21.4,18.5$. HRMS calcd. (ESI) $\mathrm{m} / \mathrm{z}$ for $\mathrm{C}_{16} \mathrm{H}_{17} \mathrm{NNaO}:[\mathrm{M}+\mathrm{Na}]^{+} 262.1202$, found: 262.1205 .

Chiral HPLC (Chiralpak IG, 4.6x250 mm; $5 \mu \mathrm{L}$, hexane/isopropanol = 85/15, flow $1.0 \mathrm{~mL} / \mathrm{min}, 254 \mathrm{~nm}$ ): $\mathrm{t}_{\mathrm{R}}=7.89 \mathrm{~min}$ (major), $\mathrm{t}_{\mathrm{R}}=9.13 \mathrm{~min}$ (minor).
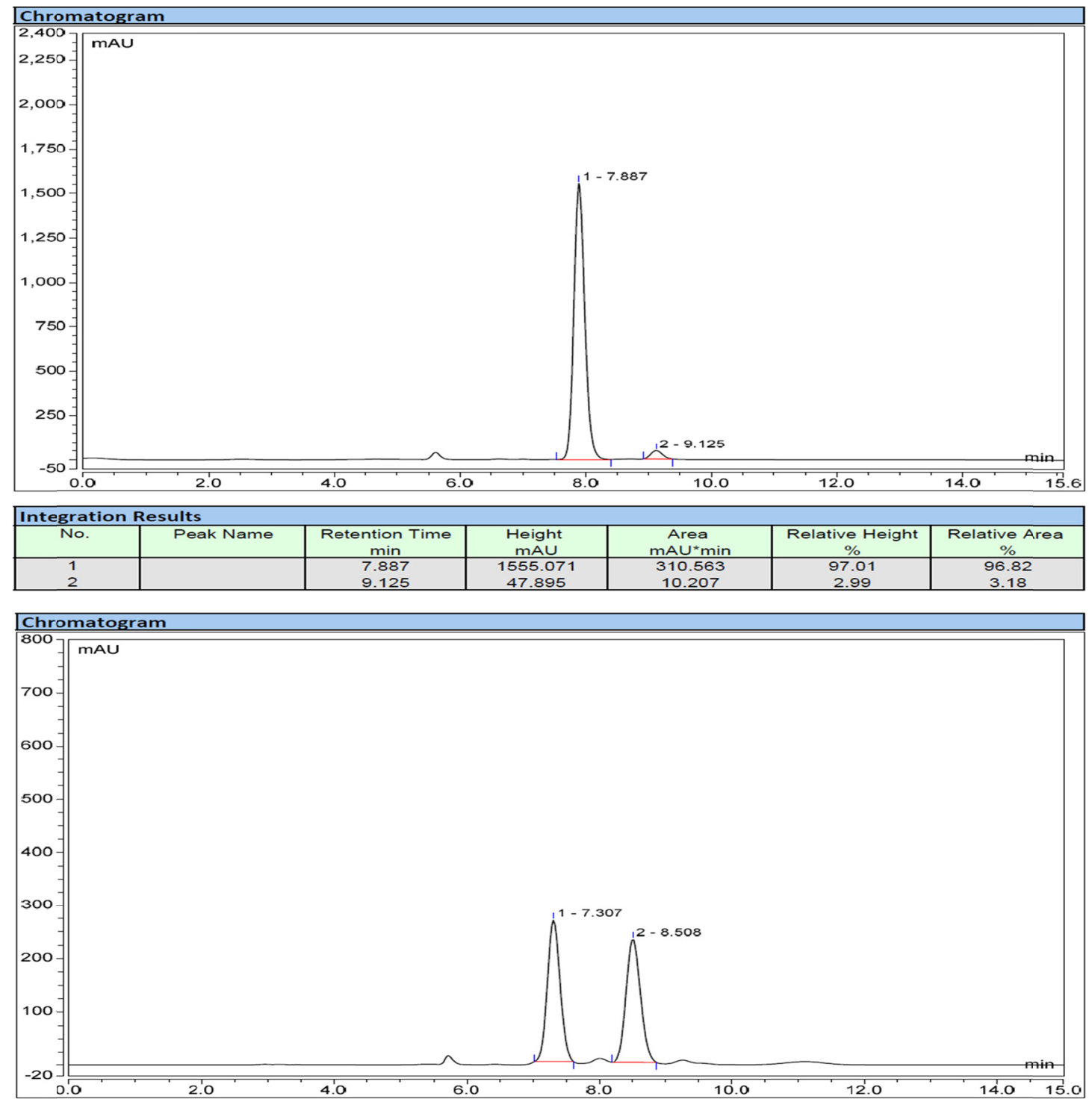

\begin{tabular}{|c|c|c|c|c|c|c|}
\hline Integration Results & \multicolumn{9}{|c|}{$\begin{array}{c}\text { Area } \\
\text { Noight } \\
\text { ma }\end{array}$} & Peak Name & $\begin{array}{c}\text { Retention Time } \\
\text { min }\end{array}$ & $\begin{array}{c}\text { Relative Height } \\
\%\end{array}$ & $\begin{array}{c}\text { Relative Area } \\
\%\end{array}$ \\
\hline 1 & & 7.307 & 265.882 & 59.384 & 53.50 & 50.27 \\
2 & & 8.508 & 231.102 & 58.748 & 46.50 & 49.73 \\
\hline
\end{tabular}




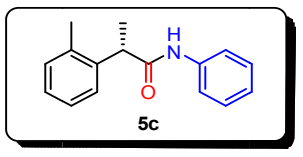

\section{(S)-N-phenyl-2-(o-tolyl)propanamide}

93\% yield, b/1 > 99:1, ee $=90 \%,[\alpha] \mathrm{D}^{17}=+17.3\left(\mathrm{c}=1.6, \mathrm{CHCl}_{3}\right) .{ }^{1} \mathrm{H}$ NMR $(400$ $\left.\mathrm{MHz}, \mathrm{CDCl}_{3}\right) \delta 7.44-7.34(\mathrm{~m}, 3 \mathrm{H}), 7.28-7.23(\mathrm{~m}, 5 \mathrm{H}), 7.06(\mathrm{t}, J=7.6 \mathrm{~Hz}, 1 \mathrm{H}), 6.92(\mathrm{~s}$, $1 \mathrm{H}), 3.93(\mathrm{q}, J=7.2 \mathrm{~Hz}, 1 \mathrm{H}), 2.37(\mathrm{~s}, 3 \mathrm{H}), 1.61(\mathrm{~d}, J=7.2 \mathrm{~Hz}, 3 \mathrm{H}) ;{ }^{13} \mathrm{C}$ NMR $(101$ $\left.\mathrm{MHz}, \mathrm{CDCl}_{3}\right) \delta 172.6,138.8,137.8,136.4,131.1,128.9,127.6,127.00,126.96,124.2$, 119.7, 44.7, 19.6, 17.6. HRMS calcd. (ESI) $\mathrm{m} / \mathrm{z}$ for $\mathrm{C}_{16} \mathrm{H}_{17} \mathrm{NNaO}$ : $[\mathrm{M}+\mathrm{Na}]^{+} 262.1202$, found: 262.1199 .

Chiral HPLC (Chiralpak IG, 4.6x250 mm; $5 \mu \mathrm{L}$, hexane/isopropanol $=90 / 10$, flow $1.0 \mathrm{~mL} / \mathrm{min}, 254 \mathrm{~nm}$ ): $\mathrm{t}_{\mathrm{R}}=8.22 \mathrm{~min}$ (major), $\mathrm{t}_{\mathrm{R}}=9.75 \mathrm{~min}$ (minor).

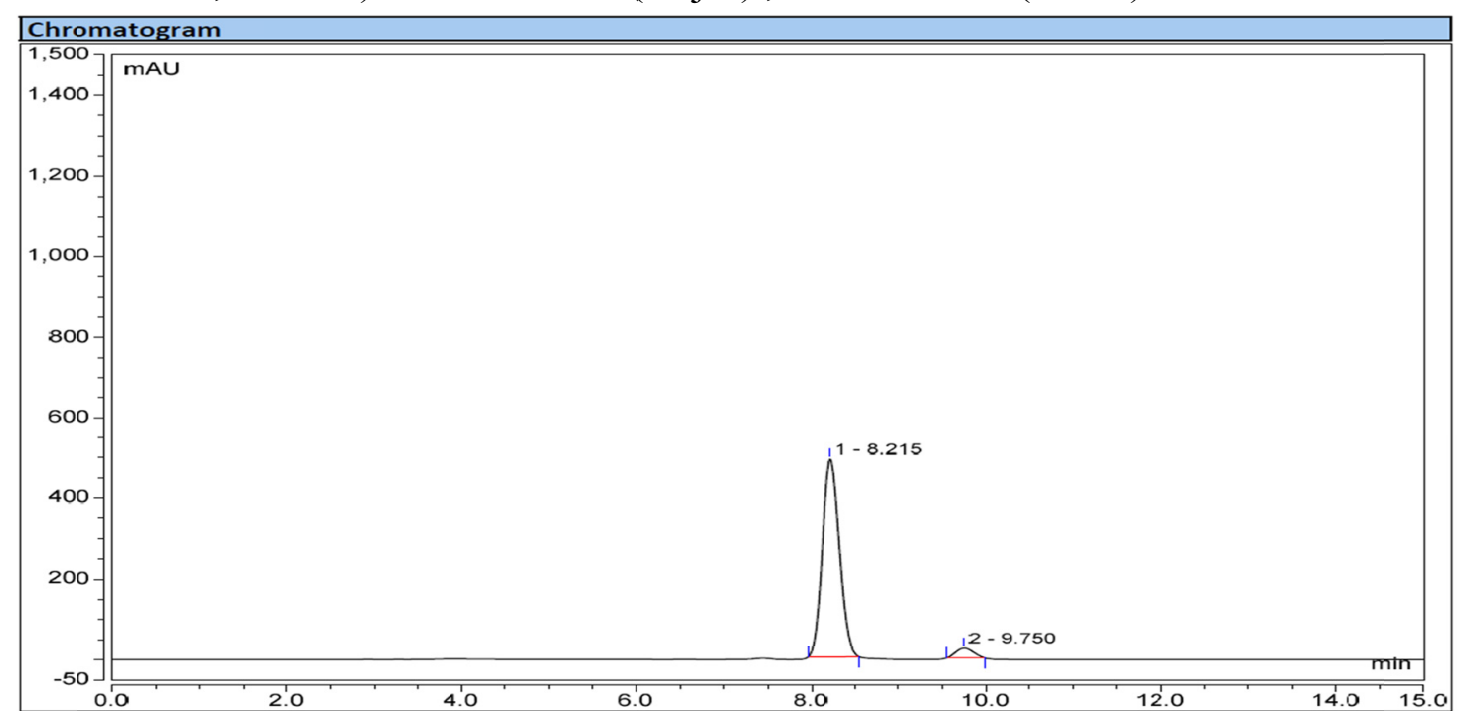

\begin{tabular}{|c|c|c|c|c|c|c|}
\hline \multicolumn{9}{|l|}{ Integration Results } & \multicolumn{5}{|c|}{ Reak Name } & $\begin{array}{c}\text { Retention Time } \\
\text { min }\end{array}$ & $\begin{array}{c}\text { Height } \\
\text { mAU }\end{array}$ & $\begin{array}{c}\text { Area } \\
\text { mAU*min }\end{array}$ & $\begin{array}{c}\text { Relative Height } \\
\%\end{array}$ & $\begin{array}{c}\text { Relative Area } \\
\%\end{array}$ \\
\hline No. & Peak & 4.215 & 489.707 & 11.664 & 95.22 & 95.14 \\
2 & & 9.750 & 24.589 & 5.706 & 4.78 & 4.86 \\
\hline
\end{tabular}

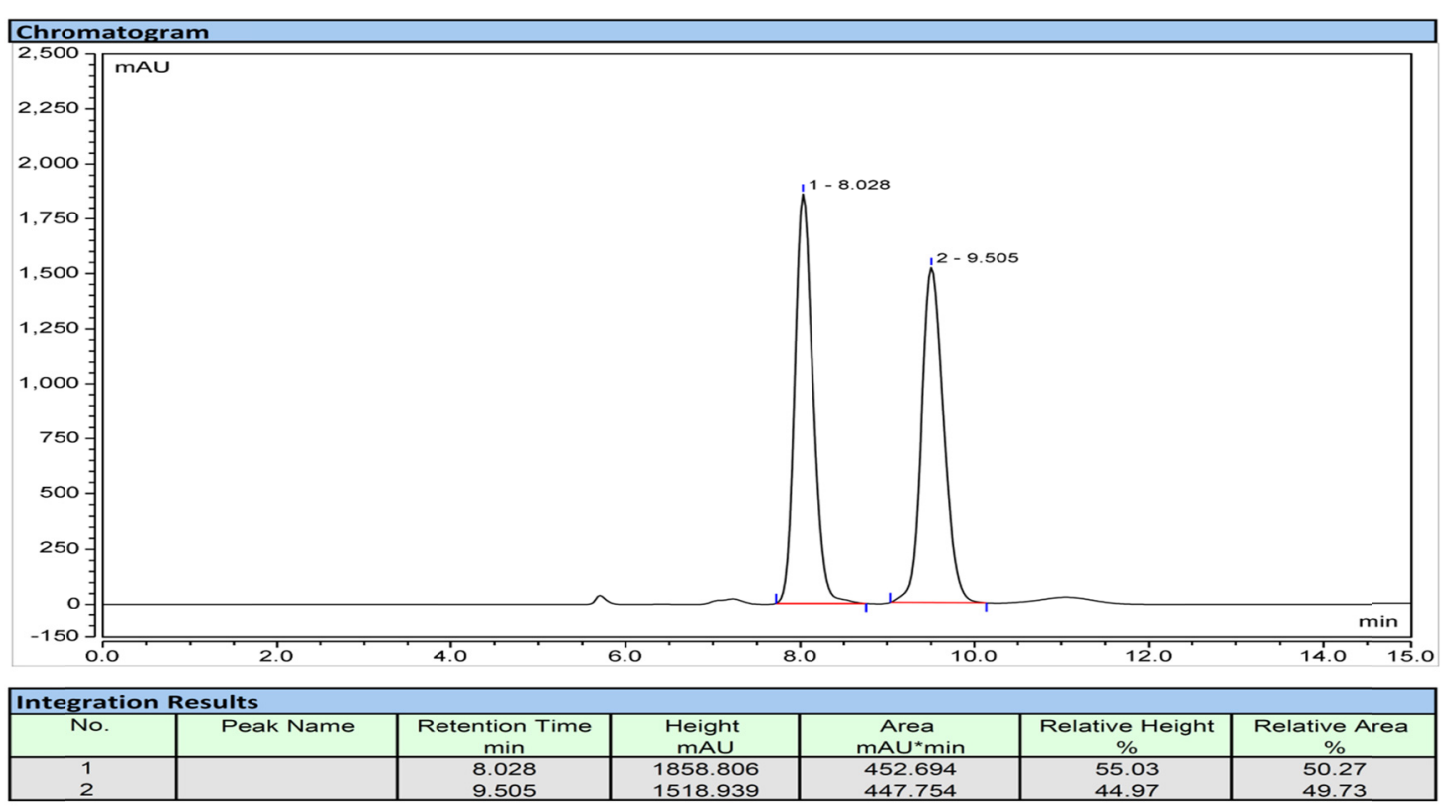




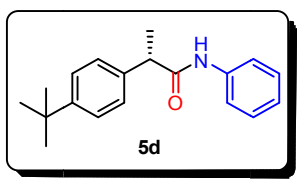

\section{(S)-2-(4-(tert-butyl)phenyl)- $N$-phenylpropanamide}

93\% yield, b/1 > 99: 1 , ee $=95 \%,[\alpha] \mathrm{D}^{16}=+26.6\left(\mathrm{c}=2.0, \mathrm{CHCl}_{3}\right) .{ }^{1} \mathrm{H}$ NMR $(400$ $\left.\mathrm{MHz}, \mathrm{CDCl}_{3}\right) \delta$ 7.43-7.37 (m, 4H), 7.29-7.23 (m, 4H), $7.19(\mathrm{~s}, 1 \mathrm{H}), 7.015(\mathrm{t}, J=7.6 \mathrm{~Hz}$, $1 \mathrm{H}), 3.68$ (q, $J=7.2 \mathrm{~Hz}, 1 \mathrm{H}), 1.58(\mathrm{~d}, J=7.2 \mathrm{~Hz}, 3 \mathrm{H}), 1.32(\mathrm{~s}, 9 \mathrm{H}) ;{ }^{13} \mathrm{C}$ NMR $(151$ $\left.\mathrm{MHz}, \mathrm{CDCl}_{3}\right) \delta 172.7,150.3,137.9,137.7,128.8,127.3,125.9,124.1,119.7,47.5$, 34.4, 31.3, 18.5. HRMS calcd. (ESI) $\mathrm{m} / \mathrm{z}$ for $\mathrm{C}_{19} \mathrm{H}_{23} \mathrm{NNaO}$ : $[\mathrm{M}+\mathrm{Na}]^{+} 304.1672$, found: 304.1678 .

Chiral HPLC (Chiralpak IG, 4.6x250 mm; $5 \mu \mathrm{L}$, hexane/isopropanol = 90/10, flow $1.0 \mathrm{~mL} / \mathrm{min}, 254 \mathrm{~nm}$ ): $\mathrm{t}_{\mathrm{R}}=7.86 \mathrm{~min}$ (major), $\mathrm{t}_{\mathrm{R}}=10.22 \mathrm{~min}$ (minor).
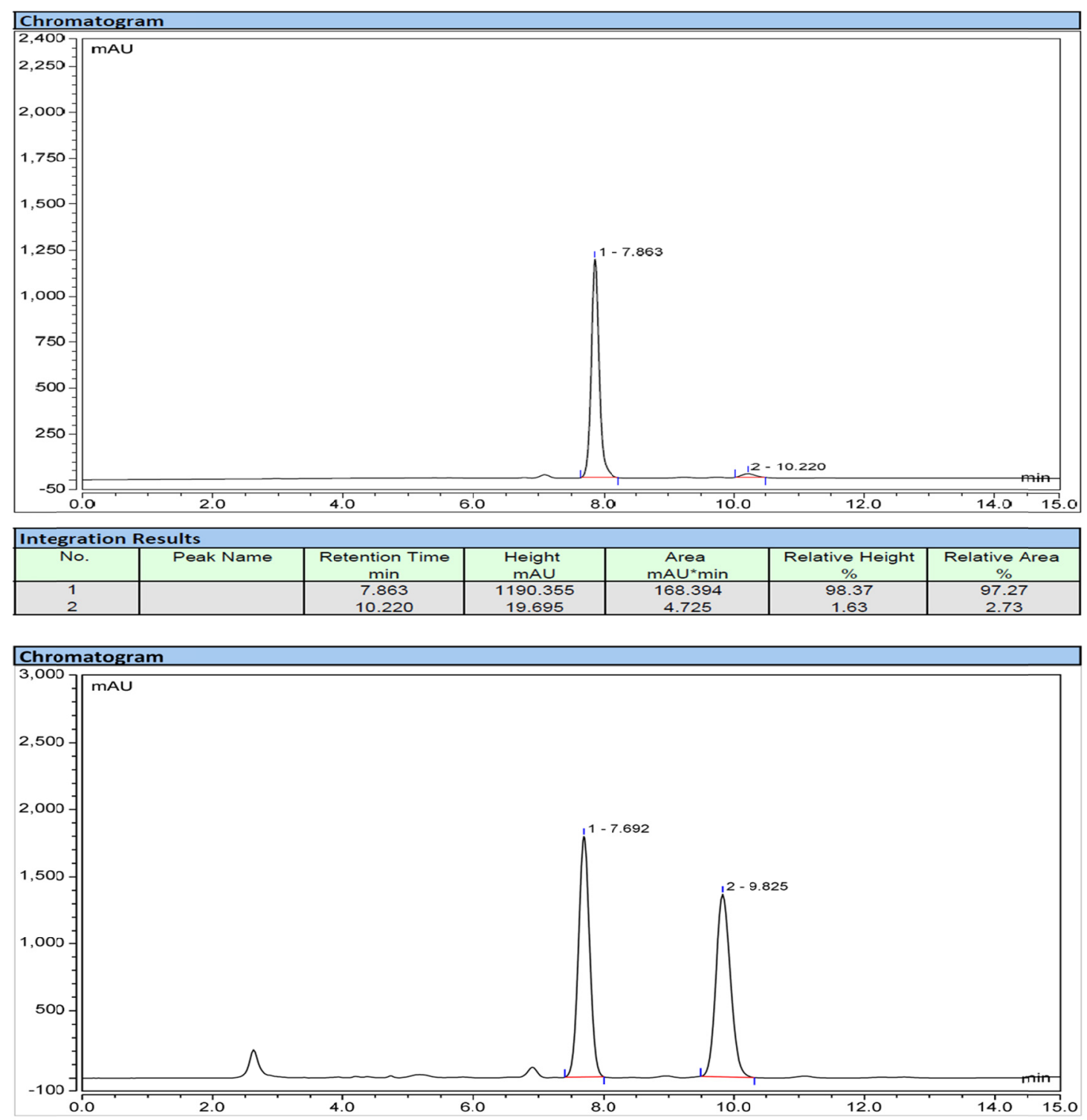

\begin{tabular}{|c|c|c|c|c|c|c|}
\hline Integration Results & \multicolumn{10}{|c|}{ Relative Height } \\
$\%$ & $\begin{array}{c}\text { Relative Area } \\
\%\end{array}$ \\
\hline No. & Peak Name & $\begin{array}{c}\text { Retention Time } \\
\text { min }\end{array}$ & $\begin{array}{c}\text { Height } \\
\text { mAU }\end{array}$ & $\begin{array}{c}\text { Area } \\
\text { mAU*min }\end{array}$ & $\begin{array}{c}\text { Rela } \\
\%\end{array}$ & 50.41 \\
2 & & 7.692 & 1791.203 & 355.931 & 56.82 & 43.18 \\
\hline
\end{tabular}




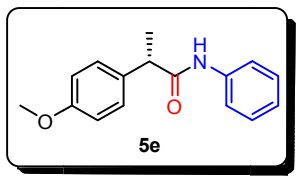

\section{(S)-2-(4-methoxyphenyl)- $N$-phenylpropanamide}

93\% yield, b/1 > 99:1, ee $=91 \%,[\alpha] \mathrm{D}^{20}=+63.4\left(\mathrm{c}=1.5, \mathrm{CHCl}_{3}\right) .{ }^{1} \mathrm{H}$ NMR $(600$ $\left.\mathrm{MHz}, \mathrm{CDCl}_{3}\right) \delta 7.56(\mathrm{~s}, 1 \mathrm{H}), 7.43(\mathrm{~d}, J=8.4 \mathrm{~Hz}, 2 \mathrm{H}), 7.28-7.18(\mathrm{~m}, 4 \mathrm{H}), 7.03(\mathrm{t}, J=7.2$ $\mathrm{Hz}, 1 \mathrm{H}), 6.86$ (d, $J=8.4 \mathrm{~Hz}, 2 \mathrm{H}), 3.77$ (s, 3H), 3.66 (q, $J=7.2 \mathrm{~Hz}, 1 \mathrm{H}), 1.53$ (d, $J=7.2$ $\mathrm{Hz}, 3 \mathrm{H}) ;{ }^{13} \mathrm{C} \mathrm{NMR}\left(151 \mathrm{MHz}, \mathrm{CDCl}_{3}\right) \delta 172.9,158.8,137.9,132.9,128.7,128.6$, 124.1, 119.8, 114.3, 55.2, 46.9, 18.5. HRMS calcd. (ESI) $\mathrm{m} / \mathrm{z}$ for $\mathrm{C}_{16} \mathrm{H}_{17} \mathrm{NNaO}_{2}$ : $[\mathrm{M}+\mathrm{Na}]^{+} 278.1152$, found: 278.1151 .

Chiral HPLC (Chiralpak IG, 4.6x250 mm; $5 \mu \mathrm{L}$, hexane/isopropanol $=85 / 15$, flow $1.0 \mathrm{~mL} / \mathrm{min}, 254 \mathrm{~nm}$ ): $\mathrm{t}_{\mathrm{R}}=12.67 \mathrm{~min}$ (major), $\mathrm{t}_{\mathrm{R}}=16.59 \mathrm{~min}$ (minor).

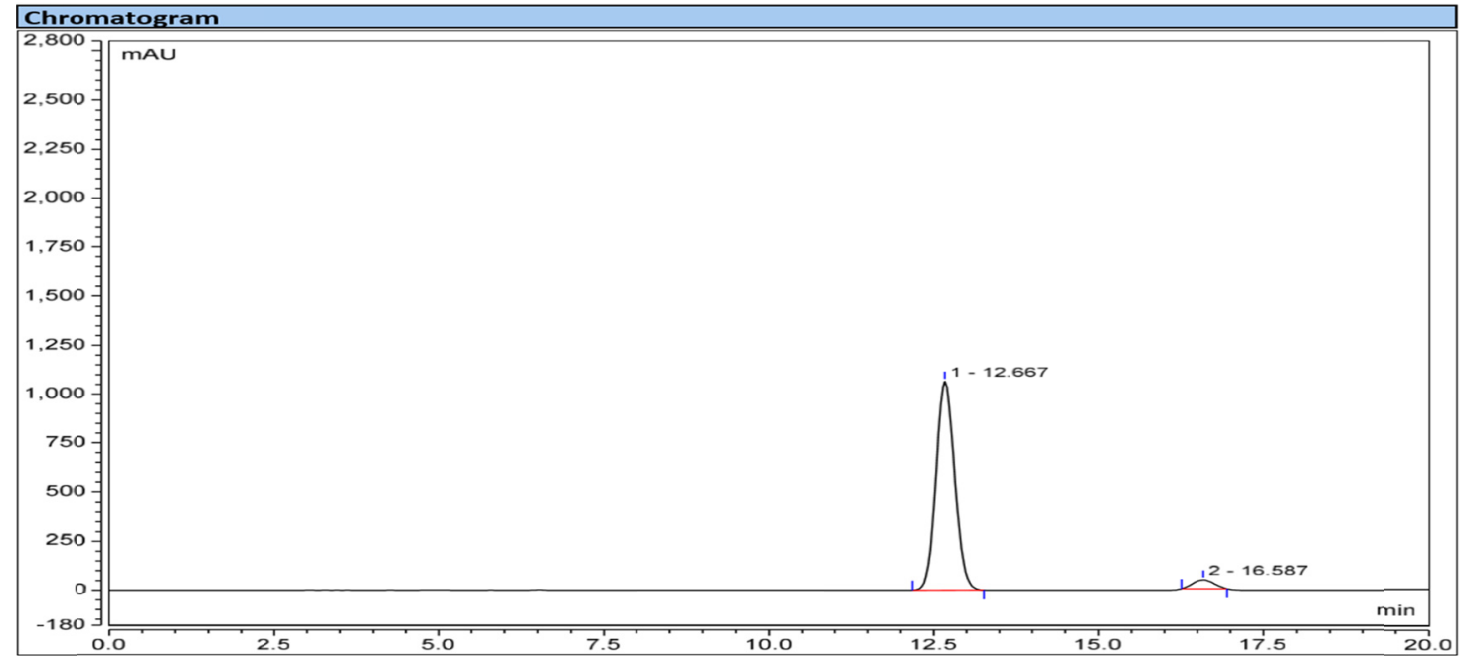

\begin{tabular}{|c|c|c|c|c|c|c|}
\hline \multicolumn{7}{|c|}{ Integration Results } \\
\hline No. & Peak Name & $\begin{array}{c}\text { Retention Time } \\
\text { min }\end{array}$ & $\begin{array}{c}\text { Height } \\
\text { mAU }\end{array}$ & $\begin{array}{c}\text { Area } \\
\text { mAU*min }\end{array}$ & $\begin{array}{c}\text { Relative Height } \\
\%\end{array}$ & $\begin{array}{c}\text { Relative Area } \\
\%\end{array}$ \\
\hline $\begin{array}{l}1 \\
2\end{array}$ & & $\begin{array}{l}12.667 \\
16.587 \\
\end{array}$ & $\begin{array}{c}1065.070 \\
45.341\end{array}$ & $\begin{array}{c}350.571 \\
16.698\end{array}$ & $\begin{array}{c}95.92 \\
4.08\end{array}$ & $\begin{array}{c}95.45 \\
4.55\end{array}$ \\
\hline
\end{tabular}

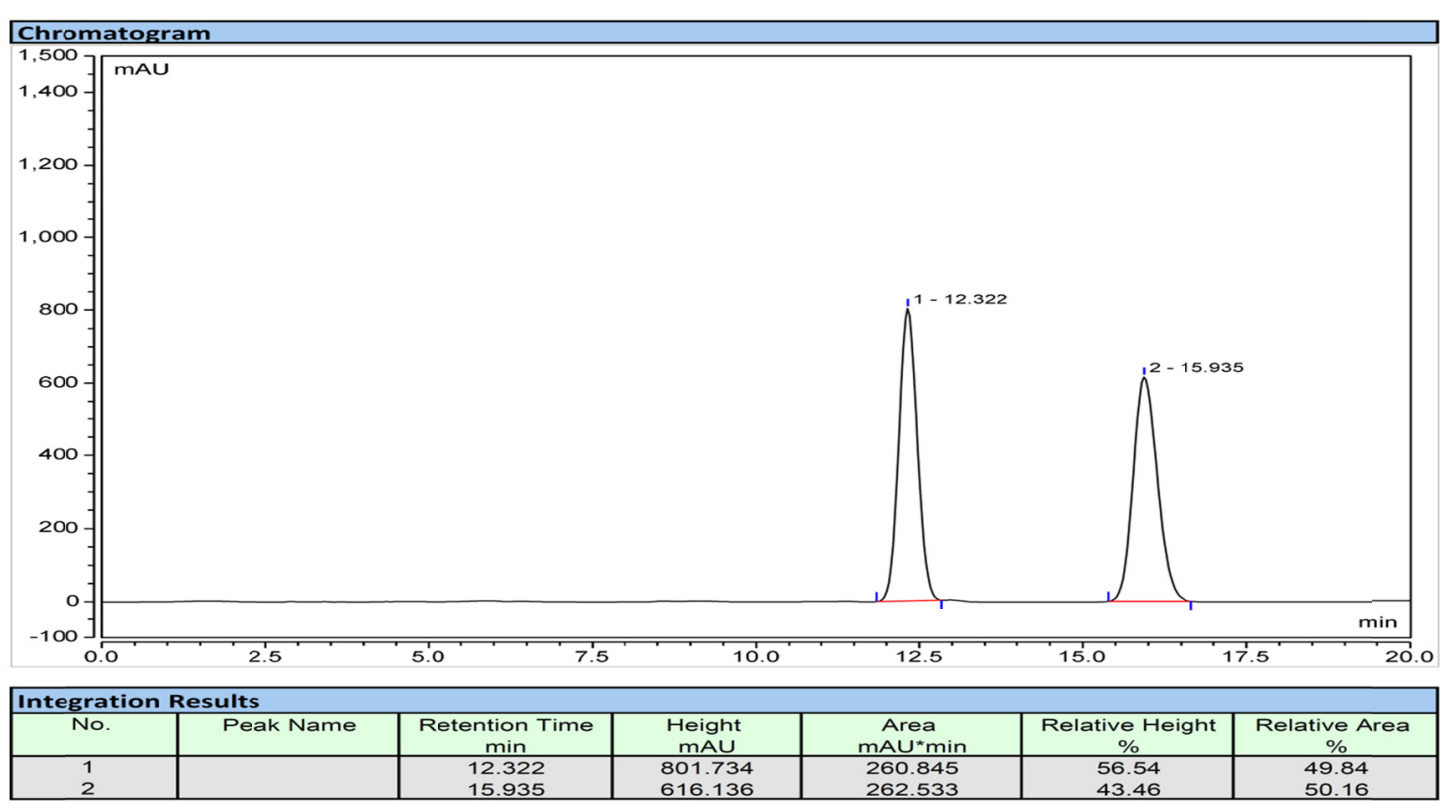




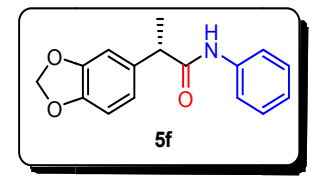

\section{(S)-2-(benzo[d][1,3] dioxol-5-yl)- $N$-phenylpropanamide}

$98 \%$ yield, b/1 > 99:1, ee $=90 \%,[\alpha] \mathrm{D}^{17}=+17.4\left(\mathrm{c}=1.7, \mathrm{CHCl}_{3}\right) .{ }^{1} \mathrm{H}$ NMR $(400$ $\left.\mathrm{MHz}, \mathrm{CDCl}_{3}\right) \delta 7.43(\mathrm{~d}, J=7.2 \mathrm{~Hz}, 2 \mathrm{H}), 7.31(\mathrm{~s}, 1 \mathrm{H}), 7.28-7.24(\mathrm{~m}, 2 \mathrm{H}), 7.06(\mathrm{t}, J=7.2$ $\mathrm{Hz}, 1 \mathrm{H}), 6.85$ (s, 1H), 6.81-6.77 (m, 2H), 5.95 (d, $J=0.8 \mathrm{~Hz}, 2 \mathrm{H}), 3.63$ (q, $J=7.2 \mathrm{~Hz}$, $1 \mathrm{H}), 1.54(\mathrm{~d}, J=7.2 \mathrm{~Hz}, 3 \mathrm{H}) ;{ }^{13} \mathrm{C} \mathrm{NMR}\left(101 \mathrm{MHz}, \mathrm{CDCl}_{3}\right) \delta 172.4,148.2,146.9$, 137.8, 134.6, 128.8, 124.2, 120.9, 119.7, 108.5, 107.9, 101.1, 47.6, 18.6. HRMS calcd. (ESI) $\mathrm{m} / \mathrm{z}$ for $\mathrm{C}_{16} \mathrm{H}_{15} \mathrm{NNaO}_{3}:[\mathrm{M}+\mathrm{Na}]^{+}$292.0944, found: 292.0942 .

Chiral HPLC (Chiralpak IG, 4.6x250 mm; $5 \mu \mathrm{L}$, hexane/isopropanol = 90/10, flow $1.0 \mathrm{~mL} / \mathrm{min}, 254 \mathrm{~nm}$ ): $\mathrm{t}_{\mathrm{R}}=14.76 \mathrm{~min}$ (major), $\mathrm{t}_{\mathrm{R}}=17.03 \mathrm{~min}$ (minor).

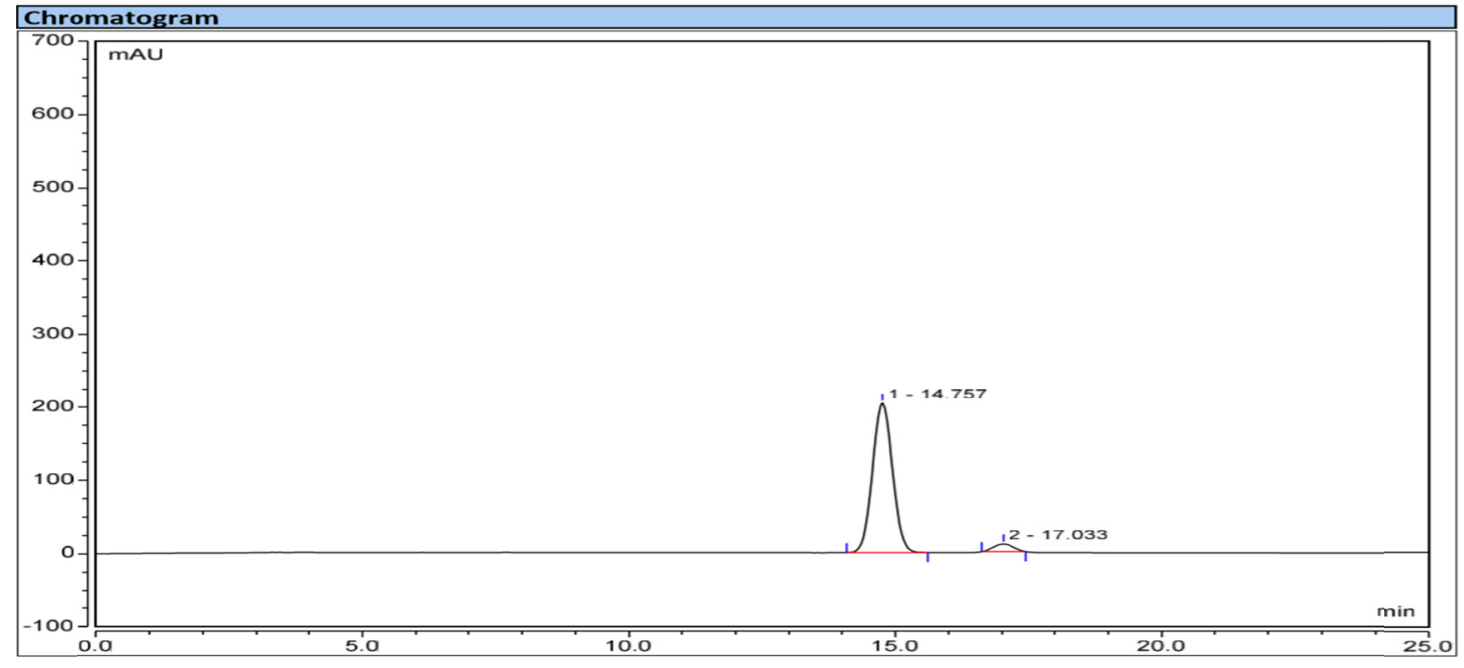

\begin{tabular}{|c|c|c|c|c|c|c|}
\hline Integration Results & \multicolumn{5}{|c|}{ Relative Height } \\
\hline No. & Peak Name & $\begin{array}{c}\text { Retention Time } \\
\text { min }\end{array}$ & $\begin{array}{c}\text { Height } \\
\text { mAU }\end{array}$ & $\begin{array}{c}\text { Area } \\
\text { mAU*min }\end{array}$ & $\begin{array}{c}\text { Relate Area } \\
\%\end{array}$ \\
\hline 1 & & 14.757 & 203.743 & 88.268 & 95.08 & 94 \\
2 & & 17.033 & 10.532 & 4.671 & 4.92 & 5.03 \\
\hline
\end{tabular}

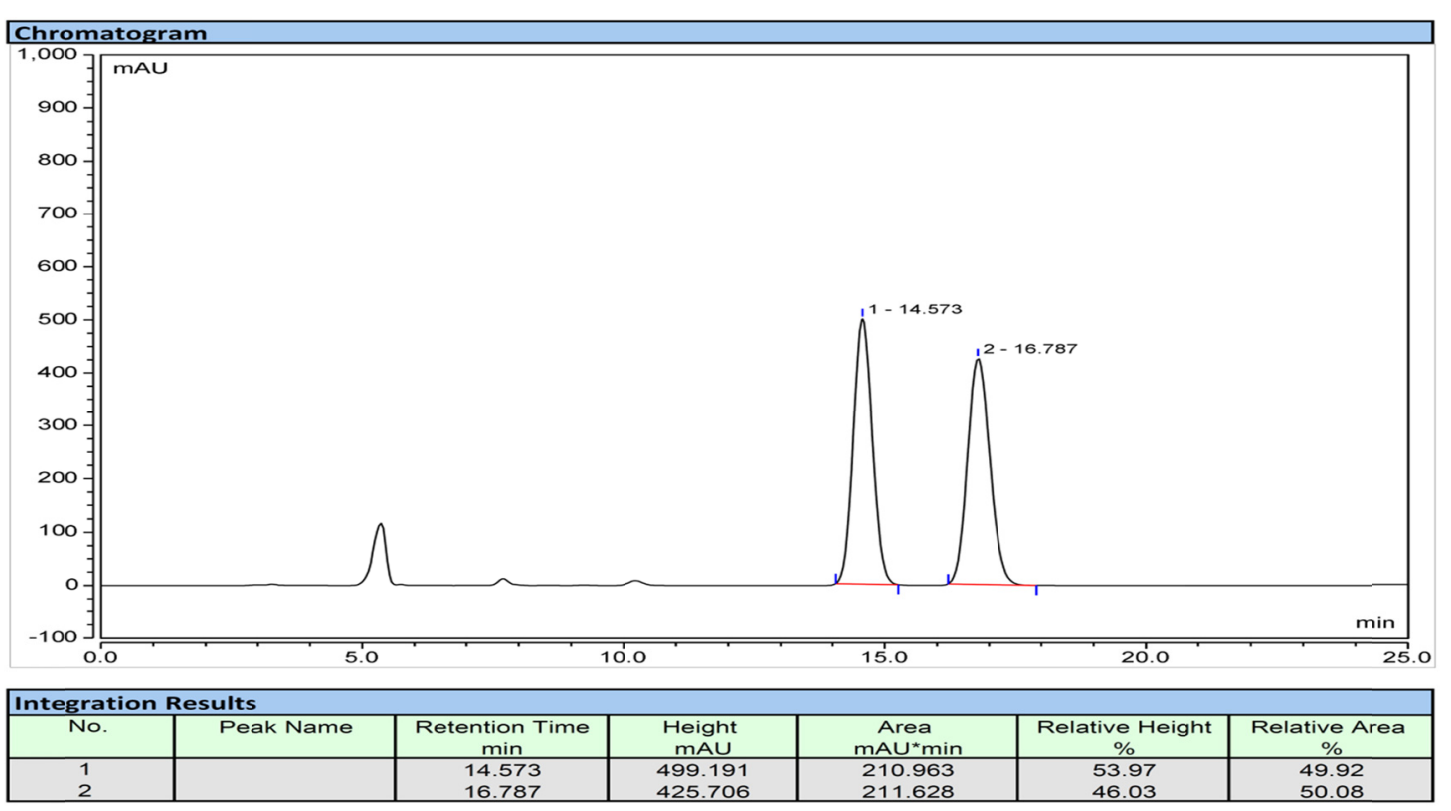




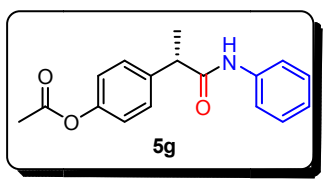

\section{(S)-4-(1-oxo-1-(phenylamino)propan-2-yl)phenyl acetate}

$77 \%$ yield, $\mathrm{b} / \mathrm{l}>99: 1$, ee $=92 \%,[\alpha] \mathrm{D}^{20}=+12.9\left(\mathrm{c}=1.3, \mathrm{CHCl}_{3}\right) .{ }^{1} \mathrm{H}$ NMR $(600$ $\left.\mathrm{MHz}, \mathrm{CDCl}_{3}\right) \delta 7.51(\mathrm{~s}, 1 \mathrm{H}), 7.42(\mathrm{~d}, J=8.4 \mathrm{~Hz}, 2 \mathrm{H}), 7.33(\mathrm{~d}, J=6.6 \mathrm{~Hz}, 2 \mathrm{H}), 7.24(\mathrm{t}$, $J=6.6 \mathrm{~Hz}, 2 \mathrm{H}), 7.06-7.04(\mathrm{~m}, 3 \mathrm{H}), 3.67(\mathrm{q}, J=7.2 \mathrm{~Hz}, 1 \mathrm{H}), 2.28(\mathrm{~s}, 3 \mathrm{H}), 1.52(\mathrm{~d}, J=$ $7.2 \mathrm{~Hz}, 3 \mathrm{H}) ;{ }^{13} \mathrm{C} \mathrm{NMR}\left(151 \mathrm{MHz}, \mathrm{CDCl}_{3}\right) \delta 172.2,169.5,149.8,138.5,137.8,128.8$, 128.6, 124.2, 122.0, 120.0, 47.2, 21.0, 18.7. HRMS calcd. (ESI) $\mathrm{m} / \mathrm{z}$ for $\mathrm{C}_{17} \mathrm{H}_{17} \mathrm{NNaO}_{3}$ : $[\mathrm{M}+\mathrm{Na}]^{+}$306.1101, found: 306.1101.

Chiral HPLC (Chiralpak IG, 4.6x250 mm; $5 \mu \mathrm{L}$, hexane/isopropanol = 90/10, flow $1.0 \mathrm{~mL} / \mathrm{min}, 254 \mathrm{~nm}$ ): $\mathrm{t}_{\mathrm{R}}=20.16 \mathrm{~min}$ (major), $\mathrm{t}_{\mathrm{R}}=23.75 \mathrm{~min}$ (minor).
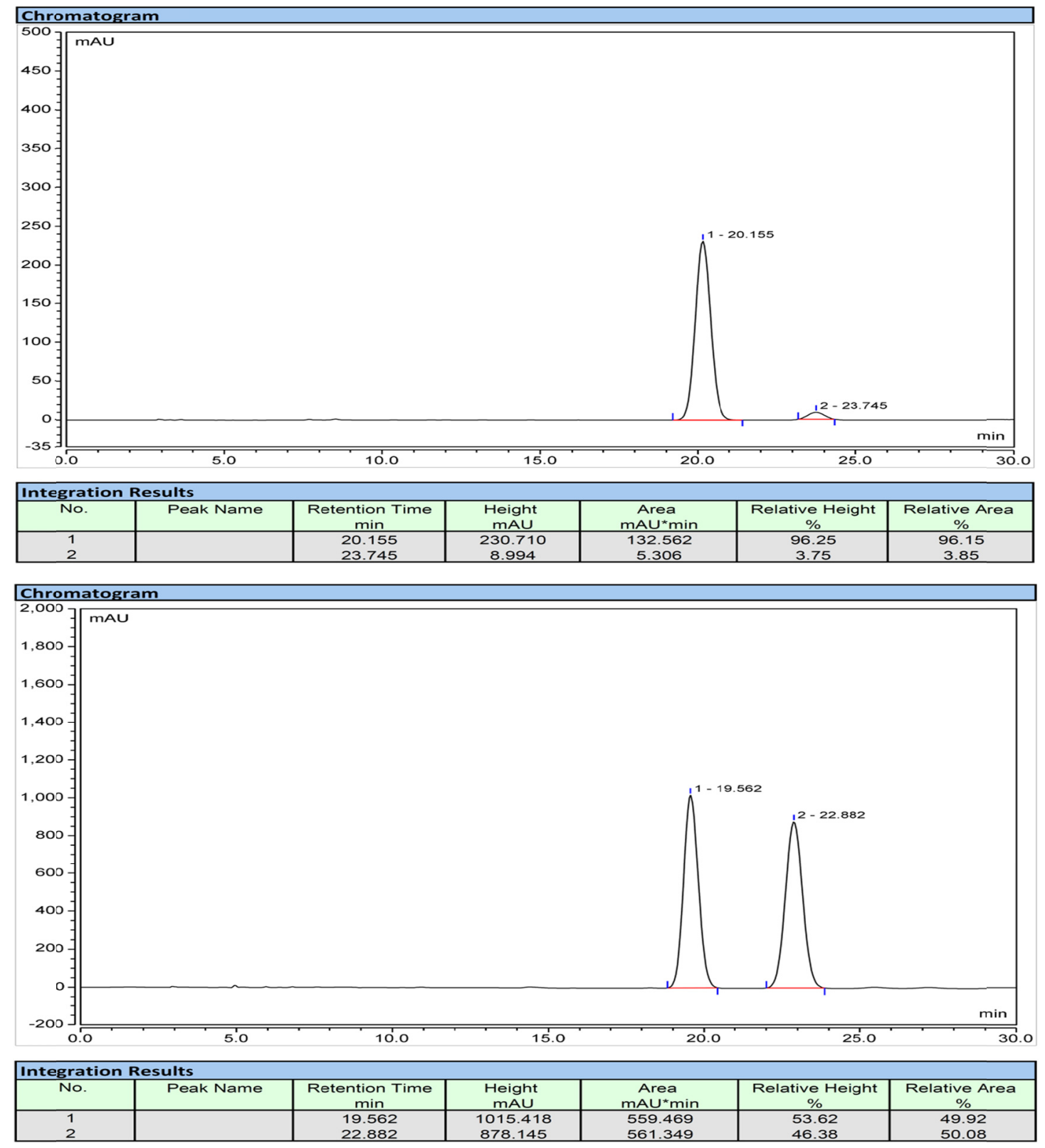


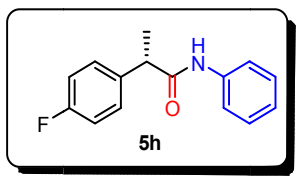

(S)-2-(4-fluorophenyl)- $N$-phenylpropanamide

$81 \%$ yield, $\mathrm{b} / \mathrm{l}>99: 1, \mathrm{ee}=91 \%,[\alpha] \mathrm{D}^{20}=+42.8\left(\mathrm{c}=1.3, \mathrm{CHCl}_{3}\right) .{ }^{1} \mathrm{H}$ NMR $(400$ $\left.\mathrm{MHz}, \mathrm{CDCl}_{3}\right) \delta 7.42(\mathrm{~d}, J=8.0 \mathrm{~Hz}, 2 \mathrm{H}), 7.35-7.32(\mathrm{~m}, 2 \mathrm{H}), 7.27(\mathrm{~d}, J=7.2 \mathrm{~Hz}, 2 \mathrm{H})$, 7.09-7.03 (m, 4H), $3.69(\mathrm{q}, J=7.2 \mathrm{~Hz}, 1 \mathrm{H}), 1.57(\mathrm{~d}, J=7.2 \mathrm{~Hz}, 3 \mathrm{H}) ;{ }^{13} \mathrm{C}$ NMR $(101$ $\left.\mathrm{MHz}, \mathrm{CDCl}_{3}\right) \delta 172.5,162.0(\mathrm{~d}, J=247.1 \mathrm{~Hz}), 137.7,136.7(\mathrm{~d}, J=3.0 \mathrm{~Hz}), 129.1(\mathrm{~d}, J$ $=8.0 \mathrm{~Hz}), 128.8,124.3,120.0,115.7(\mathrm{~d}, J=21.4 \mathrm{~Hz}), 47.0,18.7 ;{ }^{19} \mathrm{~F}$ NMR $(376 \mathrm{MHz}$, $\left.\mathrm{CDCl}_{3}\right) \delta-114.89$. HRMS calcd. (ESI) $\mathrm{m} / \mathrm{z}$ for $\mathrm{C}_{15} \mathrm{H}_{14} \mathrm{FNNaO}$ : $[\mathrm{M}+\mathrm{Na}]^{+}$266.0952, found: 266.0951 .

Chiral HPLC (Chiralpak IG, 4.6x250 mm; $5 \mu \mathrm{L}$, hexane/isopropanol = 90/10, flow $1.0 \mathrm{~mL} / \mathrm{min}, 254 \mathrm{~nm}$ ): $\mathrm{t}_{\mathrm{R}}=9.69 \min$ (major), $\mathrm{t}_{\mathrm{R}}=11.93 \mathrm{~min}$ (minor).
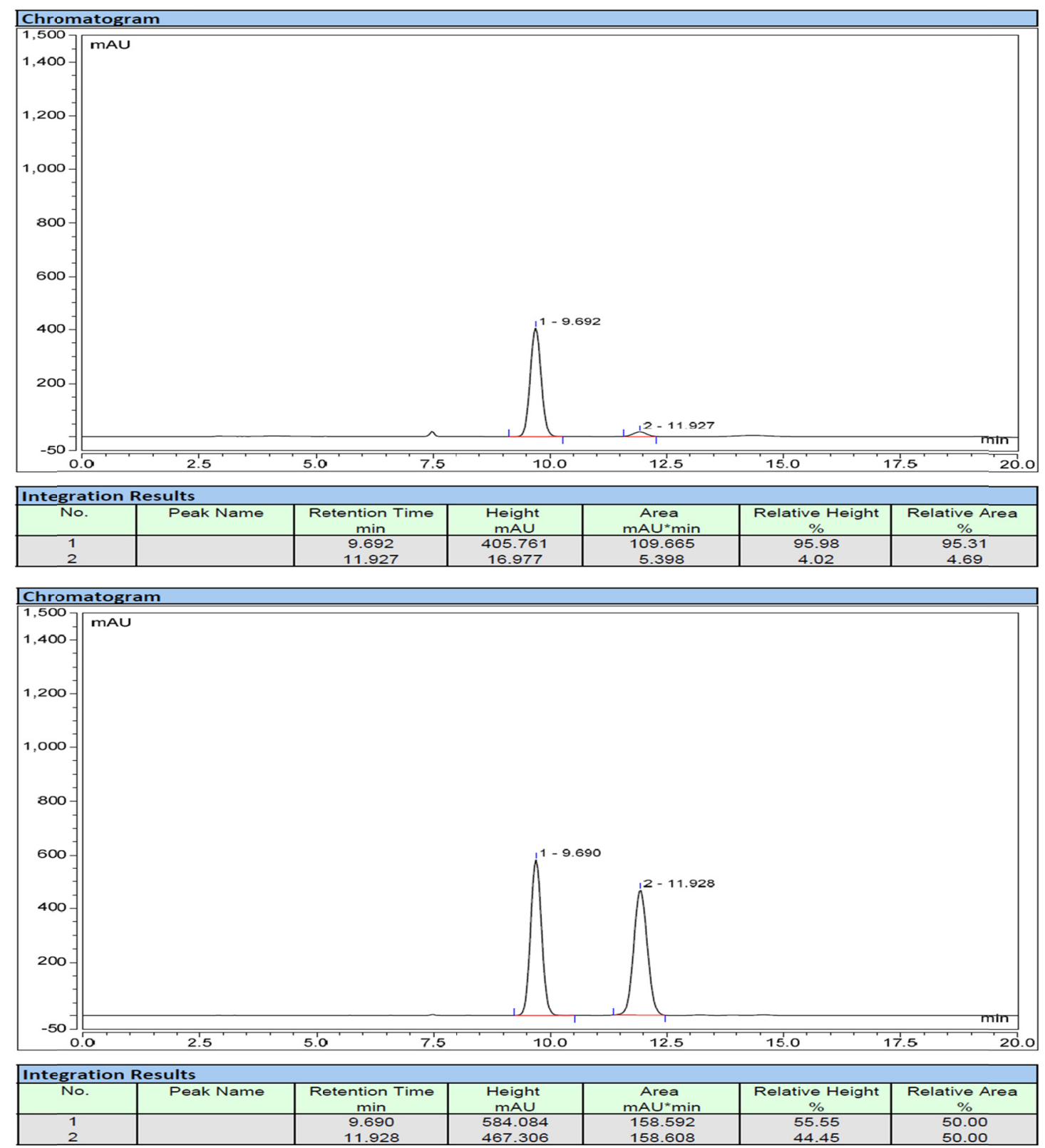


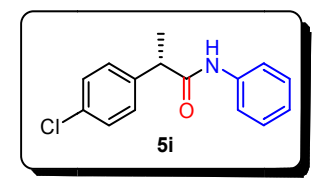

\section{(S)-2-(4-chlorophenyl)- $N$-phenylpropanamide}

$77 \%$ yield, $\mathrm{b} / 1>99: 1, \mathrm{ee}=90 \%,[\alpha] \mathrm{D}^{20}=+73.5\left(\mathrm{c}=1.7, \mathrm{CHCl}_{3}\right) .{ }^{1} \mathrm{H} \mathrm{NMR}(400 \mathrm{MHz}$, $\left.\mathrm{CDCl}_{3}\right) \delta 7.46(\mathrm{~d}, J=7.6 \mathrm{~Hz}, 2 \mathrm{H}), 7.37-7.26(\mathrm{~m}, 7 \mathrm{H}), 7.11(\mathrm{t}, J=7.6 \mathrm{~Hz}, 1 \mathrm{H}), 3.70(\mathrm{q}$, $J=7.2 \mathrm{~Hz}, 1 \mathrm{H}), 1.59(\mathrm{~d}, J=7.2 \mathrm{~Hz}, 3 \mathrm{H}) ;{ }^{13} \mathrm{C} \mathrm{NMR}\left(151 \mathrm{MHz}, \mathrm{CDCl}_{3}\right) \delta 171.9,139.4$, 137.7, 133.4, 129.2, 129.01, 128.97, 124.5, 119.8, 47.5, 18.8. HRMS calcd. (ESI) m/z for $\mathrm{C}_{15} \mathrm{H}_{14} \mathrm{ClNNaO}$ : [M+Na] $]^{+} 282.0656$, found: 282.0659 .

Chiral HPLC (Chiralpak IG, 4.6x250 mm; $5 \mu \mathrm{L}$, hexane/isopropanol = 90/10, flow $1.0 \mathrm{~mL} / \mathrm{min}, 254 \mathrm{~nm}$ ): $\mathrm{t}_{\mathrm{R}}=10.74 \mathrm{~min}$ (major), $\mathrm{t}_{\mathrm{R}}=13.99 \mathrm{~min}$ (minor).

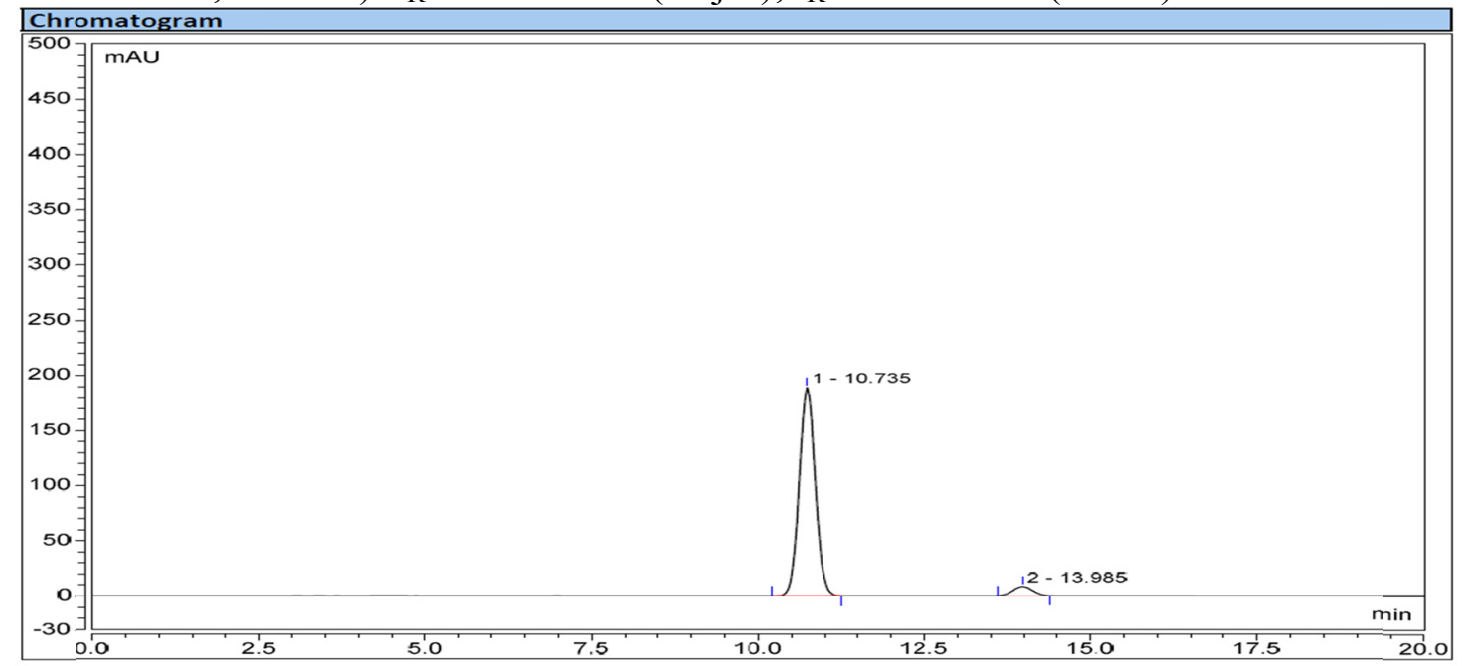

\begin{tabular}{|c|c|c|c|c|c|c|}
\hline \multicolumn{9}{|l|}{ Integration Results } \\
\hline No. & Peak Name & $\begin{array}{c}\text { Retention Time } \\
\text { min }\end{array}$ & $\begin{array}{c}\text { Height } \\
\text { mAU }\end{array}$ & $\begin{array}{c}\text { Area } \\
\text { mAU*min }\end{array}$ & $\begin{array}{c}\text { Relative Height } \\
\%\end{array}$ & $\begin{array}{c}\text { Relative Area } \\
\%\end{array}$ \\
\hline 1 & & 10.735 & 188.974 & 52.787 & 95.75 & 94.86 \\
2 & & 13.985 & 8.387 & 2.862 & 4.25 & 5.14 \\
\hline
\end{tabular}

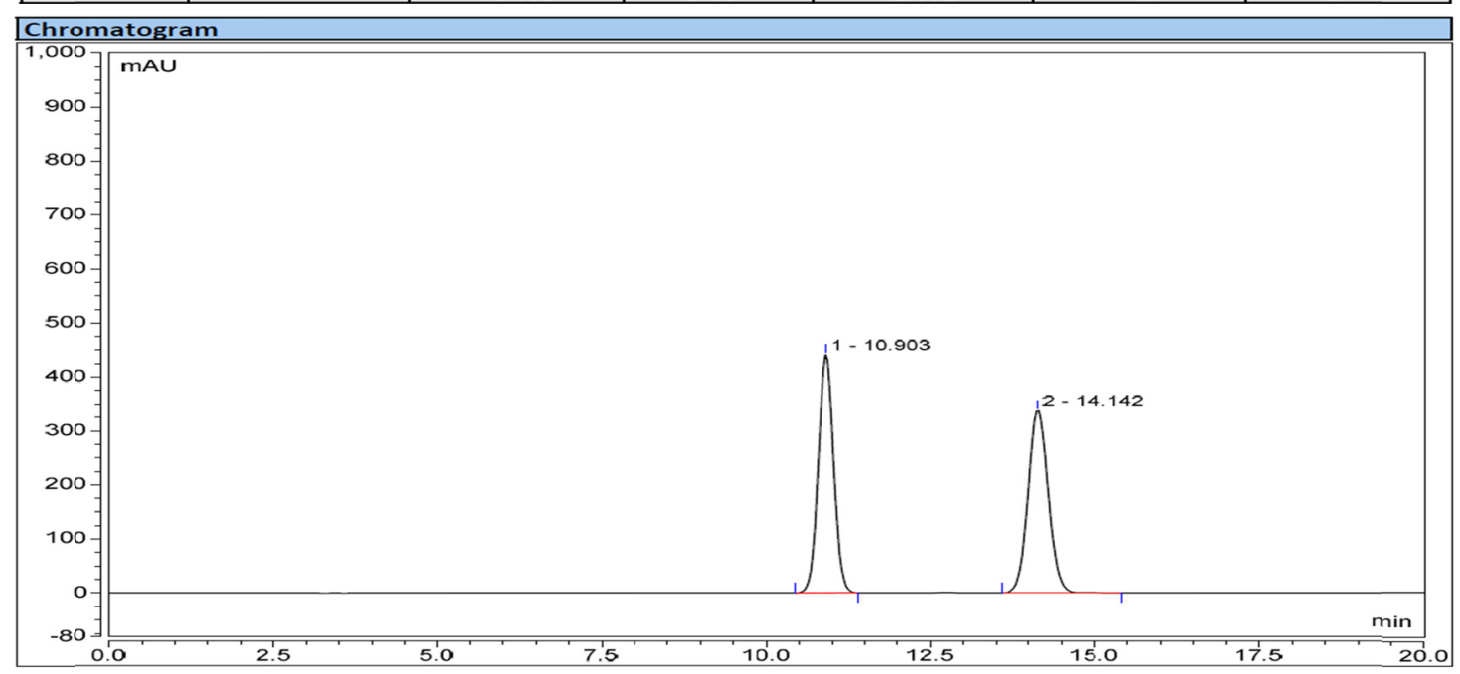

\begin{tabular}{|c|c|c|c|c|c|c|}
\hline \multicolumn{9}{|c|}{ Integration Results } \\
\hline No. & Peak Name & $\begin{array}{c}\text { Retention Time } \\
\text { min }\end{array}$ & $\begin{array}{c}\text { Height } \\
\text { mAU }\end{array}$ & $\begin{array}{c}\text { Area } \\
\text { mAU*min }\end{array}$ & $\begin{array}{c}\text { Relative Height } \\
\%\end{array}$ & $\begin{array}{c}\text { Relative Area } \\
\%\end{array}$ \\
\hline 1 & & 10.903 & 441.800 & 120.039 & 56.59 & 49.89 \\
2 & & 14.142 & 338.912 & 120.572 & 43.41 & 50.11 \\
\hline
\end{tabular}




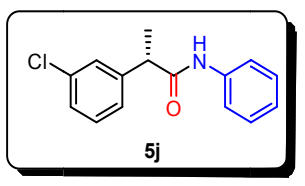

\section{(S)-2-(3-chlorophenyl)- $N$-phenylpropanamide}

$65 \%$ yield, $\mathrm{b} / 1>99: 1, \mathrm{ee}=93 \%,[\alpha] \mathrm{D}^{19}=+75.8\left(\mathrm{c}=1.1, \mathrm{CHCl}_{3}\right) .{ }^{1} \mathrm{H}$ NMR $(400$ $\left.\mathrm{MHz}, \mathrm{CDCl}_{3}\right) \delta 7.47(\mathrm{~d}, J=8.0 \mathrm{~Hz}, 2 \mathrm{H}), 7.39(\mathrm{~s}, 1 \mathrm{H}), 7.35-7.33(\mathrm{~m}, 1 \mathrm{H}), 7.32-7.28(\mathrm{~m}$, $5 \mathrm{H}), 7.11(\mathrm{t}, J=7.2 \mathrm{~Hz}, 1 \mathrm{H}), 3.69(\mathrm{q}, J=7.2 \mathrm{~Hz}, 1 \mathrm{H}), 1.59(\mathrm{~d}, J=6.8 \mathrm{~Hz}, 3 \mathrm{H}) ;{ }^{13} \mathrm{C}$ NMR $\left(101 \mathrm{MHz}, \mathrm{CDCl}_{3}\right) \delta 171.6,142.9,137.7,134.7,130.2,128.9,127.8,127.6$, 125.7, 124.4, 119.9, 47.6, 18.6. HRMS calcd. (ESI) m/z for $\mathrm{C}_{15} \mathrm{H}_{14} \mathrm{CINNaO}:[\mathrm{M}+\mathrm{Na}]^{+}$ 282.0656, found: 282.0655 .

Chiral HPLC (Chiralpak IG, 4.6x250 mm; $5 \mu \mathrm{L}$, hexane/isopropanol = 90/10, flow $1.0 \mathrm{~mL} / \mathrm{min}, 254 \mathrm{~nm}$ ): $\mathrm{t}_{\mathrm{R}}=6.27 \mathrm{~min}$ (major), $\mathrm{t}_{\mathrm{R}}=7.15 \mathrm{~min}$ (minor).
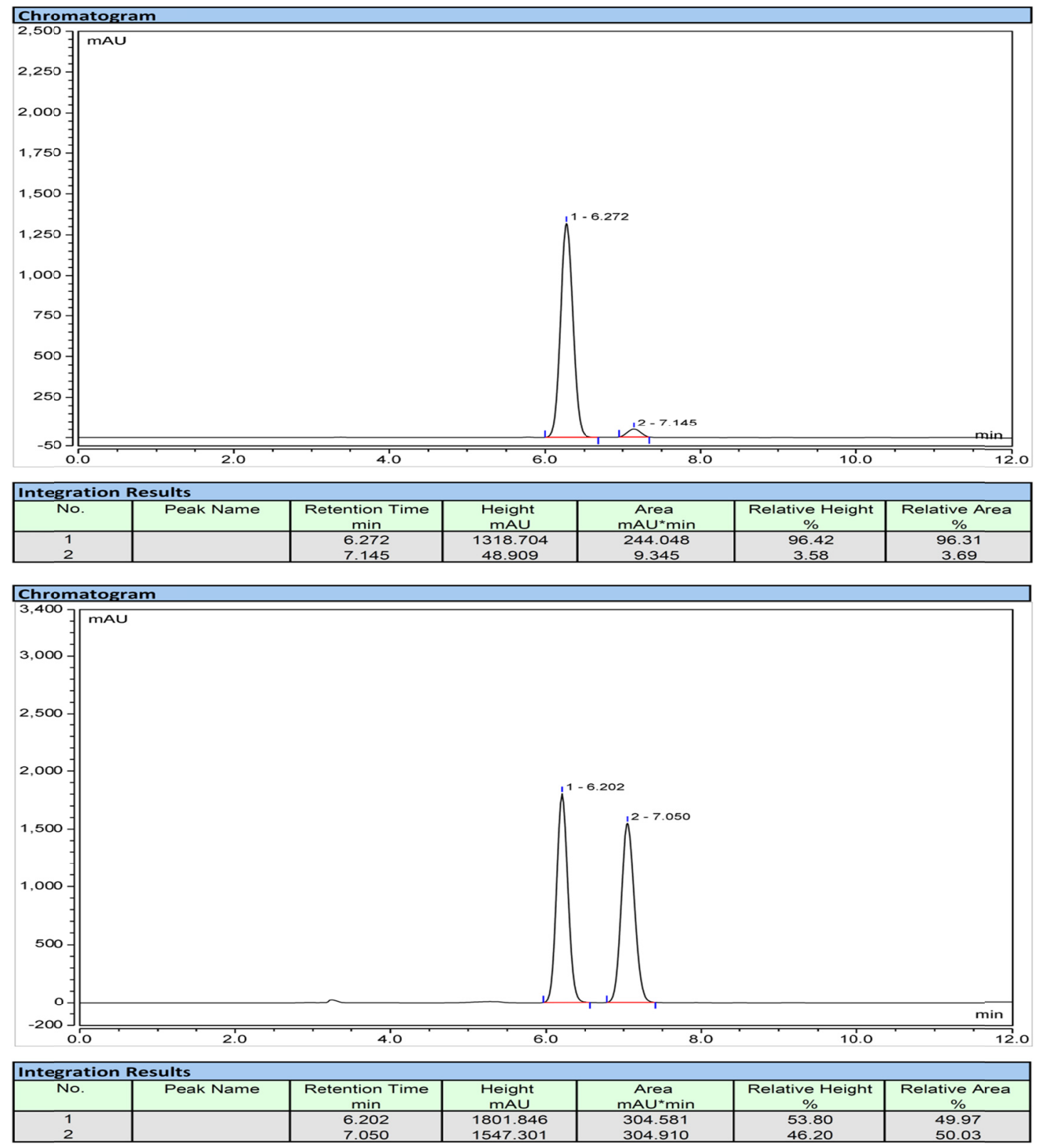


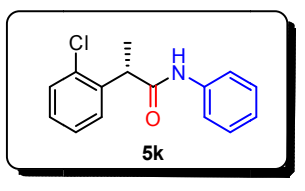

\section{(S)-2-(2-chlorophenyl)- $N$-phenylpropanamide}

$73 \%$ yield, $\mathrm{b}: 1>99: 1, \mathrm{ee}=94 \% .[\alpha] \mathrm{D}^{20}=+34.6\left(\mathrm{c}=1.1, \mathrm{CHCl}_{3}\right) .{ }^{1} \mathrm{H}$ NMR $(600$ $\mathrm{MHz}, \mathrm{CDCl} 3) \delta$ 7.48-7.38 (m, 5H), 7.28-7.19 (m, 4H), $7.05(\mathrm{t}, J=7.2 \mathrm{~Hz}, 1 \mathrm{H}), 4.22$ $(\mathrm{q}, J=7.2 \mathrm{~Hz}, 1 \mathrm{H}), 1.56(\mathrm{~d}, J=7.2 \mathrm{~Hz}, 3 \mathrm{H}) ;{ }^{13} \mathrm{C} \mathrm{NMR}\left(151 \mathrm{MHz}, \mathrm{CDCl}_{3}\right) \delta 171.3$, $138.4,137.8,133.4,129.7,128.8,128.54,128.49$, 127.6, 124.2, 119.8, 43.8, 17.3. HRMS calcd. (ESI) m/z for $\mathrm{C}_{15} \mathrm{H}_{14} \mathrm{ClNNaO}$ : $[\mathrm{M}+\mathrm{Na}]^{+} 282.0656$, found: 282.0661 .

Chiral HPLC (Chiralpak IG, 4.6x250 mm; $5 \mu \mathrm{L}$, hexane/isopropanol = 90/10, flow $1.0 \mathrm{~mL} / \mathrm{min}, 254 \mathrm{~nm}$ ): $\mathrm{t}_{\mathrm{R}}=11.95 \mathrm{~min}$ (major), $\mathrm{t}_{\mathrm{R}}=16.18 \mathrm{~min}$ (minor).
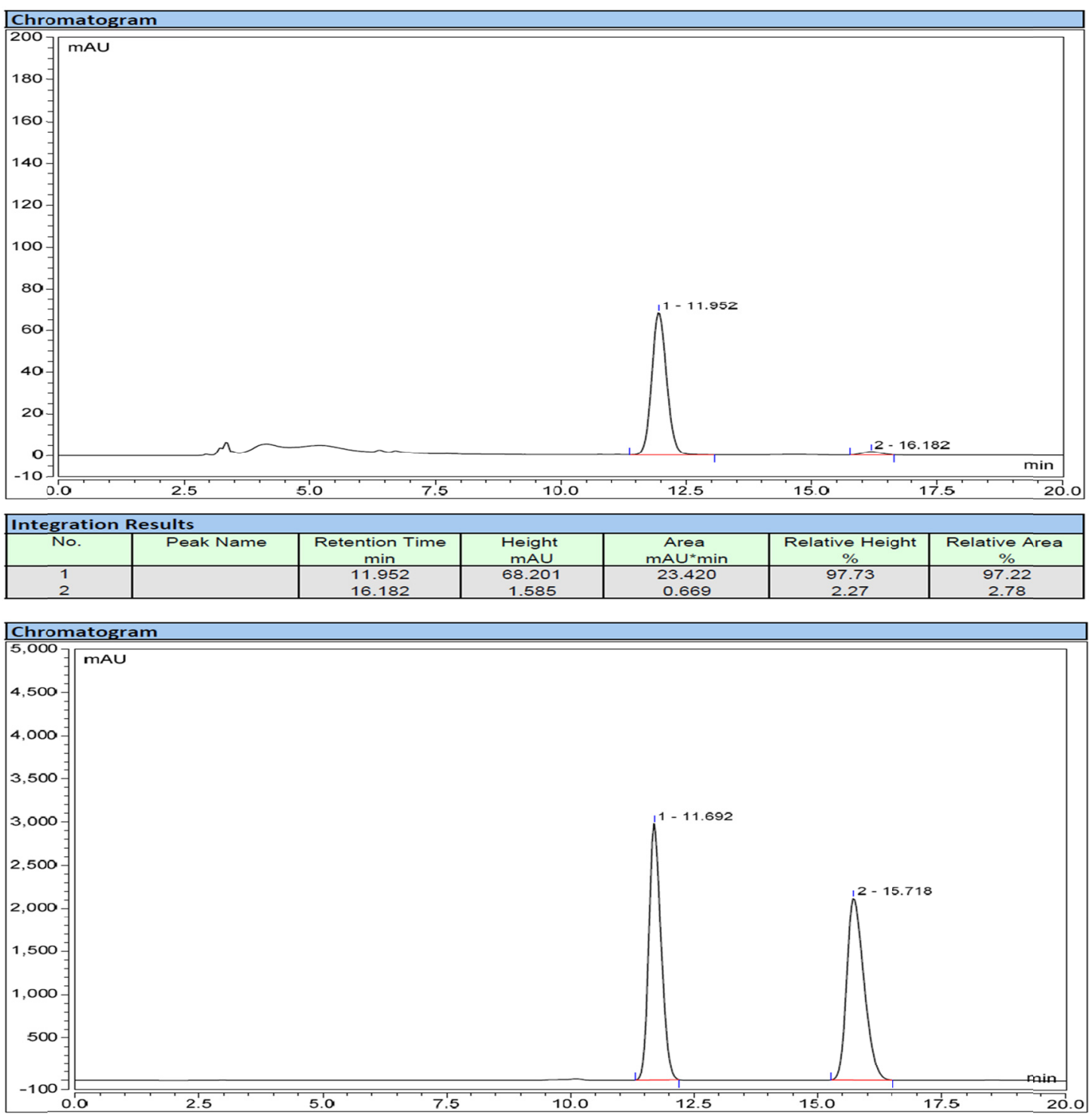

\begin{tabular}{|c|c|c|c|c|c|c|}
\hline \multicolumn{9}{|l|}{ Integration Results } \\
\hline No. & Peak Name & $\begin{array}{c}\text { Retention Time } \\
\text { min }\end{array}$ & $\begin{array}{c}\text { Height } \\
\text { mAU }\end{array}$ & $\begin{array}{c}\text { Area } \\
\text { mAU*min }\end{array}$ & $\begin{array}{c}\text { Relative Height } \\
\%\end{array}$ & $\begin{array}{c}\text { Relative Area } \\
\%\end{array}$ \\
\hline 1 & & 11.692 & 2977.203 & 854.301 & 58.45 & 50.14 \\
2 & & 15.718 & 2116.002 & 849.684 & 41.55 & 49.86 \\
\hline
\end{tabular}




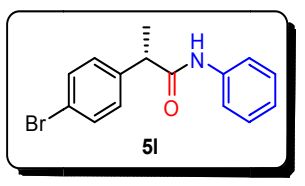

\section{(S)-2-(4-bromophenyl)- $N$-phenylpropanamide}

$82 \%$ yield, $\mathrm{b} / 1>99: 1$, ee $=94 \%,[\alpha] \mathrm{D}^{17}=+14.7\left(\mathrm{c}=1.3, \mathrm{CHCl}_{3}\right) .{ }^{1} \mathrm{H}$ NMR $(400$ $\left.\mathrm{MHz}, \mathrm{CDCl}_{3}\right) \delta 7.48(\mathrm{~d}, J=8.4 \mathrm{~Hz}, 2 \mathrm{H}), 7.42(\mathrm{~d}, J=7.8 \mathrm{~Hz}, 2 \mathrm{H}), 7.29-7.23(\mathrm{~m}, 4 \mathrm{H})$, $7.17(\mathrm{~s}, 1 \mathrm{H}), 7.08$ (t, $J=7.2 \mathrm{~Hz}, 1 \mathrm{H}), 3.65$ (q, $J=7.2 \mathrm{~Hz}, 1 \mathrm{H}), 1.56(\mathrm{~d}, J=7.2 \mathrm{~Hz}, 3 \mathrm{H})$; ${ }^{13} \mathrm{C} \mathrm{NMR}\left(151 \mathrm{MHz}, \mathrm{CDCl}_{3}\right) \delta 171.7,139.9,137.7,132.1,129.3,128.9,124.4,121.4$, 119.8, 47.5, 18.7. HRMS calcd. (ESI) $\mathrm{m} / \mathrm{z}$ for $\mathrm{C}_{15} \mathrm{H}_{14} \mathrm{BrNNaO}$ : $[\mathrm{M}+\mathrm{Na}]^{+}$326.0151, found: 326.0157 .

Chiral HPLC (Chiralpak IG, 4.6x250 mm; $5 \mu \mathrm{L}$, hexane/isopropanol = 90/10, flow $1.0 \mathrm{~mL} / \mathrm{min}, 254 \mathrm{~nm}$ ): $\mathrm{t}_{\mathrm{R}}=11.123 \mathrm{~min}$ (major), $\mathrm{t}_{\mathrm{R}}=15.26 \mathrm{~min}$ (minor).
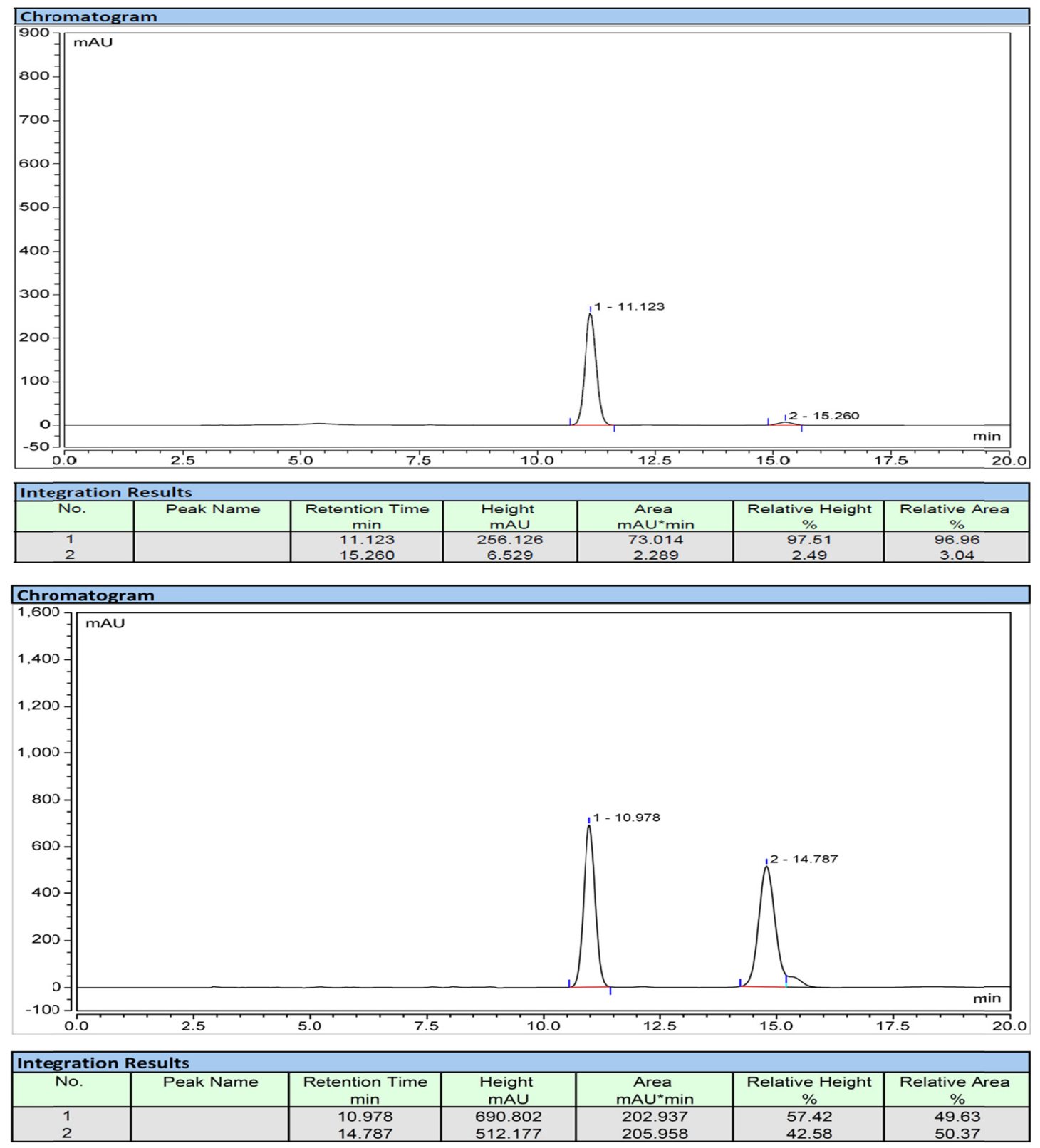


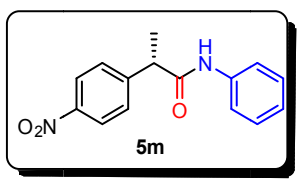

\section{(S)-2-(4-nitrophenyl)- $N$-phenylpropanamide}

$62 \%$ yield, b/1 > 99:1, 90\% ee, $[\alpha] \mathrm{D}^{20}=+27.8\left(\mathrm{c}=1.3, \mathrm{CHCl}_{3}\right) .{ }^{1} \mathrm{H}$ NMR $(400$ $\left.\mathrm{MHz}, \mathrm{CDCl}_{3},\right) \delta 8.19(\mathrm{~d}, J=8.4 \mathrm{~Hz}, 2 \mathrm{H}), 7.55(\mathrm{~d}, J=8.4 \mathrm{~Hz}, 2 \mathrm{H}), 7.46(\mathrm{~d}, J=8.0 \mathrm{~Hz}$, $2 \mathrm{H}), 7.36-7.27(\mathrm{~m}, 3 \mathrm{H}), 7.11(\mathrm{t}, J=7.2 \mathrm{~Hz}, 1 \mathrm{H}), 3.80(\mathrm{q}, J=7.2 \mathrm{~Hz}, 1 \mathrm{H}), 1.61(\mathrm{~d}, J=$ $7.2 \mathrm{~Hz}, 3 \mathrm{H}) ;{ }^{13} \mathrm{C} \mathrm{NMR}\left(151 \mathrm{MHz}, \mathrm{CDCl}_{3}\right) \delta 170.7,148.3,147.2,137.4,129.0,128.5$, 124.7, 124.1, 119.9, 47.9, 19.0. HRMS calcd. (ESI) $\mathrm{m} / \mathrm{z}$ for $\mathrm{C}_{15} \mathrm{H}_{14} \mathrm{~N}_{2} \mathrm{NaO}_{3}$ : $[\mathrm{M}+\mathrm{Na}]^{+}$ 291.0978, found: 291.0973.

Chiral HPLC (Chiralpak IG, 4.6x250 mm; $5 \mu \mathrm{L}$, hexane/isopropanol $=85 / 15$, flow $1.0 \mathrm{~mL} / \mathrm{min}, 254 \mathrm{~nm}$ ): $\mathrm{t}_{\mathrm{R}}=31.31 \mathrm{~min}$ (major), $\mathrm{t}_{\mathrm{R}}=43.75 \mathrm{~min}$ (minor).
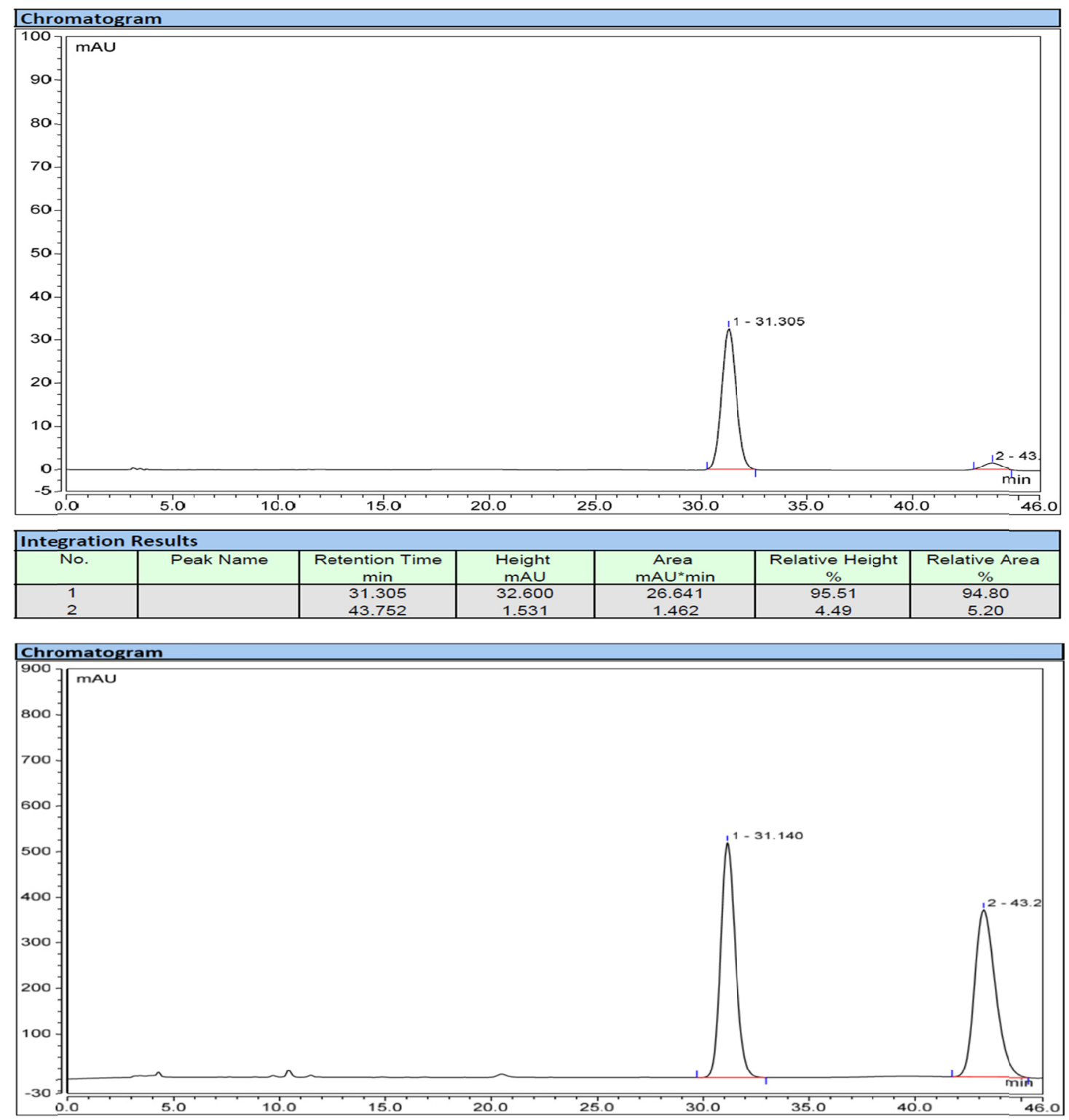

\begin{tabular}{|c|c|c|c|c|c|c|}
\hline \multicolumn{9}{|l|}{ Integration Results } \\
\hline No. & Peak Name & $\begin{array}{c}\text { Retention Time } \\
\text { min }\end{array}$ & $\begin{array}{c}\text { Height } \\
\text { mAU }\end{array}$ & $\begin{array}{c}\text { Area } \\
\text { mAUmin }\end{array}$ & $\begin{array}{c}\text { Relative Height } \\
\%\end{array}$ & $\begin{array}{c}\text { Relative Area } \\
\%\end{array}$ \\
\hline 1 & & 31.140 & 515.260 & 420.312 & 58.35 & 50.21 \\
2 & & 43.215 & 367.767 & 416.842 & 41.65 & 49.79 \\
\hline
\end{tabular}




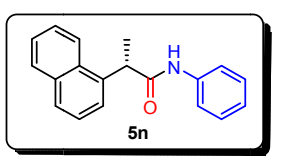

\section{(S)-2-(naphthalen-1-yl)- $N$-phenylpropanamide}

$81 \%$ yield, $\mathrm{b} / \mathrm{l}>99: 1$, ee $=90 \%,[\alpha] \mathrm{D}^{20}=+15.9\left(\mathrm{c}=1.1, \mathrm{CHCl}_{3}\right) .{ }^{1} \mathrm{H}$ NMR $(400$ $\left.\mathrm{MHz}, \mathrm{CDCl}_{3}\right) \delta 8.10-8.08(\mathrm{~m}, 1 \mathrm{H}), 7.91-7.88(\mathrm{~m}, 1 \mathrm{H}), 7.83(\mathrm{~d}, J=8.4 \mathrm{~Hz}, 1 \mathrm{H})$, 7.57-7.47 (m, 4H), $7.31(\mathrm{~d}, J=7.6 \mathrm{~Hz}, 2 \mathrm{H}), 7.21(\mathrm{t}, J=8.8,2 \mathrm{H}), 7.02(\mathrm{t}, J=7.6,2 \mathrm{H})$, 4.45 (q, $J=7.2 \mathrm{~Hz}, 1 \mathrm{H}), 1.75$ (d, $J=7.2 \mathrm{~Hz}, 3 \mathrm{H}) ;{ }^{13} \mathrm{C} \mathrm{NMR}\left(101 \mathrm{MHz}, \mathrm{CDCl}_{3}\right) \delta 172.8$, 137.7, 136.5, 134.1, 131.4, 129.1, 128.8, 128.4, 126.8, 126.0, 125.7, 125.1, 124.2, 123.1, 119.8, 44.6, 17.9. HRMS calcd. (ESI) $\mathrm{m} / \mathrm{z}$ for $\mathrm{C}_{19} \mathrm{H}_{17} \mathrm{NNaO}$ : $[\mathrm{M}+\mathrm{Na}]^{+} 298.1202$, found: 298.1206 .

Chiral HPLC (Chiralpak IG, 4.6x250 mm; $5 \mu \mathrm{L}$, hexane/isopropanol = 90/10, flow $1.0 \mathrm{~mL} / \mathrm{min}, 254 \mathrm{~nm}$ ): $\mathrm{t}_{\mathrm{R}}=18.32 \mathrm{~min}$ (minor), $\mathrm{t}_{\mathrm{R}}=19.44 \min$ (major).

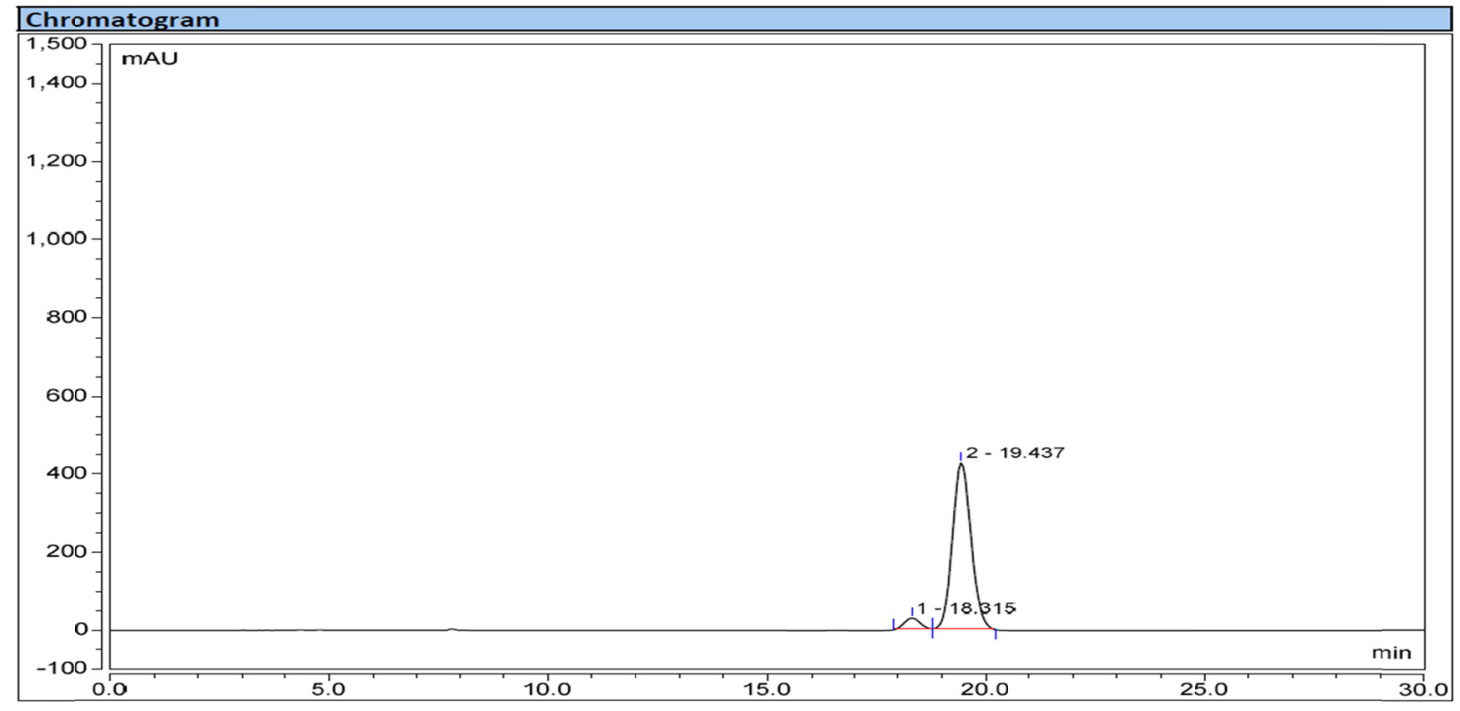

\begin{tabular}{|c|c|c|c|c|c|c|}
\hline \multicolumn{9}{|l|}{ Integration Results } \\
\hline No. & Peak Name & $\begin{array}{c}\text { Retention Time } \\
\text { min }\end{array}$ & $\begin{array}{c}\text { Height } \\
\text { mAU }\end{array}$ & $\begin{array}{c}\text { Area } \\
\text { mAU*min }\end{array}$ & $\begin{array}{c}\text { Relative Height } \\
\%\end{array}$ & $\begin{array}{c}\text { Relative Area } \\
\%\end{array}$ \\
\hline 1 & & 18.315 & 27.276 & 10.552 & 6.05 & 4.84 \\
2 & & 19.437 & 423.683 & 207.457 & 93.95 & 95.16 \\
\hline
\end{tabular}

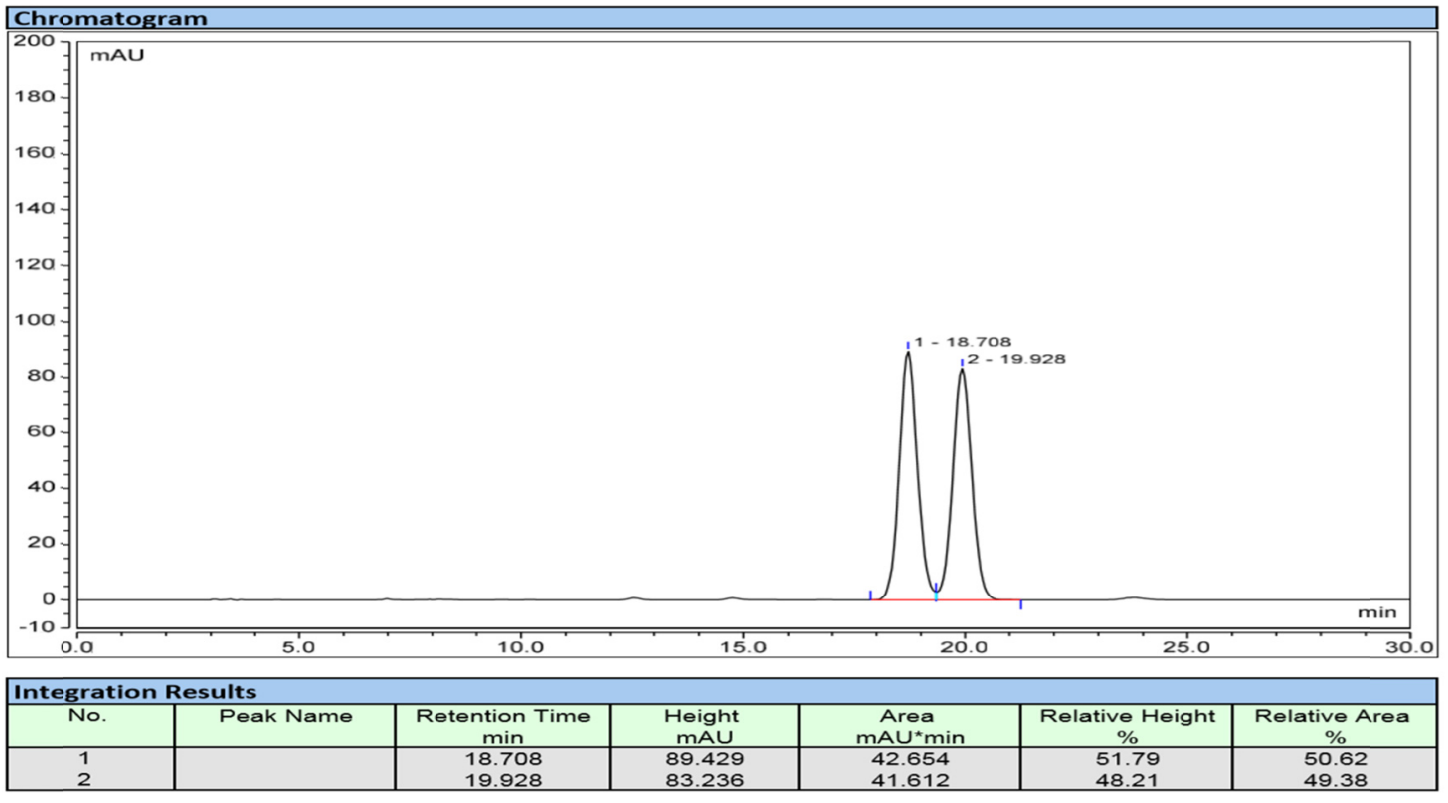




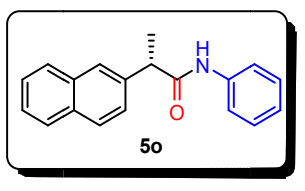

\section{(S)-2-(naphthalen-2-yl)- $N$-phenylpropanamide}

96\% yield, $\mathrm{b} / 1>99: 1, \mathrm{ee}=90 \%,[\alpha] \mathrm{D}^{20}=+68.3\left(\mathrm{c}=1.6, \mathrm{CHCl}_{3}\right) .{ }^{1} \mathrm{H}$ NMR $(400$ $\left.\mathrm{MHz}, \mathrm{CDCl}_{3}\right) \delta$ 7.85-7.80 (m, 4H), 7.49-7.45 (m, 3H), $7.41(\mathrm{~d}, J=4.0 \mathrm{~Hz}, 2 \mathrm{H})$, 7.24-7.19 (m, 3H), 7.04 (t, $J=4.0 \mathrm{~Hz}, 1 \mathrm{H}), 3.88(\mathrm{q}, J=7.2 \mathrm{~Hz}, 1 \mathrm{H}), 1.67$ (d, $J=7.2 \mathrm{~Hz}$, $3 \mathrm{H}) ;{ }^{13} \mathrm{C}$ NMR $\left(151 \mathrm{MHz}, \mathrm{CDCl}_{3}\right) \delta 172.2,138.4,137.8,133.6,132.7,129.0,128.8$, 127.8, 127.7, 126.4, 126.1, 125.6, 124.2, 119.7, 48.2, 18.5. HRMS calcd. (ESI) $\mathrm{m} / \mathrm{z}$ for $\mathrm{C}_{19} \mathrm{H}_{17} \mathrm{NNaO}$ : [M+Na] $]^{+} 298.1202$, found: 298.1197 .

Chiral HPLC (Chiralpak IG, 4.6x250 mm; $5 \mu \mathrm{L}$, hexane/isopropanol = 90/10, flow $1.0 \mathrm{~mL} / \mathrm{min}, 254 \mathrm{~nm}$ ): $\mathrm{t}_{\mathrm{R}}=8.40 \mathrm{~min}$ (major), $\mathrm{t}_{\mathrm{R}}=10.17 \mathrm{~min}$ (minor).

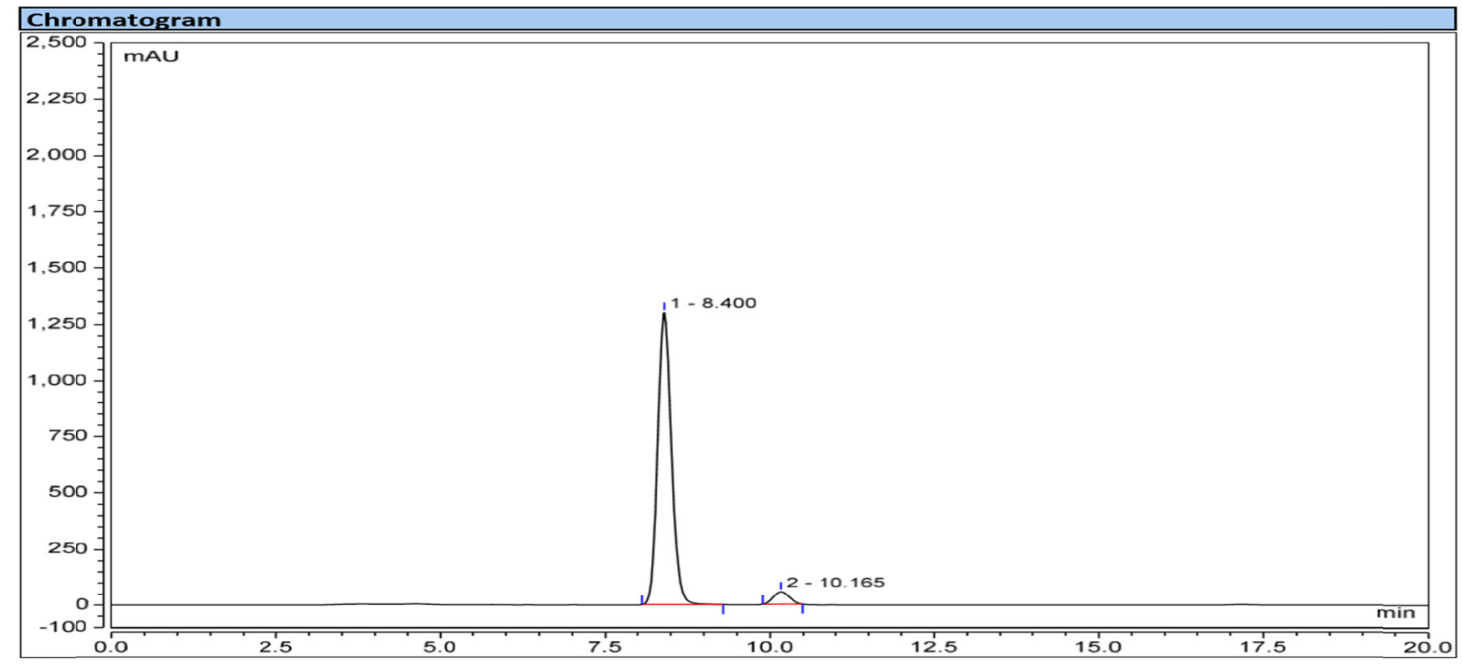

\begin{tabular}{|c|c|c|c|c|c|c|}
\hline \multicolumn{7}{|c|}{ Integration Results } \\
\hline No. & Peak Name & $\begin{array}{c}\text { Retention Time } \\
\text { min }\end{array}$ & $\begin{array}{l}\text { Height } \\
\text { mAU }\end{array}$ & $\begin{array}{c}\text { Area } \\
\text { mAU*min }\end{array}$ & $\begin{array}{c}\text { Relative Height } \\
\%\end{array}$ & $\begin{array}{c}\text { Relative Area } \\
\%\end{array}$ \\
\hline $\begin{array}{l}1 \\
2\end{array}$ & & $\begin{array}{c}8.400 \\
10.165 \\
\end{array}$ & $\begin{array}{c}1301.819 \\
57.995\end{array}$ & $\begin{array}{l}313.709 \\
16.085\end{array}$ & $\begin{array}{c}95.74 \\
4.26\end{array}$ & $\begin{array}{c}95.12 \\
4.88 \\
\end{array}$ \\
\hline
\end{tabular}

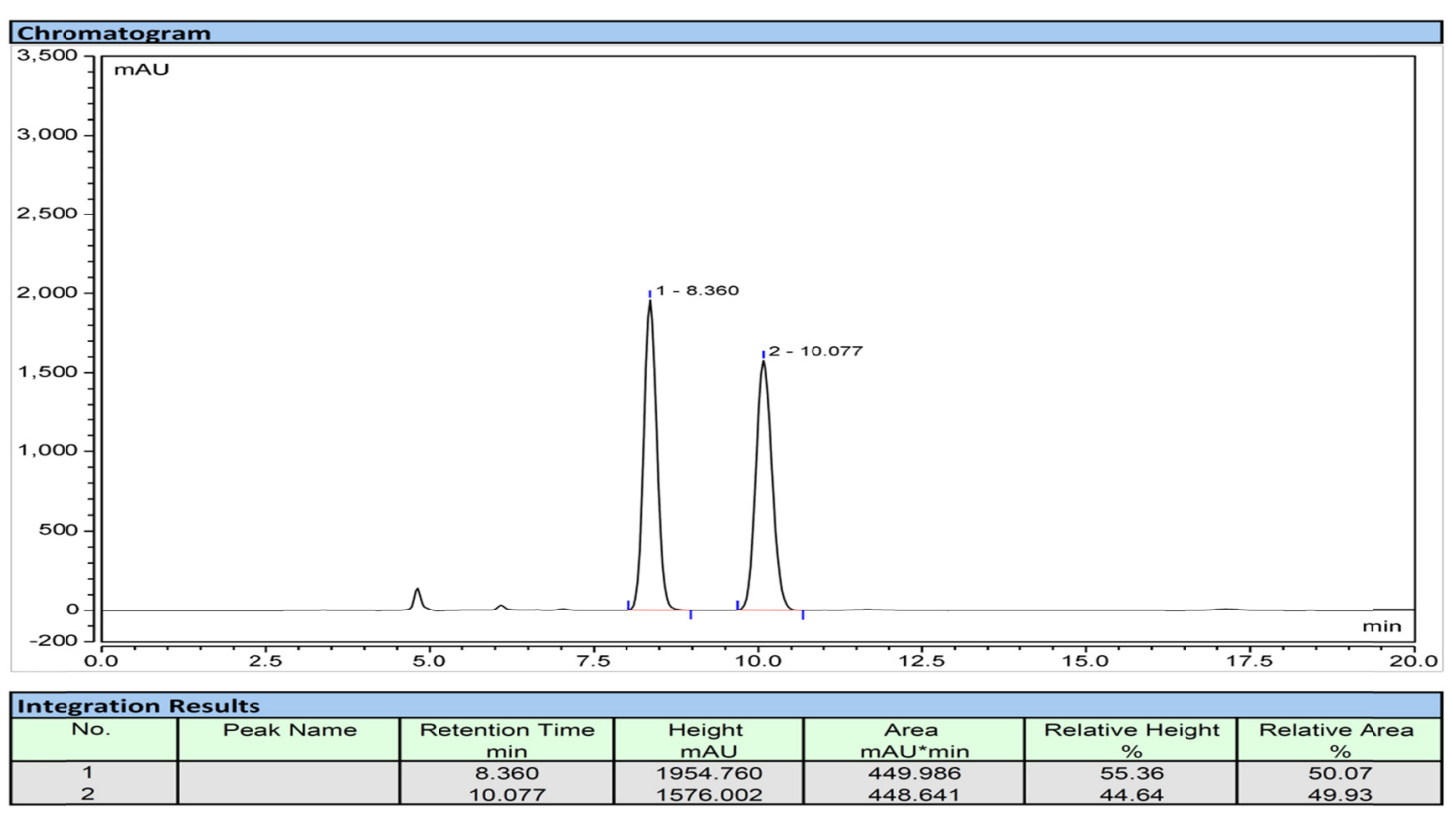




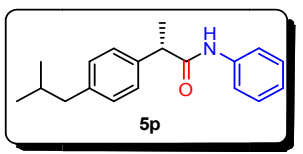

\section{(S)-2-(4-isobutylphenyl)- $N$-phenylpropanamide}

$98 \%$ yield, b/1 > 99:1, ee $=90 \%,[\alpha] \mathrm{D}^{16}=+42.0\left(\mathrm{c}=1.7, \mathrm{CHCl}_{3}\right) .{ }^{1} \mathrm{H}$ NMR $(400$ $\left.\mathrm{MHz}, \mathrm{CDCl}_{3}\right) \delta 7.42(\mathrm{~d}, J=8.0 \mathrm{~Hz}, 2 \mathrm{H}), 7.34(\mathrm{~s}, 1 \mathrm{H}), 7.26-7.22(\mathrm{~m}, 4 \mathrm{H}), 7.13(\mathrm{~d}, J=$ $7.6 \mathrm{~Hz}, 2 \mathrm{H}), 7.04$ (t, $J=7.6 \mathrm{~Hz}, 1 \mathrm{H}), 3.69$ (q, $J=7.2 \mathrm{~Hz}, 1 \mathrm{H}), 2.46(\mathrm{~d}, J=7.2 \mathrm{~Hz}, 2 \mathrm{H})$, 1.90-1.80 (m, 1H), $1.56(\mathrm{~d}, J=7.2 \mathrm{~Hz}, 3 \mathrm{H}), 0.90(\mathrm{~d}, J=6.4 \mathrm{~Hz}, 6 \mathrm{H}) ;{ }^{13} \mathrm{C}$ NMR $(101$ $\left.\mathrm{MHz}, \mathrm{CDCl}_{3}\right) \delta 172.7,140.9,138.0,137.9,129.7,128.8,127.3,124.1,119.7,47.6$, 44.9, 30.1, 22.3, 18.5. HRMS calcd. (ESI) $\mathrm{m} / \mathrm{z}$ for $\mathrm{C}_{19} \mathrm{H}_{23} \mathrm{NNaO}$ : $[\mathrm{M}+\mathrm{Na}]^{+} 304.1672$, found: 304.1676 .

Chiral HPLC (Chiralpak IG, 4.6x250 mm; $5 \mu \mathrm{L}$, hexane/isopropanol $=85 / 15$, flow $1.0 \mathrm{~mL} / \mathrm{min}, 254 \mathrm{~nm}$ ): $\mathrm{t}_{\mathrm{R}}=6.47 \mathrm{~min}$ (major), $\mathrm{t}_{\mathrm{R}}=7.78 \mathrm{~min}$ (minor).
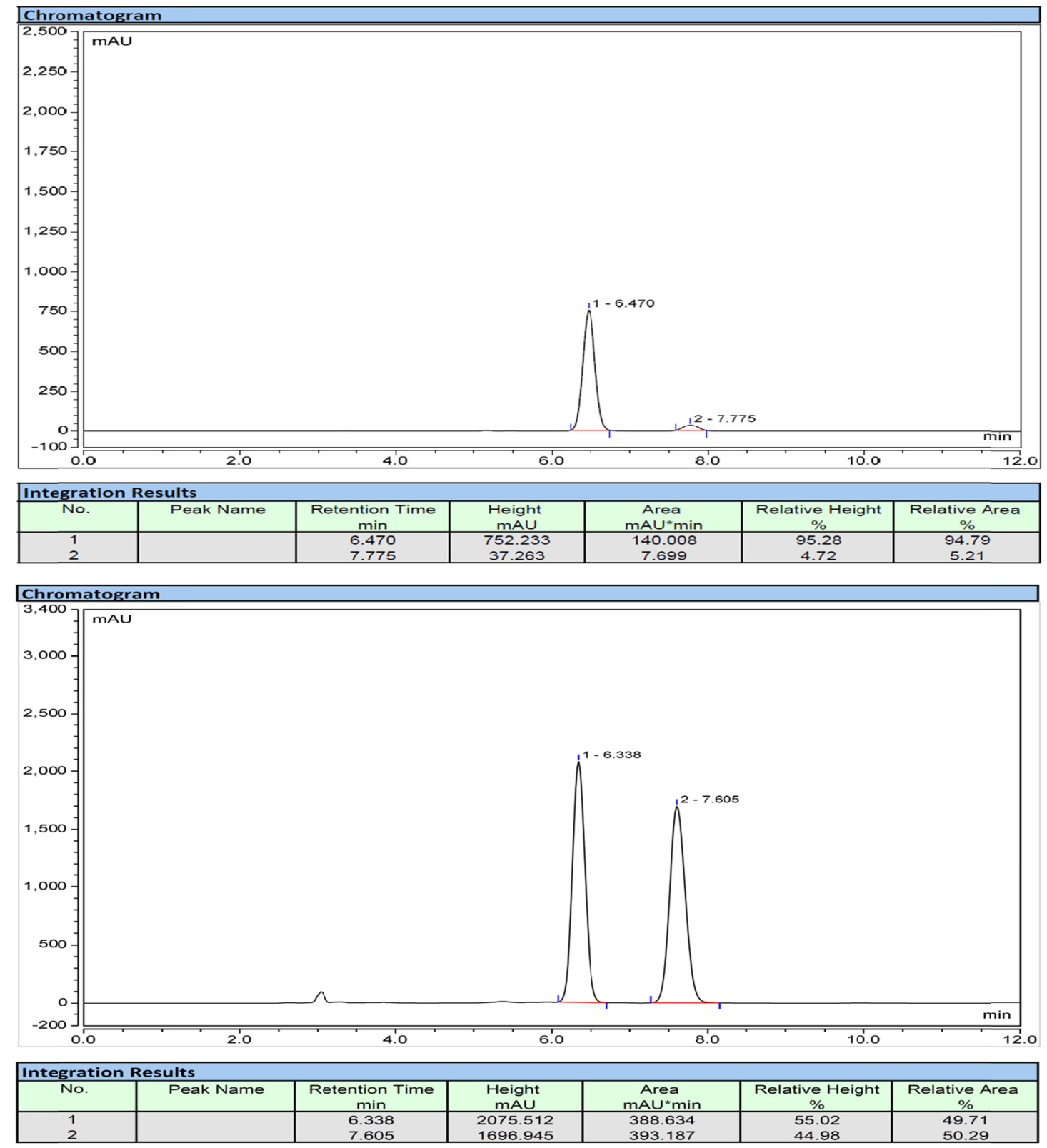


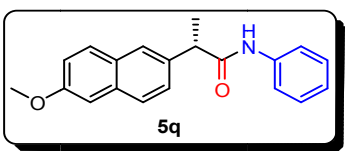

\section{(S)-2-(6-methoxynaphthalen-2-yl)- $N$-phenylpropanamide}

91\% yield, b:1 $>99: 1$, ee $=90 \%,[\alpha] \mathrm{D}^{18}=+48.2\left(\mathrm{c}=0.9, \mathrm{CHCl}_{3}\right) .{ }^{1} \mathrm{H}$ NMR $(400$ $\left.\mathrm{MHz}, \mathrm{CDCl}_{3}\right) \delta 7.71(\mathrm{t}, J=10.2 \mathrm{~Hz}, 3 \mathrm{H}), 7.42-7.39(\mathrm{~m}, 3 \mathrm{H}), 7.25-7.23(\mathrm{~m}, 3 \mathrm{H})$, 7.18-7.15 (m, 1H), 7.13-7.11 (m, 1H), $7.04(\mathrm{t}, J=7.2 \mathrm{~Hz}, 1 \mathrm{H}), 3.90(\mathrm{~s}, 3 \mathrm{H}), 3.83(\mathrm{q}, J=$ $7.2 \mathrm{~Hz}, 1 \mathrm{H}), 1.64$ (d, $J=7.2 \mathrm{~Hz}, 3 \mathrm{H}) ;{ }^{13} \mathrm{C} \mathrm{NMR}\left(101 \mathrm{MHz}, \mathrm{CDCl}_{3}\right) \delta 172.5,157.8$, $137.8,136.0,133.8,129.2,129.0,128.8,127.8,126.3,126.1,124.2,119.7,119.2$, 105.6, 55.3, 47.9, 18.5. HRMS calcd. (ESI) $\mathrm{m} / \mathrm{z}$ for $\mathrm{C}_{20} \mathrm{H}_{19} \mathrm{NNaO}_{2}$ : $[\mathrm{M}+\mathrm{Na}]^{+}$328.1308, found: 328.1307 .

Chiral HPLC (Chiralpak IG, 4.6x250 mm; $5 \mu \mathrm{L}$, hexane/isopropanol $=90 / 10$, flow $1.0 \mathrm{~mL} / \mathrm{min}, 254 \mathrm{~nm}$ ): $\mathrm{t}_{\mathrm{R}}=17.46 \mathrm{~min}$ (major), $\mathrm{t}_{\mathrm{R}}=21.81 \mathrm{~min}$ (minor).
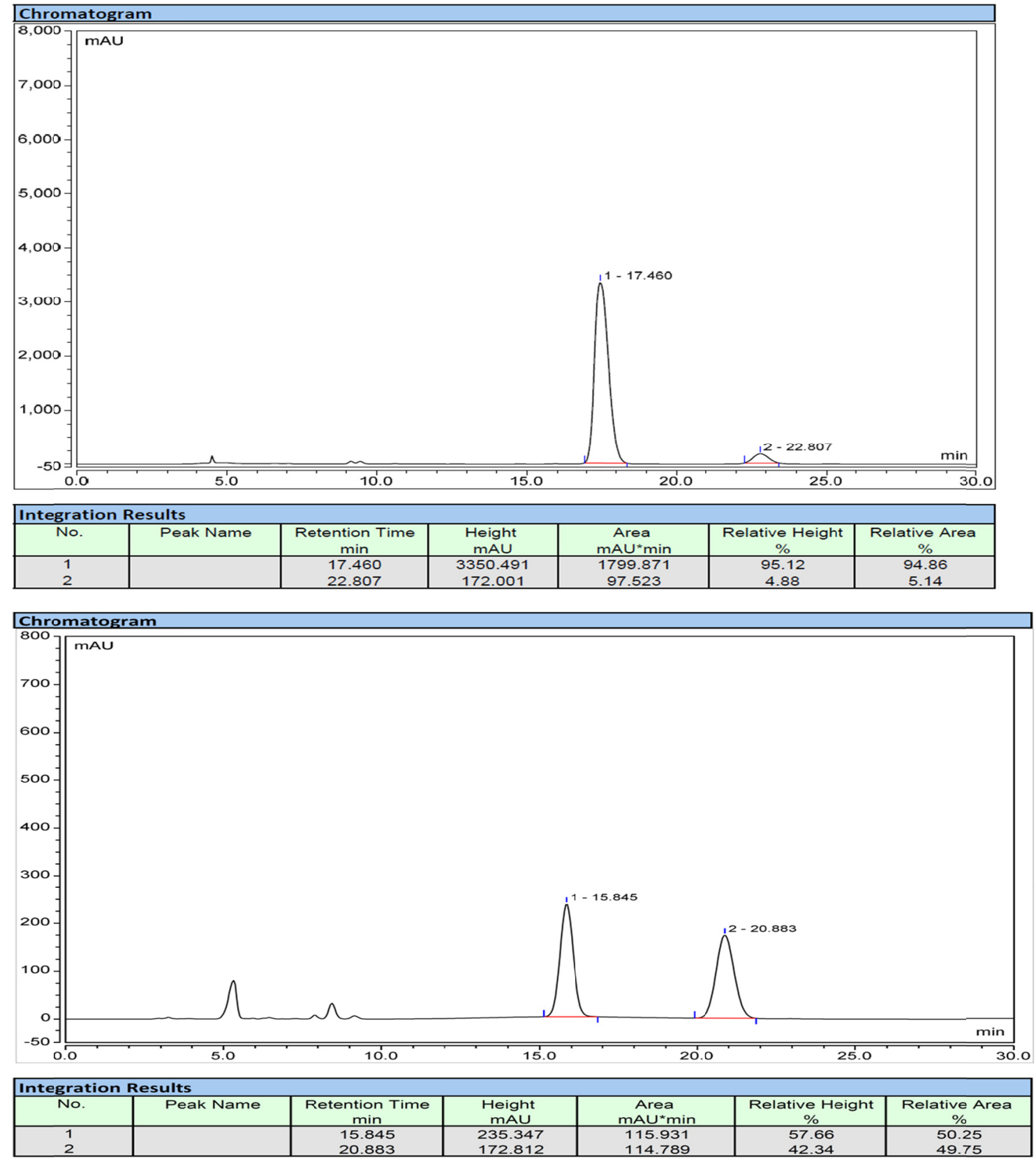


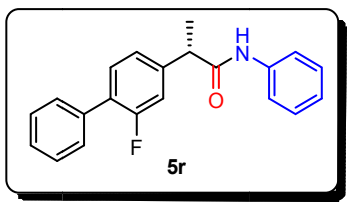

(S)-2-(2-fluoro-[1,1'-biphenyl]-4-yl)- $N$-phenylpropanamide

$83 \%$ yield, b:1 $>99: 1$, ee $=92 \%,[\alpha] \mathrm{D}^{19}=+29.6\left(\mathrm{c}=1.4, \mathrm{CHCl}_{3}\right) .{ }^{1} \mathrm{H}$ NMR $(400$

$\mathrm{MHz}, d^{6}$-DMSO) $\delta 10.14(\mathrm{~s}, 1 \mathrm{H}), 7.61(\mathrm{~d}, J=7.6 \mathrm{~Hz}, 2 \mathrm{H}), 7.54-7.52(\mathrm{~m}, 2 \mathrm{H}), 7.49-7.44$ (m, 3H), 7.40-7.38 (m, 1H), 7.33-7.27 (m, 4H), 7.03 (t, $J=7.6 \mathrm{~Hz}, 1 \mathrm{H}), 3.91$ (q, $J=7.2$ $\mathrm{Hz}, 1 \mathrm{H}), 1.46(\mathrm{~d}, J=7.2 \mathrm{~Hz}, 3 \mathrm{H}) ;{ }^{13} \mathrm{C} \mathrm{NMR}\left(101 \mathrm{MHz}, d^{6}\right.$-DMSO) $\delta 171.7,158.9$ (d, $J$ $=244.6 \mathrm{~Hz}), 143.7$ (d, $J=7.6 \mathrm{~Hz}), 139.1,134.9,130.7$ (d, $J=3.5 \mathrm{~Hz}), 128.7,128.6$, $127.8,126.6(\mathrm{~d}, J=13.3 \mathrm{~Hz}), 123.8,123.4,119.2,114.9(\mathrm{~d}, J=23.1 \mathrm{~Hz}), 45.5,18.4 ;{ }^{19} \mathrm{~F}$ NMR (376 MHz, $\mathrm{CDCl}_{3}$ ) $\delta$-113.67. HRMS calcd. (ESI) $\mathrm{m} / \mathrm{z}$ for $\mathrm{C}_{21} \mathrm{H}_{18} \mathrm{FNNaO}$ : $[\mathrm{M}+\mathrm{Na}]^{+}$342.1265, found: 342.1265 .

Chiral HPLC (Chiralpak IG, 4.6x250 mm; $5 \mu \mathrm{L}$, hexane/isopropanol $=90 / 10$, flow $1.0 \mathrm{~mL} / \mathrm{min}, 254 \mathrm{~nm}$ ): $\mathrm{t}_{\mathrm{R}}=8.42 \min$ (major), $\mathrm{t}_{\mathrm{R}}=10.95 \mathrm{~min}$ (minor).

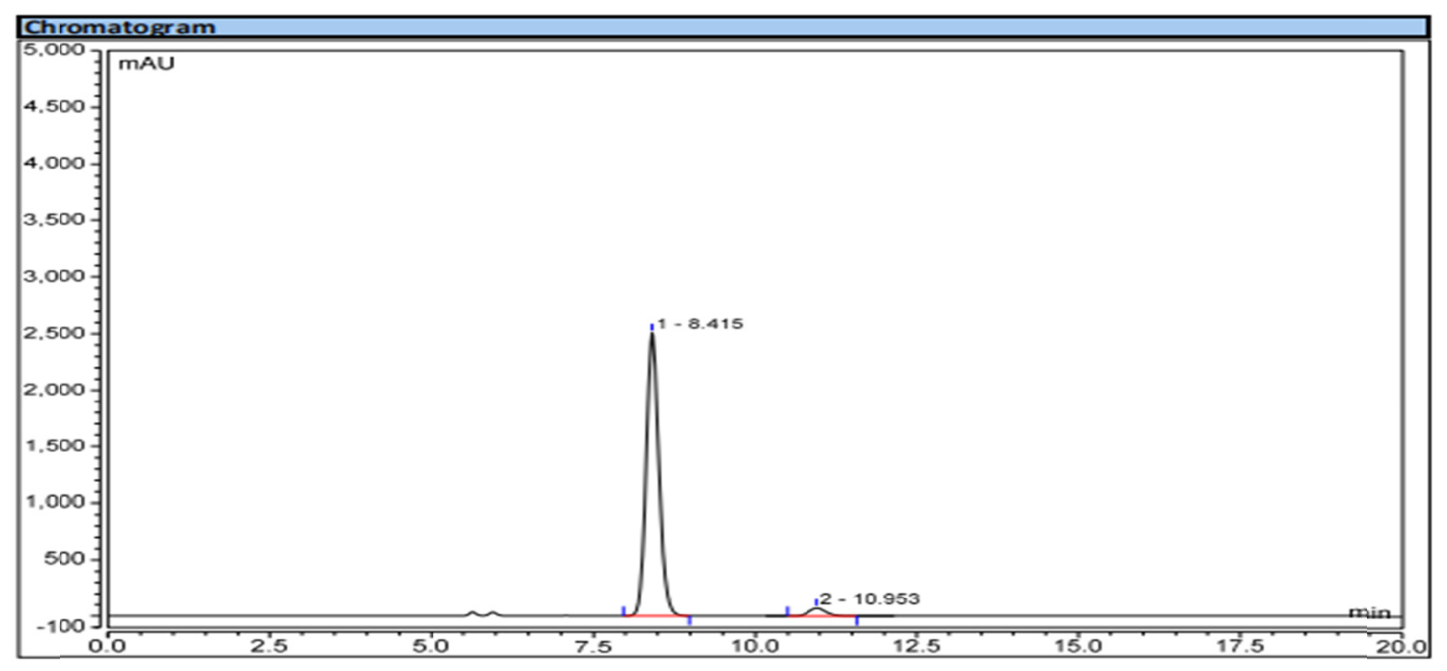

\begin{tabular}{|c|c|c|c|c|c|c|}
\hline \multicolumn{9}{|c|}{ Integration Results } & \multicolumn{5}{|c|}{ Area } \\
\hline No. & Peak Name & $\begin{array}{c}\text { Retention Time } \\
\text { min }\end{array}$ & $\begin{array}{c}\text { Height } \\
\text { mAU }\end{array}$ & $\begin{array}{c}\text { Areative Height } \\
\text { mAU*min }\end{array}$ & $\begin{array}{c}\text { Relative Area } \\
\%\end{array}$ \\
\hline 1 & & 8.415 & 2505.441 & 569.846 & 97.30 & 96.21 \\
2 & & 10.953 & 69.463 & 22.448 & 2.70 & 3.79 \\
\hline
\end{tabular}

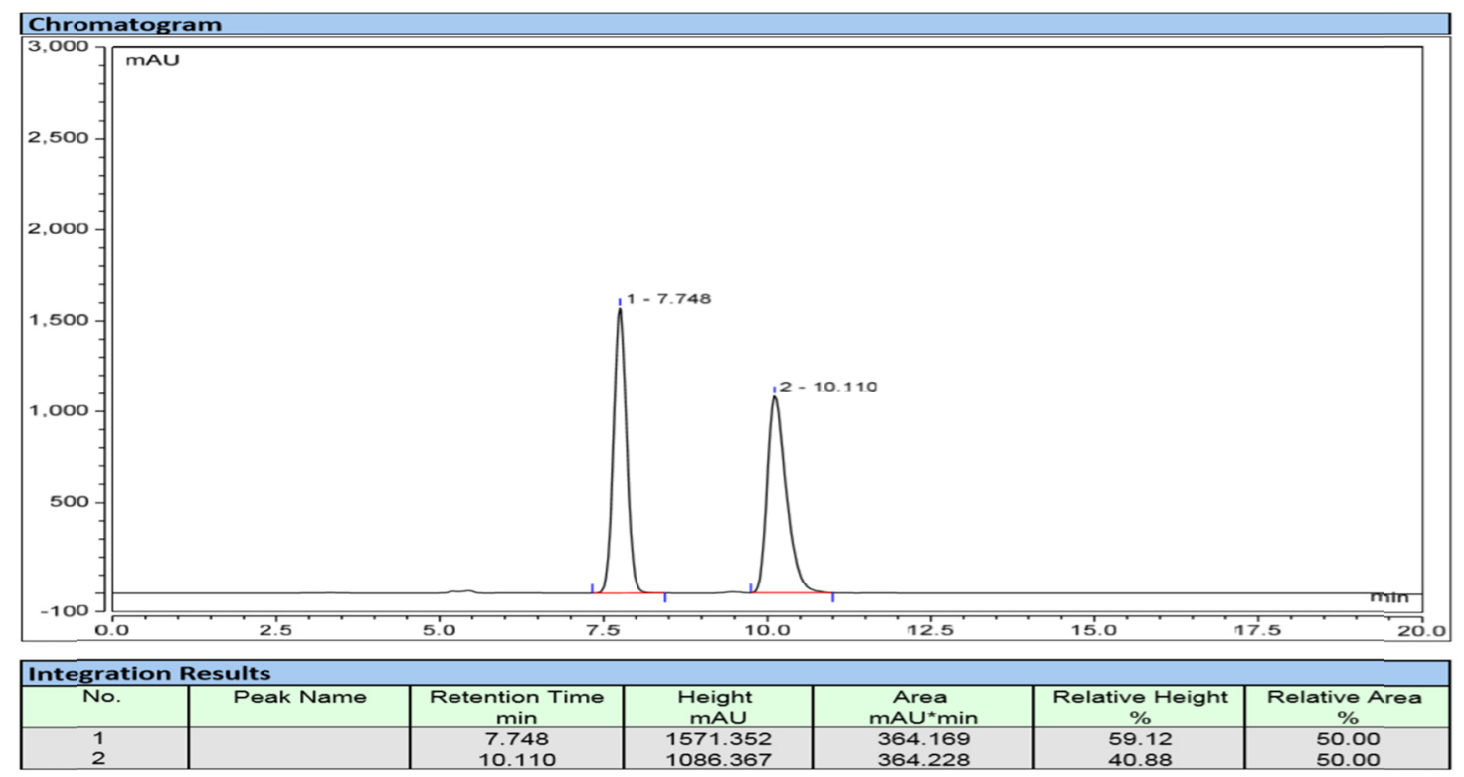




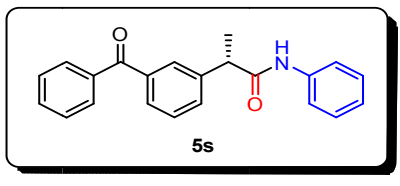

(S)-2-(3-benzoylphenyl)- $N$-phenylpropanamide

$76 \%$ yield, b:1 $>99: 1$, ee $=93 \%,[\alpha] \mathrm{D}^{19}=+36.7\left(\mathrm{c}=1.1, \mathrm{CHCl}_{3}\right) .{ }^{1} \mathrm{H}$ NMR $(400$ $\left.\mathrm{MHz}, \mathrm{CDCl}_{3}\right) \delta 7.84(\mathrm{~s}, 1 \mathrm{H}), 7.80(\mathrm{~d}, J=7.6 \mathrm{~Hz}, 2 \mathrm{H}), 7.71-7.66(\mathrm{~m}, 2 \mathrm{H}), 7.61(\mathrm{t}, J=7.2$ $\mathrm{Hz}, 1 \mathrm{H}), 7.49$ (t, $J=7.2 \mathrm{~Hz}, 6 \mathrm{H}), 7.29$ (t, $J=8.8 \mathrm{~Hz}, 2 \mathrm{H}), 7.10$ (t, $J=7.2 \mathrm{~Hz}, 1 \mathrm{H}), 3.80$ $(\mathrm{q}, J=7.2 \mathrm{~Hz}, 1 \mathrm{H}), 1.62(\mathrm{~d}, J=6.8 \mathrm{~Hz}, 3 \mathrm{H}) ;{ }^{13} \mathrm{C} \mathrm{NMR}\left(101 \mathrm{MHz}, \mathrm{CDCl}_{3}\right) \delta 196.6$, $171.7,141.5,138.2$, 137.8, 137.3, 132.6, 131.4, 130.1, 129.3, 129.2, 128.9, 128.4, 124.4, 119.8, 47.9, 18.8. HRMS calcd. (ESI) $\mathrm{m} / \mathrm{z}$ for $\mathrm{C}_{22} \mathrm{H}_{19} \mathrm{NNaO}_{2}$ : $[\mathrm{M}+\mathrm{Na}]^{+}$ 352.1308, found: 352.1301 .

Chiral HPLC (Chiralpak IG, 4.6x250 mm; $5 \mu \mathrm{L}$, hexane/isopropanol = 90/10, flow $1.0 \mathrm{~mL} / \mathrm{min}, 254 \mathrm{~nm}$ ): $\mathrm{t}_{\mathrm{R}}=17.41 \mathrm{~min}$ (major), $\mathrm{t}_{\mathrm{R}}=19.43 \min$ (minor).

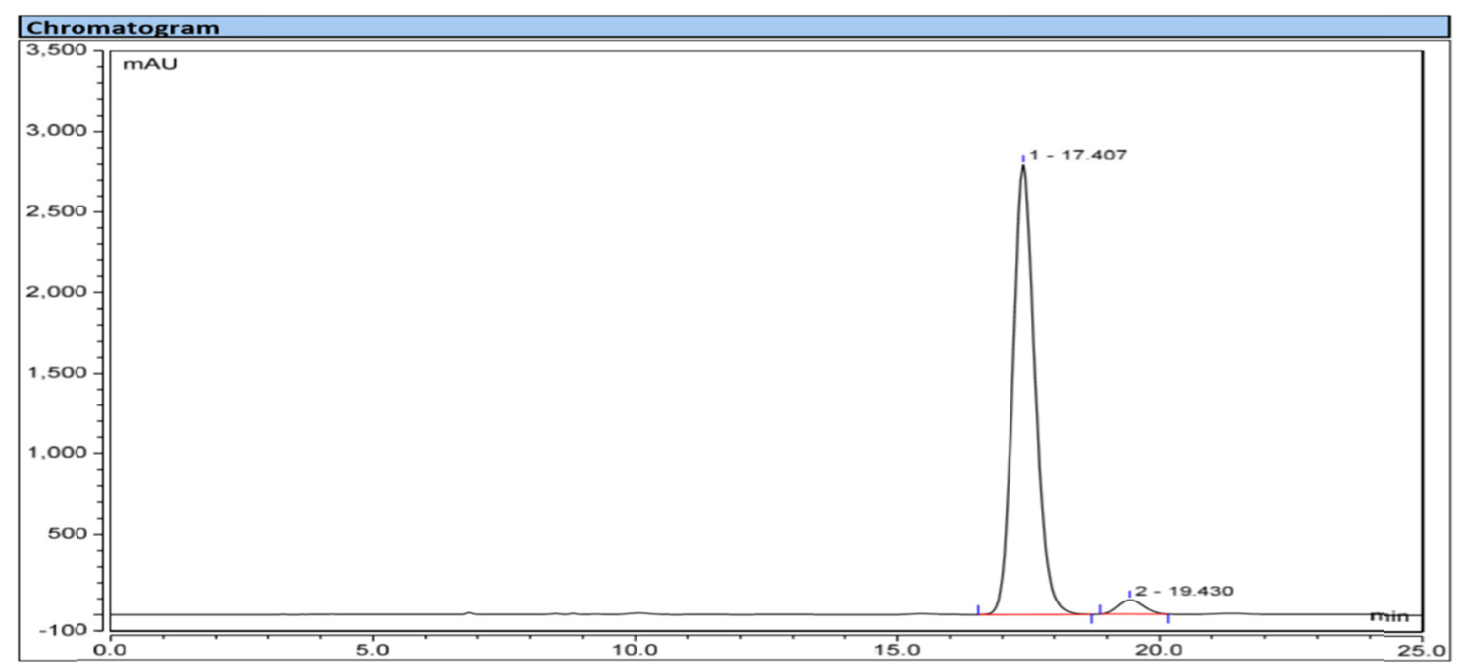

\begin{tabular}{|c|c|c|c|c|c|c|}
\hline \multicolumn{7}{|c|}{ Integration Results } \\
\hline No. & Peak Name & $\begin{array}{c}\text { Retention Time } \\
\text { min }\end{array}$ & $\begin{array}{c}\text { Height } \\
\text { mAU }\end{array}$ & $\begin{array}{c}\text { Area } \\
\text { mA } U^{\star} \text { min }\end{array}$ & $\begin{array}{c}\text { Relative Height } \\
\%\end{array}$ & $\begin{array}{c}\text { Relative Area } \\
\%\end{array}$ \\
\hline $\begin{array}{l}1 \\
2\end{array}$ & & $\begin{array}{l}17.407 \\
19.430\end{array}$ & $\begin{array}{c}2796.465 \\
89.570\end{array}$ & $\begin{array}{c}1383.747 \\
49.780\end{array}$ & $\begin{array}{c}96.90 \\
3.10\end{array}$ & $\begin{array}{c}96.53 \\
3.47\end{array}$ \\
\hline
\end{tabular}

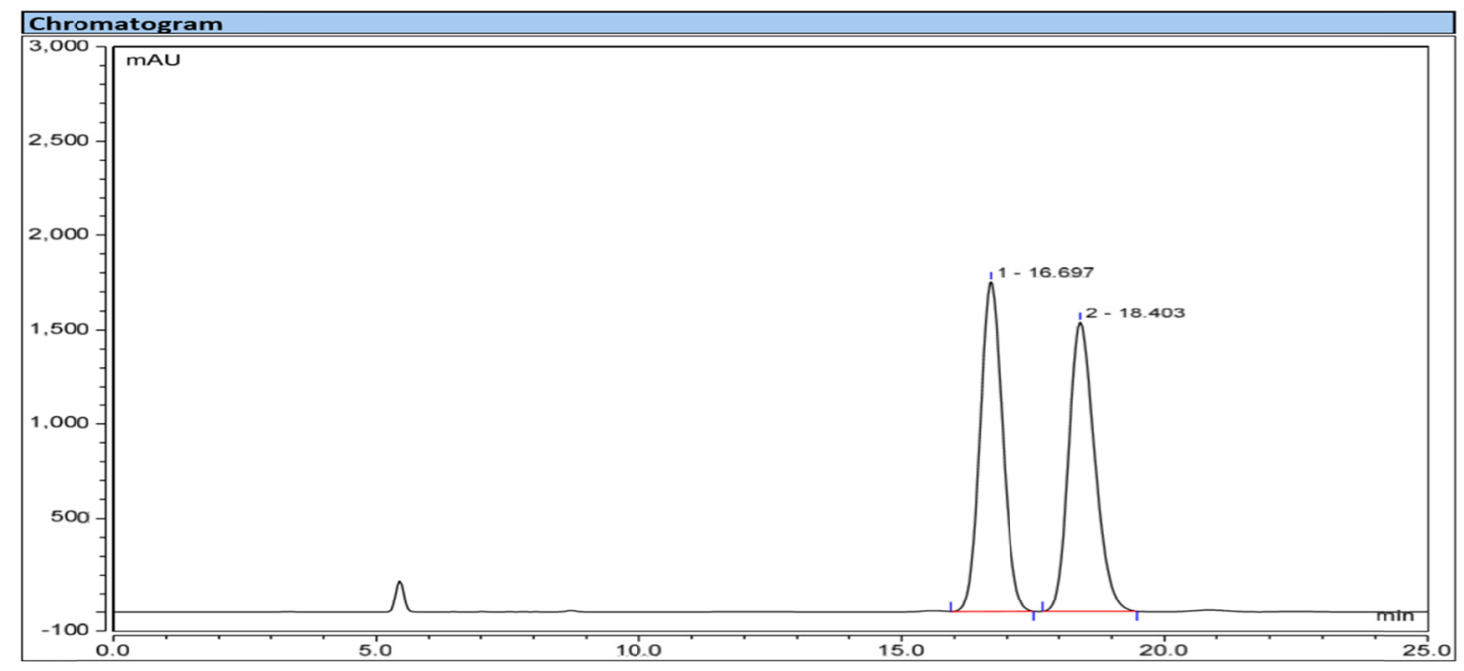

\begin{tabular}{|c|c|c|c|c|c|c|}
\hline Integration Results & \multicolumn{9}{|c|}{$\begin{array}{c}\text { Area } \\
\text { Inteight } \\
\text { No. }\end{array}$} & Peak Name & $\begin{array}{c}\text { Retention Time } \\
\text { min }\end{array}$ & $\begin{array}{c}\text { mAU } \\
\text { AUU }^{*} \text { min }\end{array}$ & $\begin{array}{c}\text { Relative Height } \\
\%\end{array}$ & $\begin{array}{c}\text { Relative Area } \\
\%\end{array}$ \\
\hline 1 & & 16.697 & 1748.702 & 876.270 & 53.20 & 49.98 \\
2 & & 18.403 & 1538.075 & 877.115 & 46.80 & 50.02 \\
\hline
\end{tabular}




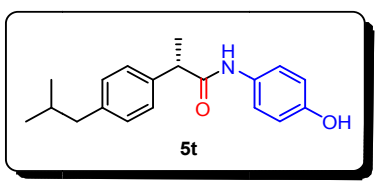

(S)- $N$-(4-hydroxyphenyl)-2-(4-isobutylphenyl)propanamide

$73 \%$ yield, b:1 > 99:1, ee $=90 \%,[\alpha] \mathrm{D}^{19}=+54.1\left(\mathrm{c}=1.2, \mathrm{CHCl}_{3}\right) .{ }^{1} \mathrm{H}$ NMR $(400$ MHz, d'-DMSO) $\delta 9.81(\mathrm{~s}, 1 \mathrm{H}), 9.19(\mathrm{~s}, 1 \mathrm{H}), 7.41(\mathrm{~d}, J=8.4 \mathrm{~Hz}, 2 \mathrm{H}), 7.33(\mathrm{~d}, J=7.6$ $\mathrm{Hz}, 2 \mathrm{H}), 7.13(\mathrm{~d}, J=8.0 \mathrm{~Hz}, 2 \mathrm{H}), 6.71(\mathrm{~d}, J=8.8 \mathrm{~Hz}, 2 \mathrm{H}), 3.78$ (q, $J=6.8 \mathrm{~Hz}, 1 \mathrm{H})$, $2.43(\mathrm{~d}, J=7.2 \mathrm{~Hz}, 2 \mathrm{H}), 1.86-1.80(\mathrm{~m}, 1 \mathrm{H}), 1.42$ (d, $J=6.8 \mathrm{~Hz}, 3 \mathrm{H}), 0.88$ (d, $J=6.8$ $\mathrm{Hz}, 6 \mathrm{H}) ;{ }^{13} \mathrm{C}$ NMR (101 MHz, $d^{6}$-DMSO) $\delta 171.6,153.2,139.4,139.3,130.9,128.8$, 126.9, 120.8, 115.0, 45.4, 44.2, 29.6, 22.2, 18.7. HRMS calcd. (ESI) $\mathrm{m} / \mathrm{z}$ for $\mathrm{C}_{19} \mathrm{H}_{23} \mathrm{NNaO}_{2}$ : [M+Na] 320.1621 , found: 320.1625 .

Chiral HPLC (Chiralpak IB-N5, 4.6x250 mm; $5 \mu \mathrm{L}$, hexane/isopropanol $=85 / 15$, flow $1.0 \mathrm{~mL} / \mathrm{min}, 254 \mathrm{~nm}$ ): $\mathrm{t}_{\mathrm{R}}=10.66 \mathrm{~min}$ (major), $\mathrm{t}_{\mathrm{R}}=15.98 \mathrm{~min}$ (minor).
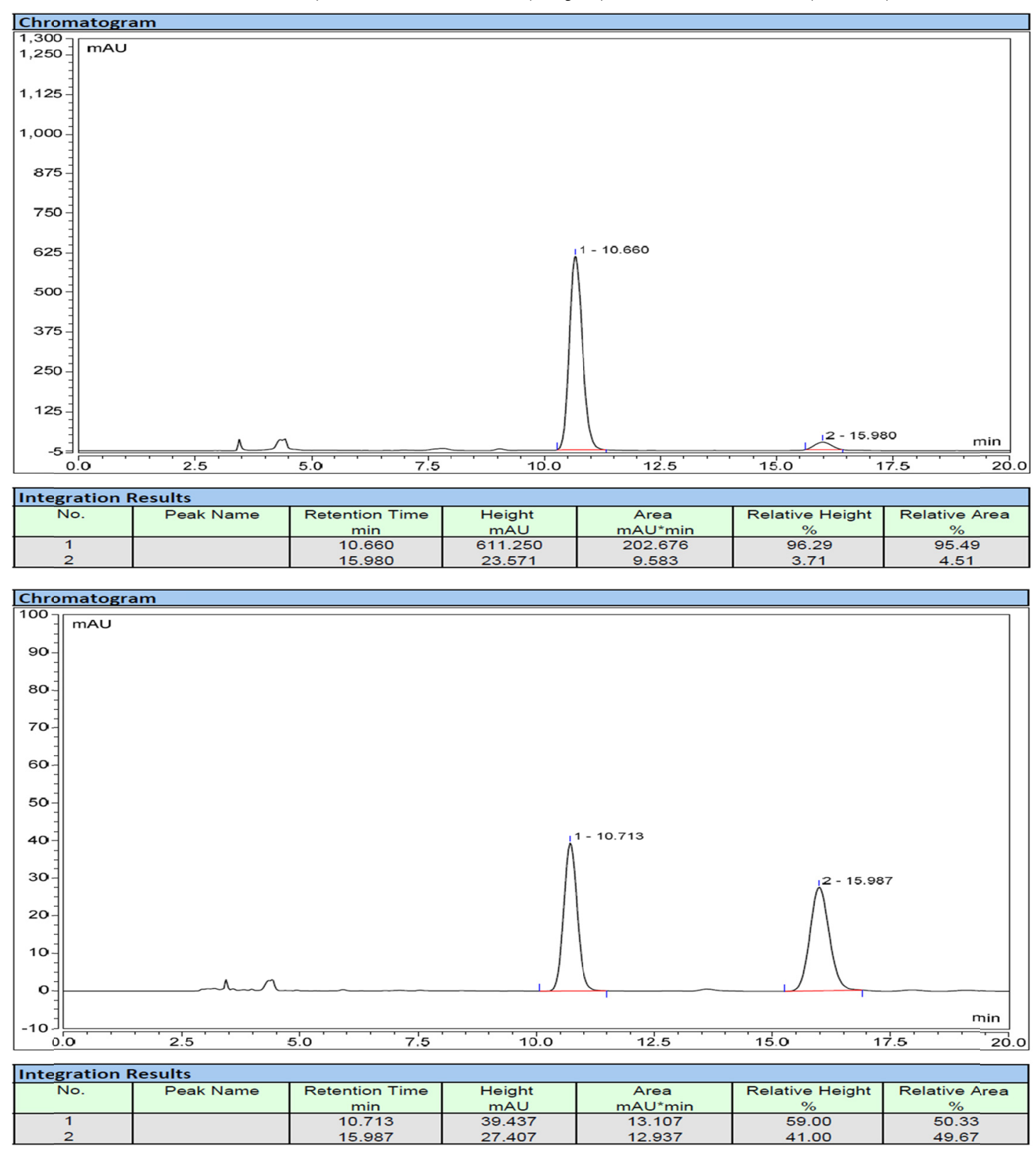


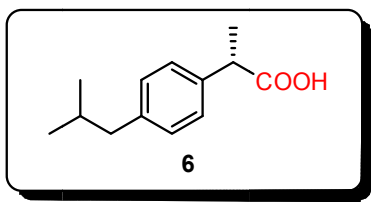

(S)-2-(4-isobutylphenyl)propanoic acid

93\% yield, b:1 $>99: 1$, ee $=90 \%,[\alpha] \mathrm{D}^{18}=+57.4\left(\mathrm{c}=0.9, \mathrm{CHCl}_{3}\right) .{ }^{1} \mathrm{H}$ NMR $(400$ $\left.\mathrm{MHz}, \mathrm{CDCl}_{3}\right) \delta 7.21$ (d, $\left.J=8.0 \mathrm{~Hz}, 2 \mathrm{H}\right), 7.09$ (d, $\left.J=8.0 \mathrm{~Hz}, 2 \mathrm{H}\right), 3.70$ (q, $J=7.2 \mathrm{~Hz}$, $1 \mathrm{H}), 2.44(\mathrm{~d}, J=7.2 \mathrm{~Hz}, 2 \mathrm{H}), 1.87-1.81(\mathrm{~m}, 1 \mathrm{H}), 1.49(\mathrm{~d}, J=7.2 \mathrm{~Hz}, 3 \mathrm{H}), 0.89$ (d, $J=$ $6.8 \mathrm{~Hz}, 6 \mathrm{H}) ;{ }^{13} \mathrm{C}$ NMR $\left(101 \mathrm{MHz}, \mathrm{CDCl}_{3}\right) \delta 181.5,140.8,136.9,129.4,127.3,45.02$, 44.97, 30.2, 22.4, 18.1. HRMS calcd. (ESI) $\mathrm{m} / \mathrm{z}$ for $\mathrm{C}_{13} \mathrm{H}_{19} \mathrm{O}_{2}:[\mathrm{M}+\mathrm{H}]^{+} 207.1380$, found:207.1386.

The 6 was treated with diazomethane. The resulting ester was compared to the racemic 6. Chiral HPLC (Chiralpak OJ-H, $4.6 \times 250 \mathrm{~mm} ; 5 \mu \mathrm{L}$, hexane/isopropanol $=$ $98 / 2$, flow $1.0 \mathrm{~mL} / \mathrm{min}, 254 \mathrm{~nm}$ ): $\mathrm{t}_{\mathrm{R}}=4.81 \mathrm{~min}$ (minor), $\mathrm{t}_{\mathrm{R}}=5.09 \mathrm{~min}$ (major).
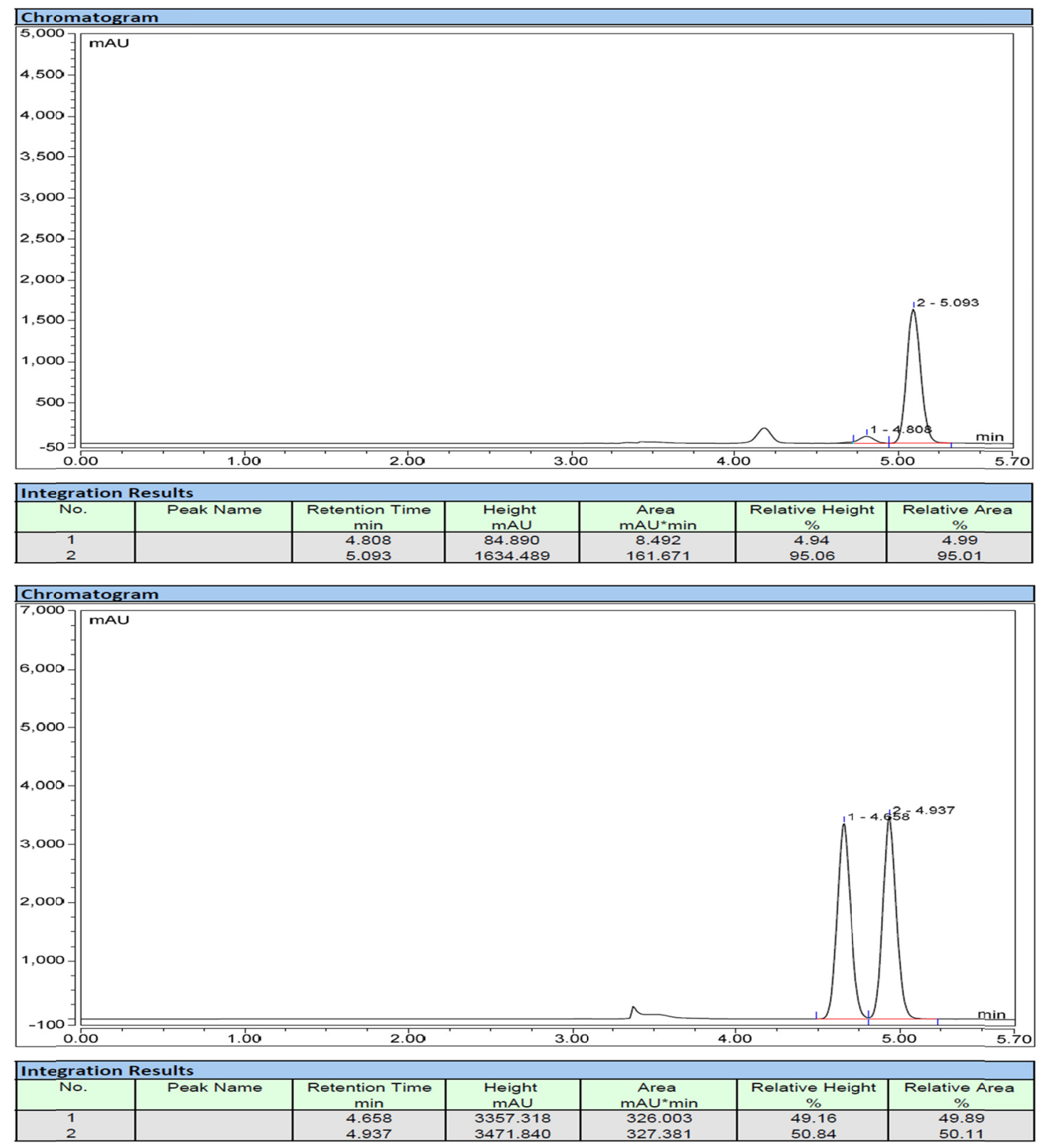


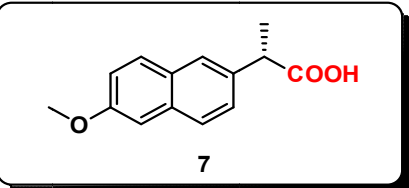

(S)-2-(6-methoxynaphthalen-2-yl)propanoic acid

$96 \%$ yield, b:1 $>99: 1$, ee $=90 \%,[\alpha] \mathrm{D}^{18}=-39.6\left(\mathrm{c}=1.2, \mathrm{CHCl}_{3}\right) .{ }^{1} \mathrm{H}$ NMR $(400$ $\left.\mathrm{MHz}, \mathrm{CDCl}_{3}\right) \delta 7.69(\mathrm{~s}, 1 \mathrm{H}), 7.67(\mathrm{~s}, 2 \mathrm{H}), 7.40(\mathrm{dd}, J=8.8,2.0 \mathrm{~Hz}, 1 \mathrm{H}), 7.12(\mathrm{dt}, J=$ 8.8, $2.4 \mathrm{~Hz}, 1 \mathrm{H}), 7.09$ (d, $J=2.4 \mathrm{~Hz}, 1 \mathrm{H}), 3.89$ (s, 3H), 3.85 (q, $J=7.2 \mathrm{~Hz}, 1 \mathrm{H}), 1.57$ $(\mathrm{d}, J=7.2 \mathrm{~Hz}, 1 \mathrm{H}) ;{ }^{13} \mathrm{C} \mathrm{NMR}\left(101 \mathrm{MHz}, \mathrm{CDCl}_{3}\right) \delta 180.9,157.6,134.8,133.8,129.3$, $128.8,127.2,126.2,126.1,119.0,105.5,55.3,45.2,18.1$. HRMS calcid. (ESI) m/z for $\mathrm{C}_{14} \mathrm{H}_{13} \mathrm{O}_{3}$ : [M-H] 229.0859 , found:229.0863.

The 7 was treated with diazomethane. The resulting ester was compared to the racemic 7. Chiral HPLC (Chiralpak iH, 4.6x250 mm; $5 \mu \mathrm{L}$, hexane/isopropanol = 98/2, flow $1.0 \mathrm{~mL} / \mathrm{min}, 254 \mathrm{~nm}$ ): $\mathrm{t}_{\mathrm{R}}=6.67 \mathrm{~min}$ (minor), $\mathrm{t}_{\mathrm{R}}=7.92 \mathrm{~min}$ (major).
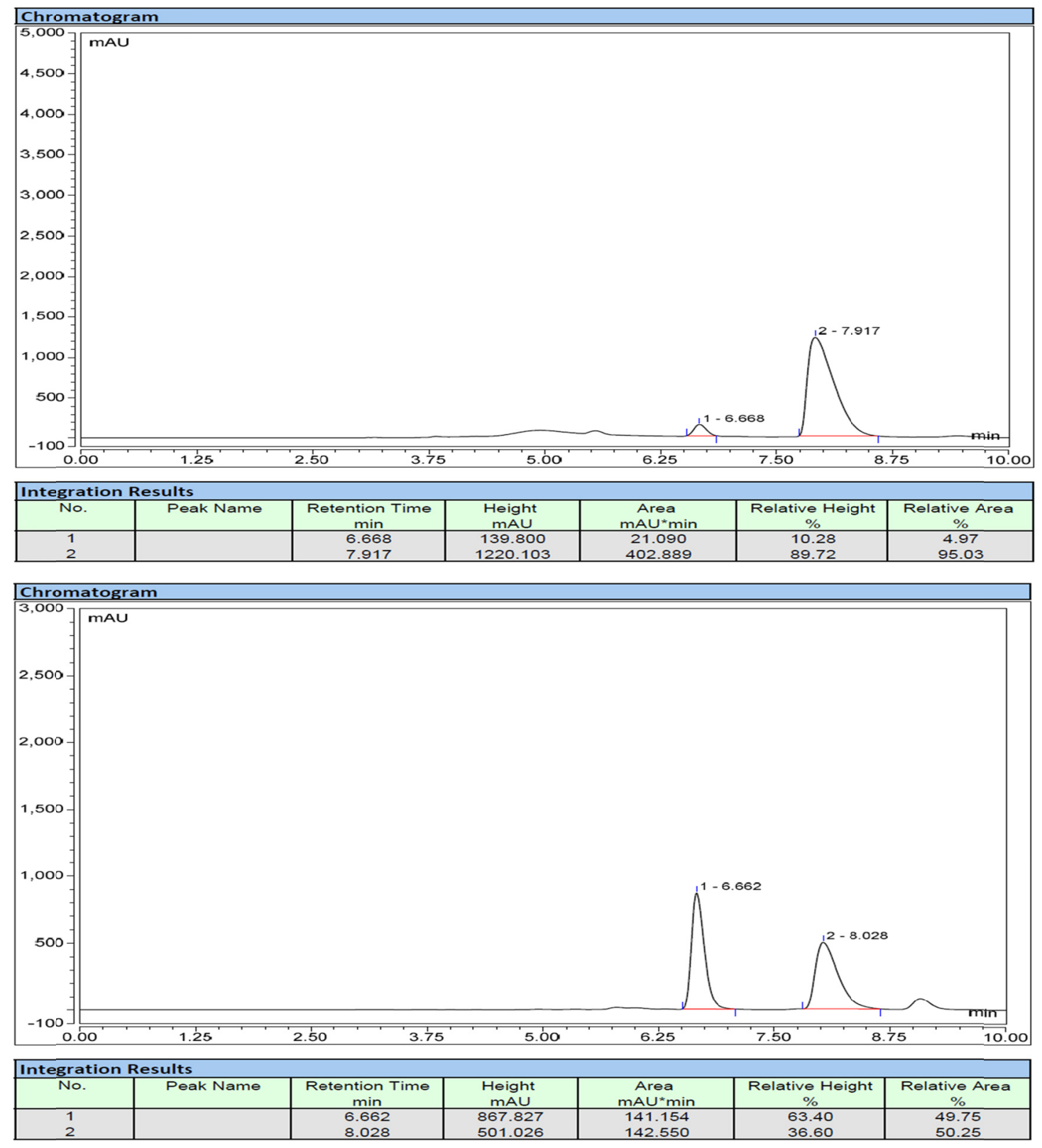


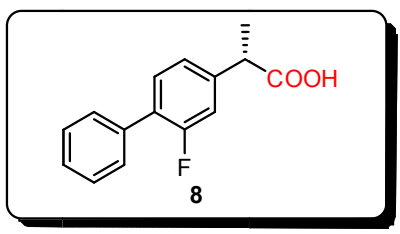

(S)-2-(2-fluoro-[1,1'-biphenyl]-4-yl)propanoic acid

$88 \%$ yield, b:1 $>99: 1$, ee $=92 \%,[\alpha] \mathrm{D}^{18}=+44.2\left(\mathrm{c}=1.0, \mathrm{CHCl}_{3}\right) .{ }^{1} \mathrm{H}$ NMR $(400$ $\left.\mathrm{MHz}, \mathrm{CDCl}_{3}\right) \delta 8.64(\mathrm{~s}, 1 \mathrm{H}), 7.52(\mathrm{~d}, J=8.0 \mathrm{~Hz}, 2 \mathrm{H}), 7.44-7.35(\mathrm{~m}, 4 \mathrm{H}), 7.19-7.14$ $(\mathrm{m}, 2 \mathrm{H}), 3.76(\mathrm{q}, J=7.2 \mathrm{~Hz}, 1 \mathrm{H}), 1.54(\mathrm{~d}, J=7.2 \mathrm{~Hz}, 3 \mathrm{H}) ;{ }^{13} \mathrm{C}$ NMR $(101 \mathrm{MHz}$, $\left.\mathrm{CDCl}_{3}\right) \delta 177.8,159.6(\mathrm{~d}, J=249.2 \mathrm{~Hz}), 141.7(\mathrm{~d}, J=7.8 \mathrm{~Hz}), 135.5,130.7(\mathrm{~d}, J=$ $4.0 \mathrm{~Hz}), 128.9(\mathrm{~d}, J=2.9 \mathrm{~Hz}), 128.4,127.8(\mathrm{~d}, J=13.5 \mathrm{~Hz}), 127.6,123.6(\mathrm{~d}, J=3.3$ $\mathrm{Hz}), 115.3(\mathrm{~d}, J=23.7 \mathrm{~Hz}), 44.9,18.1 ;{ }^{19} \mathrm{~F} \mathrm{NMR}\left(376 \mathrm{MHz}, \mathrm{CDCl}_{3}\right) \delta-117.34$. HRMS calcd. (ESI) $\mathrm{m} / \mathrm{z}$ for $\mathrm{C}_{15} \mathrm{H}_{12} \mathrm{FO}_{2}$ : [M-H] 243.0816, found: 243.0821 .

The 8 was treated with diazomethane. The resulting ester was compared to the racemic 8. Chiral HPLC (Chiralpak OJ-H, 4.6x250 mm; $5 \mu \mathrm{L}$, hexane/isopropanol $=$ $98 / 2$, flow $1.0 \mathrm{~mL} / \mathrm{min}, 254 \mathrm{~nm}$ ): $\mathrm{t}_{\mathrm{R}}=20.50 \mathrm{~min}$ (minor), $\mathrm{t}_{\mathrm{R}}=24.63 \mathrm{~min}$ (major).
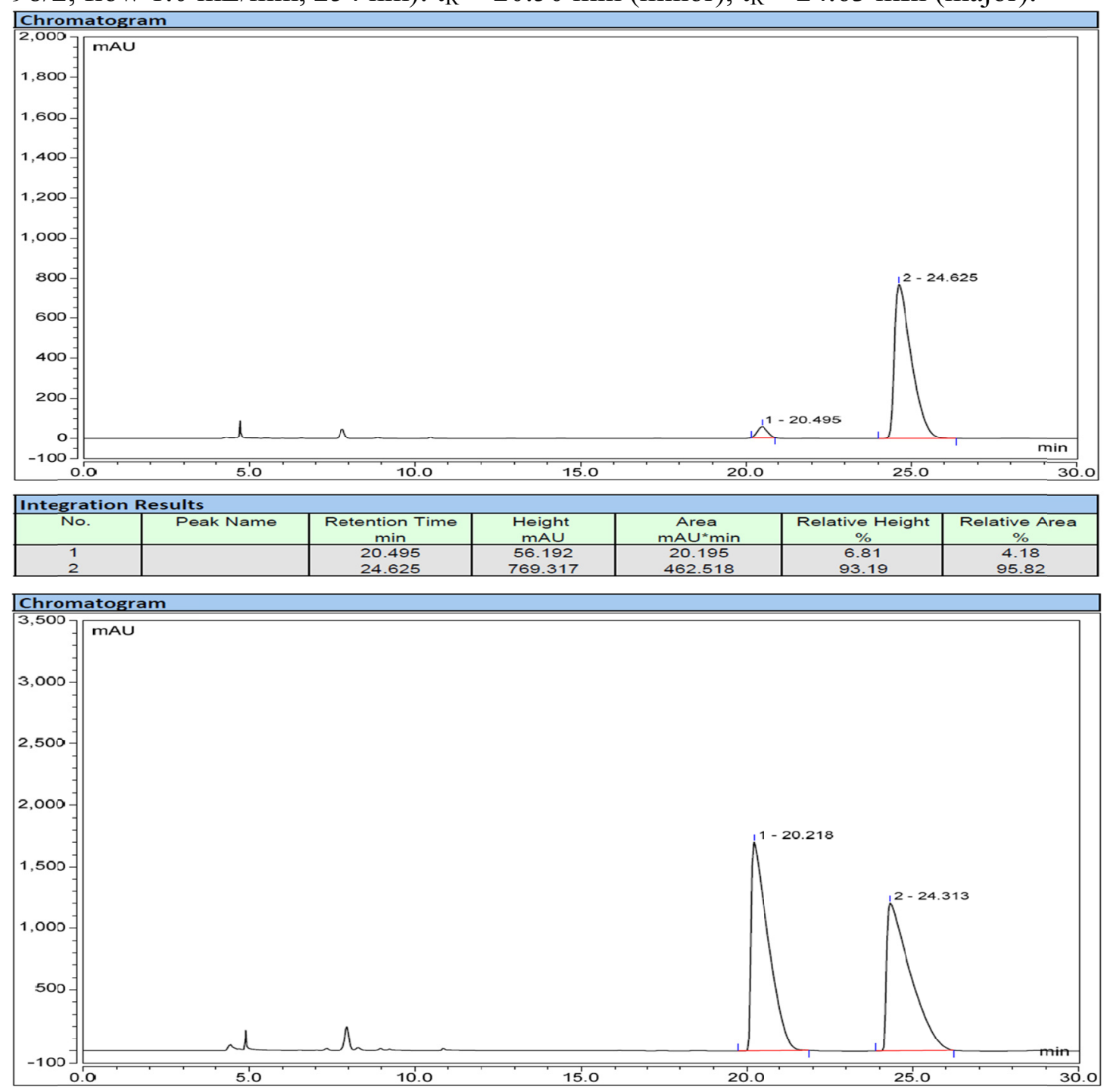

\begin{tabular}{|c|c|c|c|c|c|c|}
\hline Integration Results & \multicolumn{5}{|c|}{ Relative Height } \\
\hline No. & Peak Name & $\begin{array}{c}\text { Retention Time } \\
\text { min }\end{array}$ & $\begin{array}{c}\text { Height } \\
\text { mAU }\end{array}$ & $\begin{array}{c}\text { Area } \\
\text { mAU*min }\end{array}$ & $\begin{array}{c}\text { Relave Area } \\
\%\end{array}$ \\
\hline 1 & & 20.218 & 1703.739 & 1037.923 & 58.58 & 50.83 \\
2 & & 24.313 & 1204.710 & 1003.859 & 41.42 & 49.17 \\
\hline
\end{tabular}




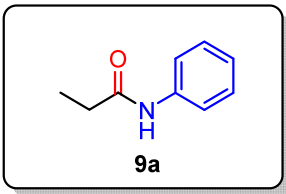

\section{$N$-phenylpropionamide}

93\% yield. ${ }^{1} \mathrm{H}$ NMR $\left(400 \mathrm{MHz}, \mathrm{CDCl}_{3}\right) \delta 7.73(\mathrm{~s}, 1 \mathrm{H}), 7.52(\mathrm{~d}, J=7.6 \mathrm{~Hz}, 2 \mathrm{H})$, $7.29(\mathrm{t}, J=7.6 \mathrm{~Hz}, 2 \mathrm{H}), 7.08(\mathrm{t}, J=7.6 \mathrm{~Hz}, 1 \mathrm{H}), 2.37(\mathrm{q}, J=7.6 \mathrm{~Hz}, 2 \mathrm{H}), 1.22(\mathrm{t}, J=$ $7.6 \mathrm{~Hz}, 3 \mathrm{H}) ;{ }^{13} \mathrm{C} \mathrm{NMR}\left(101 \mathrm{MHz}, \mathrm{CDCl}_{3}\right) \delta 172.4,138.0,128.8,124.1,119.9,30.6$, 9.7. HRMS calcd. (ESI) $\mathrm{m} / \mathrm{z}$ for $\mathrm{C}_{9} \mathrm{H}_{11} \mathrm{NNaO}$ : $[\mathrm{M}+\mathrm{Na}]^{+}$172.0733, found: 172.0730 .

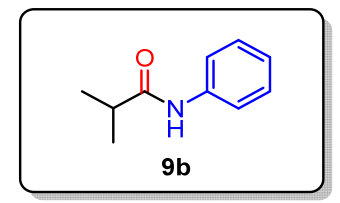

\section{$N$-phenylisobutyramide}

90\% yield, b:1 =91:9. ${ }^{1} \mathrm{H}$ NMR (400 MHz, $\left.\mathrm{CDCl}_{3}\right) \delta 7.53(\mathrm{~d}, J=8.0 \mathrm{~Hz}, 2 \mathrm{H})$, $7.46(\mathrm{~s}, 1 \mathrm{H}), 7.29$ (t, $J=7.2 \mathrm{~Hz}, 2 \mathrm{H}), 7.08(\mathrm{t}, J=7.2 \mathrm{~Hz}, 1 \mathrm{H}), 2.54-2.47$ (m, 1H), 1.23 $(\mathrm{d}, J=6.8 \mathrm{~Hz}, 6 \mathrm{H}) ;{ }^{13} \mathrm{C} \mathrm{NMR}\left(101 \mathrm{MHz}, \mathrm{CDCl}_{3}\right) \delta 175.4,138.0,128.9,124.1,119.8$, 36.6, 19.6. HRMS calcd. (ESI) $\mathrm{m} / \mathrm{z}$ for $\mathrm{C}_{10} \mathrm{H}_{13} \mathrm{NNaO}$ : $[\mathrm{M}+\mathrm{Na}]^{+} 186.0889$, found: 186.0887 . 


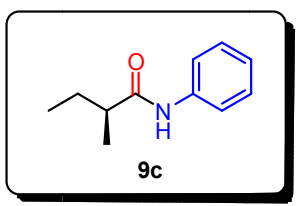

\section{(S)-2-methyl- $N$-phenylbutanamide}

$91 \%$ yield, b:1 $=83: 17$, ee $=16 \% .{ }^{1} \mathrm{H}$ NMR $\left(400 \mathrm{MHz}, \mathrm{CDCl}_{3}\right) \delta 7.54(\mathrm{~d}, J=8.0$ $\mathrm{Hz}, 2 \mathrm{H}), 7.31(\mathrm{t}, J=7.6 \mathrm{~Hz}, 2 \mathrm{H}), 7.26(\mathrm{~s}, 1 \mathrm{H}), 7.10(\mathrm{t}, J=7.2 \mathrm{~Hz}, 1 \mathrm{H}), 2.26(\mathrm{q}, J=$ $6.8 \mathrm{~Hz}, 1 \mathrm{H}), 1.81-1.72(\mathrm{~m}, 1 \mathrm{H}), 1.55-1.48(\mathrm{~m}, 1 \mathrm{H}), 1.23(\mathrm{~d}, J=6.8 \mathrm{~Hz}, 3 \mathrm{H}), 0.96(\mathrm{t}, J$ $=7.6 \mathrm{~Hz}, 3 \mathrm{H}) ;{ }^{13} \mathrm{C} \mathrm{NMR}\left(101 \mathrm{MHz}, \mathrm{CDCl}_{3}\right) \delta 174.8138 .0,129.0,124.1,119.8,44.3$, 27.4, 17.5, 11.9. HRMS calcd. (ESI) $\mathrm{m} / \mathrm{z}$ for $\mathrm{C}_{11} \mathrm{H}_{15} \mathrm{NO}$ : $[\mathrm{M}+\mathrm{Na}]^{+} 200.1046$, found: 200.1042 .

Chiral HPLC (Chiralpak IG, 4.6x250 mm; $5 \mu \mathrm{L}$, hexane/isopropanol $=95 / 5$, flow $1.0 \mathrm{~mL} / \mathrm{min}, 254 \mathrm{~nm}$ ): $\mathrm{t}_{\mathrm{R}}=16.42 \min$ (major), $\mathrm{t}_{\mathrm{R}}=17.12 \mathrm{~min}$ (minor).

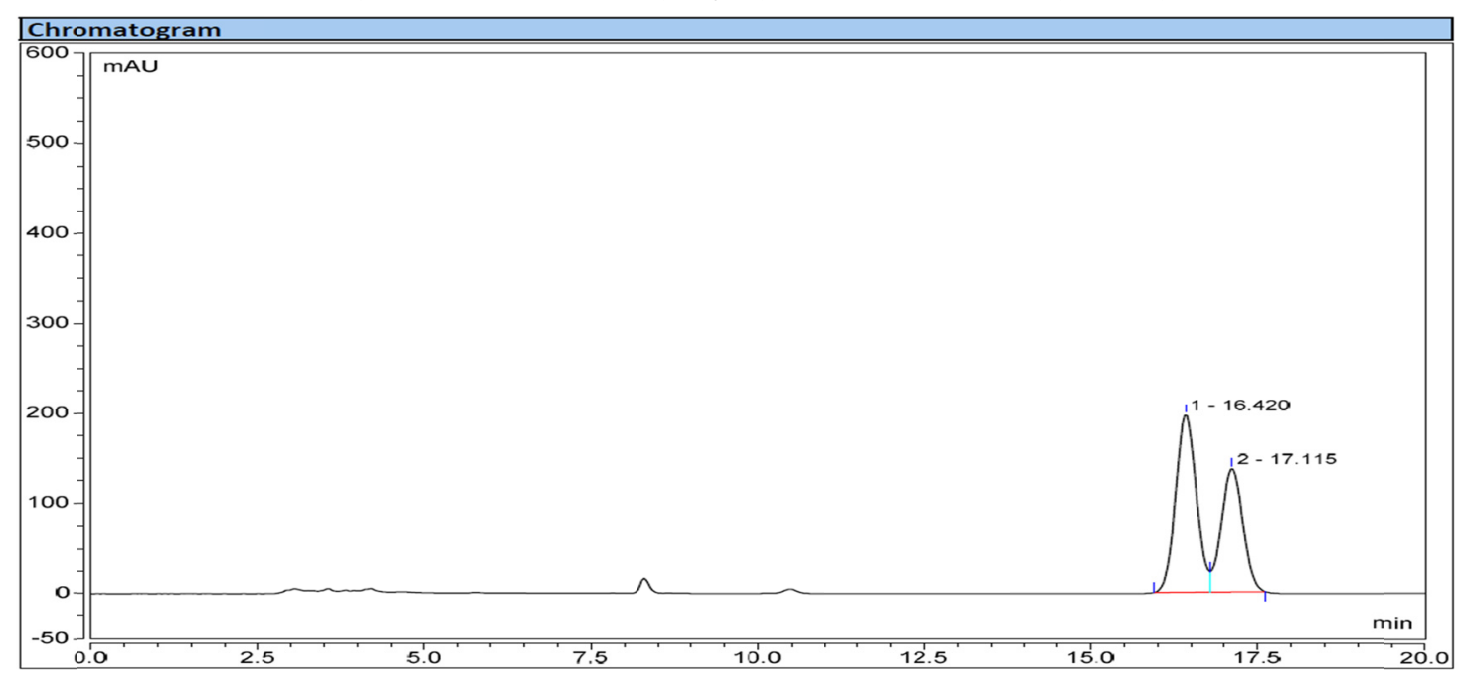

\begin{tabular}{|c|c|c|c|c|c|c|}
\hline \multicolumn{7}{|c|}{\begin{tabular}{|l|} 
Integration Results \\
\end{tabular}} \\
\hline No. & Peak Name & $\begin{array}{c}\text { Retention Time } \\
\text { min }\end{array}$ & $\begin{array}{c}\text { Height } \\
\text { mAU }\end{array}$ & $\begin{array}{c}\text { Area } \\
\text { mAU*min }\end{array}$ & $\begin{array}{c}\text { Relative Height } \\
\%\end{array}$ & $\begin{array}{c}\text { Relative Area } \\
\%\end{array}$ \\
\hline 1 & & 16.420 & 197.374 & 71.201 & 59.05 & 58.09 \\
\hline 2 & & 17.115 & 136.899 & 51.374 & 40.95 & 41.91 \\
\hline
\end{tabular}

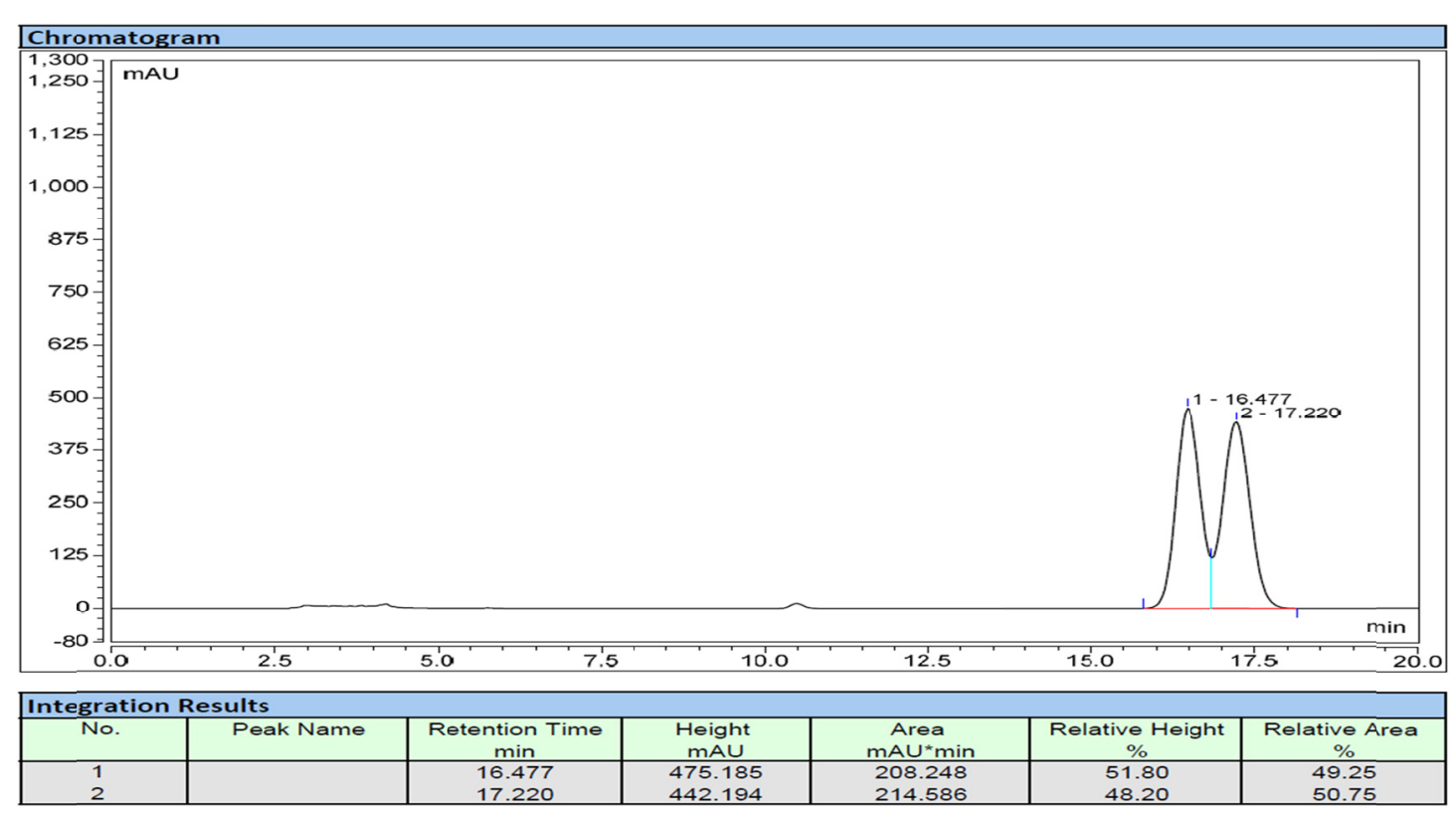




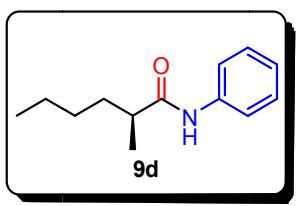

(S)-2-methyl- $N$-phenylhexanamide

$87 \%$ yield, b:1 $=84: 16$, ee $=18 \% .{ }^{1} \mathrm{H}$ NMR $\left(400 \mathrm{MHz}, \mathrm{CDCl}_{3}\right) \delta 7.69(\mathrm{~s}, 1 \mathrm{H})$, 7.55 (d, $J=7.6 \mathrm{~Hz}, 2 \mathrm{H}), 7.28$ (t, $J=7.6 \mathrm{~Hz}, 2 \mathrm{H}), 7.08$ (t, $J=7.2 \mathrm{~Hz}, 1 \mathrm{H}), 2.39-2.31$ $(\mathrm{m}, 1 \mathrm{H}), 1.77-1.68(\mathrm{~m}, 1 \mathrm{H}), 1.46-1.39(\mathrm{~m}, 1 \mathrm{H}), 1.31-1.30(\mathrm{~m}, 4 \mathrm{H}), 1.20(\mathrm{~d}, J=6.8 \mathrm{~Hz}$, $3 \mathrm{H}), 0.87(\mathrm{t}, J=6.8 \mathrm{~Hz}, 3 \mathrm{H}) ;{ }^{13} \mathrm{C}$ NMR $\left(101 \mathrm{MHz}, \mathrm{CDCl}_{3}\right) \delta 175.3,138.1,128.8$, $124.0,119.9,42.5,34.1,29.6,22.7,17.9,13.9$. HRMS calcd. (ESI) $\mathrm{m} / \mathrm{z}$ for $\mathrm{C}_{13} \mathrm{H}_{19} \mathrm{NNaO}:[\mathrm{M}+\mathrm{Na}]^{+} 228.1359$, found: 228.1356 .

Chiral HPLC (Chiralpak IG, 4.6x250 mm; $5 \mu \mathrm{L}$, hexane/isopropanol $=95 / 5$, flow $1.0 \mathrm{~mL} / \mathrm{min}, 254 \mathrm{~nm}$ ): $\mathrm{t}_{\mathrm{R}}=15.60 \mathrm{~min}$ (major), $\mathrm{t}_{\mathrm{R}}=16.33 \mathrm{~min}$ (minor).

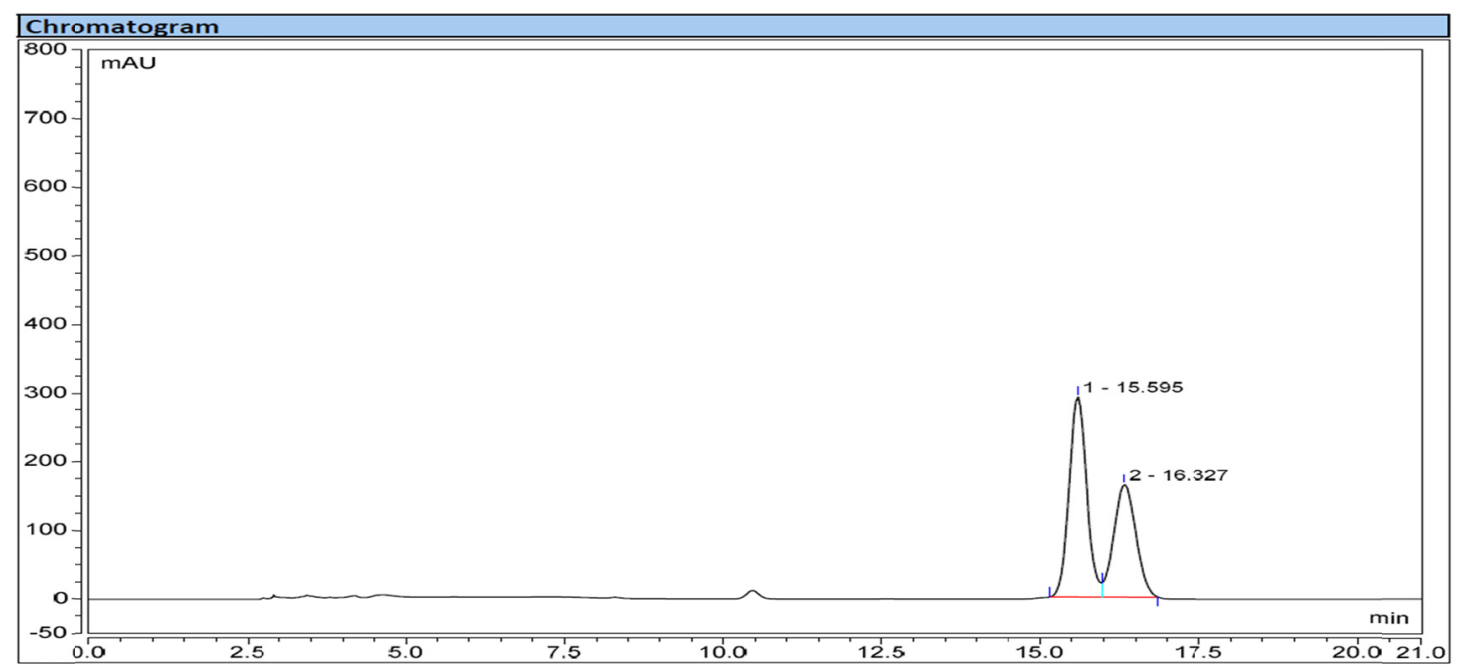

\begin{tabular}{|c|c|c|c|c|c|c|}
\hline Integration Results & \multicolumn{9}{|c|}{$\begin{array}{c}\text { Area } \\
\text { No. }\end{array}$} & Peak Name & $\begin{array}{c}\text { Retention Time } \\
\text { min }\end{array}$ & $\begin{array}{c}\text { Height } \\
\text { mAU }\end{array}$ & $\begin{array}{c}\text { Relative Height } \\
\text { mA }\end{array}$ & $\begin{array}{c}\text { Relative Area } \\
\%\end{array}$ \\
\hline 1 & & 15.595 & 292.293 & 93.555 & 64.20 & 58.85 \\
2 & & 16.327 & 162.997 & 65.410 & 35.80 & 41.15 \\
\hline
\end{tabular}

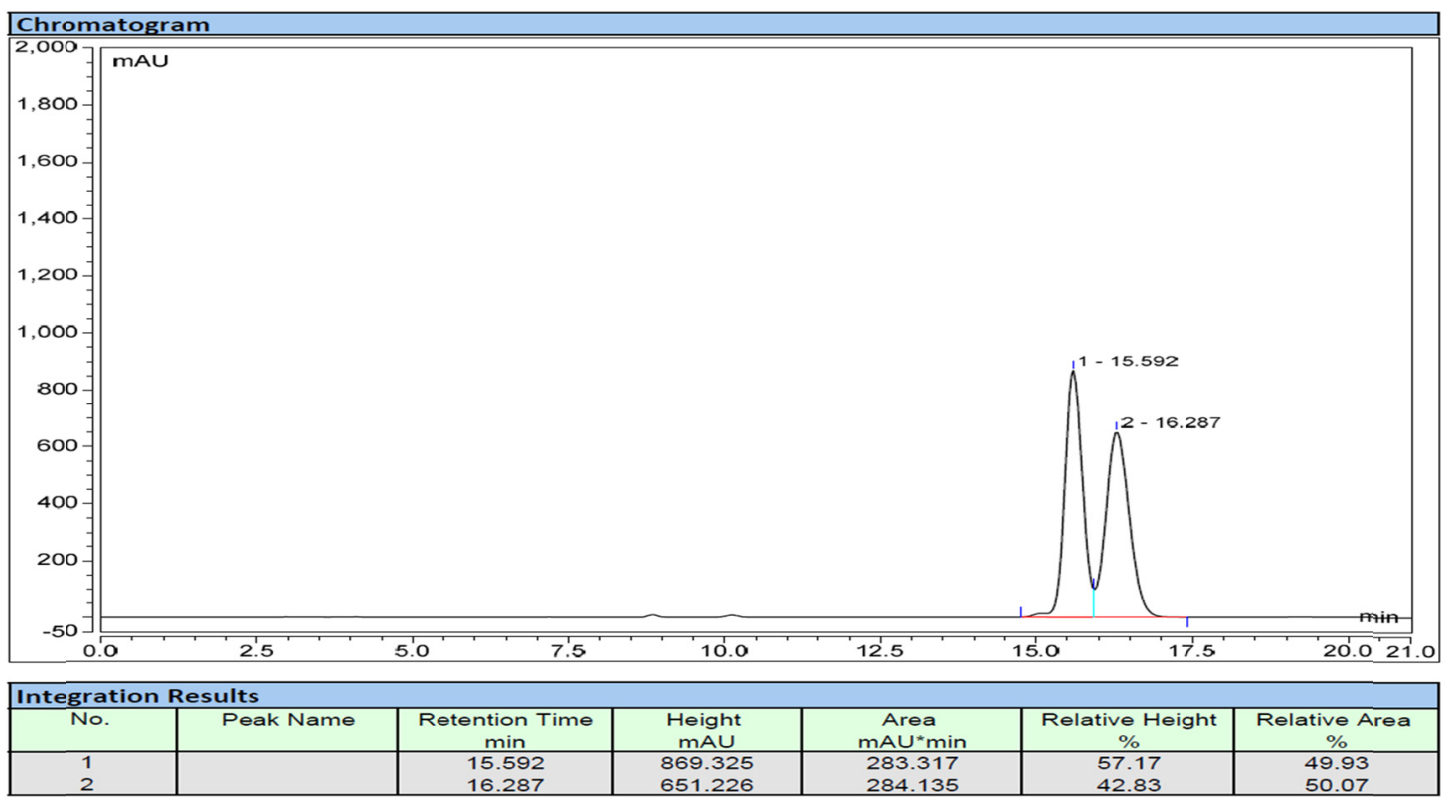




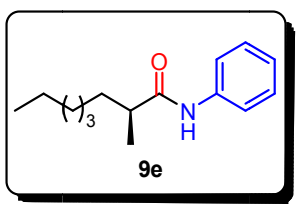

\section{(S)-2-methyl- $N$-phenyloctanamide}

$86 \%$ yield, b:1 = 86:14, ee $=19 \% .{ }^{1} \mathrm{H}$ NMR $\left(400 \mathrm{MHz}, \mathrm{CDCl}_{3}\right) \delta 7.72(\mathrm{~s}, 1 \mathrm{H})$, $7.55(\mathrm{~d}, J=7.6 \mathrm{~Hz}, 2 \mathrm{H}), 7.28(\mathrm{t}, J=7.6 \mathrm{~Hz}, 2 \mathrm{H}), 7.07(\mathrm{t}, J=7.2 \mathrm{~Hz}, 1 \mathrm{H}), 2.35(\mathrm{dd}, J$ $=14.0,7.2 \mathrm{~Hz}, 1 \mathrm{H}), 1.75-1.68(\mathrm{~m}, 1 \mathrm{H}), 1.44-1.38(\mathrm{~m}, 1 \mathrm{H}), 1.26-1.91(\mathrm{~s}, 8 \mathrm{H}), 1.20(\mathrm{~d}$, $\left.J=6.8 \mathrm{~Hz}, 3 \mathrm{H}), 0.86(\mathrm{t}, J=6.4 \mathrm{~Hz}, 3 \mathrm{H}) ;{ }^{13} \mathrm{C} \mathrm{NMR}\left(101 \mathrm{MHz}, \mathrm{CDCl}_{3}\right)\right) \delta 175.3,138.1$, $128.8,124.0,119.9,42.5,34.4,31.7,29.3,27.4,22.6,17.9,14.0$. HRMS calcd. (ESI) $\mathrm{m} / \mathrm{z}$ for $\mathrm{C}_{15} \mathrm{H}_{23} \mathrm{NNaO}:[\mathrm{M}+\mathrm{Na}]^{+} 256.1672$, found: 256.1675 .

Chiral HPLC (Chiralpak IG, 4.6x250 mm; $5 \mu \mathrm{L}$, hexane/isopropanol $=90 / 10$, flow $1.0 \mathrm{~mL} / \mathrm{min}, 254 \mathrm{~nm}$ ): $\mathrm{t}_{\mathrm{R}}=7.56 \mathrm{~min}$ (major), $\mathrm{t}_{\mathrm{R}}=8.13 \mathrm{~min}$ (minor).

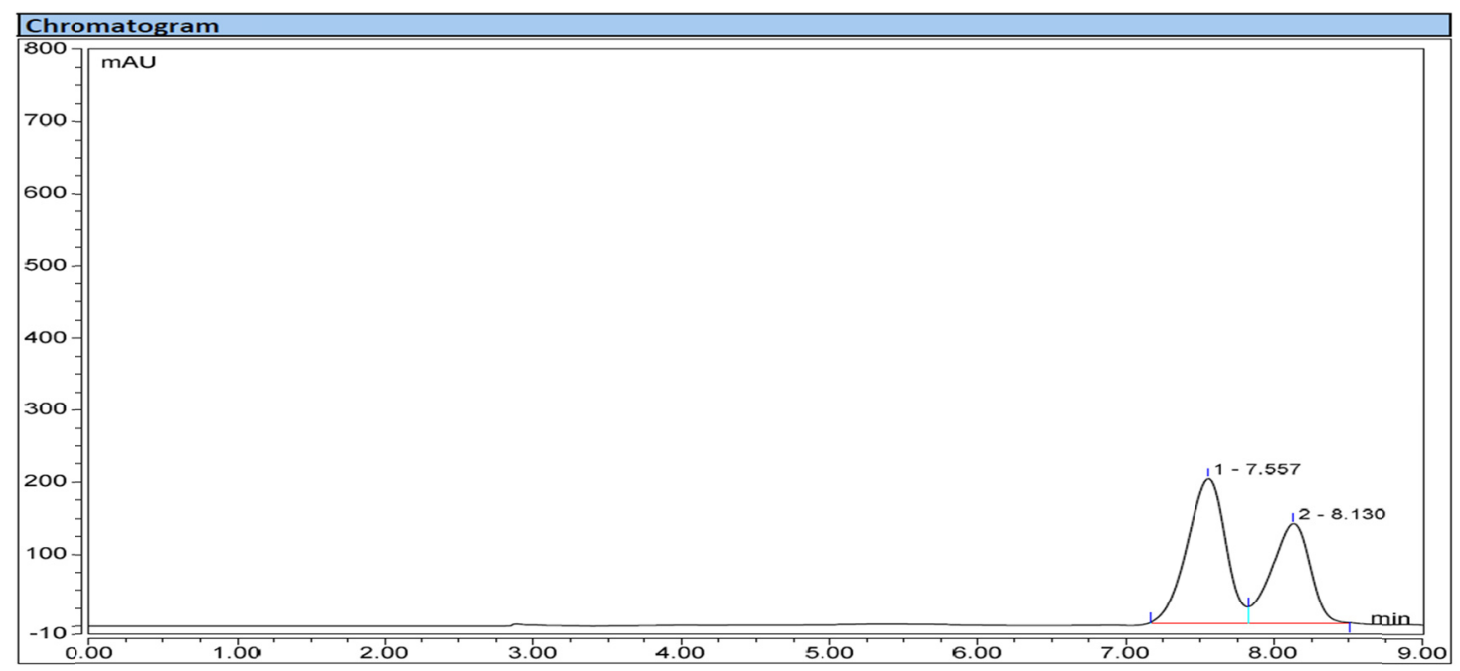

\begin{tabular}{|c|c|c|c|c|c|c|}
\hline \multicolumn{9}{|l|}{ Integration Results } \\
\hline No. & Peak Name & $\begin{array}{c}\text { Retention Time } \\
\text { min }\end{array}$ & $\begin{array}{c}\text { Height } \\
\text { mAU }\end{array}$ & $\begin{array}{c}\text { Area } \\
\text { mAU*min }\end{array}$ & $\begin{array}{c}\text { Relative Height } \\
\%\end{array}$ & $\begin{array}{c}\text { Relative Area } \\
\%\end{array}$ \\
\hline 1 & & 7.557 & 200.667 & 60.939 & 59.06 & 59.37 \\
2 & & 8.130 & 139.129 & 41.697 & 40.94 & 40.63 \\
\hline
\end{tabular}

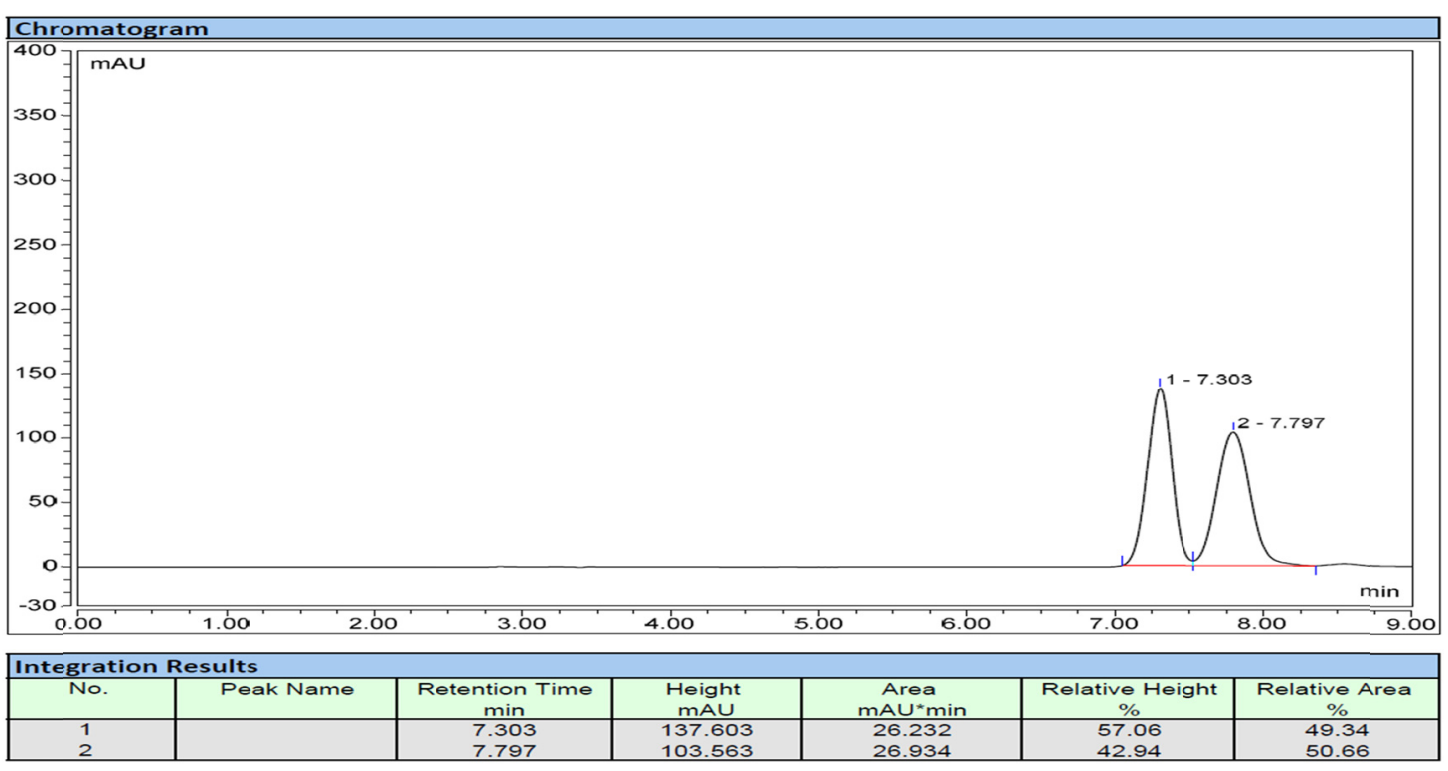




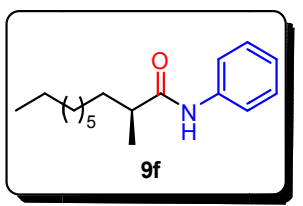

\section{(S)-2-methyl- $N$-phenyldecanamide}

$82 \%$ yield, b:1 $=88: 12$, ee $=16 \% .{ }^{1} \mathrm{H}$ NMR $\left(400 \mathrm{MHz}, \mathrm{CDCl}_{3}\right) \delta 7.77(\mathrm{~s}, 1 \mathrm{H})$, 7.55 (d, $J=8.0 \mathrm{~Hz}, 2 \mathrm{H}), 7.28$ (t, $J=7.6 \mathrm{~Hz}, 2 \mathrm{H}), 7.07$ (t, $J=7.2 \mathrm{~Hz}, 1 \mathrm{H}), 2.40-2.32$ $(\mathrm{m}, 1 \mathrm{H}), 1.76-1.67(\mathrm{~m}, 1 \mathrm{H}), 1.44-1.38(\mathrm{~m}, 1 \mathrm{H}), 1.28-1.24(\mathrm{~m}, 12 \mathrm{H}), 1.20(\mathrm{~d}, J=6.8$ $\mathrm{Hz}, 3 \mathrm{H}), 0.87(\mathrm{t}, J=6.4 \mathrm{~Hz}, 3 \mathrm{H}) ;{ }^{13} \mathrm{C}$ NMR $\left(101 \mathrm{MHz}, \mathrm{CDCl}_{3}\right) \delta 175.4,138.1,128.8$, 124.0, 120.0, 42.4, 34.4, 31.8, 29.6, 29.4, 29.2, 27.5, 22.6, 17.9, 14.0. HRMS calcd. (ESI) $\mathrm{m} / \mathrm{z}$ for $\mathrm{C}_{17} \mathrm{H}_{27} \mathrm{NNaO}$ : $[\mathrm{M}+\mathrm{Na}]^{+} 284.1985$, found: 284.1986 .

Chiral HPLC (Chiralpak IG, 4.6x250 mm; $5 \mu \mathrm{L}$, hexane/isopropanol $=90 / 10$, flow $1.0 \mathrm{~mL} / \mathrm{min}, 254 \mathrm{~nm}$ ): $\mathrm{t}_{\mathrm{R}}=15.48 \mathrm{~min}$ (major), $\mathrm{t}_{\mathrm{R}}=16.97 \mathrm{~min}$ (minor).
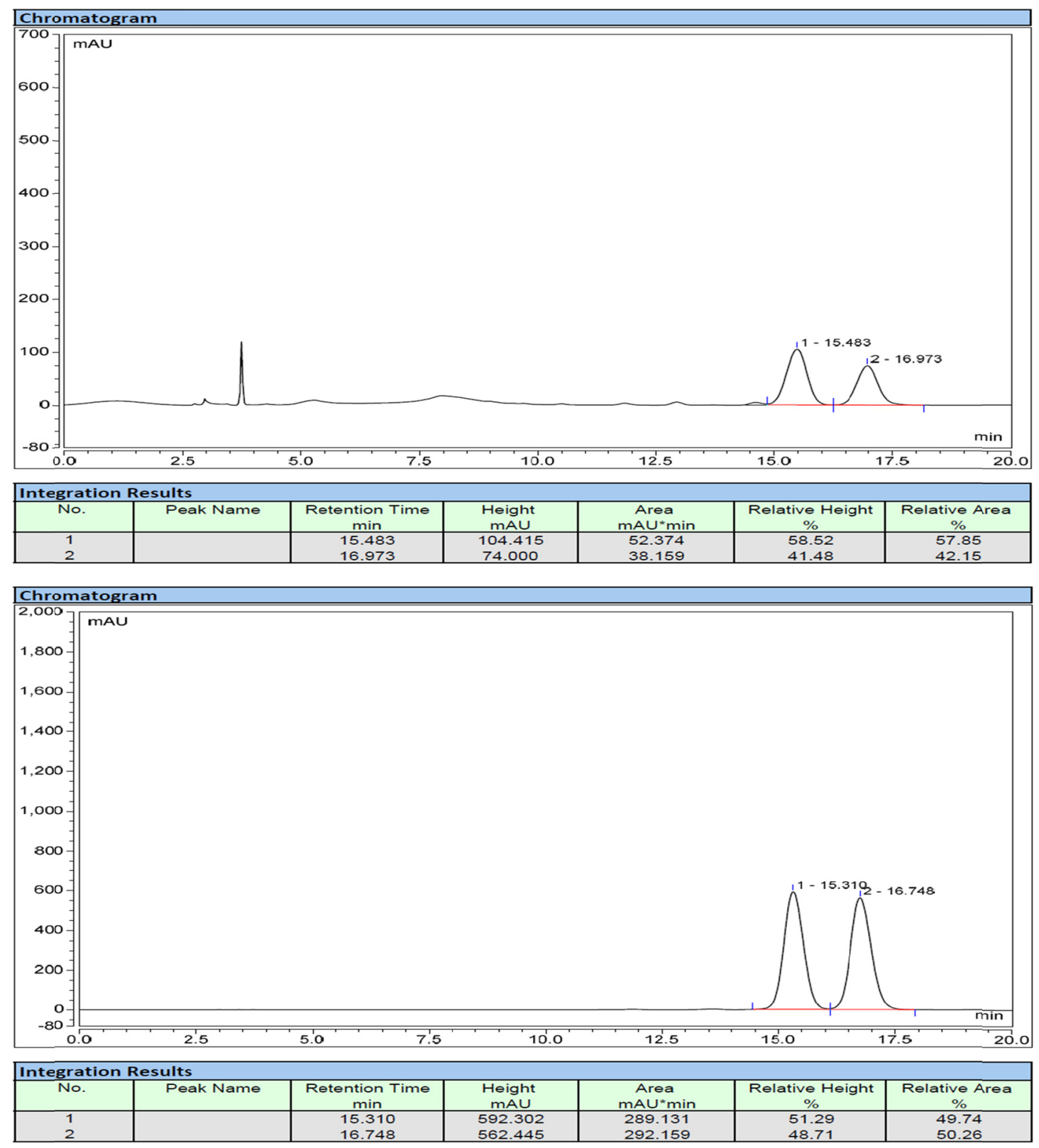


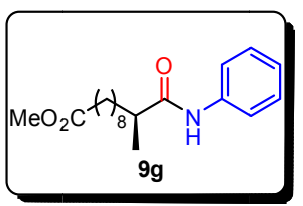

methyl (S)-10-methyl-11-oxo-11-(phenylamino)undecanoate

$83 \%$ yield, b:1 $=91: 9$, ee $=20 \% .{ }^{1} \mathrm{H}$ NMR $\left(400 \mathrm{MHz}, \mathrm{CDCl}_{3}\right) \delta 7.74(\mathrm{~s}, 1 \mathrm{H}), 7.56$ (d, $J=8.0 \mathrm{~Hz}, 2 \mathrm{H}), 7.29$ (d, $J=8.0 \mathrm{~Hz}, 2 \mathrm{H}), 7.08(\mathrm{t}, J=7.2 \mathrm{~Hz}, 1 \mathrm{H}), 3.66(\mathrm{~s}, 3 \mathrm{H})$, $2.35(\mathrm{dd}, J=14.0,7.2 \mathrm{~Hz}, 1 \mathrm{H}), 2.29$ (t, $J=7.6 \mathrm{~Hz}, 2 \mathrm{H}), 1.75-1.68(\mathrm{~m}, 1 \mathrm{H}), 1.61-1.58$ $(\mathrm{m}, 2 \mathrm{H}), 1.45-1.38(\mathrm{~m}, 1 \mathrm{H}), 1.34-1.24(\mathrm{~m}, 8 \mathrm{H}), 1.20(\mathrm{~d}, J=6.8 \mathrm{~Hz}, 3 \mathrm{H}) ;{ }^{13} \mathrm{C}$ NMR $\left(101 \mathrm{MHz}, \mathrm{CDCl}_{3}\right) \delta 175.2,174.4,138.1,128.8,123.9,119.8,51.4,42.4,34.3,34.0$, 29.4, 29.1, 29.0, 28.9, 27.4, 24.8, 17.9. HRMS calcd. (ESI) $\mathrm{m} / \mathrm{z}$ for $\mathrm{C}_{19} \mathrm{H}_{29} \mathrm{NNaO}_{3}$ : $[\mathrm{M}+\mathrm{Na}]^{+}$342.2040, found: 342.2038 .

Chiral HPLC (Chiralpak IG, 4.6x250 mm; $5 \mu \mathrm{L}$, hexane/isopropanol $=90 / 10$, flow 1.0 $\mathrm{mL} / \mathrm{min}, 254 \mathrm{~nm}$ ): $\mathrm{t}_{\mathrm{R}}=17.16 \min$ (major), $\mathrm{t}_{\mathrm{R}}=19.92 \mathrm{~min}$ (minor).
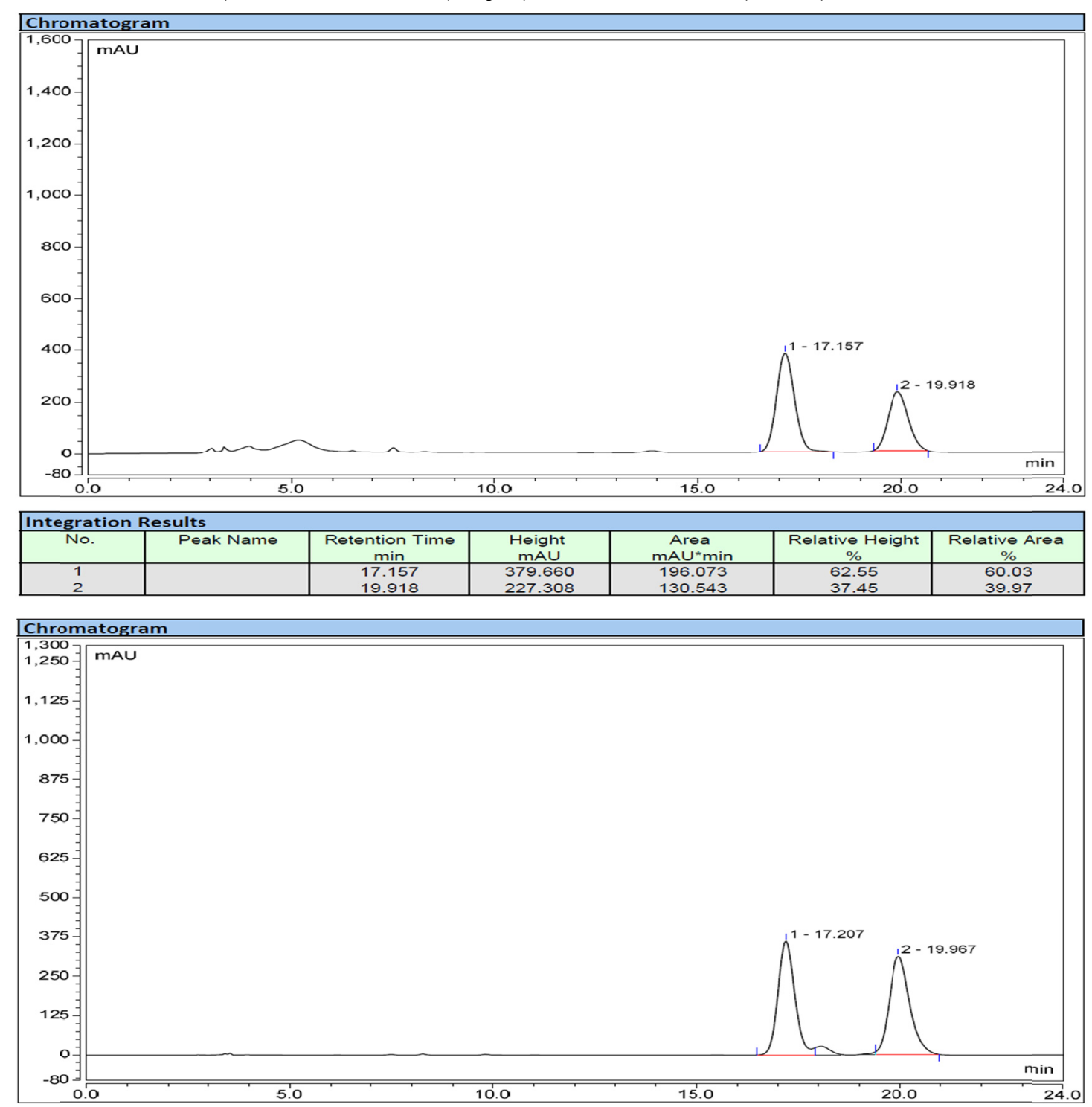

\begin{tabular}{|c|c|c|c|c|c|c|}
\hline \multicolumn{9}{|l|}{ Integration Results } \\
\hline No. & Peak Name & $\begin{array}{c}\text { Retention Time } \\
\text { min }\end{array}$ & $\begin{array}{c}\text { Height } \\
\text { mAU }\end{array}$ & $\begin{array}{c}\text { Area } \\
\text { mAU*min }\end{array}$ & $\begin{array}{c}\text { Relative Height } \\
\%\end{array}$ & $\begin{array}{c}\text { Relative A.rea } \\
\%\end{array}$ \\
\hline 1 & & 17.207 & 360.758 & 173.038 & 53.76 & 49.02 \\
2 & & 19.967 & 310.287 & 179.959 & 46.24 & 50.98 \\
\hline
\end{tabular}




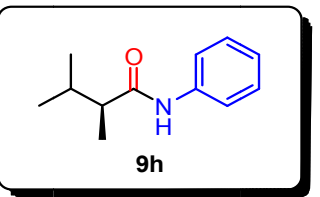

(S)-2,3-dimethyl-N-phenylbutanamide

$74 \%$ yield, b: $1=59: 41$, ee $=57 \% .{ }^{1} \mathrm{H}$ NMR $\left(400 \mathrm{MHz}, \mathrm{CDCl}_{3}\right) \delta 7.54(\mathrm{~d}, J=8.0$ $\mathrm{Hz}, 2 \mathrm{H}), 7.39$ (s, 1H), 7.30 (t, $J=8.0 \mathrm{~Hz}, 2 \mathrm{H}), 7.09$ (t, J = 7.2 Hz, 1H), 2.13-2.00 (m, $1 \mathrm{H}), 2.00-1.89(\mathrm{~m}, 1 \mathrm{H}), 1.20(\mathrm{~d}, J=6.8 \mathrm{~Hz}, 3 \mathrm{H}), 0.97(\mathrm{dd}, J=9.2,6.4 \mathrm{~Hz}, 6 \mathrm{H}) ;{ }^{13} \mathrm{C}$ NMR $\left(101 \mathrm{MHz}, \mathrm{CDCl}_{3}\right) \delta 174.8,138.0,128.9,124.1,119.9,49.5,31.6,21.1,19.5$, 15.0. HRMS calcd. (ESI) $\mathrm{m} / \mathrm{z}$ for $\mathrm{C}_{12} \mathrm{H}_{17} \mathrm{NNaO}$ : $[\mathrm{M}+\mathrm{Na}]^{+}$214.1202, found: 214.1204 .

Chiral HPLC (Chiralpak IG, 4.6x250 mm; $5 \mu \mathrm{L}$, hexane/isopropanol $=95 / 5$, flow $1.0 \mathrm{~mL} / \mathrm{min}, 254 \mathrm{~nm}$ ): $\mathrm{t}_{\mathrm{R}}=15.41 \mathrm{~min}$ (major), $\mathrm{t}_{\mathrm{R}}=17.13 \mathrm{~min}$ (minor).
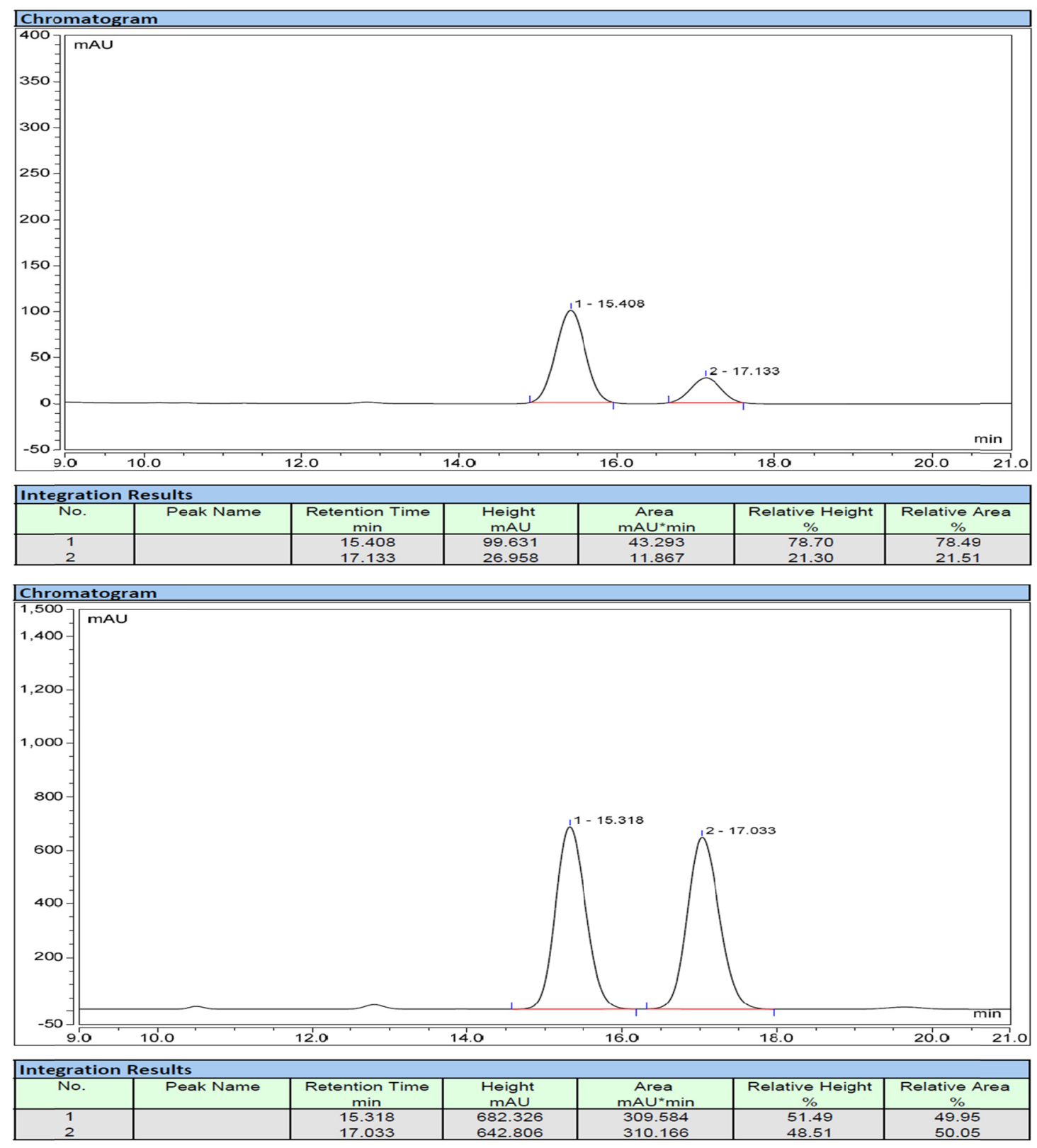


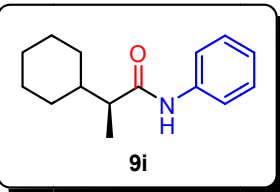

(S)-2-cyclohexyl- $N$-phenylpropanamide

$90 \%$ yield, b:1 $=62: 38$, ee $=63 \%,{ }^{1} \mathrm{H}$ NMR $\left(400 \mathrm{MHz}, \mathrm{CDCl}_{3}\right) \delta 7.59(\mathrm{~d}, J=8.0$ $\mathrm{Hz}, 2 \mathrm{H}), 7.37-7.31(\mathrm{~m}, 3 \mathrm{H}), 7.14(\mathrm{t}, J=7.6 \mathrm{~Hz}, 1 \mathrm{H}), 2.16-2.09(\mathrm{~m}, 1 \mathrm{H}), 1.90$ (d, $J=$ $12.4 \mathrm{~Hz}, 1 \mathrm{H}), 1.80-1.59(\mathrm{~m}, 5 \mathrm{H}), 1.34-1.28(\mathrm{~m}, 2 \mathrm{H}), 1.25(\mathrm{~d}, J=6.8 \mathrm{~Hz}, 3 \mathrm{H})$, 1.19-0.93 (m, 3H); ${ }^{13} \mathrm{C}$ NMR $\left(101 \mathrm{MHz}, \mathrm{CDCl}_{3}\right) \delta 174.8,137.9,128.9,124.1,119.8$, 48.8, 41.2, 31.5, 30.0, 26.3, 26.22, 26.21, 14.93. HRMS calcd. (ESI) $\mathrm{m} / \mathrm{z}$ for $\mathrm{C}_{15} \mathrm{H}_{21} \mathrm{NNaO}:[\mathrm{M}+\mathrm{Na}]^{+} 254.1515$, found: 254.1514 .

Chiral HPLC (Chiralpak IG, 4.6x250 mm; $5 \mu \mathrm{L}$, hexane/isopropanol = 90/10, flow $1.0 \mathrm{~mL} / \mathrm{min}, 254 \mathrm{~nm}$ ): $\mathrm{t}_{\mathrm{R}}=19.43 \mathrm{~min}$ (major), $\mathrm{t}_{\mathrm{R}}=21.82 \mathrm{~min}$ (minor).

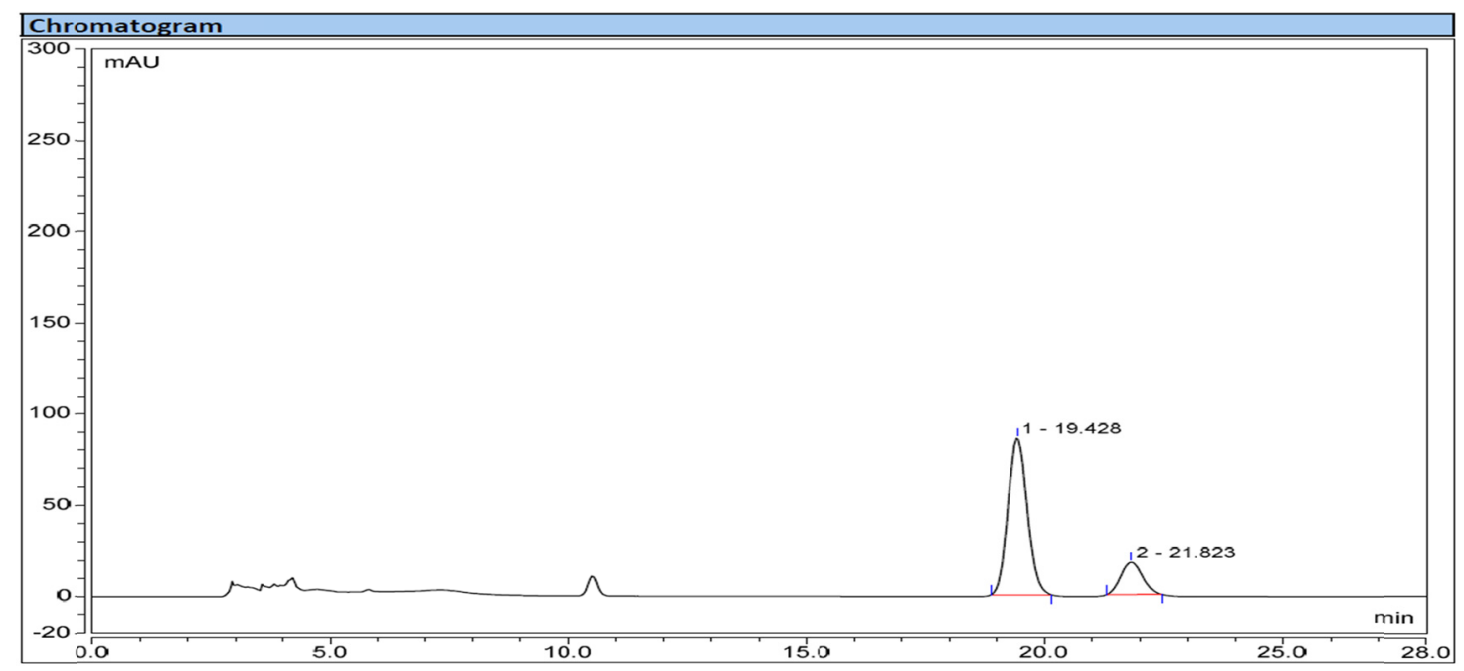

\begin{tabular}{|c|c|c|c|c|c|c|}
\hline Integration Results & \multicolumn{9}{c|}{$\begin{array}{c}\text { Area } \\
\text { No. }\end{array}$} & Peak Name & $\begin{array}{c}\text { Retention Time } \\
\text { min }\end{array}$ & $\begin{array}{c}\text { Height } \\
\text { mAU }\end{array}$ & $\begin{array}{c}\text { Relative Height } \\
\text { mAU*min }\end{array}$ & $\begin{array}{c}\text { Relative Area } \\
\%\end{array}$ \\
\hline 1 & & 19.428 & 85.696 & 40.518 & 82.74 & 81.30 \\
2 & & 21.823 & 17.874 & 9.320 & 17.26 & 18.70 \\
\hline
\end{tabular}

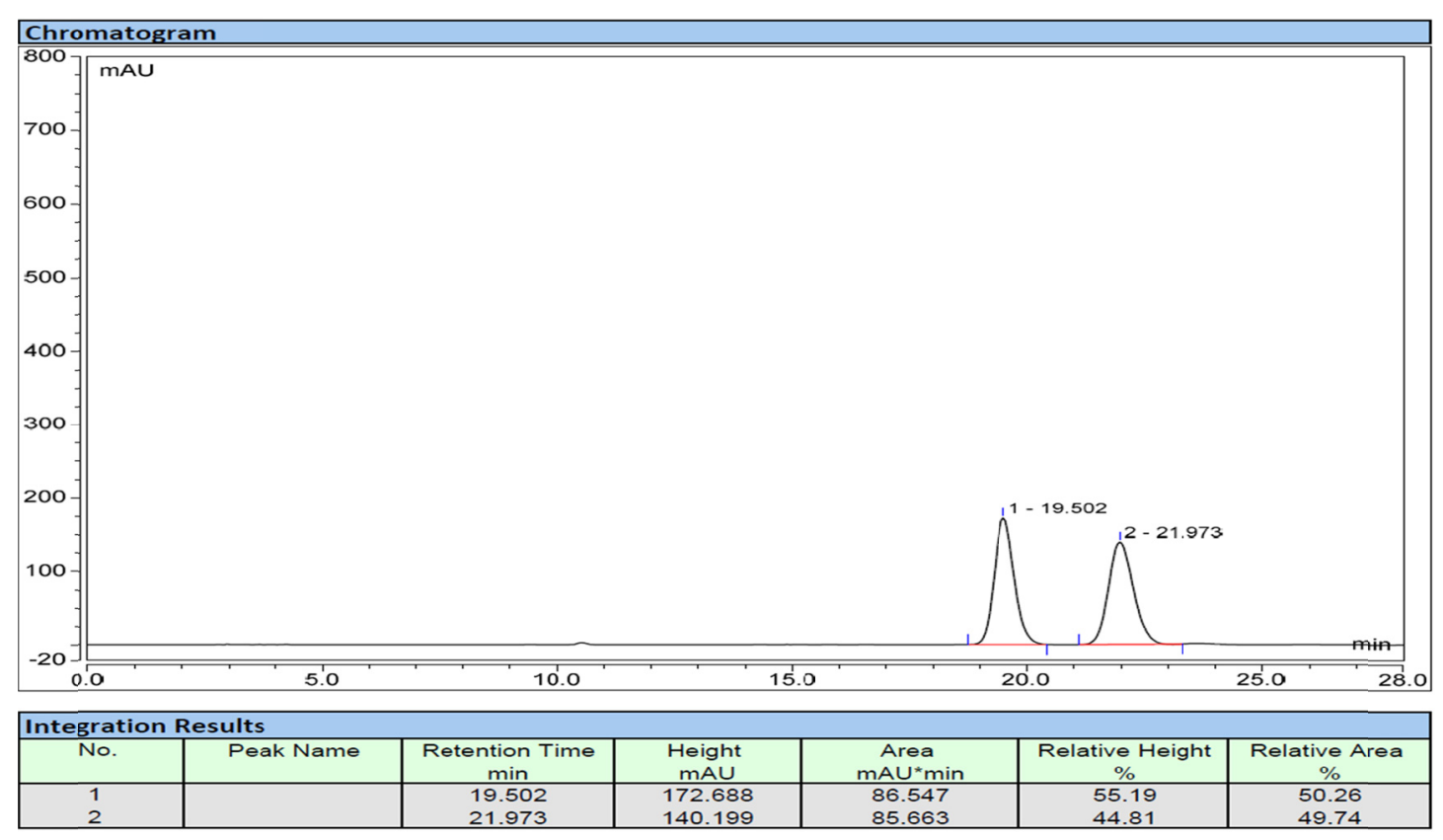


7. X-ray Structure of compound 3p

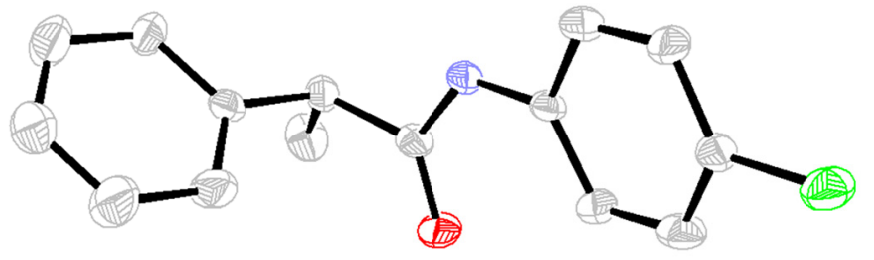

\begin{tabular}{|c|c|}
\hline \multirow{2}{*}{ Bond precision } & $\mathrm{C}-\mathrm{C}=0.0074 \mathrm{~A}$ \\
\hline & Wavelength $=0.71073$ \\
\hline \multirow{3}{*}{ Cell } & $\mathrm{a}=35.208(9)$ \\
\hline & $\mathrm{b}=8.244(2)$ \\
\hline & $\mathrm{c}=9.711(3) \quad \gamma=90$ \\
\hline Temperature & $296 \mathrm{~K}$ \\
\hline Volume & $2818.7(13)$ \\
\hline Space group & P 21212 \\
\hline Sum formula & $\mathrm{C} 15 \mathrm{H} 14 \mathrm{Cl} \mathrm{N} \mathrm{O}$ \\
\hline $\mathrm{Mr}$ & 259.72 \\
\hline $\mathrm{Dx}, \mathrm{g} \mathrm{cm}^{-3}$ & 1.224 \\
\hline $\mathrm{Z}$ & 8 \\
\hline $\mathrm{Mu}(\mathrm{mm}-1)$ & 0.259 \\
\hline F000 & 1088.0 \\
\hline $\mathrm{h}, \mathrm{k}, \operatorname{lmax}$ & $43,10,12$ \\
\hline Nref & 5684 \\
\hline Tmin,Tmax & $0.619,0.745$ \\
\hline Correction method= \# Reported T Limits & $\operatorname{Tmin}=0.619 \quad T \max =0.745$ \\
\hline \multicolumn{2}{|l|}{ AbsCorr $=$ MULTT-SCAN } \\
\hline Data completeness & 0.99 \\
\hline Theta(max) & 26.322 \\
\hline $\mathrm{R}$ (reflections) & $0.0537(3468)$ \\
\hline wR2(reflections) & $0.1243(5684)$ \\
\hline $\mathrm{S}$ & 0.969 \\
\hline Npar & 327 \\
\hline \multirow{2}{*}{ Bond precision } & $\mathrm{C}-\mathrm{C}=0.0074 \mathrm{~A}$ \\
\hline & Wavelength $=0.71073$ \\
\hline
\end{tabular}




\section{Copies of ${ }^{1} \mathrm{H}$ and ${ }^{13} \mathrm{C}$ NMR spectra}

${ }^{1} \mathrm{H}$ NMR (400 MHz, $\mathrm{CDCl}_{3}$ ) of compound 3a

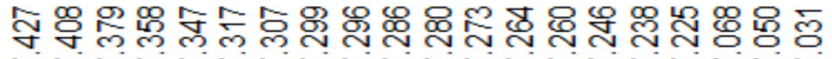

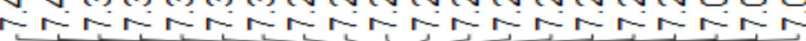

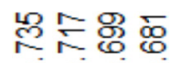

लंखंल

幽

음
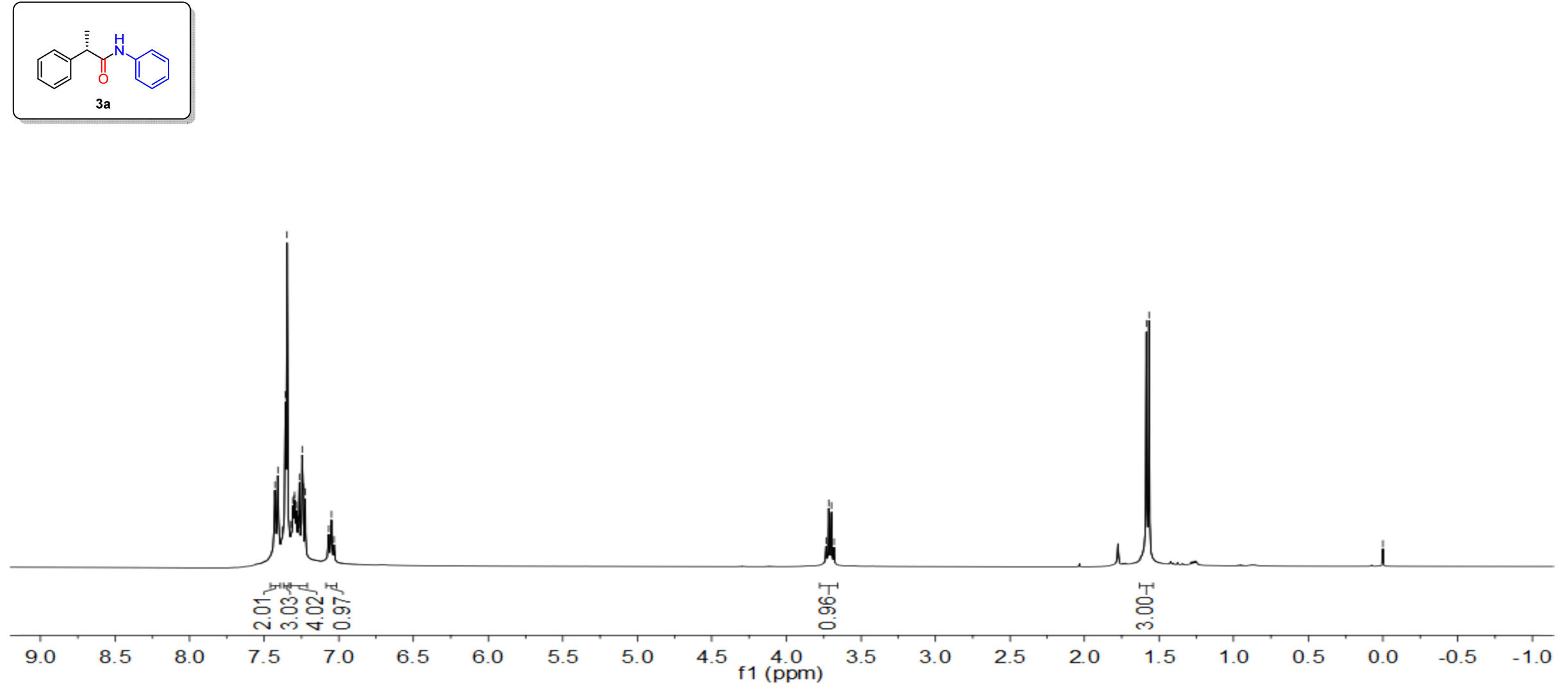
${ }^{13} \mathrm{C}$ NMR $\left(101 \mathrm{MHz}, \mathrm{CDCl}_{3}\right)$ of compound 3a
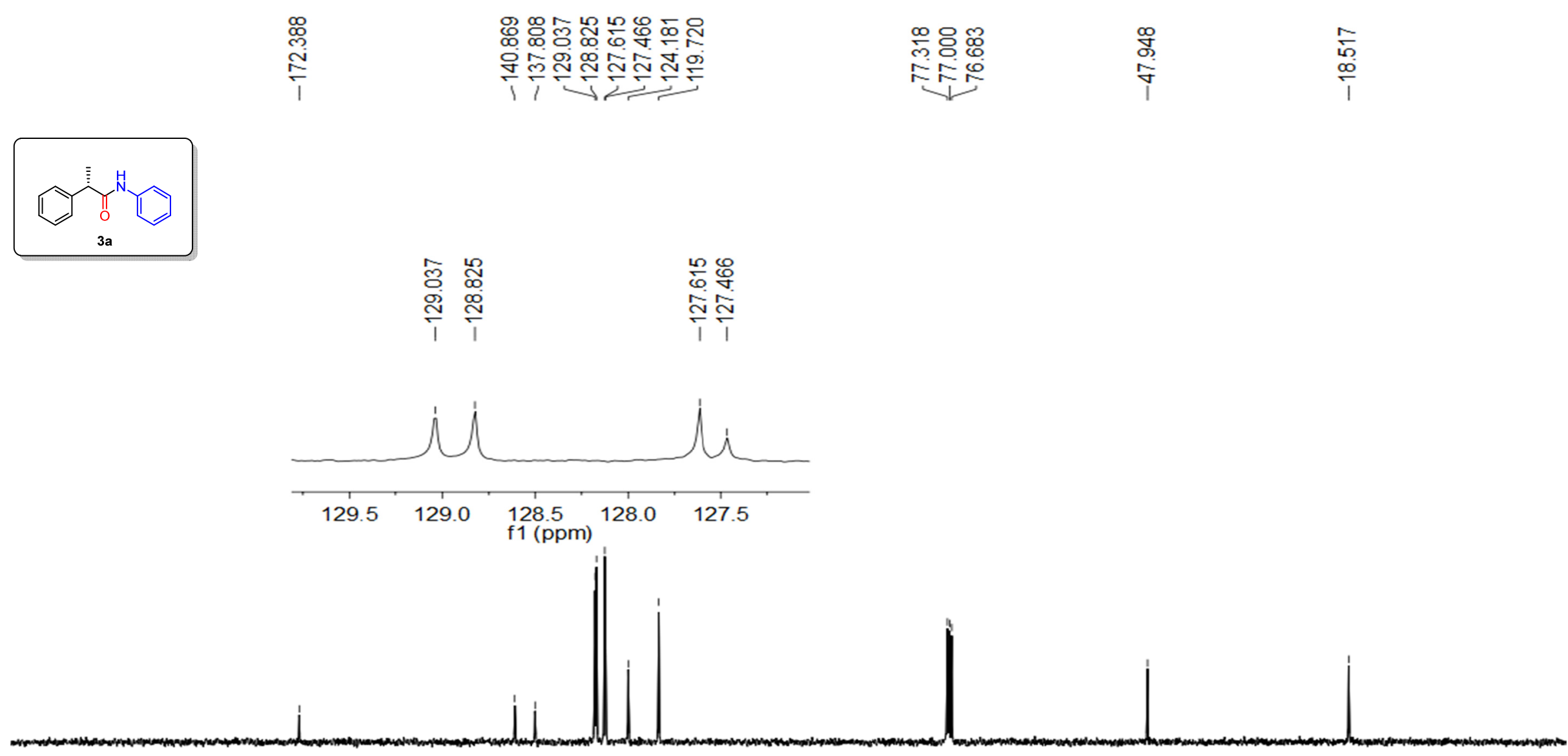

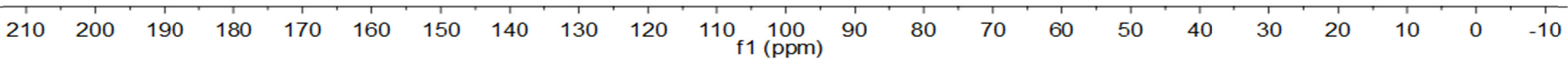


${ }^{1} \mathrm{H}$ NMR (400 MHz, $\mathrm{CDCl}_{3}$ ) of compound $\mathbf{3 b}$

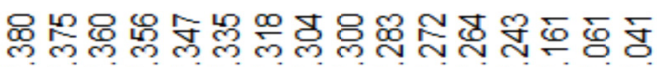

NNNNNNN

도용

نंखिं

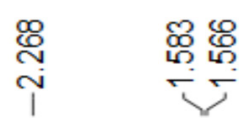

응
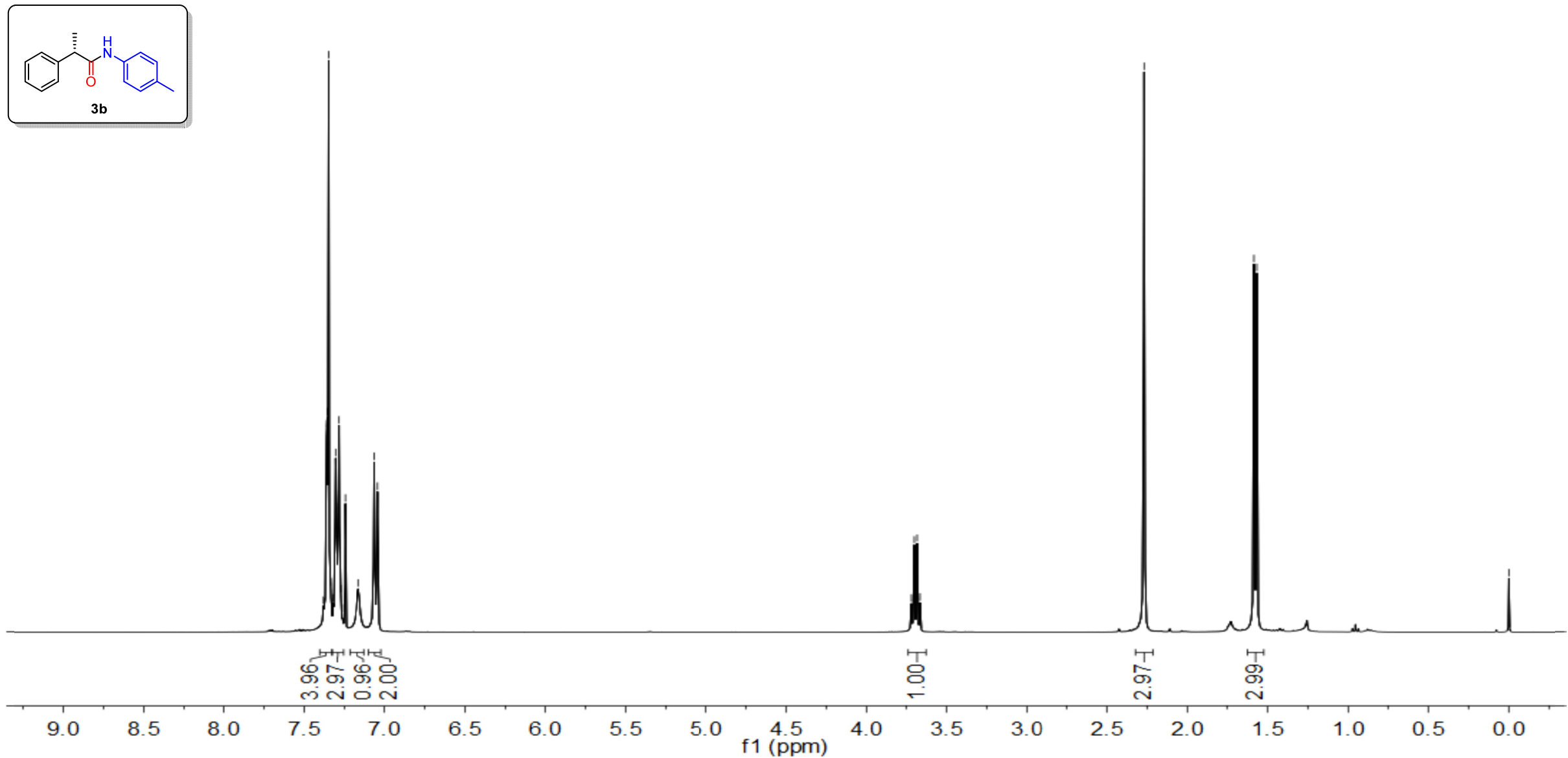
${ }^{13} \mathrm{C}$ NMR (151 MHz, $\mathrm{CDCl}_{3}$ ) of compound $\mathbf{3 b}$
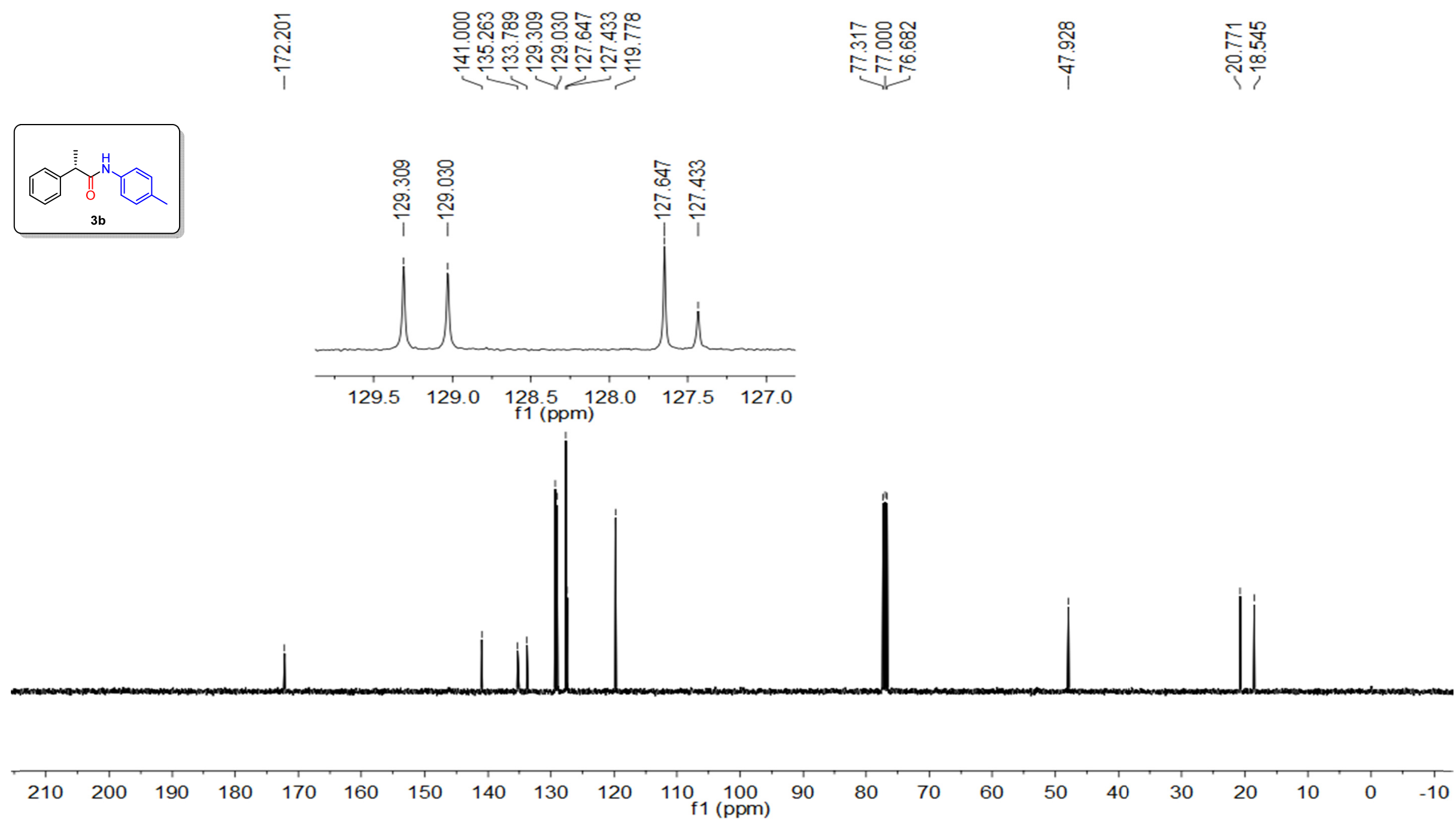
${ }^{1} \mathrm{H}$ NMR (400 MHz, $\mathrm{CDCl}_{3}$ ) of compound $\mathbf{3 c}$

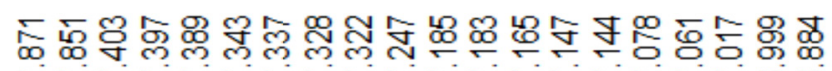

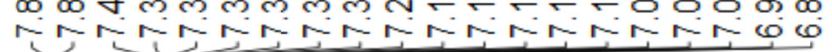

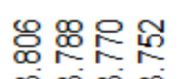

लंखिं

똥

$\stackrel{\infty}{\leftarrow} \stackrel{0}{-}$

응
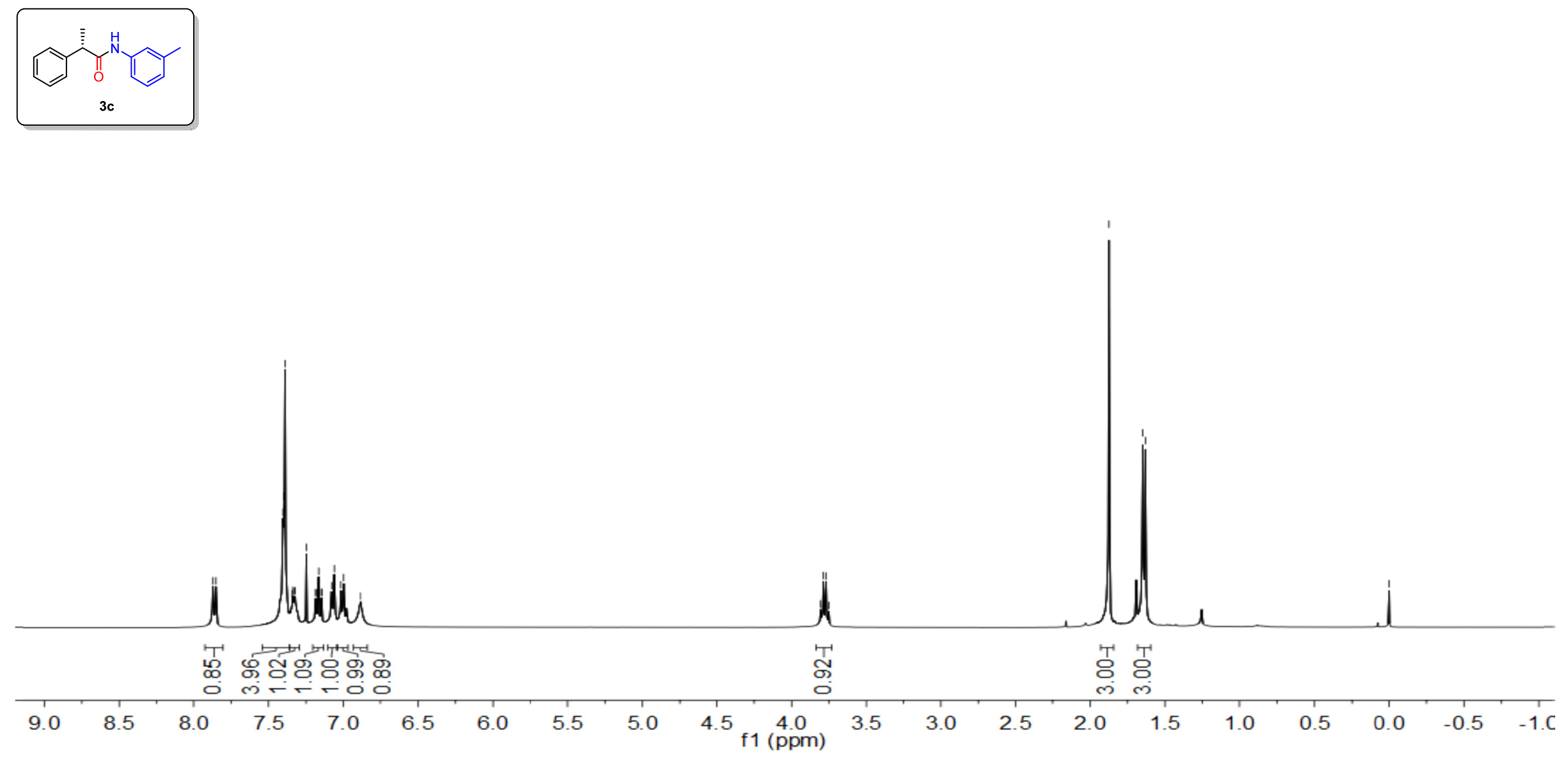
${ }^{13} \mathrm{C}$ NMR (151 MHz, $\mathrm{CDCl}_{3}$ ) of compound 3c

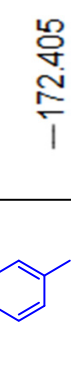

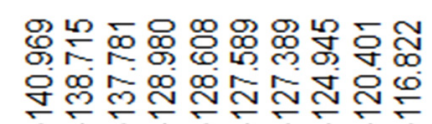

돈

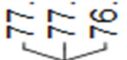

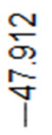

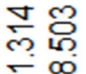

ते
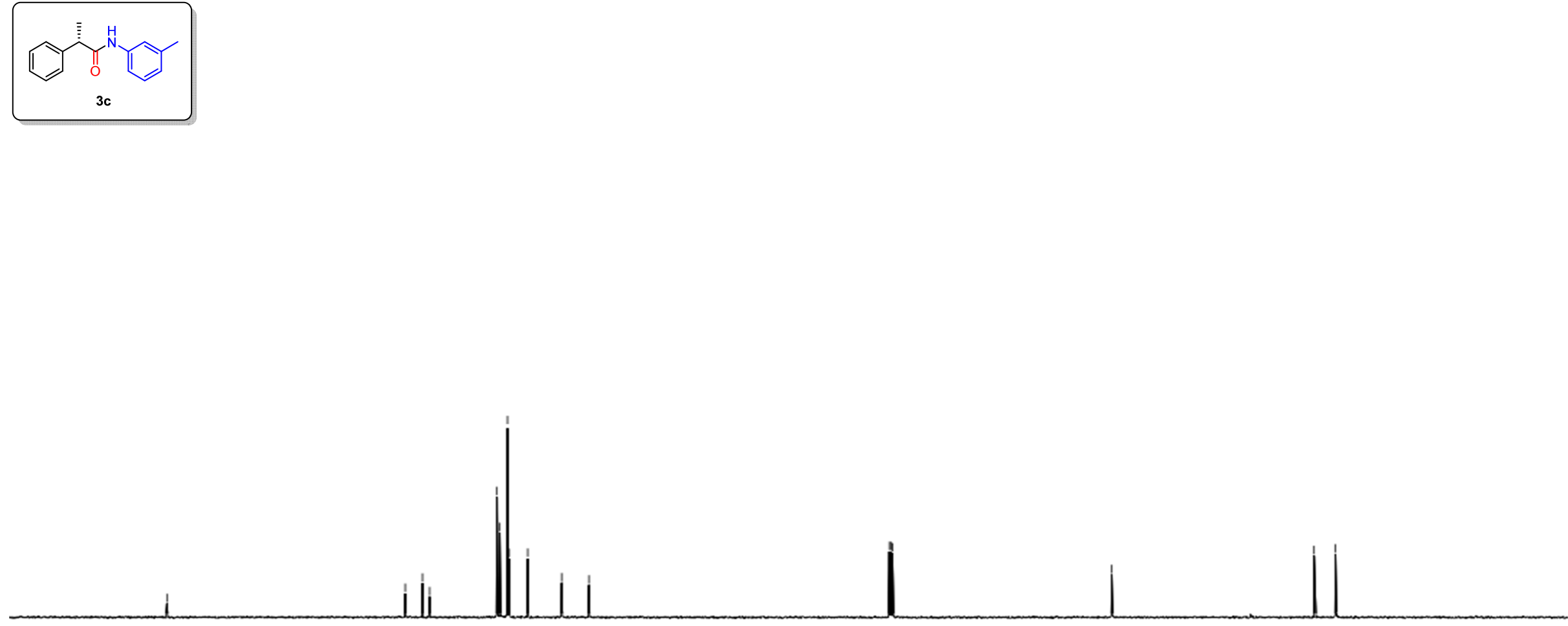
${ }^{1} \mathrm{H}$ NMR (400 MHz, $\mathrm{CDCl}_{3}$ ) of compound 3d

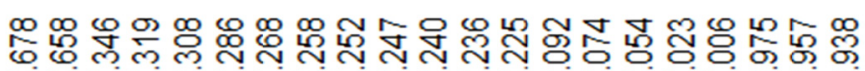

这吢禺禺

लंखु

高员

옹
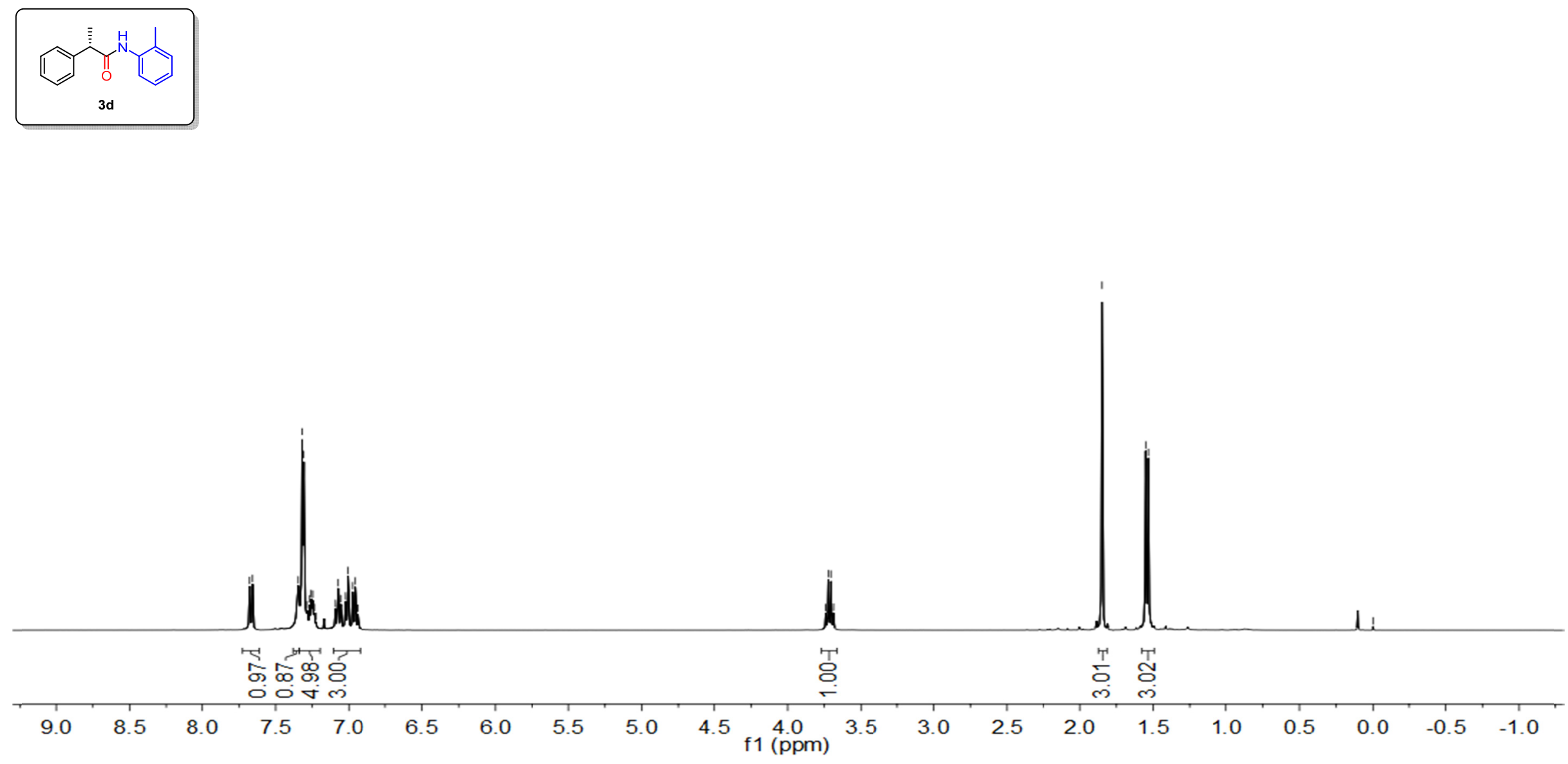
${ }^{13} \mathrm{C}$ NMR $\left(151 \mathrm{MHz}, \mathrm{CDCl}_{3}\right)$ of compound $\mathbf{3 c}$

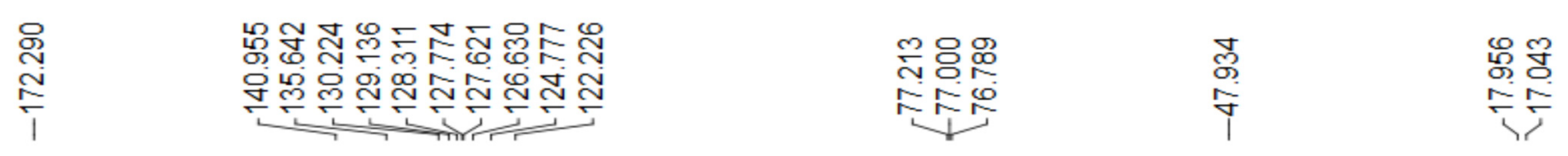
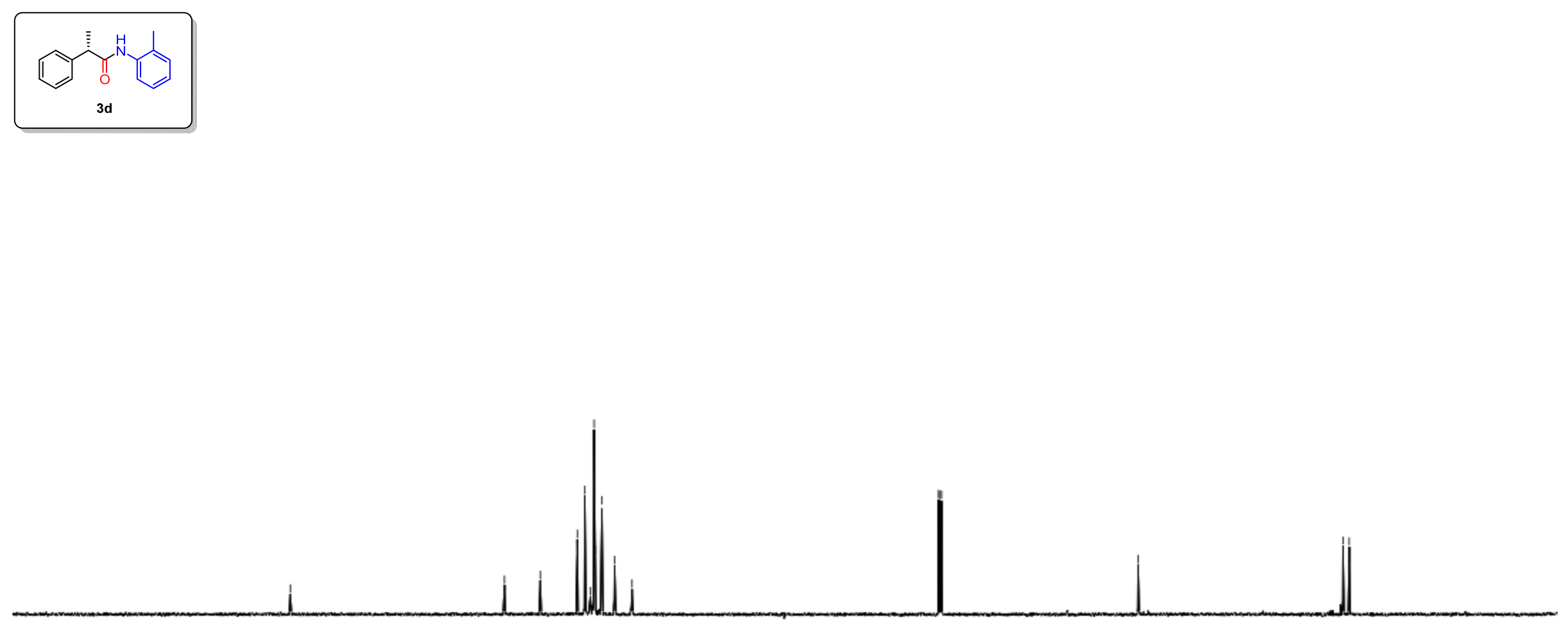

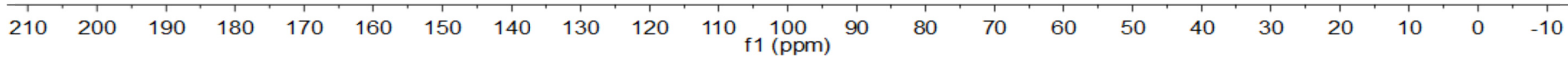


${ }^{1} \mathrm{H}$ NMR (600 MHz, $\mathrm{CDCl}_{3}$ ) of compound 3e

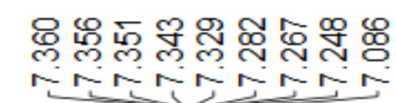

돈은 8

लिल

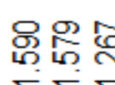

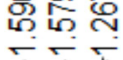
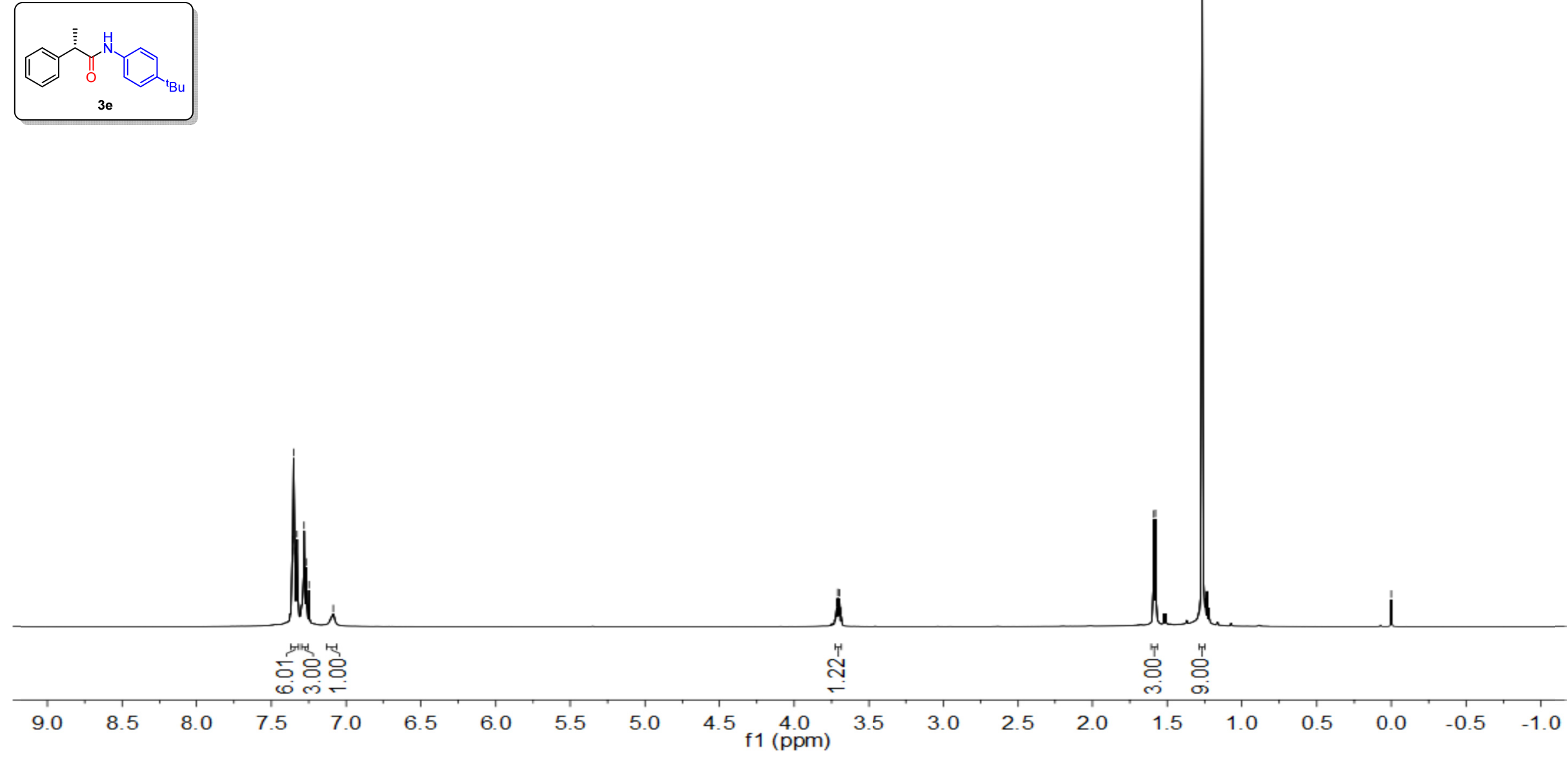
${ }^{13} \mathrm{C}$ NMR (151 MHz, $\mathrm{CDCl}_{3}$ ) of compound 3e

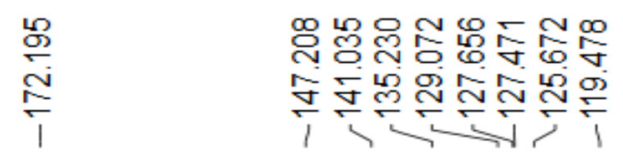

은

Nㅗㅇㅛ

ণิ
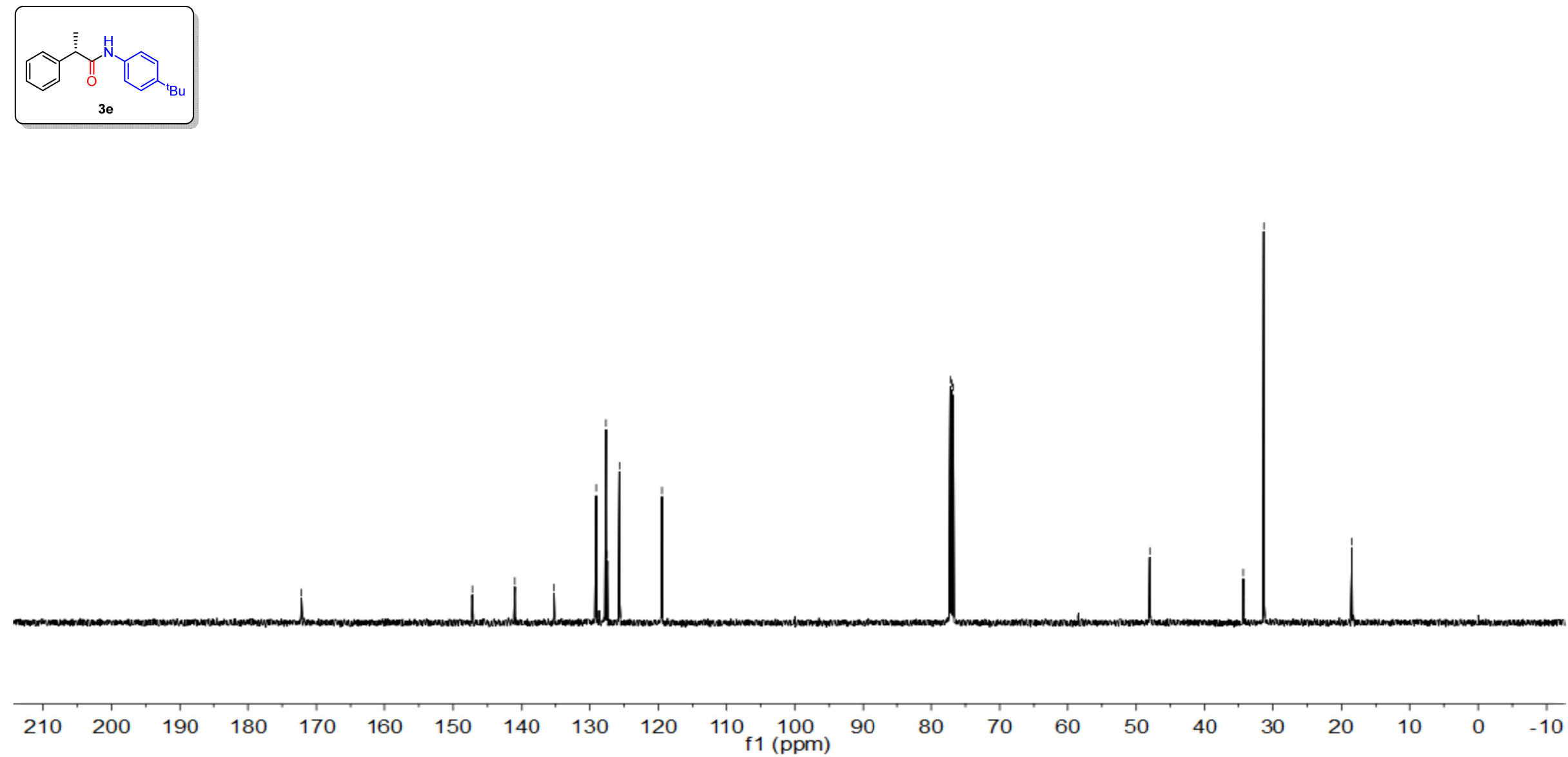
${ }^{1} \mathrm{H}$ NMR (400 MHz, $\mathrm{CDCl}_{3}$ ) of compound $\mathbf{3 f}$

守等 ํㅝㅁ

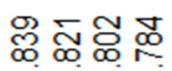

نَm

守

응
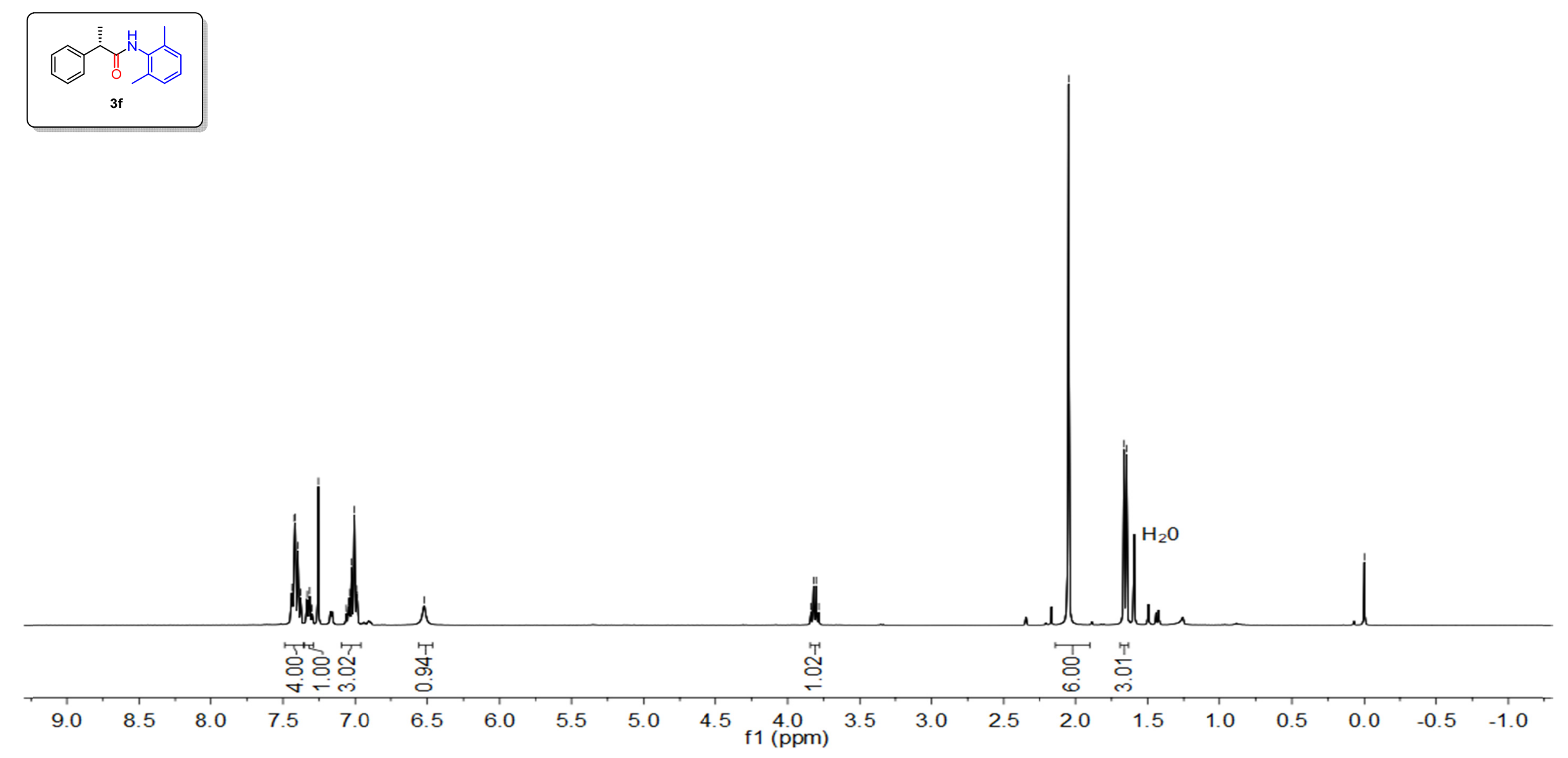
${ }^{13} \mathrm{C}$ NMR (101 MHz, $\mathrm{CDCl}_{3}$ ) of compound $\mathbf{3 f}$

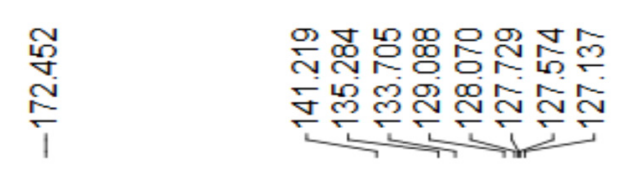

둥응

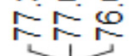

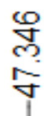

$\underset{8}{\forall}$

$\stackrel{\infty}{i}$
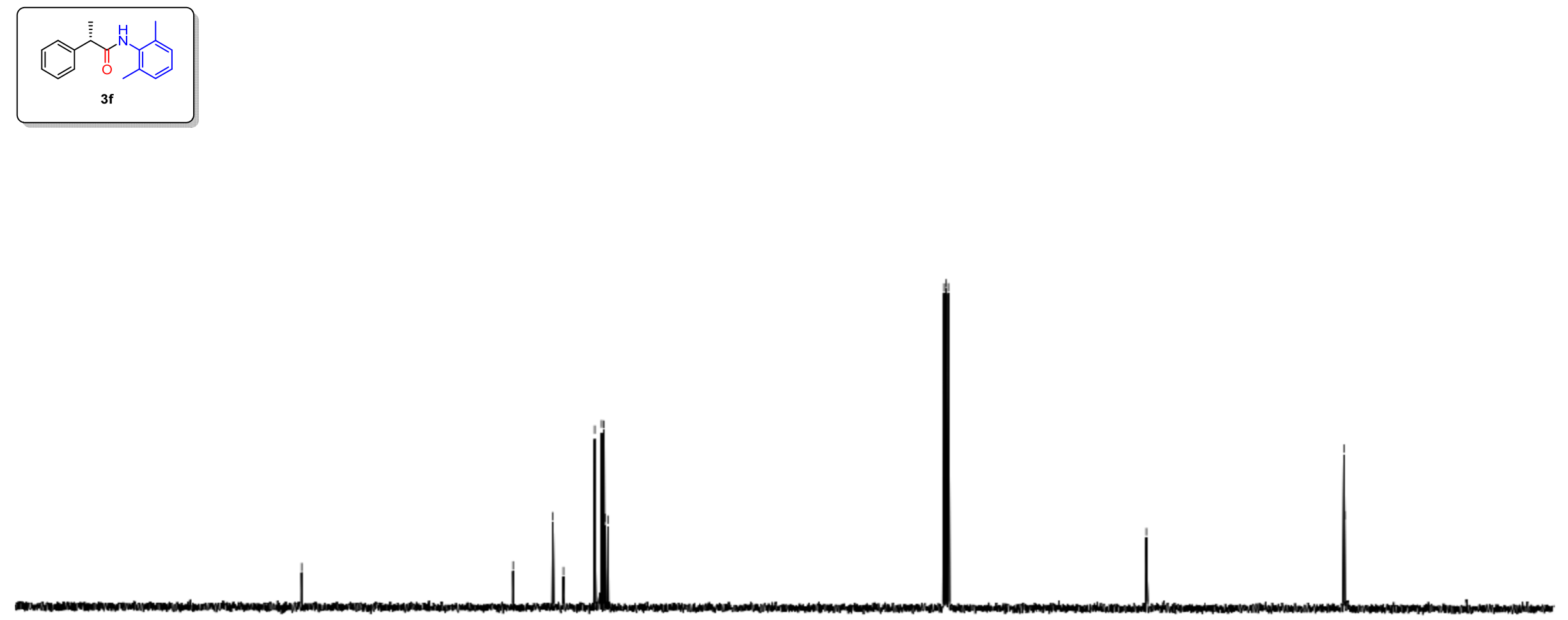

$\begin{array}{llllllllllllllllllllllll}210 & 200 & 190 & 180 & 170 & 160 & 150 & 140 & 130 & 120 & 110 & 100 & 90 & 80 & 70 & 60 & 50 & 40 & 30 & 20 & 10 & 0 & -10\end{array}$ 
${ }^{1} \mathrm{H}$ NMR (400 MHz, $\mathrm{CDCl}_{3}$ ) of compound $\mathbf{3 g}$

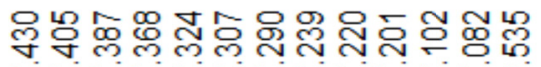

(

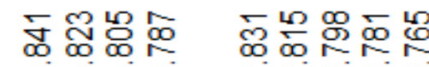

ن

電

웅
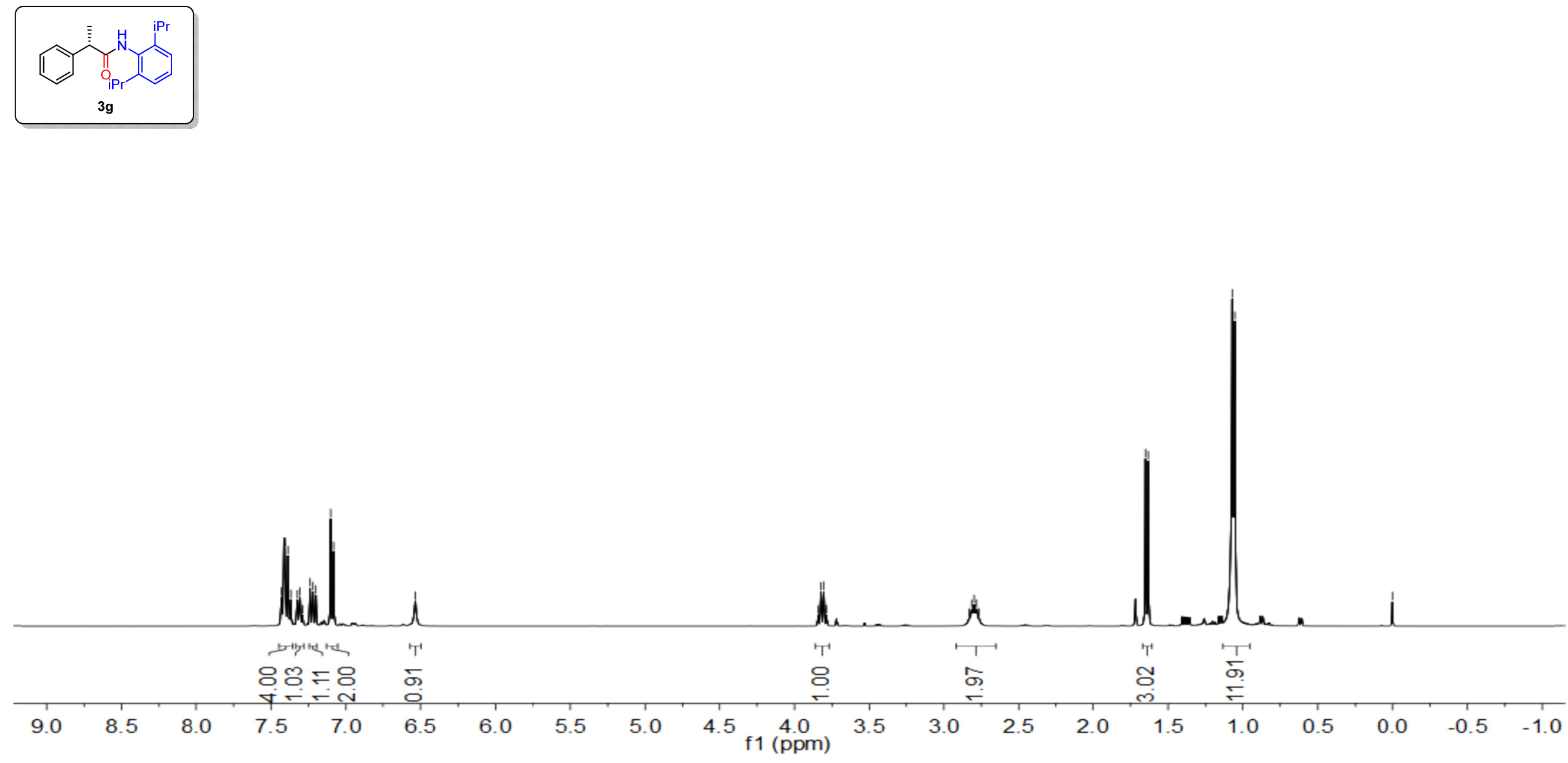
${ }^{13} \mathrm{C} \mathrm{NMR}\left(151 \mathrm{MHz}, \mathrm{CDCl}_{3}\right)$ of compound $\mathbf{3 g}$

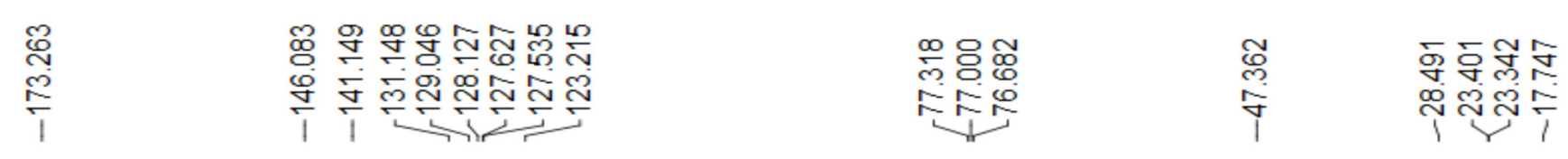
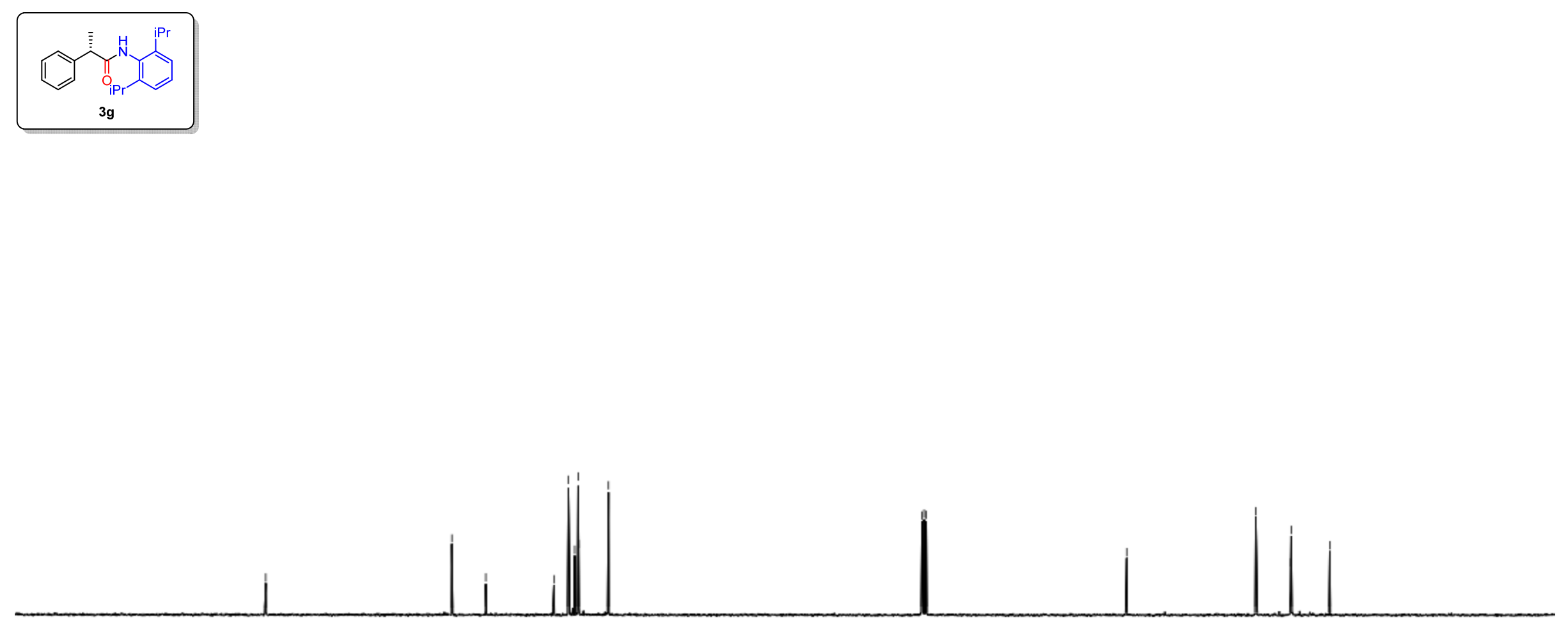

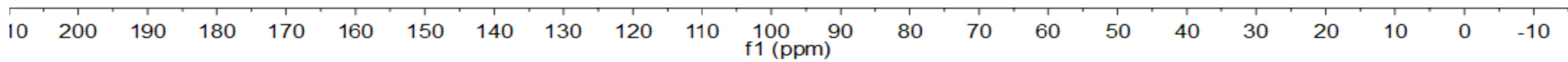


${ }^{1} \mathrm{H}$ NMR (400 MHz, $\mathrm{CDCl}_{3}$ ) of compound $\mathbf{3 h}$

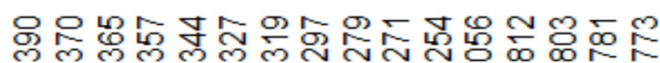

Ninr

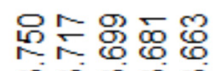

लिखंल

马ำ

음
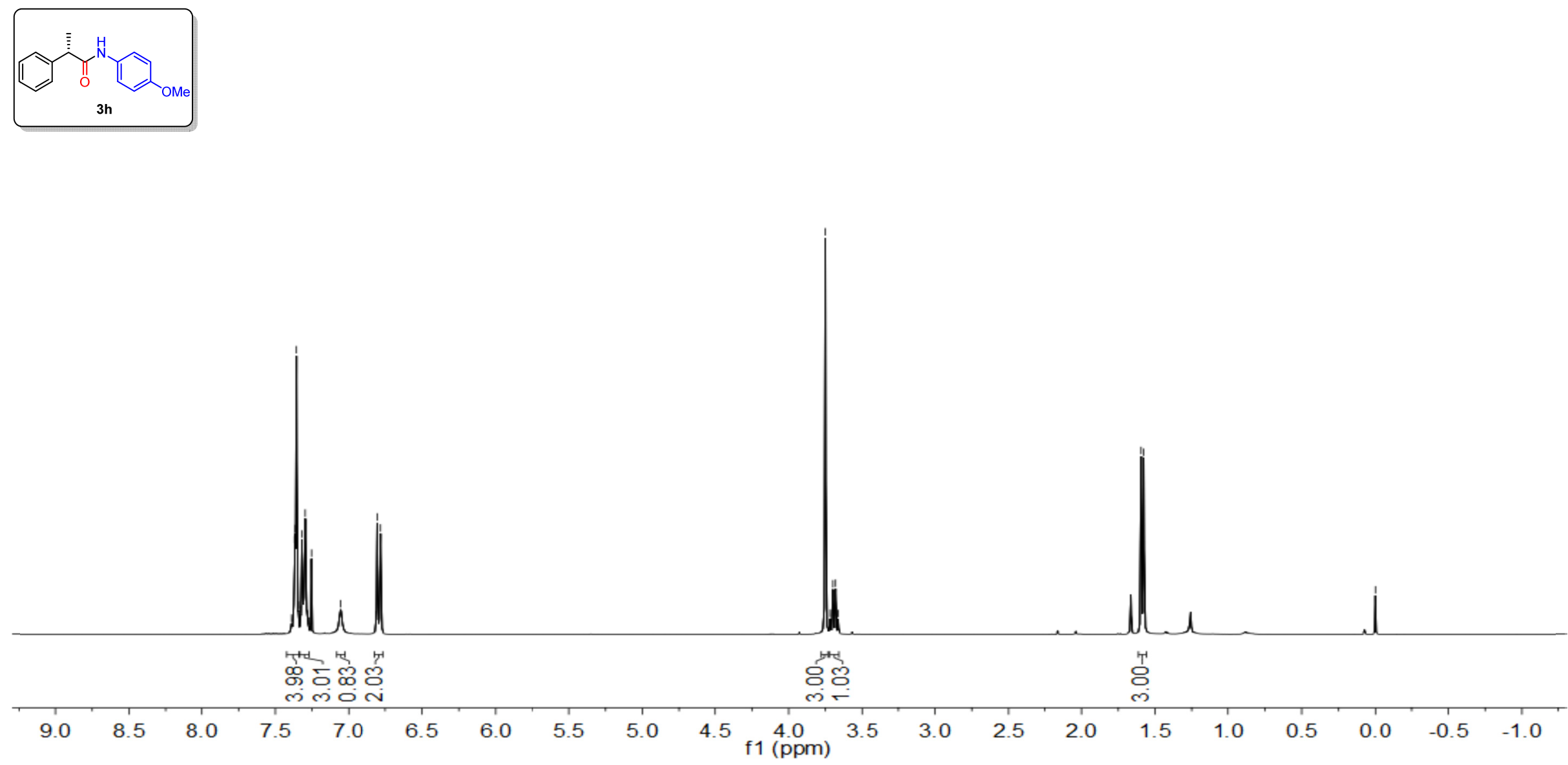
${ }^{13} \mathrm{C}$ NMR (101 MHz, $\mathrm{CDCl}_{3}$ ) of compound $\mathbf{3 h}$

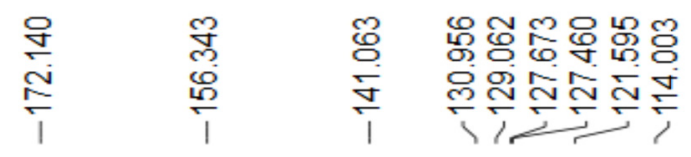

응 용

ANE

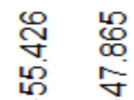

$\underset{\substack{\infty \\ \infty}}{\infty}$
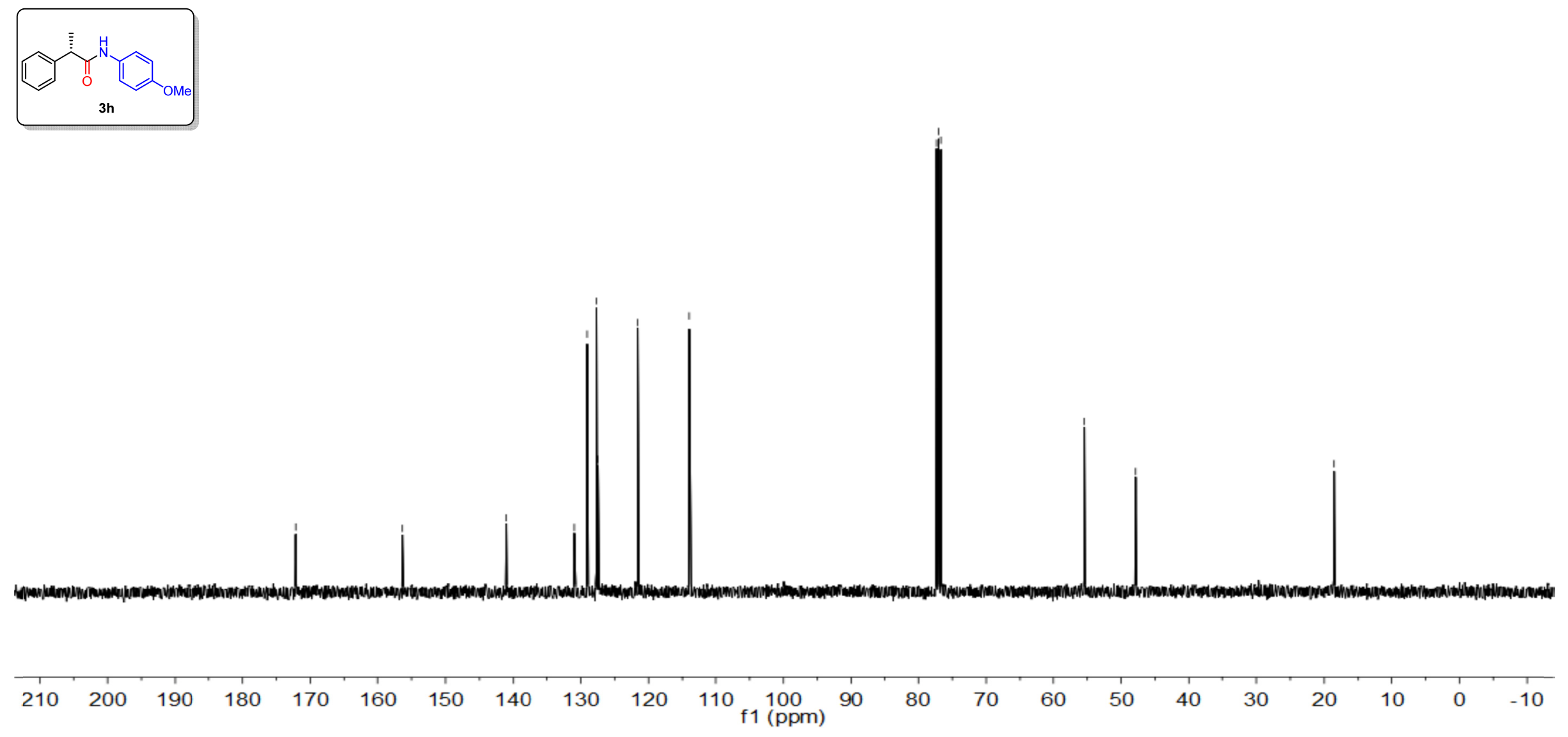
${ }^{1} \mathrm{H}$ NMR $\left(600 \mathrm{MHz}, \mathrm{CDCl}_{3}\right.$ ) of compound 3i

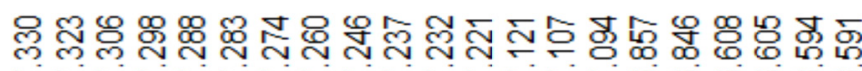

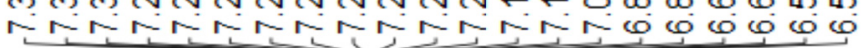

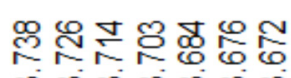

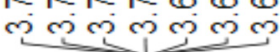

مْ

옹
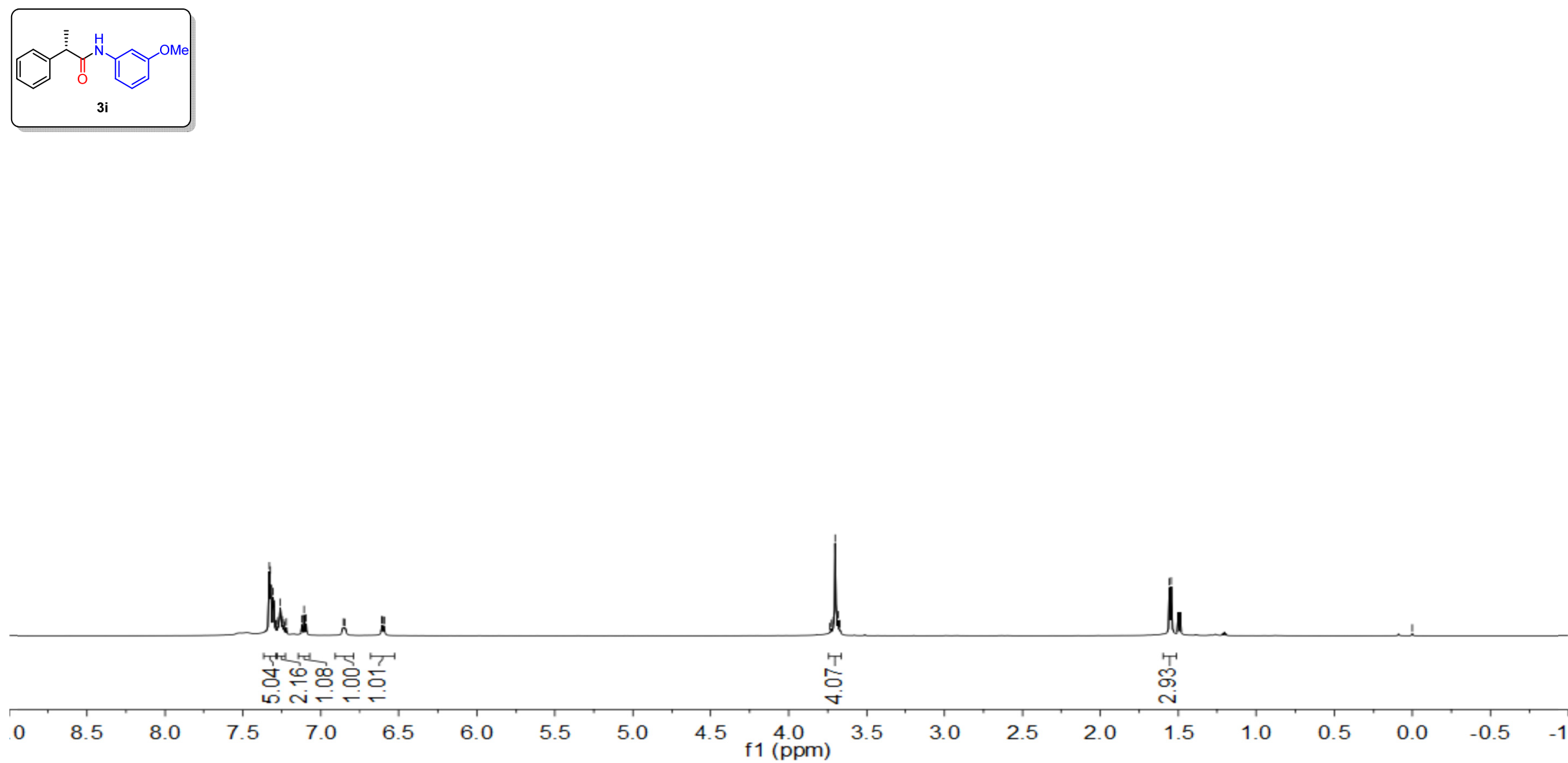
${ }^{13} \mathrm{C}$ NMR $\left(151 \mathrm{MHz}, \mathrm{CDCl}_{3}\right)$ of compound $\mathbf{3 i}$

\begin{tabular}{|c|c|c|c|c|c|}
\hline 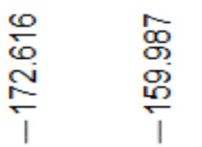 & 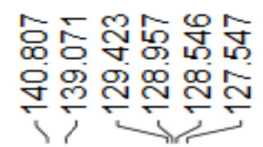 & 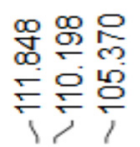 & 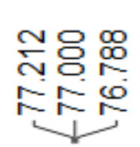 & 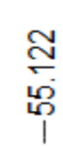 & $\begin{array}{l}\text { 只 } \\
0 \\
\text { † }\end{array}$ \\
\hline
\end{tabular}
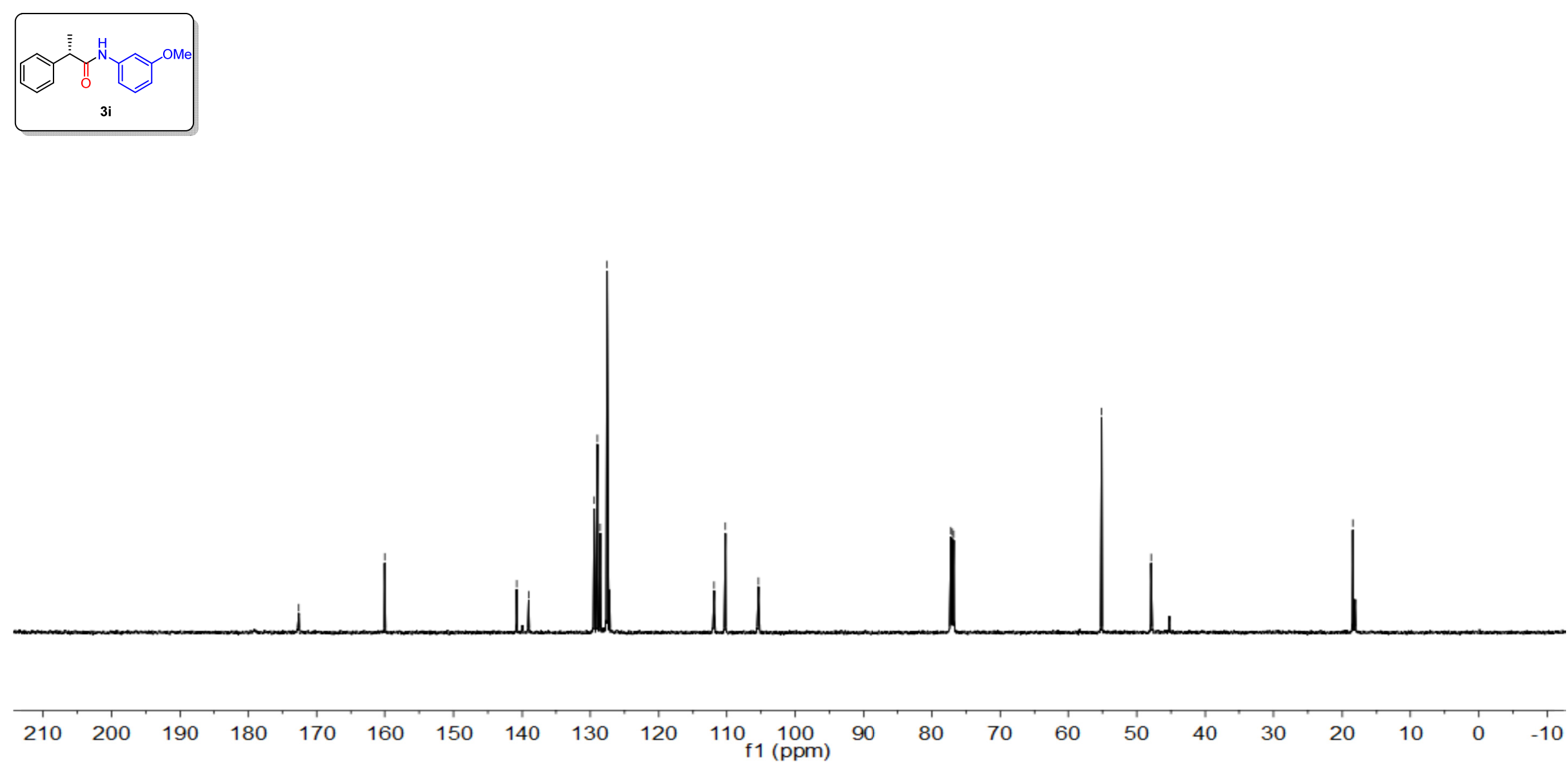
${ }^{1} \mathrm{H}$ NMR $\left(600 \mathrm{MHz}, \mathrm{CDCl}_{3}\right)$ of compound $\mathbf{3 j}$

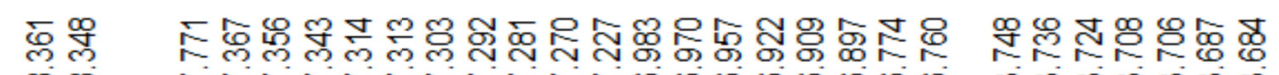

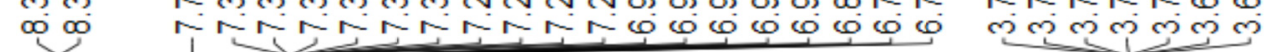
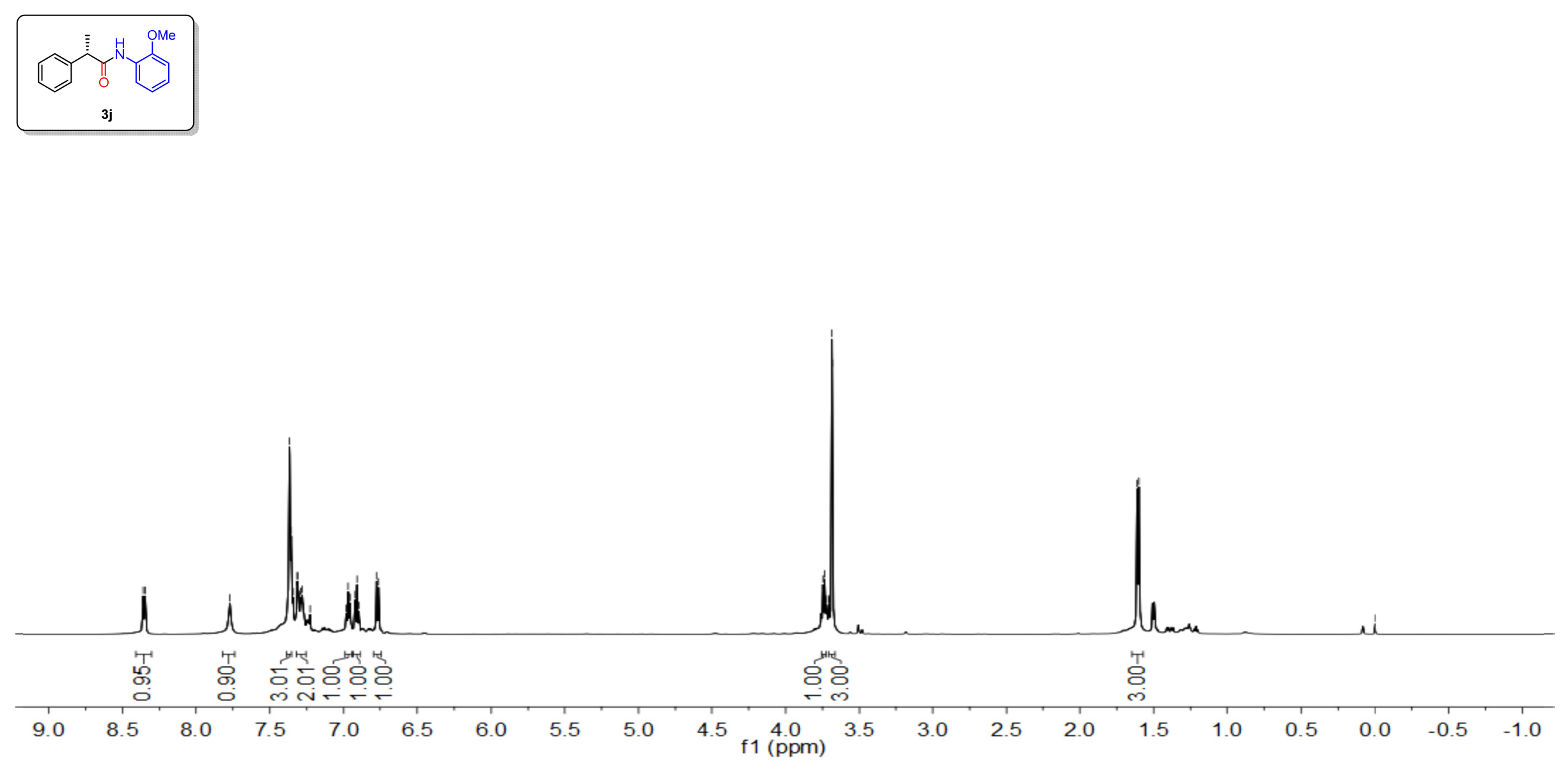
${ }^{13} \mathrm{C}$ NMR $\left(151 \mathrm{MHz}, \mathrm{CDCl}_{3}\right)$ of compound $\mathbf{3 j}$
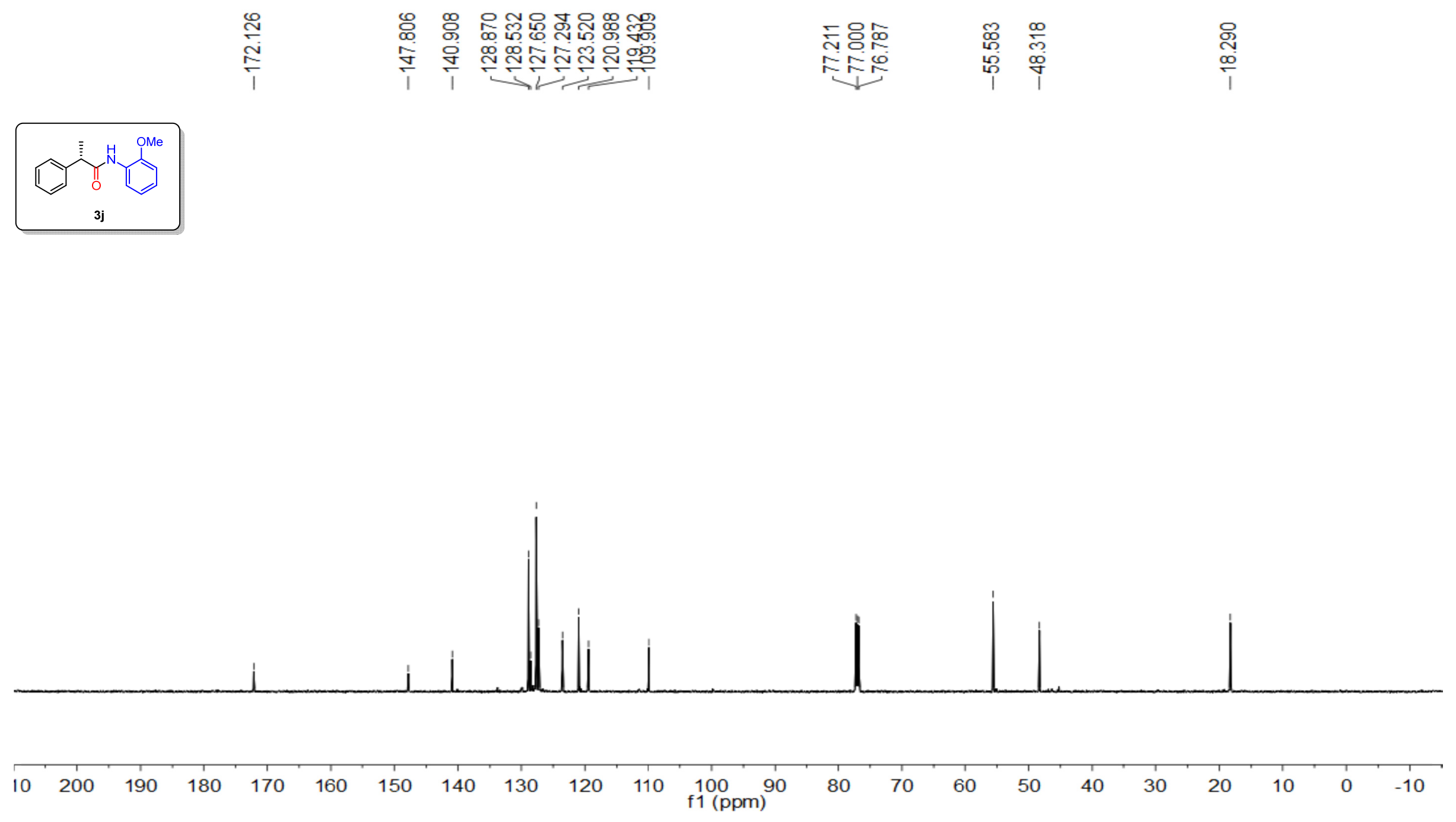
${ }^{1} \mathrm{H}$ NMR (400 MHz, $\mathrm{CDCl}_{3}$ ) of compound $\mathbf{3 k}$

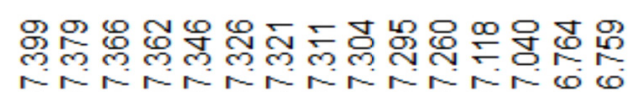

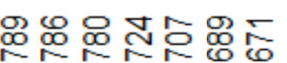

लिल लल

象

응
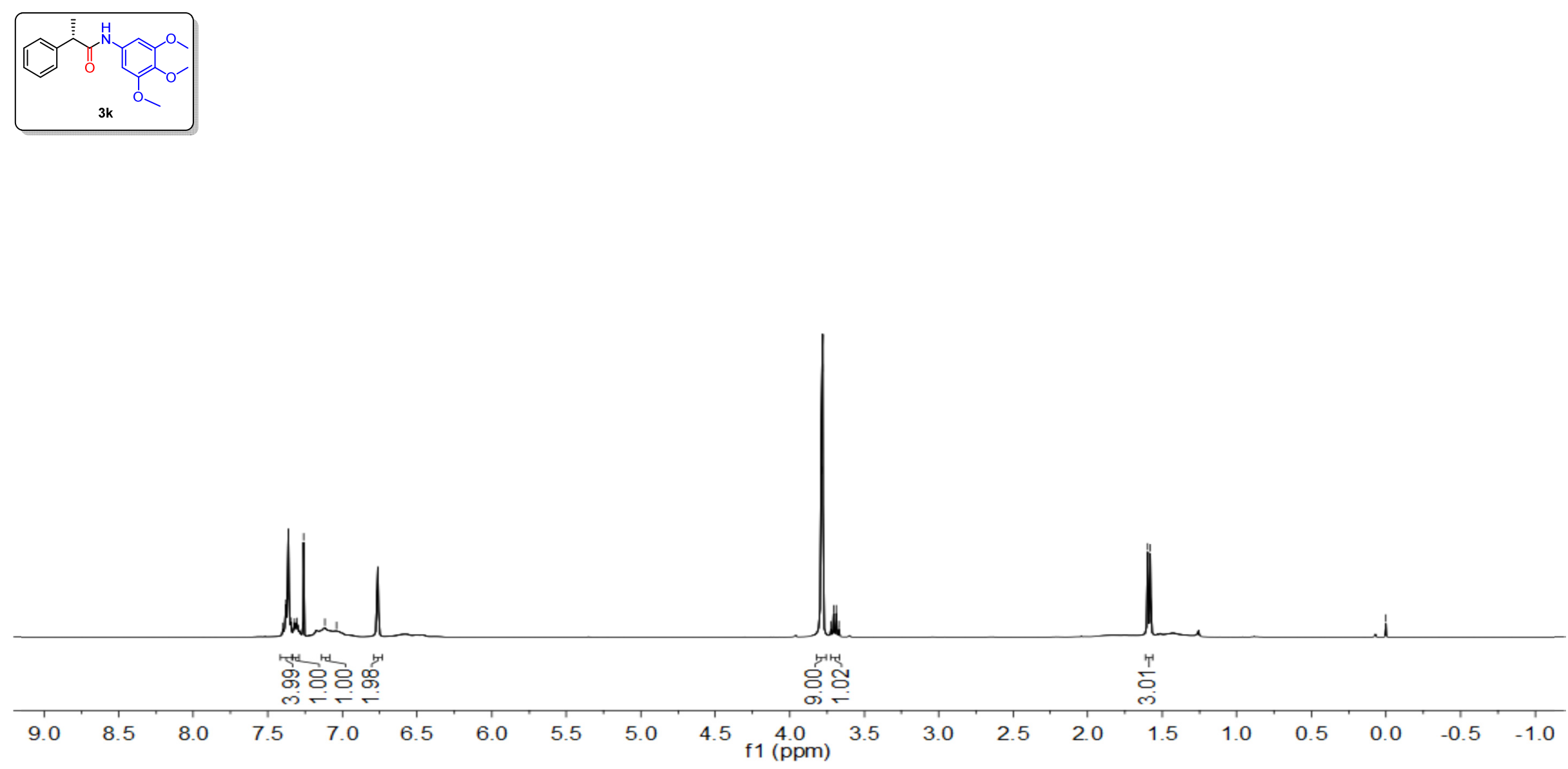
${ }^{13} \mathrm{C}$ NMR (101 MHz, $\left.\mathrm{CDCl}_{3}\right)$ of compound 3k

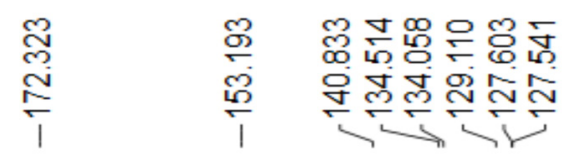

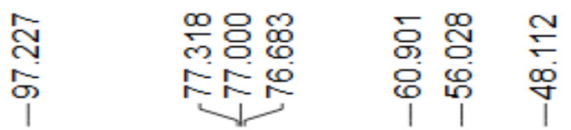

$\underset{\substack{\infty \\ \infty}}{\infty}$
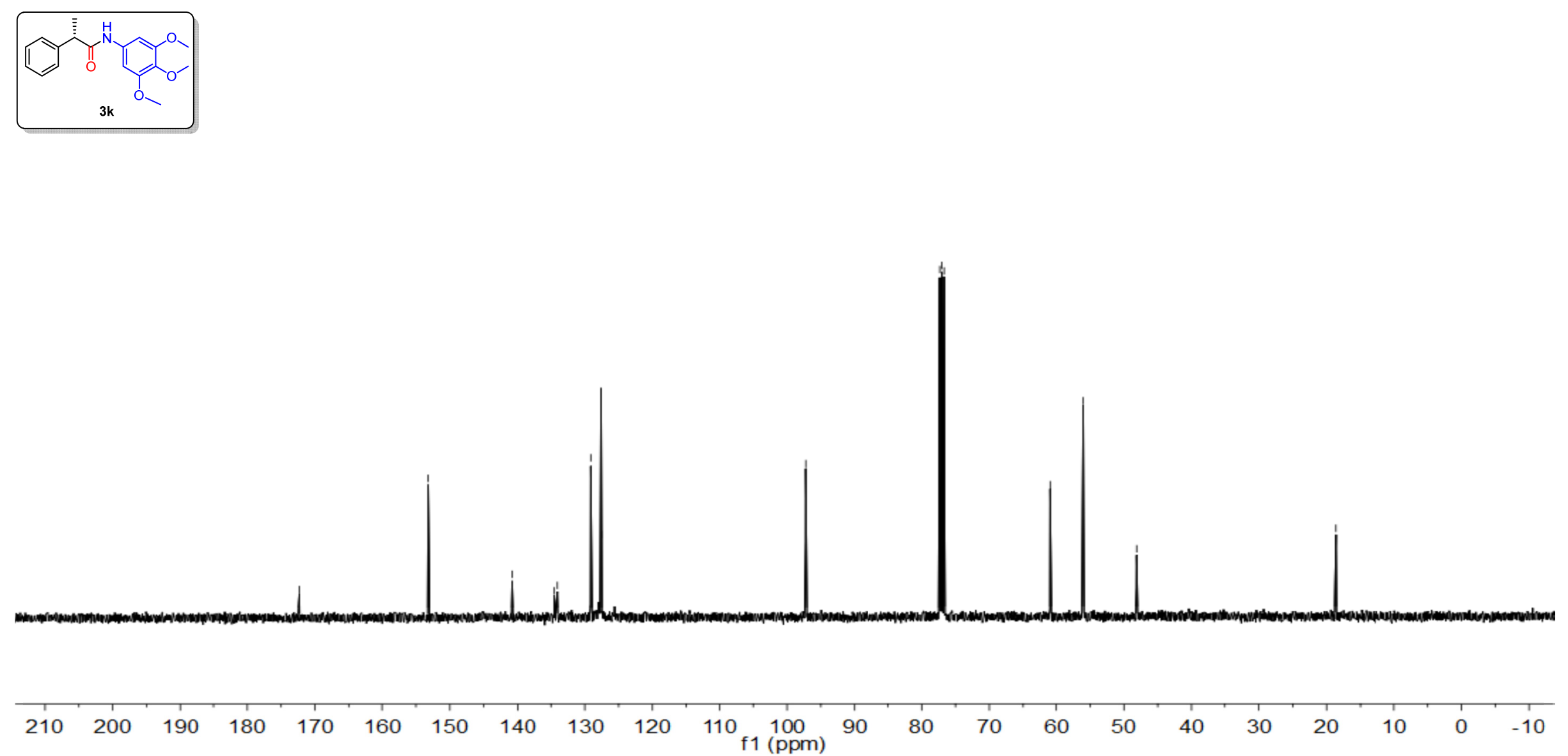
${ }^{1} \mathrm{H}$ NMR (400 MHz, $d^{6}$-DMSO) of compound 31

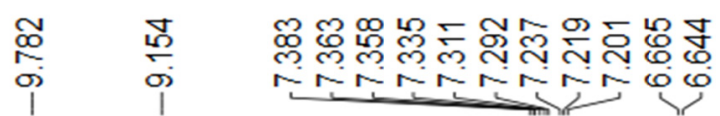

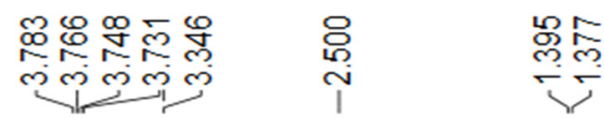
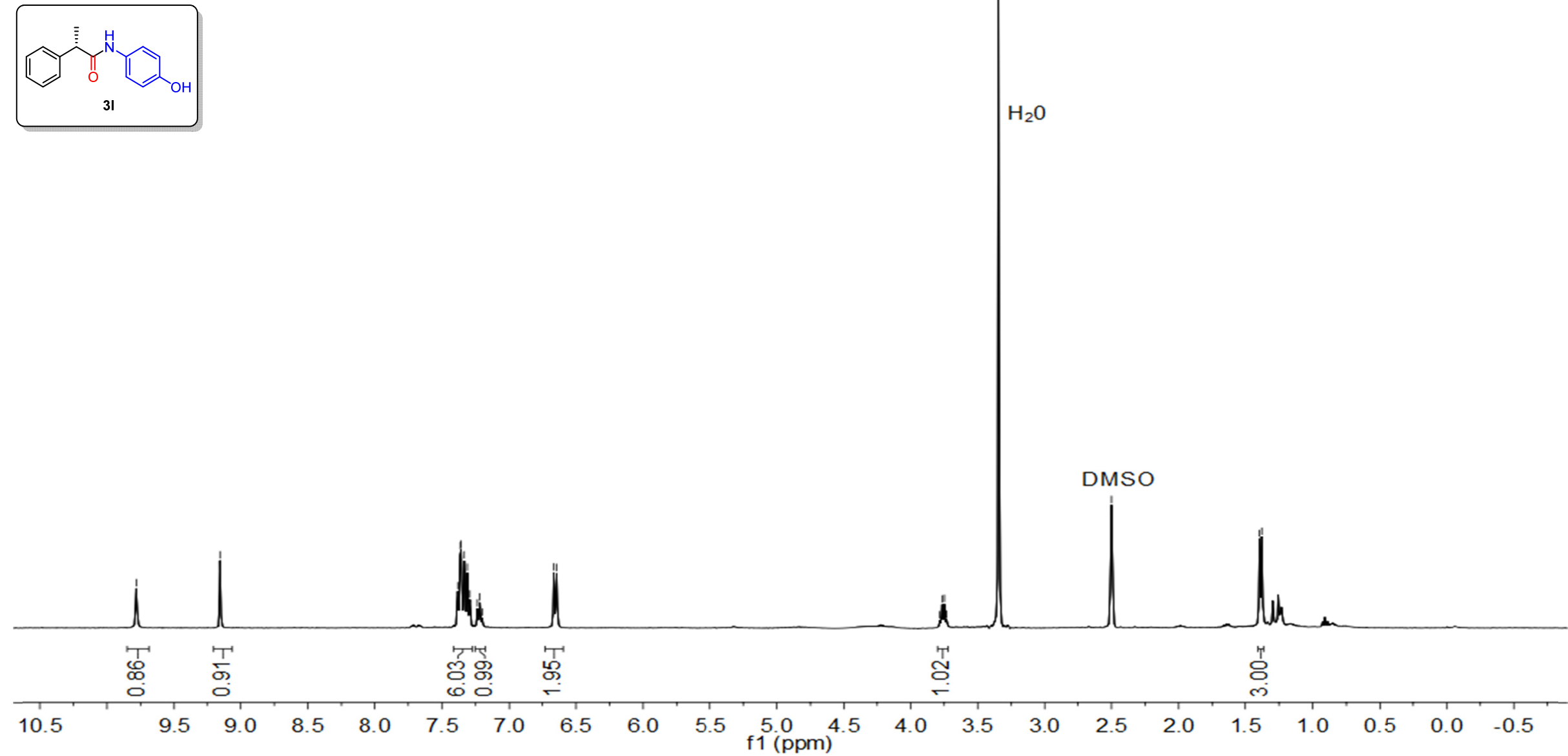
${ }^{13} \mathrm{C}$ NMR (101 MHz, $d^{6}$-DMSO) of compound 3I

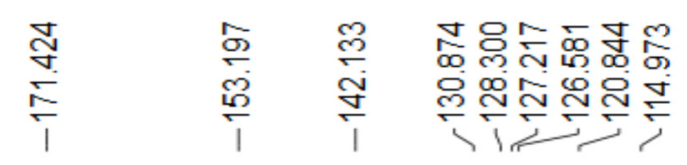

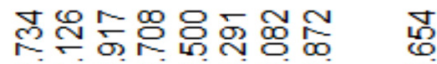

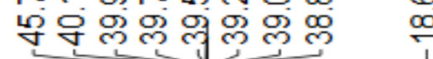
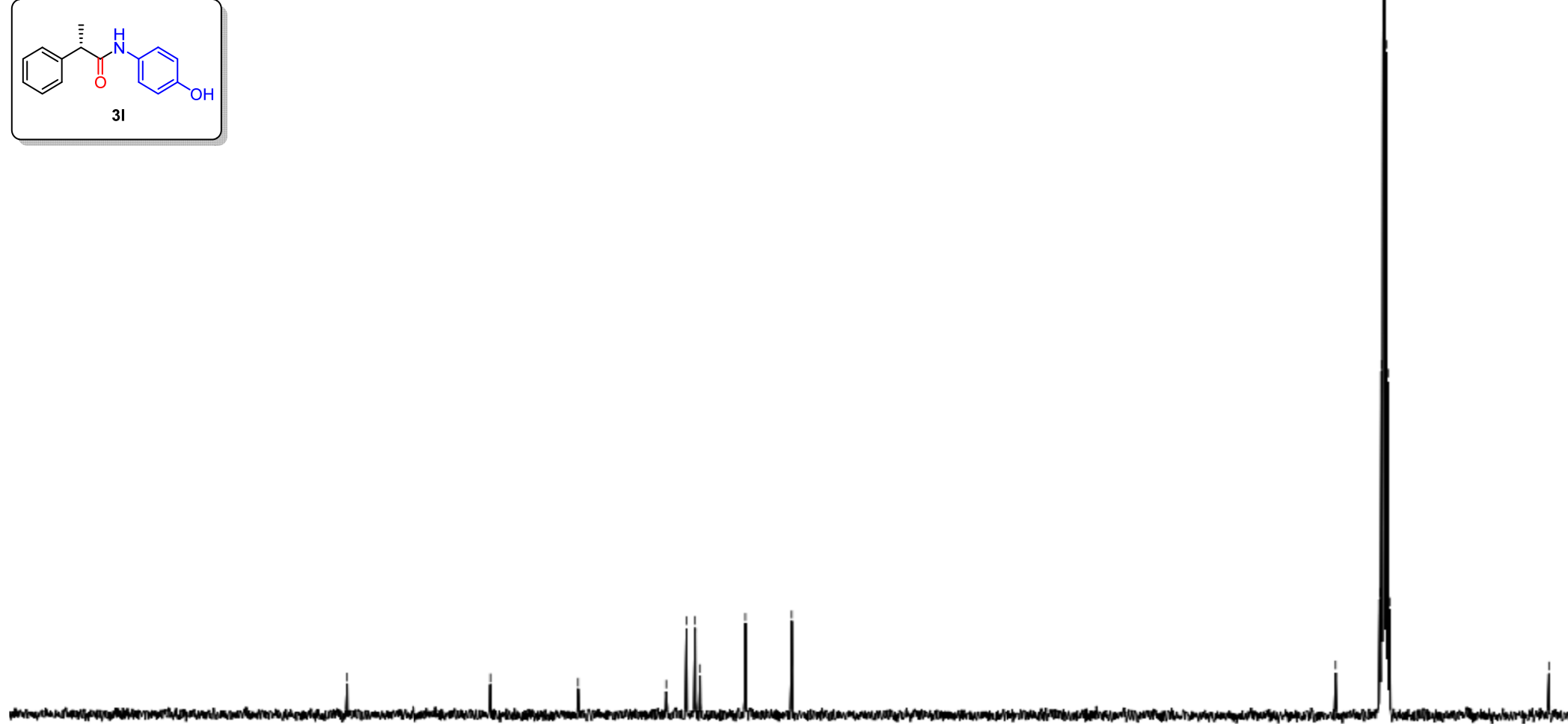

$\begin{array}{llllllllllllllllllllllll}210 & 200 & 190 & 180 & 170 & 160 & 150 & 140 & 130 & 120 & 110 & 100 & 90 & 80 & 70 & 60 & 50 & 40 & 30 & 20 & 10 & 0 & -10\end{array}$


${ }^{1} \mathrm{H}$ NMR (400 MHz, $\mathrm{CDCl}_{3}$ ) of compound $\mathbf{3 m}$

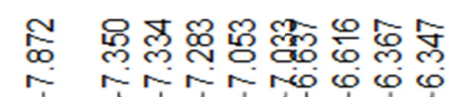
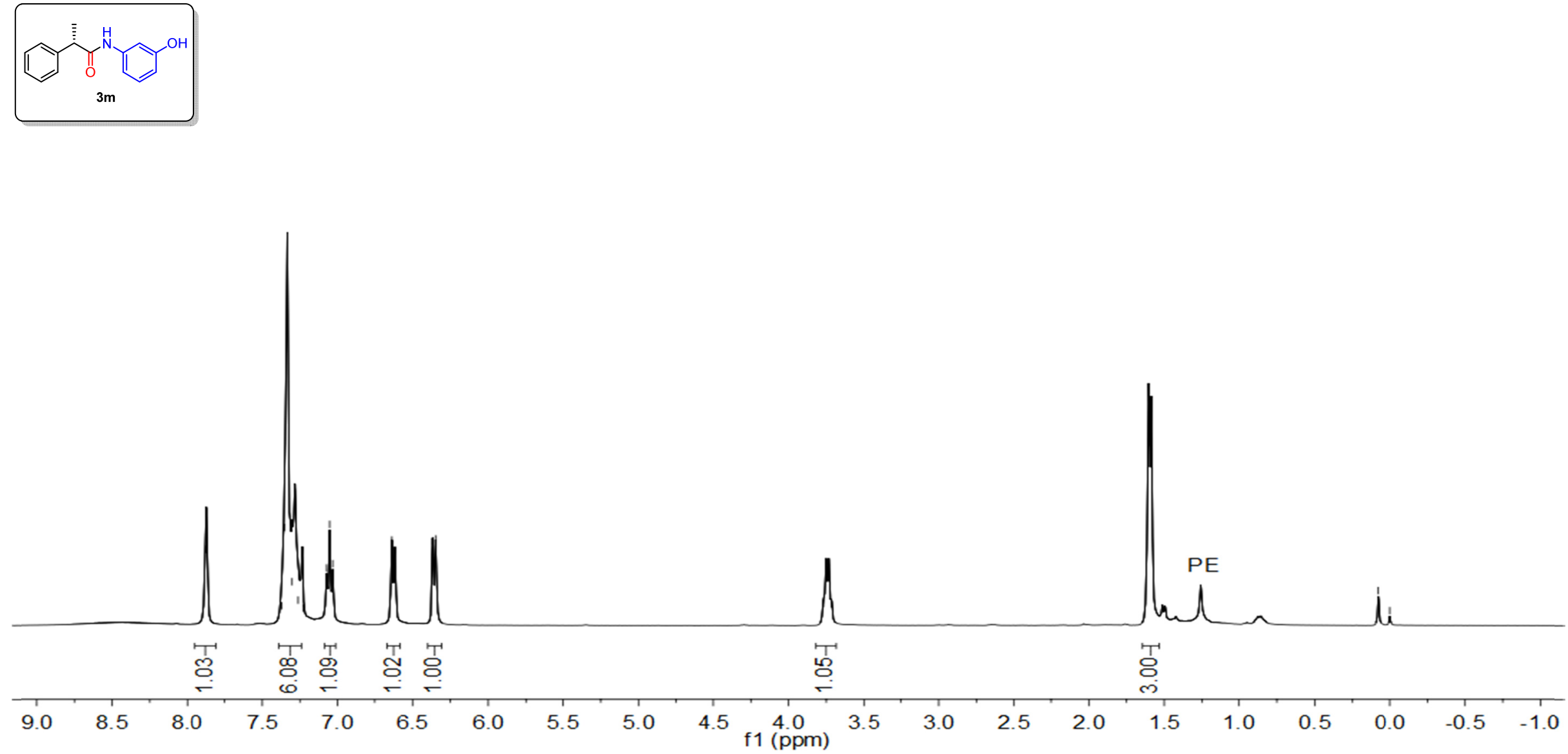
${ }^{13} \mathrm{C}$ NMR (101 MHz, $\mathrm{CDCl}_{3}$ ) of compound 3m

\begin{tabular}{|c|c|c|c|c|}
\hline 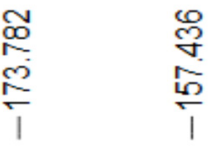 & 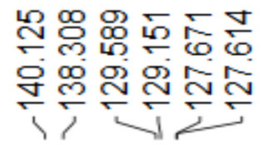 & 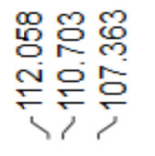 & 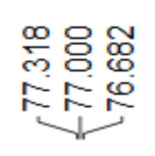 & $\begin{array}{l}\text { 令 } \\
\text { ơ } \\
\text { jo }\end{array}$ \\
\hline
\end{tabular}
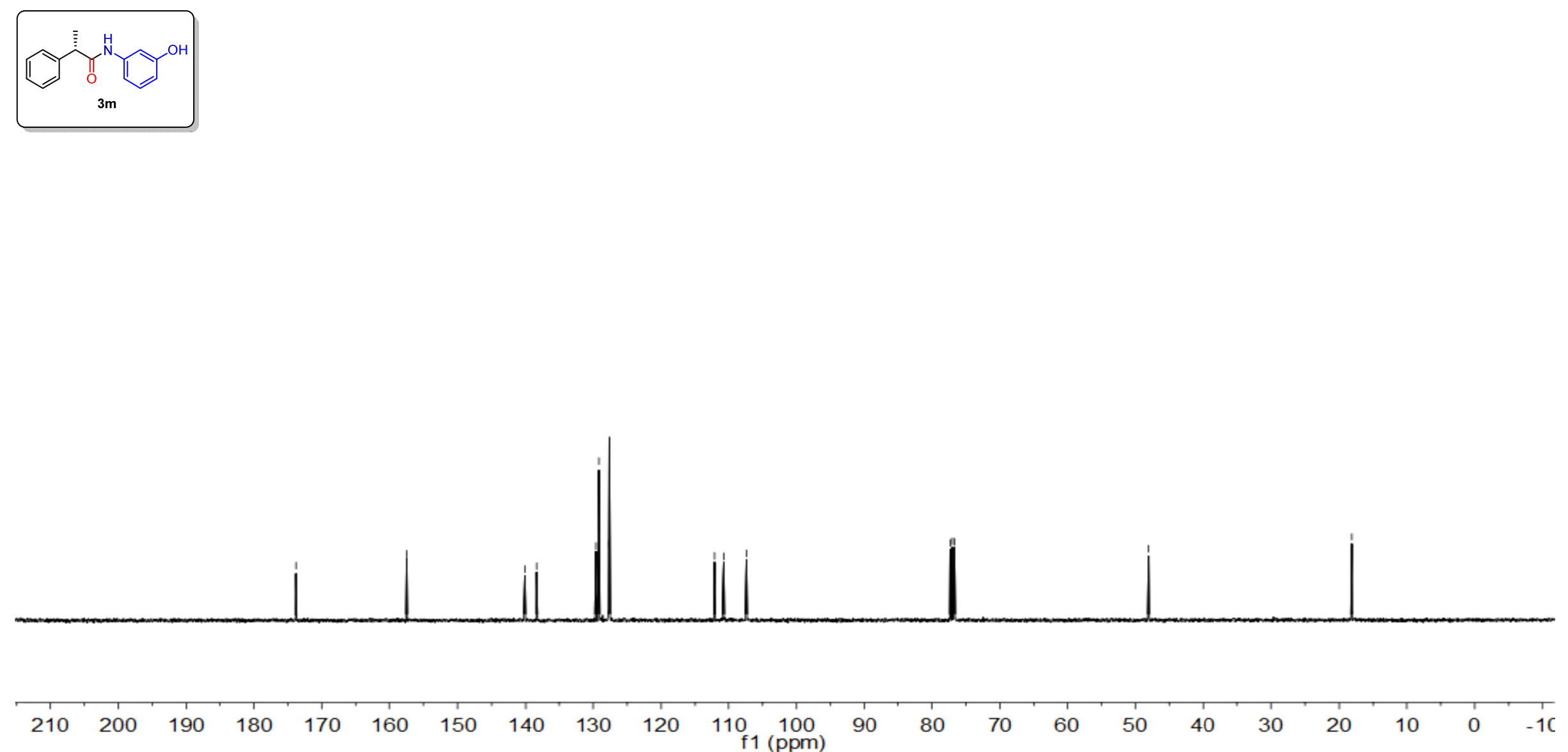
${ }^{1} \mathrm{H}$ NMR (400 MHz, $d^{6}$-DMSO) of compound 3n

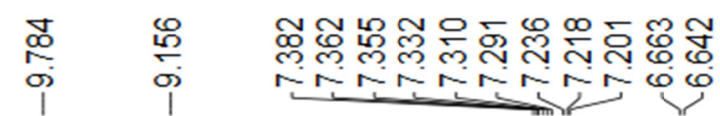
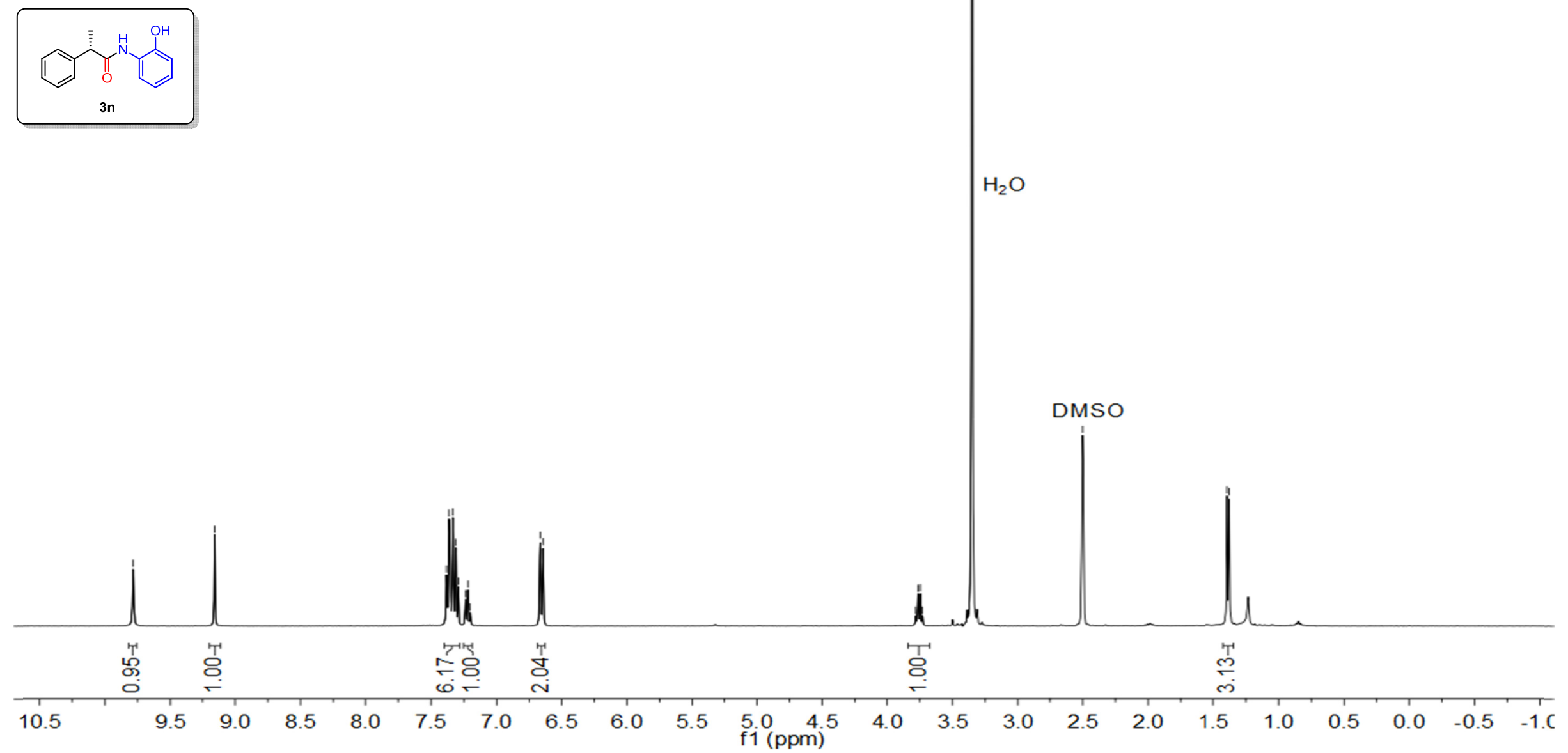
${ }^{1} \mathrm{H}$ NMR (600 MHz, $\mathrm{CDCl}_{3}$ ) of compound 3 o

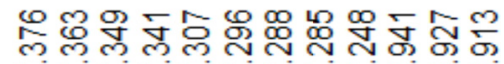

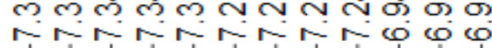

웅 $\frac{0}{6}$

ن

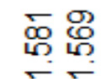

응
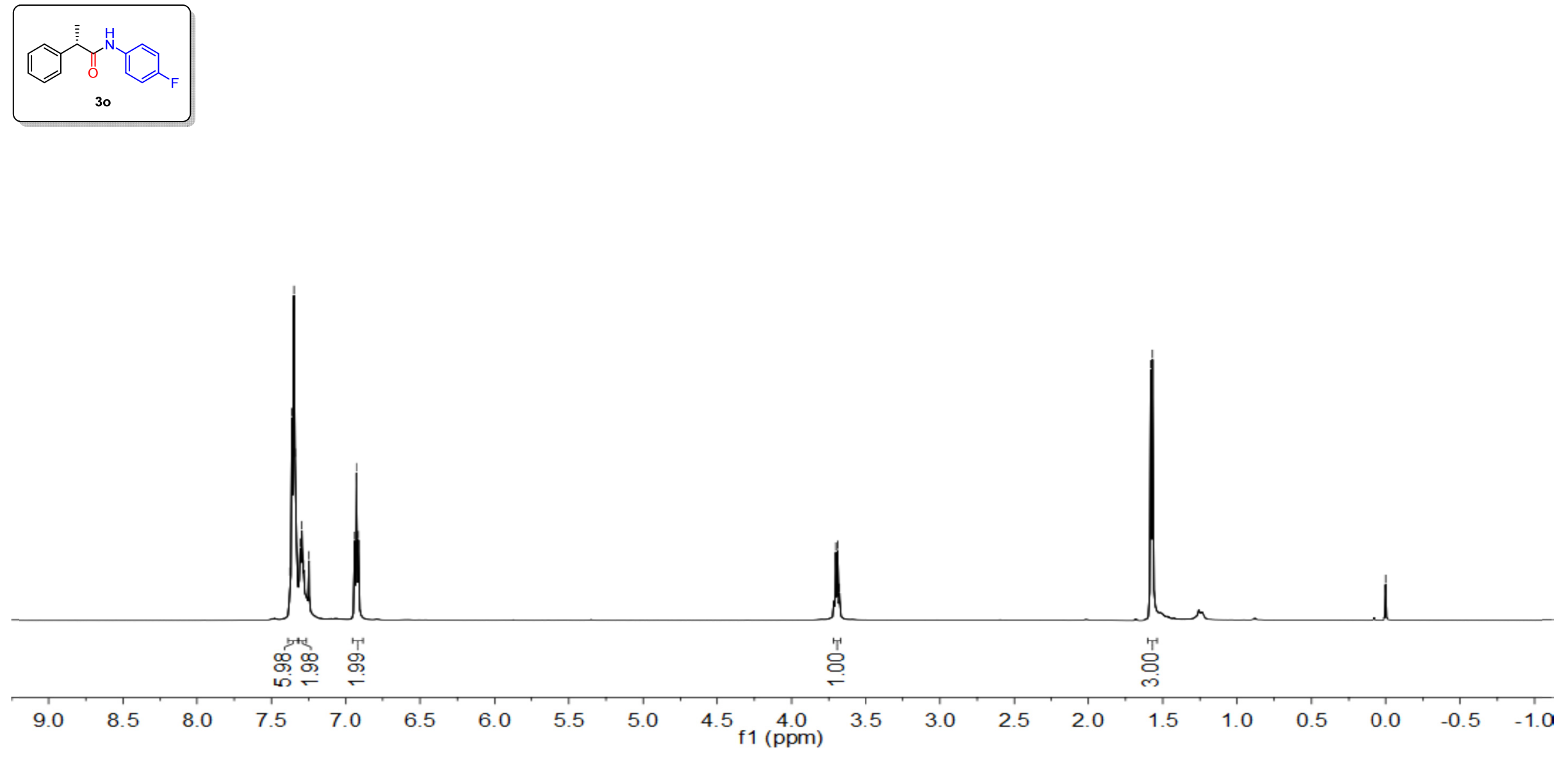
${ }^{13} \mathrm{C}$ NMR (151 MHz, $\left.\mathrm{CDCl}_{3}\right)$ of compound 3o

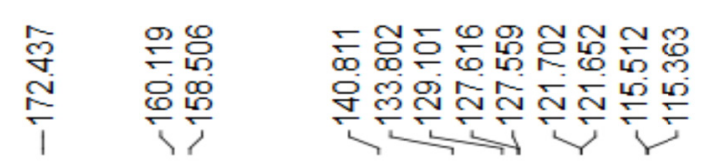

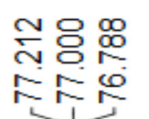

$\bar{n}$
v
†

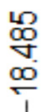
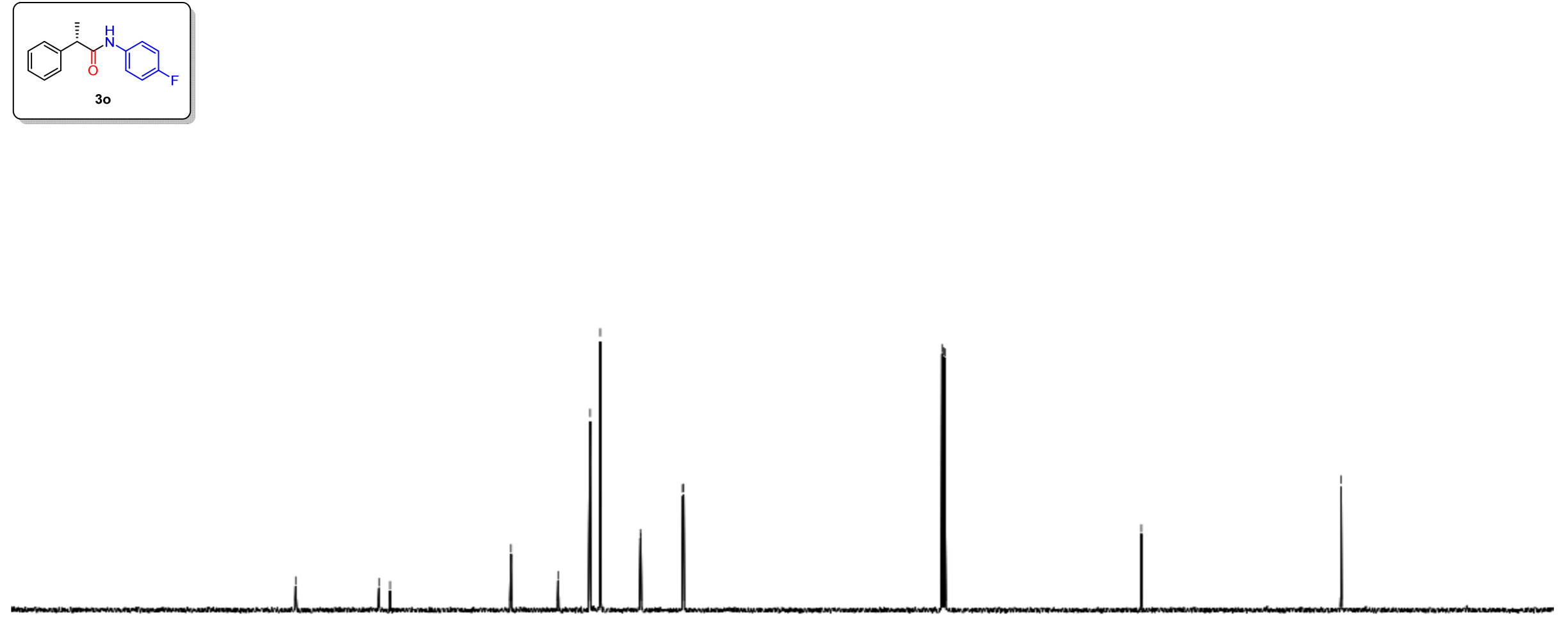

$\begin{array}{lllllllllll}210 & 200 & 190 & 180 & 170 & 160 & 150 & 140 & 130 & 120 & 110 \begin{array}{l}100 \\ \mathrm{f} 1\end{array}(\mathrm{ppm})\end{array}$ 
${ }^{19} \mathrm{~F}$ NMR (376 MHz, $\mathrm{CDCl}_{3}$ ) of compound 30

$$
\stackrel{ }{\stackrel{0}{r}}
$$
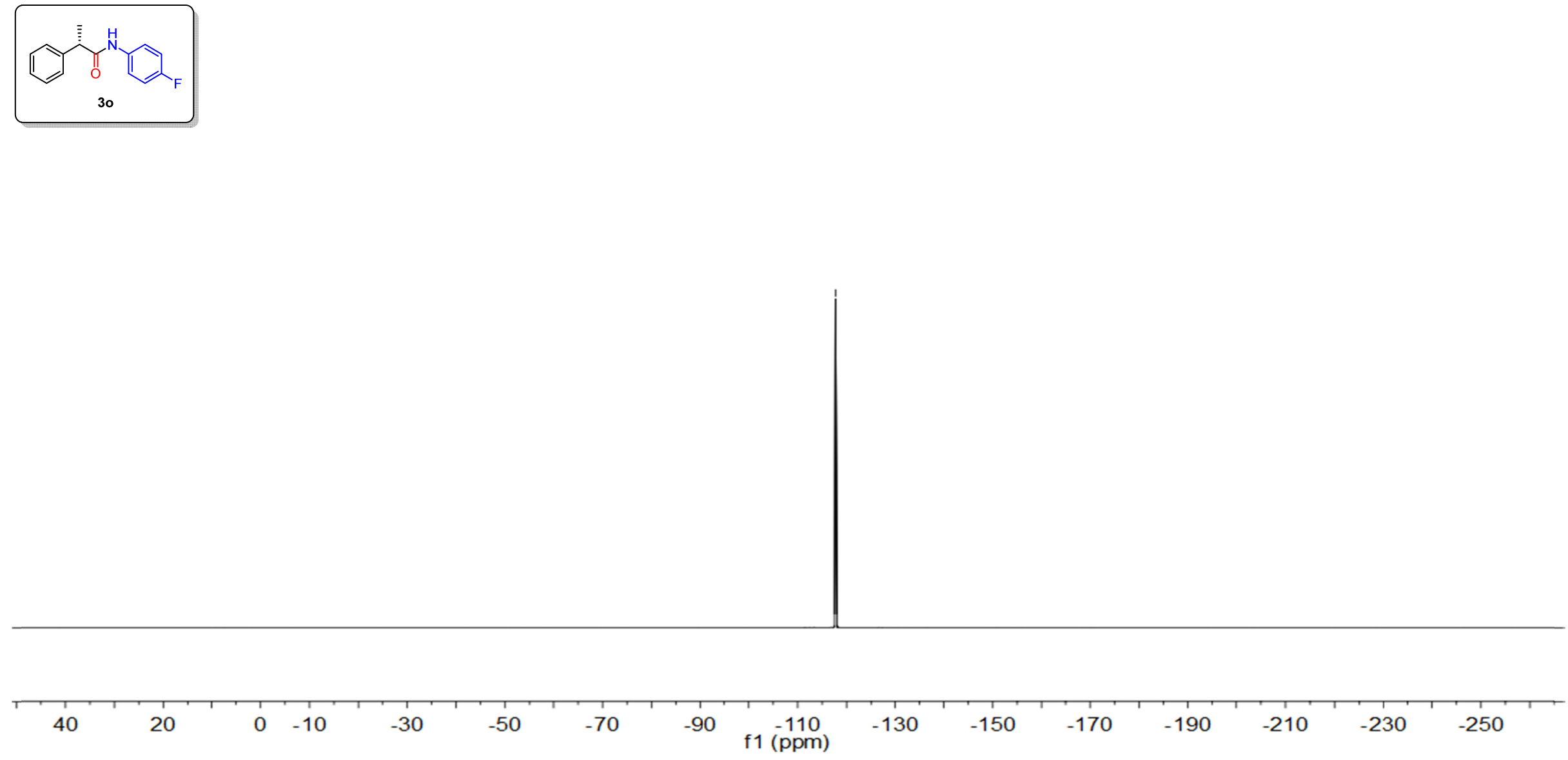
${ }^{1} \mathrm{H}$ NMR (400 MHz, $\mathrm{CDCl}_{3}$ ) of compound 3p

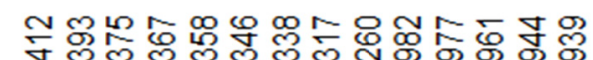
की
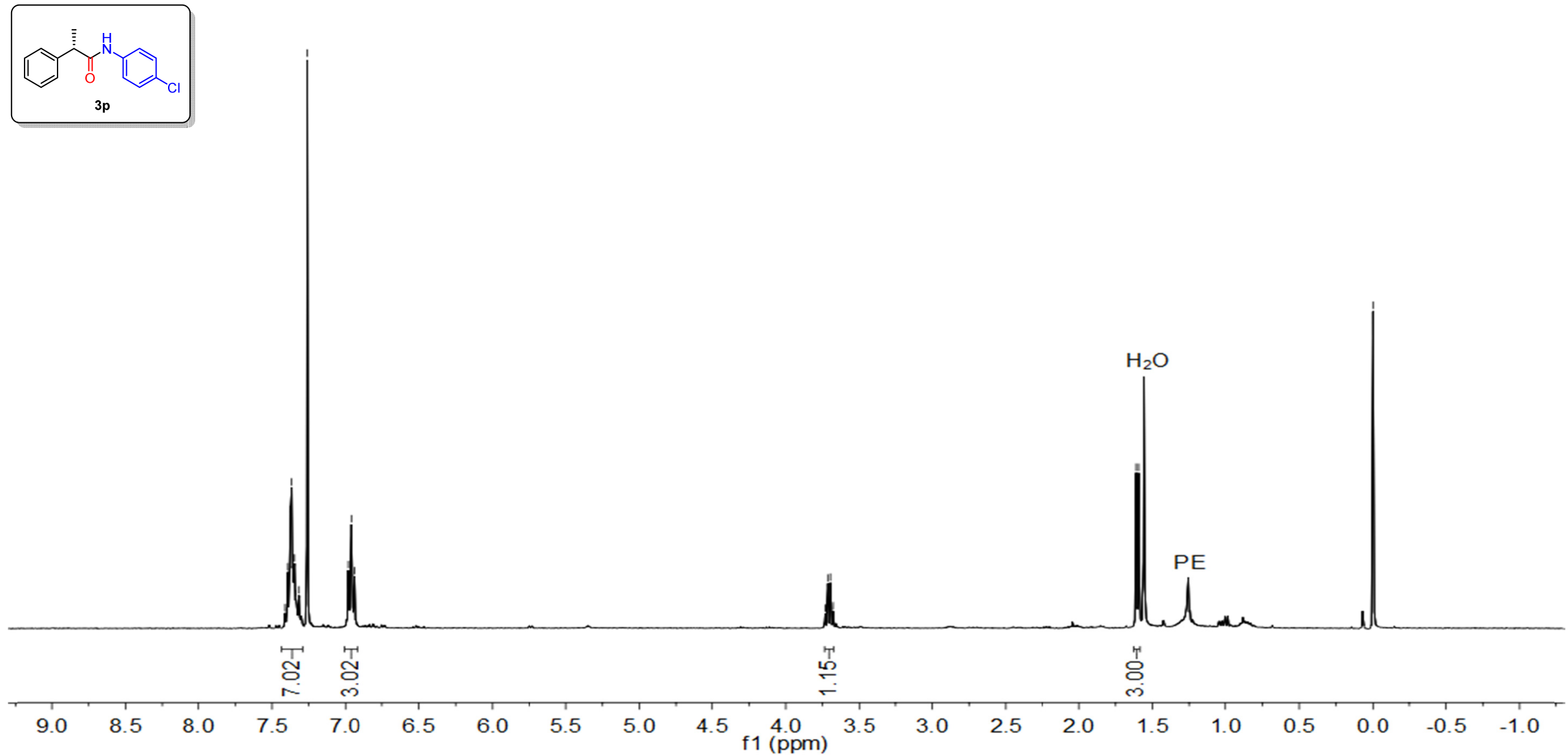
${ }^{13} \mathrm{C}$ NMR (101 MHz, $\mathrm{CDCl}_{3}$ ) of compound 3p

\begin{tabular}{|c|c|c|c|}
\hline 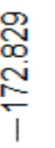 & 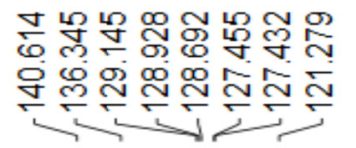 & 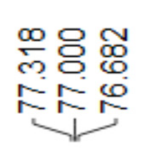 & 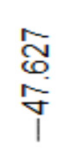 \\
\hline
\end{tabular}
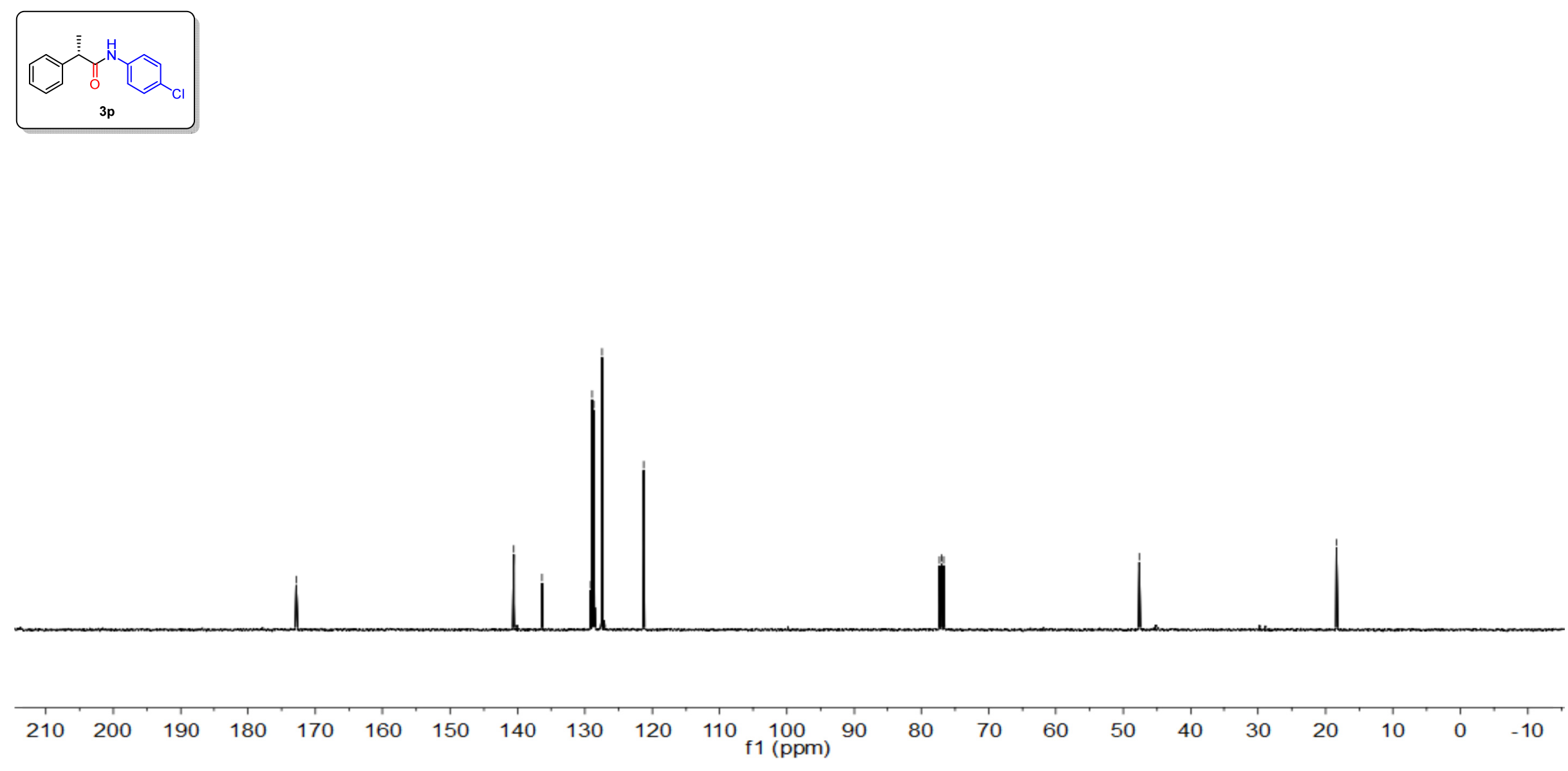
${ }^{1} \mathrm{H}$ NMR $\left(600 \mathrm{MHz}, \mathrm{CDCl}_{3}\right)$ of compound $\mathbf{3 q}$

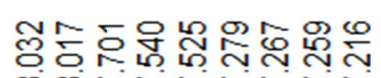

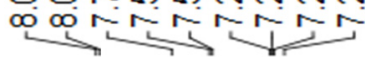

구요

लंखल

亘疍
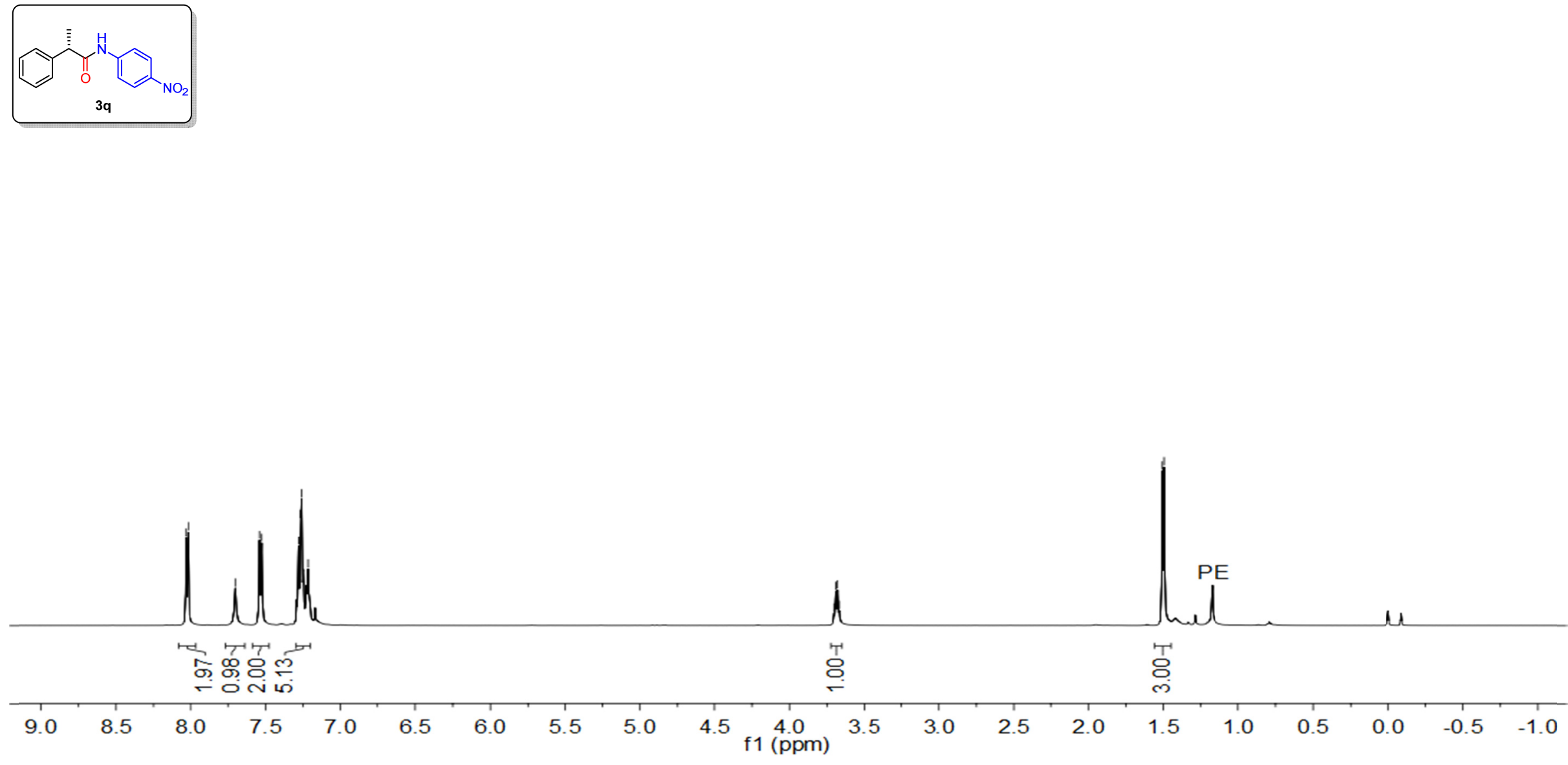
${ }^{13} \mathrm{C}$ NMR (101 MHz, $\mathrm{CDCl}_{3}$ ) of compound $\mathbf{3 q}$

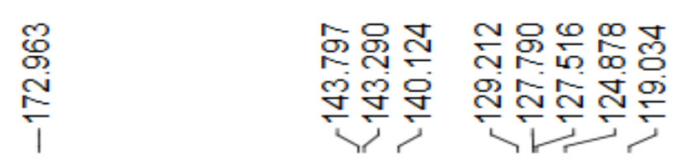

ำㅇㅇㅇ

는

$\underset{\substack{\text { ơ } \\ \text { o }}}{\stackrel{0}{1}}$

$\frac{\infty}{1}$
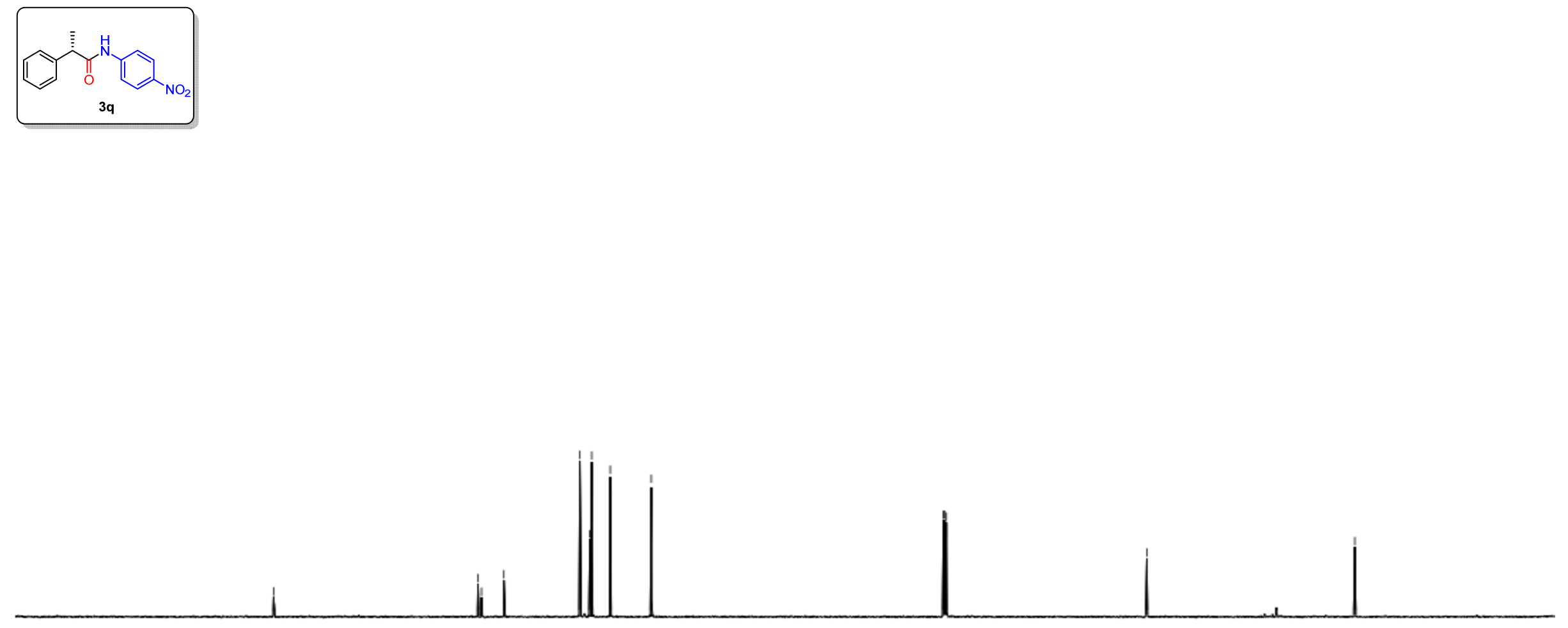

\begin{tabular}{lllllllllllllllllllllll}
\hline 10 & 200 & 190 & 180 & 170 & 160 & 150 & 140 & 130 & 120 & 110 & $\begin{array}{c}100 \\
\mathrm{f} 1(\mathrm{ppm})\end{array}$ & 90 & 80 & 70 & 60 & 50 & 40 & 30 & 20 & 10 & 0 & -1
\end{tabular} 
${ }^{1} \mathrm{H}$ NMR (400 MHz, $\mathrm{CDCl}_{3}$ ) of compound 3r

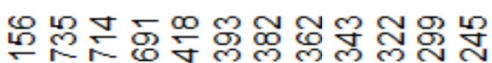

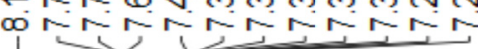
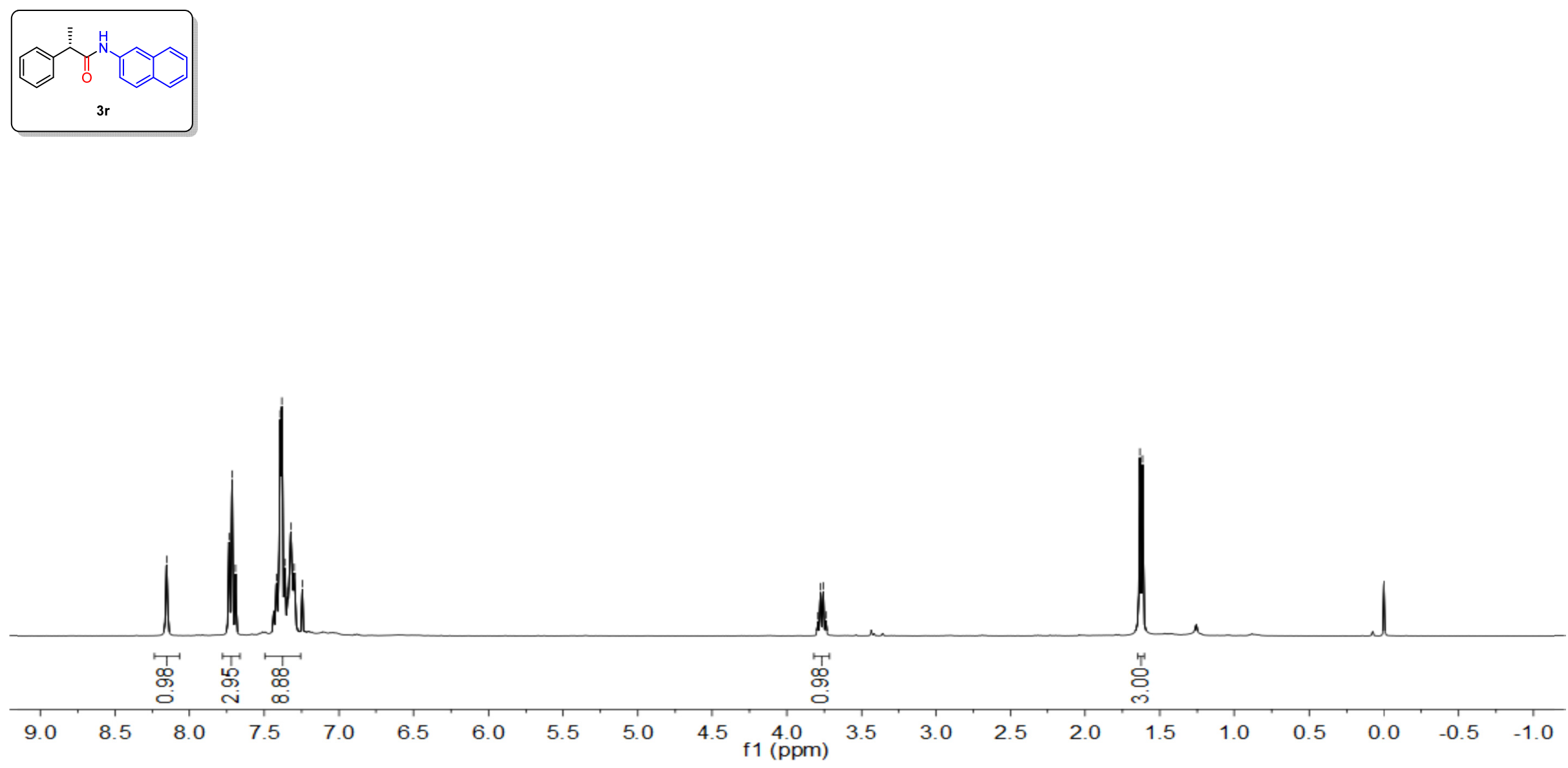
${ }^{13} \mathrm{C}$ NMR (101 MHz, $\mathrm{CDCl}_{3}$ ) of compound $\mathbf{3 r}$

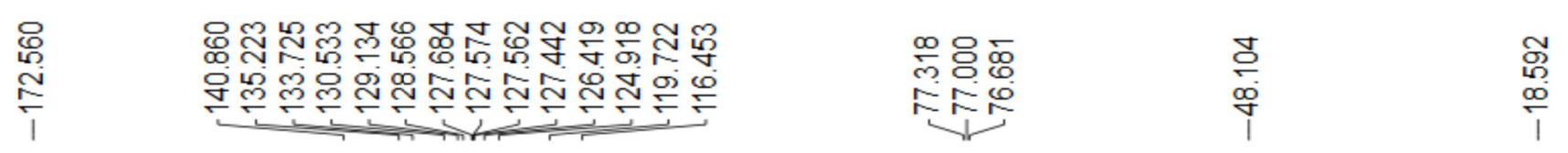
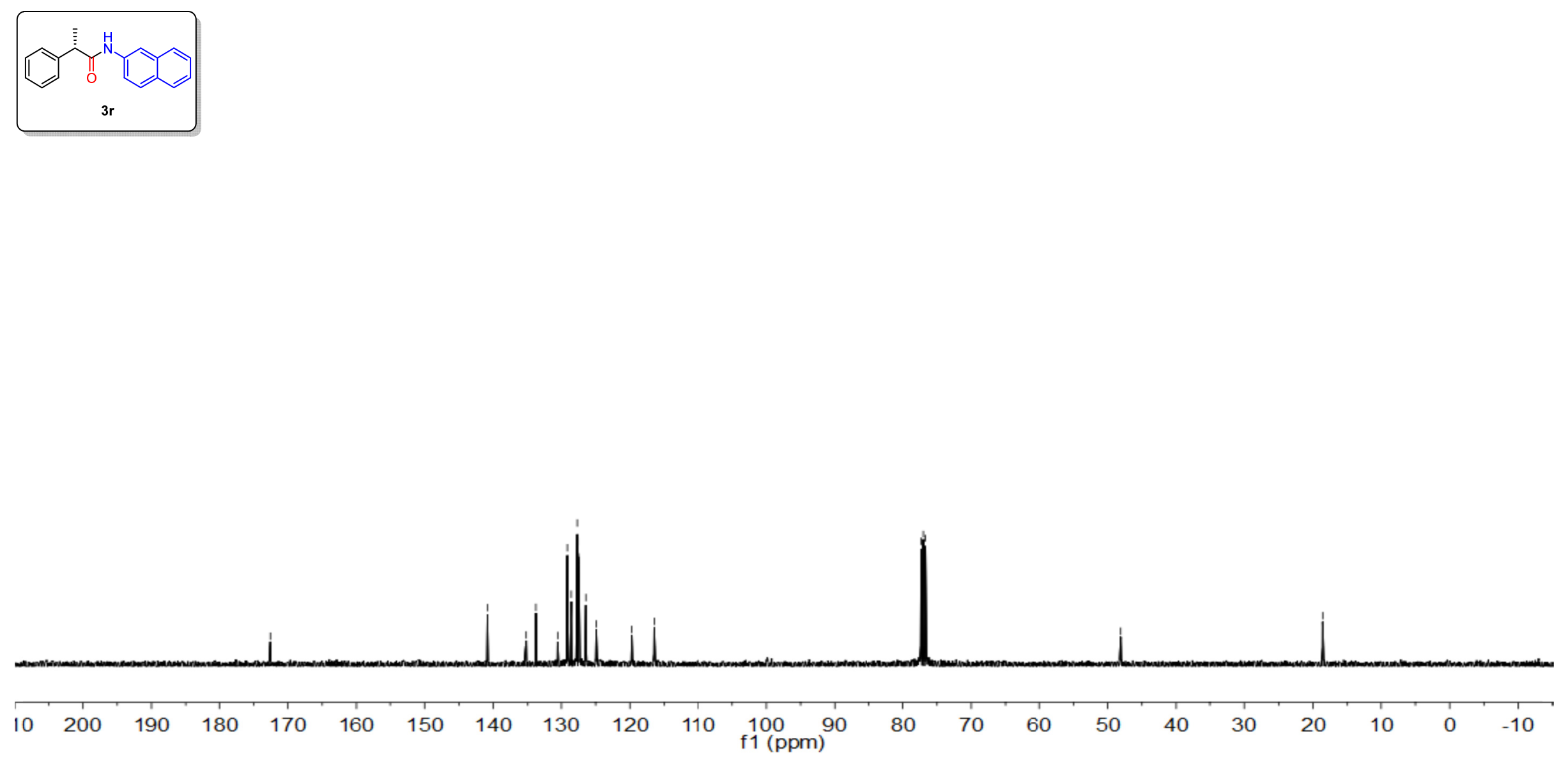
${ }^{1} \mathrm{H}$ NMR (400 MHz, $\mathrm{CDCl}_{3}$ ) of compound 3s

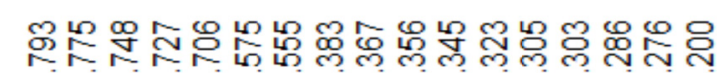
rarrarrar
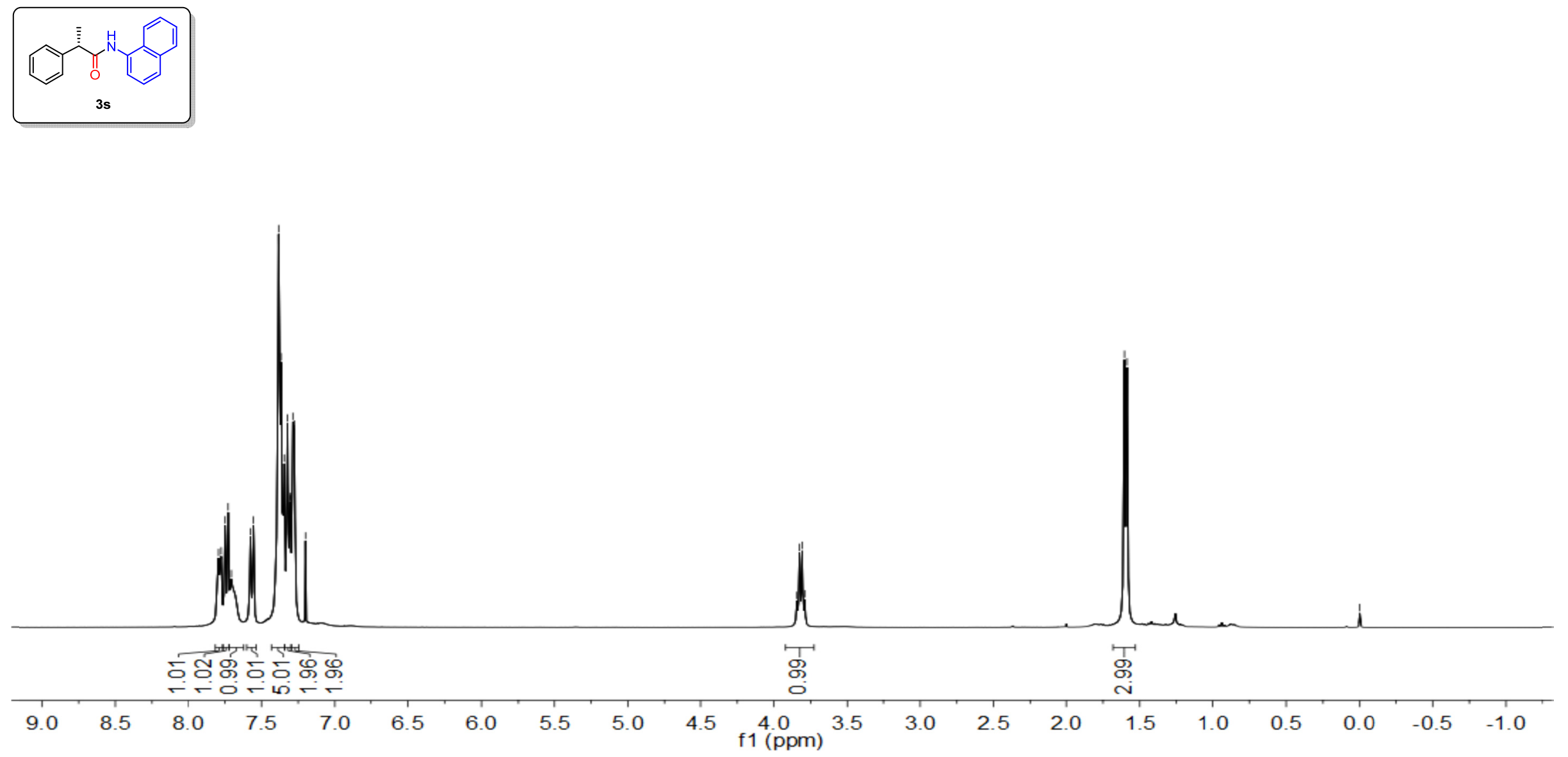
${ }^{13} \mathrm{C}$ NMR (151 MHz, $\mathrm{CDCl}_{3}$ ) of compound 3s
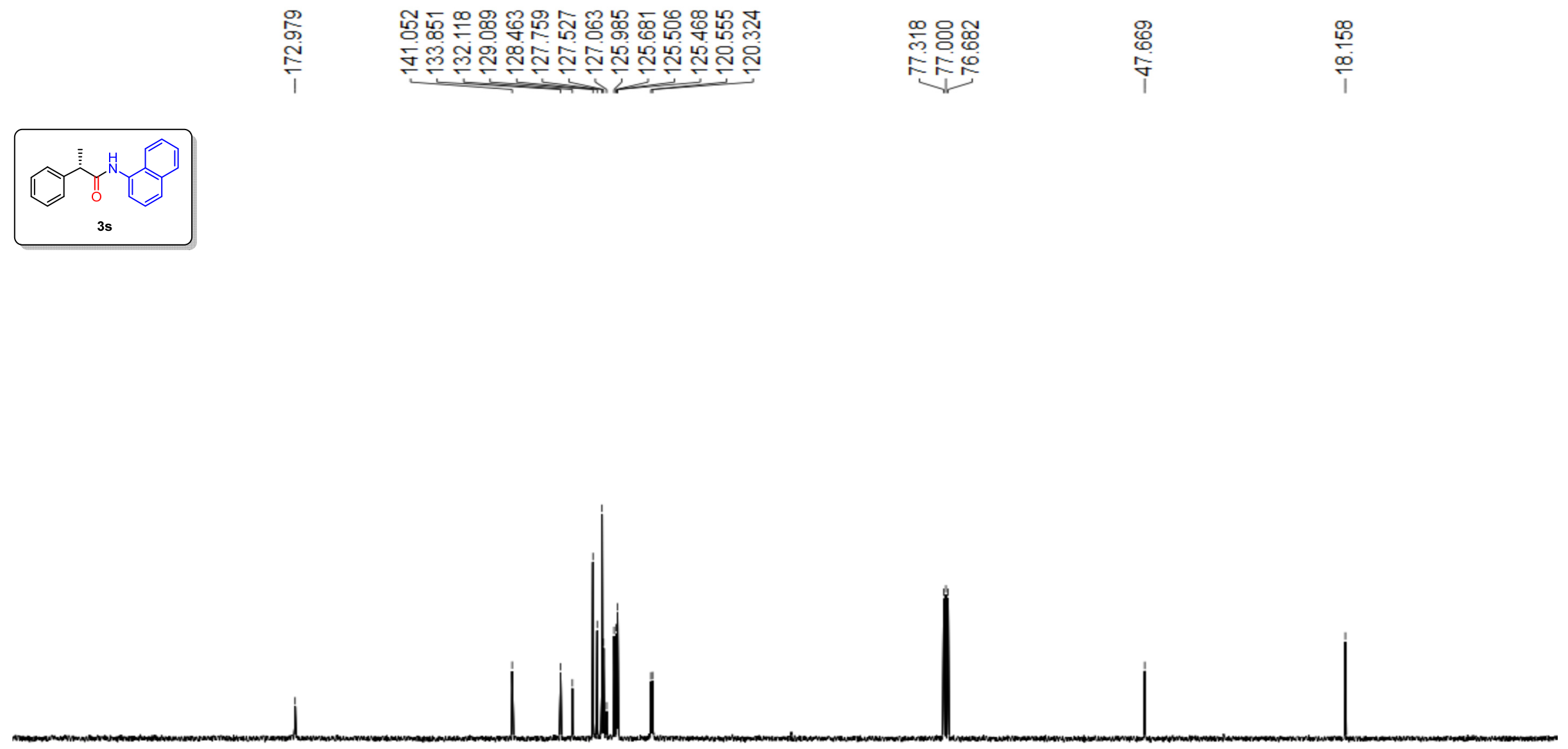

$\begin{array}{llllllllllll}210 & 200 & 190 & 180 & 170 & 160 & 150 & 140 & 130 & 120 & 110 & 100 \\ \mathrm{f} 1(\mathrm{ppm})\end{array}$ 
${ }^{1} \mathrm{H}$ NMR $\left(600 \mathrm{MHz}, \mathrm{CDCl}_{3}\right)$ of compound $\mathbf{3 t}$

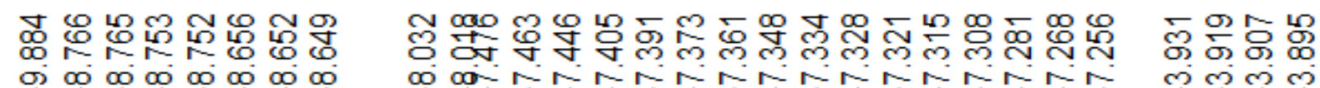

लिल्ल

$\overbrace{\substack{0 \\ 0}}^{-1}$

음
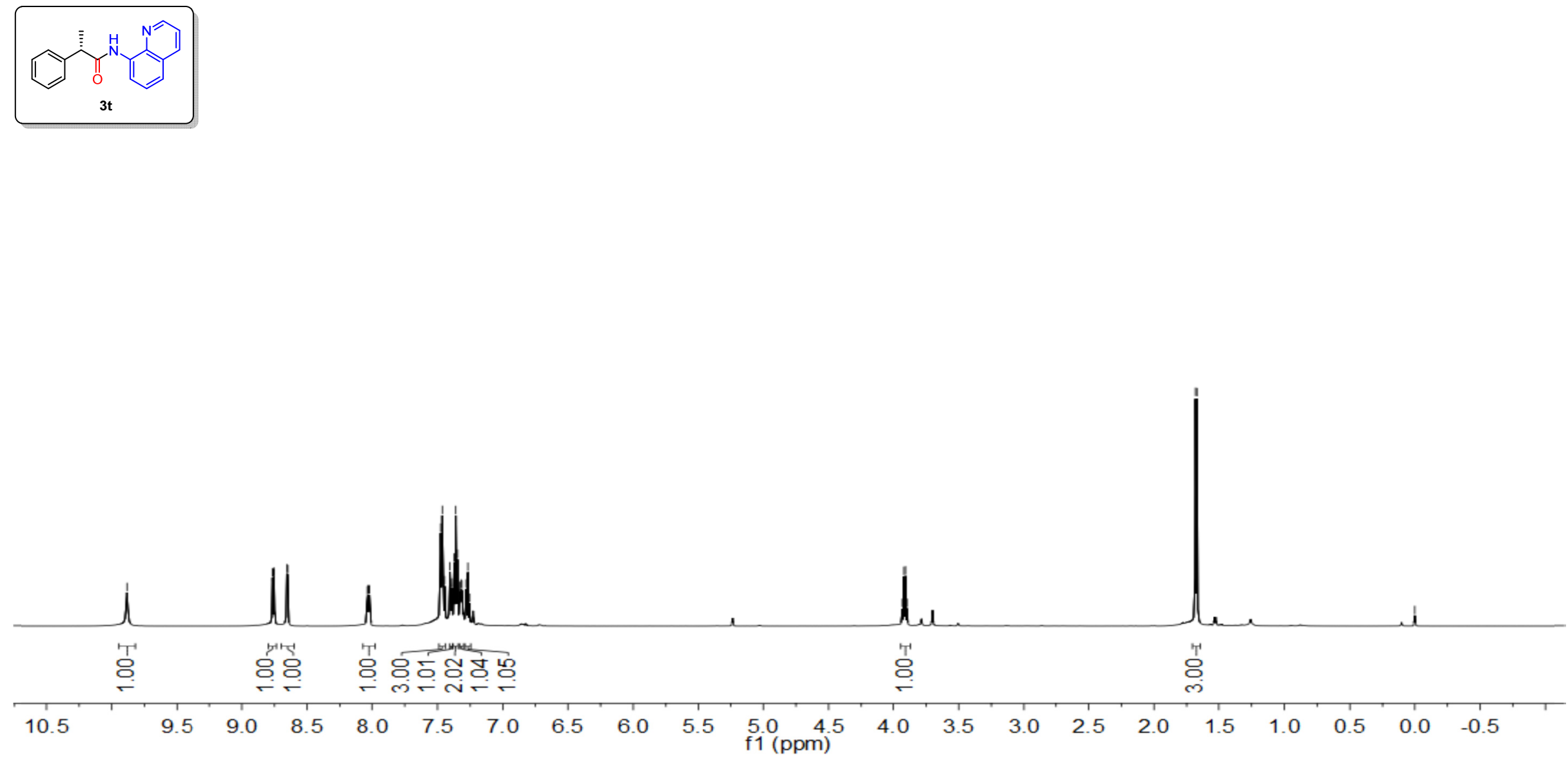
${ }^{13} \mathrm{C}$ NMR (151 MHz, $\mathrm{CDCl}_{3}$ ) of compound $\mathbf{3 t}$

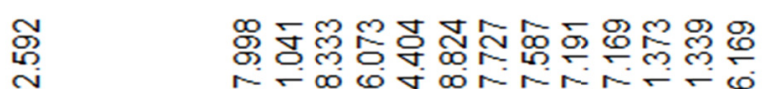

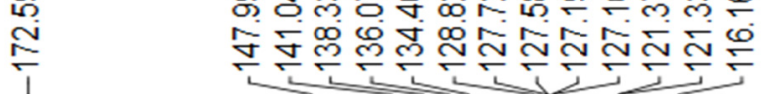

둥ㅇㅇ욤

Fin

in

$\underset{1}{\frac{0}{10}}$
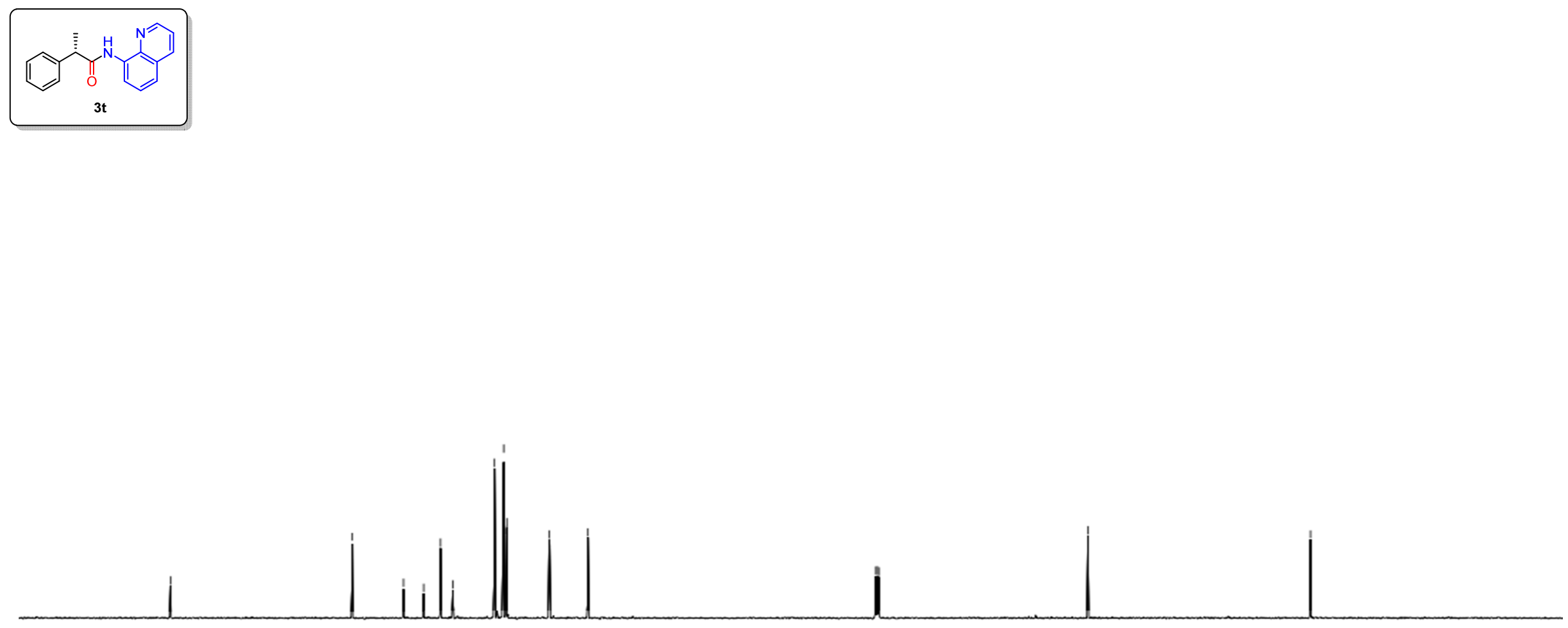
${ }^{1} \mathrm{H}$ NMR (400 MHz, $\mathrm{CDCl}_{3}$ ) of compound $4 \mathbf{a}$

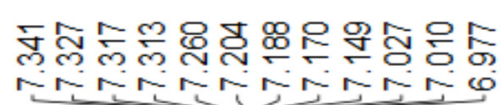

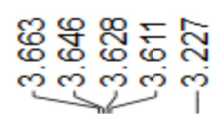

昂

응
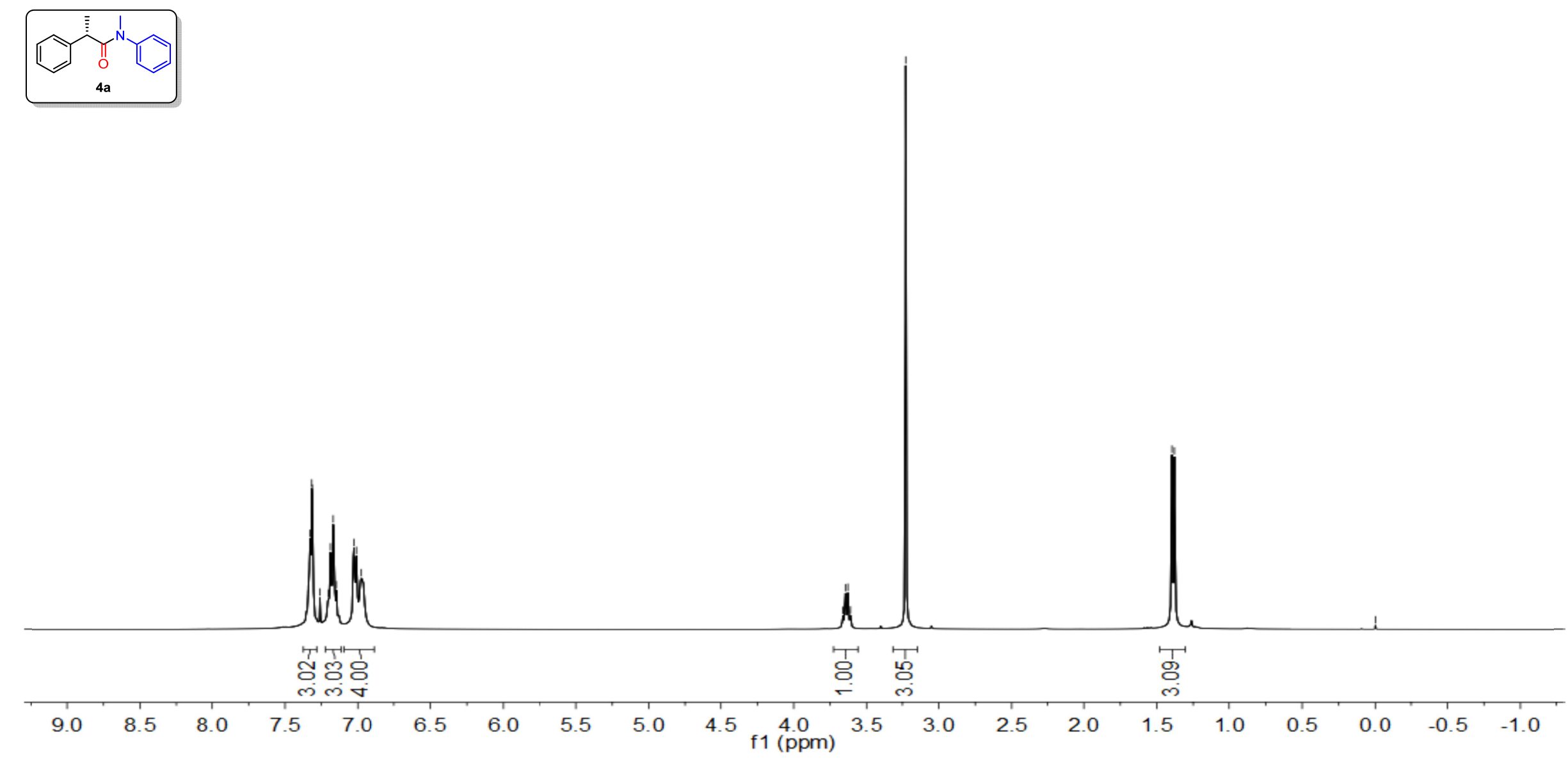
${ }^{13} \mathrm{C}$ NMR (101 MHz, $\mathrm{CDCl}_{3}$ ) of compound 4a

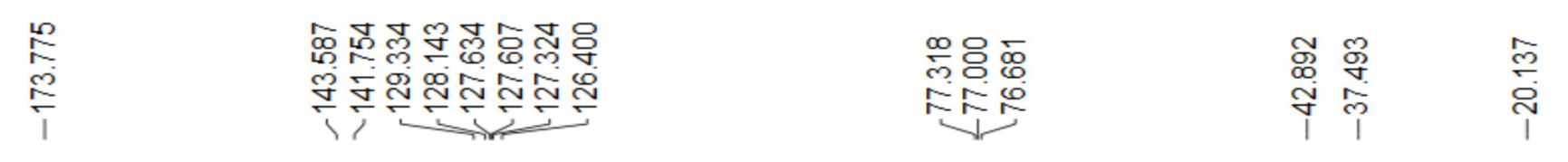
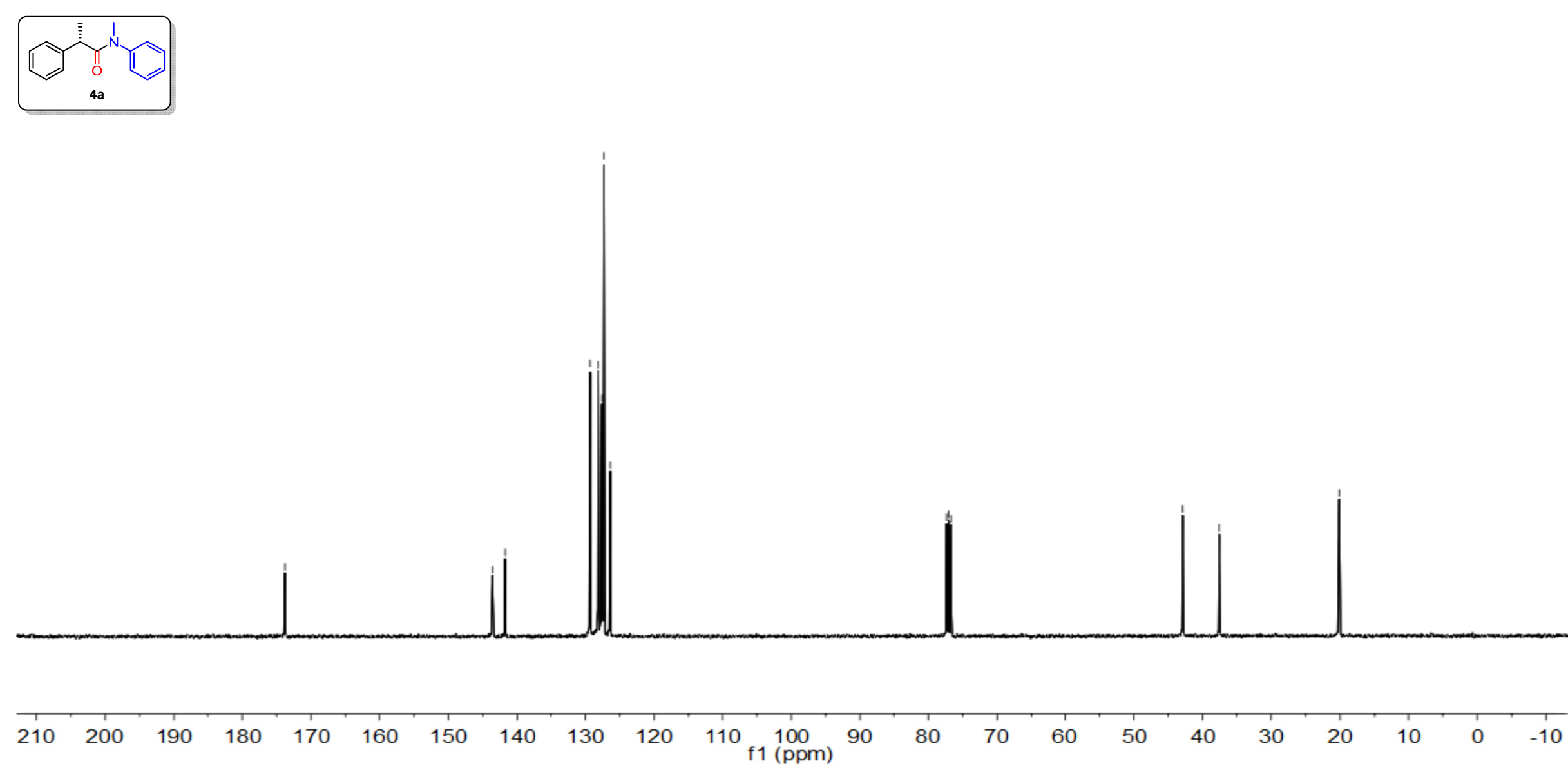
${ }^{1} \mathrm{H}$ NMR (400 MHz, $\mathrm{CDCl}_{3}$ ) of compound $\mathbf{4 b}$

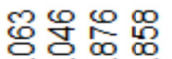

$$
\begin{aligned}
& \text { 武监 }
\end{aligned}
$$

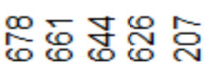

लिल

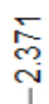

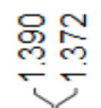

응

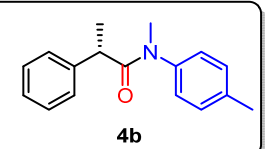

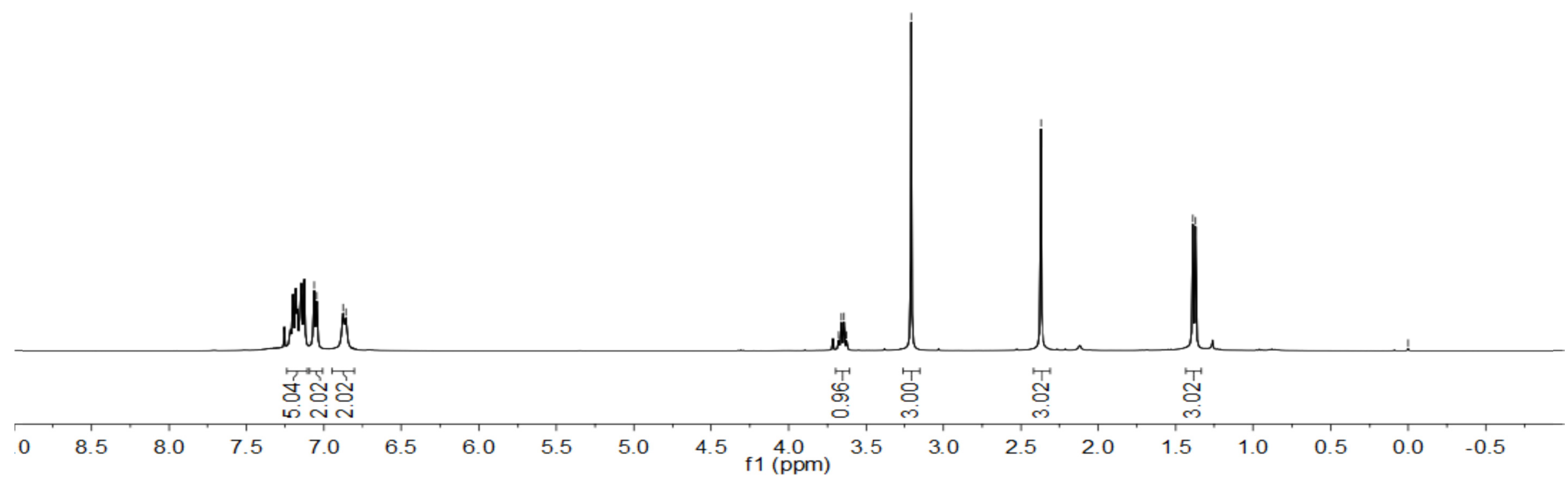


${ }^{13} \mathrm{C}$ NMR $\left(101 \mathrm{MHz}, \mathrm{CDCl}_{3}\right)$ of compound $\mathbf{4 b}$

荥
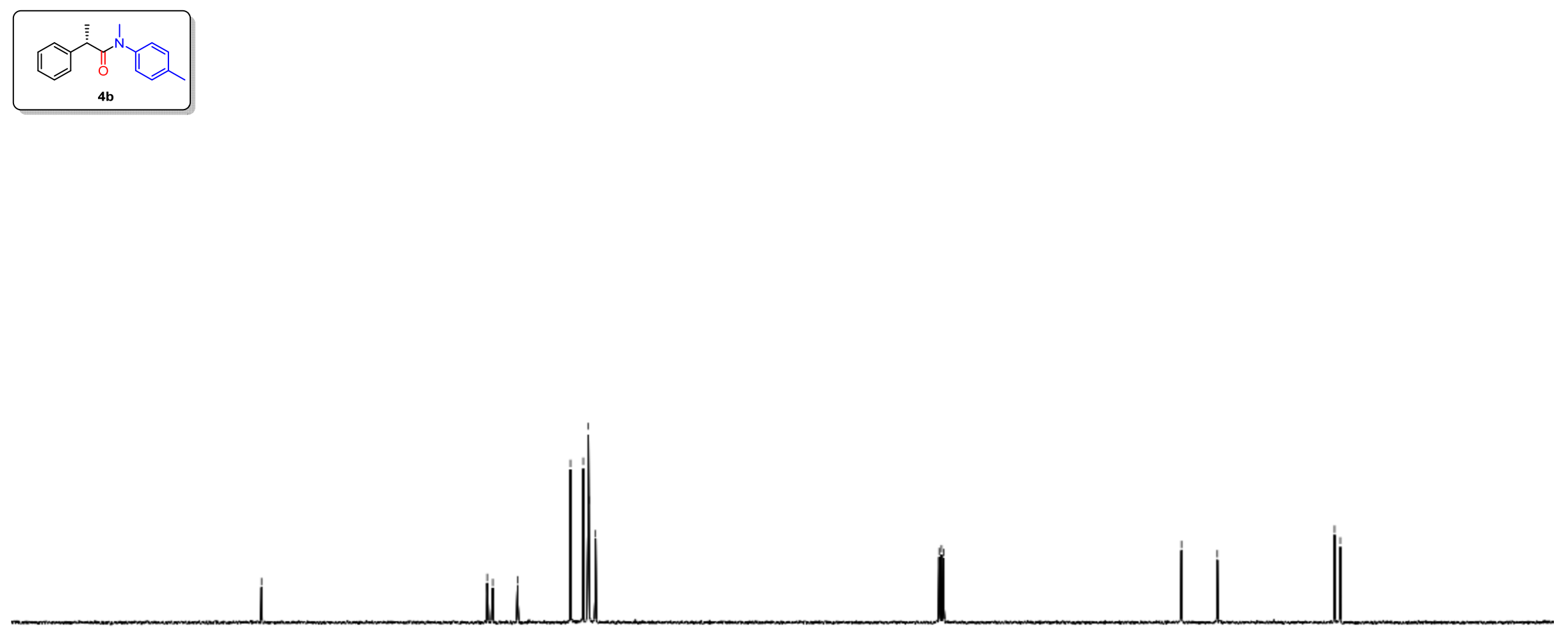

$110 \quad 100$ f1 $(\mathrm{ppm})$

80

$70 \quad 60$

50

$40 \quad 30$

20 $10 \stackrel{-1}{10}$ 
${ }^{1} \mathrm{H}$ NMR (400 MHz, $\mathrm{CDCl}_{3}$ ) of compound $\mathbf{4 c}$

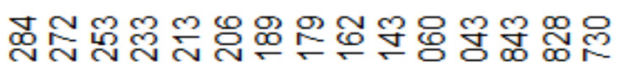

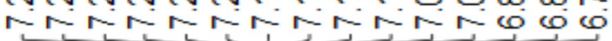

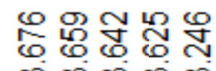

लंखुल

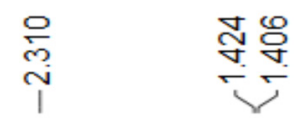
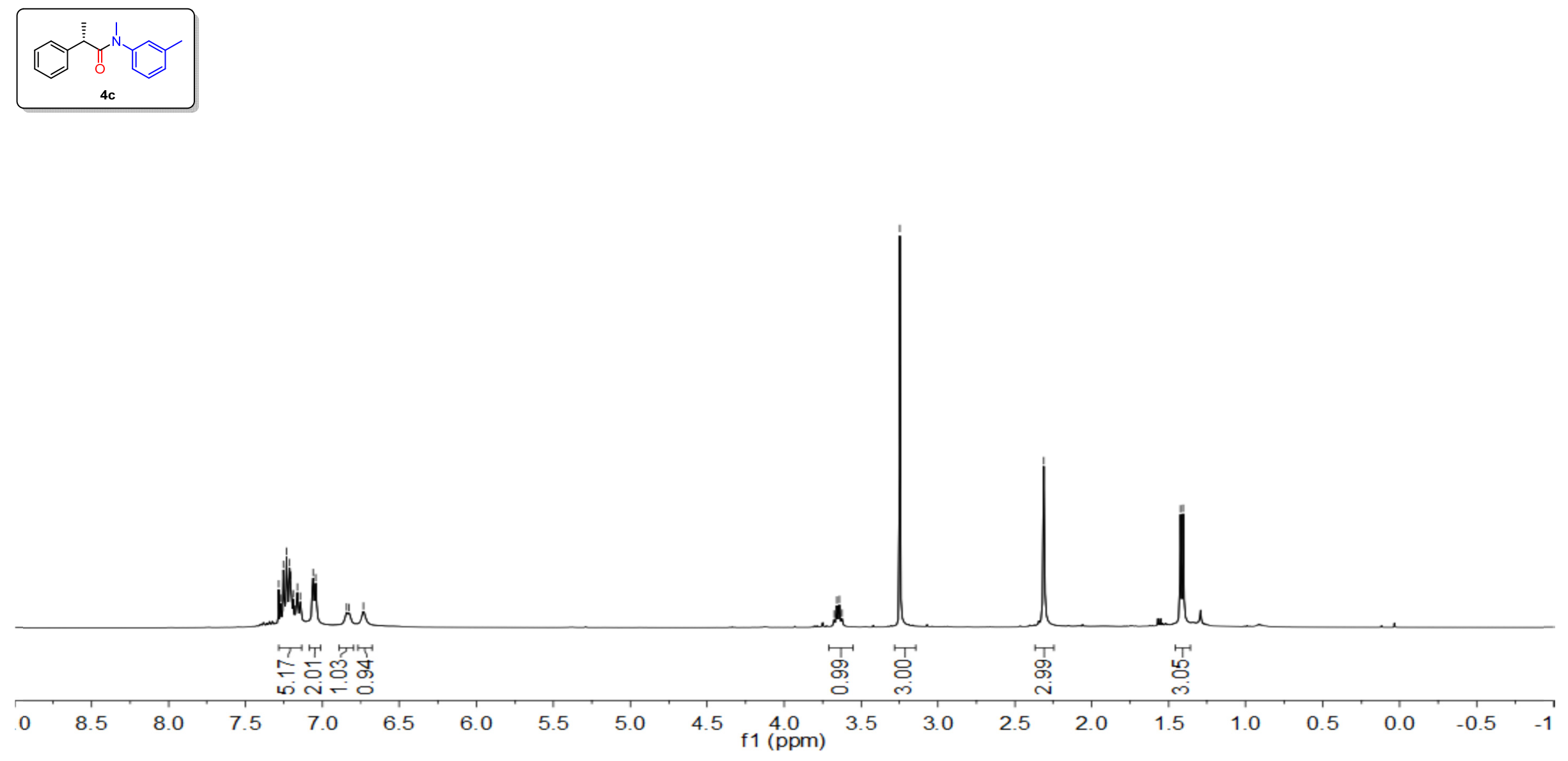
${ }^{13} \mathrm{C}$ NMR (101 MHz, $\left.\mathrm{CDCl}_{3}\right)$ of compound $4 \mathbf{c}$

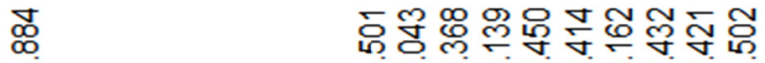

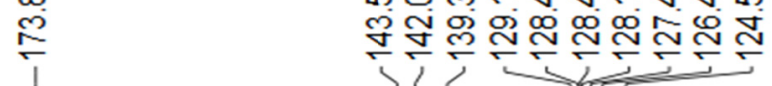

동 용

준

芒

辛

d: 莳

กับ
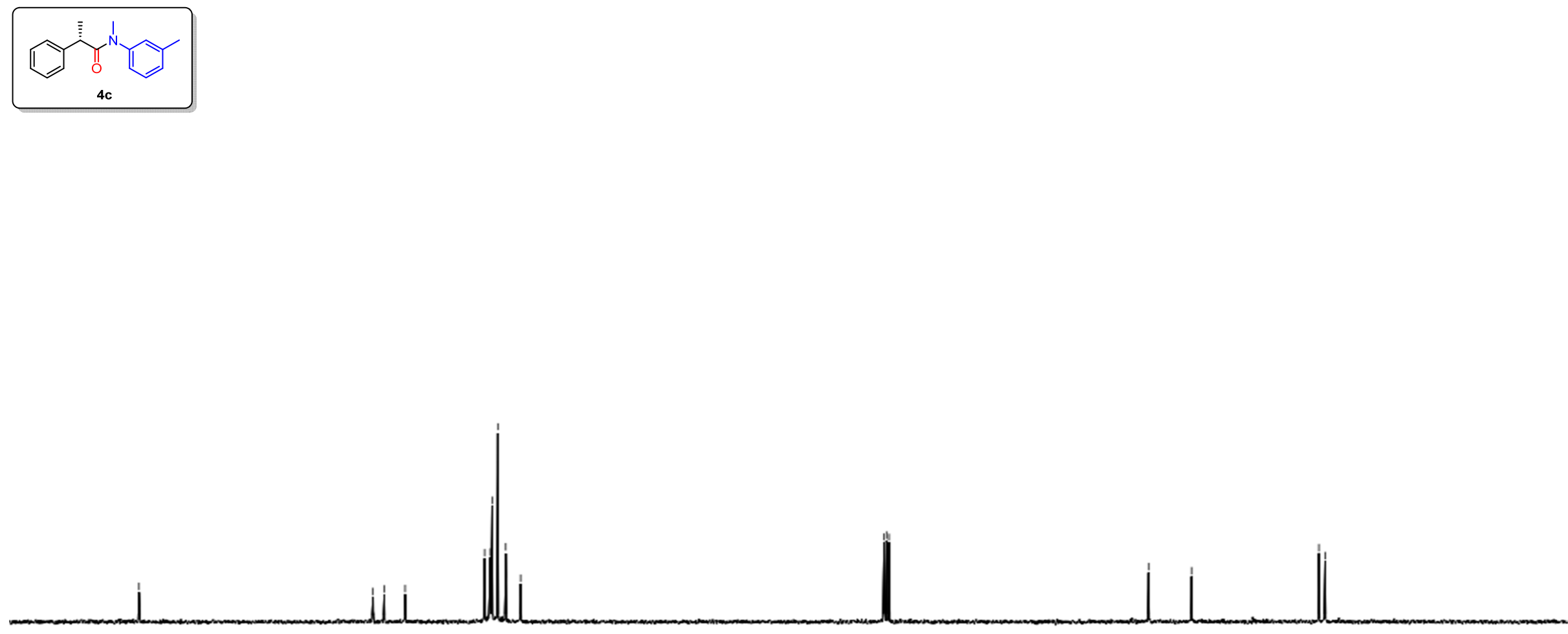

$90 \quad 180-170 \quad 160 \quad 150-140$

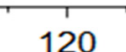

110

10090 90
$\mathrm{f} 1(\mathrm{ppm})$

$80 \quad 70 \quad 60$

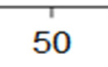

$40 \quad 30$ $20 \quad 10 \quad 0 \quad-11$ 
${ }^{1} \mathrm{H}$ NMR (400 MHz, $\mathrm{CDCl}_{3}$ ) of compound 4d

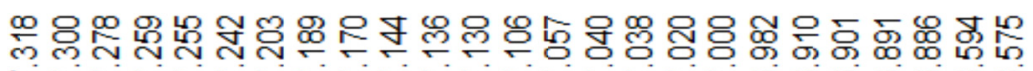

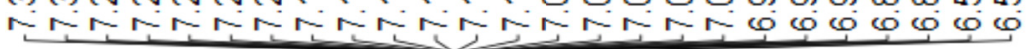

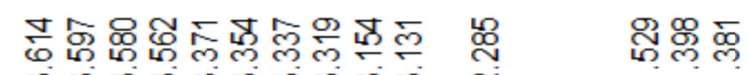

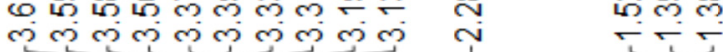

응
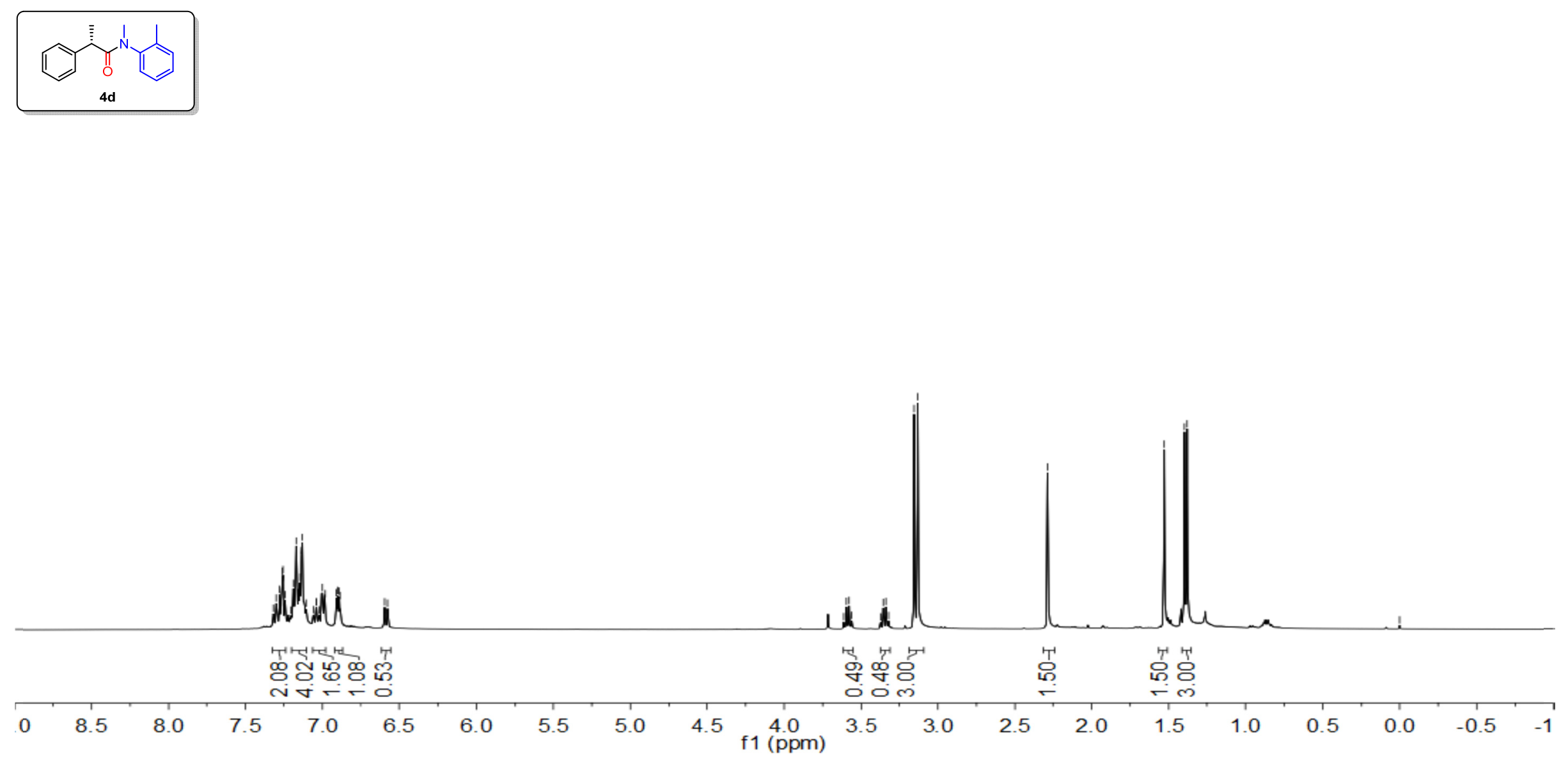
${ }^{13} \mathrm{C}$ NMR (101 MHz, $\mathrm{CDCl}_{3}$ ) of compound 4d

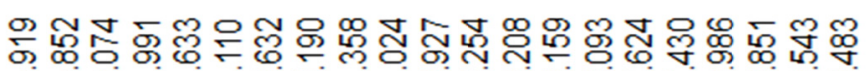

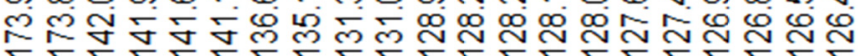
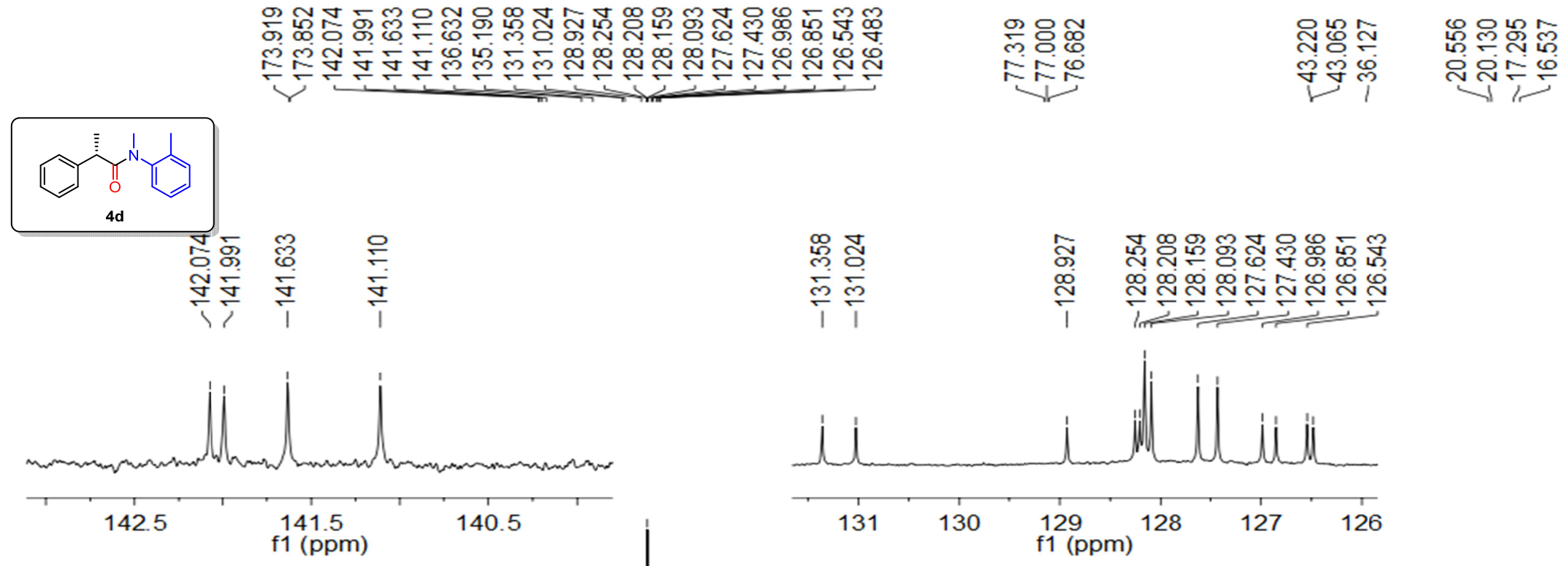

102

20019

180

170

160

$\begin{array}{lll}150 & 140 \quad 130\end{array}$

120

10100

100
$\mathrm{f} 1(\mathrm{ppm})$

80

$70 \quad 60$

$50 \quad 40$

30

$20 \quad 10$

0 -10 
${ }^{1} \mathrm{H}$ NMR (400 MHz, $\mathrm{CDCl}_{3}$ ) of compound $4 \mathbf{e}$

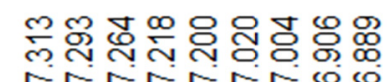

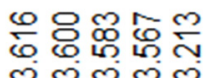
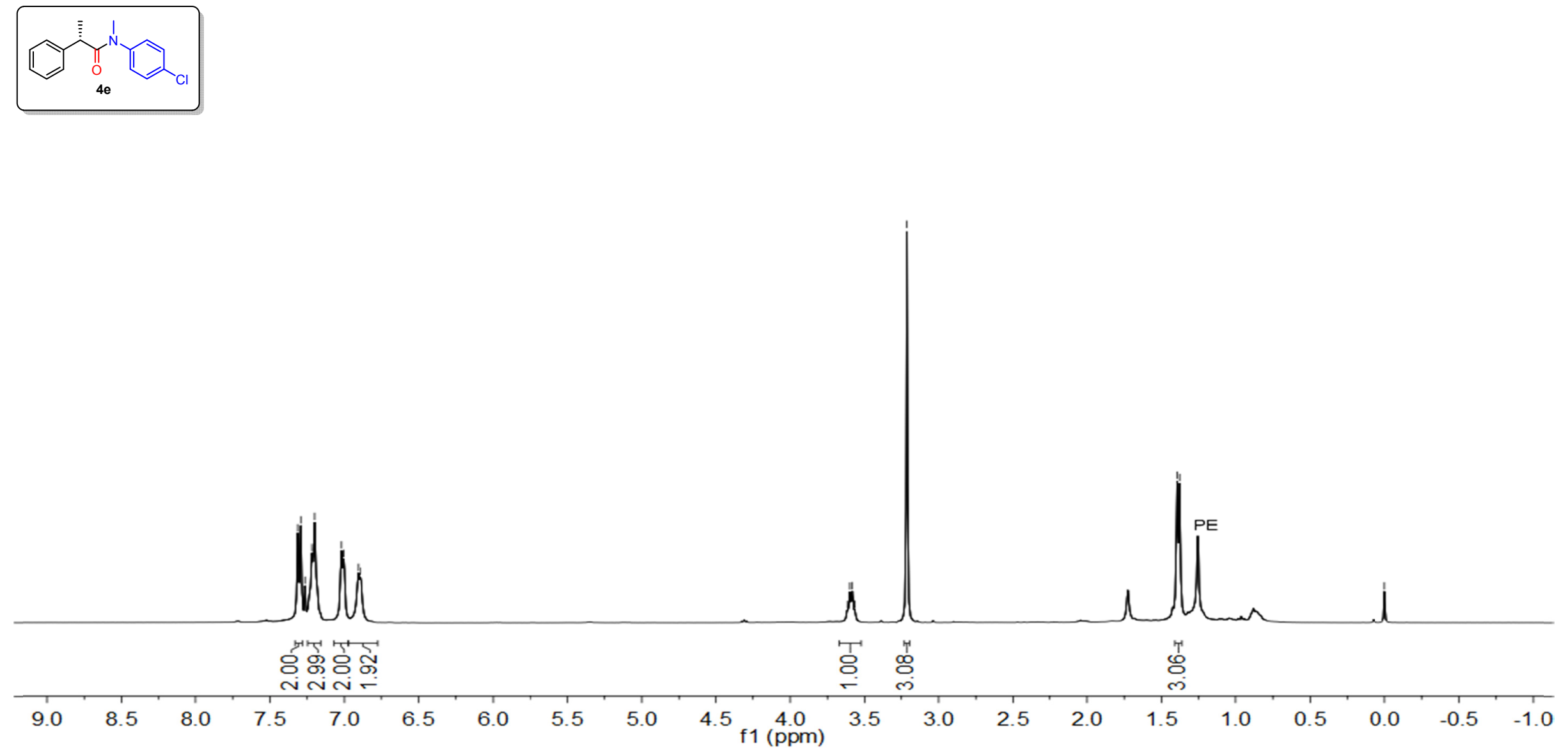
${ }^{13} \mathrm{C}$ NMR (101 MHz, $\mathrm{CDCl}_{3}$ ) of compound $4 \mathbf{e}$

\begin{tabular}{|c|c|c|c|}
\hline 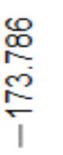 & 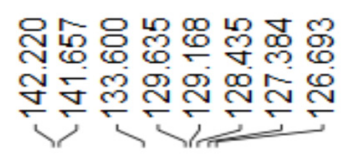 & $\begin{array}{l}\infty 8 \infty \\
m 80 \\
N=6\end{array}$ & 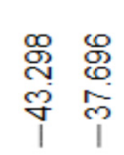 \\
\hline
\end{tabular}
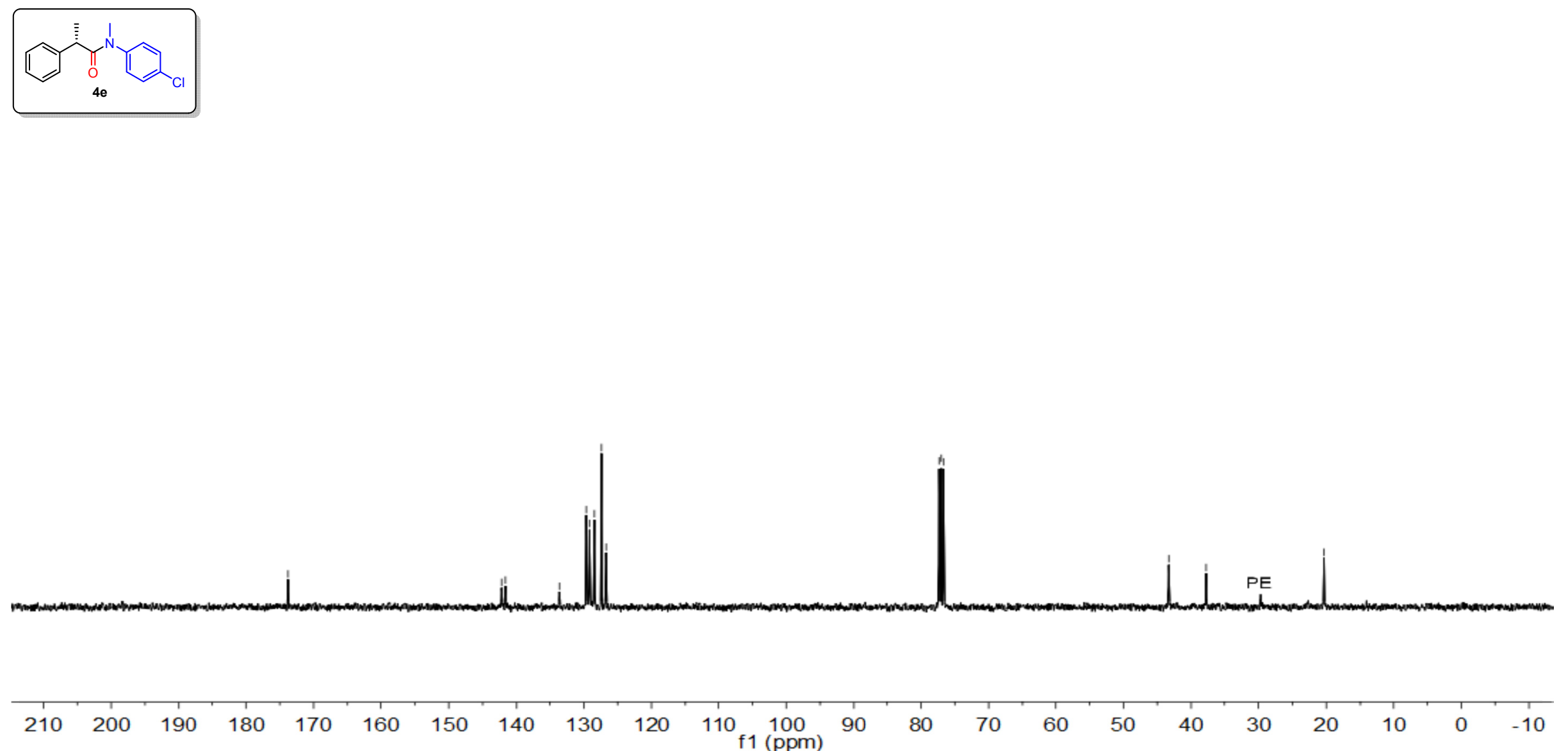
${ }^{1} \mathrm{H}$ NMR (400 MHz, $\mathrm{CDCl}_{3}$ ) of compound $\mathbf{4 f}$

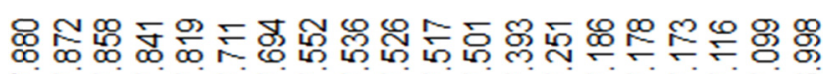

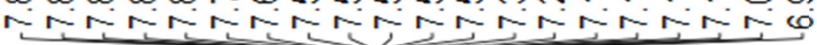

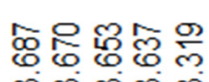

रंलुलं

꼴

○̊․․
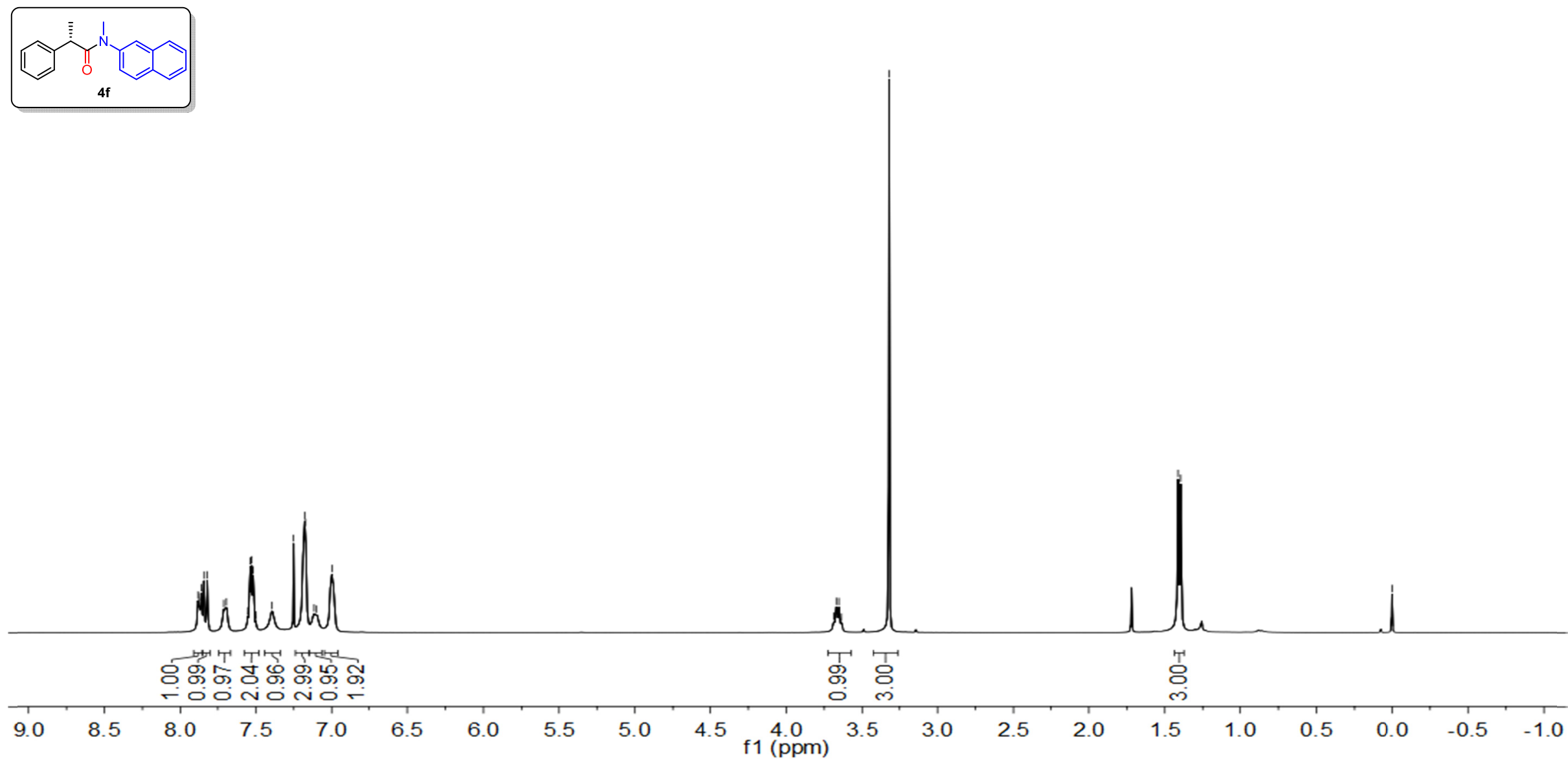
${ }^{13} \mathrm{C}$ NMR $\left(101 \mathrm{MHz}, \mathrm{CDCl}_{3}\right)$ of compound $\mathbf{4 f}$
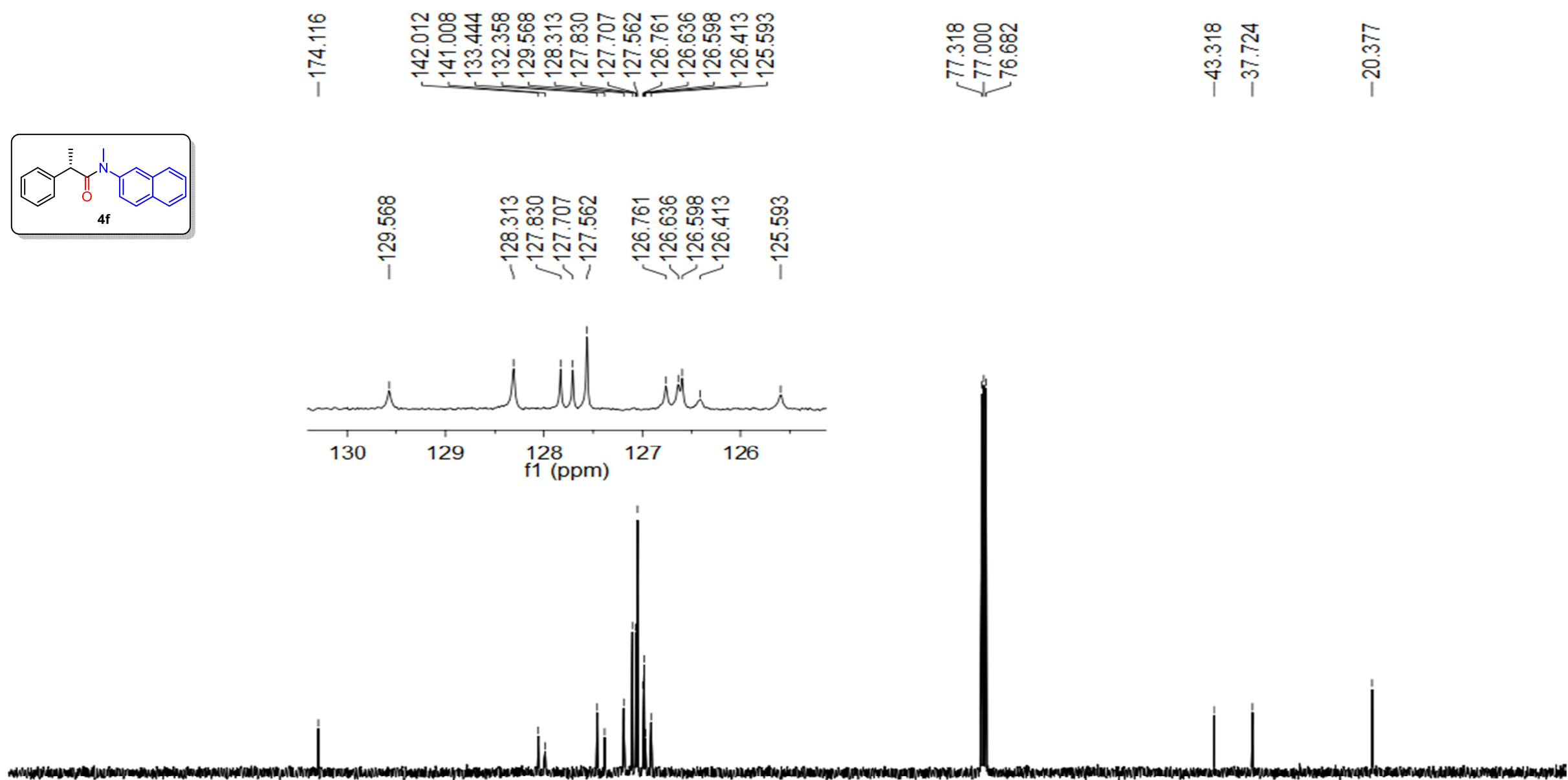

$\begin{array}{lllllllllll}210 & 200 & 190 & 180 & 170 & 160 & 150 & 140 & 130 & 120 & \begin{array}{l}110 \\ \mathrm{f} 1(\mathrm{ppm})\end{array}\end{array}$ 
${ }^{1} \mathrm{H}$ NMR (600 MHz, $\mathrm{CDCl}_{3}$ ) of compound $\mathbf{4 g}$

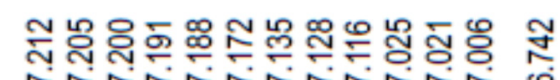

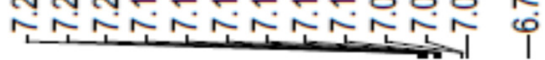

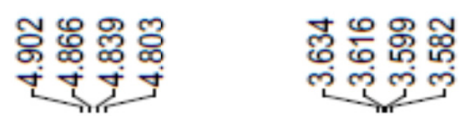

守莳
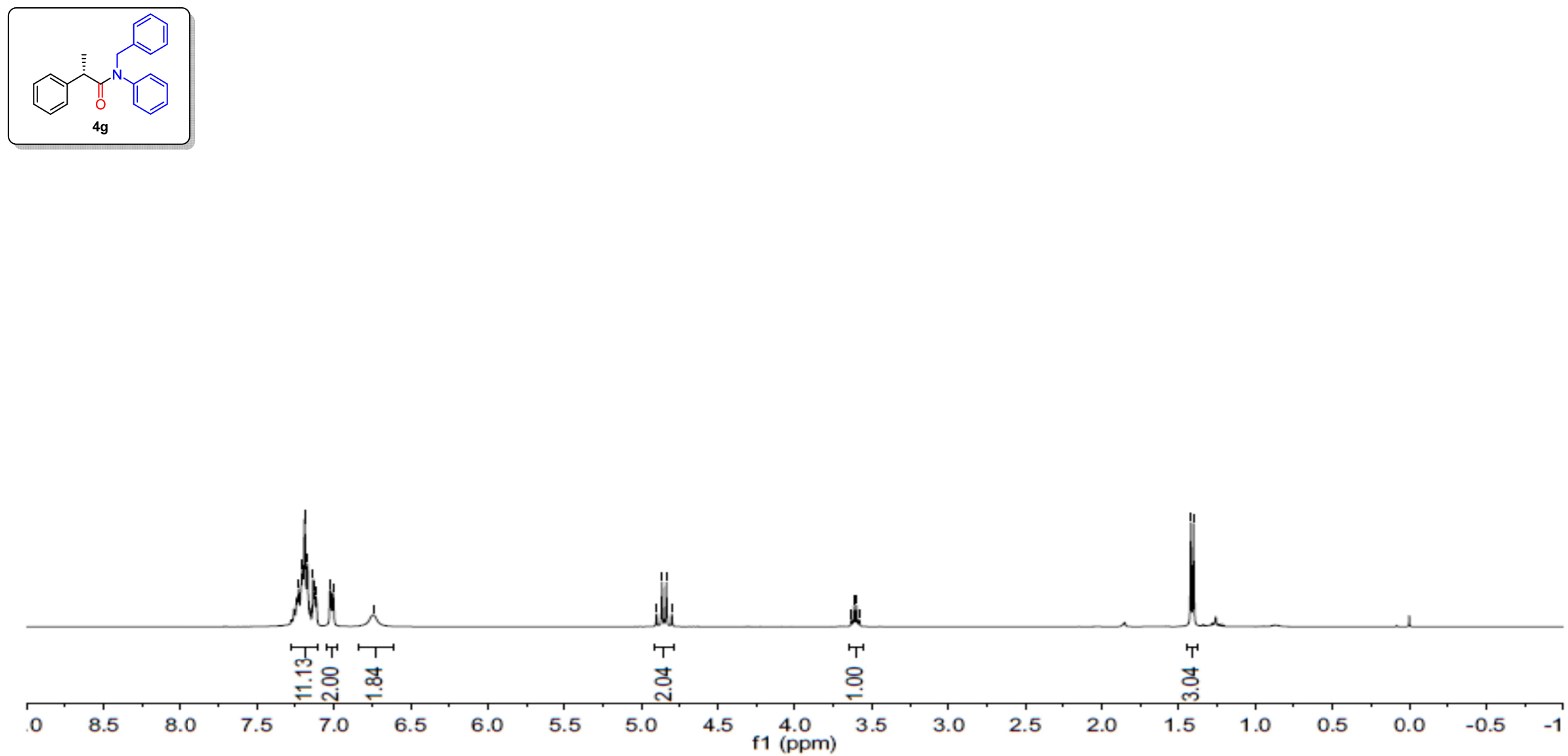

S126 
${ }^{13} \mathrm{C}$ NMR (151 MHz, $\mathrm{CDCl}_{3}$ ) of compound $\mathbf{4 g}$
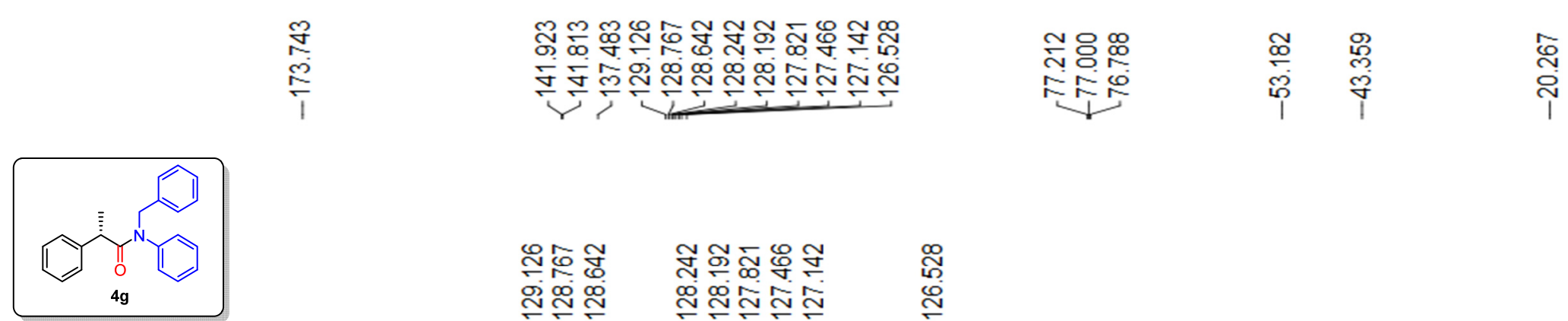

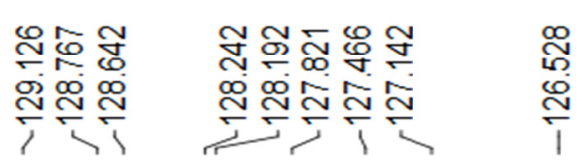
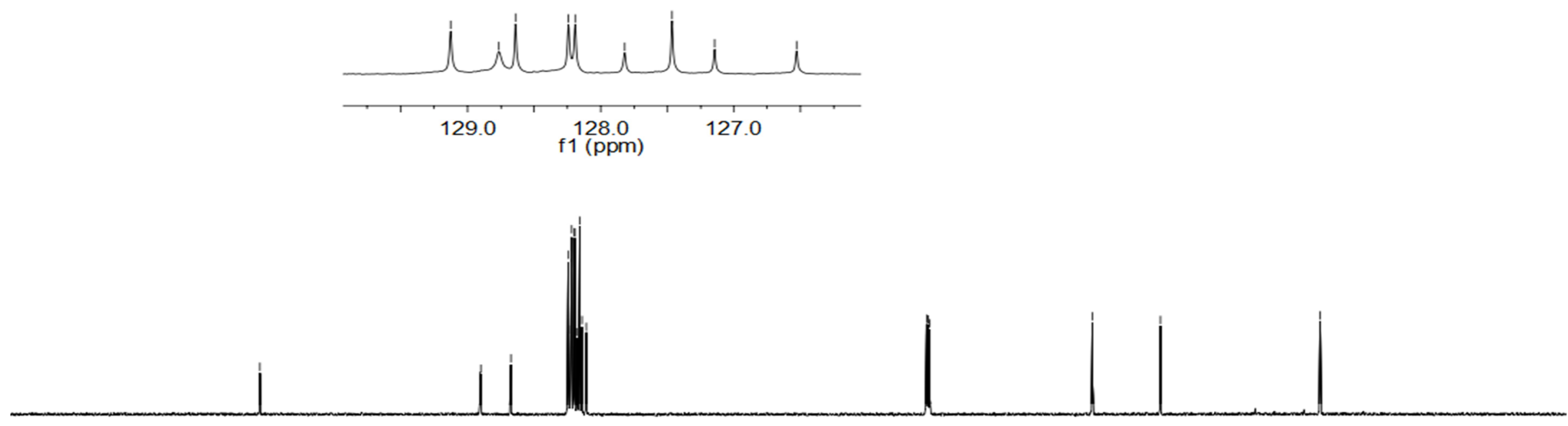

200190

$180 \quad 170$

160

150

$140 \quad 130$

$120 \quad 110$

10090

80

$70 \quad 60$

$50 \quad 40$

$30 \quad 20$

$10 \quad 0 \quad-10$ 
${ }^{1} \mathrm{H}$ NMR (600 MHz, $\mathrm{CDCl}_{3}$ ) of compound $\mathbf{4 h}$

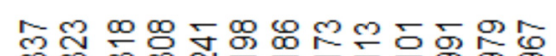

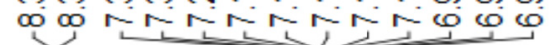

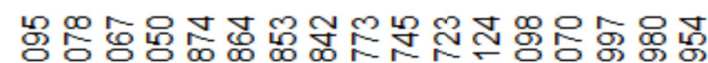

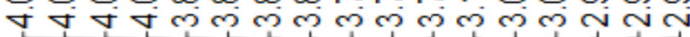

ํㅠำ

兽
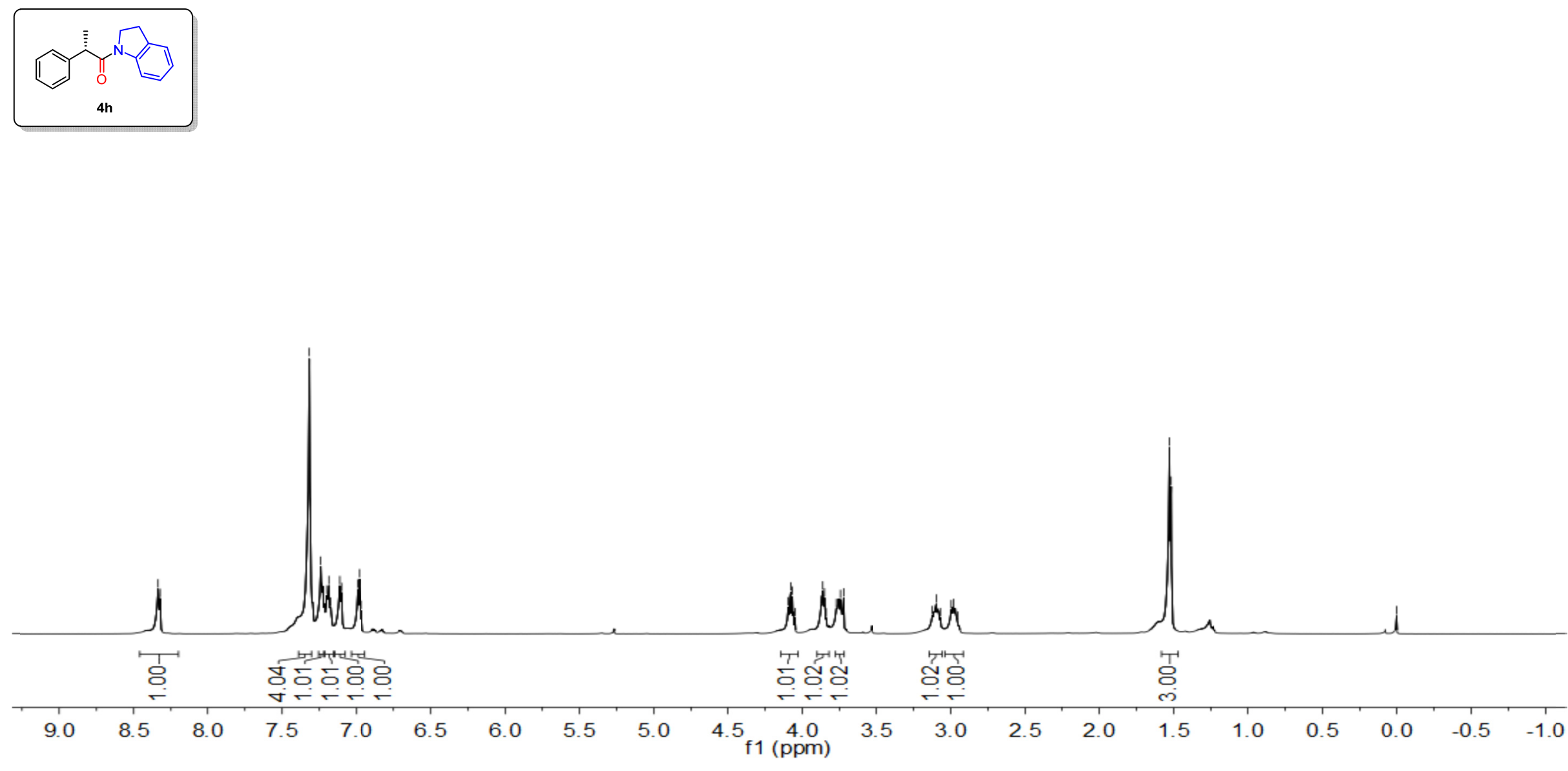

S128 
${ }^{13} \mathrm{C}$ NMR (151 MHz, $\mathrm{CDCl}_{3}$ ) of compound $\mathbf{4 h}$
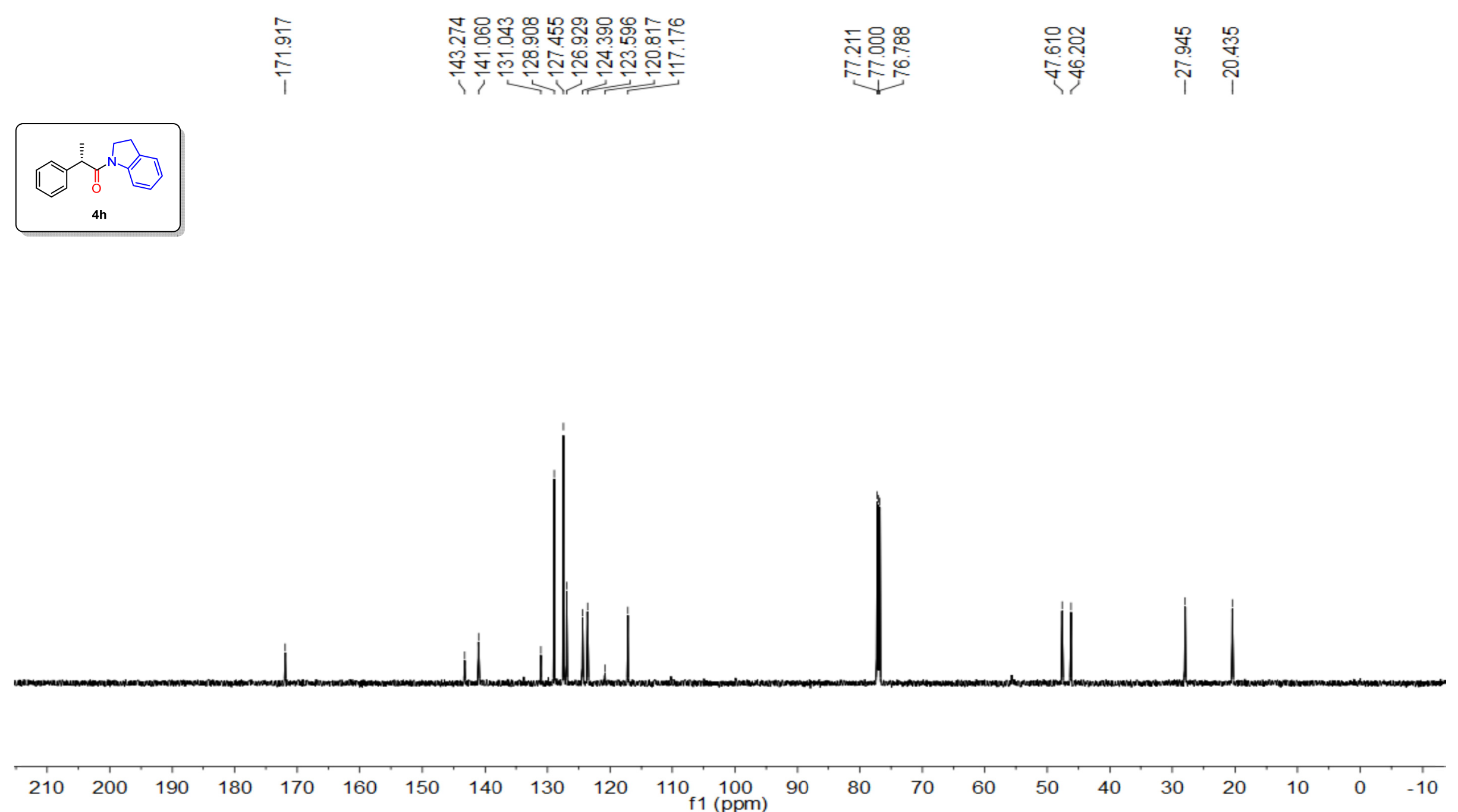
${ }^{1} \mathrm{H}$ NMR (600 MHz, $\mathrm{CDCl}_{3}$ ) of compound $\mathbf{4 i}$

$$
\text { 운은 }
$$

సี่

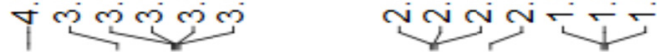
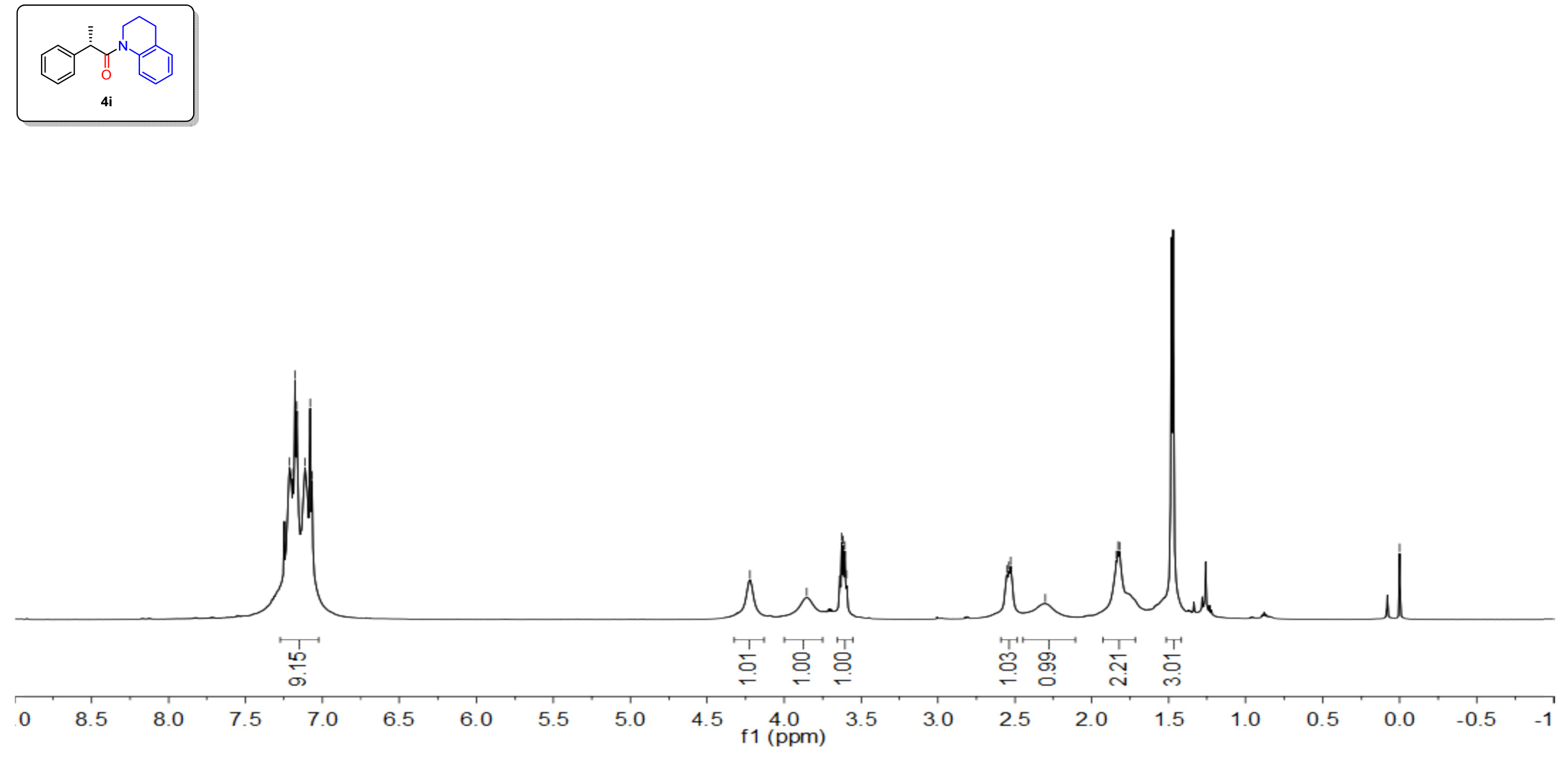
${ }^{13} \mathrm{C}$ NMR $\left(151 \mathrm{MHz}, \mathrm{CDCl}_{3}\right)$ of compound $\mathbf{4 i}$

\begin{tabular}{|c|c|c|c|}
\hline 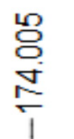 & 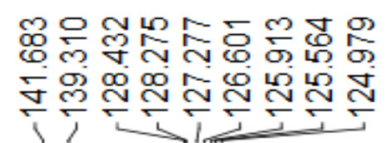 & 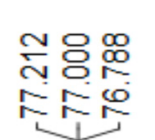 & 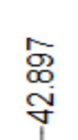 \\
\hline
\end{tabular}

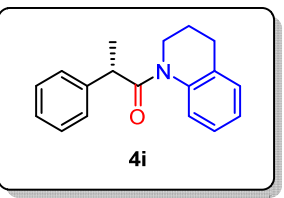

寸

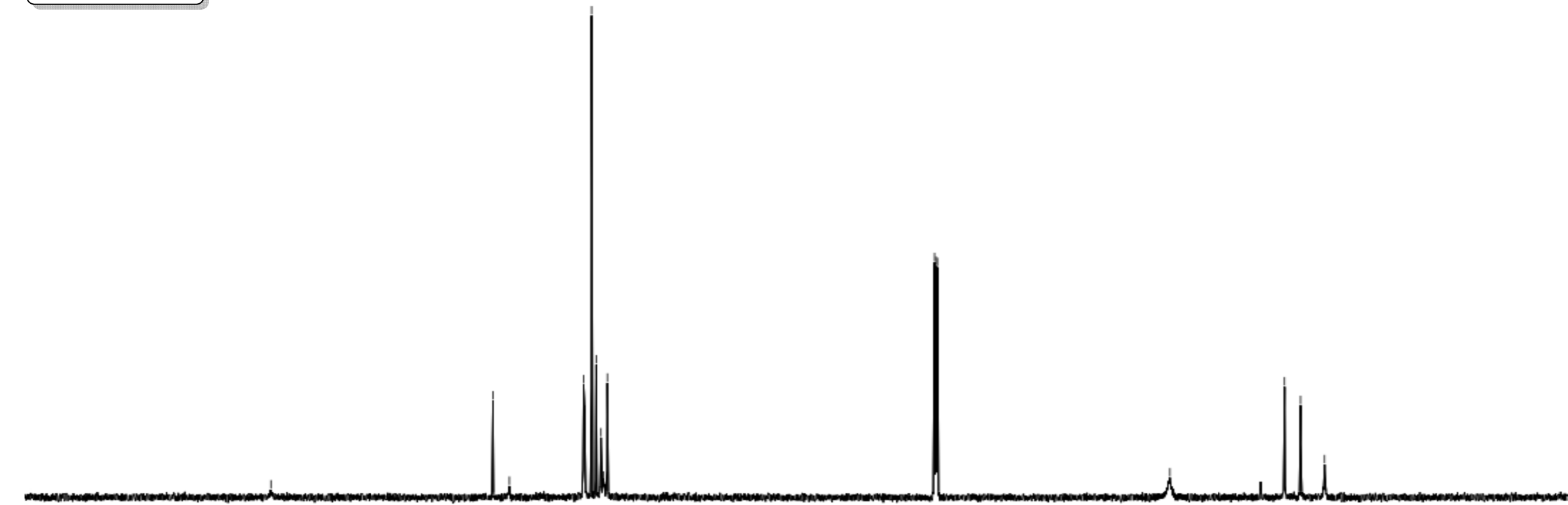

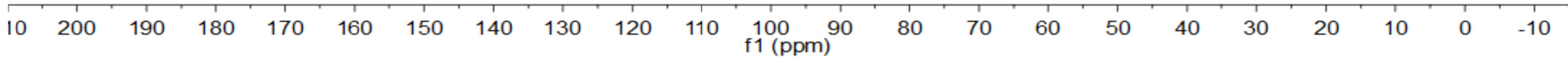


${ }^{1} \mathrm{H}$ NMR (400 MHz, $\mathrm{CDCl}_{3}$ ) of compound 5a

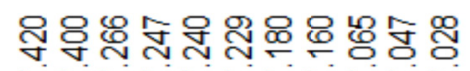

vinñNaris

$8 \stackrel{809}{6}$

लंखु

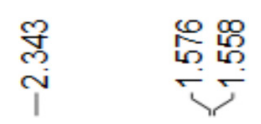

兽
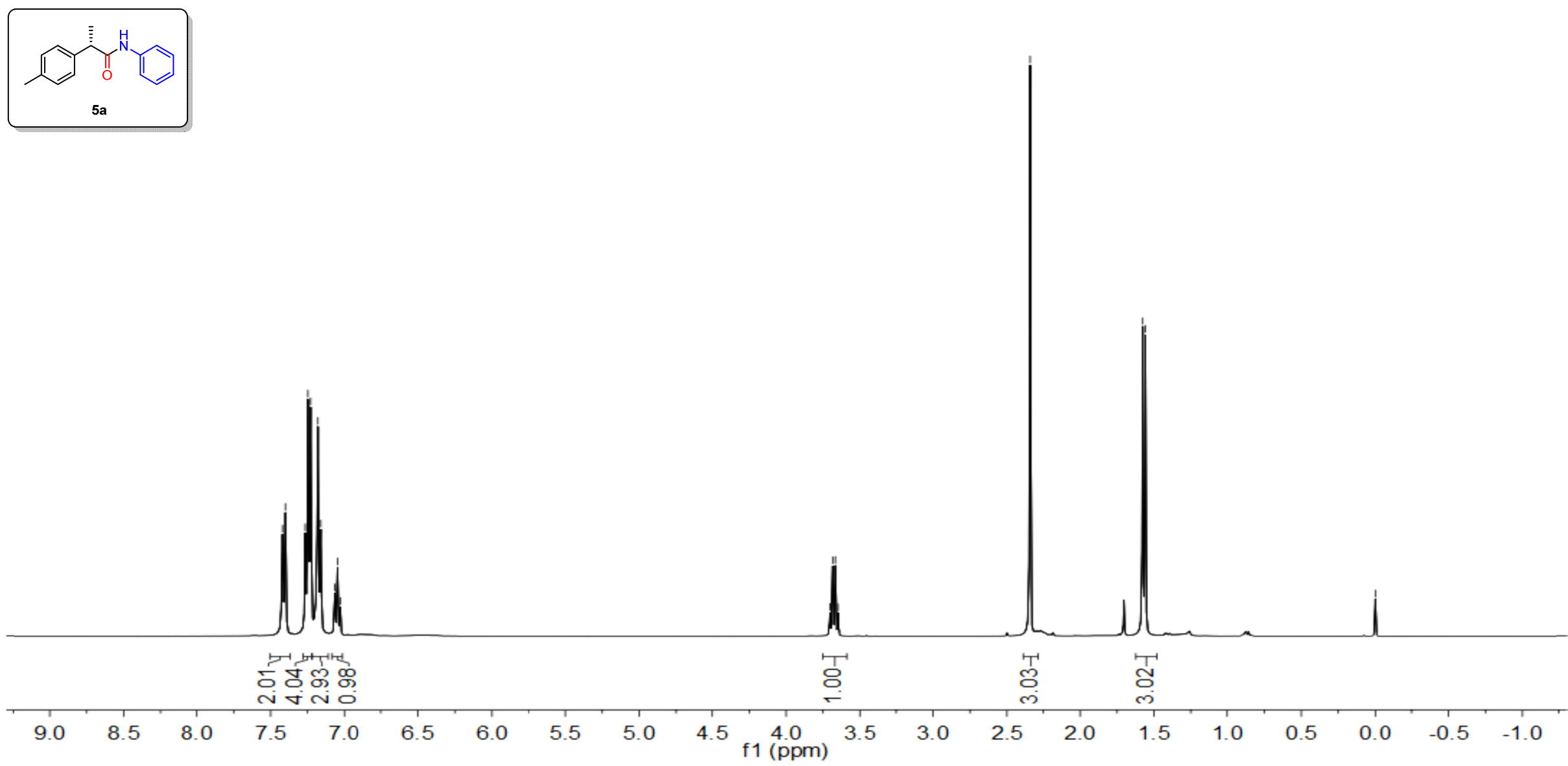
${ }^{13} \mathrm{C}$ NMR (151 MHz, $\mathrm{CDCl}_{3}$ ) of compound 5a
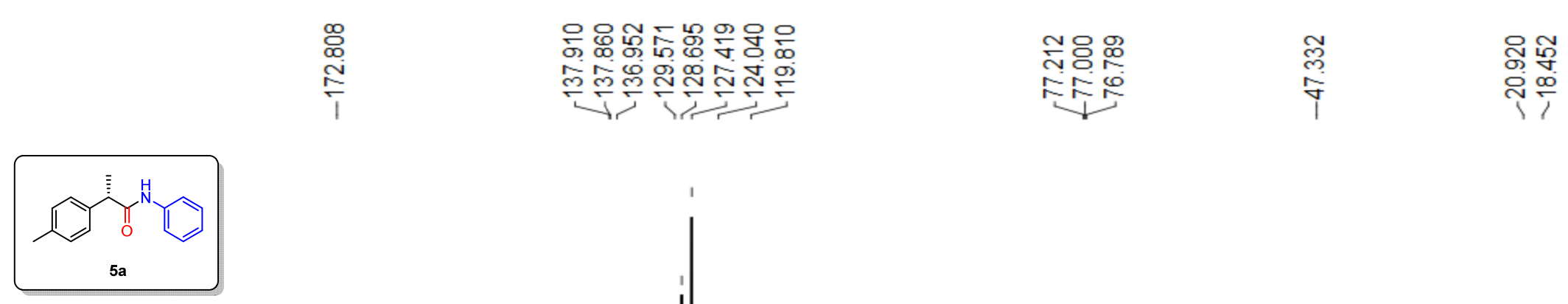

సิㅇㅇㅇ

No

유ำ

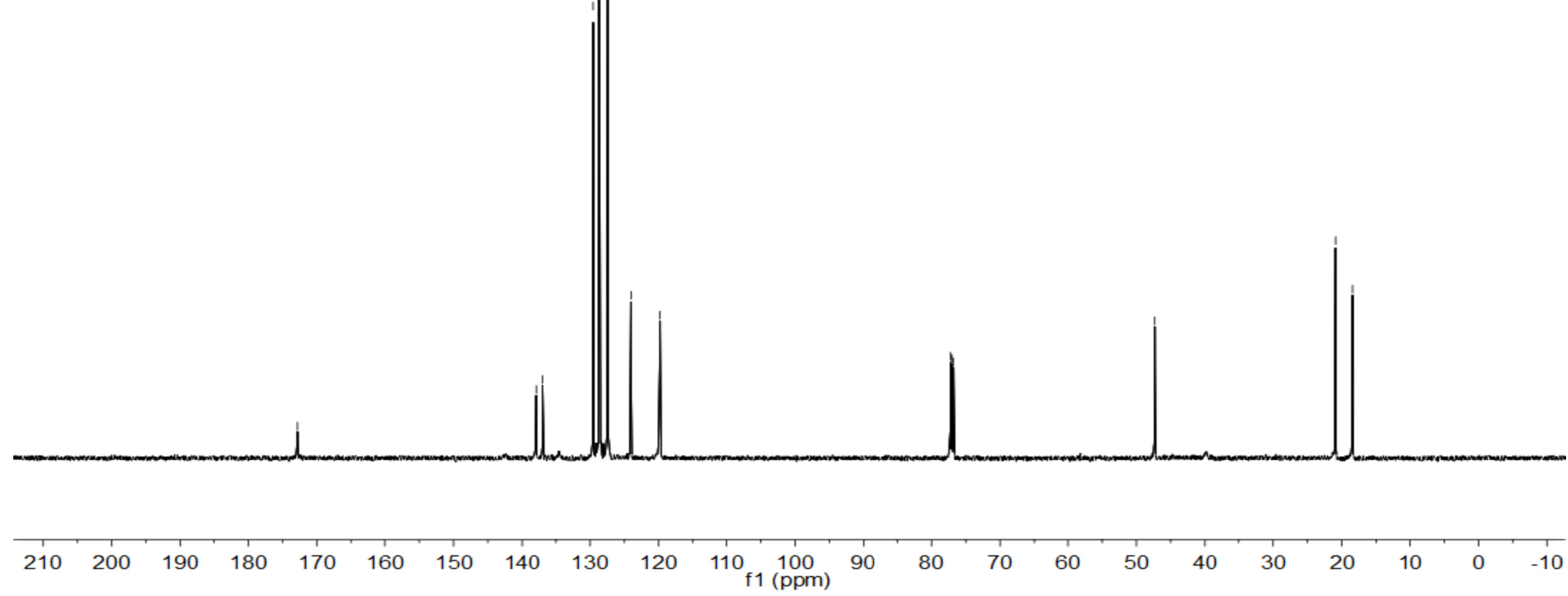


${ }^{1} \mathrm{H}$ NMR (400 MHz, $\mathrm{CDCl}_{3}$ ) of compound $\mathbf{5 b}$

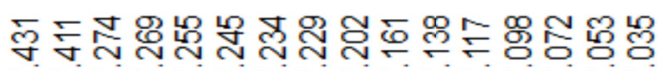

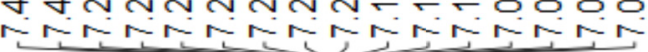

용요
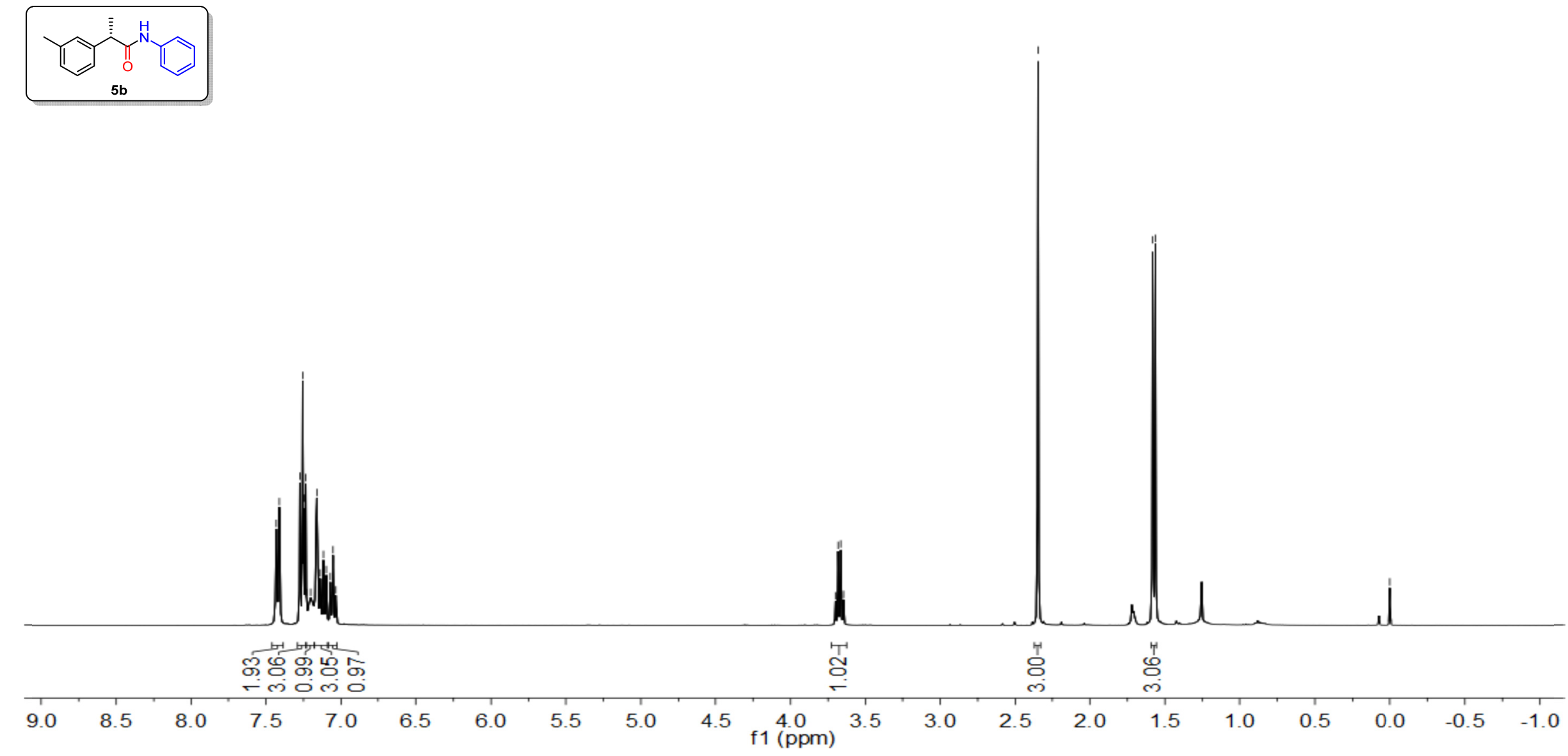
${ }^{13} \mathrm{C}$ NMR $\left(101 \mathrm{MHz}, \mathrm{CDCl}_{3}\right)$ of compound $\mathbf{5 b}$
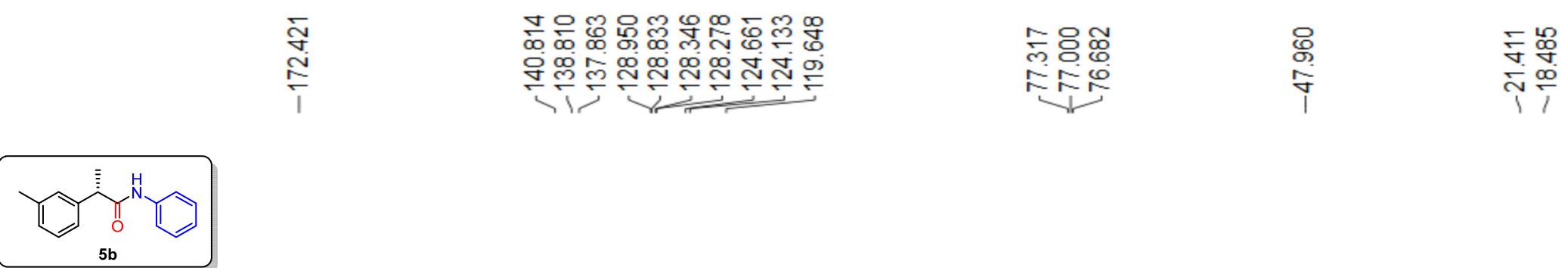

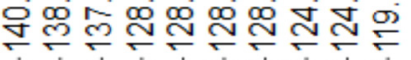

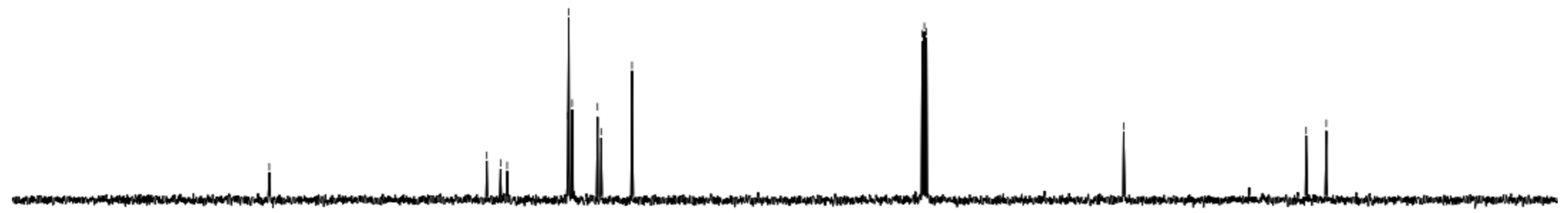

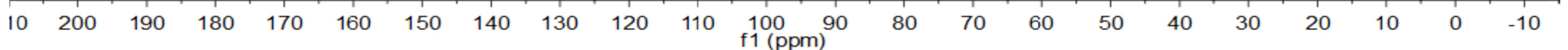


${ }^{1} \mathrm{H}$ NMR (400 MHz, $\mathrm{CDCl}_{3}$ ) of compound $\mathbf{5 c}$

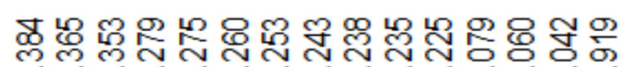
NMNNN
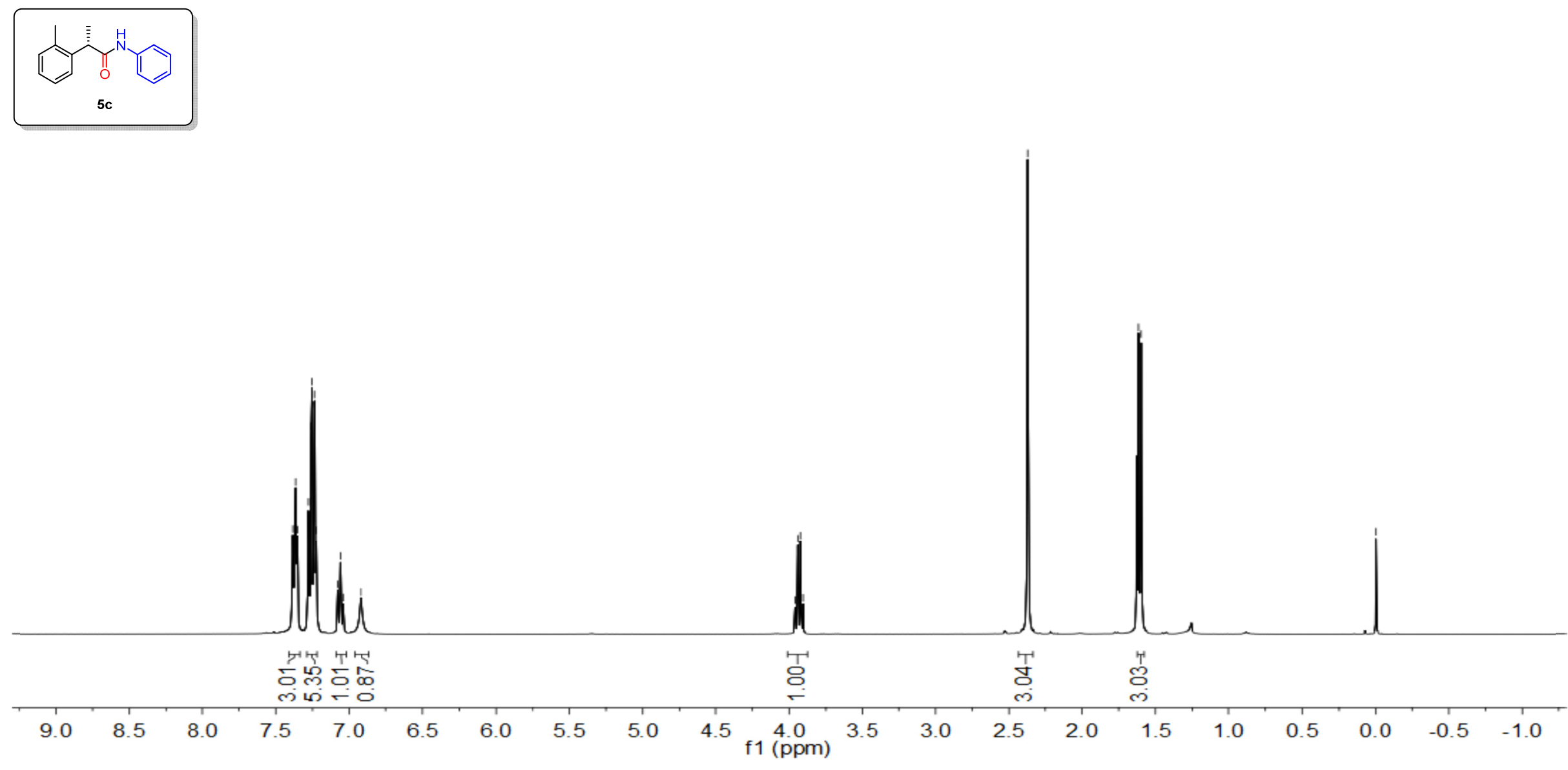
${ }^{13} \mathrm{C}$ NMR $\left(101 \mathrm{MHz}, \mathrm{CDCl}_{3}\right)$ of compound $\mathbf{5 c}$
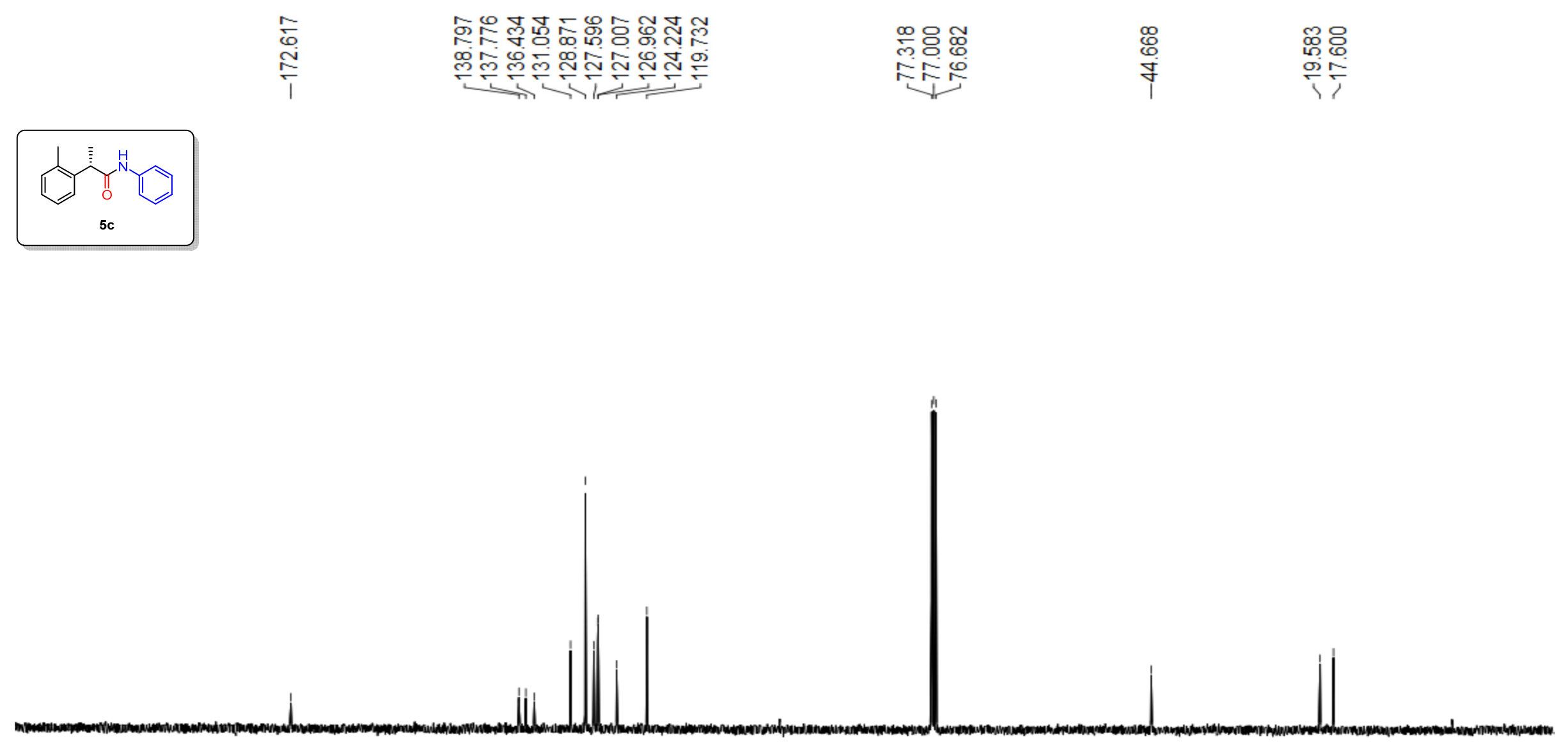

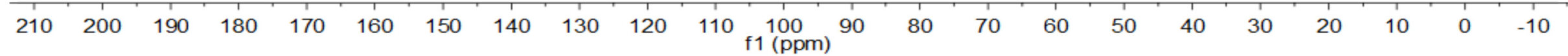


${ }^{1} \mathrm{H}$ NMR (400 MHz, $\mathrm{CDCl}_{3}$ ) of compound 5d

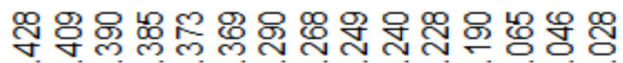

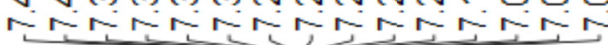

도용 लिखल
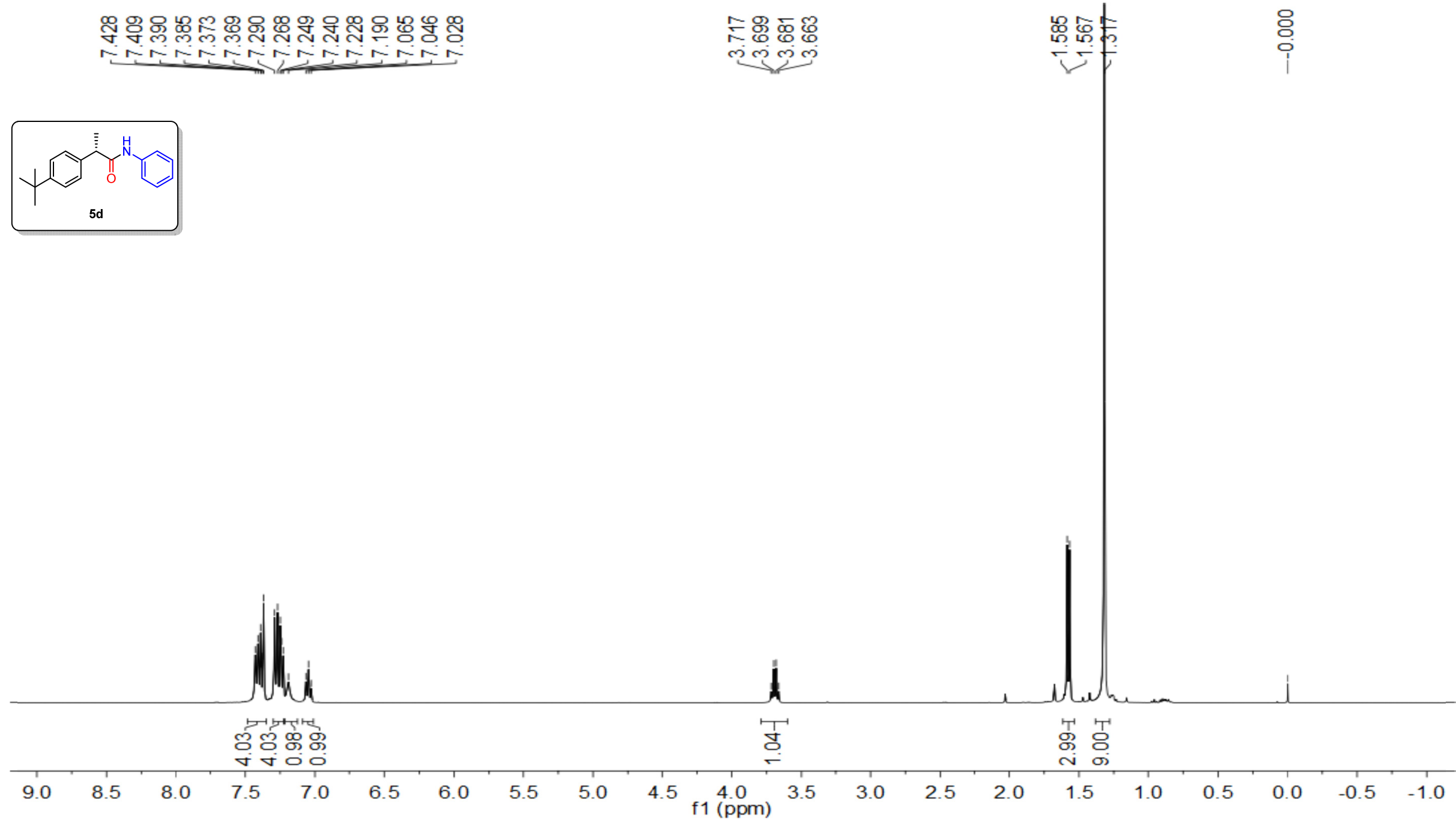
${ }^{13} \mathrm{C}$ NMR $\left(151 \mathrm{MHz}, \mathrm{CDCl}_{3}\right)$ of compound 5d

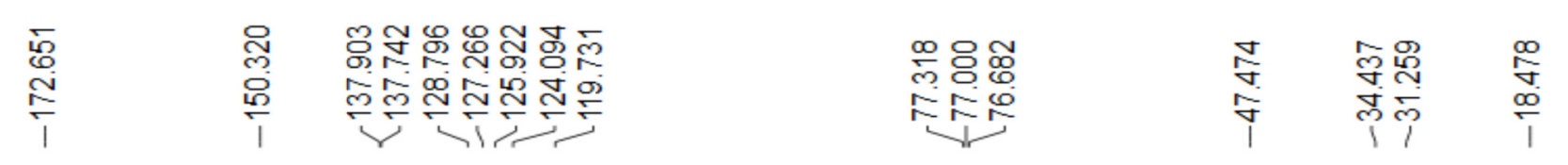
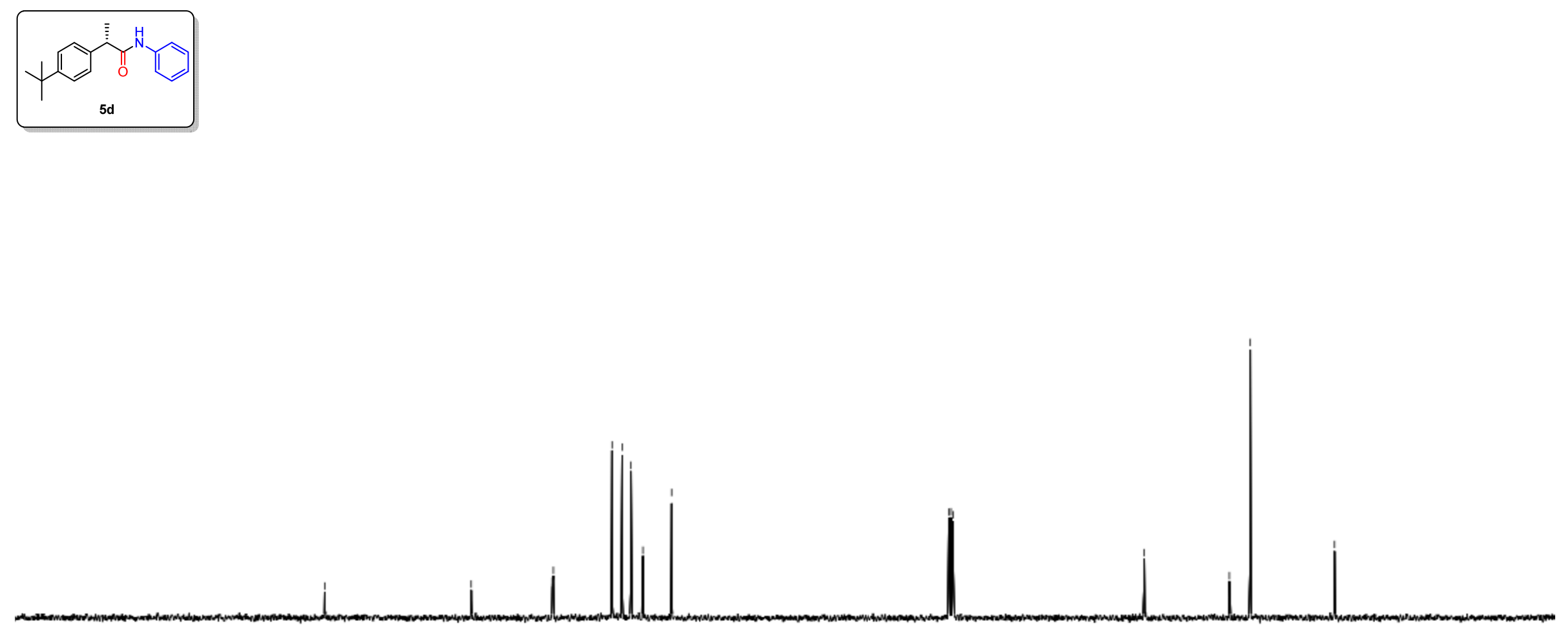

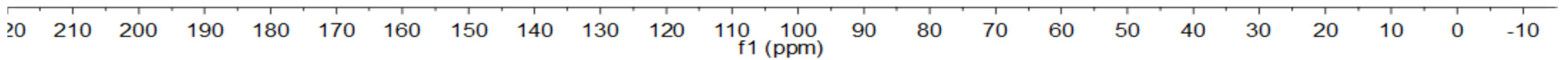


${ }^{1} \mathrm{H}$ NMR (600 MHz, $\mathrm{CDCl}_{3}$ ) of compound $\mathbf{5 e}$

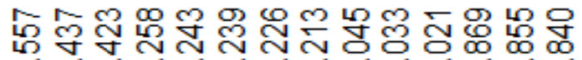
年0

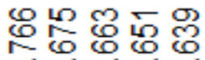

लंखंल

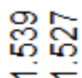

응
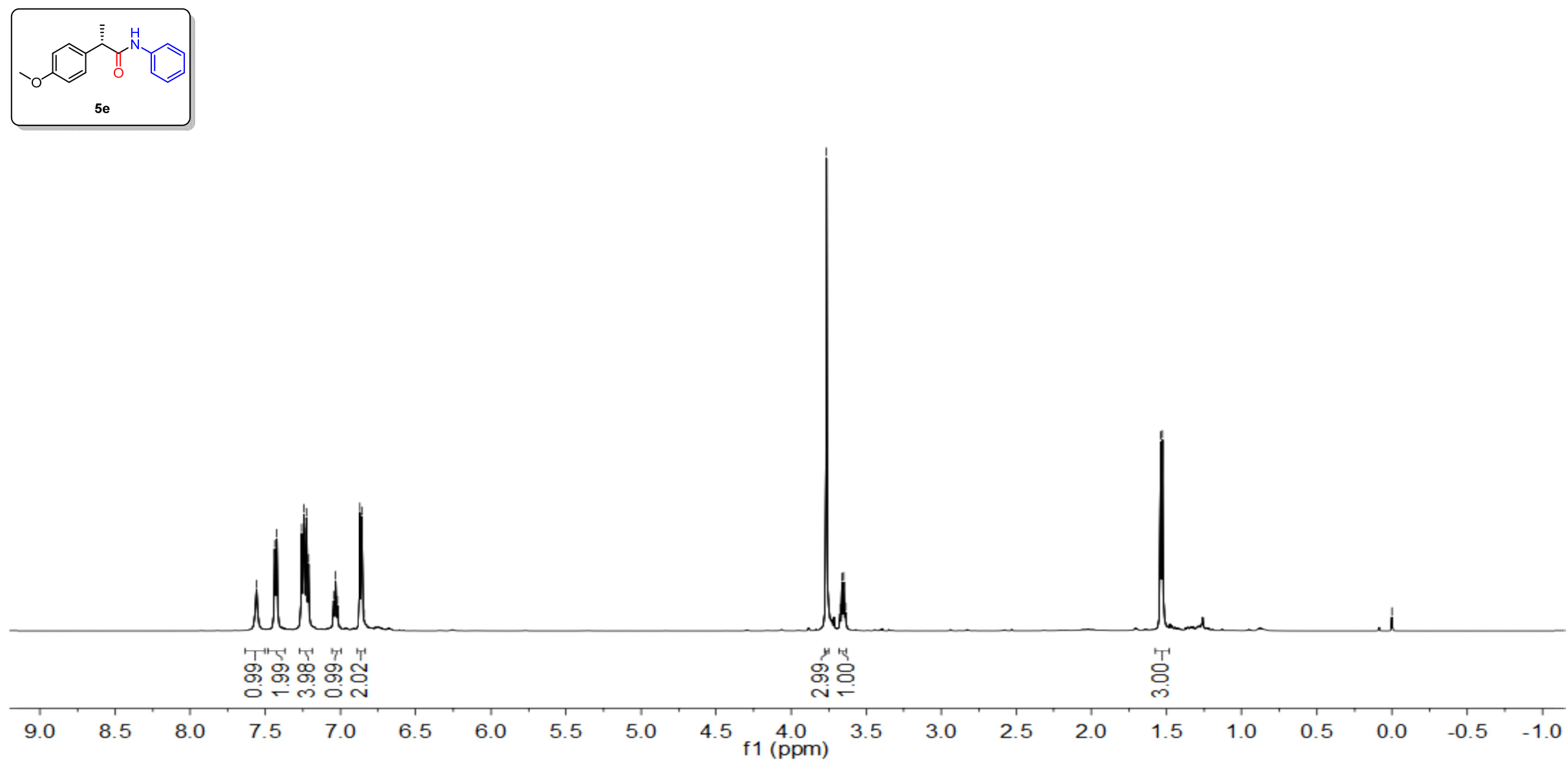
${ }^{13} \mathrm{C}$ NMR (151 MHz, $\mathrm{CDCl}_{3}$ ) of compound $\mathbf{5 e}$

\begin{tabular}{|c|c|c|}
\hline 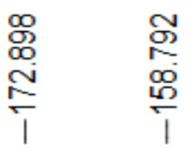 & 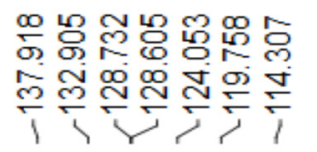 & 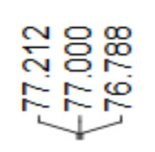 \\
\hline
\end{tabular}
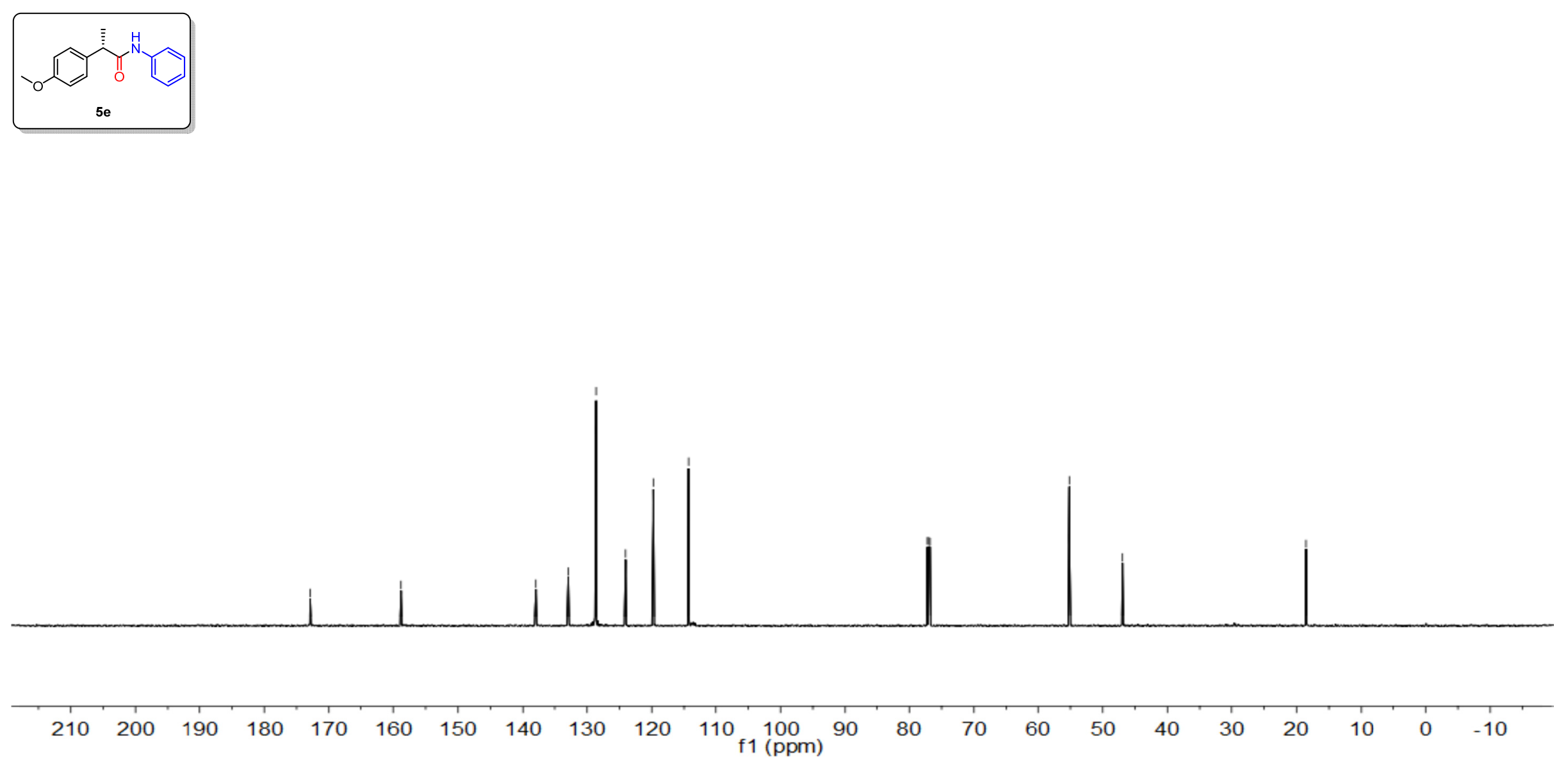
${ }^{1} \mathrm{H}$ NMR $\left(400 \mathrm{MHz}, \mathrm{CDCl}_{3}\right)$ of compound $\mathbf{5 f}$

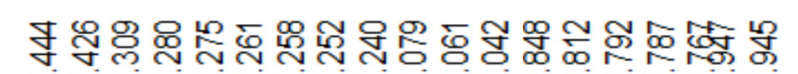

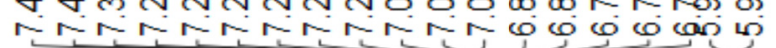

蛋

रांग

电虾

Wִ

응
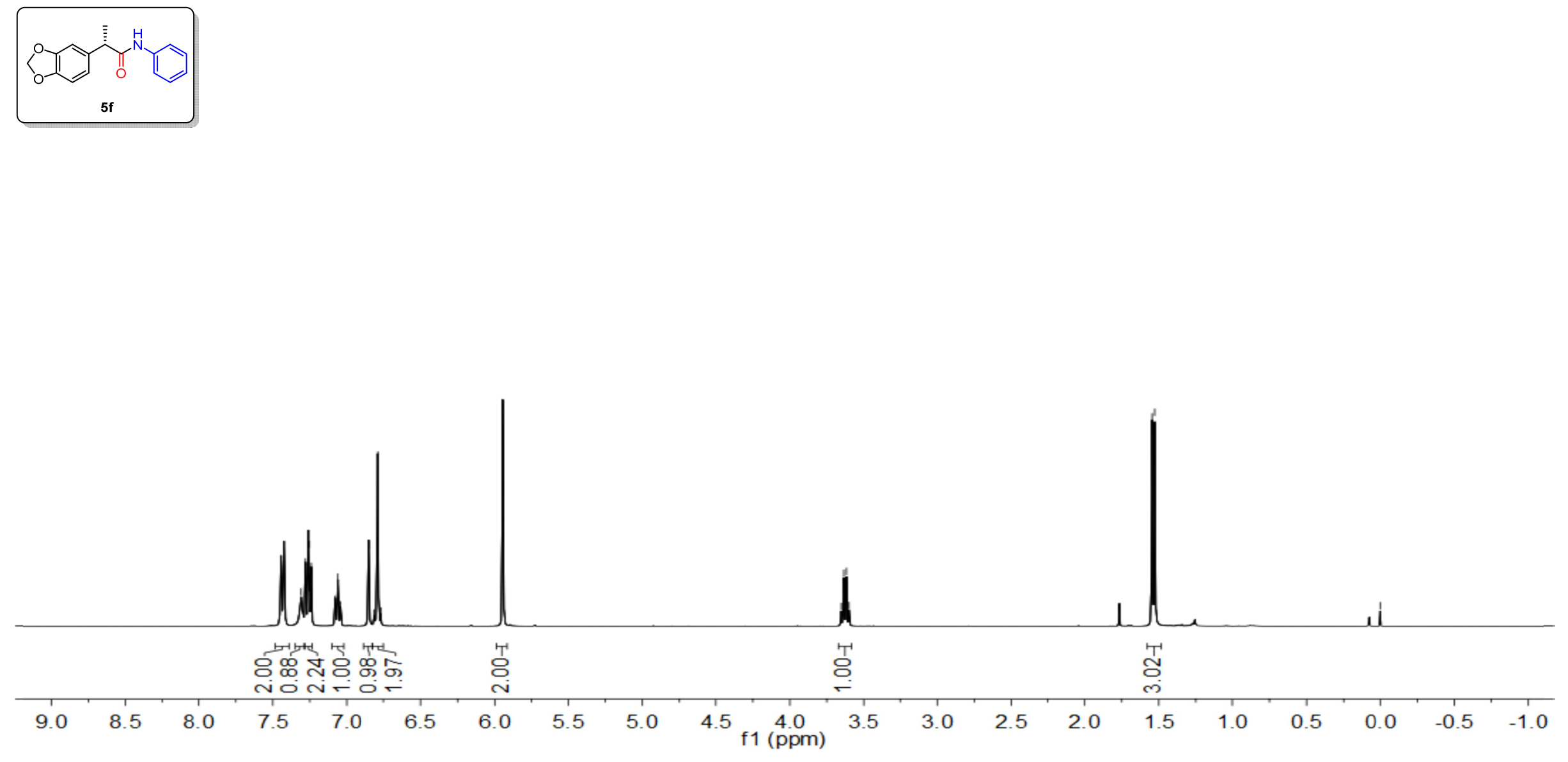
${ }^{13} \mathrm{C}$ NMR (101 MHz, $\left.\mathrm{CDCl}_{3}\right)$ of compound $\mathbf{5 f}$

\begin{tabular}{|c|c|c|c|c|}
\hline 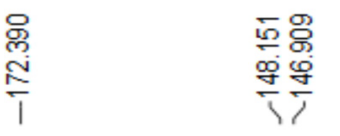 & 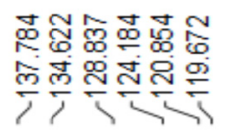 & 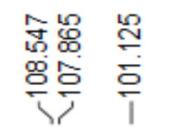 & 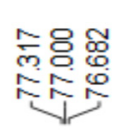 & $\begin{array}{l}\text { 只 } \\
\text { f }\end{array}$ \\
\hline
\end{tabular}
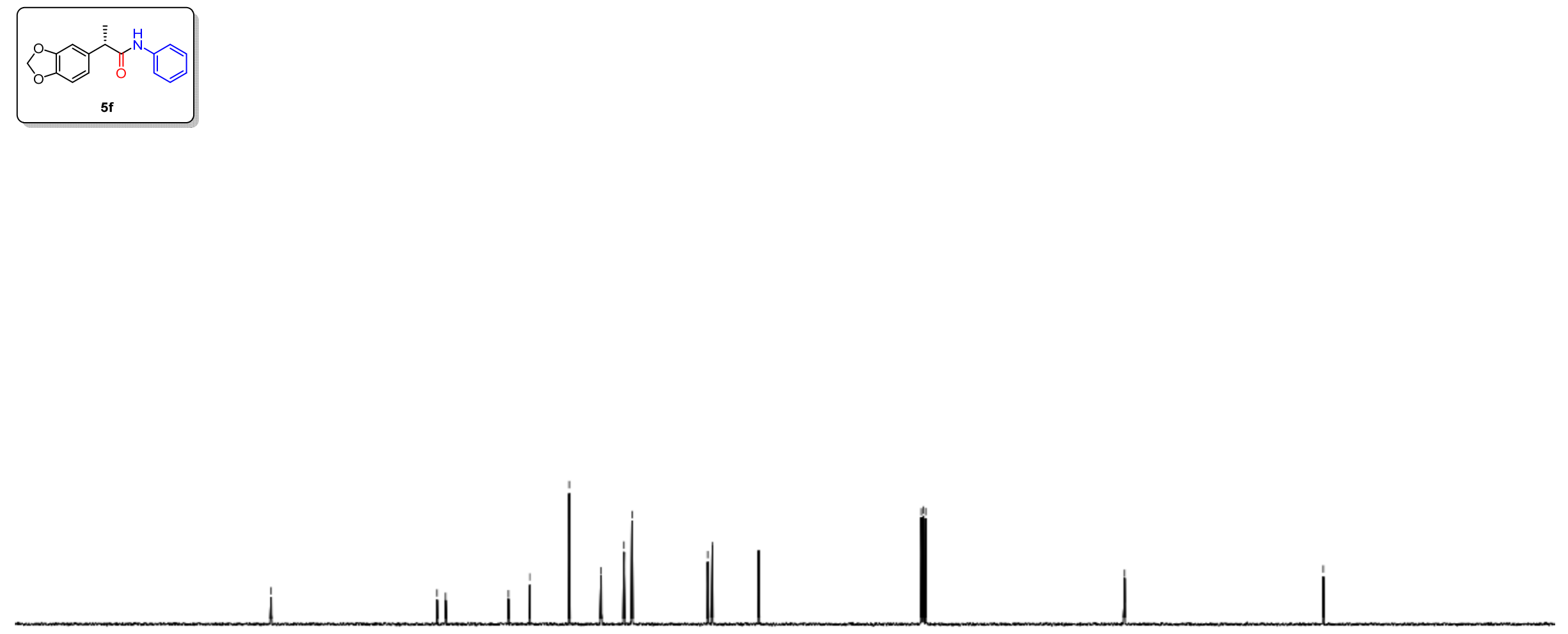

$200 \quad 190 \quad 180 \quad 170$ 
${ }^{1} \mathrm{H}$ NMR (600 MHz, $\mathrm{CDCl}_{3}$ ) of compound $\mathbf{5 g}$

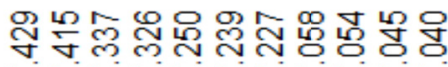

잉

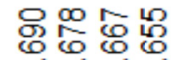

लंख

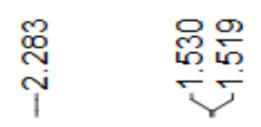

웅
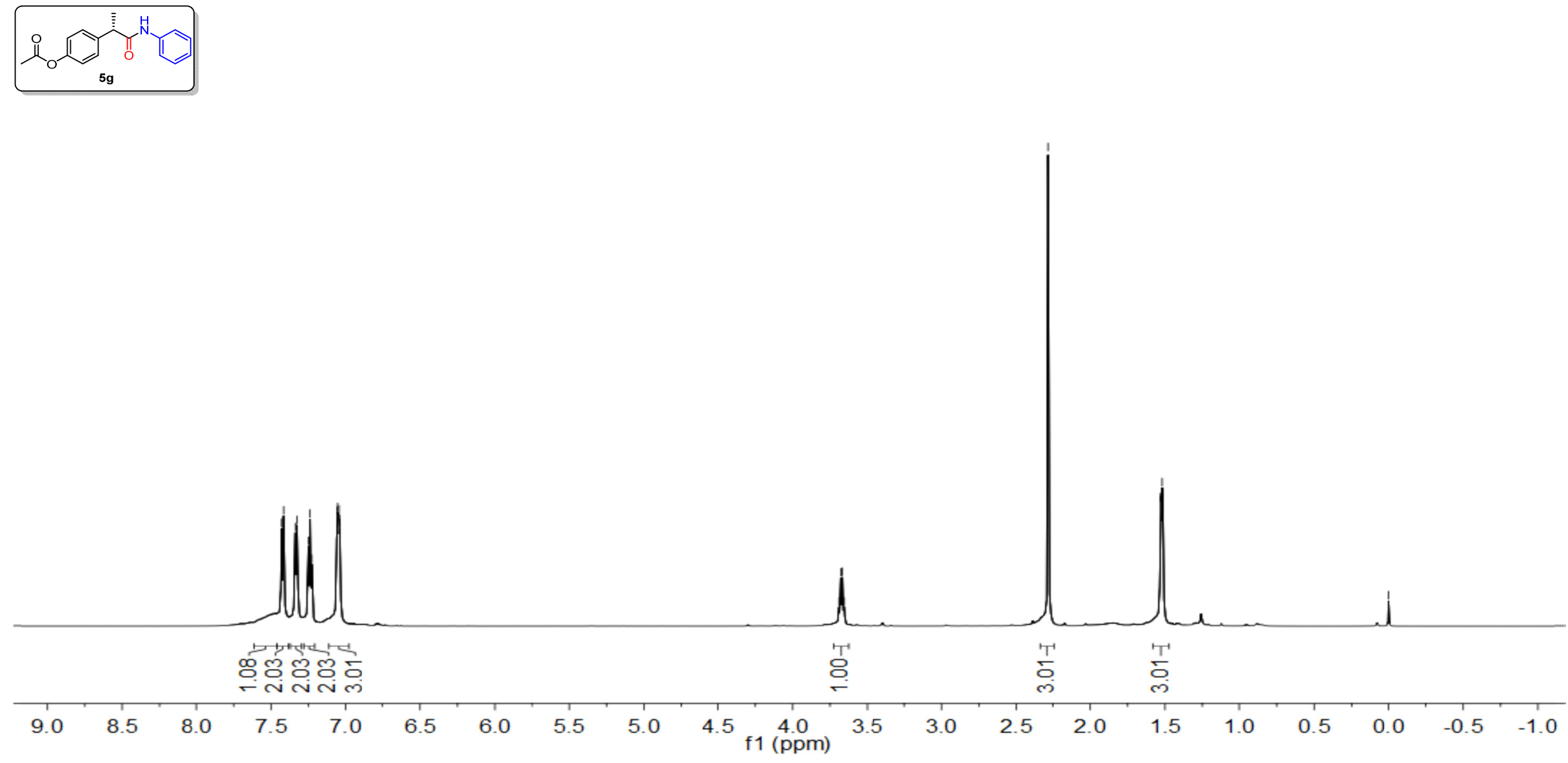

S144 
${ }^{13} \mathrm{C} \mathrm{NMR}\left(151 \mathrm{MHz}, \mathrm{CDCl}_{3}\right)$ of compound $\mathbf{5 g}$
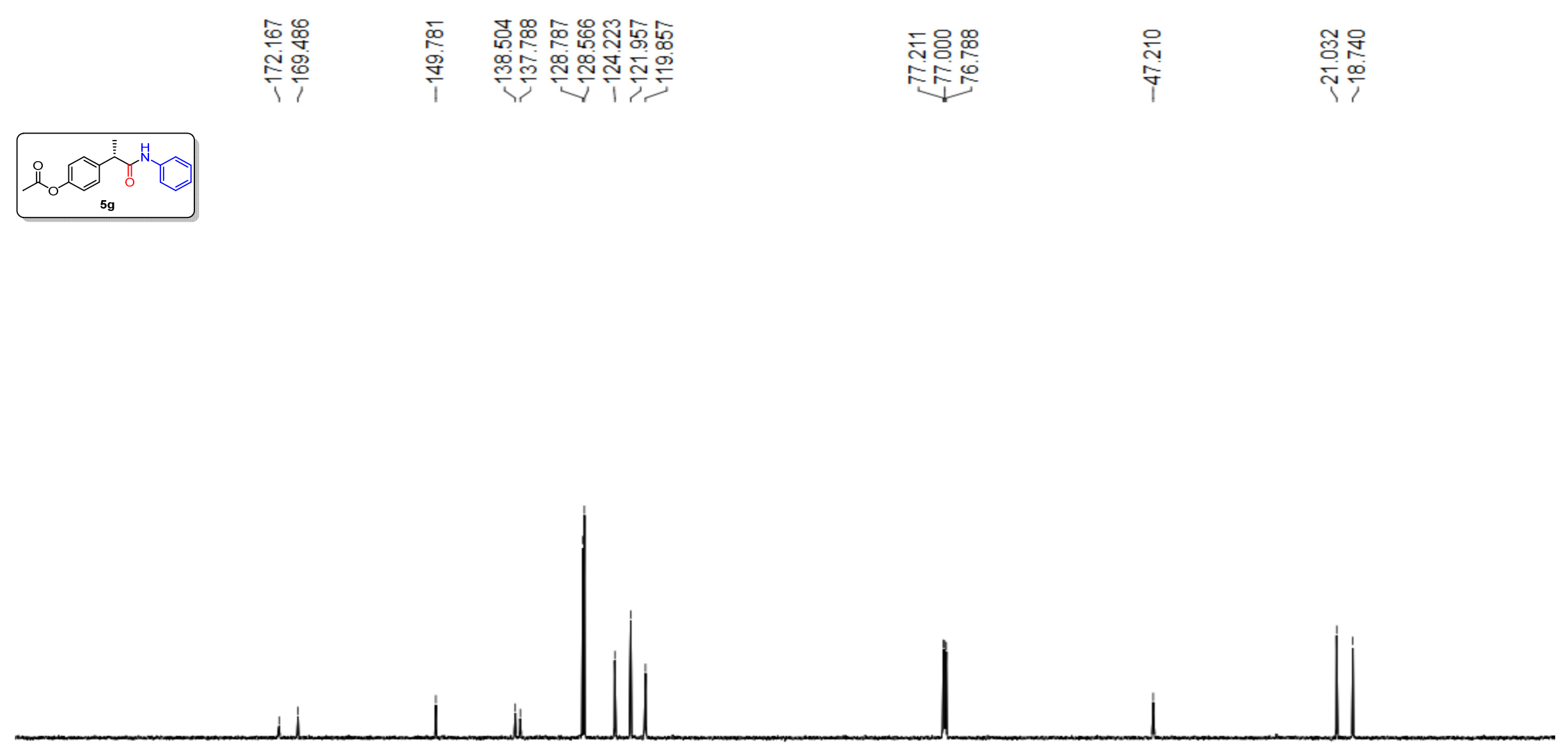

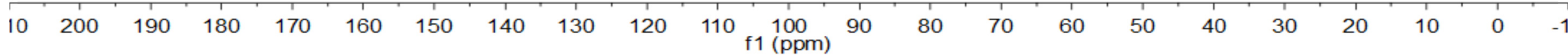


${ }^{1} \mathrm{H}$ NMR (400 MHz, $\mathrm{CDCl}_{3}$ ) of compound $\mathbf{5 h}$

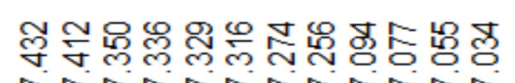

Niñ

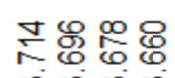

लिख

赵

乐

응
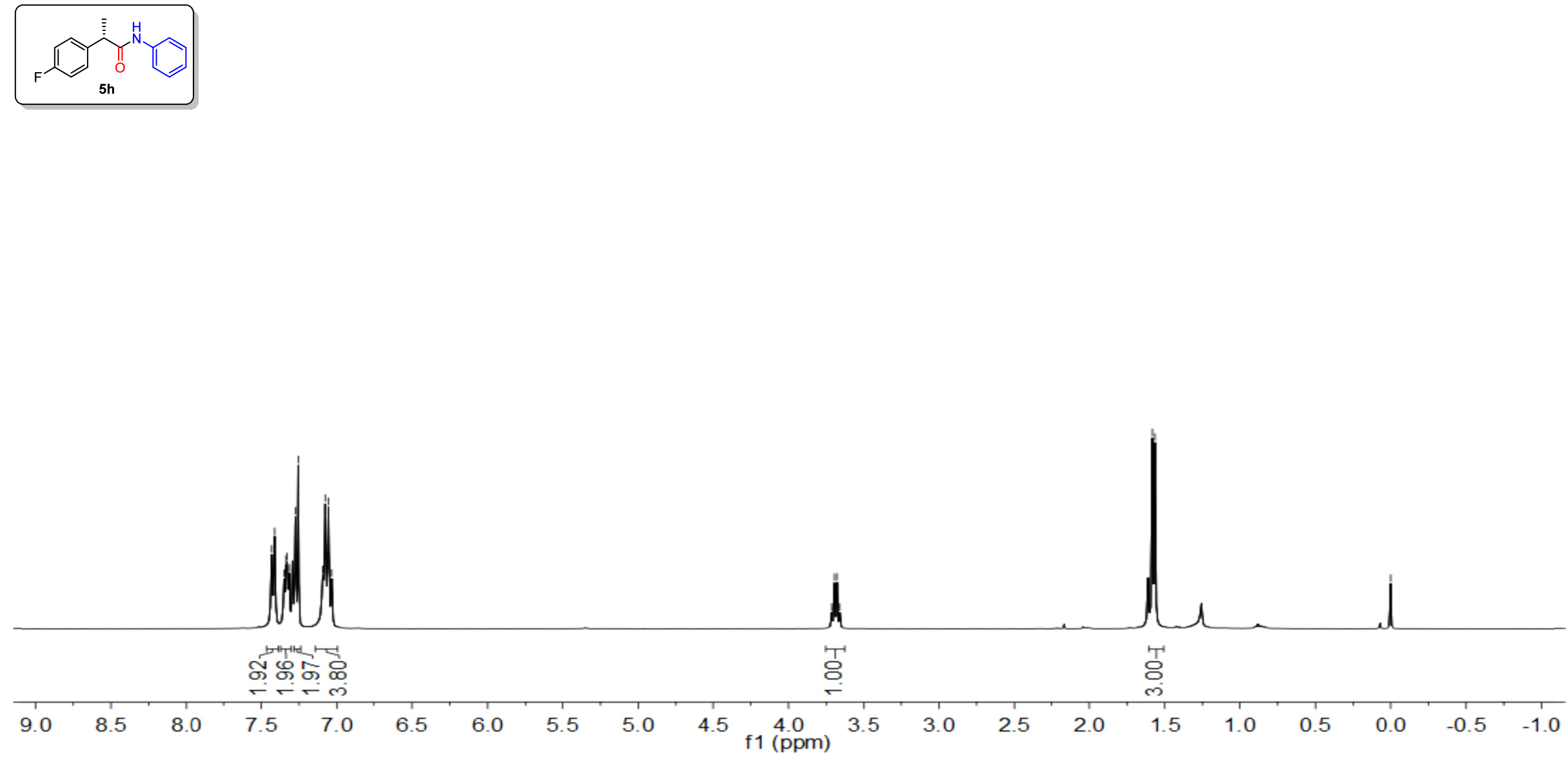

S146 
${ }^{13} \mathrm{C}$ NMR (101 MHz, $\mathrm{CDCl}_{3}$ ) of compound $\mathbf{5 h}$
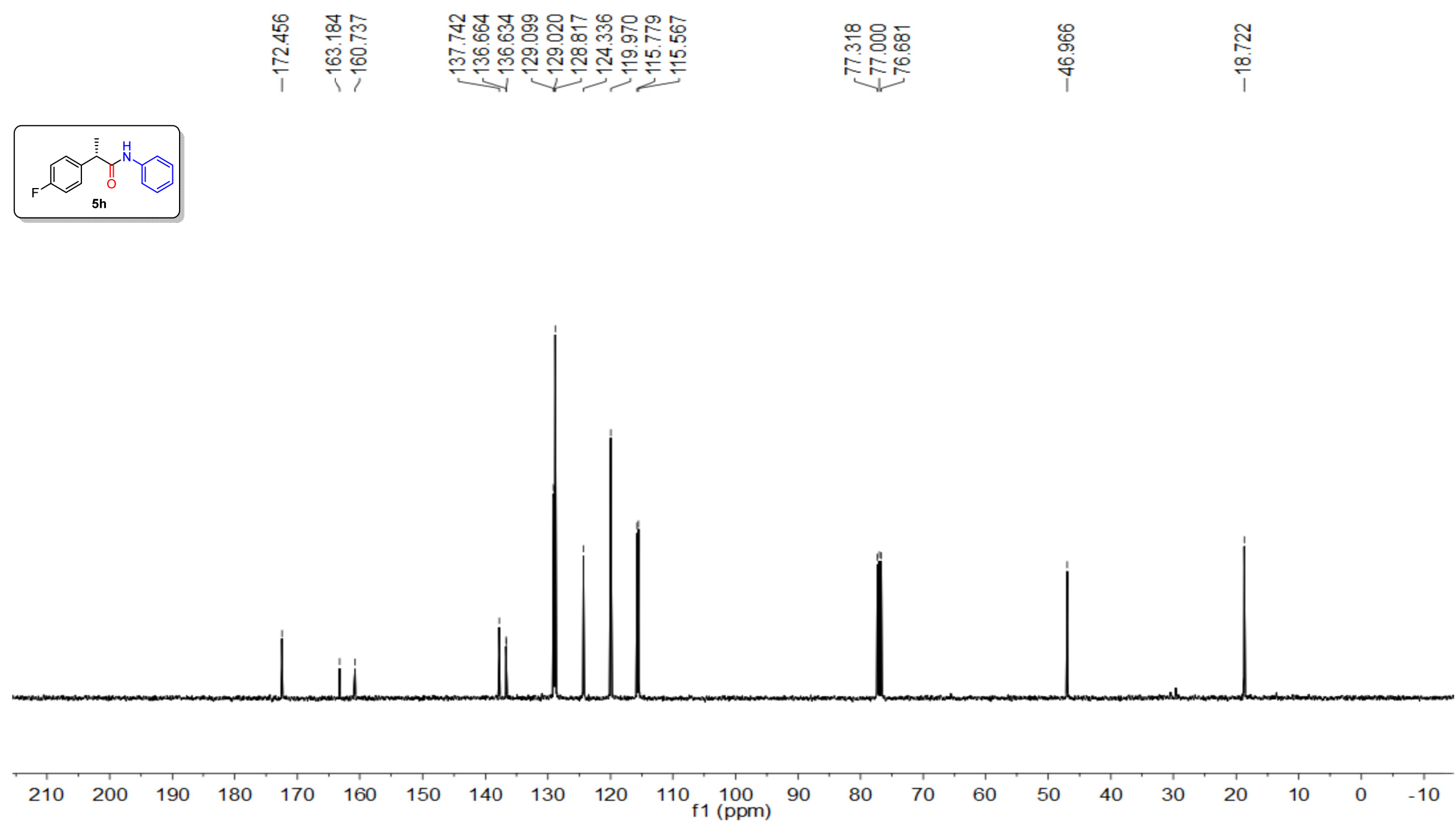
${ }^{19} \mathrm{~F}$ NMR (376 MHz, $\mathrm{CDCl}_{3}$ ) of compound $\mathbf{5 h}$

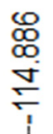
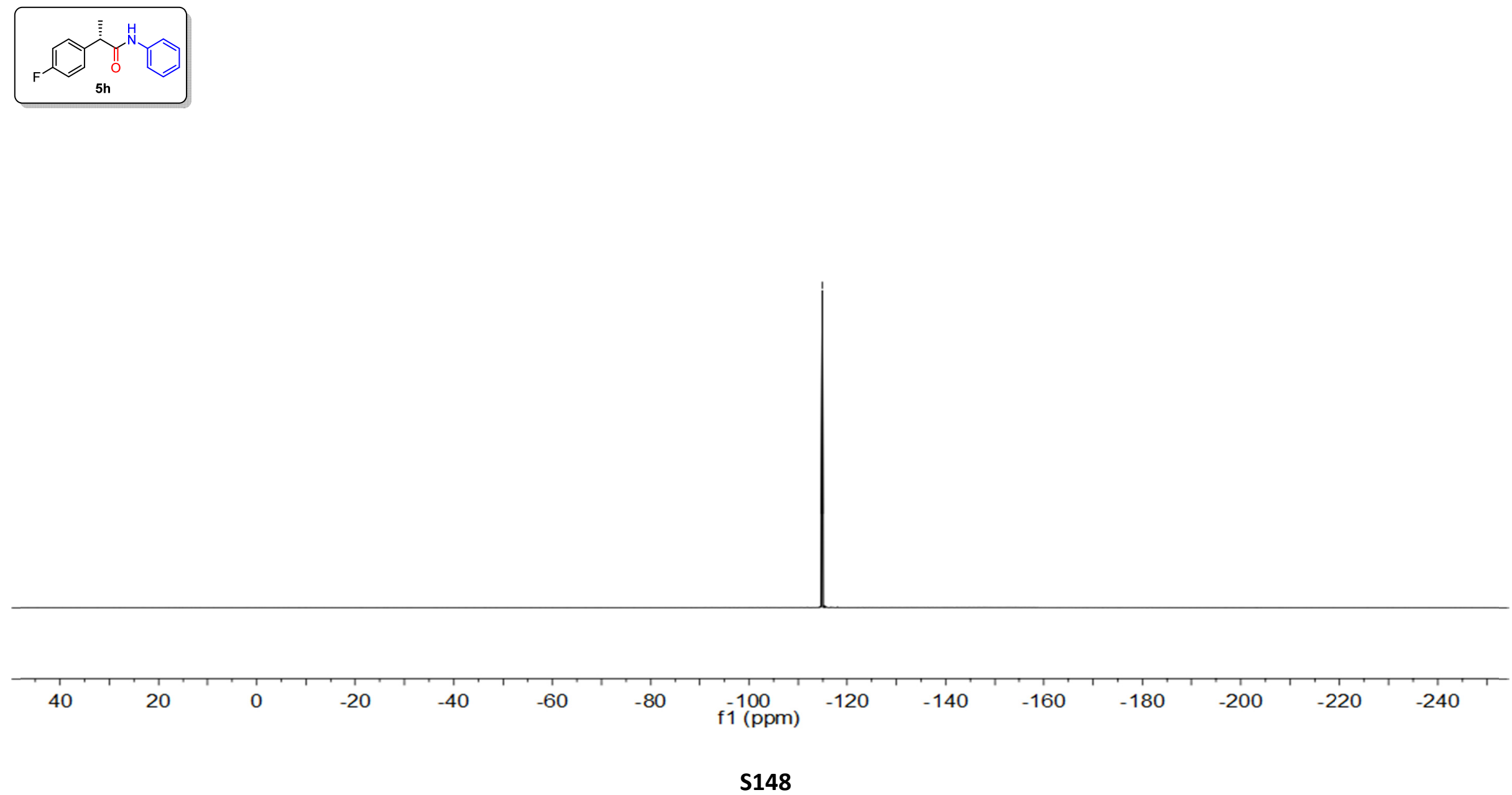
${ }^{1} \mathrm{H}$ NMR (400 MHz, $\mathrm{CDCl}_{3}$ ) of compound $\mathbf{5 i}$

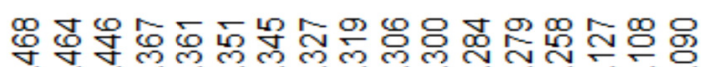

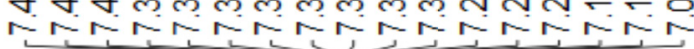
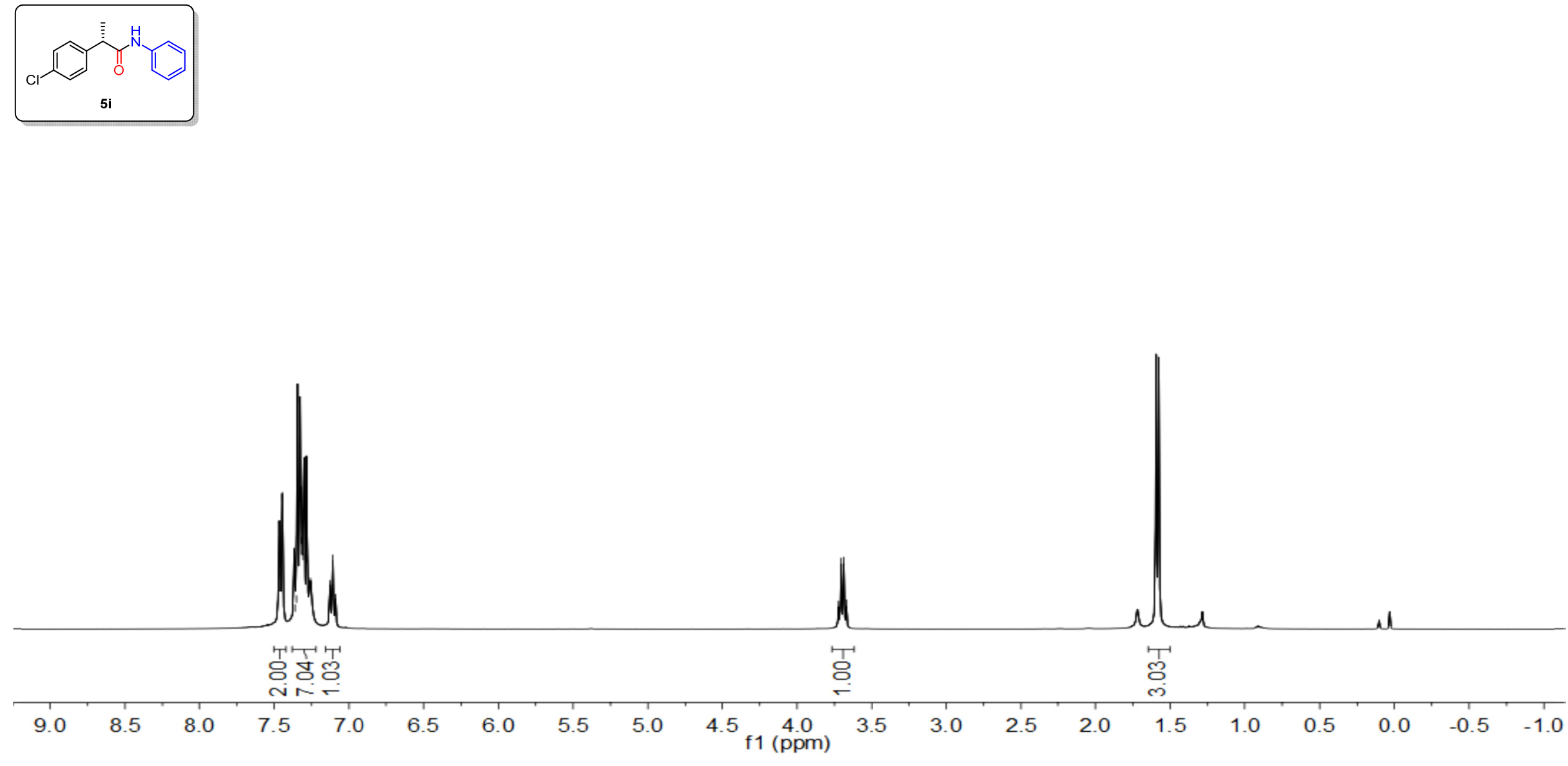
${ }^{13} \mathrm{C}$ NMR $\left(151 \mathrm{MHz}, \mathrm{CDCl}_{3}\right)$ of compound $\mathbf{5 i}$

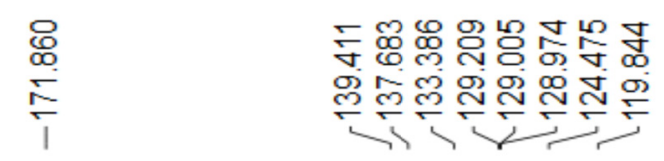

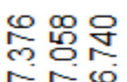

단
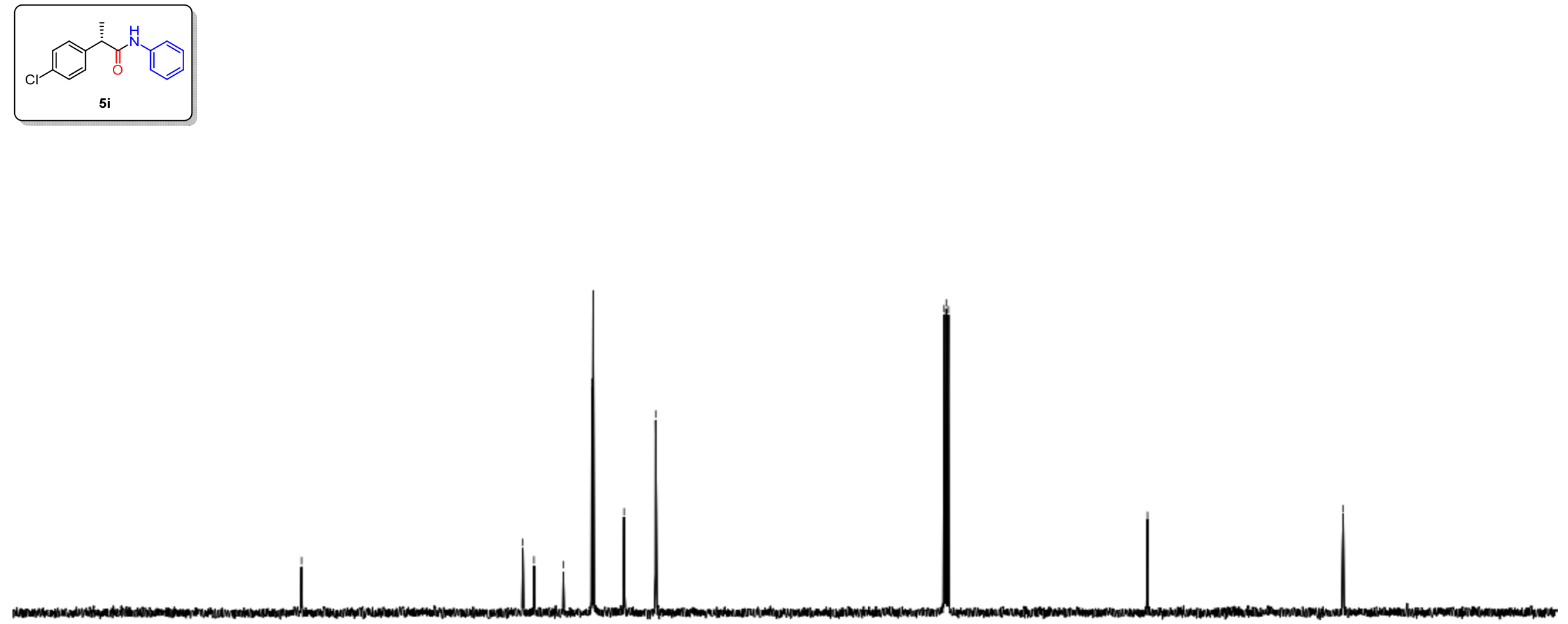

$\begin{array}{lllllllllll}210 & 200 & 190 & 180 & 170 & 160 & 150 & 140 & 130 & 120 & 110 \begin{array}{l}100 \\ \mathrm{f} 1\end{array}(\mathrm{ppm})\end{array}$

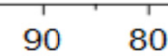

7060

$50 \quad 40$

30

20

10

0 10 
${ }^{1} \mathrm{H}$ NMR (400 MHz, $\mathrm{CDCl}_{3}$ ) of compound 5j

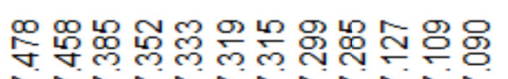

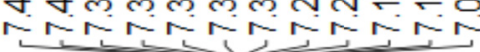

조용

लिख

象
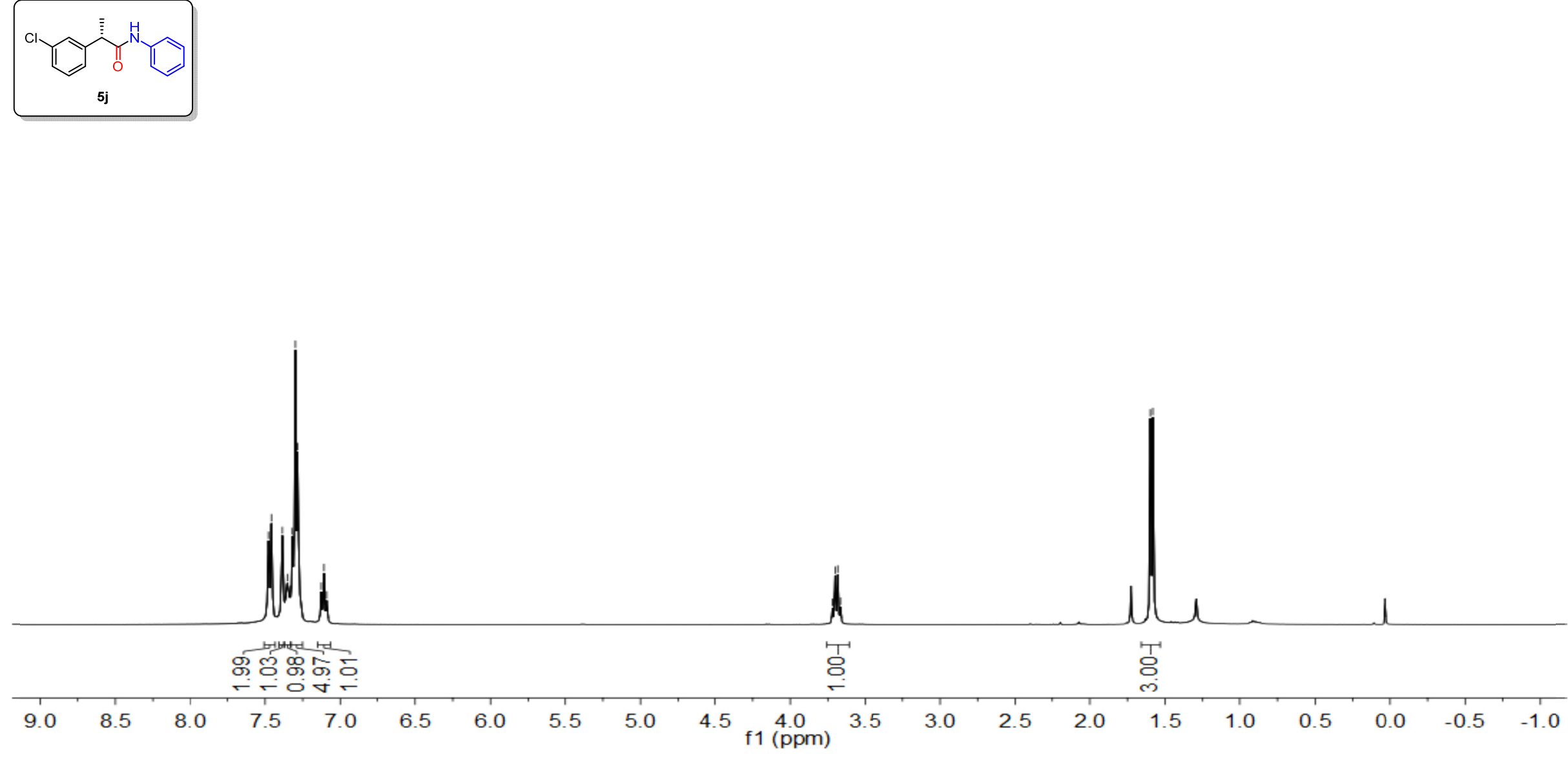
${ }^{13} \mathrm{C}$ NMR $\left(101 \mathrm{MHz}, \mathrm{CDCl}_{3}\right)$ of compound $\mathbf{5 j}$
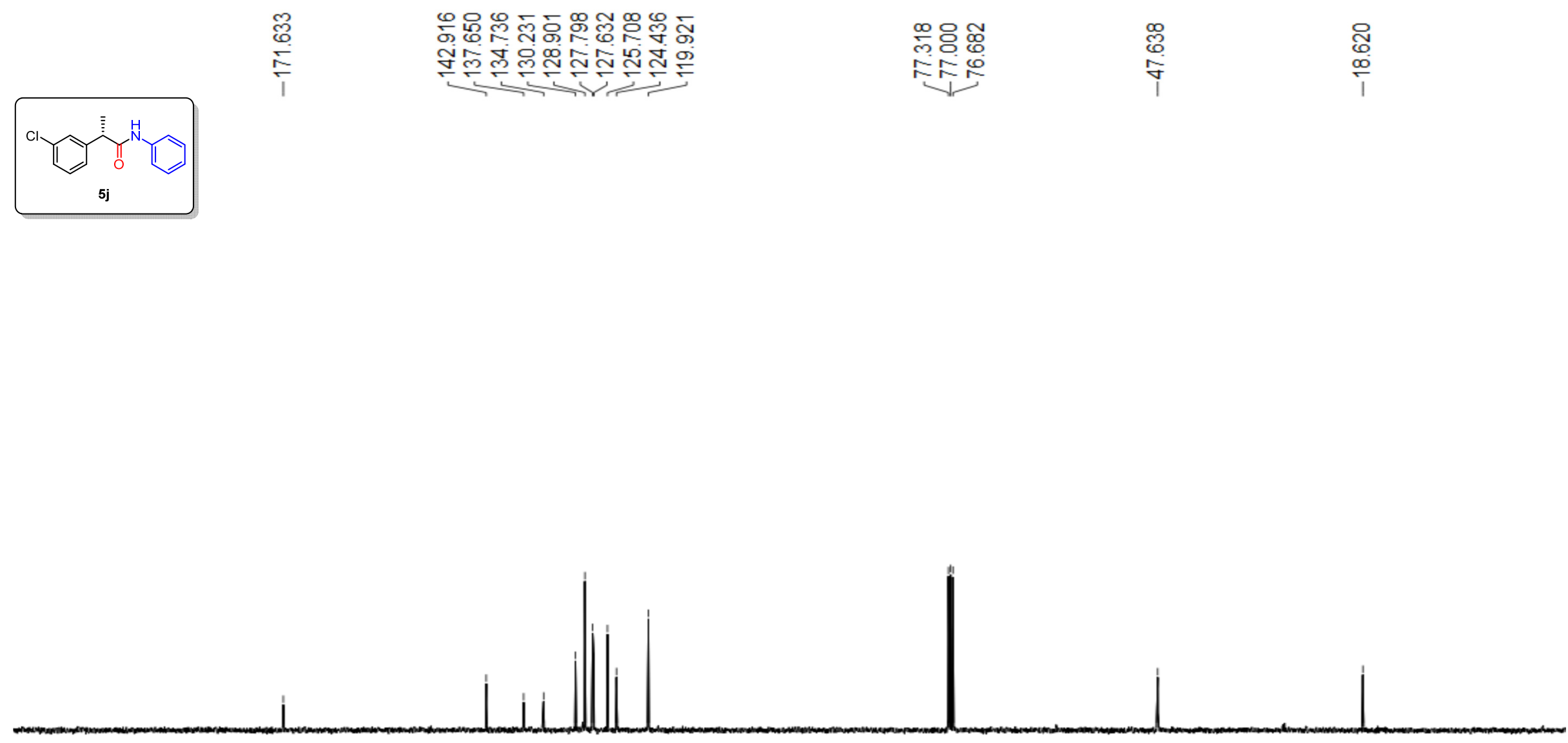

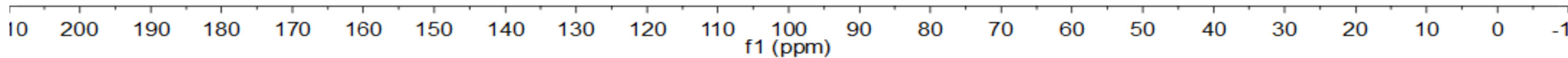


${ }^{1} \mathrm{H}$ NMR $\left(600 \mathrm{MHz}, \mathrm{CDCl}_{3}\right.$ ) of compound $\mathbf{5 k}$

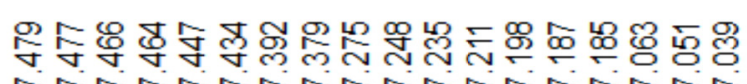

Ninn.

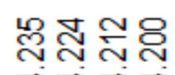

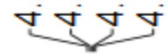

串

兽
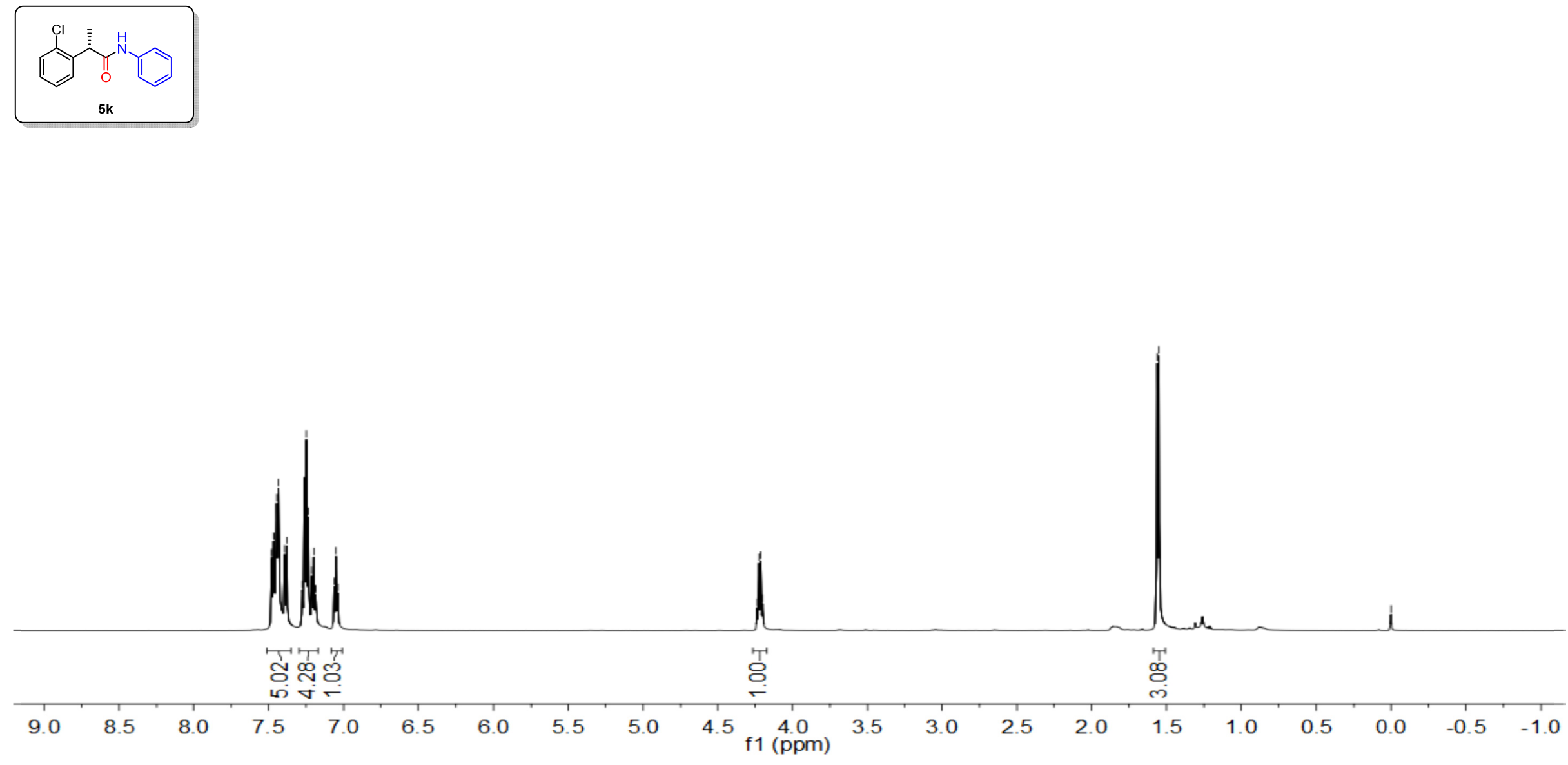
${ }^{13} \mathrm{C}$ NMR (151 MHz, $\mathrm{CDCl}_{3}$ ) of compound $\mathbf{5 k}$

管

:

$\stackrel{ }{\sim} \mathrm{O}_{\mathrm{O}}^{\infty}$

Fin

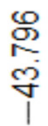

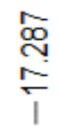
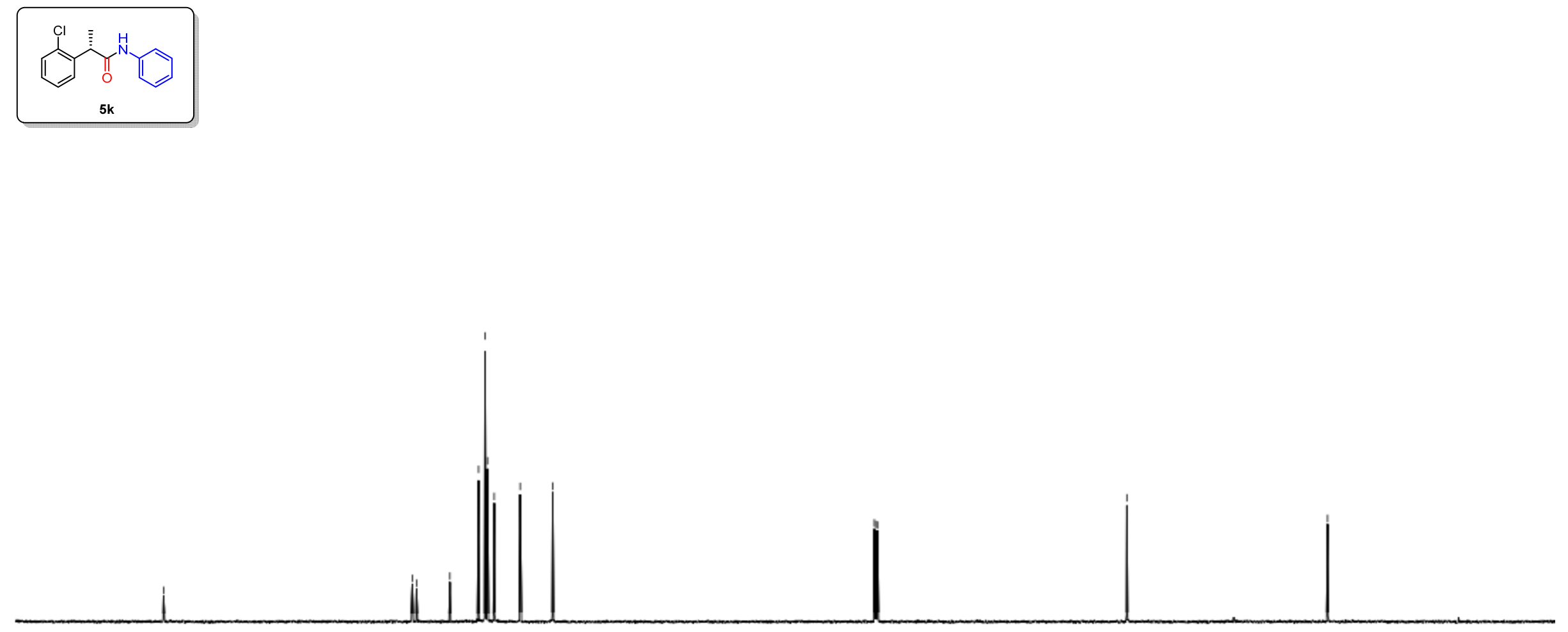

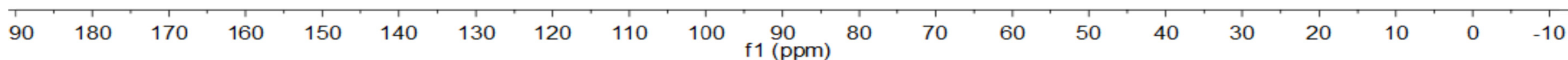


${ }^{1} \mathrm{H}$ NMR (400 MHz, $\mathrm{CDCl}_{3}$ ) of compound $\mathbf{5 l}$

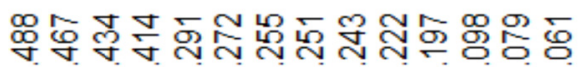

N-Nind

토워

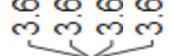

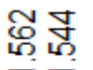

党

兽
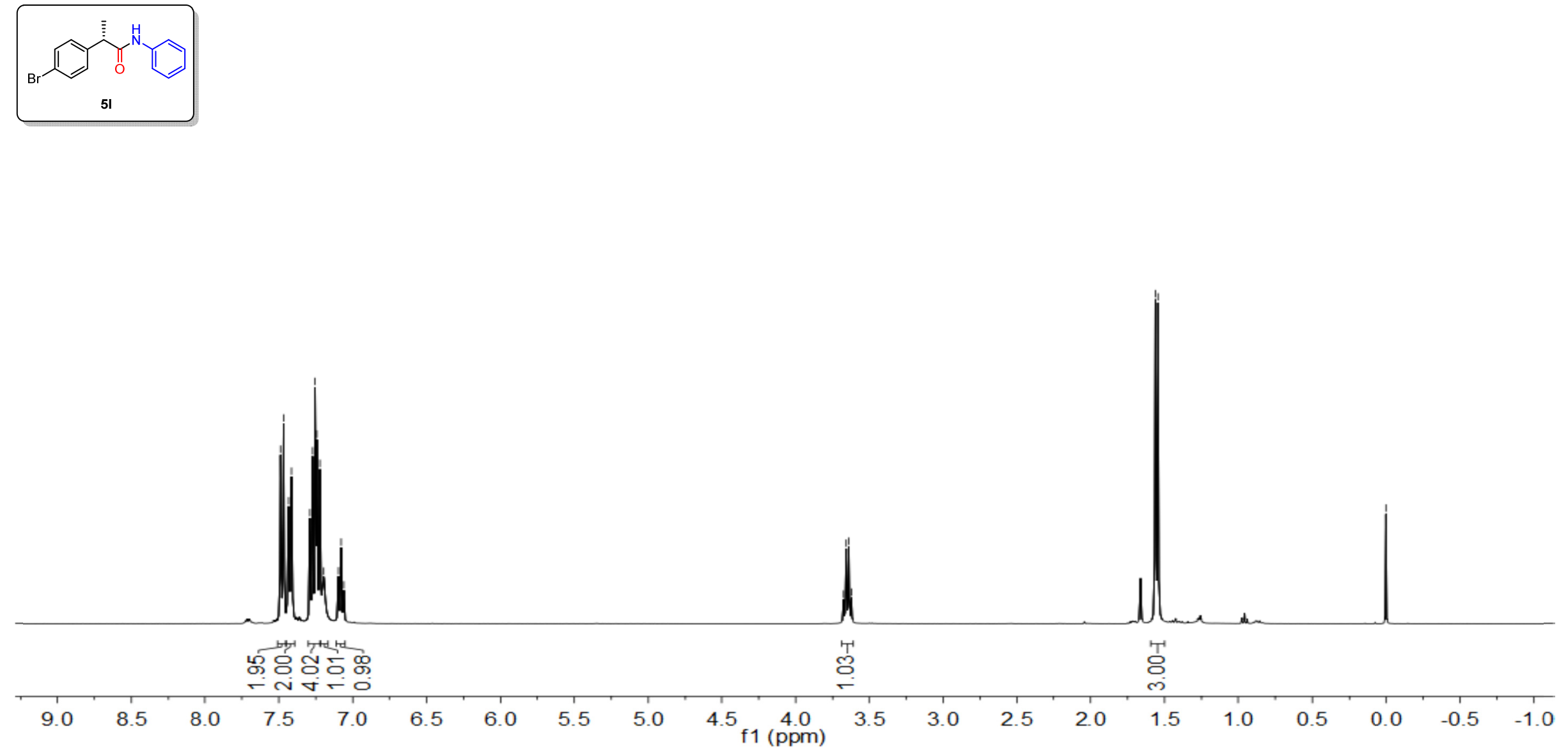

S155 
${ }^{13} \mathrm{C}$ NMR (151 MHz, $\left.\mathrm{CDCl}_{3}\right)$ of compound $\mathbf{5 l}$
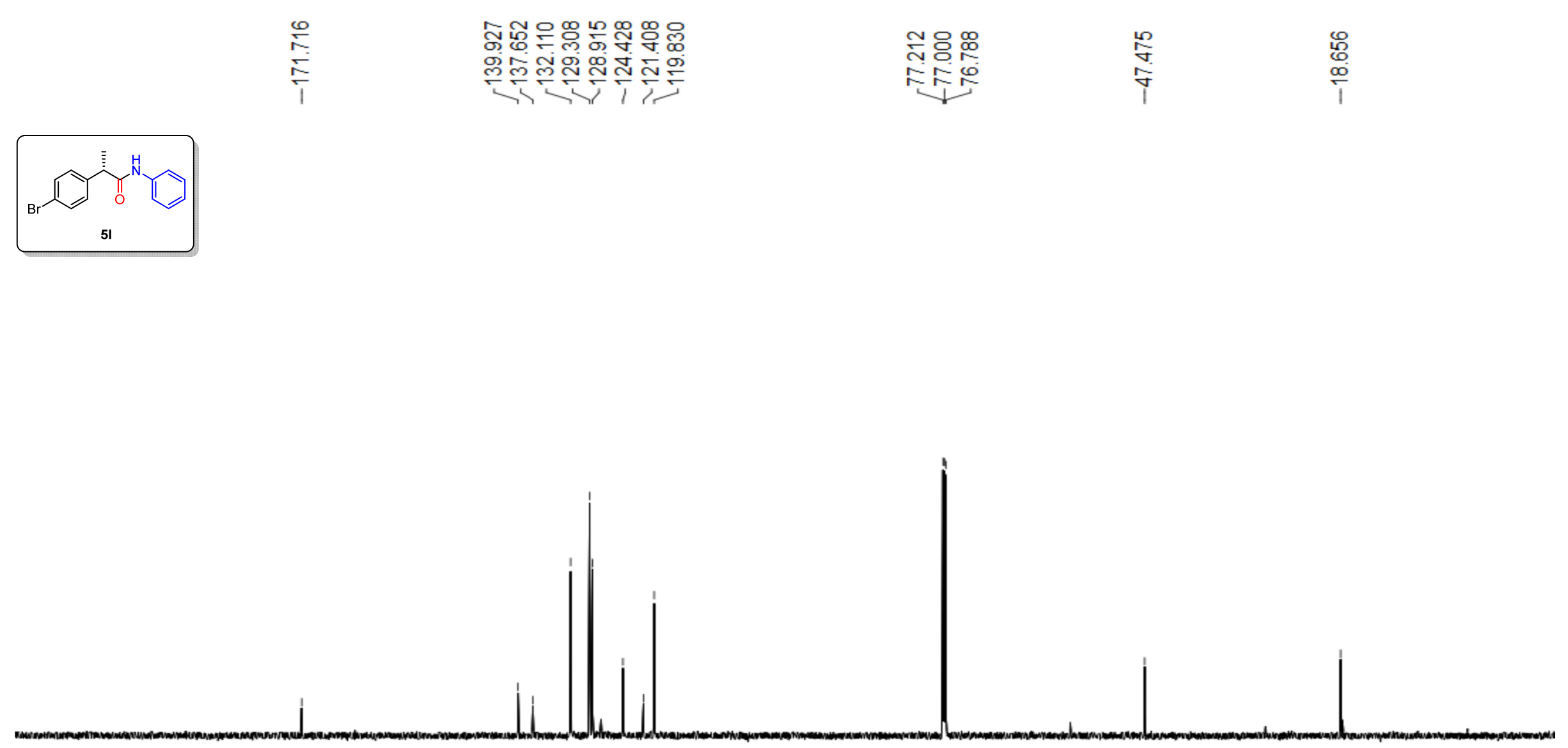

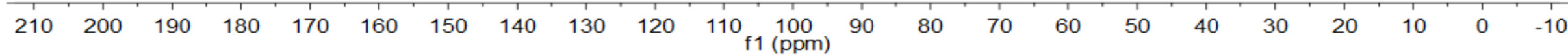


${ }^{1} \mathrm{H}$ NMR (400 MHz, $\mathrm{CDCl}_{3}$ ) of compound $\mathbf{5 m}$

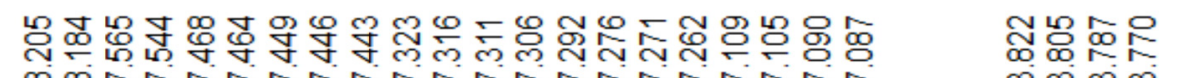

o on

สุ้

응
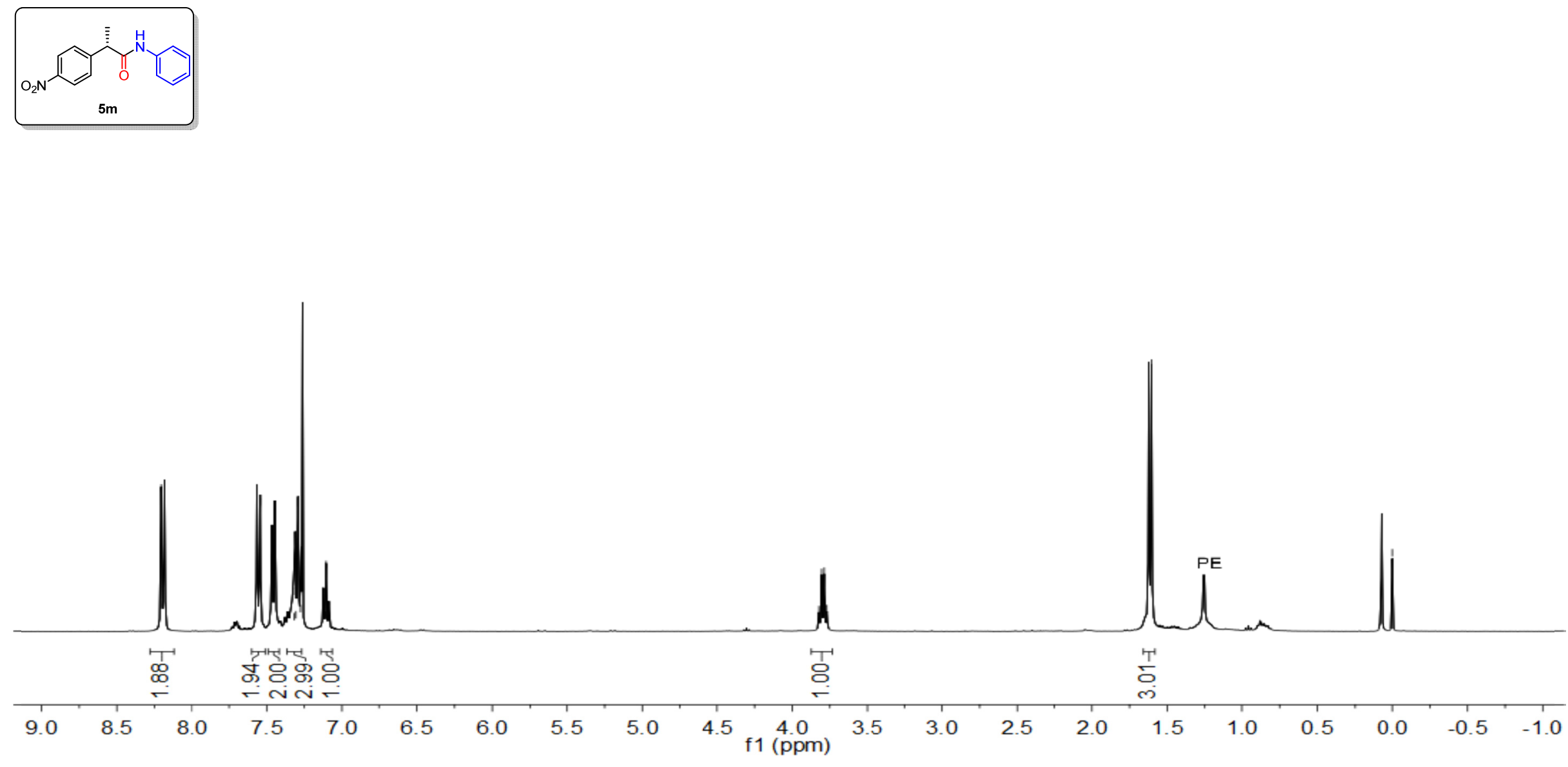

S157 
${ }^{13} \mathrm{C}$ NMR (151 MHz, $\mathrm{CDCl}_{3}$ ) of compound $\mathbf{5 m}$
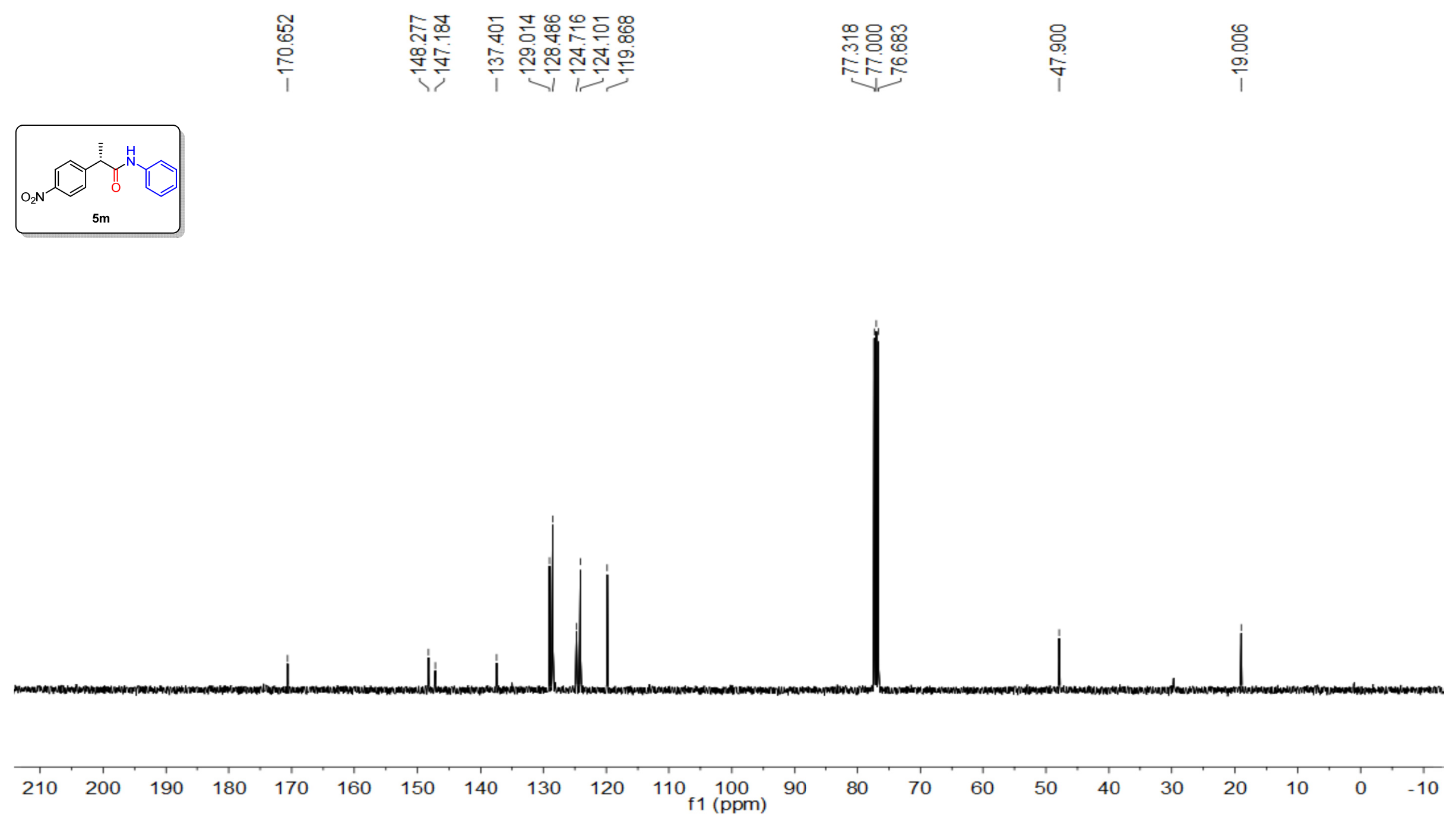
${ }^{1} \mathrm{H}$ NMR (400 MHz, $\mathrm{CDCl}_{3}$ ) of compound $\mathbf{5 n}$

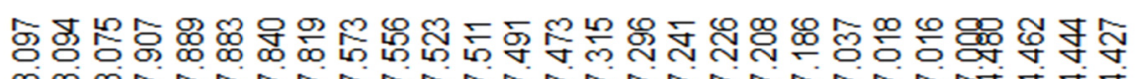

我 00 r.

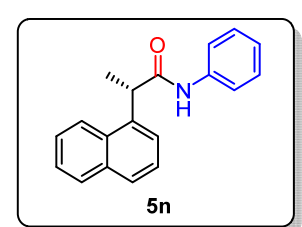

里电

음

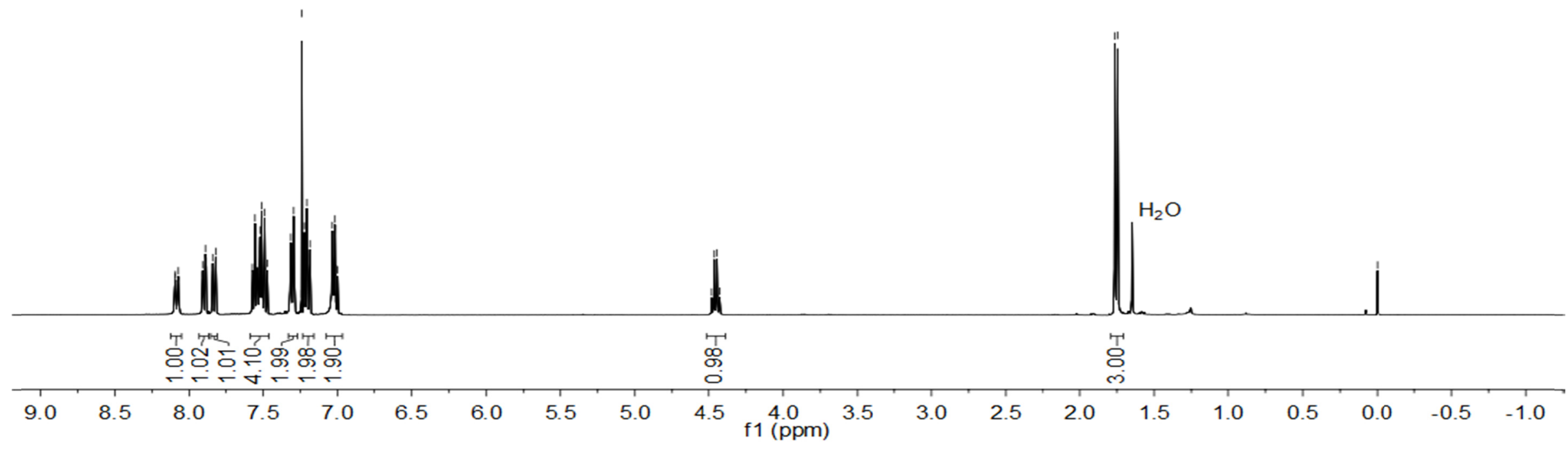


${ }^{13} \mathrm{C}$ NMR (101 MHz, $\mathrm{CDCl}_{3}$ ) of compound 5n
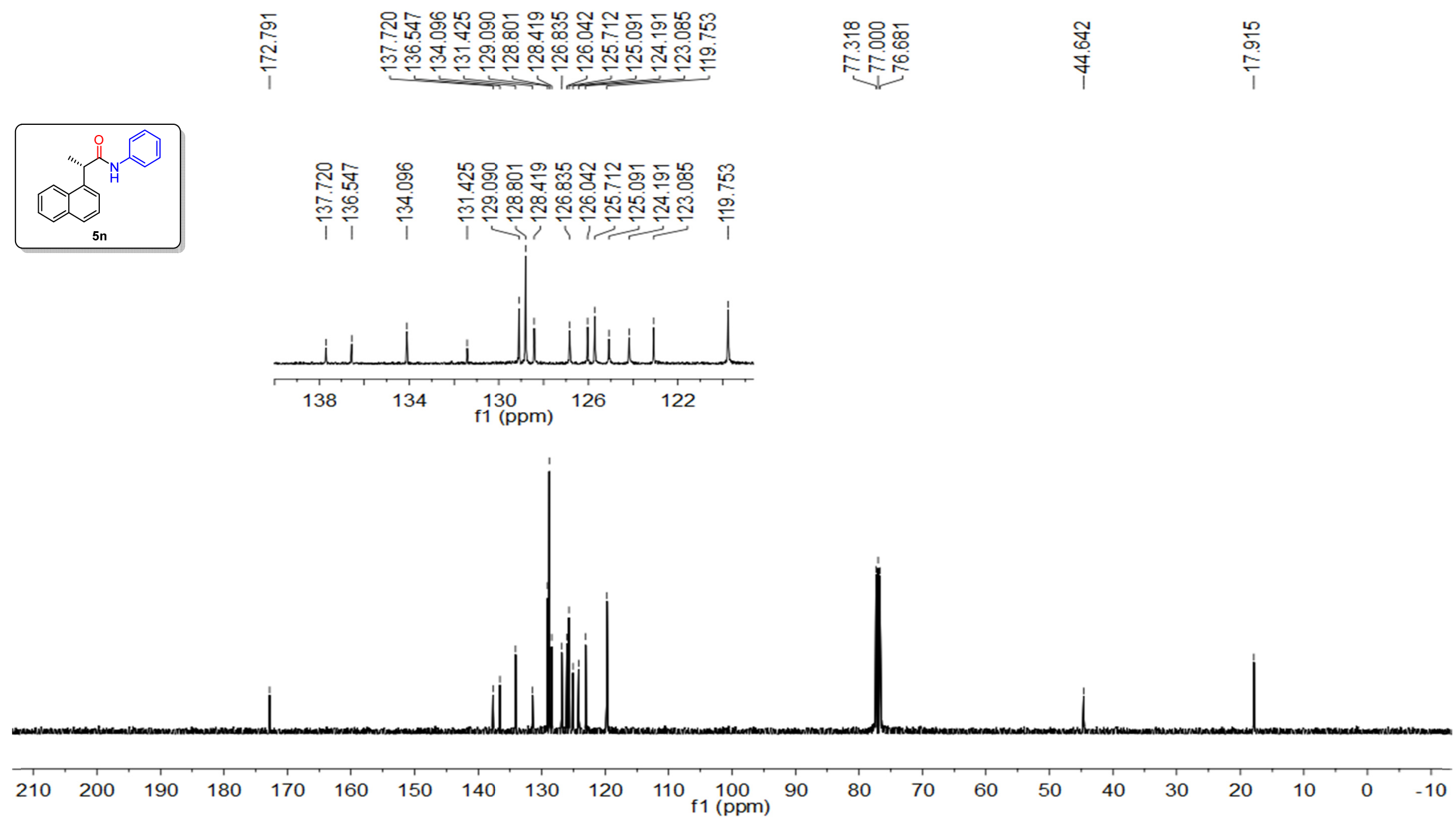
${ }^{1} \mathrm{H}$ NMR (400 MHz, $\mathrm{CDCl}_{3}$ ) of compound 50

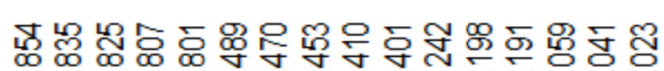

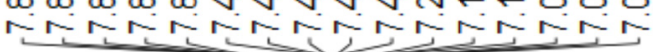

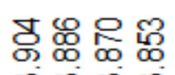

लालm

$6 \%$

这

옹
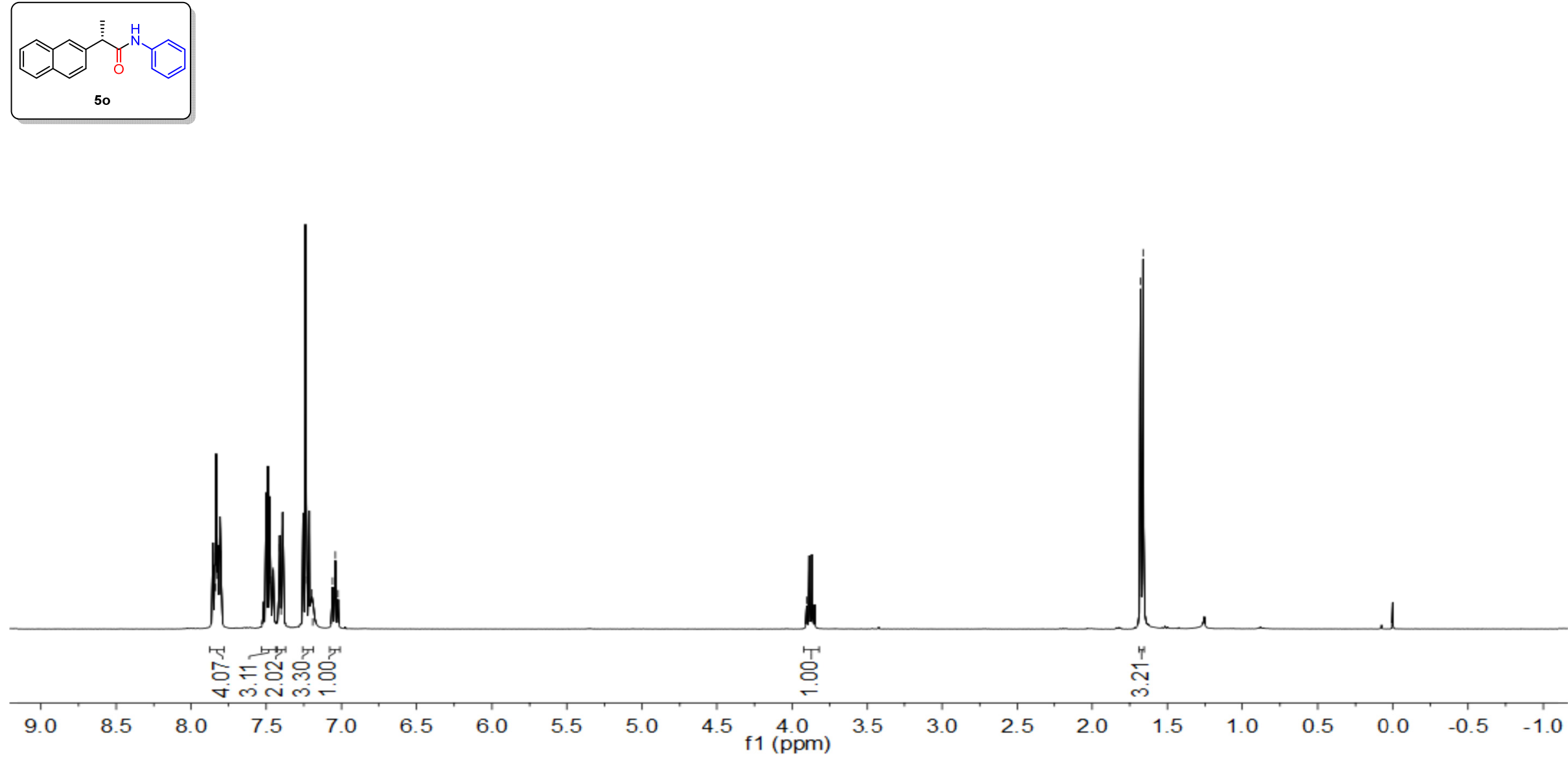
${ }^{13} \mathrm{C}$ NMR (151 MHz, $\mathrm{CDCl}_{3}$ ) of compound 50

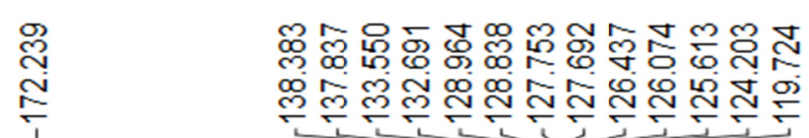

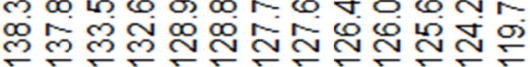

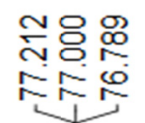

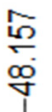

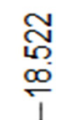
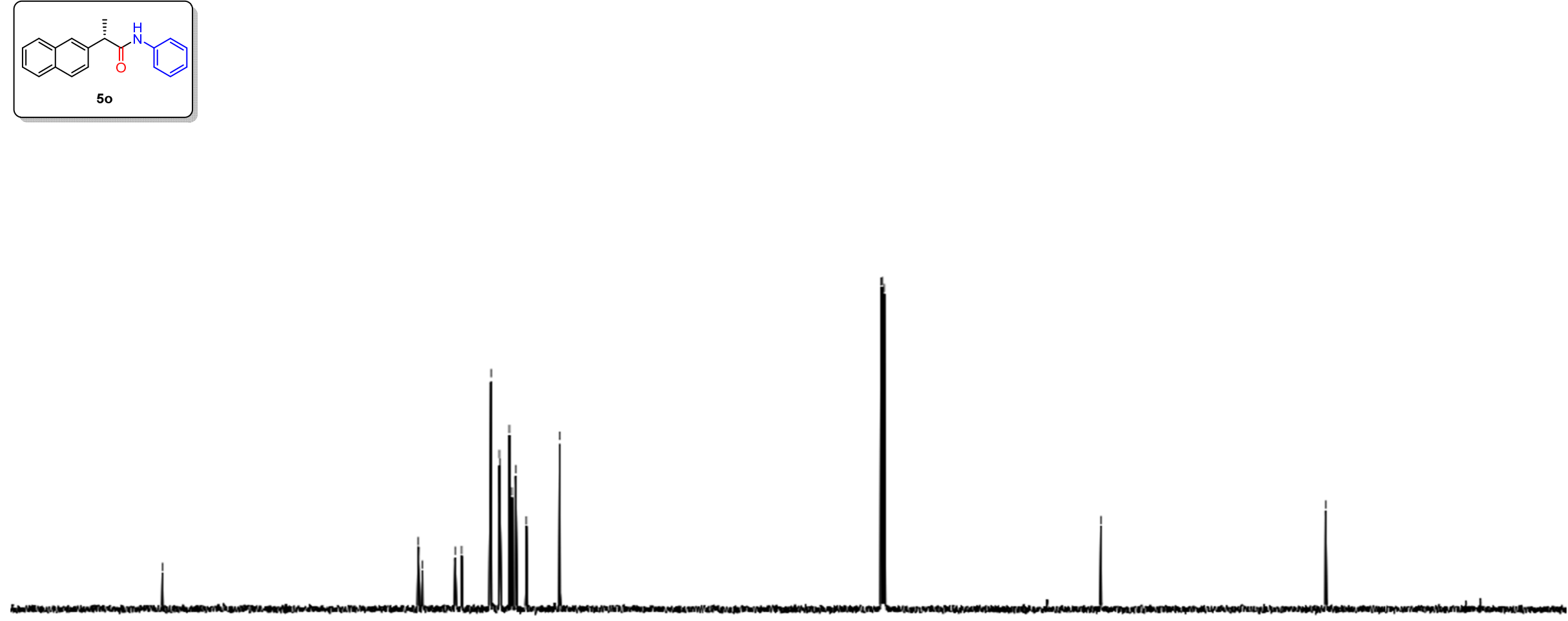
${ }^{1} \mathrm{H}$ NMR (400 MHz, $\mathrm{CDCl}_{3}$ ) of compound 5p

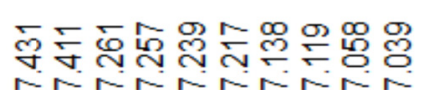
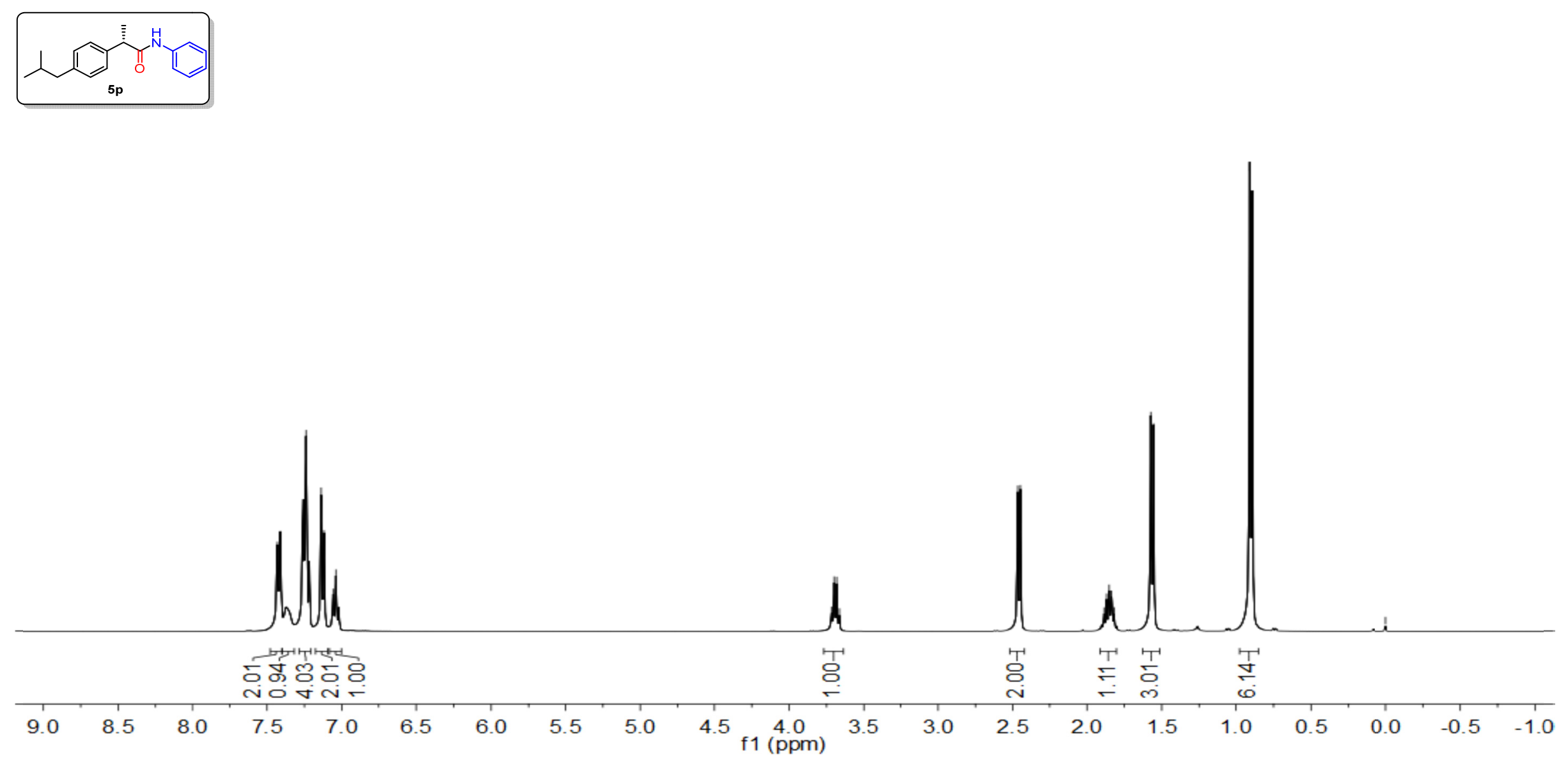
${ }^{13} \mathrm{C}$ NMR (101 MHz, $\left.\mathrm{CDCl}_{3}\right)$ of compound 5p

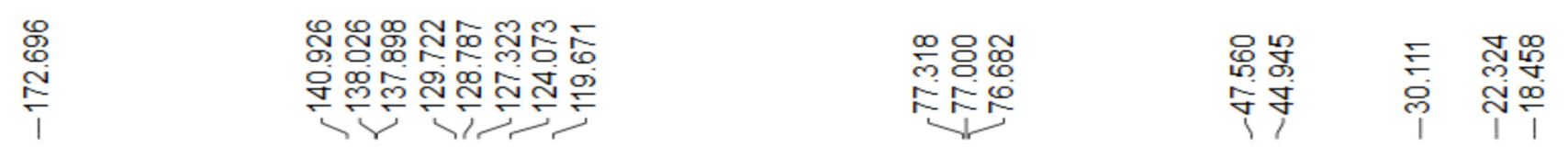
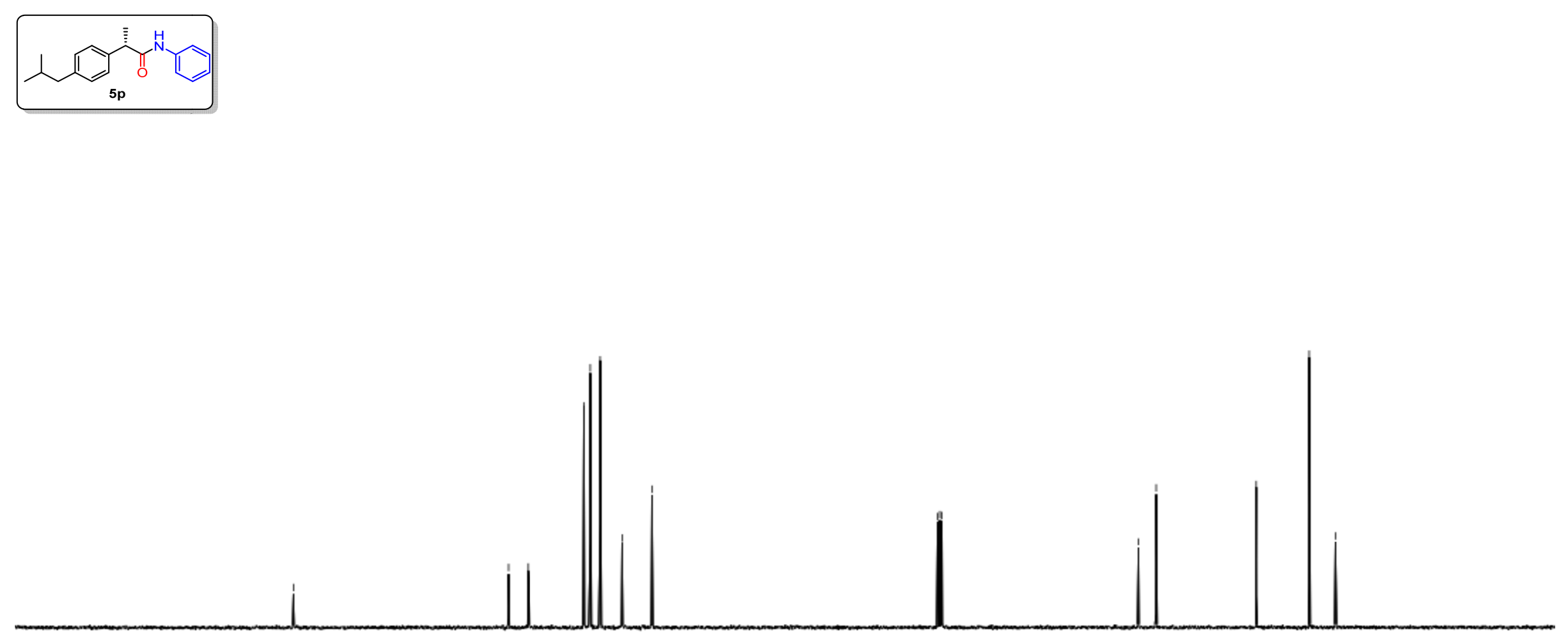

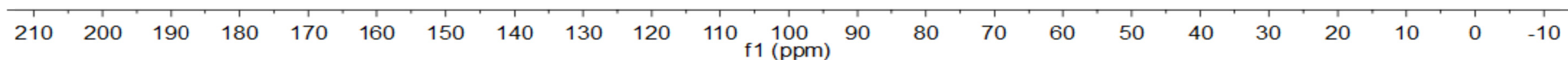


${ }^{1} \mathrm{H}$ NMR (400 MHz, $\mathrm{CDCl}_{3}$ ) of compound $\mathbf{5 q}$

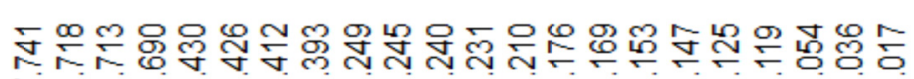

象赵

ल

思㐫

i

응
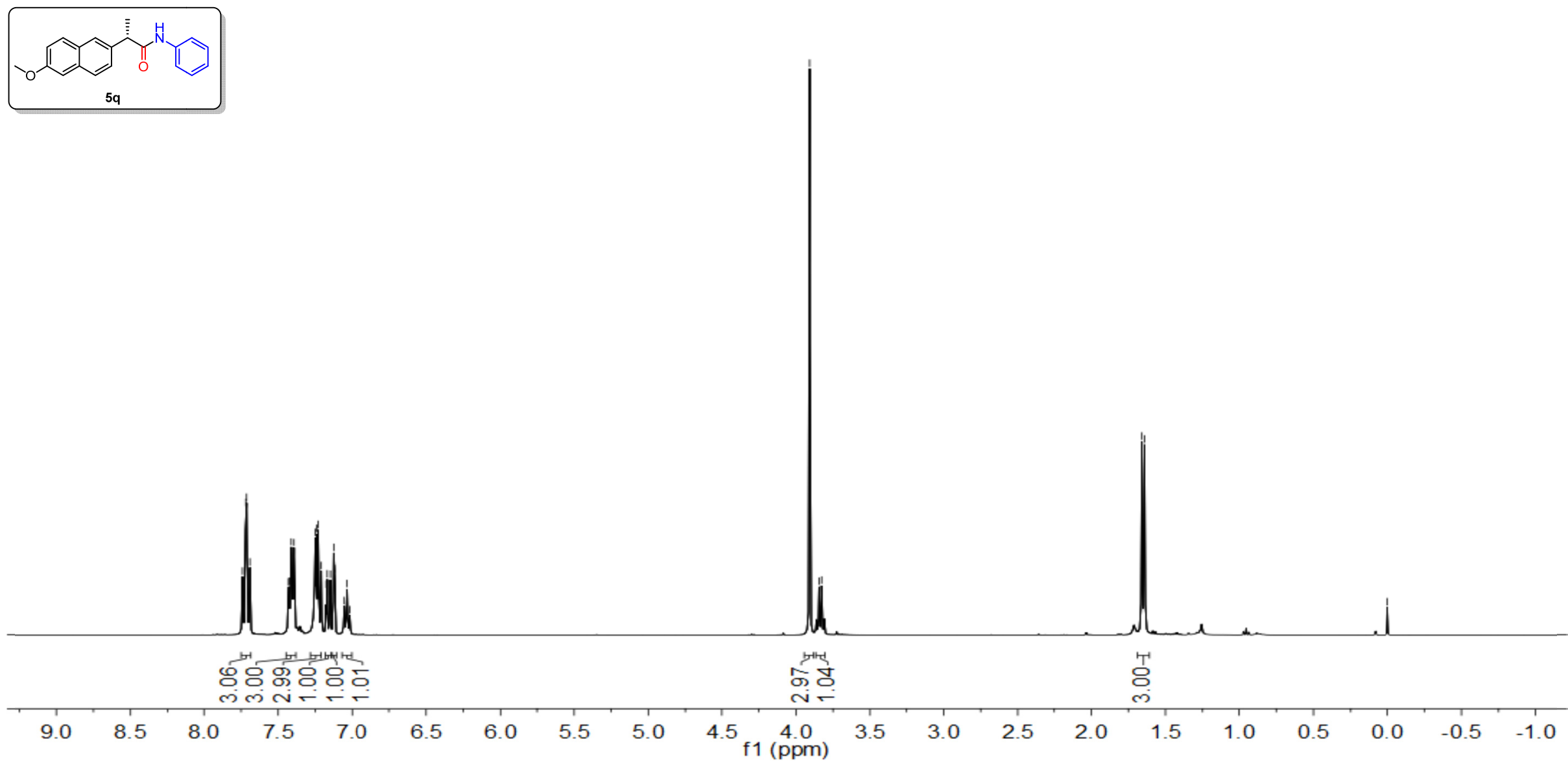

S165 
${ }^{13} \mathrm{C}$ NMR $\left(101 \mathrm{MHz}, \mathrm{CDCl}_{3}\right)$ of compound $\mathbf{5 q}$

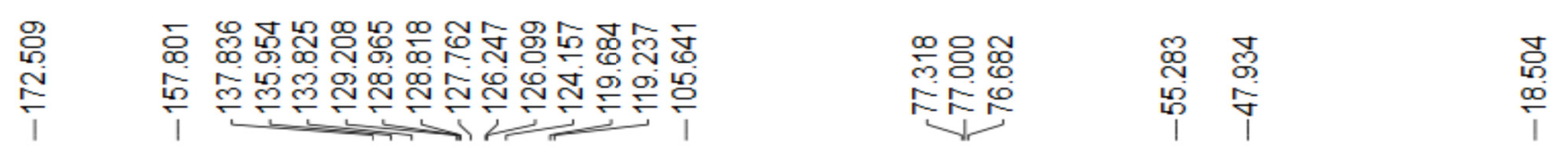

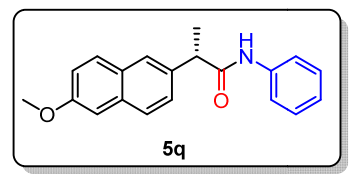

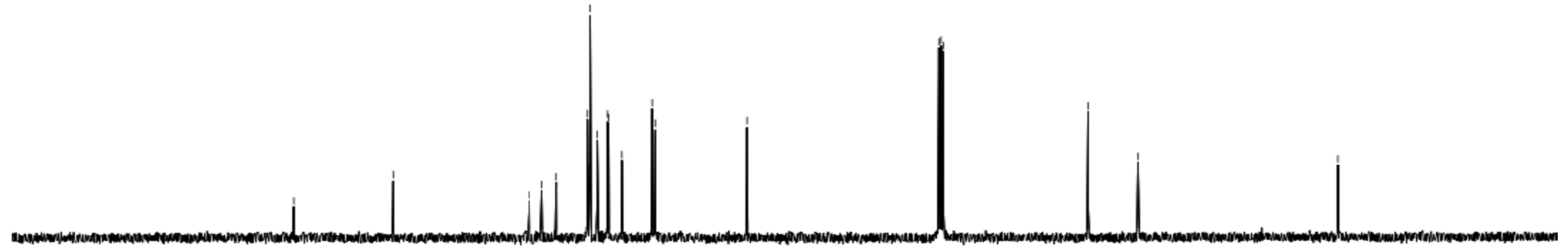

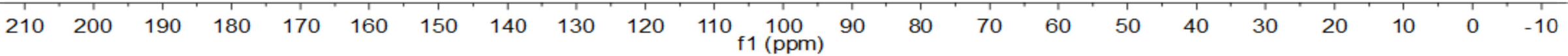


${ }^{1} \mathrm{H}$ NMR (400 MHz, $d^{6}$-DMSO) of compound $\mathbf{5 r}$

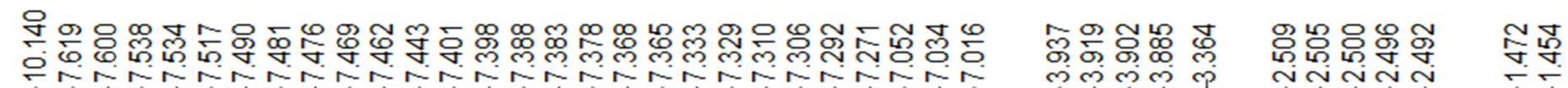

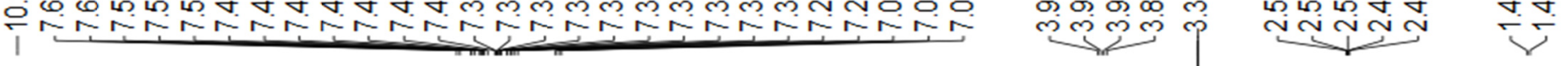

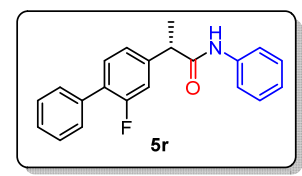

$\mathrm{H}_{2} \mathrm{O}$

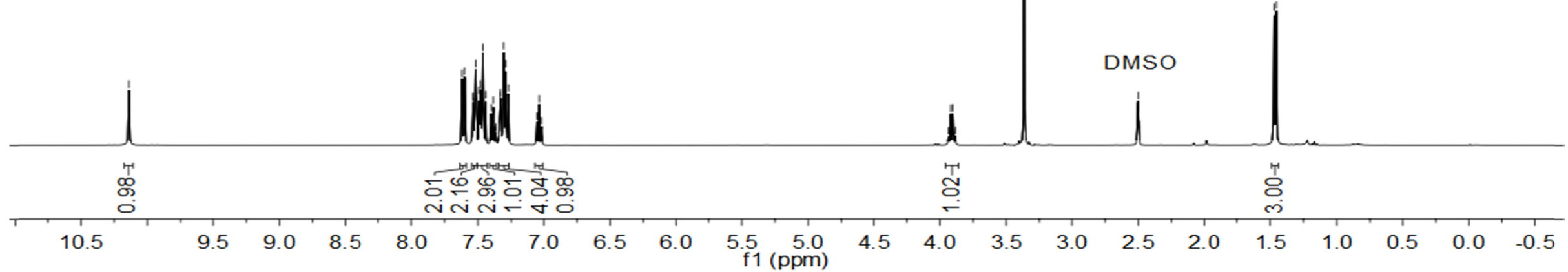

S167 
${ }^{13} \mathrm{C}$ NMR (101 MHz, $d^{6}$-DMSO) of compound $\mathbf{5 r}$

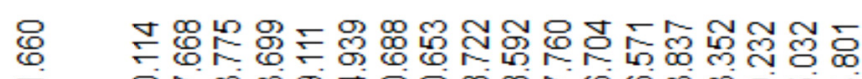

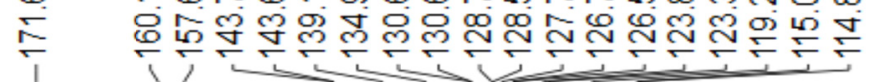

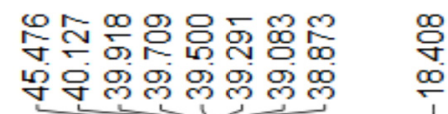
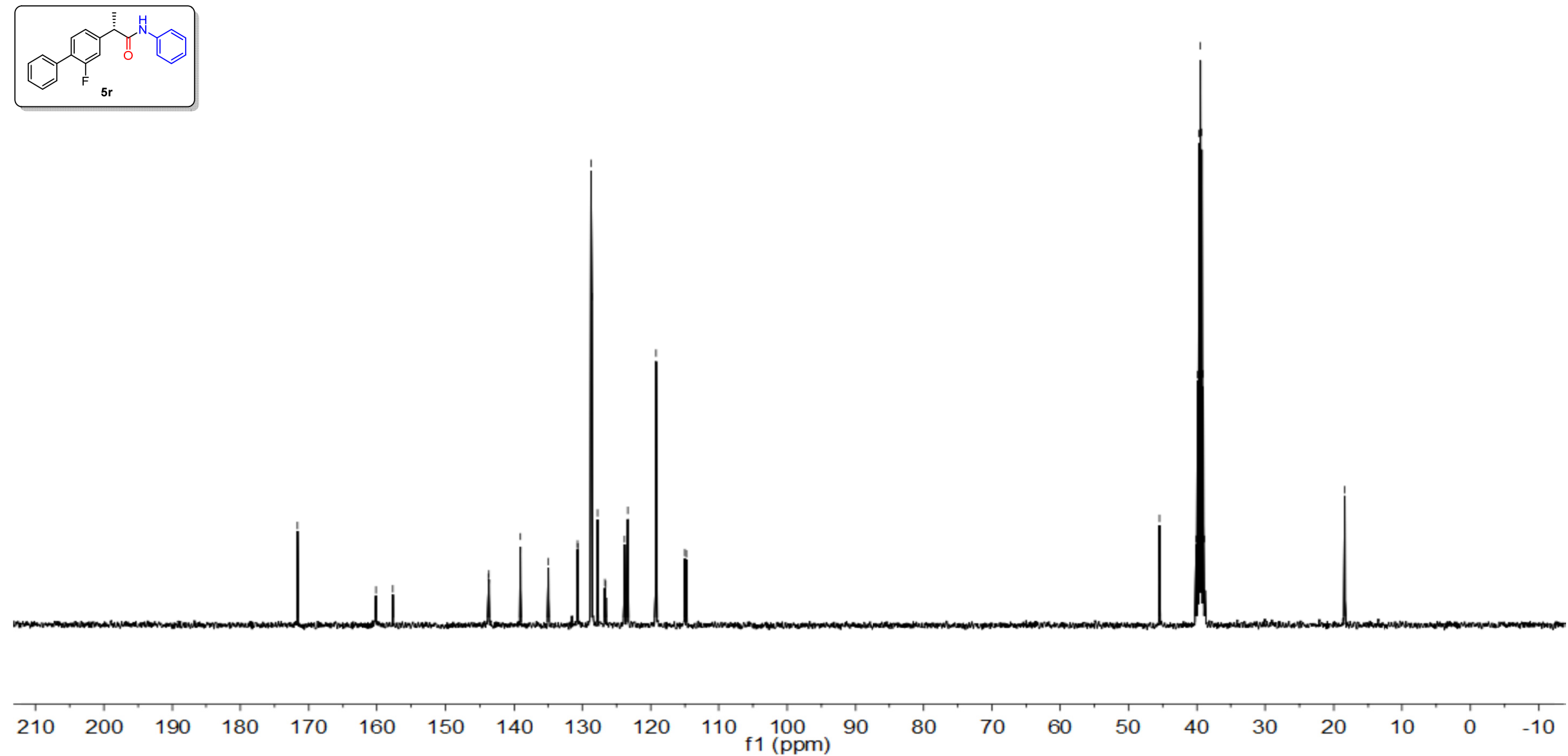
${ }^{19} \mathrm{~F}$ NMR (376 MHz, $d^{6}$-DMSO) of compound $\mathbf{5 r}$

$$
\underset{i}{\stackrel{0}{6}}
$$
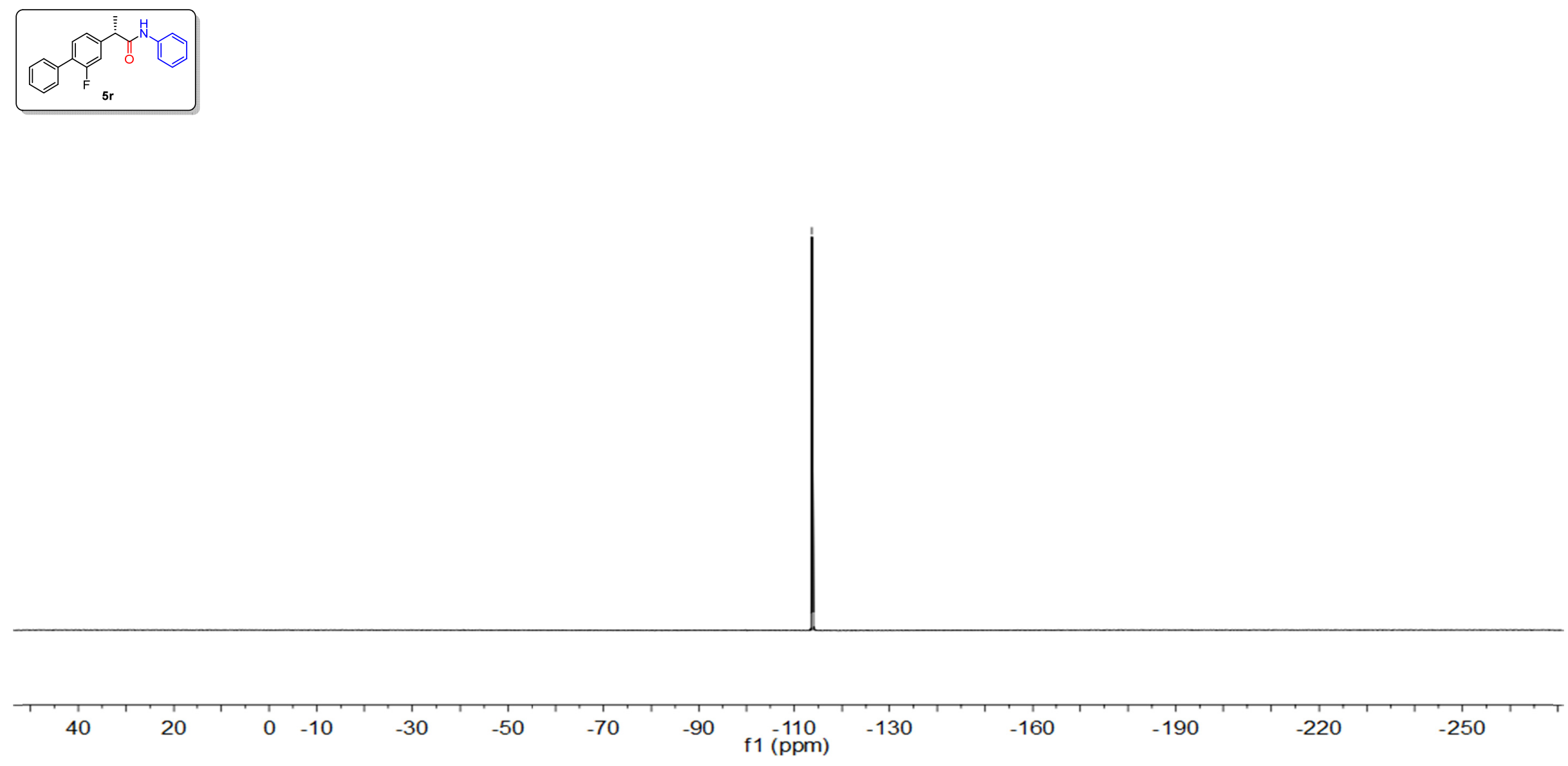
${ }^{1} \mathrm{H}$ NMR (400 MHz, $\mathrm{CDCl}_{3}$ ) of compound $\mathbf{5 s}$

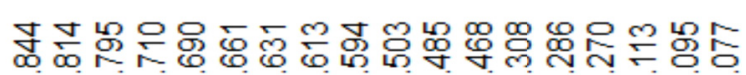

marmon
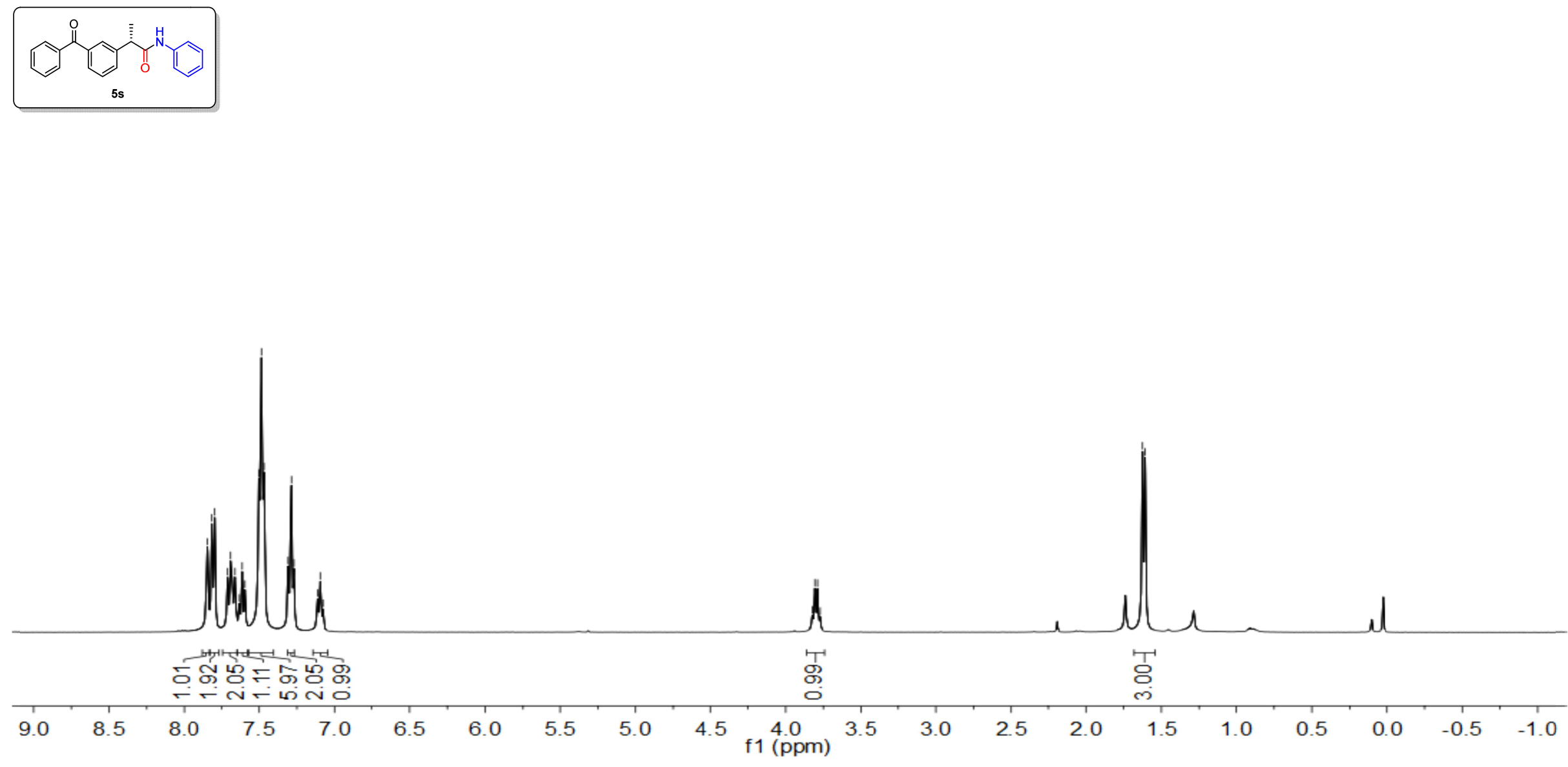
${ }^{13} \mathrm{C}$ NMR $\left(101 \mathrm{MHz}, \mathrm{CDCl}_{3}\right)$ of compound $\mathbf{5 s}$

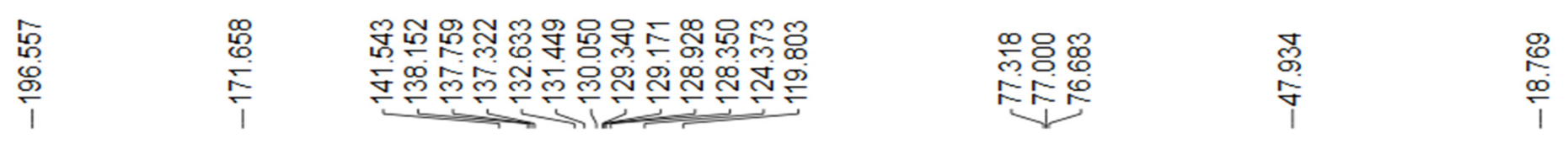
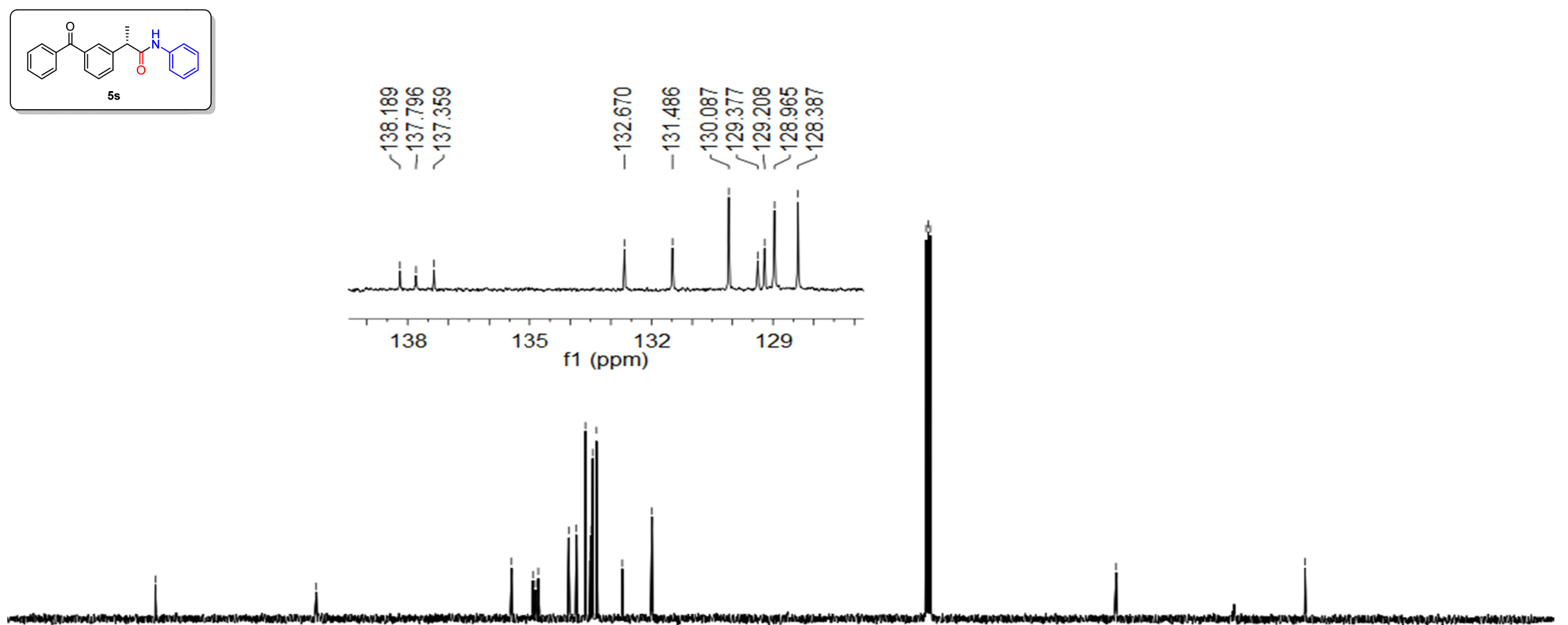

$\begin{array}{lllllllllll}210 & 200 & 190 & 180 & 170 & 160 & 150 & 140 & 130 & 120 & 110 \begin{array}{c}100 \\ \mathrm{f} 1(\mathrm{ppm})\end{array}\end{array}$

80

$70 \quad 60$

$50 \quad 40$

30

2010

$0 \quad-10$

S171 
${ }^{1} \mathrm{H}$ NMR (400 MHz, $d^{6}$-DMSO) of compound $\mathbf{5 t}$

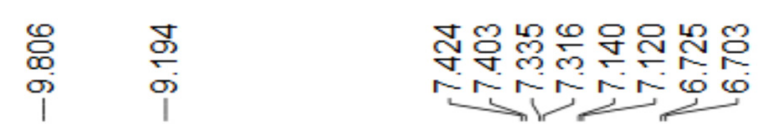

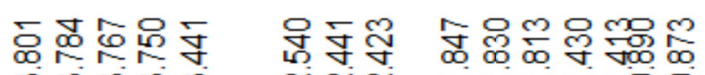

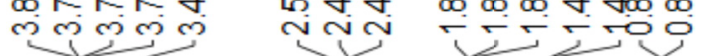
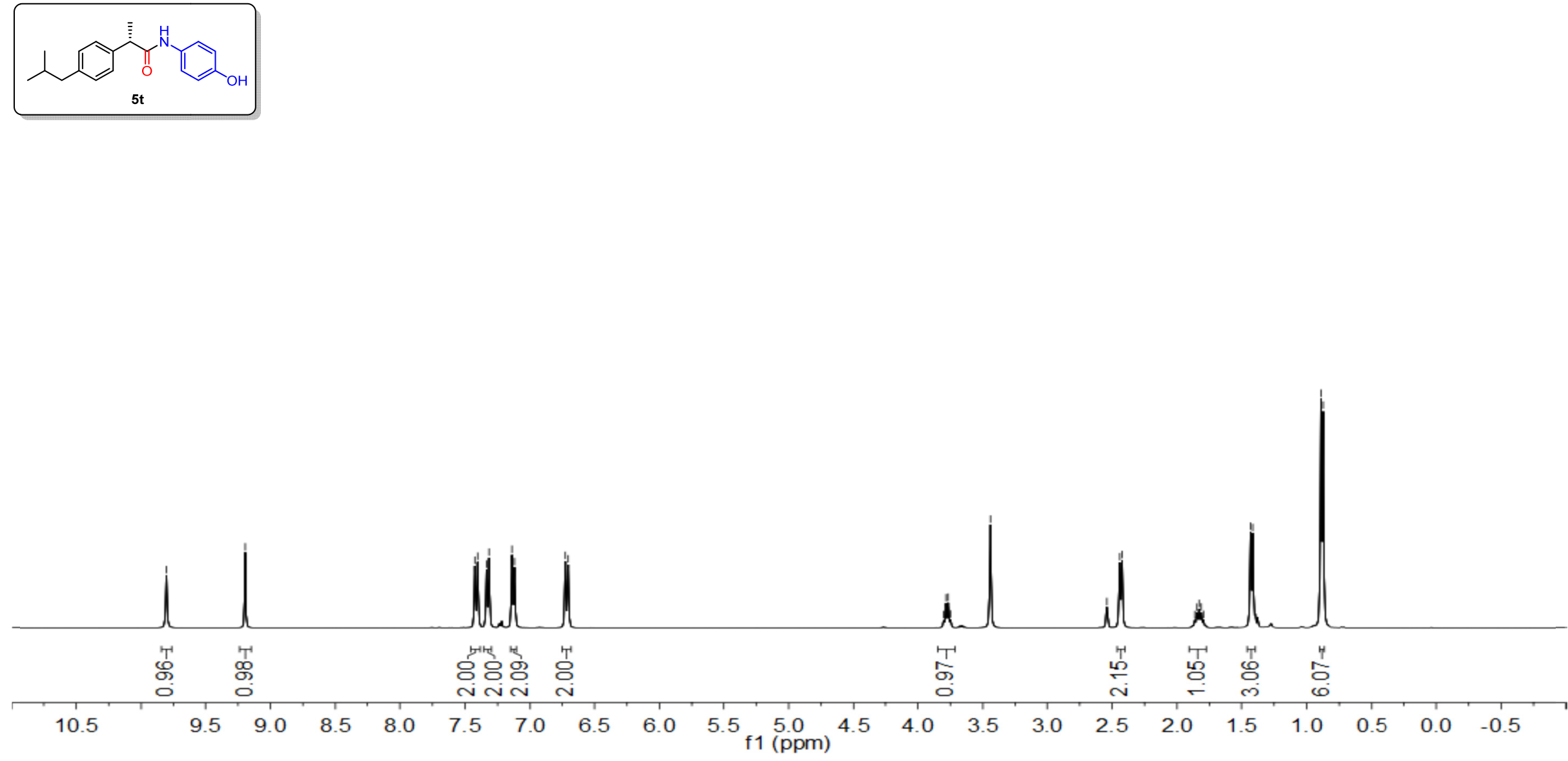
${ }^{13} \mathrm{C}$ NMR (101 MHz, $d^{6}$-DMSO) of compound $\mathbf{5 t}$

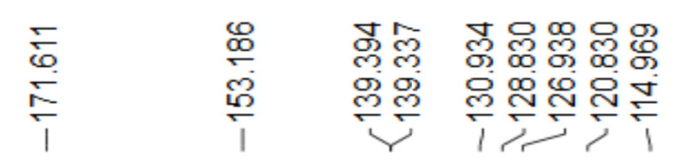

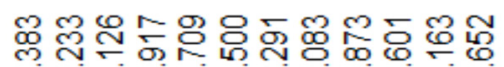

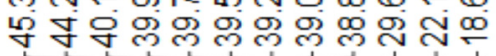

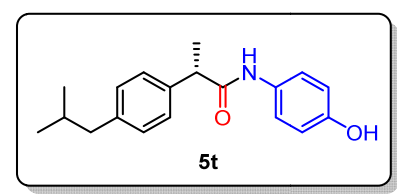

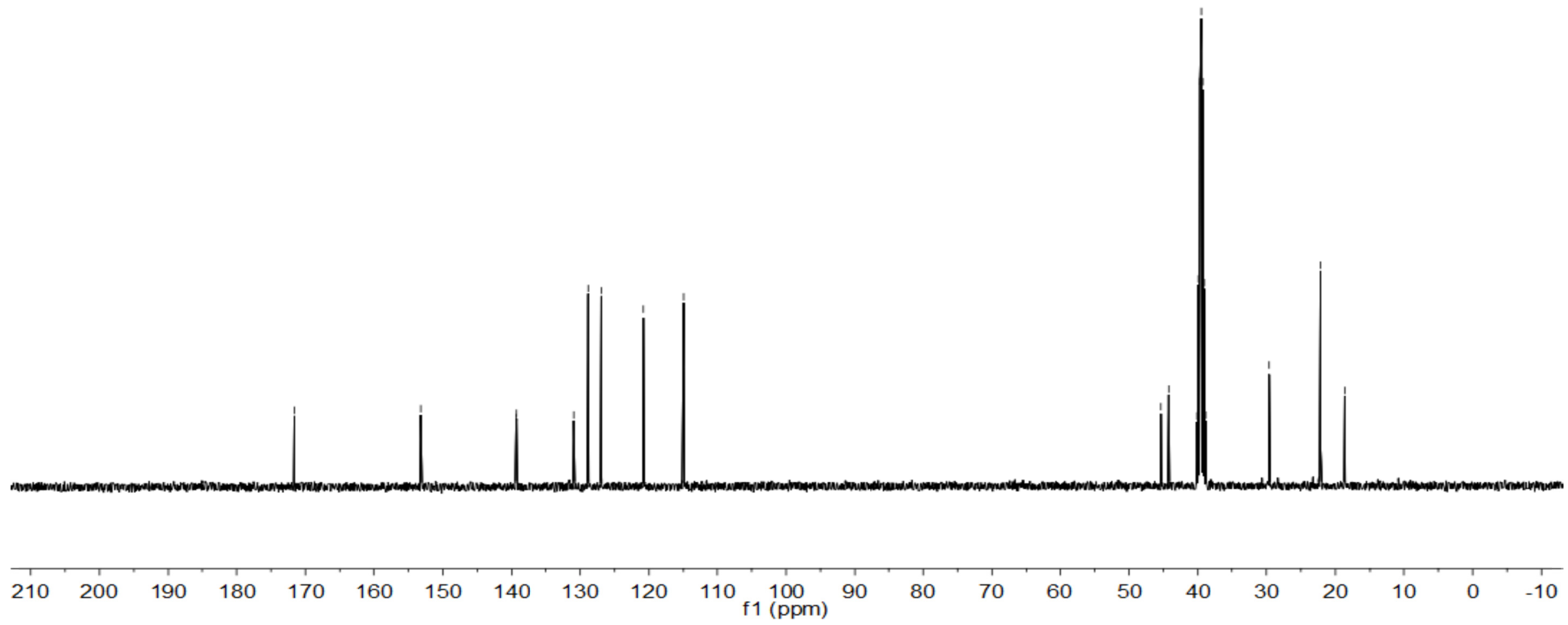


${ }^{1} \mathrm{H}$ NMR (400 MHz, $\mathrm{CDCl}_{3}$ ) of compound 6

ูํํํํำ

Niv

농요용
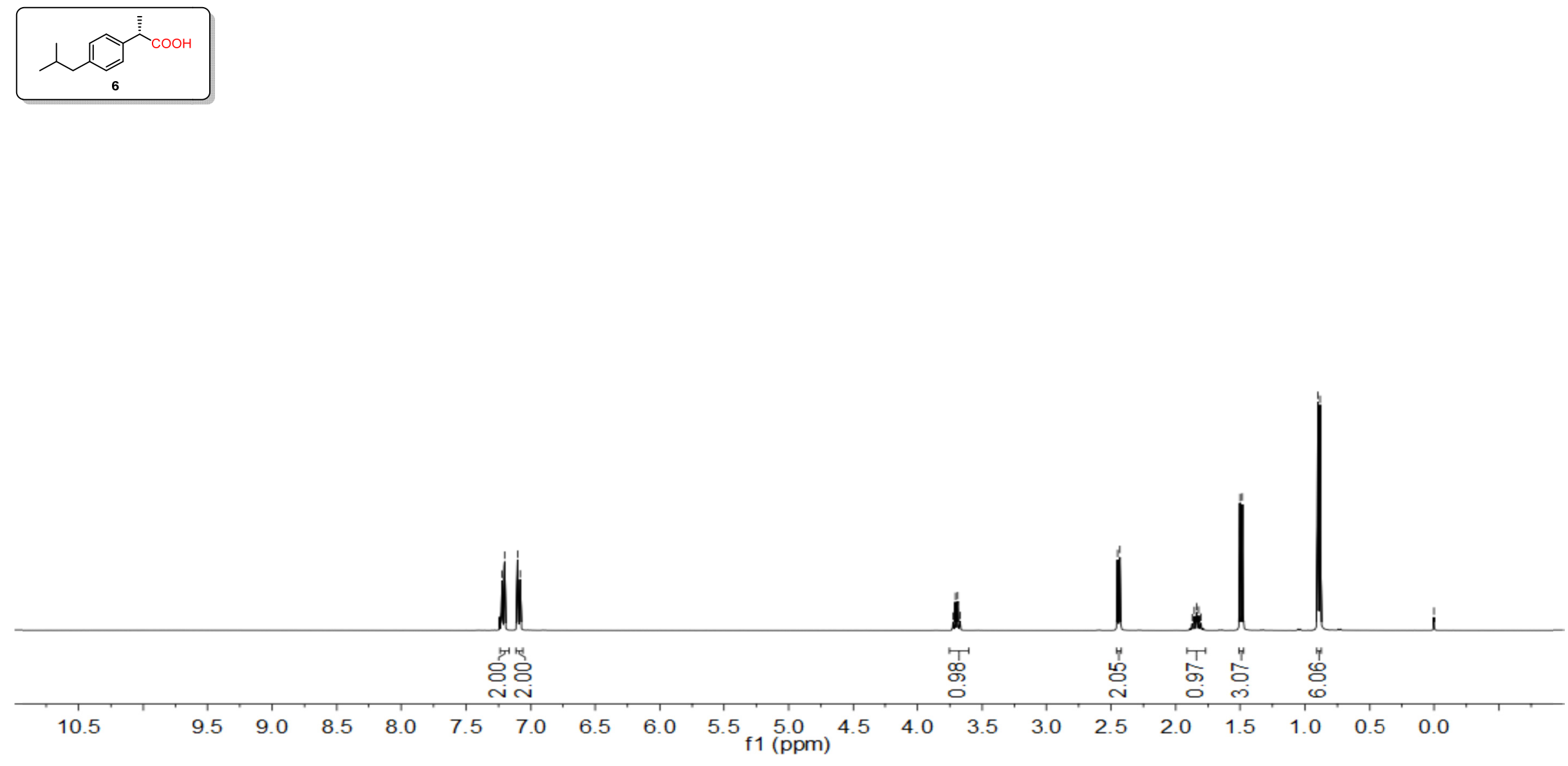
${ }^{13} \mathrm{C}$ NMR $\left(101 \mathrm{MHz}, \mathrm{CDCl}_{3}\right)$ of compound 6
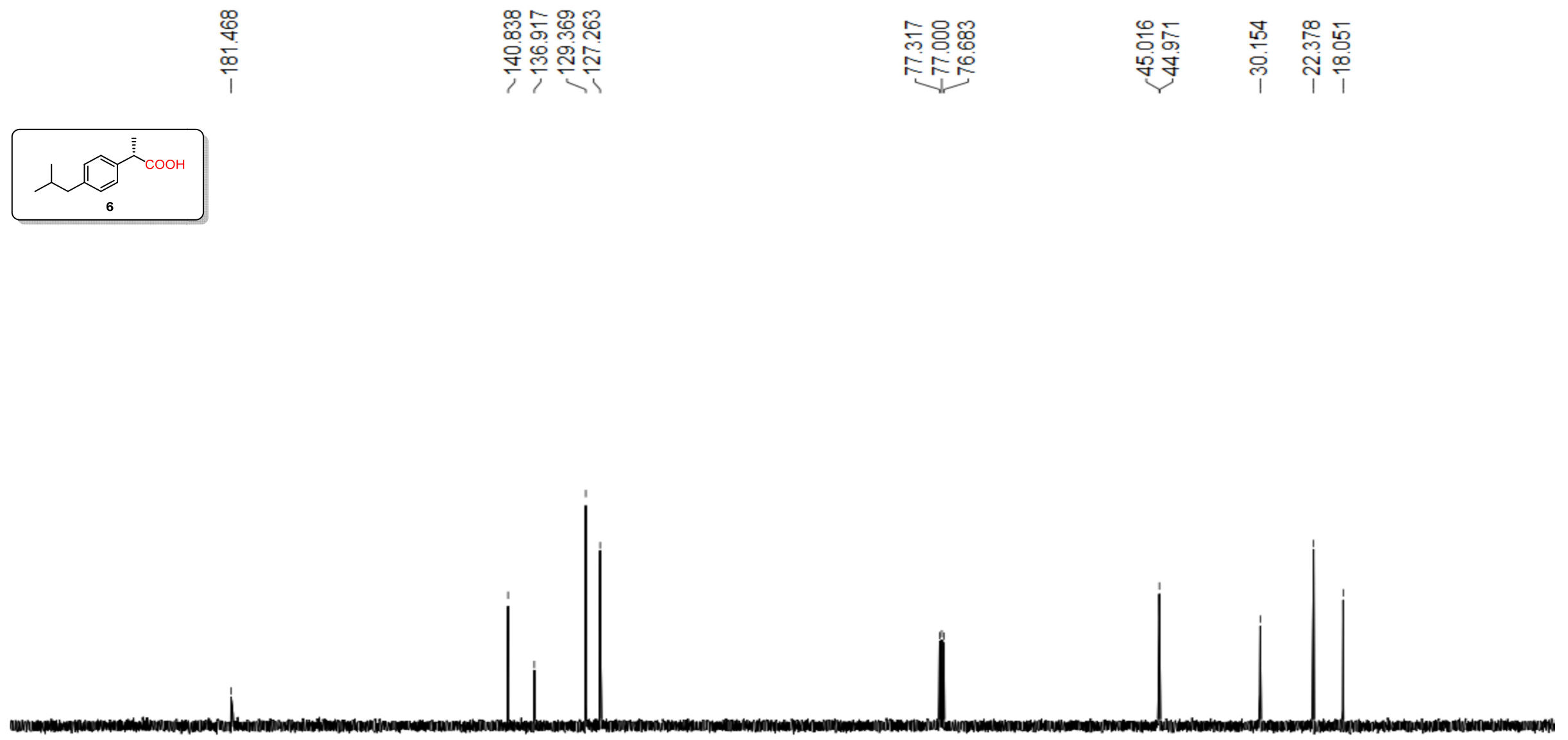
${ }^{1} \mathrm{H}$ NMR (400 MHz, $\mathrm{CDCl}_{3}$ ) of compound 7

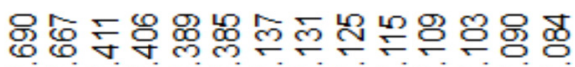

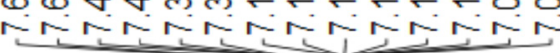

œ

लंखल

串

옹
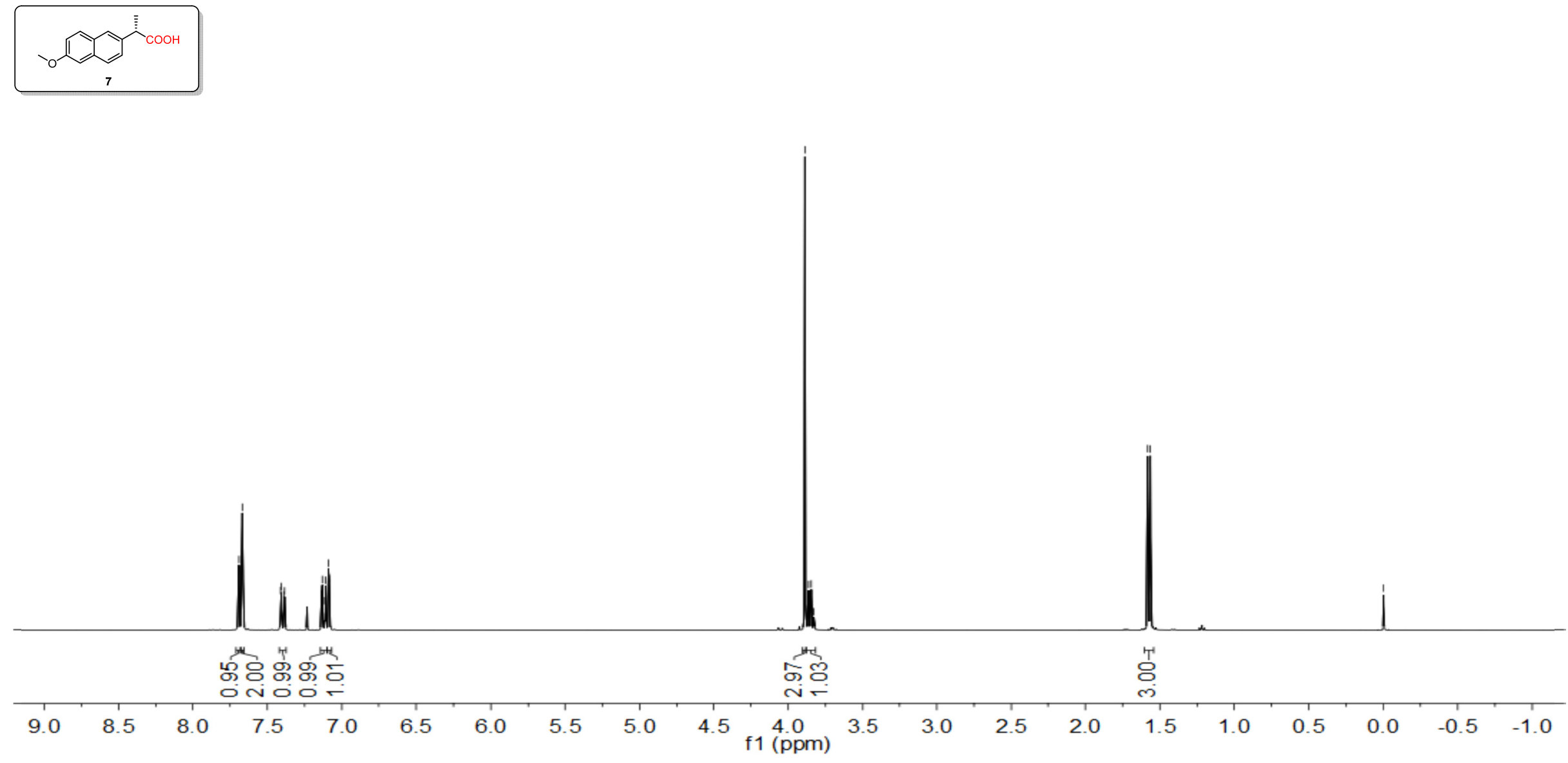

S176 
${ }^{13} \mathrm{C}$ NMR (101 MHz, $\left.\mathrm{CDCl}_{3}\right)$ of compound 7

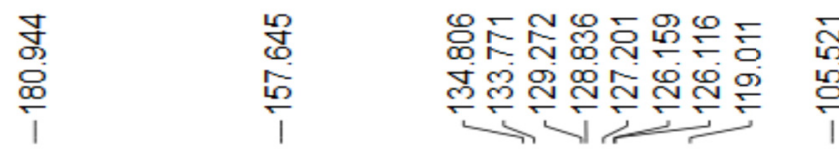

帘

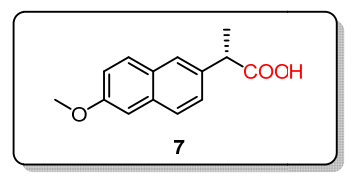

응

FE

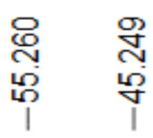

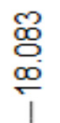

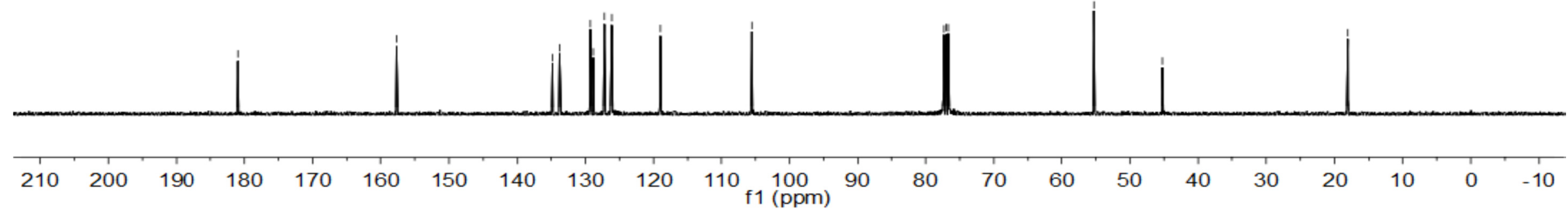


${ }^{1} \mathrm{H}$ NMR (400 MHz, $\mathrm{CDCl}_{3}$ ) of compound 8

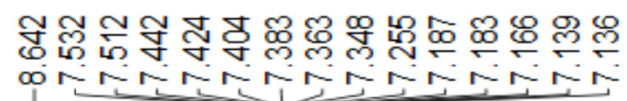

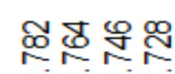

गल लि

承

응
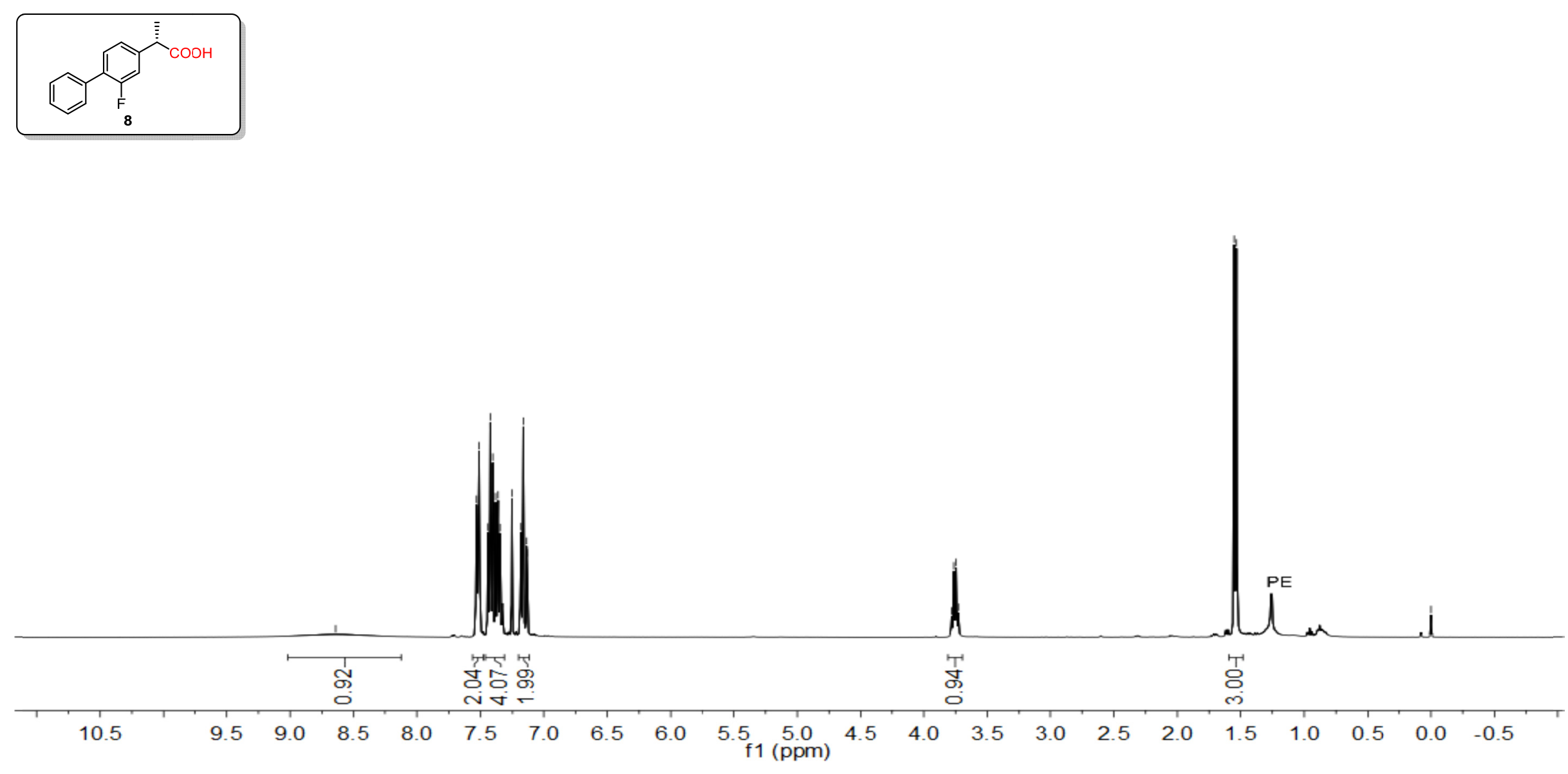
${ }^{13} \mathrm{C}$ NMR $\left(101 \mathrm{MHz}, \mathrm{CDCl}_{3}\right)$ of compound 8

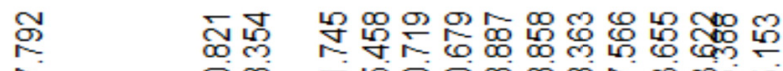

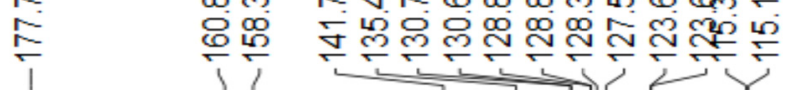

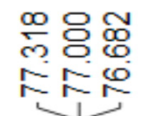

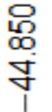

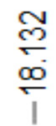
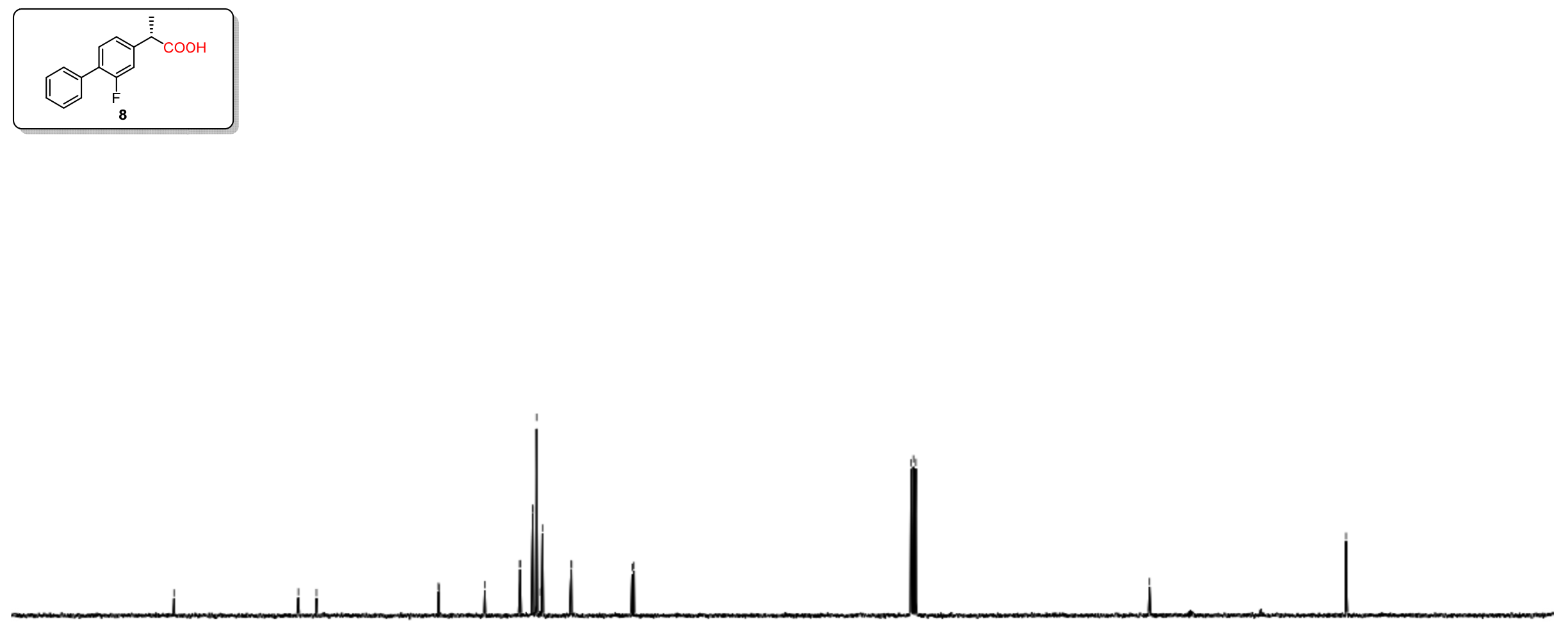

) $190 \quad 180 \quad 170 \quad 160$

$130 \quad 120$

$110 \quad 100 \quad 90$

80

$70 \quad 60$

50

40

30

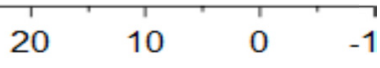


${ }^{19} \mathrm{~F}$ NMR (376 MHz, $\mathrm{CDCl}_{3}$ ) of compound 8

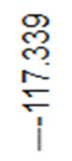
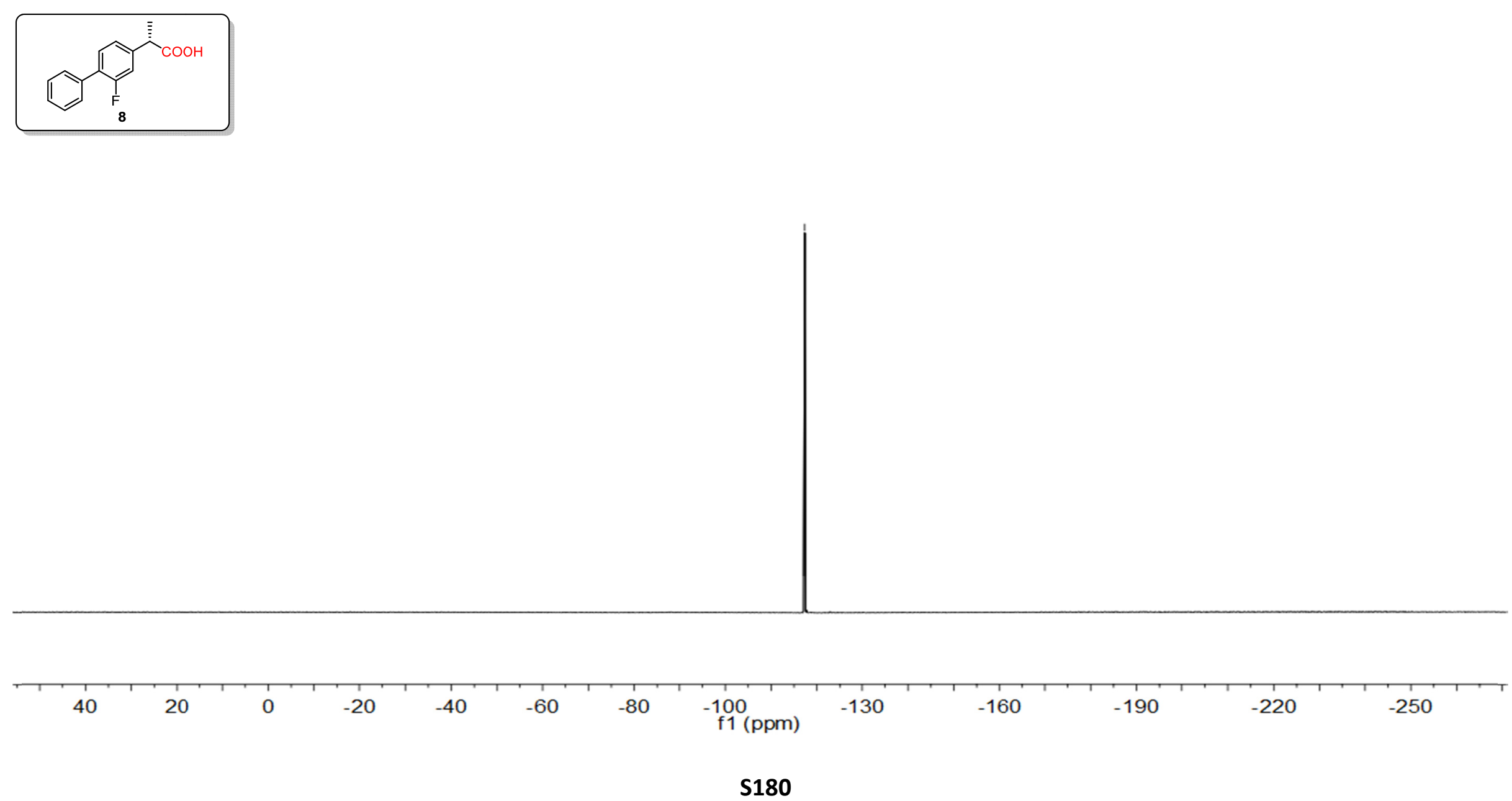
${ }^{1} \mathrm{H}$ NMR (400 MHz, $\mathrm{CDCl}_{3}$ ) of compound 9a

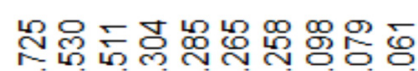

N-n

rarrarrar

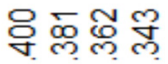

거서

ํำ

$\underbrace{2}$

兽
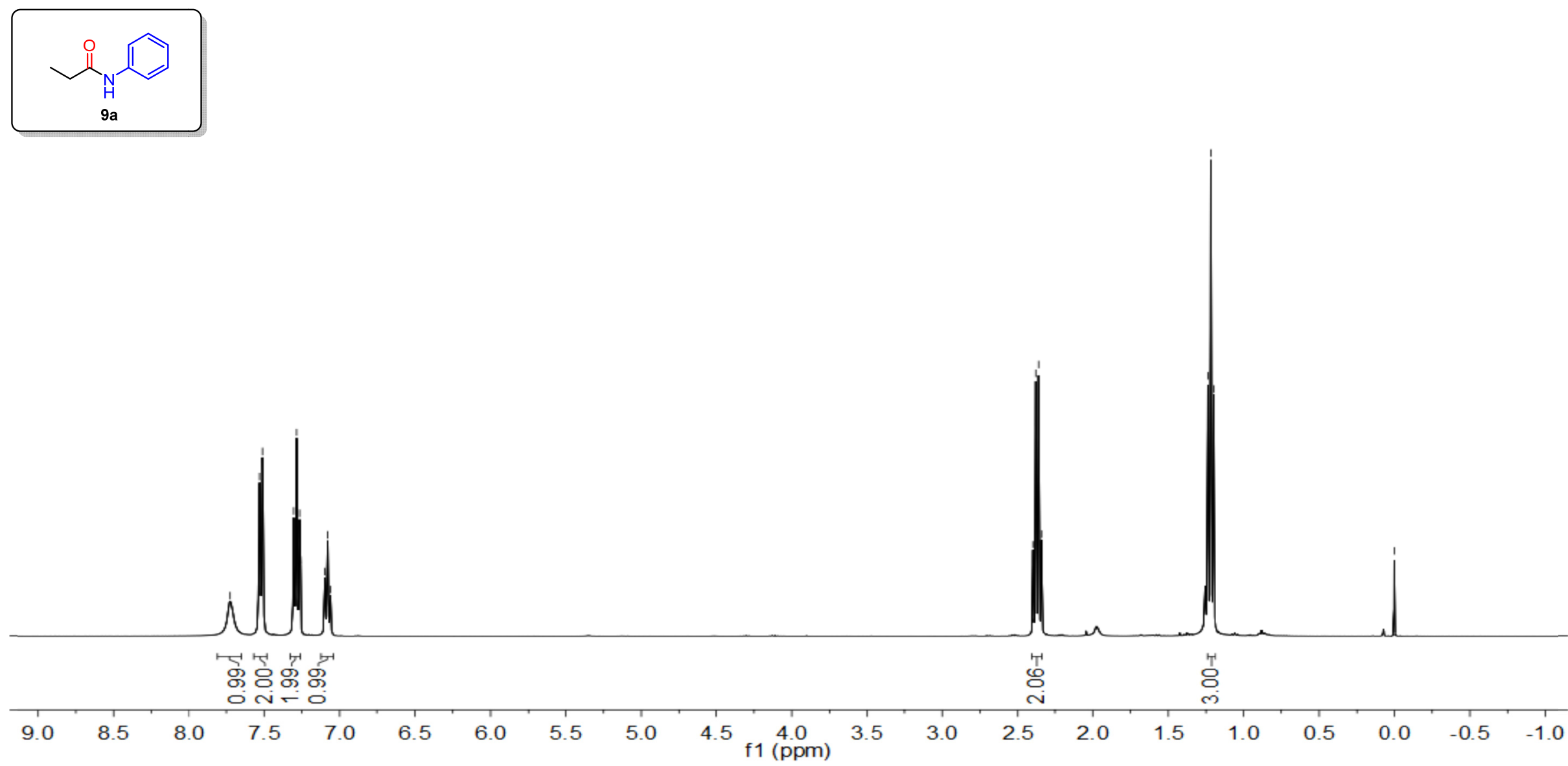

S181 
${ }^{13} \mathrm{C}$ NMR (101 MHz, $\mathrm{CDCl}_{3}$ ) of compound 9a

\begin{tabular}{|c|c|c|c|c|}
\hline 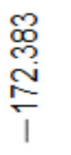 & $\begin{array}{l}\mathscr{\mathscr { O }} \\
\frac{\mathrm{g}}{\mathrm{m}} \\
\frac{\mathrm{m}}{1}\end{array}$ & 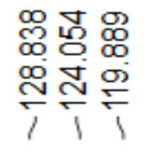 & 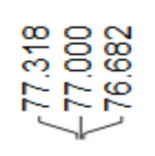 & $\begin{array}{l}\text { D } \\
\text { م. } \\
\text { م) } \\
1\end{array}$ \\
\hline
\end{tabular}
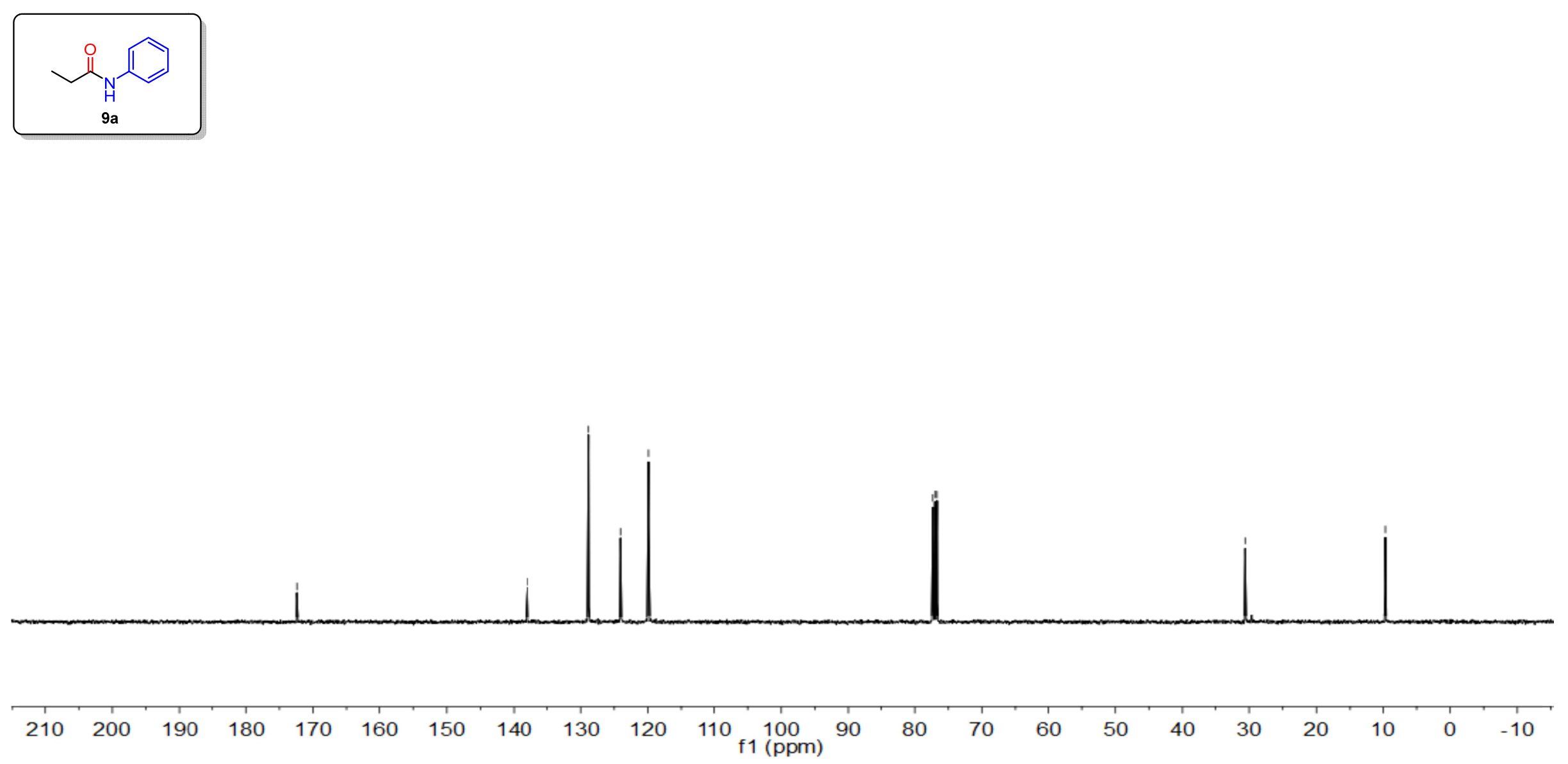
${ }^{1} \mathrm{H}$ NMR (400 MHz, $\mathrm{CDCl}_{3}$ ) of compound $9 \mathbf{b}$

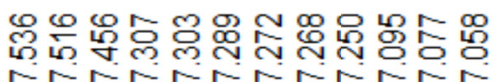

rnn-Nan

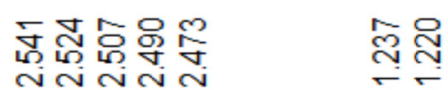

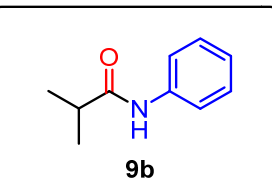

$9 b$

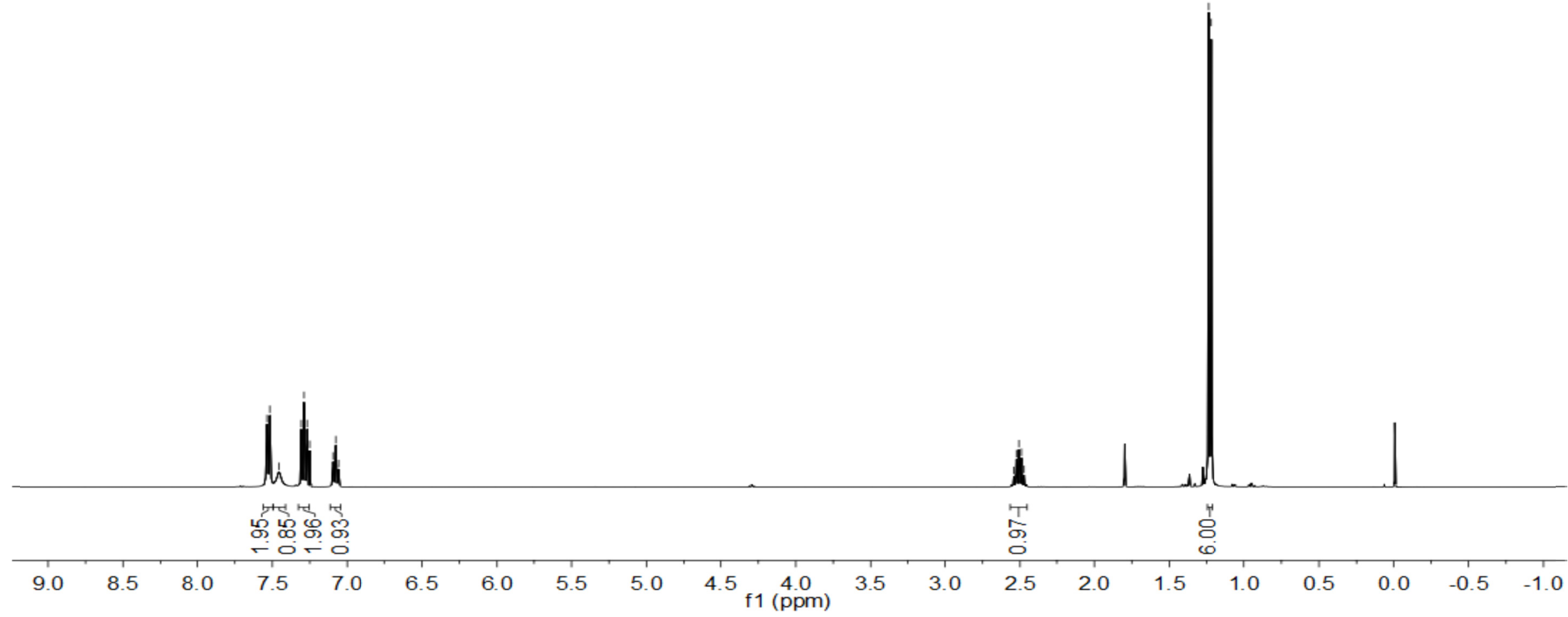


${ }^{13} \mathrm{C}$ NMR $\left(101 \mathrm{MHz}, \mathrm{CDCl}_{3}\right)$ of compound $\mathbf{9 b}$

\begin{tabular}{|c|c|c|c|c|}
\hline$F$ & 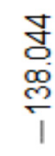 & 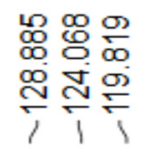 & 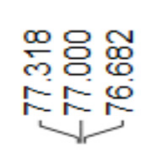 & $\begin{array}{l} \pm \\
\infty \\
\infty \\
0 \\
0 \\
0 \\
1\end{array}$ \\
\hline
\end{tabular}
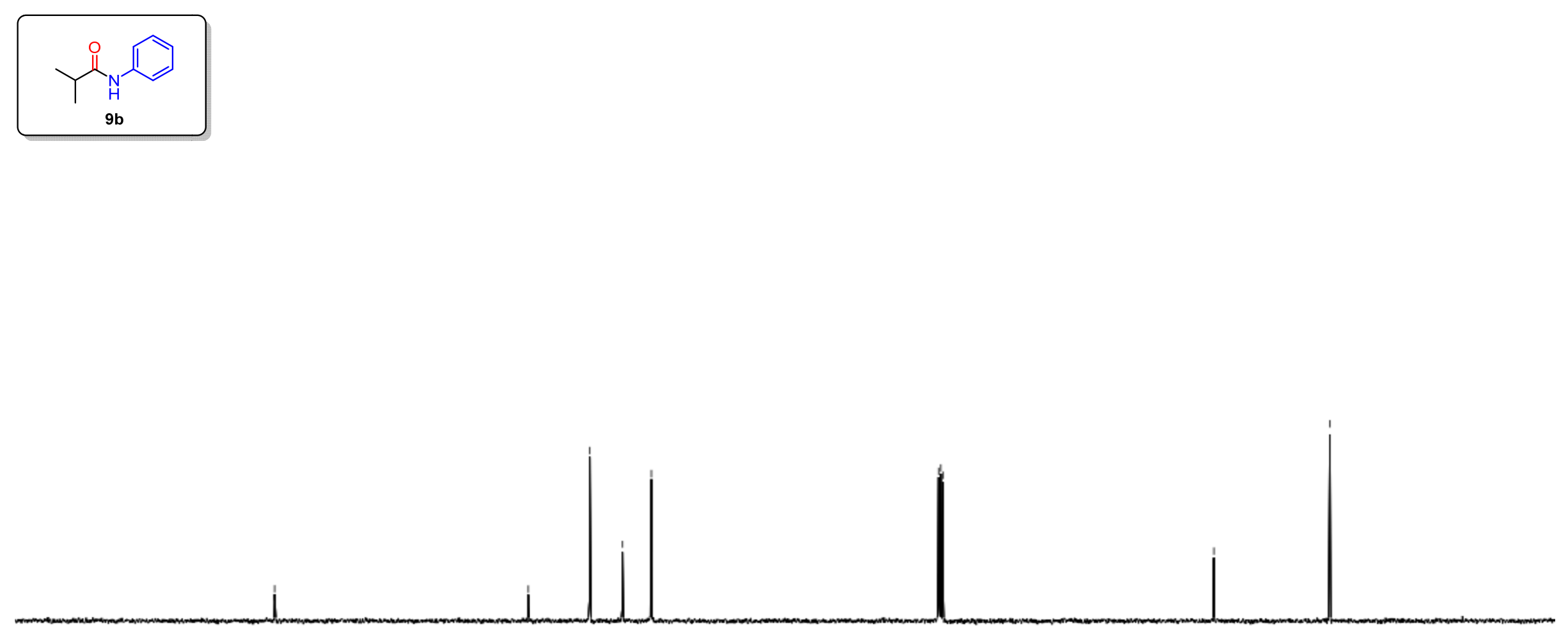

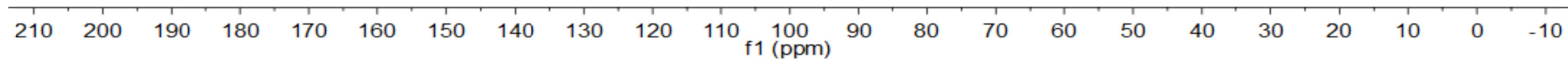


${ }^{1} \mathrm{H}$ NMR (400 MHz, $\mathrm{CDCl}_{3}$ ) of compound 9c

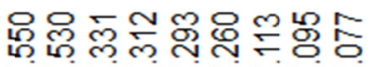

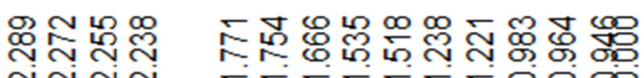

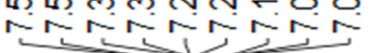

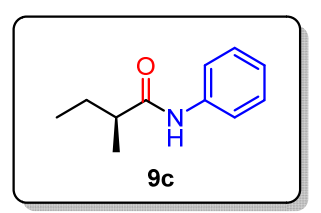

55 5590

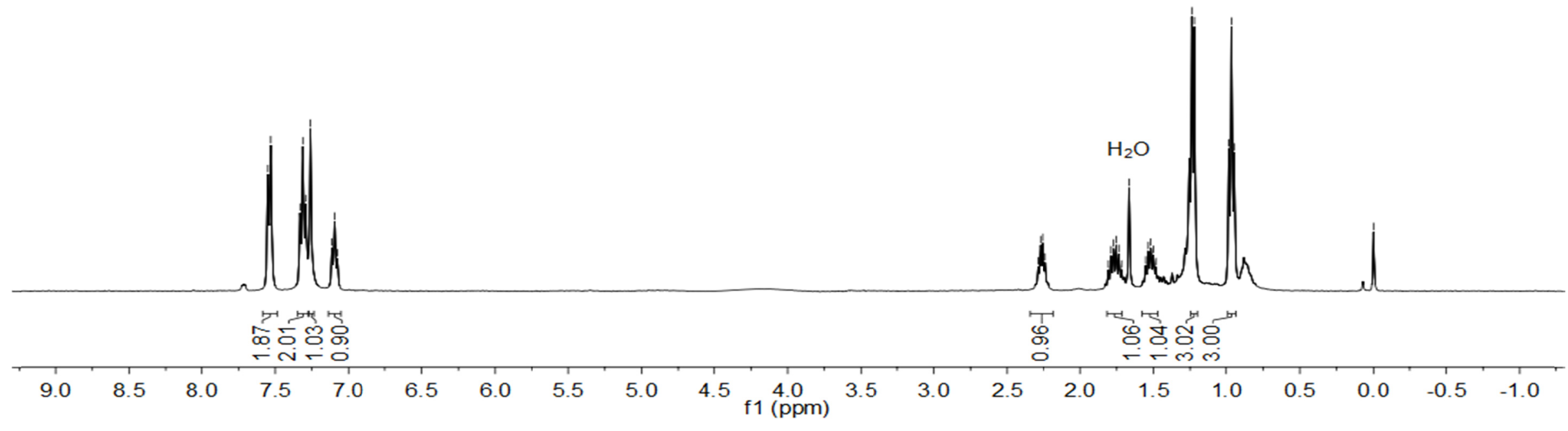

S185 
${ }^{13} \mathrm{C}$ NMR (101 MHz, $\mathrm{CDCl}_{3}$ ) of compound 9c

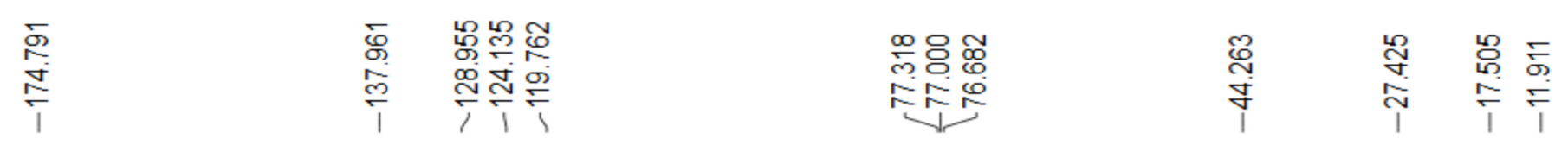
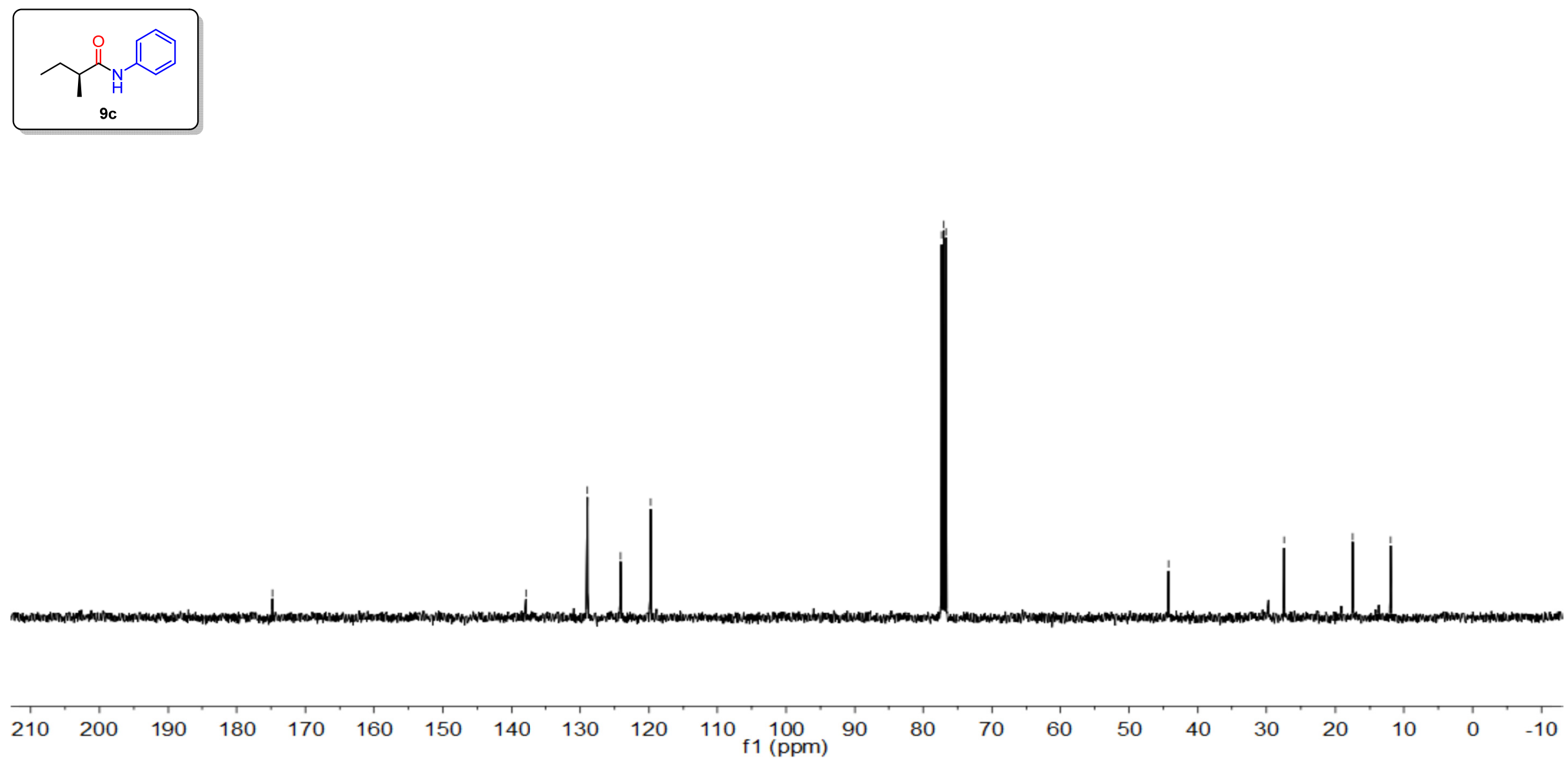
${ }^{1} \mathrm{H}$ NMR (400 MHz, $\mathrm{CDCl}_{3}$ ) of compound 9d

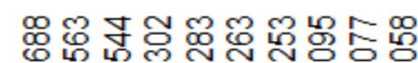

NNMNrNon

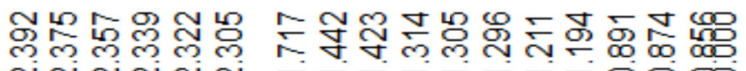

लNलNNN
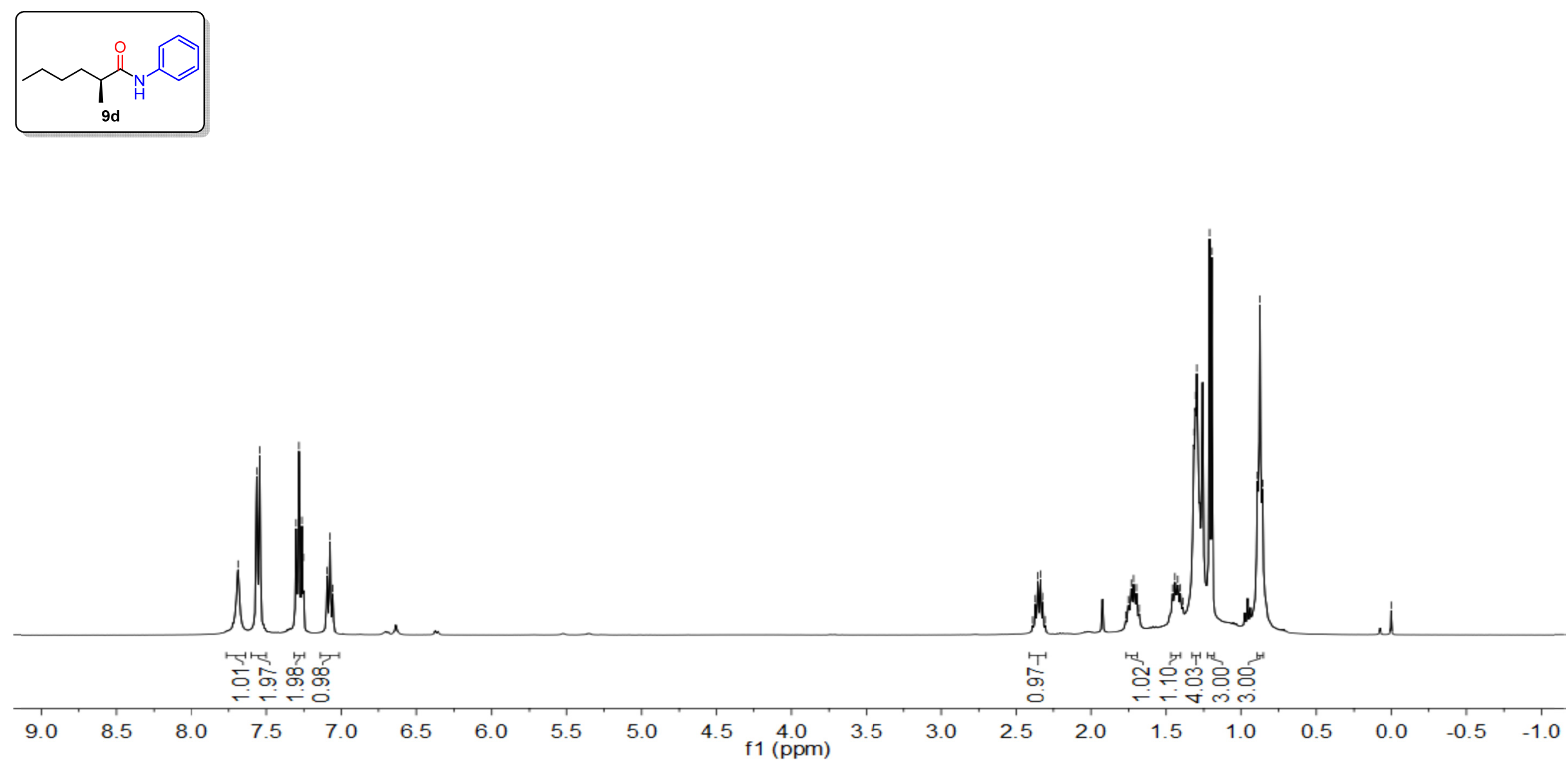
${ }^{13} \mathrm{C}$ NMR (101 MHz, $\left.\mathrm{CDCl}_{3}\right)$ of compound 9d
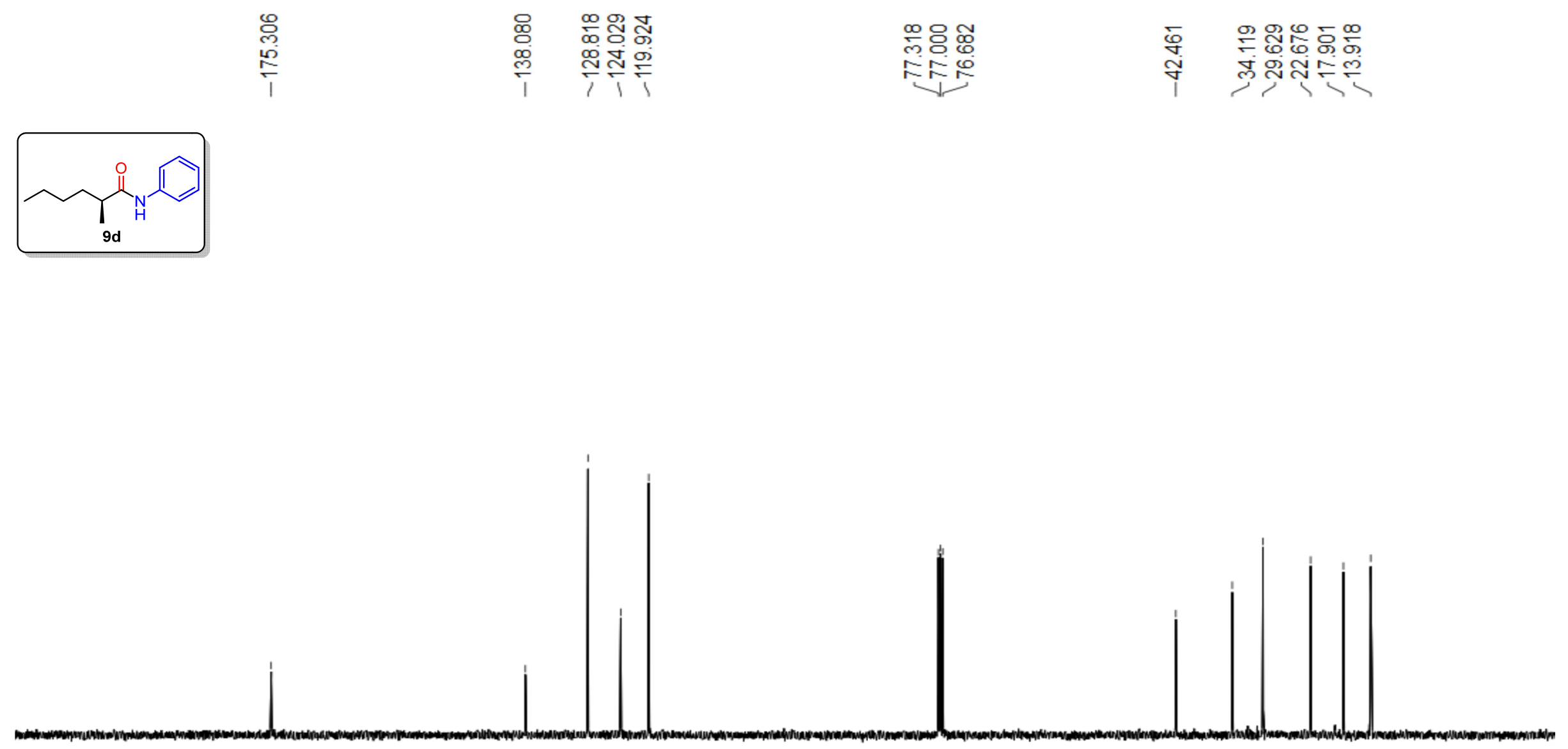

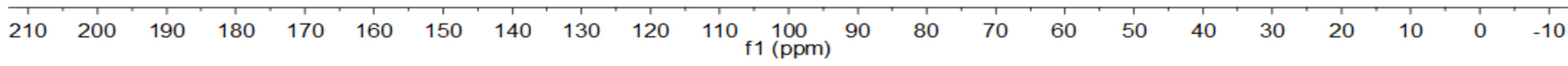


${ }^{1} \mathrm{H}$ NMR (400 MHz, $\mathrm{CDCl}_{3}$ ) of compound 9e

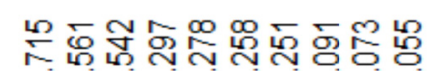

r.inN

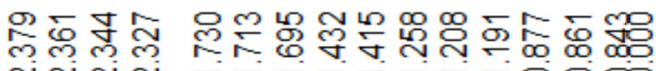

Niñ
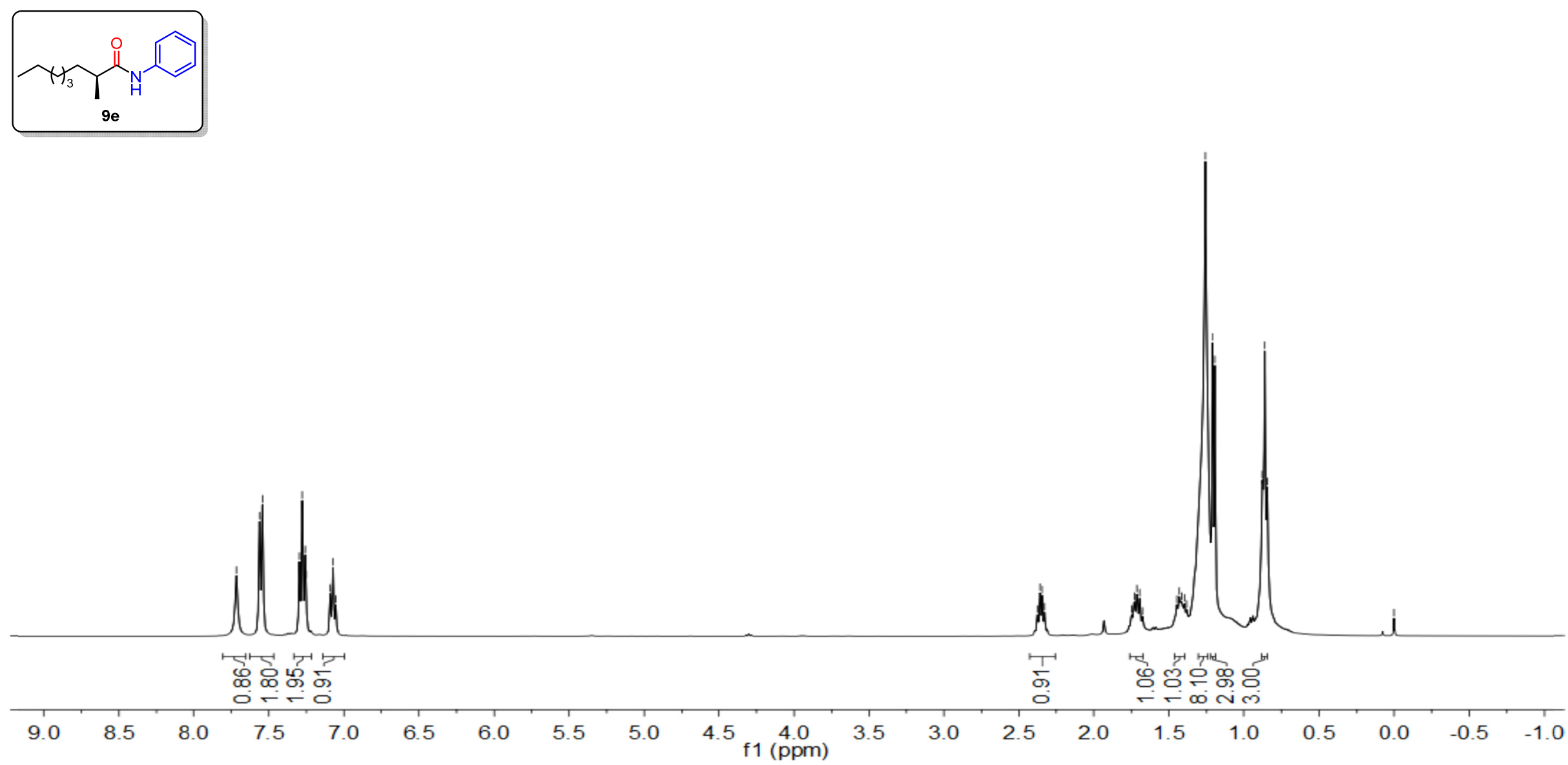

S189 
${ }^{13} \mathrm{C}$ NMR (101 MHz, $\mathrm{CDCl}_{3}$ ) of compound 9e

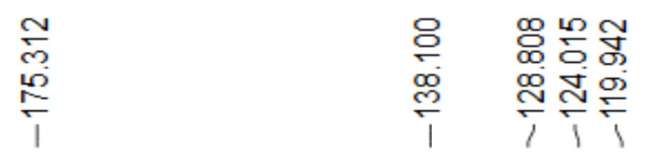

응 응

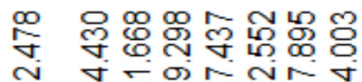

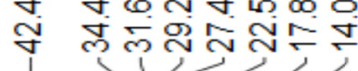
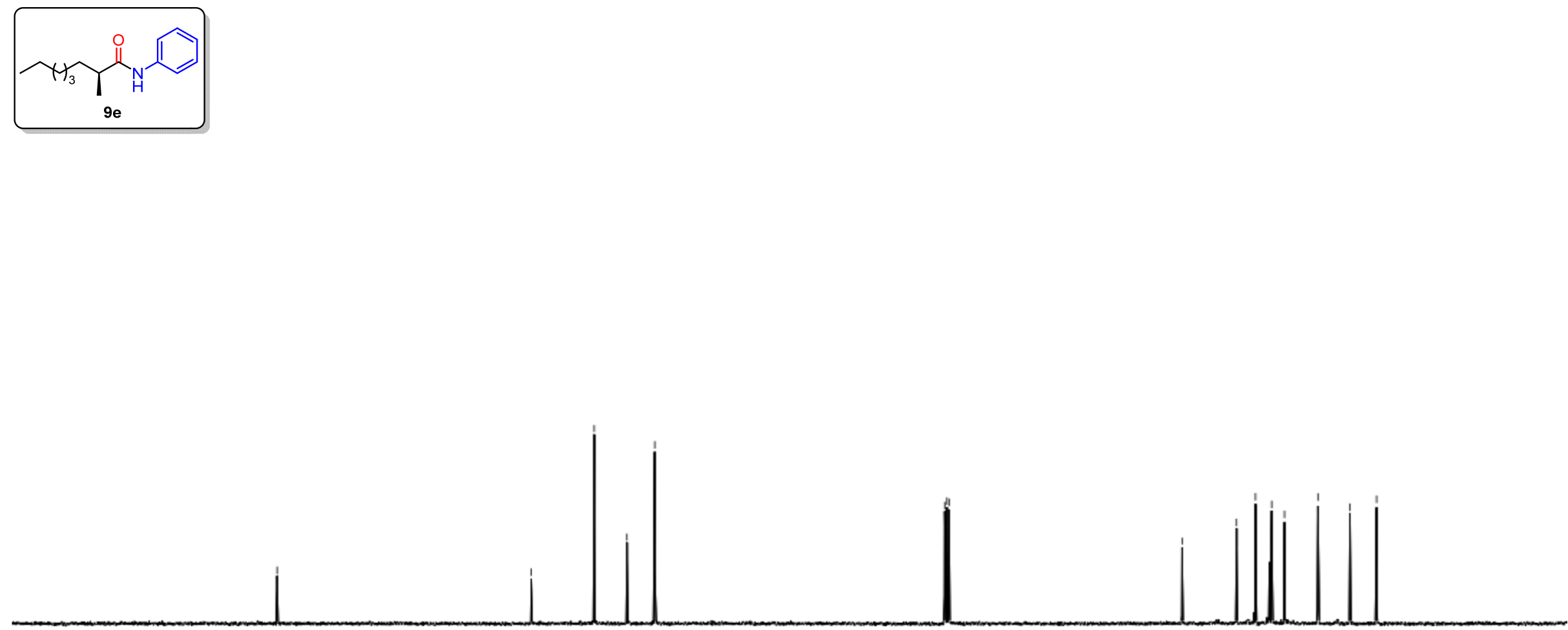

$\begin{array}{lllllllllll}210 & 200 & 190 & 180 & 170 & 160 & 150 & 140 & 130 & 120 & 110 \begin{array}{l}100 \\ \mathrm{f} 1(\mathrm{ppm})\end{array}\end{array}$ 
${ }^{1} \mathrm{H}$ NMR (400 MHz, $\mathrm{CDCl}_{3}$ ) of compound $9 \mathbf{f}$

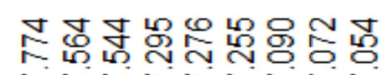

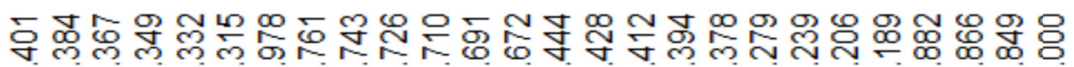

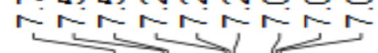

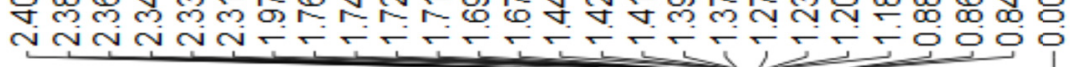
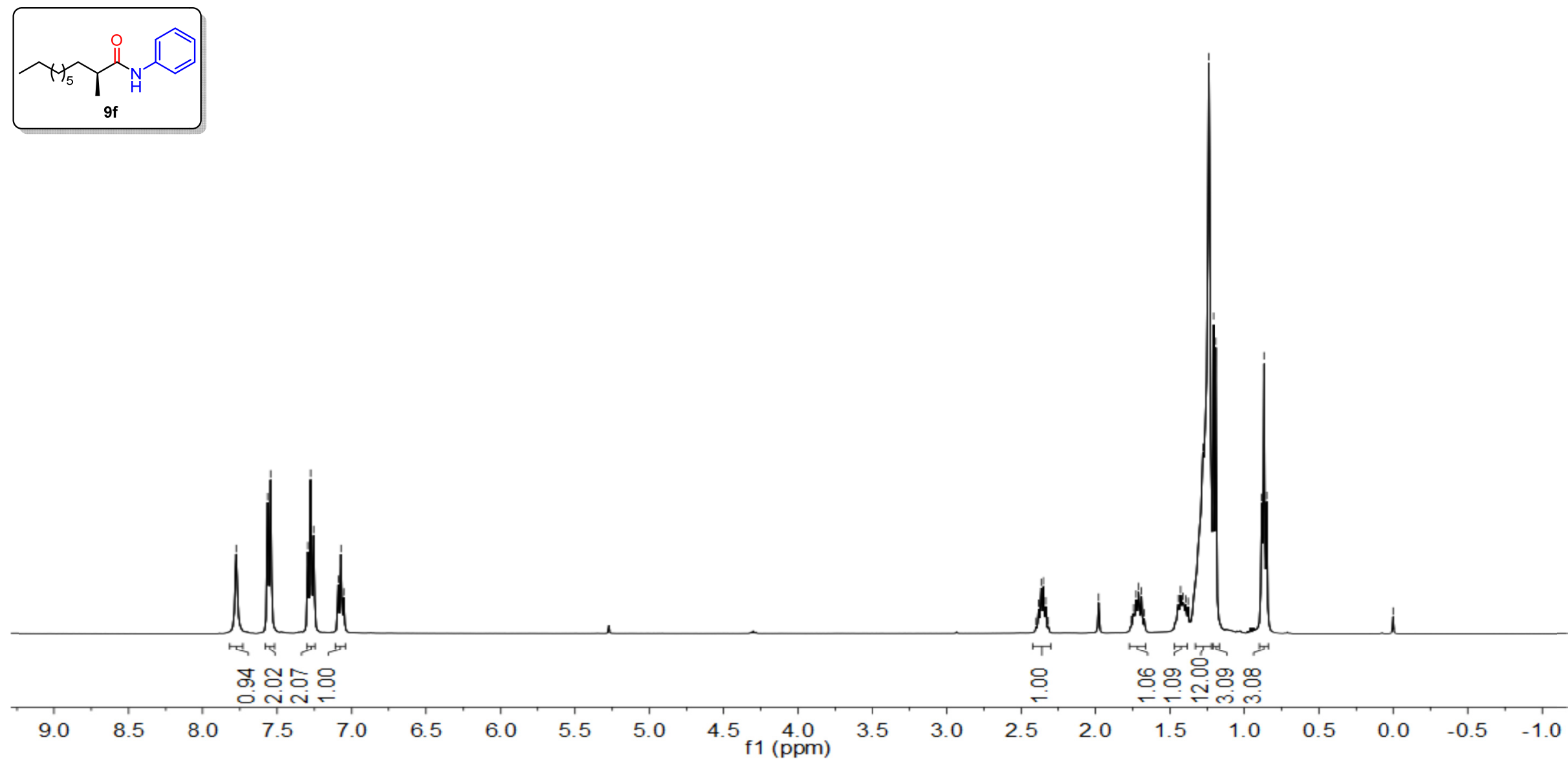
${ }^{13} \mathrm{C}$ NMR (101 MHz, $\mathrm{CDCl}_{3}$ ) of compound $9 f$

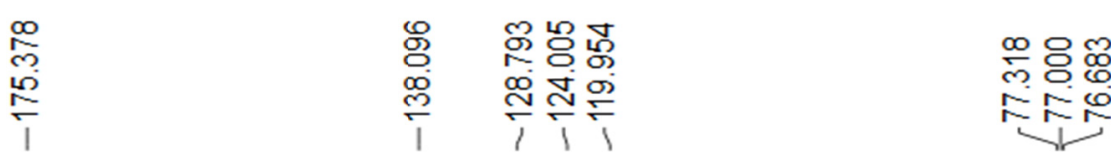

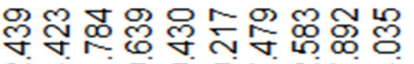

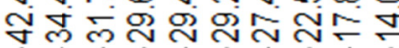
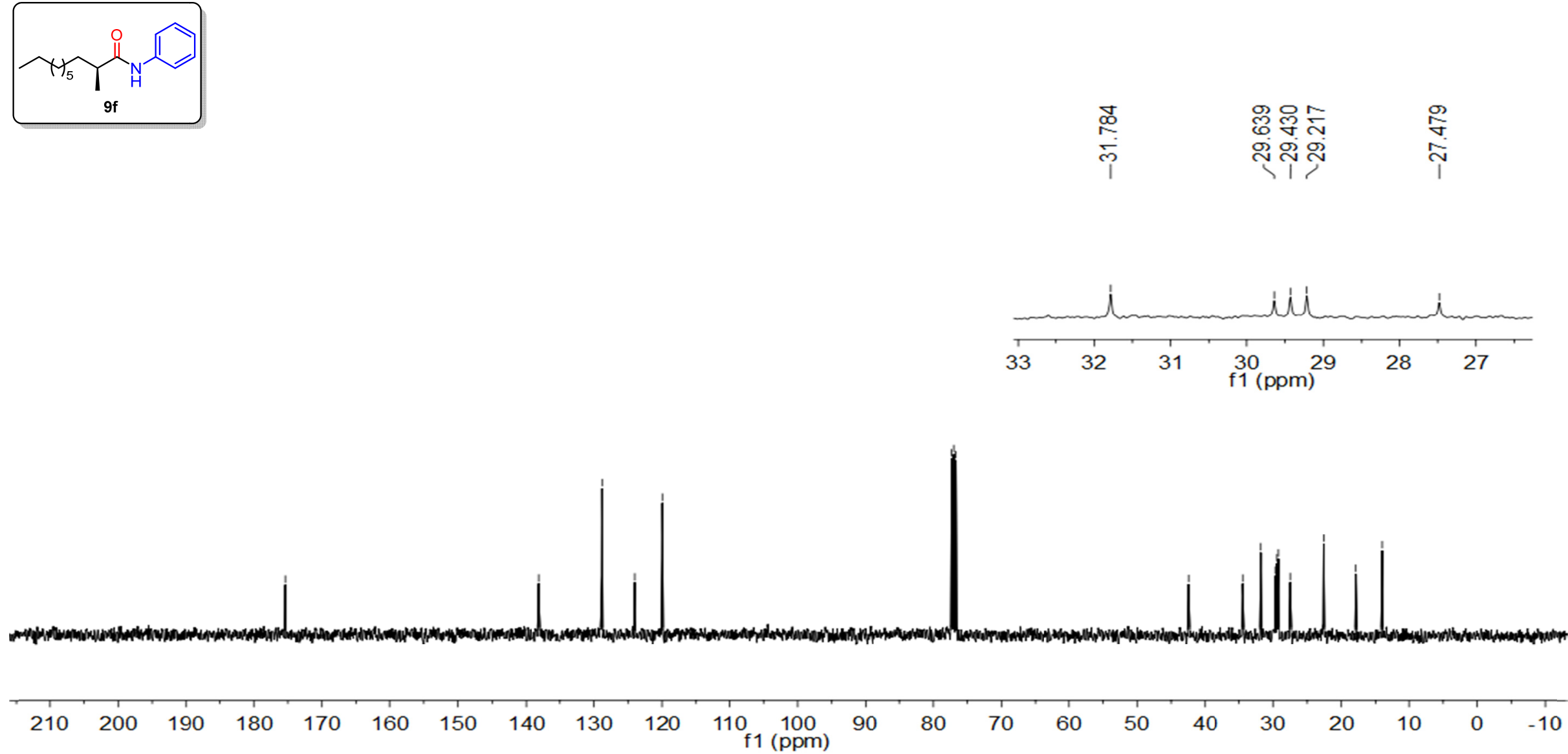
${ }^{1} \mathrm{H}$ NMR (400 MHz, $\mathrm{CDCl}_{3}$ ) of compound $\mathbf{9 g}$

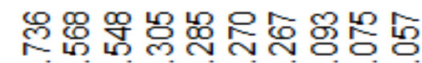

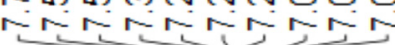

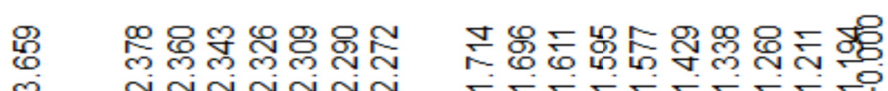

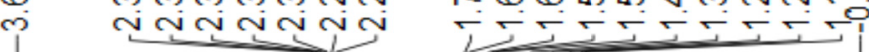
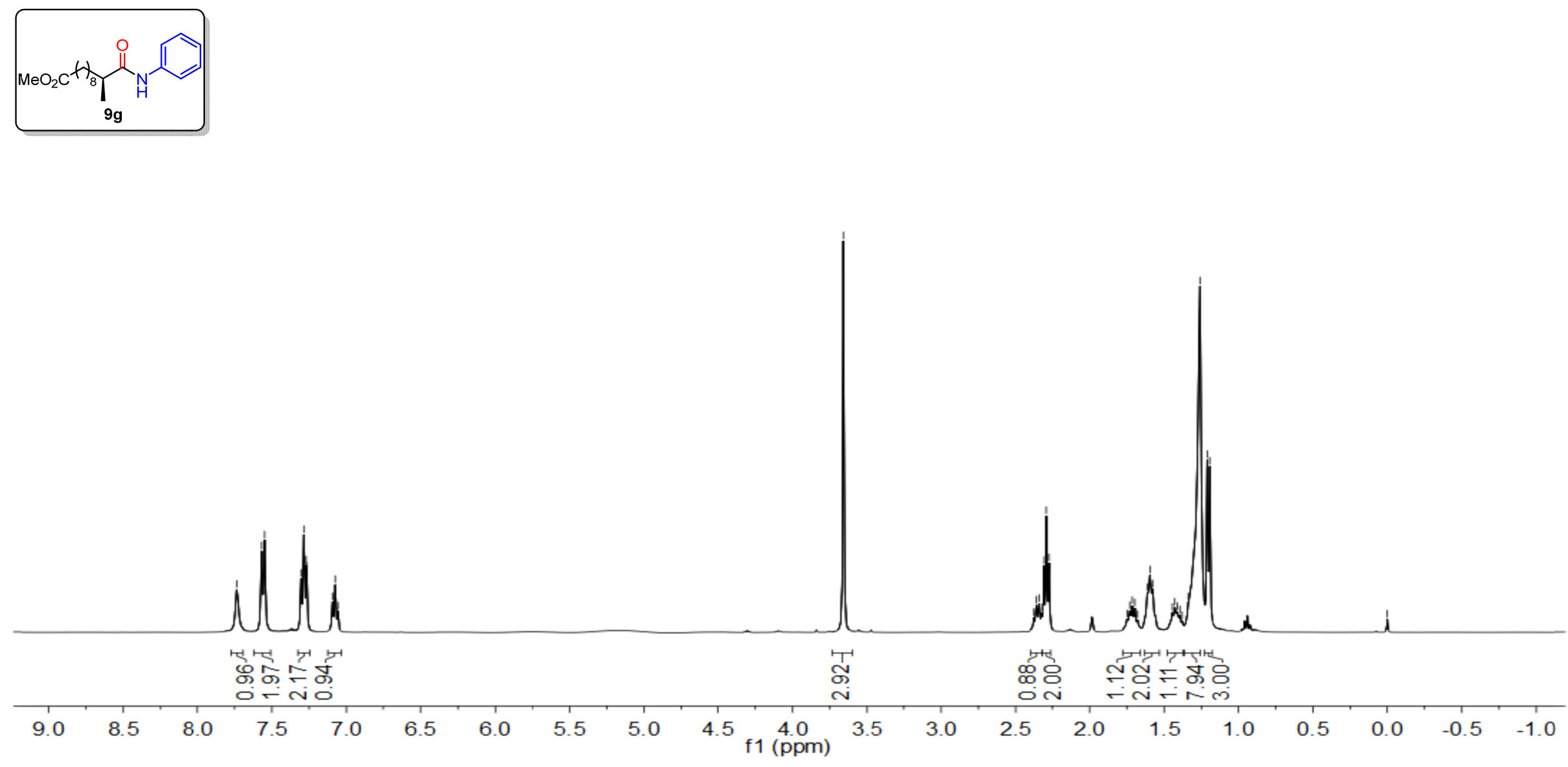
${ }^{13} \mathrm{C}$ NMR (101 MHz, $\left.\mathrm{CDCl}_{3}\right)$ of compound 9g

突怘
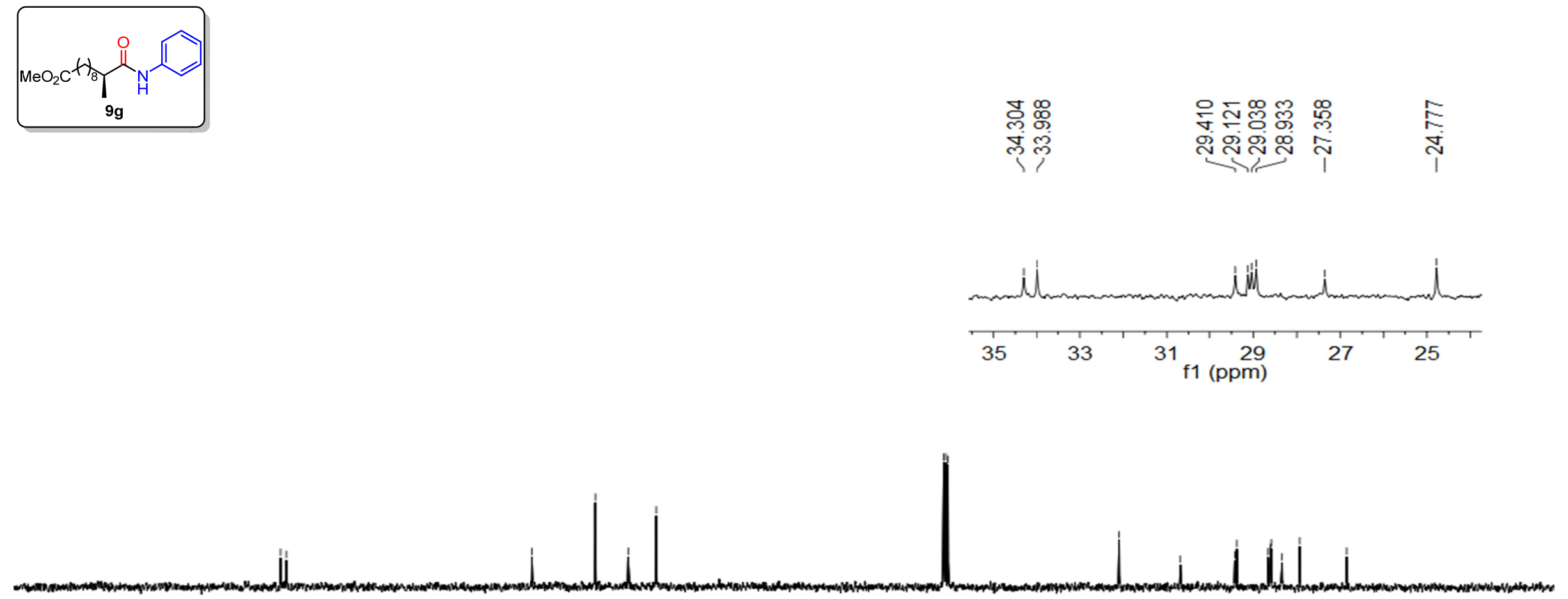

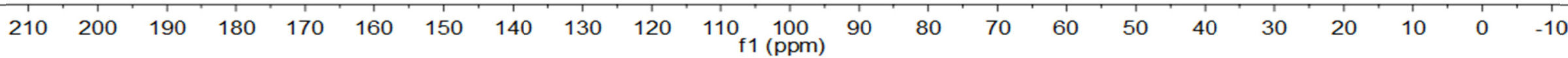


${ }^{1} \mathrm{H}$ NMR (400 MHz, $\mathrm{CDCl}_{3}$ ) of compound $\mathbf{9 h}$

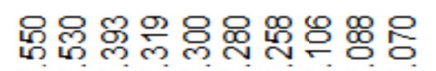

NiñNaris

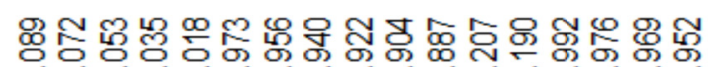

nNNNMTH

兽
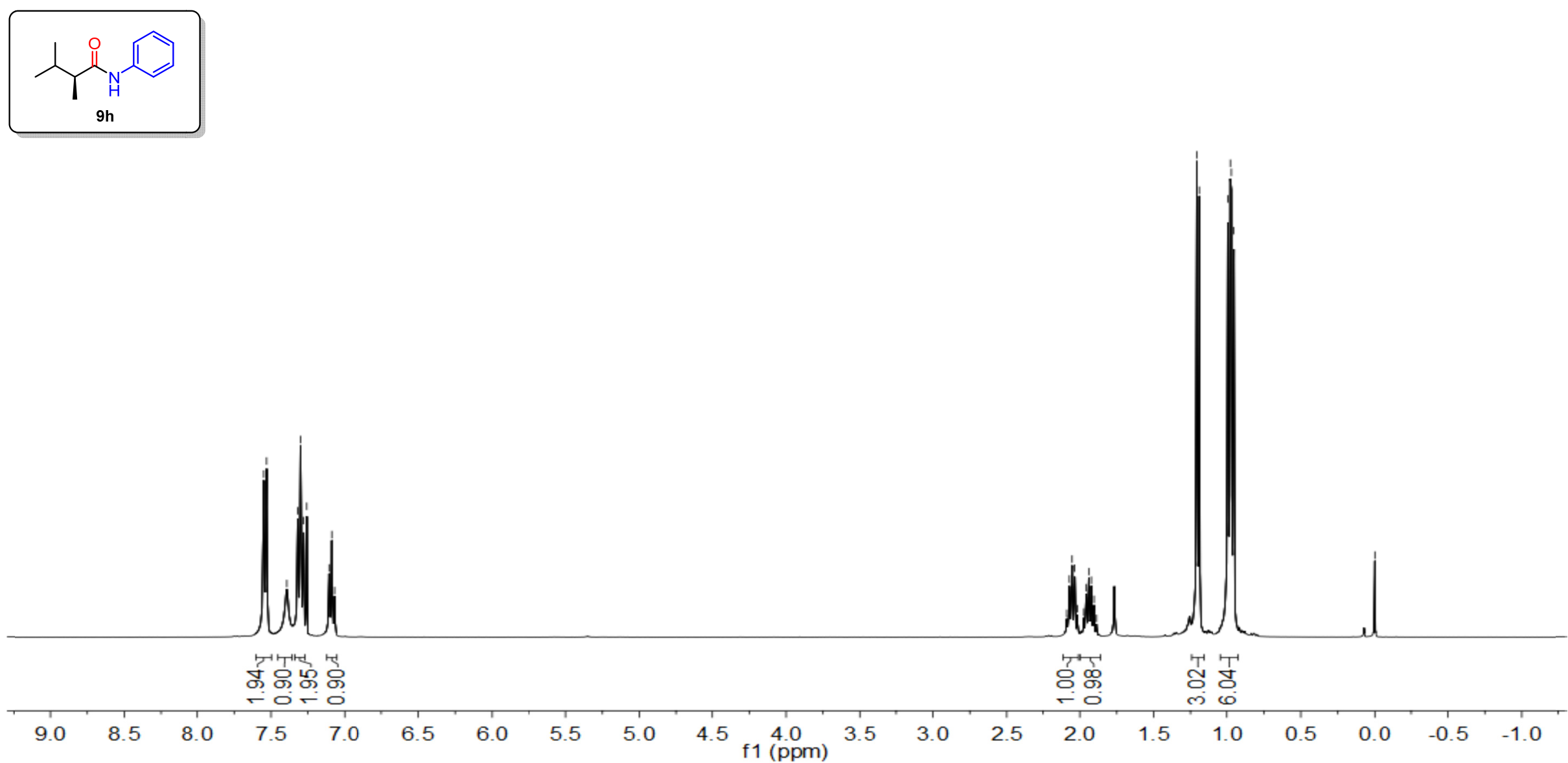

S195 
${ }^{13} \mathrm{C}$ NMR $\left(101 \mathrm{MHz}, \mathrm{CDCl}_{3}\right)$ of compound $\mathbf{9 h}$

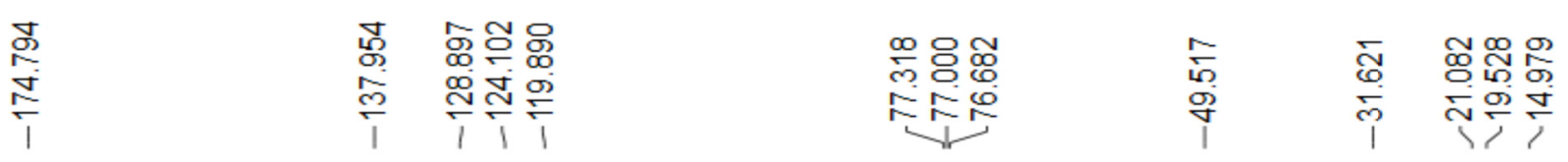
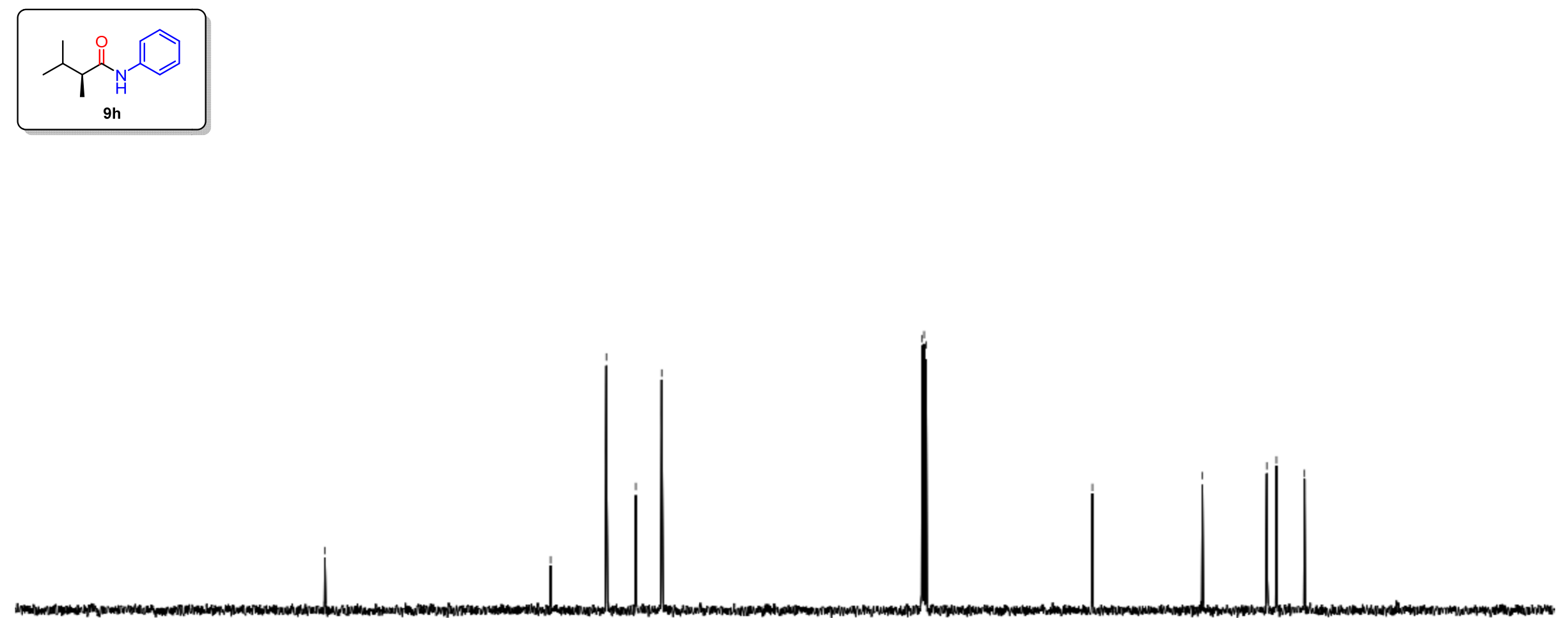

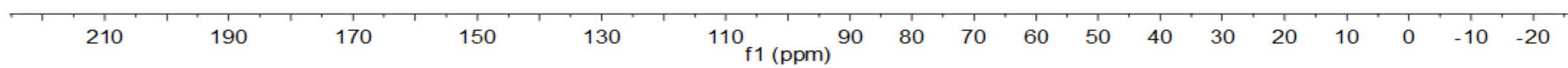


${ }^{1} \mathrm{H}$ NMR (400 MHz, $\mathrm{CDCl}_{3}$ ) of compound $\mathbf{9 i}$

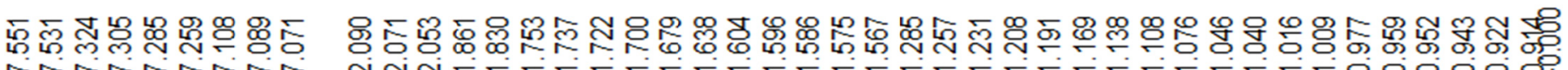

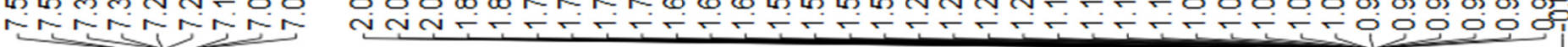
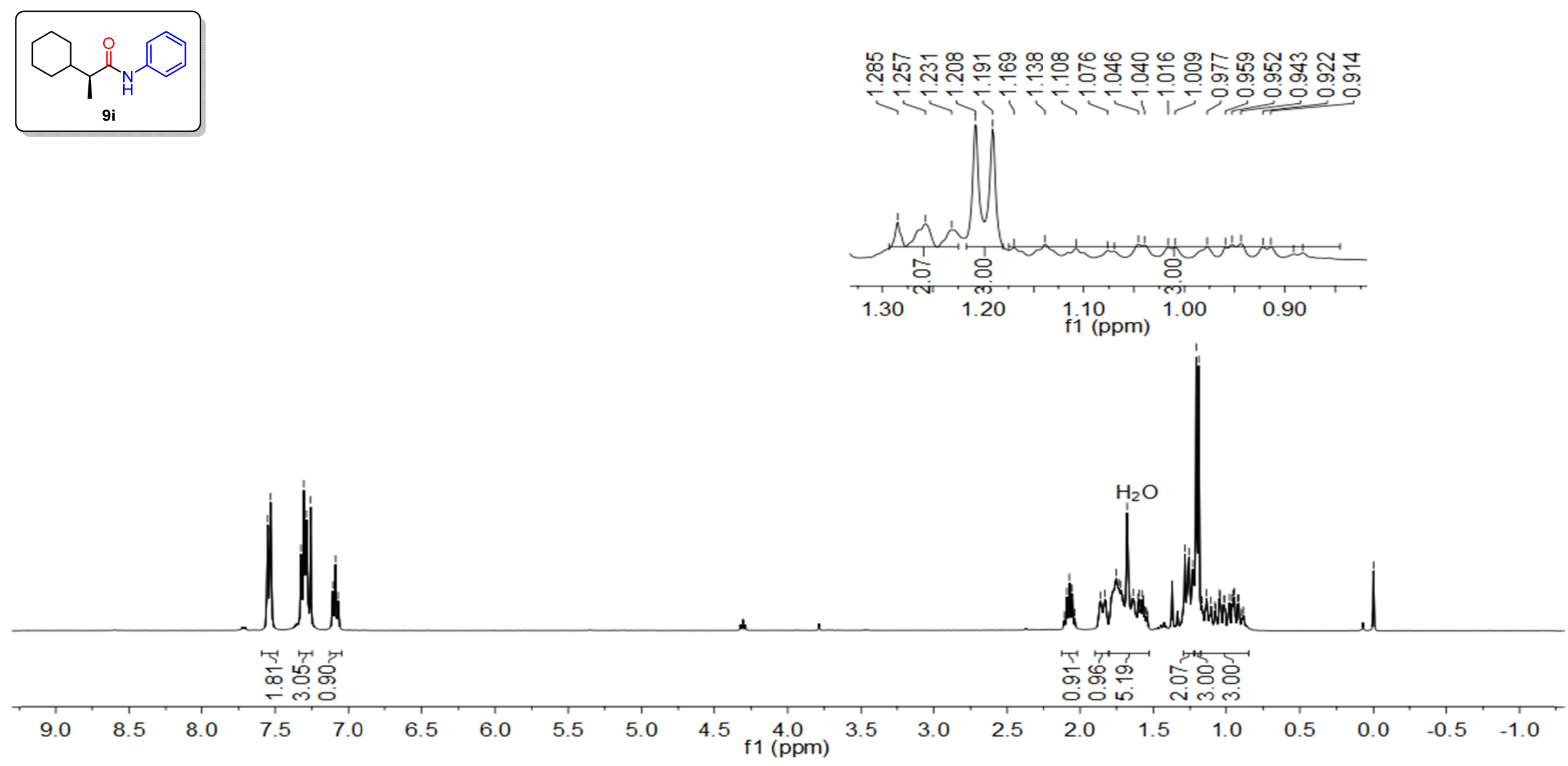
${ }^{13} \mathrm{C}$ NMR (101 MHz, $\left.\mathrm{CDCl}_{3}\right)$ of compound 9i

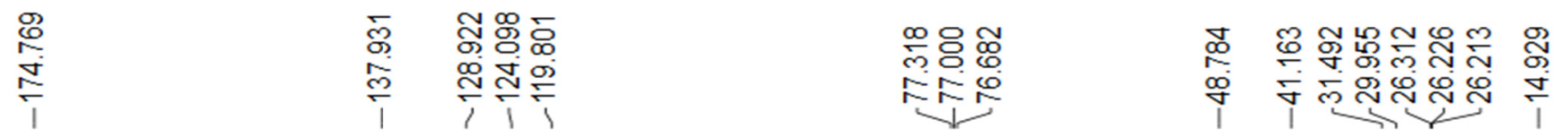
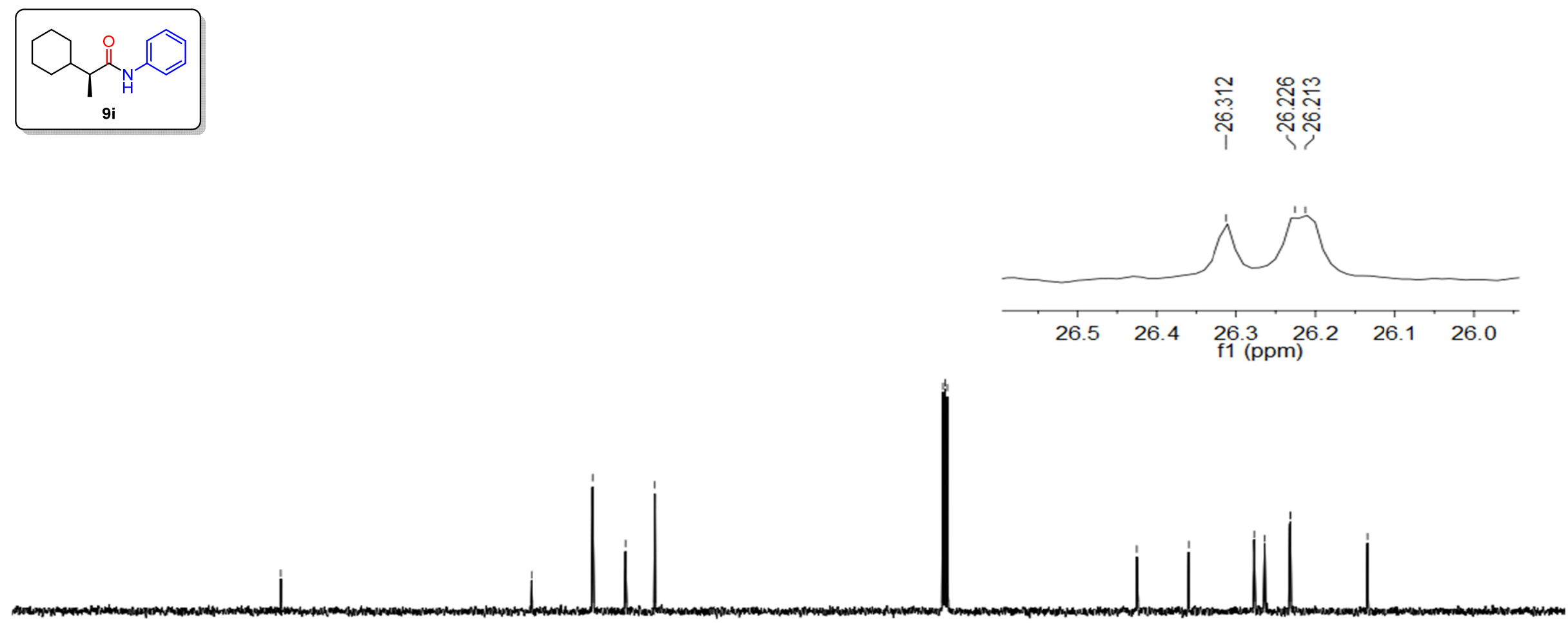

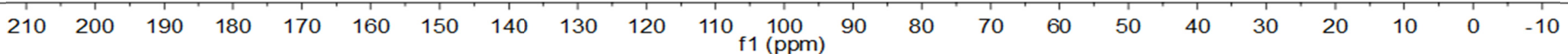

